\title{
Ecotoxicological characterization of micropollutants and wastewater samples from conventional biological and advanced wastewater treatment
}

\author{
Dissertation \\ zur Erlangung des Doktorgrades \\ der Naturwissenschaften \\ vorgelegt beim Fachbereich Biowissenschaften \\ der Johann Wolfgang Goethe-Universität \\ in Frankfurt am Main
}

von

Aennes Abbas

Heidelberg (2021)

(D30) 
vom Fachbereich Biowissenschaften der Johann Wolfgang Goethe-Universität als Dissertation angenommen

\section{Dekan:}

\section{Prof. Dr. Sven Klimpel}

Institut für Ökologie, Evolution und Diversität Integrative Parasitologie und Tierphysiologie Johann Wolfgang Goethe-Universität Max-von-Laue-Str. 13

D-60439 Frankfurt am Main

\section{Gutachter:}

Prof. Dr. Jörg OehImann

Institut für Ökologie, Evolution und Diversität Aquatische Ökotoxikologie Johann Wolfgang Goethe-Universität Max-von-Laue-Str. 13 D-60438 Frankfurt am Main

Prof. Dr. Henner Hollert

Institut für Ökologie, Evolution und Diversität Evolutionsökologie und Umwelttoxikologie Johann Wolfgang Goethe-Universität Max-von-Laue-Str. 13 D-60438 Frankfurt am Main 
Ecotoxicological characterization of micropollutants and wastewater samples from conventional biological and advanced treatment technologies

Aennes Abbas 
(C) Aennes Abbas

March 2021 


\section{Table of content}

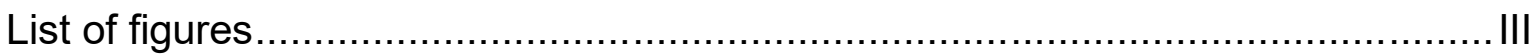

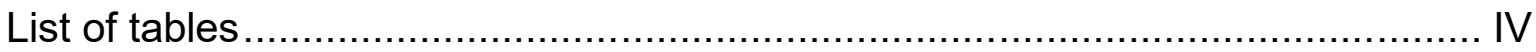

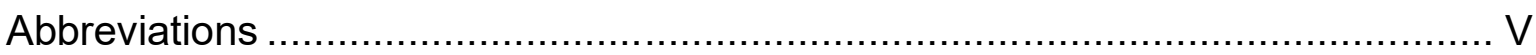

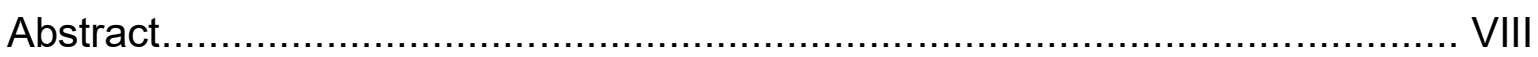

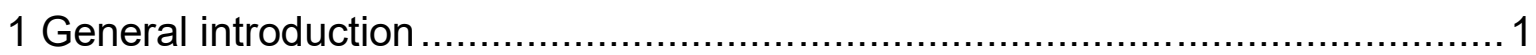

1.1 Urban water cycle and the emission of wastewater ............................... 1

1.2 Wastewater treatment plants as point sources for aquatic pollution .............2

1.2.1 Environmental behavior and biological impacts of wastewater-borne (micro)pollutants

1.2.2 Advanced wastewater treatment technologies for the additional removal of (micro)pollutants and residual toxicity ................................................ 8

1.3 Ecotoxicological characterization of water and wastewater quality by in vitro and in vivo bioassays ............................................................................. 13

1.4 Caenorhabditis elegans in ecotoxicological research ............................ 17

1.4.1 Ecology and establishment as biological model organism..................17

1.4.2 Development and reproduction ............................................... 18

1.4.3 Xenobiotic metabolism.............................................................. 19

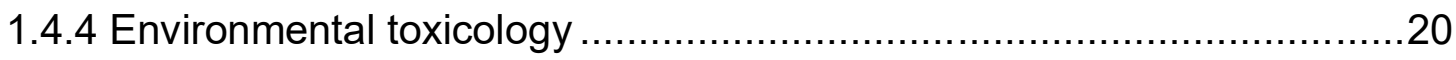

1.5 Integration of this thesis into the present state of research ......................22

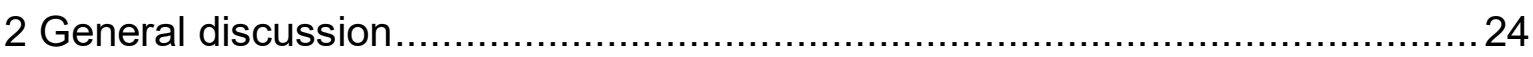

2.1 Optimising sample preparation for in vitro bioassays ............................24

2.1.1 Sample acidification ............................................................ 24

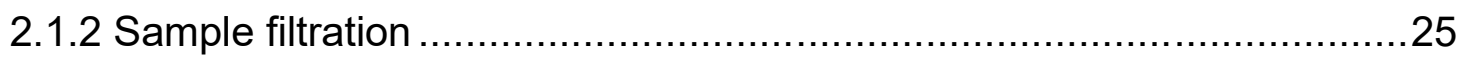

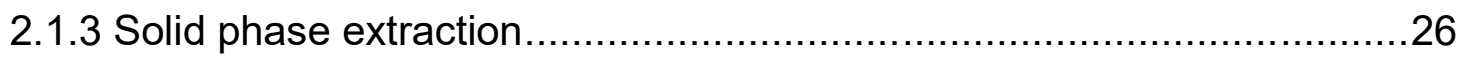

2.2 In vitro bioassays as environmental monitoring tools - case study of a water protection region in Southwest Germany ............................................... 32

2.3 In vivo bioassays for the ecotoxicological assessment of wastewater - the case of Caenorhabditis elegans....

2.3.1 Development and reproduction as human and environmentally relevant endpoints

2.3.2 CYP-35As as biomarker of (micro)pollutant exposure and contaminated environmental samples

2.3.3 Functional ecotoxicogenomics, mechanistic ecotoxicology and novel endpoints for (micro)pollutant and (waste)water quality assessment

2.4 Integrated wastewater quality evaluation - case study of a pilot WWTP equipped with AWWT technologies 
Annex

A.1 What you extract is what you see: Optimising the preparation of water and wastewater samples for in vitro bioassays .............................................. ii

Declaration (paper A.1) ............................................................ ii

Author accepted manuscript (paper A.1) ........................................... iv

Supplementary information (paper A.1) ............................................ liv

A.2 Ecotoxicological impacts of surface water and wastewater from conventional and advanced treatment technologies on brood size, larval length and cytochrome P450 (35A3) expression in Caenorhabditis elegans ................. Ixxvii

Declaration (paper A.2) ............................................................... Ixxviii

Author accepted manuscript (paper A.2) ......................................... Ixxix

Supplementary information (paper A.2) …........................................ Cxviii

A.3 Post-treatment of ozonated wastewater with activated carbon and biofiltration compared to membrane bioreactors: Toxicity removal in vitro and in Potamopyrgus antipodarum ........................................................... cxix

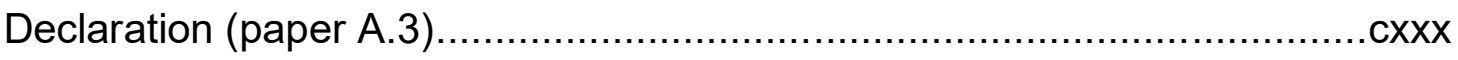

Author accepted manuscript (paper A.3) ...........................................xxi

Supplementary information (paper A.3) ...................................... clxxxvi

A.4 Zusammenfassung (German summary) .........................................c.

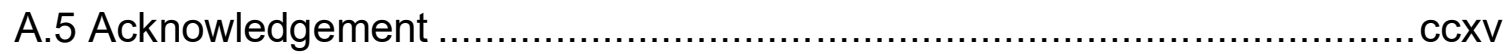

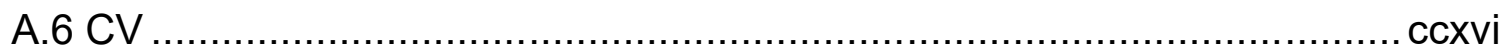

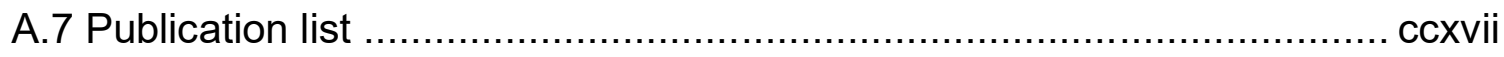




\section{List of figures}

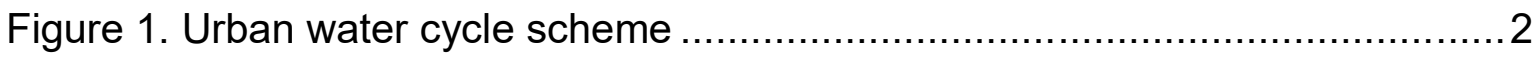

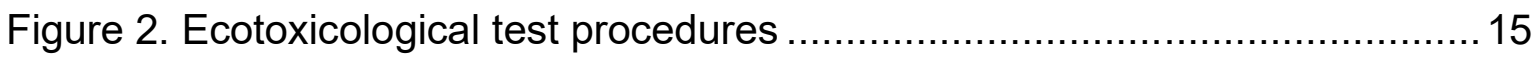

Figure 3. C. elegans life cycle and scanning electron microscope image .............18

Figure 4. Detoxification in an intestinal cell of $C$. elegans .................................20

Figure 5. Two SPE methods designed as parallel and sequential protocols optimised for in vitro bioassays .28

Figure 6. Estrogenic activity in extracted hospital wastewater (HOS) and a WWTP influent (INF) quantified as mean 17ß-estradiol equivalents (ng E-EQ/L) \pm standard error of the mean (SEM)

Figure 7. Anti-estrogenic activities in aqueous samples quantified as mean 4hydroxytamoxifen equivalents (mg OHT-EQ/L) \pm SEM.

Figure 8. Estrogenic (left) and anti-estrogenic (right) activities in raw (dark grey bars) and biologically-treated (white bars) wastewater samples from three WWTPs (using activated sludge) presented as mean 17ß-estradiol (ng E-EQ/L) or 4hydroxytamoxifen ( $\mathrm{mg} O H T-E Q / L)$ equivalents \pm SEM, respectively

Figure 9. Anti-estrogenic activities presented as mean 4-hydroxytamoxifen equivalents ( $\mathrm{mg} \mathrm{OHT}-\mathrm{EQ} / \mathrm{L}) \pm \mathrm{SEM}$ of aqueous grab samples taken from water intakes (1-6) and wells (1-2) in the model region in October $2013(n=8)$

Figure 10. Expression of cyp-35A3::GFP in transgenic C. elegans of the Bag phenotype

Figure 11. Reproductive toxicity of $\beta-N F(1-5 \mathrm{mg} / \mathrm{L})$, surface water $(\mathrm{SW})$ and WWTP effluent (EFF) samples to $C$. elegans

Figure 12. Expression of cyp-35A3::GFP in transgenic C. elegans after exposure to $\beta-\mathrm{NF}$

Figure 13. Down-regulation ( $0-72 \mathrm{~h}$ ) of cyp-35A3::GFP in transgenic $C$. elegans after $24 \mathrm{~h}$ exposure to $1 \mathrm{mg} \beta-\mathrm{NF} / \mathrm{L}$

Figure 14. Two in silico models of hCYP-2C8 (human ortholog of ceCYP-35A3) .50

Figure 15. Mechanistic scheme of a hypothetical DART pathway in C. elegans .61

Figure 16. Wastewater collection basins at the pilot WWTP in Hessen, Germany installed during on site testing

Figure 17. Impacts of WWTP influent (INF-4), effluent (EFF-4), ozonated effluent $\left(\mathrm{EFF}+\mathrm{O}_{3}\right)$ and $\mathrm{GAC} / \mathrm{BF}$-filtered ozonated effluent $\left(\mathrm{O}_{3}+\mathrm{GAC}, \mathrm{O}_{3}+\mathrm{GAC}_{\mathrm{a}}, \mathrm{O}_{3}+\mathrm{BF}\right.$, $\mathrm{O}_{3}+\mathrm{BF}_{\mathrm{a}}$ ) samples on the reproduction (brood size) of $C$. elegans 


\section{List of tables}

Table 1. Selected C. elegans cyp-35A3 inducers in the scientific literature 47

Table 2. Selected (non-genome wide) studies on effects and apparent MoAs of environmental chemicals/samples in C. elegans

Table 3. Wastewater quality evaluation matrix based on the TransRisk project....69

Table 4. Ecotoxicological effects of corresponding WWTP effluents (EFF), ozonated effluents $\left(\mathrm{EFF}+\mathrm{O}_{3}\right)$ and three types of post-treatment: $A$ ) activated carbon $\left.\left(\mathrm{O}_{3}+\mathrm{AC}\right), \mathrm{B}\right)$ biological treatment $\left(\mathrm{O}_{3}+\mathrm{B}\right)$ and $\left.\mathrm{C}\right) \mathrm{BAC}$ as "hybrid" of $\left.\mathrm{A}\right)$ and B) $\left(\mathrm{O}_{3}+\mathrm{BAC}\right)$ reported in the literature .73 


\section{Abbreviations}

\begin{tabular}{|c|c|}
\hline$\mu \mathrm{g}$ & microgram \\
\hline$\cdot \mathrm{OH}$ & hydroxyl radical \\
\hline $\mathrm{ABC}$ & adenosine triphosphate binding cassette \\
\hline $\begin{array}{l}\text { AChE } \\
\text { AhR }\end{array}$ & $\begin{array}{l}\text { acetylcholinesterase } \\
\text { aryl hydrocarbon receptor }\end{array}$ \\
\hline AHTN & 7-acetyl-1,1,3,4,4,6-hexamethyl-1,2,3,4-tetrahydro naphthalene \\
\hline AOP & adverse outcome pathway \\
\hline ATP & adenosine triphosphate \\
\hline AWWT & advanced wastewater treatment \\
\hline BAC & biologically activated carbon \\
\hline BAT & best available technology \\
\hline BBP & benzyl butyl phthalate \\
\hline BF & biological filtration \\
\hline$\beta-N F$ & beta-naphthoflavone \\
\hline BMU & Bundesministerium für Umwelt, Naturschutz und nukleare Sicherheit \\
\hline BOD & biological oxygen demand \\
\hline BPA & bisphenol A \\
\hline BPS & bisphenol S \\
\hline BLAST & basic local alignment search tool \\
\hline ceCYP-35A3 & Caenorhabditis elegans cytochrome P450 isoform 35A3 (protein) \\
\hline cep-1 & transcription factor (Caenorhabditis elegans), human p53 ortholog \\
\hline COD & chemical oxygen demand \\
\hline CYP & cytochrome P450 \\
\hline daf-16 & abnormal dauer formation 16 (gene) \\
\hline DART & developmental and reproductive toxicity \\
\hline DDT & dichlordiphenyltrichlorethan \\
\hline DEB & dynamic energy budget \\
\hline DEHP & di(2-ethylhexyl)phthalate \\
\hline DEP & diethyl phthalate \\
\hline DNA & deoxyribonucleic acid \\
\hline DOC & dissolved organic carbon \\
\hline E1 & estrone \\
\hline E2 & $17 \beta$-estradiol \\
\hline EC & effect concentration \\
\hline $\mathrm{EC}_{50}$ & median effect concentration \\
\hline EDA & effect directed analysis \\
\hline EDC & endocrine disrupting chemical \\
\hline EE2 & 17a-ethinylestradiol \\
\hline EFF & wastewater treatment plant effluent \\
\hline
\end{tabular}




\begin{tabular}{|c|c|}
\hline EQS & environmental quality standard \\
\hline EPA & Environmental Protection Agency \\
\hline EROD & ethoxyresorufin-O-deethylase \\
\hline $\mathrm{F}_{1}$ & filial generation 1 \\
\hline $\mathrm{F}_{2}$ & filial generation 2 \\
\hline GAC & granulated activated carbon \\
\hline$g c s-1$ & glutamylcysteine synthetase (gene) \\
\hline GST & glutathione-s-transferase (protein) \\
\hline GW & groundwater \\
\hline hCYP-2C8 & human cytochrome P450 isoform 2C8 (protein) \\
\hline HHCB & $\begin{array}{l}\text { 1,3,4,6,7,8-hexahydro-4,6,6,7,8,8-hexamethylcyclopenta- } \gamma-2 \text { - } \\
\text { benzopyran }\end{array}$ \\
\hline HRT & hydraulic retention time \\
\hline HSP & heat shock protein \\
\hline hus-1 & DNA damage checkpoint protein \\
\hline ICI 182780 & fulvestrant \\
\hline INF & wastewater treatment plant influent \\
\hline ISO & International Organization for Standardization \\
\hline L1 & larval stage 1 (Caenorhabditis elegans) \\
\hline L2 & larval stage 2 (Caenorhabditis elegans) \\
\hline L3 & larval stage 3 (Caenorhabditis elegans) \\
\hline L4 & larval stage 4 (Caenorhabditis elegans) \\
\hline LC & liquid chromatography \\
\hline LCA & life cycle assessment \\
\hline LOEC & lowest observed effect concentration \\
\hline LOQ & limit of quantification \\
\hline LVSPE & large volume solid phase extraction \\
\hline MEC & measured environmental concentration \\
\hline MoA & mode of (toxic) action \\
\hline n.a. & not applicable \\
\hline n.d. & not determined \\
\hline NC & negative control \\
\hline NDMA & N-Nitrosodimethylamine \\
\hline NHR & nuclear hormone receptor \\
\hline NOM & natural organic matter \\
\hline $\mathrm{O}_{3}$ & ozone (in the context of wastewater ozonation) \\
\hline p53 & human phosphoprotein 53 (tumor suppressor protein) \\
\hline PAC & powdered activated carbon \\
\hline $\mathrm{PAH}$ & polycyclic aromatic hydrocarbon \\
\hline PC & positive control \\
\hline PCB & polychlorinated biphenyl \\
\hline PGP & P-glycoprotein \\
\hline
\end{tabular}




\begin{tabular}{|c|c|}
\hline PNEC & predicted no effect concentration \\
\hline POP & persistent organic pollutant \\
\hline PPCP & pharmaceuticals and personal care products \\
\hline$P_{\text {total }}$ & total phosphorus \\
\hline QSAR & quantitative structure activity relationships \\
\hline RNAi & ribonucleic acid interference \\
\hline ROS & reactive oxygen species \\
\hline RTG & rainbow trout gonad \\
\hline SC & solvent control \\
\hline SDR & short-chain dehydrogenase \\
\hline SEM & standard error of the mean \\
\hline SKN-1 & transcription factor skinhead-1 \\
\hline SMX & sulfamethoxazole \\
\hline SOD & superoxide dismutase \\
\hline SPE & solid phase extraction \\
\hline SW & surface water \\
\hline TCC & triclocarban \\
\hline TCS & triclosan \\
\hline TIE & toxicity identification evaluation \\
\hline TN & total nitrogen \\
\hline TP & transformation product \\
\hline TSS & total suspended solids \\
\hline UGT & uridine 5'-diphospho-glucuronosyltransferase \\
\hline WET & whole effluent toxicity \\
\hline WFD & water framework directive \\
\hline WW & wastewater \\
\hline WWTP & wastewater treatment plant \\
\hline YAAS & yeast anti-androgen screen \\
\hline YAES & yeast anti-estrogen screen \\
\hline YAS & yeast androgen screen \\
\hline YES & yeast estrogen screen \\
\hline YG7108 & Salmonella typhimurium YG7108 strain \\
\hline
\end{tabular}




\section{Abstract}

Clean water is fundamental to human health and ecosystem integrity. However, water quality deteriorates due to novel anthropogenic pollutants present at microgram per liter concentrations in urban water cycles (termed micropollutants). Wastewater treatment plants (WWTP) have been identified as major point sources for aquatic (micro-)pollutants. Chemical and ecotoxicological analyses have shown that conventional biological WWTPs do not fully remove micropollutants and associated toxicities, which is often because of mobile, polar and/or recalcitrant compounds. To minimize possible environmental risks, advanced wastewater treatment (AWWT) technologies could be a promising mitigation measure. Multiple processes are therefore being developed and evaluated such as ozonation and ozonation followed by granulated activated carbon (GAC) or biological filtration.

Assessing the performance of these combined AWWTs was the focus the TransRisk project. Within this project, this thesis accomplished four major goals. Firstly, the preparation of (waste)water samples was optimised for in vitro bioassays. Acidification, filtration and solid phase extraction (SPE) were tested for their impact on environmentally relevant in vitro endocrine activities, mutagenicity, genotoxicity and cytotoxicity. Significantly different outcomes of these assays were detected comparing neutral and acidified samples. Sample filtration had a lesser impact, but in some cases retention of particle-bound compounds could have caused significant toxicity losses. Out of three SPE sorbents the Telos C18/ENV at sample pH 2.5 extracted highest toxicity, some undetected in aqueous samples. These results indicate that sample preparation needs to be optimised for specific sample matrices and bioassays to avoid false-positive or -negative detects in effect-based analyses.

Secondly, the above listed in vitro toxicities were monitored in a protected region for drinking water production in South-West Germany (2012-2015). Out of 30 sampling sites surface water and groundwater were the least polluted. Nonetheless, a few groundwater samples induced high anti-estrogenic activity that prompted further monitoring. The latter included a waterworks in which no toxicity was detected. Hospital wastewater also had elevated in vitro toxicities and hospitals are, thus, relevant intervention points for source control. The biological WWTPs were effective in removing most of the detected toxicity, and the selected bioassays proved to be pertinent tools for water quality assessment and prioritisation of pollution hotspots. 
Thirdly, the in vivo bioassay ISO10872 based on Caenorhabditis elegans ( $C$. elegans) was adapted for this thesis. Using this model, a median effect concentration $\left(E_{50}\right)$ for reproductive toxicity of the polycyclic aromatic hydrocarbon $\beta$-naphthoflavone ( $\beta$-NF) of $114 \mu \mathrm{g} / \mathrm{L}$ was computed which is slightly lower than reported in the scientific literature. $\beta$-NF induced cyp-35A3::GFP (a biomarker in transgenic animals) in a time and concentration dependent manner ( $\leq 21.3-24$ fold above controls). $\beta$-NF spiked wastewater samples supported earlier hypotheses on particle-bound pollutants. Reproductive toxicity $(96 \mathrm{~h})$ and cyp-35A3 induction $(24 \mathrm{~h})$ of biologically treated and/or ozonated wastewater extracts and growth promoting effects of GAC/biologically filtered ozonated wastewater extracts were observed. This suggested the presence of residual bioactive/toxic chemicals not included in the targeted chemical analysis. It also highlighted the importance of integrating multiple (apical and molecular) endpoints in wastewater assessments.

Fourthly, five in vitro and the adapted $C$. elegans bioassay were integrated into a wastewater quality evaluation (developed within TransRisk). Out of the five AWWT options, ozonation (at $1 \mathrm{~g} \mathrm{O}_{3 \text {,applied }} / \mathrm{g} \mathrm{DOC}, \mathrm{HRT} \sim 18 \mathrm{~min}$ ) combined with nonaerated GAC filtration was rated most effective for toxicity removal. All five AWWTs largely removed estrogenic and (anti-)androgenic activities, but not anti-estrogenic activity and mutagenicity, which even increased during ozonation. This has been observed in related studies and points towards toxic transformation products. These results also emphasized the need for implementing an effective post-treatment for ozonation. The results from a parallel in vivo study with Lumbriculus variegatus and Potamopyrgus antipodarum conducted on site at the WWTP (using flow through systems) were in accordance with the $C$. elegans results. In this context, $C$. elegans can be further implemented as sensitive, feasible and ecologically relevant model. In conclusion, this thesis shows how optimised sample preparation, long-term (in vitro) environmental monitoring, sensitive and ecologically relevant (in vivo) bioassays as well as innovative evaluation concepts, are pivotal in improving the removal of micropollutants and their toxicities with AWWTs. Future research should further develop and evaluate measures at sewer systems, conventional biological, tertiary and other advanced treatment technologies, as well as sociopolitical strategies (e.g., source control or natural conservation) and restoration projects. The effect-based tools optimised in this thesis will support assessing their success. 


\section{General introduction}

\subsection{Urban water cycle and the emission of wastewater}

Along human history natural water cycles are becoming increasingly interconnected with anthropogenic activities, infrastructures and emissions. While in $201454 \%$ of the world population resided in urban areas, this rate was predicted to increase to 65\% until 2050 (Brooks et al. 2020, World Health Organization 2016). This growth will also lead to massive expansion and intensification of water usage and demand, conveying key challenges to global water and wastewater management. These challenges are associated with other major environmental concerns such as climate change, resource depletion and habitat/biodiversity impacts (e.g., Rockström et al. 2009, Stamm et al. 2016).

Water represents the largest mass influx into urban areas (Philip et al. 2011). Due to the high demand on water, $4.5 \times 10^{12} \mathrm{~m}^{3}$ of water is withdrawn from freshwater resources every year. This approximates to one third of the total existing amount (Jackson et al. 2001). Fresh water is mainly extracted from groundwater and surface water, whereby its extraction relies on various qualitative, quantitative, economic and regulatory aspects. To produce drinking water it is essential to remove waterborne pathogens and contaminants. Depending on the initial water quality this requires basic (e.g., boiling for disinfection) to advanced (e.g., reverse osmosis) treatment processes. The application range of (treated) water is extremely versatile. Households mostly use tap and bottled water, while consuming $7 \%$ of the total extracted water. Industry needs $22 \%$, while the largest amount $(65 \%)$ is consumed by agriculture (Jackson et al. 2001). Figure 1 illustrates main water usage sites and wastewater fluxes. Wastewater is generated where water usage is accompanied by degradation of the provided quality. Wastewater in urban areas is generally collected via drainage networks and sewer systems that merge into wastewater treatment plants (WWTP). In WWTPs influent wastewater is treated by elaborate processes (multi-barrier principle), because WWTP effluents have to fulfill regulated quality standards to be permissible for discharge into receiving water bodies. The main goal of wastewater treatment thereby is that no biological/ecological risks are posed to humans and the environment (Tchobanoglous and Burton 1991). Once this requirement is fulfilled the water cycle can begin anew. 


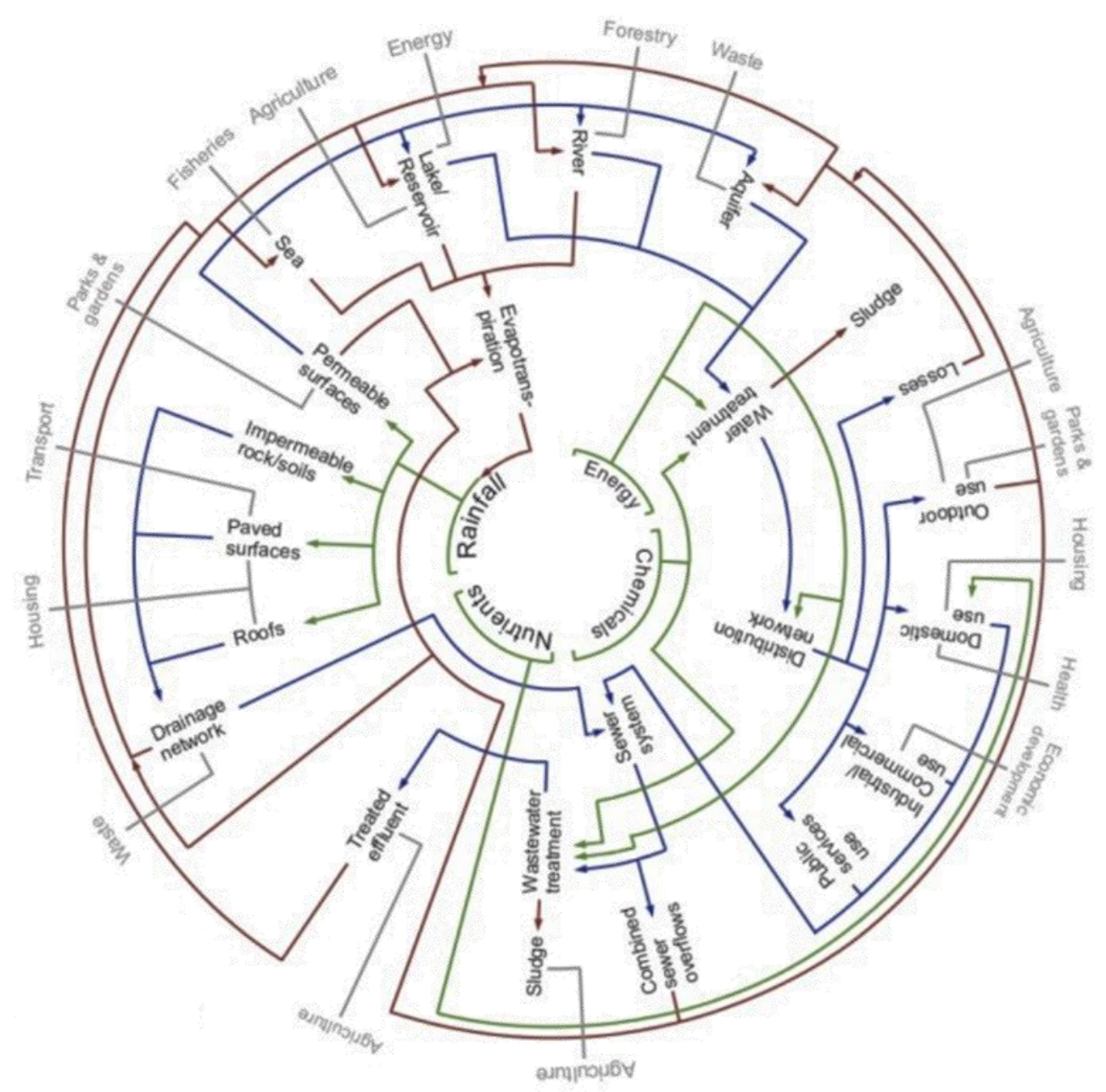

Figure 1. Urban water cycle scheme indicating major entities including inputs (green lines), flows (blue lines), outputs (brown lines) and impacting factors (grey lines, Philip et al. 2011)

\subsection{Wastewater treatment plants as point sources for aquatic pollution}

In 2012 approximately half of European surface waters did not match a "good ecological status" (European Commission 2012, European Parliament and Council 2000). Large parts of surface water pollution is caused by WWTPs (European Commission 2012, Malaj et al. 2014, Pal et al. 2010, Triebskorn et al. 2017, 2019). Their contribution will likely increase along the ongoing urbanization and growing world population (compare 1.1). Raw (untreated) municipal wastewater generally consists of $99 \%$ water and $1 \%$ waste. The waste fraction contains nutrients, (dissolved) organic matter, bacteria and other particulate matter (including nanoand microplastic) as well as toxic pollutants (e.g., Bruni et al. 2019, Tchobanoglous and Burton 1991, Triebskorn et al. 2019, Stamm et al. 2016). Due to its hazard 
potential wastewater is treated by series of physical, chemical and biological WWTP processes that categorize into primary, secondary and where applicable tertiary and advanced treatment. Each of these stages (further) reduces the content of specific wastewater constituents. Preliminary treatment excludes grease, oil, debris, grit and odor before primary treatment separates out floating and settling materials (e.g., in primary clarifiers). Secondary treatment mainly reduces organic matter and suspended solids; most commonly by activated sludge processes (Prasse et al. 2015). Tertiary treatment aims at enhanced denitrification and removal of phosphorus, bacteria and virus, by coagulation, flocculation, precipitation and/or filtration. Waste sludge is mostly withdrawn during primary settling and/or the biological stage. It has to undergo separate treatment in digesters, centrifuges or presses that prepare it for final disposal (e.g., by incineration) or reuse (compare 'peak phosphorous', Cordell et al. 2009). Treated wastewater either becomes discharged into surface waters or marine environments or may be allocated for reuse depending on its final quality (e.g., in industry, irrigation or prospectively reclaimed water, Tchobanoglous and Burton 1991).

Wastewater quality has been characterized by physical parameters, such as odor, turbidity or TSS (compare figure 16), chemical parameters such as $\mathrm{pH}$, salinity, nitrogen (e.g., total nitrogen (TN) computed as sum concentration of $\mathrm{NH}_{4}{ }^{+}, \mathrm{NO}_{2}{ }^{-}$and $\mathrm{NO}_{3}{ }^{-}$), phosphorus (e.g., total phosphorus $\left(\mathrm{P}_{\text {total }}\right)$ ), sulfate/sulfides $\left(\mathrm{SO}_{4}{ }^{2-} / \mathrm{S}^{2-}\right)$, biological/chemical oxygen demand (BOD/COD), dissolved organic carbon (DOC), often extended by adsorbable organic halides (AOX) or benzene, toluene, xylenes (BTEX), and biological parameters such as bacteria, viruses or parasites. Several of these parameters constitute, contribute or indicate aquatic pollution, if released at excessive amounts into water bodies. Most countries thus regulate their discharge through setting permissible limits (e.g., European Council 1991, US EPA 2002). Other parameters such as the $\mathrm{BOD}_{5}$, which refers to the $\mathrm{BOD}$ of $a$ wastewater sample within a 5 day incubation period, are used as quantitative indicators of the contained biodegradable compounds. Despite that these parameters represent "sums" of relevant physicochemical attributes/chemicals specific (micro)pollutants may not be captured. For this reason, several regulations have included so called priority pollutants based on their toxicity and other environmentally relevant criteria such as persistence (European Parliament and 
Council 2000, US EPA 1972). Priority pollutants that have to be monitored such as heavy metals, polycyclic aromatic hydrocarbon (PAH), different pesticides, endocrine disrupting chemicals (EDCs) and industrial chemicals, whose usage has to be restricted over the next decades. Moreover, if compliance with directives (e.g., Urban Waste Water Directive, European Council 1991) is not fulfilled, such as permissible discharge limits, immediate actions should be taken (e.g., technical WWTP and/or source control measures). In addition, several contaminants have been placed onto watch lists to become priority pollutants such as of the water framework directive (WFD, European Parliament and Council 2013), or are part of additional regulation such as for industrial wastewaters (e.g., indirect emissions).

Nonetheless, the vast majority of known and emerging pollutants remains unregulated as well as incompletely removed during conventional biological wastewater treatment (compare 1.2.1). This circumstance has defined WWTP as major point sources for (micro)pollutants and potentially associated risks in aquatic ecosystems. Several of these pollutants were repeatedly made responsible for adverse effects on different aquatic species (1.3) and regionally conflicted with environmental quality standards (EQS, a presently non-legislative ecotoxicological complementation to chemical permissible limits). Although EQS proved to be useful in protecting aquatic organisms to certain extend, they have been defined for only a few compounds up to present (Escher et al. 2018, Malaj et al. 2014, Tousova et al. 2017). In addition to these challenges, most pollutants do not fully mineralize during different wastewater treatments, triggering the emission of unknown TPs. TPs may show higher toxicity than their parental compound (Boxall et al. 2004, Cao et al. 2020b, Cwiertny et al. 2014, Knoop et al. 2018, Larcher et al. 2012, Ma et al. 2019, Schlüter-Vorberg et al. 2015). The overall situation has thus initiated numerous research projects on the detection and characterization of wastewater-borne (micro)pollutants/TPs (1.2.1) and has strongly challenged WWTP to further reduce emissions such as by technical upgrades (1.2.2).

\subsubsection{Environmental behavior and biological impacts of wastewater-borne (micro)pollutants}

Concerns in the late 1960s focused on WWTP as causes for eutrophication and declining fish populations (e.g., Karlson et al. 2002, Pihl et al. 1991). Thereafter, the discharge of so called macro-pollutants (including nutrients, organic matter, salts 
and acids) was brought under control (Schwarzenbach et al. 2006). In the 1980 s the attention shifted to hazardous organic chemicals. Persistent organic pollutants (POPs), such as PAH or polychlorinated biphenyls (PCBs), but also heavy metals, were widely investigated and regulated (UNEP 2005). Through ugrading WWTPs (e.g., enhancing biodegradation and sludge adsorption) and source control measures (e.g., at industrial sites) many POPs were reduced to levels of lesser concern (Prasse et al. 2015, Völker et al. 2019). In the last two decades instrumental chemistry has advanced in a way that organic pollutants occurring at trace concentrations could be reliably detected. According to their concentration in the nanogram to microgram per liter range these pollutants were termed "micropollutants" (Kümmerer 2011). Micropollutants have been classified (Kümmerer 2011, Ribeiro et al. 2015) according to:

- Their anthropogenic function such as pharmaceuticals and personal care products (PPCPs), biocides, nutrient-related or industrial chemicals (such as brominated flame retardants or surfactants).

- Their chemical structures such as phenols, phthalates or polyhalogenated compounds involving perfluorinated compounds or brominated diphenhyl ethers.

- Their mode of action (MoA) such as EDCs or anti-neoplastics.

Many micropollutants show (pseudo)persistent characteristics, a high mobility in the water cycle and/or low sorption rate (De Baat et al. 2020, Reemtsma et al. 2016, Richardson 2009). Several micropollutants were thus detected at nanogram per liter concentrations in groundwater (Heberer 2002) and raw drinking water (Benotti et al. 2009). Nonpolar micropollutants often show high biodegradability, their hydrophobic properties however also facilitates accumulation to sediments and/or in aquatic biota (Brown et al. 2007, Fick et al. 2010). Munz et al. (2018) detected this phenomena for 63 (semi)polar micropollutants. Bioaccumulation phenomena generally imply further risks of trophic transfers (e.g., Park et al. 2009, Stylianou et al. 2018). Albeit significant hazards to humans could so far be prevented (such as by multi-barrier principle in the production of drinking water), ecotoxicological effects and ecological impacts are well documented in the scientific literature (e.g., Stamm et al. 2016, 1.3). The following passages shall summarize a few relevant examples hereof. 
EDCs, environmental hormones and WWTP discharges containing these compounds have been associated with reproductive impairments in wild fish and other species (Jobling et al. 1998, 2002, Sumpter 2005, Tetreault et al. 2011, Wagner et al. 2017). Although suspected causative agents, such as estrone (E1), estradiole (E2) or ethinylestradiole (EE2) are generally effectively removed during activated sludge treatments (e.g., to $83 \%, 99 \%$ and $78 \%$ for E1, E2 and EE2, Ternes et al. 1999), these compounds nonetheless indicated high potencies at very low concentrations. EE2 for instance disturbed egg fertilization and sex ratios of fat head minnows at a lowest observed effect concentration (LOEC) of $0.32 \mathrm{ng} / \mathrm{L}$ (Parrott and Blunt 2005). It was further shown to cause in situ feminization of males at longerterm exposure to 5-6 ng/L (Kidd et al. 2007). Thus their measured environmental concentrations (MECs) often range above their reported effect concentrations (EC, Quednow and Püttmann 2008). Other EDCs such as the industrial chemical nonylphenol (priority pollutant WFD) caused feminization in fish at a LOEC of $8.2 \mu \mathrm{g} / \mathrm{L}$. Despite its ban in several countries nonylphenol was detected at up to $4.1 \mathrm{\mu g} / \mathrm{L}$ in surface waters and $1 \mathrm{mg} / \mathrm{kg}$ in river sediments (Soares et al. 2008, World Health Organization 2002). The UV filter octinoxate (or 2-ethylhexyl-4methoxycinnamate) was recently added to the WFD watch list (Ramos et al. 2015, European Parliament and Council 2013). Its accumulation in sediments (at $\mu \mathrm{g} / \mathrm{kg}$ ) and in aquatic biota (up to several hundred $\mu \mathrm{g} / \mathrm{kg}$ ) is thought to be associated with toxicological effects (Kaiser et al. 2012). Markedly, only little is known about its occurrence and behavior in WWTPs (compare 1.2.2).

Low ECs were also observed for different pharmaceuticals, such as carbamazepine, diclofenac or metoprolol. Diclofenac was reported to induce tissue-specific damage in rain bow trout at a LOEC of $1 \mu \mathrm{g} / \mathrm{L}$ (Triebskorn et al. 2007). Albeit WWTP influent concentrations of diclofenac and carbamazepine are relatively low, both compounds indicated poor removal rates during activated sludge treatment (Abbas et al. 2018, Calisto and Esteves 2009) and were detected in surface waters in the microgram per liter range (Gros et al. 2006, Zhou et al. 2009). High plasma concentrations of diclofenac were quantified in rainbow trout exposed to diluted treated wastewater (Brown et al. 2007). High bioconcentration factors were quantified for other pharmaceuticals (Burns et al. 2010, Fick et al. 2010). Antibiotics, excreted by humans or livestock represent another problematic group of pharmaceuticals which 
stimulated (multi-)resistant mechanisms in sludge bacteria (Bruni et al. 2019, Rizzo et al. 2013, Triebskorn et al. 2017). Accordingly azithromycin, clarithromycin and erythromycin, detected in treated wastewaters, surface water and groundwater (up to the microgram per liter range), were included into the mentioned recent WFD watch list (European Parliament and Council 2013).

Biocides are another frequently detected pollutant group in aquatic compartments (e.g., Abbas et al. 2018). Pesticides of different classes normally occur at low concentrations in WWTP influents (e.g., < $1 \mu \mathrm{g} / \mathrm{L}$, Köck-Schulmeyer et al. 2013). Many pesticides are recalcitrant towards conventional biological wastewater treatment (Köck-Schulmeyer et al. 2013, Sadaria et al. 2016). They are also widely distributed in natural waters due to their extensive agricultural application. In addition certain neonicotinoids indicated diffusion into surface waters from groundwater (Morrissey et al. 2015). Many pesticides and their formulations/TPs have been associated with toxicity, including carcinogenicity, reproductive toxicity, hepatotoxicity and/or neurotoxicity in different non-target species (Cao et al. 2020a, Dabrowski et al. 2014, Sanchez-Bayo and Hyne 2014). Although newer generations of pesticides are equipped with comparably short environmental half-lifes, ecological and human health risks prevail. For instance several neonicotinoids discharged from WWTPs were observed to exceed regulatory threshold. However, adverse effects on aquatic invertebrates also occurred below threshold concnetrations (Münze et al. 2017). Many pesticides have thus to be stringently regulated or had to be phased out in the past (European Parliament and Council 2013, US EPA 2011). Other biocides, such as antifouling agents, are often underrepresented in regulative assessments, despite their high production volumes and potential toxicity (e.g., Dafforn et al. 2011).

Foodstuff-related chemicals, such as artificial sweeteners, are often used as chemical indicator substances due to their aquatic persistence and mobility (e.g., Abbas et al. 2018, Seitz and Winzenbacher 2017). For a number of these chemicals even health risks were reported. It was for instance observed that the food additive "E 321" butylhydroxytoluol (2,6-di-tert-butyl-4-methylphenol, BHT) produces a metabolite that apparently caused DNA damage in rat and mice (Fries and Püttmann 2004). BHT entering the aquatic environment through WWTPs was detected in surface water $(<1.6 \mu \mathrm{g} / \mathrm{L})$, groundwater $(<2.2 \mu \mathrm{g} / \mathrm{L})$ and wastewater 
$(<0.3 \mu \mathrm{g} / \mathrm{L}$, Barbosa et al. 2016). It was thus also placed on the WFD watch list (European Parliament and Council 2013). In general, more (eco)toxicological data has been obtained on BHT, its $\operatorname{TP}(\mathrm{s})$ and other nutrition-related chemicals, to stringently exclude risks to humans and the environment.

Although older generations of POPs do not (regularly) exceed permissible thresholds any more, WWTP effluents are considered as prevailing source of certain PAH and PCBs (Abdel-Shafy and Mansour 2016, Forsgren 2015). Their emission is probably linked to their on-going industrial application and high production volumes (e.g., Bergqvist et al. 2006, Blanchard et al. 2004). Many hydrophobic POPs "escape" wastewater treatment bound to residual TSS (e.g., Marttinen et al. 2003, McLaggan et al. 2012). In surface waters they often accumulate in sediment sinks that are documented to exert toxic effects to bottom-dwelling organisms (e.g., Ahlf et al. 2002). Due to this fact sediments were integrated as one of the monitoring targets (besides the water phase and aquatic biota) of the WFD (European Parliament and Council 2008). Other than point sources, diffuse emissions including those from registered contamination sites or resulting from man-made disasters such as oil spills (Kim et al. 2019c), represent relevant sources of hazardous POPs.

\subsubsection{Advanced wastewater treatment technologies for the additional removal of (micro)pollutants and residual toxicity}

In response to the growing awareness about residual (micro)pollutants and toxicity in conventional-biological WWTP effluents, advanced wastewater treatment (AWWT) technologies were proposed as mitigation measure (e.g., Barbosa et al. 2016, Rizzo et al. 2019, Völker et al. 2019). Predominant AWWT technologies under investigation are advanced oxidation processes (such as ozonation or UV treatments), adsorptive technologies (such as powdered or granulated activated carbon), pressure-driven membranes (such as ultrafiltration or reverse osmosis), ion exchangers and air stripping techniques (Tchobanoglous and Burton 1991).

Advanced oxidation processes are classified by producing reactive oxidizing species. These specimens are mainly hydroxyl radicals $(\cdot \mathrm{OH})$ such as formed from hydrogen peroxide $\left(\mathrm{H}_{2} \mathrm{O}_{2}\right)$ or from ozone $\left(\mathrm{O}_{3}\right)$. The latter two are directly introduced into the wastewater matrix. Hydroxyl radicals and ozone unspecifically react with wastewater constituents leading to stepwise degradation/transformation of the 
attacked compounds/TPs (Schindler-Wildhaber et al. 2015). Advanced oxidation processes can be installed as pretreatment step for enhancing micropollutant biodegradability in subsequent treatment stages or as post-treatment step for oxidizing residual micropollutants and DOC (e.g., Bougrier et al. 2007, Rizzo 2011, Yeom et al. 2002). Under optimised conditions advanced oxidation processes, such as (catalytic) ozonation, (photo-)Fenton processes, wet peroxide/air or electrochemical oxidation and photocatalysis, indicated moderate to high (compound- and matrix-specific) removal rates (e.g., Klavarioti et al. 2009, Knopp et al. 2016, Ribeiro et al. 2015). Due to this potential ozonation has already been implemented at the national scale in a few countries (Gottschalk et al. 2009, Barbosa et al. 2016). Certain wastewater constituents delimit the effectivity of advanced oxidation processes such as high natural organic matter (NOM) content scavenging reactive oxidizing species introduced into the wastewater matrix. Other drawbacks are the generation of secondary wastes (most Fenton processes), demanding process parameters (e.g., low pH for conventional Fenton processes or elevated temperatures for wet peroxide oxidation), dependency on ambient conditions (e.g., solar light for photocatalysis), and low mineralization potential (e.g., ozonation at economic ozone doses and realistic HRTs as limited by DOC content). Moreover, advanced oxidation processes can generate TPs that obligate posttreatment. Many TPs can derive from a single oxidized compound, while the total number and physicochemical properties of the resulting TPs remains difficult to predict at present. TPs are currently classified based on their origin such as the mentioned technical TPs (from AWWT). Furthermore, natural TPs involve human/bacterial metabolites or solar degradation products (Escher and Fenner 2011). Prominent examples of TPs formed during ozonation are bromate (generated in the presence of bromide) and brominated TPs (in the presence of organic matter) as well as N-Nitrosodimethylamine (NDMA, Schindler-Wildhaber et al. 2015, Wu et al. 2019).

Adsorptive wastewater treatment by granulated or powdered activated carbon (PAC) is known for its effective removal of non-polar to moderately polar micropollutants and their toxicity (Prasse et al. 2015). Due to its granular structure GAC is packed into bed filters, whereas PAC is added to the wastewater and removed by subsequent filtration after defined contact times (Boehler et al. 2012). 
(Micro)pollutant removal and the large capacity of these technologies base on the porous structure of charcoal, exhibiting a large surface that allows for hydrophobic interactions and chemical binding. Both processes have several advantages, such as a comparably low energy demand (PAC $\geq G A C$ ) and a high removal effectivity (often PAC $\geq$ ozonation, e.g., Altmann et al. 2014). Moreover, the micropollutant removal normally proceeds without the generation of TPs. Their major disadvantage is that there capacity can become saturated, once all accessible binding palces are occupied. Then, these techniques need to be regenerated such as by heating them up to very high temperatures. They also suffer from variable sorption rates towards different compounds (Prasse et al. 2015). Biologically activated carbon (BAC) is thus being tested as an alternative that demonstrated to effectively decrease concentrations of DOC, nitrogen and of a number of recalcitrant micropollutants (Chen et al. 2017, Reungoat et al. 2012). BAC systems use fixed beds filled with GAC to support the growth of bacteria, which degrade the respective (micro)pollutants. Due to their performances in terms of micropollutant and toxicity retention all three technologies (GAC, PAC and BAC) have been implemented at various scales at WWTPs (e.g., Boehler et al. 2012, Mailler et al. 2014, table 4). However, for all activated carbon processes, highly polar chemicals may not be fully removed (Prasse et al. 2015, Reemtsma et al. 2016, Völker et al. 2019). Other biologically-activated filter materials are also being examined such as expanded clay in the biological filters (BFs) used in the TransRisk project (Knopp et al. 2016, 1.5).

Membrane based technologies have widely been utilized such as in reverse osmosis to desalinate sea water or in membrane bioreactors as an alternative to activated sludge processes (Schneider et al. 2020, Tchobanoglous and Burton 1991). Lately, ultrafiltration, microfiltration or nanofiltration that are distinguished by different standard pressures and pore sizes were tested as promising AWWT technologies (e.g., for polishing final effluents or indirect potable reuse). Thereby the molecular weight cut-off and other membrane criteria determine the rates and types of retained compounds/particles (Oulton et al. 2010). Promising variations of these technologies are being developed, such as forward osmosis and membrane distillation (Tchobanoglous and Burton 1991). Although processes, such as reverse osmosis and nanofiltration can produce particle-free effluents of very high quality 
(Stalter 2010), major disadvantages are their high energy demand and production of a (highly) enriched retentates that requires independent costly treatment (PerezGonzalez et al. 2012). Membrane technologies are in a number of cases combinable with certain biotechnological applications. Enzymes, such as laccases, lignin peroxidase, manganese peroxidase, purified from funghi, bacteria or plants respectively are therefore immobilized on membranes. In addition, these enzymes can be dissolved in bioreactors, freely or mounted onto carriers (e.g., Demarche et al. 2012). In nature, they degrade/modify certain chemicals, organic matter or biomass. These functions can be also be used to degrade several hormones, phenols, plasticizers, PAH (Demarche et al. 2012, Kim and Nicell 2006), EDCs, antibiotics (Becker et al. 2017) and other pharmaceuticals in different wastewater matrices. As enzyme technologies are only recently being transferred to the treatment of wastewaters, their potential seems by far not exceeded (such as regarding the full range of micropollutant degradations and large-scale technical application).

AWWT technologies may be combined with each other, as far as economically feasible and once an individual technology alone does not provide the demanded wastewater quality. This is mostly realized by additional post-treatment such as in the combination of ozonation with sand-, activated carbon or biological filtration (Knopp et al. 2018, Prasse et al. 2015, Völker et al. 2019) and other promising process combinations (e.g., Knopp et al. 2018, Yang et al. 2017). The latter may for instance be realized by "hybrids" of technologies such as in biological membrane assisted carbon filtration (e.g., van Hege et al. 2002). AWWT have been benchmarked for their efficacy by means of chemical, ecotoxicological and microbiological analyses (compare 1.3-1.5 and 2.4). A prominent example in this regard is given by the combination of ozonation with sandfiltration or activated carbon filtrations, which demonstrated high removal rates for a large set of micropollutants, TPs as well as toxicity that withstood or was generated during ozonation (da Costa et al. 2014, Magdeburg et al. 2014, Knopp et al. 2016, Stalter et al. 2010, Wu et al. 2019, table 3-4). Other wastewater treatment systems currently under investigation as independent or post/tertiary treatments are biologically-activated filtrations (including riverbank filtration or retention soil filters), constructed wetlands and/or aquifer recharge (e.g., Li et al. 2014, Zhang et al. 
2014). Although many of these technologies and treatment processes are considered promising in further reducing wastewater-borne micropollutants and toxicity (e.g., Bundschuh et al. 2011b, Hicks et al. 2016), more interdisciplinary research is needed for their holistic evaluation and comparison. 


\subsection{Ecotoxicological characterization of water and wastewater quality by in vitro and in vivo bioassays}

Ecotoxicological methods are essential to environmental risk assessments and monitoring, as they study the biological responses of (micro)pollutants or environmental samples (e.g., Backhaus et al. 2019, Brack et al. 2017). Bioassays are mostly combined with chemical analyses to classify/identify the drivers and conditions of (micro)pollutant-based effects (e.g., Maier et al. 2016, Neale et al. 2017, Sonne et al. 2018, Stamm et al. 2016). Challenges arise from various aspects, such as the large number of contaminants in urban water cycles, their occurrence at mostly low concentrations, selecting the appropriate sampling, sample preparation and detection methods as well as breaking down the overall complexity and variability of aquatic ecosystems (Eggen et al. 2004, Stalter et al. 2013, figure 1-2). These circumstances also have to be considered when planning, conducting and evaluating environmentally relevant investigations on AWWT.

Sampling guidelines are also compiled and adapted to achieve this goal (Prasse et al. 2015, Völker et al. 2019) such as for different environmental compartments and sampling modes (e.g., grab versus composite samples or extraordinary conditions such as heavy rain falls or proximity to pollution hot spots). In all cases, proper sampling and sample handling is crucial to minimize deviation from in situ conditions (Baker and Kasprzyk-Hordern 2011, Hillebrand et al. 2013). Albeit immediate testing is imperative, storage periods of several days or weeks leading to degradation of target compounds can sometimes not be avoided. Sample freezing for instance is regularly applied to slow down physicochemical and microbial processes that take place during storage. Microorganisms are further targeted by filtration (e.g., at pore sizes $<0.2 \mu \mathrm{m}$ ) as well as by the addition of acids or preservatives. In accordance, suitable storage vessels (e.g., amber glass for organic pollutants) and conditions (e.g., storage temperature) have to be defined. Each of these techniques beholds advantages and disadvantages that have to be balanced with a higher environmental relevance of field investigations (compare below).

Untreated wastewater samples may serve for whole effluent toxicity (WET) estimations (Norberg-King et al. 2018). Dilution series must be prepared to investigate ECs related to different endpoints. Sample preparation may also 
increase sensitivity and feasibility (compare effect directed analysis, EDA, or toxicity identification evaluations, TIE). Other bioassays do not require this steps due to their high sensitivity (e.g., Backe and Field 2012, Völker et al. 2019, Escher et al. 2014, 2018). Sample preparation is moreover practiced to reduce matrix interferences and for reasons of sample stabilization. Sample extraction and enrichment are common sample preparation approaches that involve techniques such as ultrasonic, solvent, soxhlet, liquid-liquid or SPE, freeze-drying, passive sampling and purge-trapmethods (De Baat et al. 2020, Prasse et al. 2015, Zwart et al. 2018). Many of these techniques are known to behold specific selectivity and effectivity towards different chemicals and environmental matrices (compare 2.1 and Abbas et al. 2019).

Ecotoxicological methods comprise field (in situ), semi-field (such as mesocosms) and laboratory studies using in vivo (whole organisms) and in vitro (cells) bioassays. In in vitro an in vivo bioassays different organisms/cells become exposed to individual compounds, synthetic mixtures or environmental samples (e.g., Klaassen 2007, Escher et al. 2017, Stamm et al. 2016). Bioassays are thereby capable of integrating and detecting the effects of multiple pollutants also referred to as mixture toxicity. Therefore, bioassays can be used as proxy of environmental toxicities, albeit full assessment of the latter generally requires (additional) higher tier testing (figure 2). Moreover, for optimal test strategies a well-defined set of parameters need to be considered such as organismal sensitivities, targeted endpoints, expected contaminant concentrations and other exposure related conditions (Prasse et al. 2015). To assess a widest feasible range of ecotoxicological effects in vivo bioassays should ideally be conducted using species from different taxonomic groups, trophic levels (such as producers or primary/secondary consumers) or specific habitats (such as aquatic or benthic/sediment dwelling, Rizzo 2011, Schlüter-Vorberg et al. 2017, Wernersson et al. 2015). Ecotoxicological assessments thus often include invertebrates such as the water flea Daphnia magna (D. magna, Organisation for Economic Cooperation and Development, OECD 2012), plants such as the duckweed Lemna minor (L. minor, OECD 2006), and fish species such as Danio rerio (D. rerio, zebrafish, ISO 1996) or Oncorhynchus mykiss (O. mykiss, rainbow trout, OECD 1992). These tests are often adapted or modified such as in case of the fish early-life stage test. In vivo assays are often combined with in vitro test systems in so called batteries of bioassays (compare below). 

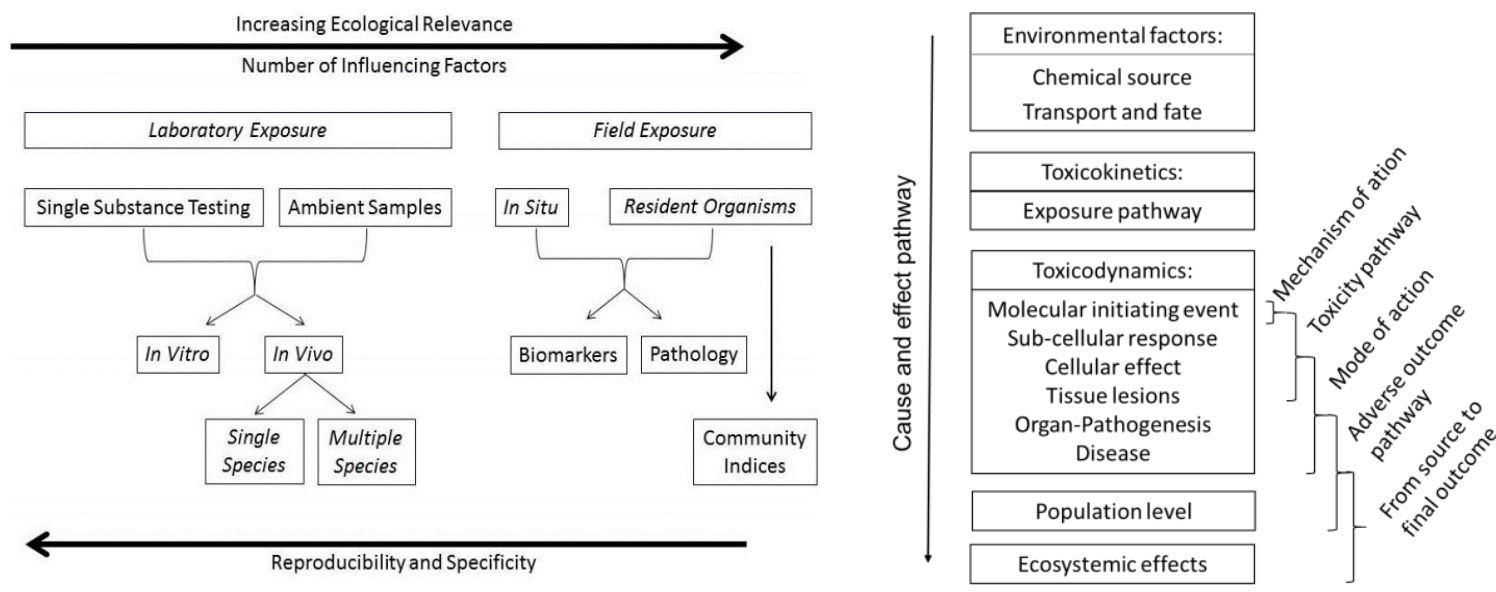

Figure 2. Ecotoxicological test procedures including their estimated ecological relevance, influencing factors and reproducibility/specificity (left, Connon et al. 2012). Each test examines different patches of toxicological cause and effect pathways (right).

In vitro bioassays utilize bacterial or eukaryotic cells (including recombinant cell lines) to screen for non-specific toxicity (e.g., cytotoxicity) as well as specific toxicity pathways / MoA (e.g., Escher et al. 2020, Rehberger et al. 2018, 2.2). In vitro systems are known for their high reproducibility and feasibility (figure 2 ) and are thus used for large-scale initiatives in predictive toxicology such as the Tox21 or ToxCast database (Brooks et al. 2020). Nonetheless they incorporate a lower predictive power regarding population-relevant effects than in vivo bioassays. Thus they are mainly used in mechanistic studies (e.g., on receptor interactions or cellular stress / metabolic responses) and MoA-based screenings of environmental samples as well as for substituting animal testing (e.g., Burgess et al. 2013, Martin et al. 2010, Escher et al. 2014, Norberg-King et al. 2018). They also became important (prospective and retrospective) monitoring tools including water and wastewater regulations (e.g., European Parliament and Council 2000, Leusch et al. 2017, Wernersson et al. 2015). A recent sound concept in this regard are effect based trigger values used as ecotoxicological (MoA-specific) complementation to EQS (Daniels et al. 2019, Escher et al. 2018, Itzel et al. 2019).

In vivo and in vitro bioassays have fundamentally shaped the current (eco)toxicological knowledge and large knowledge base on hazardous substances in the urban water cycle. A database query in the Web of Science (Clarivate Analytics, USA) searching for the term "wastewater toxicity" for instance indexed 5,547 publications referring to the topic and 410 publications containing these words 
in the title of the respective studies (Web of Science 2017). Of these publications 601 and 41 respectively were published only in 2016 , while some of the earliest date back to the 1970s (e.g., Esvelt et al. 1973). The number of relevant publications increases once keywords synonymous or related to toxicity (such as "adverse effects", "negative impacts", "hazards", "risks", "detoxification") or to wastewater (such as "WWTP discharges", "sewage treatment" or "municipal effluents") are included or if specific bioassays, endpoint, pollutant groups and treatment technologies are searched.

This exemplary database query indicates the broad research on WWTP discharges including their environmental risks. Only a fraction of these studies identified plausible causative agents of the observed effects. This circumstance is particularly complex because of the vast number of existing and newly developed chemicals that are presently not or insufficiently assessed. The authors of these studies thus generally concluded towards unknown compounds and sample constituents not covered by target chemical analyses and/or their cumulative effects to be responsible (e.g., Abbas et al. 2018, Backhaus and Karlsson 2014, Maier et al. 2016, Thrupp et al. 2018). Through these studies it also became apparent that neither the reduction of target (micro)pollutants, nor of the DOC content, may correlate to the removal of toxicity. Effect-based methods (comprising in vitro, in vivo and in situ) are thus imperative to complement on chemical analyses (compare above) and to establish the missing line(s) of evidence (Backhaus et al. 2019, De Baat et al. 2020, Leusch et al. 2014). Moreover, the development of new methods and optimisation of current test strategies as well as further integration into (waste)water-related regulations should be urged (e.g., Pal et al. 2010, Reemtsma et al. 2016, Rizzo 2011, Schwarzenbach et al. 2006, Völker et al. 2019). 


\subsection{Caenorhabditis elegans in ecotoxicological research}

\subsubsection{Ecology and establishment as biological model organism}

Caenorhabditis elegans (C. elegans) belongs to the diverse animal phylum Nematoda (roundworms). It was firstly described by Maupas (1899) who discovered it in humus rich soils in Algeria. In later years, it was also identified in freshwater habitats (i.e., Hirschmann 1952). C. elegans generally prefers temperate regions and, as a bacterivore, microorganism-rich habitats such as rotting fruits and plants (Hope 1999). Like other nematodes, it plays an important role in soil and benthic food webs (Yeates et al. 1993, Traunspurger 1997), wherein it is eaten by predating nematodes and omnivore insects. If present in unfavorable environments (compare 1.4.2 and 1.4.4) it can assume a so called dauer state. Dauer larvae ingested by invertebrates were observed to be (passively) disseminated to more distant locations (Félix and Braendle 2010). First laboratory isolates were gained early on (Nigon 1949). Since then, the growing knowledge on C. elegans reflects in more than 15,000 published articles up to 2010 , including its complete cell lineage, fully sequenced genome and other major discoveries (e.g., Chalfie 2009, Félix and Braendle 2010). Its genetic and functional correlations to humans (Corsi 2006, Kim et al. 2019c, Leung et al. 2008) were used to elucidate biochemical pathways of human diseases such as Alzheimer, Diabetes or obesity (Tejeda-Benitez and Olivero-Verbel 2016a). Similar to Drosophila melanogaster it implies typical advantages of a biological model, including a well-described morphology, small transparent body (well observable with differential interference contrast (DIC) microscopy), fast reproduction cycle, large brood size, facile cultivation, low maintenance costs (e.g., frozen stocks that can be kept for months), rather invariant development, amenability to genetic crosses and an extensive spectrum of methods (e.g., Altun and Hall 2009, Hope 1999, Sulston and Horvitz 1977). Despite these advantages a few experimental limitations exist, such as a rather impermeable cuticule, and similar to other laboratory models, the reconstruction/extrapolation of in situ environmental conditions, has to be considered when applying $C$. elegans as ecotoxicological model. Therefore, well-defined and adapted culturing practices (Hunt 2016) as well as result verification in the light of higher tier studies (compare figure 2 and chapter 2.3) have been recommended as promising way forward. 


\subsubsection{Development and reproduction}

C. elegans exists as hermaphrodites $(X X)$ and males $(X 0)$ incorporating five pairs of autosomes with $\sim 18,000$ genes. Hermaphrodites mainly self-fertilize producing genetically identical offspring. Males arise by spontaneous non-disjunction at a rate of $0.1 \%$. Mating increases the genetic variability, including a population proportion of males of up to $50 \%$ (Altun and Hall 2009). Adults can grow to an average length of $0.8-1.1 \mathrm{~mm}$ during a short life cycle of about 3.5 days (figure 3). The hermaphrodite life span counts 12-20 days under standard laboratory conditions (optimal feeding, $20^{\circ} \mathrm{C}$ ). C. elegans can survive $4-8$ times longer as dauer larvae (Cassada and Russell 1975, Golden and Riddle 1984, figure 3). Under favorable conditions adult hermaphrodites may lay up to 280-300 eggs in total (Byerly et al. 1976), but the number of fertilized eggs can increase to 1200-1400 upon mating (Hodgkin 1988). Byerly et al. (1976) observed the life cycle to be temperature dependent and a continuous temperature of $>25^{\circ} \mathrm{C}$ causes sterility.

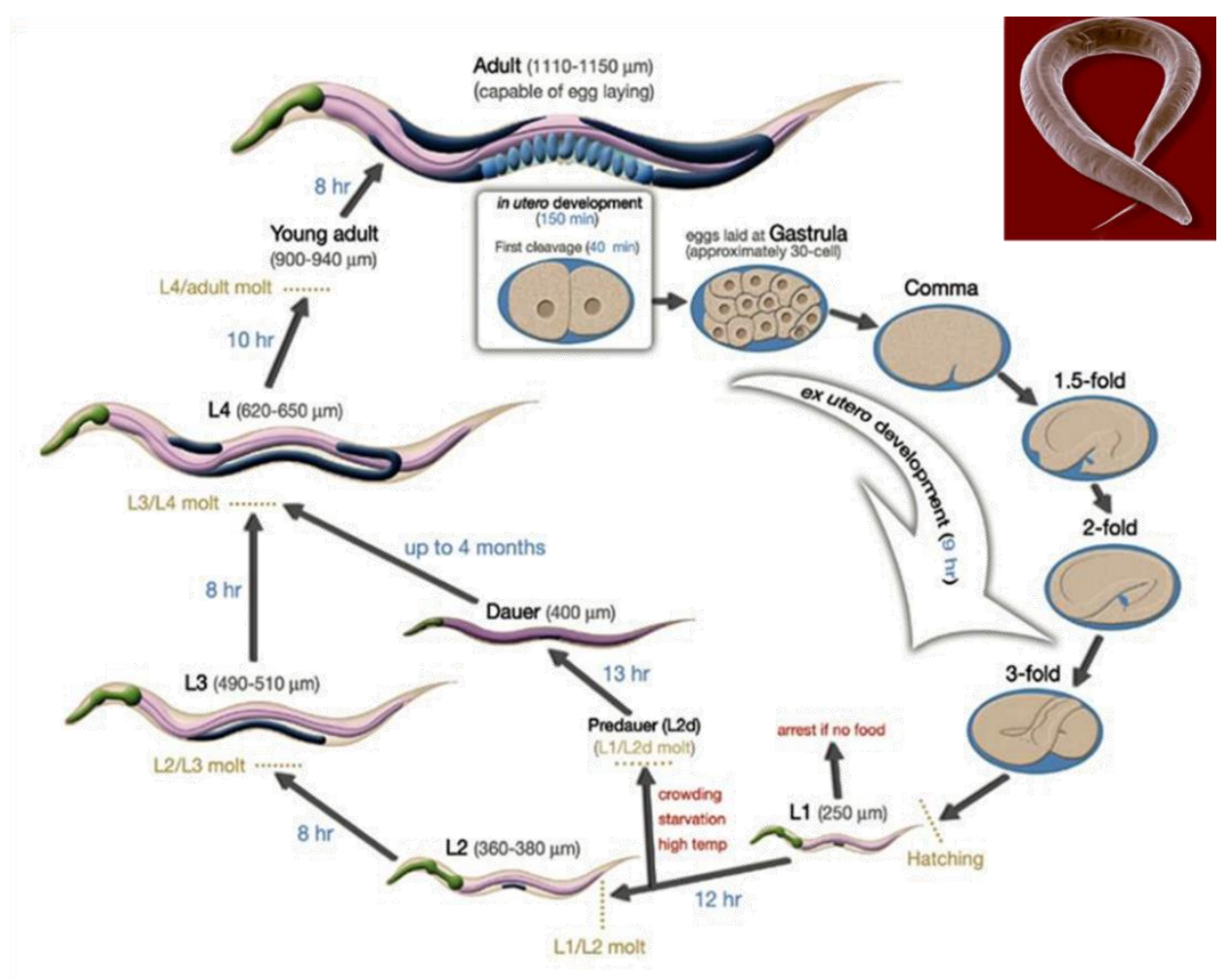

Figure 3. C. elegans life cycle (Altun and Hall 2009) and scanning electron microscope image of an adult hermaphrodite (J. Berger, Max-Planck-Institute for Developmental Biology, Germany). Illustration indicates pharynx (green), intestine (pink), proximal and distal gonad (dark blue), uterus with developing eggs (light blue) and developmental data. 
The genetics of $C$. elegans proved to be relatively amenable, which could hint on alternative ecological specializations (Félix and Braendle 2010, 1.4.4). Adult hermaphrodites count 959 somatic nuclei and approximately 2000 germ cells. Males incorporate 1031 somatic nuclei and about 1000 germ cells (Alberts et al. 2002). Somatic cells differentiate into various tissues and compartments, such as neurons, muscles, hypodermal cells, reproduction system, intestine, pharynx, excretory system and glands (Sulston and Horvitz 1977). C. elegans also implies a sensory system that reacts to various environmental signals (1.4.4). In addition, C. elegans shows a wide range of behavioral traits such as feeding, defecation, egg laying, locomotion, learning as well as social behavior (e.g., Rankin 2002, De Bono 2003).

\subsubsection{Xenobiotic metabolism}

C. elegans has a versatile xenobiotic metabolism that reflects its evolutionary adaption to heterogeneous habitats and various external stressors. This involves traits such as its sensory system that can trigger avoidance behaviors towards contaminated food or pathogenic bacteria (Sambongi et al. 1999, Lindblom and Dodd 2006). Primary exposure sites for environmental chemicals, small particles and other ingestible materials are pharynx and intestine. In contrast, the permeability of its cuticle seems rather low (Chisholm and Xu 2012). In the intracellular space xenobiotics are recognized by ligand activated transcriptional factors, such as nuclear hormone receptors (NHRs) or the maternally-inherited skinhead-1 (SKN-1), that regulate different detoxification genes (figure 4). DNA sequencing and homology analyses thereby revealed 284 genes coding for NHRs; five times more than in vertebrates. However, the majority of $C$. elegans NHRs are currently considered orphan (An and Blackwell 2003, Antebi 2006, Inoue et al. 2005, Mendelski et al. 2019, Taubert et al. 2011).

Similar to most organisms, detoxification in C. elegans proceeds through three phases. Phase 1 (transformation) is characterized by the modification of xenobiotics through cytochromes P450 (CYPs) and short-chain dehydrogenases (SDRs). Pan et al. (2016) report on $83 \mathrm{C}$. elegans and 57 human cyp genes, while the overall evolutionary diversity of cyps estimates to 39,417 isoforms in 236 species. CYPs generally change the polarity of target molecules by adding chemical groups (see 2.3.2 for examples). Phase 2 (conjugation) genes, such as glutathione-s-transferase 
(GST) or UDP-glucuronosyl-transferase (UGT) further increase the solubility of their targets. They are also responsible for directing their excretion, which is performed by transport proteins. The latter belong to phase 3 (excretion) and include adenosine triphosphate (ATP)-binding cassette (ABC) transporters, such as P-glycoproteins (PGP 1-4), and other efflux pumps (Lindblom and Dodd 2006). Modified xenobiotics that are excreted into the gut lumen are subsequently removed by defecation.

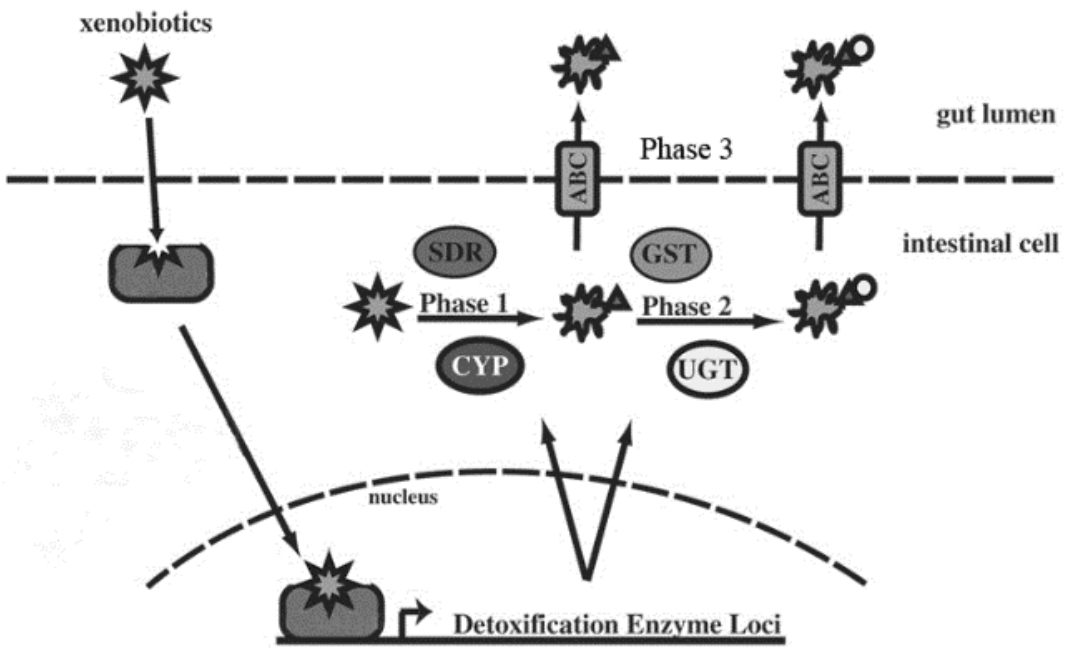

Figure 4. Detoxification in an intestinal cell of C. elegans (Lindblom and Dodd 2006)

Toxic effects of xenobiotics often go in hand with the formation of reactive intermediates, such as reactive oxygen species (ROS). Several detoxification genes protect $C$. elegans against ROS, such as GSTs, catalases, superoxide dismutases (SODs) or glutamylcysteine synthetase (e.g., gcs-1, An and Blackwell 2003, Inoue et al. 2005). Other (chemical) stress related genes are heat shock proteins (HSPs), metallothioneins (e.g., $m t l-1$ and $m t /$ ) ) or abnormal dauer formation 16 (DAF-16, Freedman et al. 1993, Morimoto 1998). The human p53 ortholog cep-1 (Rodriguez et al. 2013) is also known to participate in protective and regulatory mechanisms associated with chemical/stress responses. The complex metabolic interplay of these genes is the basis for cellular integrity and homeostasis. If detoxification capacities are exceeded damage may propagate and irreversible effects may occur.

\subsubsection{Environmental toxicology}

Early studies on environmental factors and cues investigated responses to food sources, temperatures (e.g., Klass 1977), heavy metals (e.g., Popham and Webster 1979), gamma-radiation (Yeargers 1981), UV light (Hartman and Herman 1982) and 
pharmaceuticals (e.g., Höss and Weltje 2007). These studies prepared the ground for C. elegans as a toxicological model (Leung et al. 2008, Hägerbäumer et al. 2015, Williams et al. 2017, Wilson and Kakouli-Duarte 2009) used for the assessment of anthropogenic chemicals, technical materials and environmental samples including sediments, soils, sludge or waste (table 2). C. elegans has also been involved in microcosm studies and community analyses (e.g., Hägerbäumer et al. 2015, Mueller et al. 2020a, Wang et al. 2015, Wilson and Khakouli-Duarte 2009, table 2). The nematode thereby proved to be a versatile tool for answering toxicokinetic (Burns et al. 2010, Chen et al. 2016, 2018, Kim et al. 2019a, Liu et al. 2019, Mueller et al. 2020b, Offermann et al. 2009, Ristau et al. 2015, Roh et al. 2014, 2016, Spann et al. 2015, Stylianou et al. 2018) and toxicodynamic research questions (compare below).

C. elegans studies have examined a panoply of apical endpoints including mortality (e.g., Coomans and Vanderhaeghen 1985, Williams and Dusenbery 1990), life span, reproduction (e.g., Anderson et al. 2001, Leung et al. 2010), fertility/fecundity (e.g., van Kessel 1989, Popham and Webster 1979), population growth rate (e.g., Ohba and Ishibashi 1984), growth (e.g., van Kessel 1989, Ohba and Ishibashi 1984), morphology (e.g., Ohba and Ishibashi, 1984, Popham and Webster 1979) and development (e.g., Coomans and Vanderhaeghen 1985, Ohba and Ishibashi 1984). Most apical endpoints incorporate midpoints or secondary endpoints such as life span, larval arrest or morphological defects for development (e.g., TejedaBenitez and Olivero-Verbel 2016a) and reproductive delays, decreased progeny or reduced fertility for reproduction (e.g., Tejeda-Benitez and Olivero-Verbel 2016a). Behavioral endpoints include feeding, locomotion (e.g., Williams and Dusenbery 1990, Boyd et al. 2003, Anderson et al. 2004), chemotaxis, avoidance and motility (e.g., Bargmann 2006, Li et al. 2020, Sambongi et al. 1999). These are complemented by cellular and molecular endpoints, such as apoptosis, disruption of membranes, mitochondria, chromosomal aberrations, cell cycle inhibitions, reversible and irreversible DNA damage (e.g., Allard et al. 2013, Behl et al. 2016, Leung et al. 2010), gene expression profiling (e.g., Peter et al. 1996, de Pomerai et al. 2008, Reichert and Menzel 2005, 2.3.3), energy budget (e.g., McLaggan et al. 2012). Other complementations are given by interactions of toxicity pathways such as in behavioral neurotoxicity (e.g., Williams and Dusenbery 1990, Liu et al. 2020). 


\subsection{Integration of this thesis into the present state of research}

This thesis was embedded in the research project TransRisk dealing with the "characterization, communication and minimization of risks from novel pollutants and pathogens in the water cycle" (www.transrisk-projekt.de). Anthropogenic (micro-)pollutants vastly contribute to aquatic pollution. The characterization of their realistic hazards and feasible mitigation options has become imperative. Due to their incomplete removal in conventional biological WWTPs, AWWT technologies are being implemented to (further) reduce their emission. This thesis particularly aims at the ecotoxicological evaluation of the effectivity of the AWWT processes ozonation and ozonation coupled to GAC/biological post-filtration, which was targeted in the following key challenges and knowledge gaps:

1) Sample preparation is pivotal for the accurate detection and quantification of micropollutants and toxicity (Prasse et al. 2015, Völker et al. 2019). Nonetheless, current methods are mainly optimised for chemical analyses (Escher et al. 2005, Prasse et al. 2015), but not for effect-based detections (Bistan et al. 2012, Stalter et al. 2016, Wagner and Oehlmann 2010). In our study we optimised three major methods - acidification, filtration and SPE - for in vitro analysis. In earlier studies, acidification was capable of modifying pollutants in different water matrices (Baker and Kasprzyk-Hordern 2011, US EPA 2010, Vanderford et al. 2011), filtration altered specific in vitro activities compared to untreated samples (Dagnino et al. 2010, Janex-Habibi et al. 2009, Routledge 2003) and SPE selectively enriched (micro)pollutants. This situation can cause under- or over-estimations of actual hazards. We optimised these techniques using eleven in vitro bioassays and different (waste)water matrices. Based thereon specific preparation procedures and optimised methods were recommended (3.1 and Abbas et al. 2019).

2) A long-term environmental monitoring was conducted in a protected region in Baden-Württemberg, Germany, using in vitro bioassays screening for endocrine activities, mutagenicity and genotoxicity. Up to 33 sites representative for the water cycle were sampled during five campaigns (2012-2014) including wastewater, surface water, groundwater and drinking water. In vitro bioassays were accompanied by a chemical monitoring of 92 chemical indicators (Anna Bollmann unpublished results, Seitz and Winzenbacher 2017). Main objectives were to 
determine the types and levels of ecotoxicological in vitro potentials, to localize eventual pollution hot spots, to indicate environmental and/or human hazards as well as to generate data sets for before-and-after-comparisons of WWTP upgrades (2.2). The obtained results further supported regional stakeholders (e.g., waterworks and municipalities) in their sustainable (waste)water management plans.

3) An in vivo bioassay based on ISO10872 and the model C. elegans (Haegerbaeumer et al. 2019, Höss et al. 2012, Wilson and Khakouli-Duarte 2009) was adapted for this thesis. This involved apical (reproduction and growth) and molecular endpoints (CYP-35A3 related xenobiotic metabolism in a transgenic strain, Menzel et al. 2007). Proof-of-principle experiments were conducted using the reference PAH $\beta$-NF (Forsgren 2015, Leung et al. 2010) and different (waste)water samples (aqueous, spiked and extracted) comprising the described conventional biological and AWWTs. An influence of TSS was also analysed, because particlebound pollutants may alter bioavailability (Offermann et al. 2009, Spann et al. 2015, Stylianou et al. 2018, table 2). Cumulative effects due to background contaminations were hypothesized (Backhaus and Karlsson 2014, Thrupp et al. 2018). The adapted bioassay allowed for the combined assessment of developmental and reproductive toxicity (DART) and CYP-35A3 induction (2.3 and Abbas et al. 2018). Novel test systems and strategies have been rated highly important, because standard bioassays may be insensitive in detecting relevant water quality deficiencies (Berger et al. 2016, Schwarzenbach et al. 2006, Sonne et al. 2018, Wigh et al. 2018).

4) The AWWTs ozonation and ozonation with GAC/biological post-filtration were evaluated for their (micro)pollutant/toxicity removal capacity. The ecotoxicological part of this evaluation was performed with a battery of in vitro and on-site in vivo assays previously applied in related wastewater assessments (e.g., da Costa et al. 2014, Gartiser et al. 2010, Giebner et al. 2018, Magdeburg et al. 2012, 2014, Maltby et al. 2000, Stalter et al. 2010, 2011, Triebskorn et al. 2017). This thesis thereby examined (the above mentioned) five in vitro and C. elegans bioassay for their usefulness at the laboratory-scale. The detections made by these assays were integrated into a 'wastewater quality evaluation matrix' (developed in TransRisk, Ternes et al. 2017) that rated ozonated coupled to GAC filtration as most effective AWWT option (out of five, 2.4). The evaluation concept may readily be transferred to future evaluations of WWTPs. 


\section{General discussion}

\subsection{Optimising sample preparation for in vitro bioassays}

Sample preparation methods can bias the quantification of the original toxicity of environmental samples (e.g., Neale et al. 2018). Main reasons for this are modifications/losses of sample constituents during sample processing/storage and/or interfering environmental factors (Daughton 2003, Maruya et al. 2016). This chapter discusses ways to reduce such artifacts at different preparational/analytical steps of an in vitro analysis. Bioassay-based optimisations have scarcely been performed despite their apparent relevance for realistic hazard assessment and monitoring. Characteristic challenges in this endeavor were previously studied by Bistan et al. (2012), Macova et al. (2010), Neale et al. (2015) and Stalter et al. (2016). Based thereon the present optimisation focuses on the influence of sample acidification, filtration and SPE on the outcome of eleven in vitro bioassays testing 17 (waste)water samples representative for the urban water cycle.

\subsubsection{Sample acidification}

Acidification is commonly used for stabilization of (non-)target compounds during sample storage. The present experiments focused on possible ecotoxicological discrepancies between neutral (untreated) and acidified samples that would emerge during a $24 \mathrm{~h}$ storage period. Significant differences occurred regarding different endocrine activities and cytotoxicity. The degree of this discrepancy was higher at certain sampling points (e.g., highest for raw wastewater) and in a number of in vitro bioassays (e.g., highest in the yeast anti-estrogen screen, YAES). However, no obvious reason or trend became apparent, such as sample toxicity or the specificities of the respective in vitro bioassay. From these findings it was thus concluded that 1) the added sulphuric acid can modify (bioactive) sample constituents (e.g., in an unspecific manner) and bioassay outcomes, 2) Possible sample modifications by microbes contained in neutral samples may or may not have a more severe effect on the outcome of an in vitro bioassay than those mentioned under 1). Both situations (1 and 2) thus demand further research to fully clarify the advantages/disadvantages of testing native or acidified samples, and to allow their analytical comparison to other sample stabilization techniques (such as sample filtration or sample freezing). 
To this end, neutral samples were recommended for ecotoxicological assessments, because higher toxicity was detected. It is also known that the chemical composition of samples may be altered by $\mathrm{pH}$ shifts and acid-based hydrolyses (Baker and Kasprzyk-Hordern 2011, Stalter et al. 2016, Prasse et al. 2015, Vanderford et al. 2011). Such an alteration was possibly observed by Bollmann et al. (unpublished results) who performed a non-target screening on a wastewater sample (that was also tested in this thesis, Abbas et al. 2019). In this screening the researchers detected an overlap of compounds of only $72-75 \%$ between the neutral and acidified sample aliquot. In addition, acidified samples require further preparation steps, such as neutralisation prior to bioassays, not to compromise bioassays test organisms.

\subsubsection{Sample filtration}

Filtration has several advantages such as isolation of particulate matter and/or sample sterilization (e.g., Janex-Habibi et al. 2009, McLaggan et al. 2012). Earlier bioanalytical comparisons also illustrated potential drawbacks such as significant losses of detectable endocrine activity. These losses seemed to originate from the retention of TSS-bound compounds (also hypothesized by Abbas et al. 2018) which was also suggested in earlier studies (Dagnino et al. 2010, Janex-Habibi et al. 2009, Routledge 2003, Shieh et al. 2016) that also described hydrophobic (micro)pollutants to adsorb to filter membranes. In this context $\mathrm{Ng}$ and Cao (2015) published the article "What Exactly Are You Filtering Out?" wherein they suggest to better adjust filter materials to the aims of an investigation.

In several samples the present in vitro bioassays detected higher endocrine activities in filtered samples than the corresponding unfiltered samples. This was explained as the in vitro test systems applied in this thesis can be activated and/or inhibited by agonistic or antagonistic compounds respectively. Such interactions in wastewater samples have been reported earlier (Ihara et al. 2014, Itzel et al. 2019, Rao et al. 2014, Zwart et al. 2018). Thus a selective retention of (particle-associated) antagonists during filtration could have allowed an increased receptor binding of agonist in the respective filtrates, and vice versa. In addition, agonist/antagonists could have been "washed off" from their particle-bound states during filtration. In this way they could further have altered their ratio in the filtrates. Despite these findings, most samples showed similar activity levels regarding unfiltered and filtered 
samples, which suggested that most environmental hormones and EDCs remained dissolved in the aqueous phase of the respective samples (e.g., due to their hydrophilicity /polarity, 1.2.1).

Filtration unpredictably modified the composition and in vitro activities/toxicity of (waste)water samples. This situation may lead to misinterpretations of bioassay results including under- and over-estimations of actual hazards (e.g., Burton 2000, Dagnino et al. 2010). For the effective application of filtration in ecotoxicology further compound, sample type and bioassay specific optimisations are recommended.

\subsubsection{Solid phase extraction}

Micropollutants detection often requires sample enrichment, such as by SPE, to increase quantification limits (LOQs) and for reducing matrix interferences (Prasse et al. 2015, Wagner and Oehlmann 2011, Wagner et al. 2013). SPE methods have been optimised for the recovery of different pollutant classes (Baker and KasprzykHordern 2011, Leusch et al. 2012, de Alda and Barcelo 2001) focusing on methodical parameters, such as sample pretreatment, SPE sorbents or different extraction modes (Pietrogrande and Basaglia 2007). These optimisations also involved different evaluation methods such as multivariate statistics (e.g., Harju et al. 2015, Polo et al. 2005, Yang et al. 2014). Most studies used defined standard solutions, while a few further validated their results using complex environmental samples. The present investigation focused on benchmarking six SPE methods for their recovery of endocrine activity, genotoxicity and cytotoxicity from real (waste)water samples. These methods combined three SPE sorbents (Oasis HLB, Supelco ENVI-Carb+ and Telos C18/ENV) with two extraction pH (7 and 2.5). All SPE sorbents were eluted with a methanol:acetone (1:1) mixture (figure 5). Moreover, a multivariate evaluation via the Pareto algorithm was integrated (compare Abbas et al. 2019).

The multivariate optimisation approach demonstrated that the highest endocrine activities and genotoxicity were recovered by the Telos C18/ENV sorbent. Highest cytotoxicity was obtained by the Oasis HLB sorbent. By the optimised SPE method certain endocrine activities, such as estrogenicity, (anti-)androgenicity and dioxinlike $(A h R)$ activity, were only detected after sample enrichment. In line with these results the Telos C18/ENV and Oasis HLB indicated effective recoveries of 
cytotoxicity (Escher et al. 2005, Stalter et al. 2011, 2016) and estrogenicity (Bistan et al. 2012, Wagner and Oehlmann 2011) in other optimisation studies. Regarding the putative causative agents of these activities/toxicity the composite material of the Telos C18/ENV and the amphipathic material of the Oasis HLB were previously described to extract compounds with various physicochemical properties (e.g., Leusch et al. 2012). It is also thought that the applied in vitro bioassays can be activated by heterogeneous chemical compounds. In addition, Stalter et al. (2016) suggested that the ENV material of the Telos C18/ENV and the Oasis HLB seem capable of adsorbing polar cytotoxic compounds. Wagner and Oehlmann (2011) suspected non-polar chemicals to act as causative agents of the estrogenicity that they had extracted from bottled mineral water using a C18 material. It remains to be clarified whether (and how) these results apply to the present recovery rates. Most extractions in this study were more effective at an acidic sample enrichment $\mathrm{pH}$ (Abbas et al. 2019). The multivariate Pareto evaluation however favored a neutral sample extraction $\mathrm{pH}$ and SPE by the Telos C18/ENV as optimal method. This difference resulted from the integration of the parameter cytotoxicity. The latter was rated as potential indicator of a higher overall pollutant load including environmental hormones and EDCs (compare figure 6 and further discussion).

More effective extraction at acidic pH were also obtained by Misik et al. (2011) and Stalter et al. (2016) for the endpoints mutagenicity and cytotoxicity respectively. In their study Stalter et al. (2016) compared different SPE sorbents applied to disinfected drinking water, whereas Misik et al. (2011) had extracted biologicallytreated and ozonated wastewater using a C18 sorbent. In the general context of SPE of bioactive/toxic compounds Escher et al. (2005) mention that short-term acidification may increase the recovery of weak acids and ionized (micro-)pollutants, while weak bases or zwitterions might be lost. Stalter et al. (2016) however also argue that short-term acidification (compare 2.1.1 and Abbas et al. 2019) can already degrade SPE matrices and/or modify dissolved organic matter constituents in aqueous samples. 


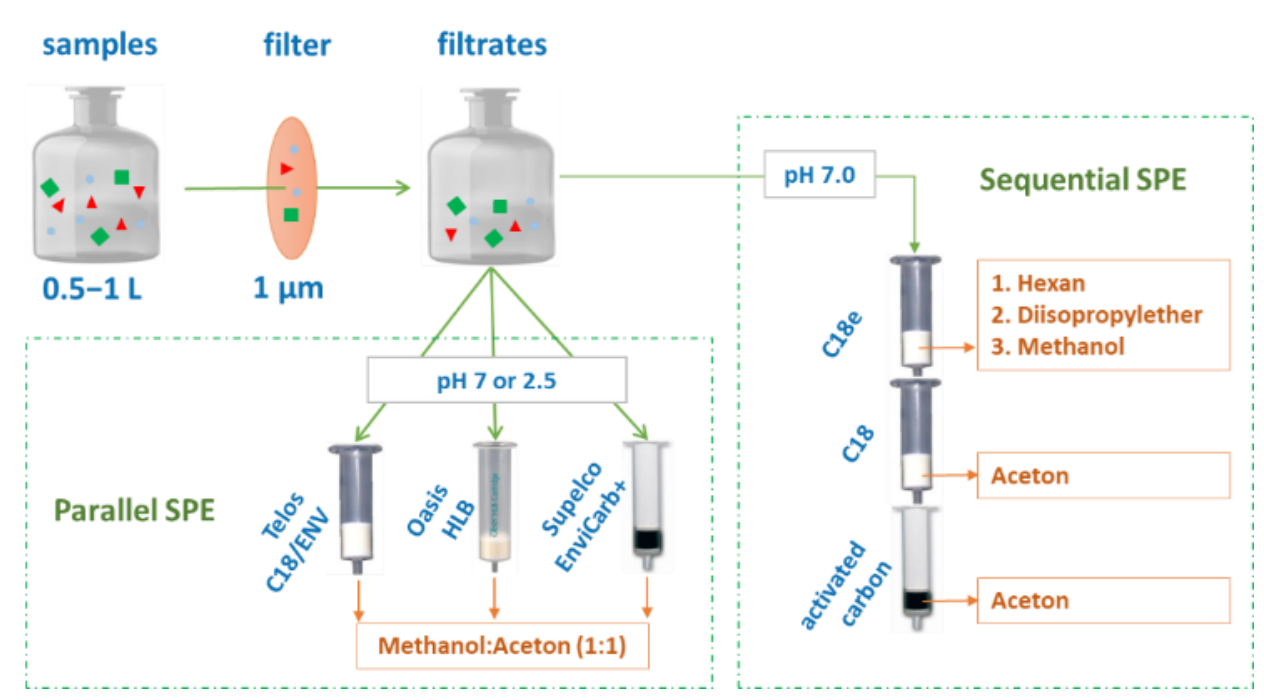

Figure 5. Two SPE methods designed as parallel and sequential protocols optimised for in vitro bioassays in the course of this thesis and within the TransRisk project.

These results point out the necessities in further optimising SPE methods for in vitro bioassay-based (as well as chemical) assessments and monitoring. Therefore, several optimisation parameters and experimental set ups described in the scientific literature seem promising. Different SPE sorbents and extraction modes (e.g., comparing eluting solvents of different polarities) improved the recovery of target compounds and TPs (e.g., Chang et al. 2009, Kern et al. 2009, Stalter et al. 2016). Bollmann et al. (unpublished results) connected three SPE cartridges (C18e, C18 and activated carbon) in a serial manner ('Sequential SPE' in figure 5) for the extraction of different (waste)water samples. Using the eluting solvents hexan, diisopropyl ether, methanol and acetone different extractions were performed. These could be used for separate (sample pre-fractionation) or combined ("total extract") analysis. In addition, these extracts were tested in the previously described in vitro bioassays. The sequential methods performed equally well than the 'Parallel SPE' method (figure 5) for most of the endocrine activities and cytotoxicity. Merging the individual solvent fractions into a "total extract" has not been compared.

Another SPE parameter that proved to be particularly relevant for bioassay analyses is the final enrichment factor at which an extract is tested. SPE enrichment factors were compared in previous studies (Escher et al. 2014, Macova et al. 2010, Tang et al. 2013). Escher et al. $(2009,2014)$ used 103 in vitro bioassays for "Benchmarking Organic Micropollutants in Wastewater" by applying a concept that compared the estrogenicity, genotoxicity and cytotoxicity of extracted wastewater 
samples along the enrichment factor required to produce an effect (compare figure 6 in this context). Extracted samples also built the basis for EDA to identify possible causative agents (Burgess et al. 2013, Brack et al. 2017, Neale et al. 2017, Schulze et al. 2017, Tang et al. 2014). Samples with low contamination level such as groundwater and certain endpoints without toxicity thresholds such as genotoxicity often require high sample enrichment factors (up to 100, Keiter et al. 2006, Stalter et al. 2016). This circumstance may further be required for extracting surface waters or wastewater from AWWT stages, due to their generally low contamination levels.

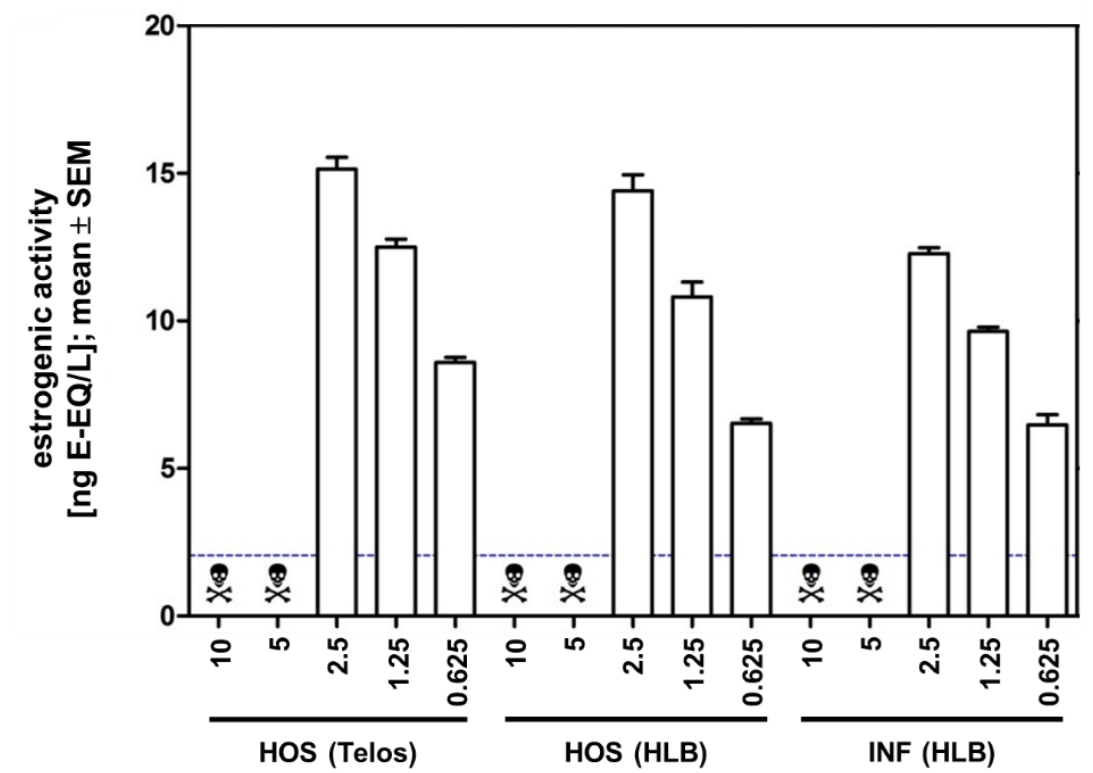

Figure 6. Estrogenic activity in extracted hospital wastewater (HOS) and a WWTP influent (INF) quantified as mean 17ß-estradiol equivalents (ng E-EQ/L) \pm standard error of the mean (SEM). Telos C18/ENV ("Telos") and Oasis HLB ("HLB") were applied as SPE sorbents. Different sample concentration factors $(0.625-10$ fold) were used to investigate concentration response relationships. In 5-10 fold concentrated samples cytotoxicity occurred $(\dot{x})$. Dashed line = limit of quantification. Unpublished result jointly obtained by $\mathrm{I}$. Schneider and A. Abbas.

Nevertheless, exhaustive sample concentration may bear the risk of introducing artifacts such as from the (co)enrichment of matrix components and/or leaching materials (Leusch et al. 2014, Macova et al. 2010). Control samples such as procedural blanks are thus imperative for any bioanalysis involving SPE extracts (Stalter et al. 2016). High concentration factors (below toxic concentrations of keeping solvents) often indicate unspecific toxicity masking other endpoints (Escher et al. 2020). In contrast, at (very) low concentration factors the investigated effects 
may be diluted out. In addition, interpolation from lower to higher enrichment factors (and vice versa) was rated rather unfeasible due to mostly non-linear concentration response relationships (figure 6). For analyzing SPE extracts the performance of preliminary range-finding experiments has thus been recommended (Tang et al. 2014, Escher et al. 2014).

Further optimisation potential was also allocated to loading modes and sample volumes. In a study by Macova et al. (2011) extraction of higher sample loading volumes $(0.5-4 \mathrm{~L})$ lowered the detection limit for cytotoxicity (Alliovibrio fisheri), estrogenicity (E-Screen), dioxin-like activity (AhR-CAFLUX) and genotoxicity (umu test) by up to 8 to 10 fold. As previously stated higher sample loading volumes may go in hand with an increased risk of artefacts and may require extended sample preparation, such as additional sample filtration. Schulze et al. (2017) developed an automated on site SPE device that can process sample volumes up to $50 \mathrm{~L}$. In their experiments such sample volumes enhanced the recovery and enrichment of (ultraJow concentrated (micro)pollutants and different in vitro activities, such as mutagenicity or (anti-)estrogenic activities. This technique (also termed large volume SPE, LVSPE) seems particularly useful for surface water and groundwater monitoring as well as investigations requiring a high number of parallel experiments on the same sample/extract. LVSPE also requires elaborate equipment and handling, such as the preparation of special circulation blanks. Automated and/or on-site SPE methods were also implemented with beneficial outcomes in several ecotoxicological studies (e.g., Henneberg et al. 2014, Prieto-Rodriguez et al. 2013).

Additional potential lies in optimising SPE methods for an enhanced recovery of volatile, (highly) polar and particle-associated compounds that are mostly lost during conventional sample processing and/or standard SPE procedures (compare below). Significantly different test results were for instance obtained in the E-Screen, once a keeping solvent was added before full evaporation of the eluting solvent (Wagner and Oehlmann 2010). This measure indicated the presence of estrogenic compounds that volatilized during evaporation without adding a keeping solvent. In an investigation by Stalter et al. (2016) solvent evaporation of wastewater extracts without solvent exchange (dimethyl sulfoxide, DMSO) diminished the recovery of cytotoxicity. These studies show how simple sample preparation steps can significantly improve the effectivity of standard SPE methods. However, according 
to Stalter et al. (2016) and others (e.g., Benner and Ternes 2009, De Baat et al. 2020), the effective extraction of volatile, (highly) polar and unknown compounds however remains one of the main challenges in optimising the preparation of water and wastewater samples.

In perspective, optimised SPE methods offer specific advantages for in vitro bioassay-based environmental assessments and monitoring. Among their important benefits are an improved preservation, enrichment, detection and identification of micropollutants and toxicity (compare 2.2-2.4). SPE methods have previously facilitated the classification of (putative) causative agents (Itzel et al. 2020) exhibiting estrogenicity (Allinson et al. 2010, Ma et al. 2005, Routledge 2003, Zhao et al. 2015), anti-estrogenicity (Tang et al. 2014), dioxin-like (Allinson et al. 2011, Ma et al. 2005) and retinoic acid-like activities (Allinson et al. 2011, Sawada et al. 2012), genotoxicity (Keiter et al. 2006) or cytotoxicity (Allinson et al. 2010, Ma et al. 2005). If the same SPE extracts are applied in parallel in selected in vitro and in vivo test systems this can provide valuable insights regarding observed physiological effects (e.g., Hugget et al. 2003, Schneider et al. 2020). Another way forward will be to compare optimised SPE methods with particular sampling or other sample extraction techniques (such as SPME, passive samplers or purge-and-trap methods) and to broaden the knowledge on their benefits, limitations and further options for optimisation. 


\subsection{In vitro bioassays as environmental monitoring tools - case study of a water protection region in Southwest Germany}

In vitro bioassays are important tools for ecotoxicological assessments and environmental monitoring. They are routinely applied to investigate unspecific toxicity as well as specific MoAs. MoA based bioassays can serve as indicators of different bioactive/toxic contaminants such as herbicides or EDCs (e.g., Escher et al. 2014, Wagner et al. 2017). The in vitro activity of these pollutants has often been verified using in vivo test systems. For certain EDCs the induction of vitellogenin served as biomarker for reproductive toxicity (e.g., Huang et al. 2016, Stalter et al. 2015). In this way in vitro and in vivo results were associated with suspected or identified compounds (compare EDA). Nevertheless, once the selected assays lack specificity and sensitivity respective toxicants may remain undetected. Clarification of causative agents thus resembles a complex task. In vitro bioassays for baseline or reactive toxicity (such as cytotoxicity) are known to integrate compounds with multiple MoAs (e.g., Escher et al. 2014, 2020, Riss and Moravec 2004, Tang et al. 2014). Although cytotoxicity has helped to draw correlations to in vivo effects (e.g., Stalter et al. 2015), several studies involving iceberg experiments demonstrated that most cytotoxic agents remained unknown (e.g., Neale et al. 2014, Tang et al. 2014). However, cytotoxicity assays proved to be beneficial tools in (high-throughput and preliminary) environmental screenings and prioritisation of pollution sites.

In the present thesis an environmental monitoring was conducted that involved six sampling campaigns at up to 30 sampling sites within a water protection region in Southwest Germany (compare Seitz and Winzenbacher 2017). Water and wastewater samples from relevant sites were screened for different endocrine activities (according to Routledge and Sumpter 1996, Stalter et al. 2011, Wagner et al. 2013), mutagenicity (based on ISO11350), genotoxicity (according to ISO13829) and cytotoxicity (occurring in these cellular test systems). The in vitro investigation was accompanied by a detailed chemical monitoring program (Anna Bollmann unpublished results, Seitz and Winzenbacher 2017) to investigate potential correlations with respective chemical indicators. In the course of this monitoring, high endocrine activities were detected in hospital, raw and to lesser extend biologically-treated wastewater with predominantly (anti-)estrogenic and partially androgenic activities. 

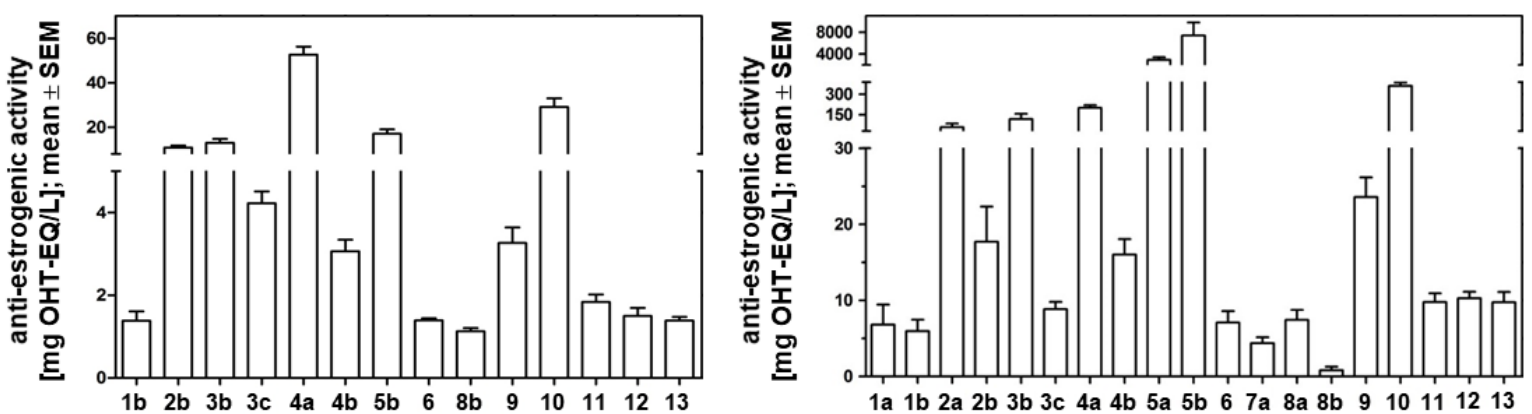

Figure 7. Anti-estrogenic activities in aqueous samples quantified as mean 4hydroxytamoxifen equivalents ( $\mathrm{mg}$ OHT-EQ/L) \pm SEM. A total of 14-19 sampling sites were analyzed in April 2012 and February 2014 respectively including WWTP influents (1a, 2a, $4 a$ ), effluents (1b, 2b, 3b, 4b), a filtered WWTP effluent (3c), hospital wastewaters (5a, 5b), surface waters $(6,12,13)$, samples from a rain water collection / retention / infiltration basin $(7 a, 8 b, 8 b)$ as well as groundwater hotspots $(9-11)$. Results jointly obtained by I. Schneider and $\mathrm{A}$. Abbas in collaboration with the TransRisk project partners.

In contrast, genotoxic and mutagenic potentials were rarely detected in raw and treated wastewater. Extracted hospital wastewater however indicated both genotoxicity and mutagenicity at increased rates. None of these toxicities including cytotoxicity was detected in surface water or groundwater throughout the entire monitoring. These results were in line with the scientific literature, whereby cytotoxicity and genotoxicity were mainly detected in extracted wastewater or (highly) polluted surface water (e.g., Baumstark-Khan et al. 2005, Escher et al. 2014, Macova et al. 2011). The fact that groundwater generally exhibits no or insignificant toxicity unless affected by landfills or contamination sites (e.g., Baun et al. 2000) was also supported by the present monitoring results. In vitro activities/toxicities were further investigated in the context of WWTP efficiencies. Their removal efficiencies were compared to identify and prioritize plausible pollution sources in the model region. All surveyed WWTPs (Langenau, Halzhausen, Steinhäule) indicated an effective removal of estrogenic, androgenic, genotoxic and mutagenic potentials (figure 8). This is of particular relevance as a few of the receiving streams of WWTPs show high wastewater fractions and flow through water protection zones of increased vulnerability. 

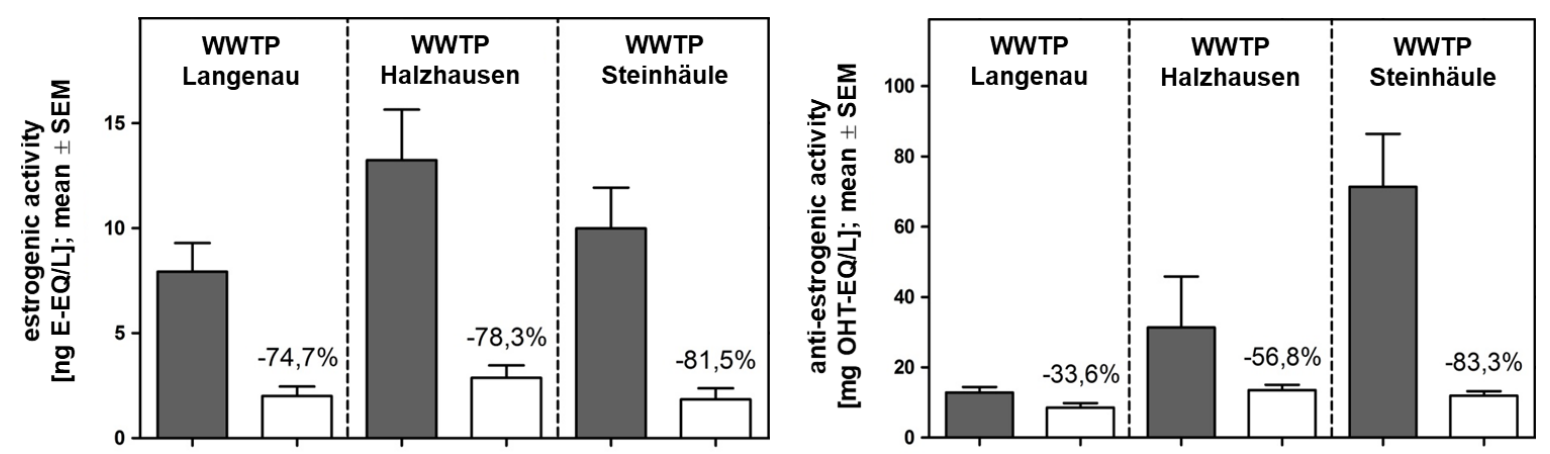

Figure 8. Estrogenic (left) and anti-estrogenic (right) activities in raw (dark grey bars) and biologically-treated (white bars) wastewater samples from three WWTPs (using activated sludge) presented as mean 17ß-estradiol (ng E-EQ/L) or 4-hydroxytamoxifen (mg OHT$E Q / L$ ) equivalents \pm SEM, respectively. Results base on five monitoring campaigns (20122014, $n=40-48$ ). Negative percent values (above white bars) represent the estimated removal rate towards the respective activity. Results jointly obtained by I. Schneider and A. Abbas.

The extensive reduction of these in vitro activities was reported for other WWTPs (e.g., Allinson et al. 2011, European Commission 2012, Giebner et al. 2018, Sawada et al. 2012). Their retention indicates the biodegradability and/or sludge adsorption of the respective (micro)pollutants during activated sludge treatments (Margot et al. 2013, Leusch et al. 2014, Dagnino et al. 2010). Notwithstanding the suitability of the applied in vitro bioassays for evaluating WWTP efficiencies, care should be taken when different test systems probing for the same endpoint are compared (e.g., AYES, E-screen, ER-calux, ER-GeneBlazer or YES for estrogenicity) and/or once different sample preparation methods were used to obtain a result (e.g., Magdeburg et al. 2014, Jia et al. 2015). Unlike the other in vitro potentials, anti-estrogenic activity seemed less effectively removed at a few investigated WWTPs (figure 8). Residual anti-estrogenic activity in treated wastewater was also observed by lhara et al. (2014), Itzel et al. (2020) and Rao et al. (2014), who stated the need for further research on this endpoint in water and wastewater assessments.

Markedly, elevated anti-estrogenic activities were recurrently recognized in one of the groundwater hotspots (sample 10 in figure 7). These hotspots are known to be impacted by a nearby landfill (Wolfram Seitz, personal communication) so that they were previously prioritized for chemical monitoring. The present ecotoxicological monitoring of these hotspots included the examination of their most proximate water intakes and wells used for the extraction of raw drinking water (figure 9). Three 
important aims were pursued in this investigation: 1) the detection of relevant in vitro activities, 2) to ensure an effective water treatment, if these activities would occur and 3) to narrow down putative causative agents in groundwater (e.g., if natural or anthropogenic chemicals) and their potential sources, such as WWTPs or agricultural run offs.

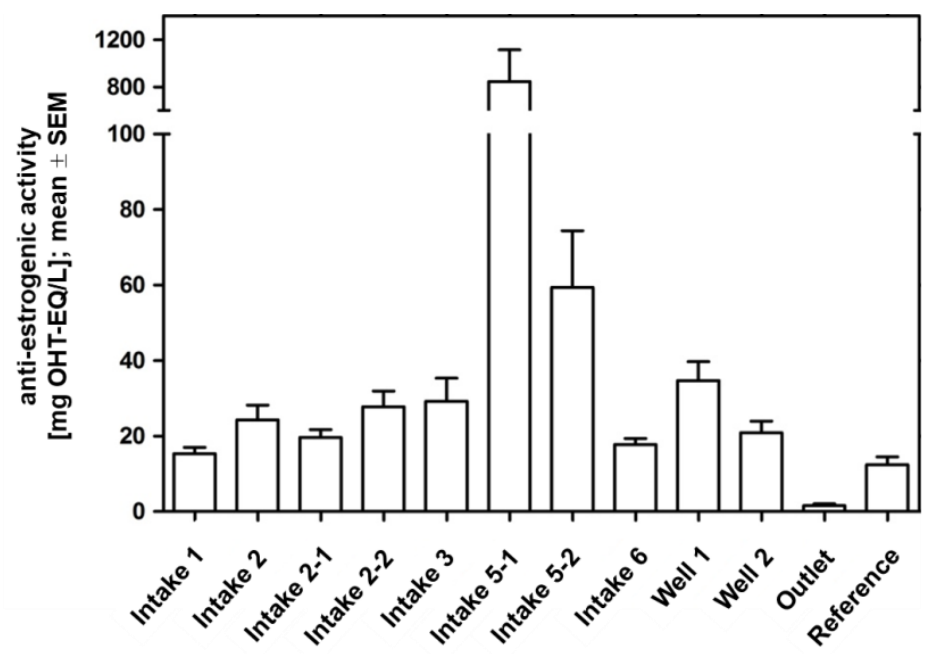

Figure 9. Anti-estrogenic activities presented as mean 4-hydroxytamoxifen equivalents (mg OHT-EQ/L) \pm SEM of aqueous grab samples taken from groundwater intakes (1-6) and groundwater wells $(1-2)$ in the model region in October $2013(n=8)$. Outlet = polished groundwater from a local waterworks. Reference = raw groundwater from large depth . Results jointly obtained by I. Schneider and A. Abbas in collaboration with the TransRisk project partners.

As an outcome, one groundwater intake (sample 5-1 and 5-2), but none of the other prioritized sampling sites indicated any relevant anti-estrogenic activity. Importantly, no activity was detected in drinking water from a supply pipe (sample 'outlet') and in groundwater used for the production of drinking water after minor treatment (sample 'well 1' and 'well 2'). These results were obtained using aqueous grab samples of the sampling campaign in October 2013 (figure 9). Samples taken during the subsequent monitoring (February 2014) from the same sampling sites served for follow up examination. For possible effect confirmation these samples were prepared by SPE. The high anti-estrogenic activity previously detected in sample 51 (figure 9) could not be confirmed (remaining at the level of the LOQ, results not shown). Moreover, the parallel chemical monitoring at these sampling sites also did not showed any relevantly increased concentrations of (micro)pollutants. 
Up to present, only a small number of chemicals are known for anti-estrogenic effects. One chemical thereof that was included in the chemical monitoring program was $1 \mathrm{H}$-benzotriazole (Abbas et al. 2018). Benzotriazole was reported for in vitro anti-estrogenicity by Harris et al. (2007) as well as to be responsible for different in vivo effects (Seeland et al. 2012). However, MECs of $1 \mathrm{H}$-benzotriazol at all monitored sites (e.g., 30-90 ng/L in the groundwater hotspots) were far below its reported ECs (e.g., several milligram per liter in the YAES). Nonetheless, Harris et al. (2007) also computed a predicted no effect concentration (PNEC) of $60 \mu \mathrm{g} / \mathrm{L}$ based on further literature data. Moreover, other antagonist of the human estrogen receptor capable of activating the YAES could have been present in the analyzed groundwater samples. Known or suspected anti-estrogens in the medical and ecotoxicological literature are for instance the anti-neoplastic drugs fulvestrant (ICI 182780, e.g., Hu et al. 1993), raloxifene (Matsumoto et al. 2004), tamoxifen and a number of its derivatives/TPs (Knoop et al. 2018, Sohoni and Sumpter 1998, Stalter et al. 2011, Zhao et al. 2011) as well as the insecticide hexachlorcyclohexan (Li et al. 2008), several flame retardants (Di Benedetto 2009, Martin Wagner personal communication, Zhang et al. 2014), the disinfectants hexachlorophene, pentachlorophenol and the vitamin K3 (Jung et al. 2004), the PAHs dibenz[a,h]anthracene, 6-hydroxy-chrysene, 2,3-benzofluorene and benzo(a)pyrene (Tran et al. 1996). However, these compounds were not expected to occur at all or at relevant concentrations in the analyzed samples. Whereas potential mixture toxicity effects remained to be investigated. A number of natural compounds have been discussed to imply anti-estrogenic potentials or to be capable of influencing the outcome of in vitro bioassay (e.g., Janosek et al. 2007, Neale et al. 2015). As substances such as humic or fulvic acids represent natural soil constituents, they seem (more) likely to occur at milligram per liter concentrations in groundwater. Follow up investigations thus seem essential to clarify on the nature of the antiestrogenic activities in aqueous and extracted samples. Unlike for groundwater, anti-estrogenic pharmaceuticals might explain the high anti-estrogenicity detected in hospital wastewater (sample $5 \mathrm{a}$ and $5 \mathrm{~b}$, figure 7 ). These compounds are regularly administered in oncology departments such as in the hospital referring to sample 5a. Patients undergoing hormone therapies may furthermore emit these pharmaceuticals into sewage collected from households. 
Hospital wastewater moreover exhibited high anti-androgenic activity. This activity may derive from anti-androgenic drugs used in cancer therapy. Regularly administered drugs in this regard are bicalutamide (e.g., Blackledge 1993), flutamide (e.g., Grover et al. 2011, Sohoni and Sumpter 1998, used as positive control in the YAAS) and its metabolite hydroxyflutamide (e.g., Kusk et al. 2011), nilutamid (e.g., Gaillard 1996), enzalutamid (e.g., Gordon et al. 2017) and 4hydroxytamoxifen (compare anti-estrogens above). Zwart et al. (2018) detected the drugs amitriptyline (anti-depressant) and celecoxib (anti-inflammatory drug) in surface water, which may also be administered in the analyzed hospital. Both compounds exhibited anti-androgenic properties. However, their individual concentrations might not reach EC in the YAAS. Other anthropogenic chemicals with experimented anti-androgenic potential are 1H-benzotriazole (Fent et al. 2014), the plasticizers bisphenol A (BPA, e.g., Li et al. 2008, Sohoni and Sumpter 1998) and benzyl butyl phthalate (BBP, Li et al. 2010, Sohoni and Sumpter 1998), the industrial chemicals nonylphenol and octylphenol (e.g., Li et al. 2010) as well as several pesticides, such as a degradation product of dichlordiphenyltrichlorethane (DDT, e.g., Laudet and Gronmeyer 2002, Sohoni and Sumpter 1998), rhexachlorcyclohexan, hexachlorobenzene ( $\mathrm{Li}$ et al. 2008), iprodione, linuron, metabolites of vinclozolin, methoxyclor and procymidone (Rempel and Schlenk 2008, Urbatzka et al. 2007). Henry and Fair (2013) investigated anti-androgenic activities for perfluorooctane sulfonate, perfluorooctanoic acid and triclosan. An EDA by Urbatzka et al. (2007) identified a number of these compounds in surface waters. The authors further concluded towards unknown anti-androgenic causative agents. Regarding the present samples non-pharmaceutical compounds such as identified by Muschket et al. (2018) and Urbatzka et al. (2007) or quantified in the course of this thesis (1H-benzotriazole, compare above) seem unlikely to occur in hospital wastewater or only at very low concentrations including their potential release from sources such as polycarbonate (BPA) or polyvinylchloride (BBP) plastic materials. It also remains to be investigated to which extend NOM constituents that were supposed to interfere with the human androgen receptor (Bittner et al. 2012) may occur in hospital wastewater (at relevant concentrations). However, due to elevated concentrations and known potencies of the mentioned anti-androgenic pharmaceutical they remained the main suspects. As all of the investigated hospitals connect to WWTPs precautionary measures seem advisable 
to verify their elimination during wastewater treatment as well as to prevent their excessive discharge into the environment, such as during heavy rain events. This may similarly pertain the genotoxic and mutagenic potentials detected in hospital wastewater.

The presented in vitro bioassay results support the scientific consensus on their usefulness in environmental monitoring and ecotoxicological assessments. Although extrapolations from in vitro to in vivo systems can be attached to uncertaincy (Henneberg et al. 2014, Huang et al. 2016, Prasse et al. 2015, Rehberger et al. 2018, Schneider et al. 2020), in vitro bioassays are nonewithstanding beneficial tools for the detection of hazard potentials and MoAbased classification of (micro)pollutants and (waste)water samples. Due to this they were furthermore integrated in a (waste)water quality evaluation concept developed in the course of the TransRisk project (2.4). In addition, several under-investigated endpoints / MoAs are presently implemented in in vitro bioassay based wastewater assessments (Daniels et al. 2019, Escher et al. 2014). These assays amongst others indicated the presence of mostly undetected EDCs (e.g., glucocorticoids or progestogens) calling for further research. 


\subsection{In vivo bioassays for the ecotoxicological assessment of wastewater - the case of Caenorhabditis elegans}

\subsubsection{Development and reproduction as human and environmentally relevant endpoints}

C. elegans is a well established DART model in human toxicology (Avila et al. 2011, Baker 2016, Harlow et al. 2016, 2018, Leung et al. 2008, Williams et al. 2017). This includes a high predictive power (Allard et al. 2013, Hunt 2016, Kim et al. 2020, Wittkowski et al. 2019). In this context, Racz et al. (2017) analyzed 31 human reproductive toxicants with 27 also showing DART in C. elegans. Harlow et al. (2016) reported on a high positive (89\%) and lower negative (25\%) predictivity regarding the estimated mammalian activity of 72 pesticides. The low negative predictivity could however be the result of recording only one endpoint (egg viability), whereas incorporating other endpoints may have likely identified further matches. Due to their relevance to humans as well as wild life DART endpoints are integral part of regulatory (and non-regulatory) toxicity assessments (Wernersson et al. 2015, Wilson and Khakouli-Duarte 2009, Wittkowski et al. 2019).

In this light, many DART inducing chemicals, including different aquatic micropollutants, were examined using $C$. elegans (compare below selection):

- The metals aluminium, barium, cadmium, chromium, lead, mercury, nickel, palladium and platinum all exhibited DART in C. elegans, as well as excessive concentrations of the essential metals cobalt, copper, iron, manganese, potassium and zinc or the metalloid arsenic (e.g., Avila et al. 2011, Schertzinger et al. 2017, Tejeda-Benitez et al. 2016a, 2016b, Wang et al. 2008a). Thereby $C$. elegans indicated similar sensitivity than D. magna and the redworm Eisenia fetida regarding several of the above cited metals (Queirós et al. 2019).

- Pesticides represent another relevant group investigated for DART involving various MoAs (Harlow et al. 2016, Tejeda-Benitez and Olivero-Verbel 2016a, table 2). Severe damage often occurred at low ECs such as for chlorpyrifos (at 3-100 $\mathrm{\mu g} / \mathrm{L}$ (Roh and Choi 2008, Ruan et al. 2012), fenoxycarb and spirotetramat (at 1.5 and $0.75 \mu \mathrm{g} / \mathrm{L}$ respectively, Xiong et al. 2017), epoxiconazole (LOEC of $0.1 \mu \mathrm{g} / \mathrm{L}$, Li et al. 2016) or lindane (at 1-100 ng/L, Yu et al. 2020). DART of other pesticides thereby included transgenerational effects 
(e.g., Lopes et al. 2008), interference with biochemical signaling pathways (e.g., Li et al. 2016) or cumulative effects (Martin et al. 2009).

- Several PPCPs were reported for DART in C. elegans such as EE2 (Höss and Weltje 2007), nicotine (e.g., Smith et al. 2013), piperazine analogs (Racz et al. 2017), the withdrawn anti-obesity drug sibutramine (Aitlhadj and Stürzenbaum 2013), tamoxifen (Höss and Weltje 2007), the anticancer drug 5-fluorouracil (ECs $\geq 0.6 \mathrm{mg} / \mathrm{L}$, Kumar et al. 2010, table 2), the banned weight loss agents clenbuterol and animal feed additive ractopamine (ECs $\geq 0.1-10 \mu \mathrm{g} / \mathrm{L}$ Zhuang et al. 2014), carbamazepine (ECs at [mg/L], Olga Kolychalow, personal communication), the antibiotic sulfamethoxazole (SMX, at environmentally relevant ECs, Yu et al. 2011, Liu et al. 2013, table 2) or the antimicrobial agents triclocarban and triclosan (LOECs of 10-100 $\mu \mathrm{g} / \mathrm{L}$ respectively, Lenz et al. 2017, table 2). The ECs of triclocarban and triclosan were thereby similar than to those of $D$. renio embryos (Sreevidya et al. 2018).

- Several environmental hormones and EDCs were positively assessed for DART in C. elegans. Albeit C. elegans is known not to incorporate an entire endocrine system, several hormonal pathways have been assumed (Höss and Weltje 2007). Related adverse effects were documented for nonylphenol (Cao et al. 2020a), di(2-ethylhexyl)phthalate (DEHP, Roh et al. 2007, Pradhan et al. 2018), its substitute diethyl phthalate (DEP, Pradhan et al. 2018), BPA (Zhou et al. 2016) and its substitute bisphenol S (BPS, Chen et al. 2016, Mersha et al. 2015). García-Espiñeira et al. (2018) showed that 11.4 and $114 \mu \mathrm{g}$ BPA / L increased body length or brood size. The authors suggested a non-linear-concentrationresponse-relationship such as established for EDCs in other species. Camacho et al. (2018) further examined an epigenetic mechanism that could have triggered reproductive dysfunctions inherited over five generations (table 2). Mersha et al. (2015) suggested a similar MoA for the structurally related BPS. In this context Chen et al. $(2016,2019)$ proposed DNA damage (BPS, Chen et al. 2016) and interference with mitochondrial cholesterol transport (BPA, Chen et al. 2019) as possible molecular initiating events (compare AOP concept, 2.3.3).

- Industrial chemicals included 2-(thiocyanomethylthio)-benzothiazol (Allard et al. 2013), different flame retardants (Behl et al. 2016, Liu et al. 2019), octachlorostyrene (Kim and Choung 2009), tributyltin (Cheng et al. 2014) and vinyl chloride (Nam and An 2010). DART was also rated more sensitive than 
lethality in assessing the toxicity of PAHs (Leung et al. 2010, Sese et al. 2009). Nonetheless, Ura et al. (2002) computed an 24 h LC 50 of $50 \mu \mathrm{g} / \mathrm{L}$ for B[a]P. Sese et al. (2009) reported a comparable $\mathrm{LC}_{50}$ of $80 \mu \mathrm{g} / \mathrm{L}$ (and an $\mathrm{EC}_{50}$ for reproduction of $59 \mu \mathrm{g} / \mathrm{L})$. The sensitivity of $C$. elegans towards $\mathrm{B}[\mathrm{a}] \mathrm{P}$ was similar to $D$. magna (Sese et al. 2009). Haegerbaeumer et al. (2018) detected an even lower LC 50 of $12.8 \mu \mathrm{g} / \mathrm{Lafter} 48 \mathrm{~h}$ exposure of $\mathrm{L} 1$ animals to $\mathrm{B}[\mathrm{a}] \mathrm{P}$. Toxicokinetic experiments with fluoranthene indicated a rapid bioaccumulation and food particles to significantly contribute to the DART of this PAH (Matthai 2009).

In the course of this thesis $\beta-N F$ was analyzed for DART in $C$. elegans (e.g., figure 10-12). A LOEC of $100 \mu \mathrm{g} / \mathrm{L}$ and an $\mathrm{EC}_{50}$ of $140 \mu \mathrm{g} / \mathrm{L}$ was determined for brood size reduction (96 h). These ECs were comparable to previous observations (ECs $\geq 273 \mu \mathrm{g} / \mathrm{L}$, Leung et al. 2010). Markedly, adult hermaphrodites exposed to high $\beta$-NF concentrations exhibited an increased bagging rate (figure 10). Mosser et al. (2011) and several of the cited authors described the Bag phenotype as good indicator of stress. Its feasibility as (eco)toxicological endpoint however remains to be clarified. Regarding the possible MoAs of $\beta-N F$ not much has been proposed, but it was experimented that $\beta-N F$ does not bind to the ortholog of the human AhR in C. elegans (Powell-Coffman et al. 1998). $\beta$-NF and PAHs may thus further be examined within ecotoxicological concepts discussed under 2.3.2-2.3.3.

In this thesis DART was further investigated for groundwater, surface water and wastewater from different WWTP using conventional biological and AWWT processes (Abbas et al. 2018, figure 11 and 17, table 3). The impacts of these samples on reproduction/growth indicated the presence of residual causative agents in WWTP effluents (discussed in further detail in Abbas et al. 2018). Thereby, DART endpoints proved to be beneficial in comparing the effectiveness of different WWTP processes (2.4). These estimations are required for reducing WWTP emissions (such as through WWTP upgrade or centralisation) and for reaching the goals of the WFD (European Parliament and Council 2008, 1.2.2). It also became apparent that chemical target analysis, including several compounds affecting the reproduction and growth of $C$. elegans, did not explain the detected effects (see 'Online Resource 3' in Abbas et al. 2018). Chemical analyses should thus be better adjusted to ecotoxicological effects and research questions (e.g., Maier et al. 2016, Neale et al. 2017, 2018). This adjustment should acknowledge (micro)pollutants 
known for their presence in wastewater as well as DART in C. elegans. Even if their MECs generally occur below their reported EC, cumulative effects may not be excluded in advance. In general, ecosystem complexity and matrix effects that can interfere with bioassay outcomes (e.g., Haitzer et al. 1999, Höss et al. 2001) should be better involved in the assessment and monitoring of (waste)water.
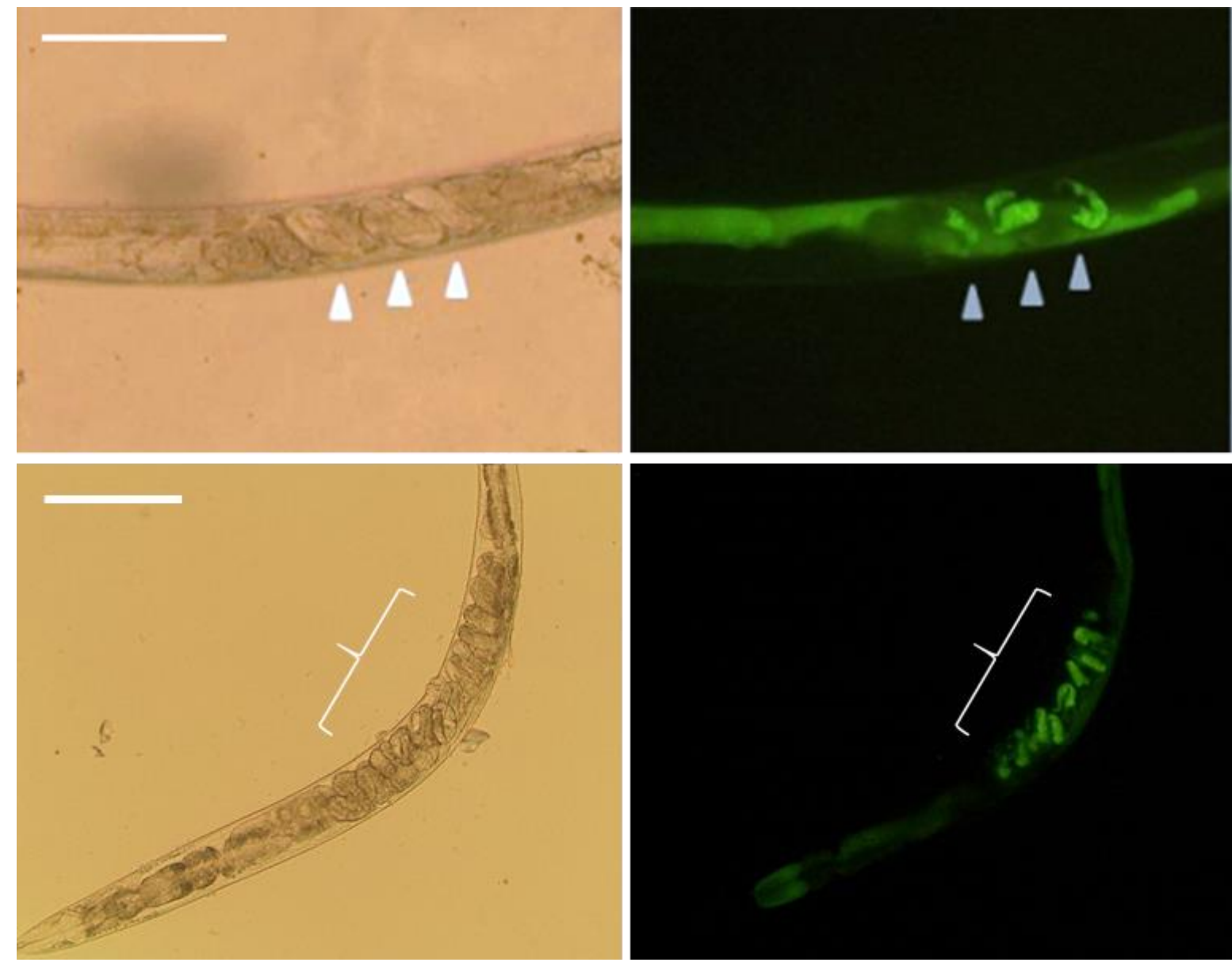

Figure 10. Expression of cyp-35A3::GFP in transgenic C. elegans of the Bag phenotype. Arrows/Brackets pointing at internally hatching larvae in adult hermaphrodites. Images (left: DIC and right: GFP) recorded after $48 \mathrm{~h}$ exposure to $\beta-N F(1 \mathrm{mg} / \mathrm{L})$. Size bar $=100 \mu \mathrm{m}$. Unpublished results jointly obtained by L. Valek and A. Abbas.

Hitchcock et al. $(1997,1998)$ investigated municipal and industrial wastewater using C. elegans. The authors concluded that discharges should undergo intensified in vivo monitoring as well as source control. Wang et al. $(2008,2010)$ performed two TIE on a paper recycling mill effluent whereby $C$. elegans was sensitive towards several TIE sample manipulations. McLaggan et al. (2012) used transgenic strains and DART endpoints to study different pollutants (such as PAHs, PCBs, EDCs and metals) extracted from sewage sludge. Wang et al. (2015) adapted the "transgenic 
approach" by combining molecular, apical, behavioral and community endpoints to investigate a natural wetland (focusing on nitrogen and phosphorous). In a study of Clavijo et al. (2016) the authors determined that physicochemical and bacteriological water parameters correlated to $<62 \%$ of the observed growth inhibitions (compare 'immunotoxicology' under 2.3.3). Kim et al. (2019c) detected DART in model crude oil samples apparently triggering the nucleotide excision repair pathway (table 2, 2.3.3). The authors suggested the PAH C3-naphthalene as major toxicity contributor and validated their results using $D$. renio (Kim et al. 2020). In addition, DART endpoints have been employed to assess the quality of river sediments (e.g., Harris et al. 2020, Höss et al. 2012, Menzel et al. 2009). For several sediment samples Duft (2004) observed a higher sensitivity and feasibility of $C$. elegans over Chironomus riparius. However, the author suggested that both assays may complement each other as well as comparisons to other sensitive species in respective bioassay batteries. Soil quality/toxicity has also been assesses using $C$. elegans DART endpoints (e.g., Graves et al. 2005, Höss et al. 2009, 2015, Höss and Römbke 2019, Peredney and Williams 2000, Roh et al. 2007, Wilson and Khakouli-Duarte 2009).

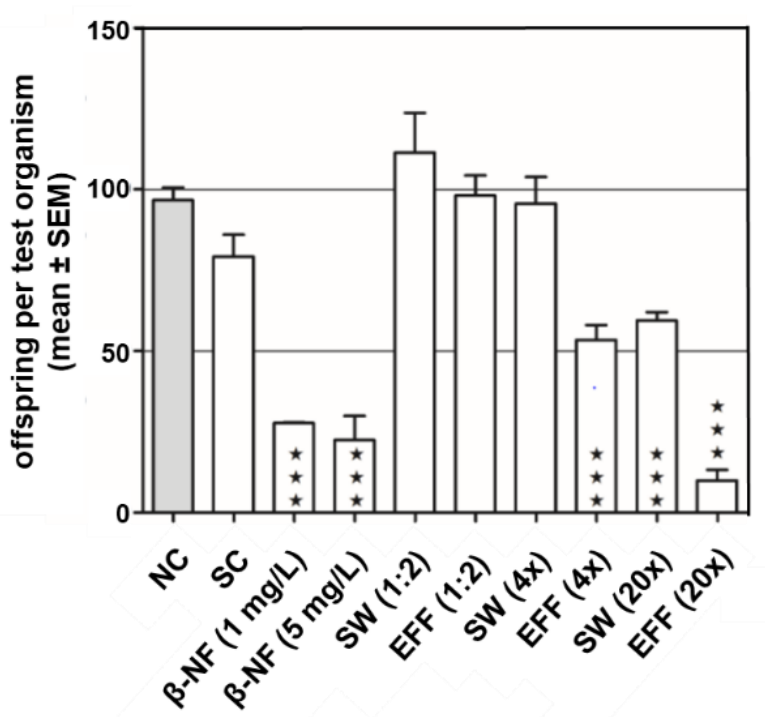

Figure 11. Reproductive toxicity of $\beta-N F(1-5 \mathrm{mg} / \mathrm{L})$, surface water (SW) and WWTP effluent (EFF) samples to $C$. elegans. Reproduction (brood size) expressed as mean offspring number per test organism \pm SEM. Aqueous (1:2) and extracted (4-20x) samples tested as $0.5,4$ and 20 fold concentrations respectively. Significant differences $\left({ }^{* * *} p<0.001\right)$ tested by 1 -way ANOVA against negative control (NC, S-media). Solvent control $(\mathrm{SC})=0.4 \%$ DMSO. $n=25-40$ per treatment group. Results jointly obtained by L. Valek and A. Abbas. 
Based on these results the development and reproduction of $C$. elegans provides sensitive and environmentally relevant endpoints for the assessment and monitoring of different environmental compartments. By implementing thorough experimental designs (such as involving a 'good C. elegans culture practice', Hunt 2016), and test strategies, such as combined with chemical analyses (e.g., Abbas et al. 2018, McLaggan et al. 2012, Wang et al. 2008b, 2015) as well as mutant and transgenic strains (compare 2.3.3), hypotheses such as on probable causative agents (Harris et al. 2020) can be tested. DART-related endpoints are furthermore important for cross-references to other species and for high throughput screens (HTS) that are witnessing a growing relevance in (eco)toxicology (Avila et al. 2011, Boyd et al. 2010, 2012, Brooks et al. 2020, Hunt 2016).

\subsubsection{CYP-35As as biomarker of (micro)pollutant exposure and contaminated environmental samples}

Biomarkers are important tools to indicate the physiological and metabolic state and its modification (Peakall 1994, Walker 1998). Protein expression is a frequent biomarker for intracellular exposures to xenobiotics (Wilson and Khakouli-Duarte 2009 , 1.4.3). Effect biomarkers detect (sub)cellular effects such as oxidative stress, DNA damage/repair, apoptosis and/or activation of detoxification (table 2, figure 15). A few biomarkers are intermediates between exposure and effect biomarkers. An example hereof are DNA damage associated genes that are expressed upon reversible and irreversible DNA manipulations. The biological relevance of changes in gene expression, however, mainly depends on the further manifestation of cellular effects and their propagation to higher levels of organization (Feder and Walser 2005, Peakall 1994, Walker 1998). Detoxification pathways in C. elegans (and other species) are frequently investigated through CYPs (Gotoh 1998, Harlow et al. 2018, Nelson et al. $1996,1.4 .3)$. Members of the $C$. elegans cyp-35A subfamily $(n=5)$ are highly inducible by various xenobiotics such as $\mathrm{PAH}, \mathrm{PCB}$ and dioxin-like pollutants (table 1-2, figure 12). Although biotransformation of organic chemicals by CYPs generally initiates their detoxification, they can also contribute to toxicity through bioactivation (e.g., Leung et al. 2010, Schäfer et al. 2009). Accordingly, knockdown/knockout of CYPs often reduced the toxicity of the respective pollutants and/or led to rescued phenotypes (Eom et al. 2014, Harlow et al. 2016, 2018, Jones et al. 2015, Min et al. 2015, Roh and Choi 2011, Schäfer et al. 2009). 


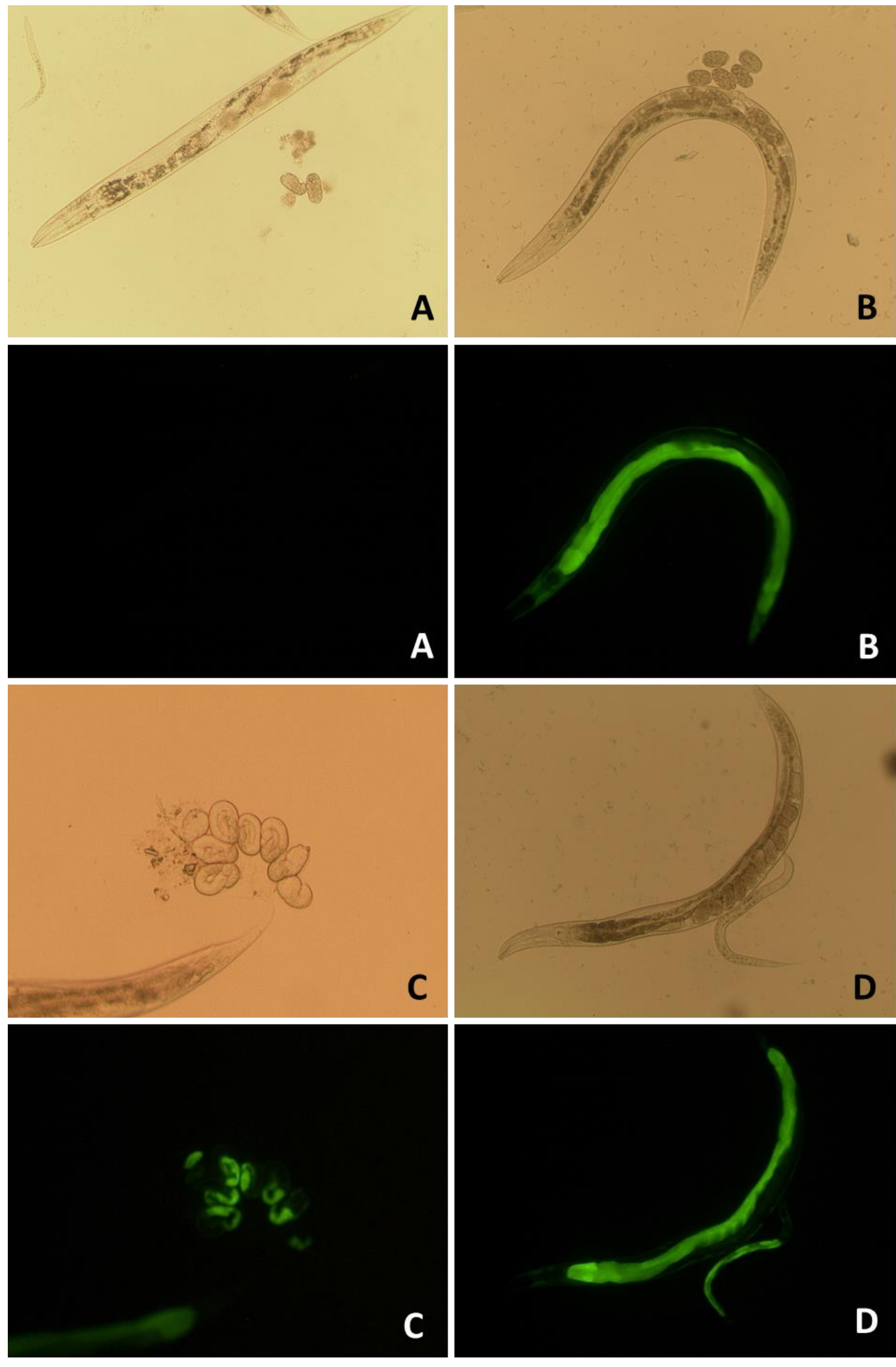

Figure 12. Expression of cyp-35A3::GFP in transgenic C. elegans. No biomarker induction was detected in unexposed control animals $(A)$ or freshly laid eggs after $24 \mathrm{~h}$ of exposure to $\beta-N F(B)$. A strong GFP signal was captured at the late embryonic (C), larval (D) and adult stage (B-D). Magnification adjusted to the respective developmental stage. DIC = panel one and three. GFP = panel two and four. Results obtained by L. Valek and A. Abbas. 
In this thesis cyp-35A2::GFP and cyp-35A3::GFP served as biomarkers of exposure to the reference PAH $\beta-N F$ (Menzel et al. 2001, 2007) as well as environmental samples possibly containing cyp-35A inducers. $\beta-N F$ induced both isoforms in a concentration-dependent manner at subacute exposures. For cyp-35A3::GFP, maximal expression levels were reached after $8 \mathrm{~h}$, whereas the earliest induction was recorded after $1 \mathrm{~h}$ of exposure to $5 \mathrm{mg} / \mathrm{L}$. An earlier detection was methodically unfeasible due to the time for translating and post-modifying the GFP (estimated to $1 \mathrm{~h}$ by David et al. 2003). PCR methods could be used to spare this time lag (Valek 2013) and to quantify the expression levels of multiple CYPs in parallel. However, these methods do not allow for microscopic (live) imaging such as performed with the cyp-35A3::GFP and other transgenic strains. By fluorescence microscopy a predominant expression location of cyp-35A3 was confirmed for the intestine (figure 12 and Abbas et al. 2018) similar to the observation by Menzel et al. (2007). The C. elegans intestine is its primary detoxification organ and also the main exposure route for various chemicals and ingested particles (Offermann et al. 2009, Wilson and Khakouli-Duarte 2009, Stylianou et al. 2018). Based on other studies overlapping substrates and substituting roles for cyp-35A subfamily members were suggested (Harlow et al. 2018, Inokuchi et al. 2014, Jones et al. 2015, Roh et al. 2014). This highlights the importance of detecting multiple CYP isoforms in ecotoxicogenomic studies. The induction of cyp-35A3::GFP was furthermore tracked throughout different developmental stages (figure 12). This result was in line with previous observations by Menzel et al. (2007). Interestingly, cyp-35A3::GFP induction was also found in internally hatching larvae of the Bag phenotype (figure 10). In the literature cyp-35A3 inducers were mostly tested at concentrations in the milligram per liter range (table 1). A few studies also analyzed concentration response relationships for CYP inducing compounds such as $\beta-N F$ (Menzel et al. 2002). Therein, Menzel et al. (2002) reported on a LOEC $0.1 \mu \mathrm{g} \beta-N F / L$ for cyp$35 A 3$ induction. In comparison, an $\mathrm{EC}_{50}(8-24 \mathrm{~h})$ of $72-79 \mu \mathrm{g} / \mathrm{L} \beta-\mathrm{NF}$ was determined in this thesis. CYP induction concentrations in the microgram per liter range were also detected for triclocarban (> $170 \mu \mathrm{g} / \mathrm{L}$, Inokuchi et al. 2014) and chlorpyrifos (60-300 $\mu \mathrm{g} / \mathrm{L}, 2-8 \mathrm{~h}$, Roh et al. 2016) speaking for the sensitivity of this biomarker. Similar to $\beta-N F$ (DART at $>100 \mu \mathrm{g} / \mathrm{L}$ ), the low induction concentrations of triclocarban conincided with high DART. In case of chlorpyrifos cyp-35A3 induction concentrations even came close to the $\mathrm{LC}_{50}(24 \mathrm{~h})$ of $300 \mu \mathrm{g} / \mathrm{L}$. In addition, 
prolonged exposure $(96 \mathrm{~h})$ at $10-100 \mu \mathrm{g} / \mathrm{L}$ was concomitant with severe developmental and reproductive defects (Roh and Choi 2008). Similar ECs of DART and cyp-35A3 induction might indicate its involvement in bioactivation (compare putative toxicodynamic and AOP concepts discussed in figure 15 and under 2.3.3).

Table 1. Selected C. elegans cyp-35A3 inducers in the scientific literature (reference given in brackets). Two out of four inducers reported by Harlow et al. (2016) are shown. 3Dmodels of compounds available at PubChem (Kim et al. 2016) and other databases

\section{Selected cyp-35A3 inducers}<smiles>O=c1cc(-c2ccccc2)oc2ccc3ccccc3c12</smiles>

$\beta-N F(P A H)$ (Menzel et al. 2001, 2007)<smiles>COc1cc(NC(C)CCCN)c2ncccc2c1</smiles>

(R)-primaquine (Menzel et al. 2001)

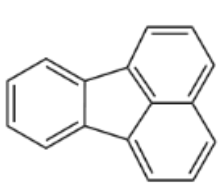

fluoranthene (PAH)

(Menzel et al. 2001)<smiles>Clc1ccc(-c2cc(Cl)c(Cl)cc2Cl)cc1Cl</smiles>

PCB52

(Menzel et al. 2001)<smiles>c1ccccc1</smiles>

benzene

(Eom et al. 2014)

lansoprazole (Menzel et al. 2001)<smiles>CCOP(=S)(OCC)Oc1nc(Cl)c(Cl)cc1Cl</smiles>

chlorpyrifos

(Roh et al. 2014, 2016)<smiles>CCOP(=S)(OCC)Oc1cc(C)nc(C(C)C)n1</smiles>

diazinon (Vinuela et al. 2010)<smiles>c1ccc2[nH]c(-c3cscn3)nc2c1</smiles>

thiabendazole (Jones et al. 2013, 2015)<smiles>O=[N+]([O-])NC1=NCCN1Cc1ccc(Cl)nc1</smiles>

imidacloprid (Jones et al. 2013)

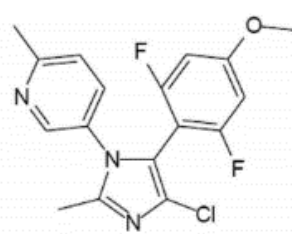

imidazole fungicide (Harlow et al. 2016)<smiles>Oc1cc(Cl)ccc1Oc1ccc(Cl)cc1Cl</smiles>

triclosan

(Inokuchi et al. 2014)<smiles>O=C(Nc1ccc(Cl)cc1)Nc1ccc(Cl)c(Cl)c1</smiles>

triclocarban (Inokuchi et al. 2014)<smiles>COc1ccc(-c2c(O)nnc(C(F)F)c2-c2ccc(Cl)nc2)c(F)c1</smiles>

pyridazine fungicide (Harlow et al. 2016)<smiles>Cn1c(=O)c2c(ncn2C)n(C)c1=O</smiles>

caffeine

(Min et al. 2015) 
Biomarkers of exposure represent relevant tools for toxicokinetic analyses (Offermann et al. 2009, Roh et al. 2016, Stylianou et al. 2018, table 2, figure 13). Toxicokinetic information such as determining bioavailable concentrations of (micro)pollutants are important such as for defining toxicity thresholds in ERA. Bioavailable concentrations also depend on the degradation and detoxification rates of the respective compounds (e.g., Roh et al. 2016, Spann et al. 2015). In this thesis it was hypothesized that decreasing internal concentrations of $\beta$-NF may coincide with a down-regulation of cyp-35A3::GFP. The down-regulation of cyp-35A3::GFP apparently depends on various exogenous factors (e.g., nominal $\beta-N F$ concentrations and exposure duration/intervals) and internal conditions (e.g., exposure routes or bioaccumulation behavior). Although in the conducted experiments internal concentrations were not directly measured (e.g., compare Chen et al. 2016 for BPA/BPS), it was found that cyp-35A3::GFP levels gradually decreased over $72 \mathrm{~h}$ after $\beta$-NF exposure at $1 \mathrm{mg} / \mathrm{L}$ for $24 \mathrm{~h}$ (figure 13). In perspective, such experiments will have to be complemented by examining the above mentioned exogenous and endogenous factors. The potential effect of these parameters on downstream effects such as DART are thereby of particular interest. Moreover, the degradation kinetics of GFP itself may have to be involved such as by comparing reports on the half-life of the fluorescence protein (e.g., $26 \mathrm{~h}$, Corish and Tyler-Smith 1999) with the present conditions.

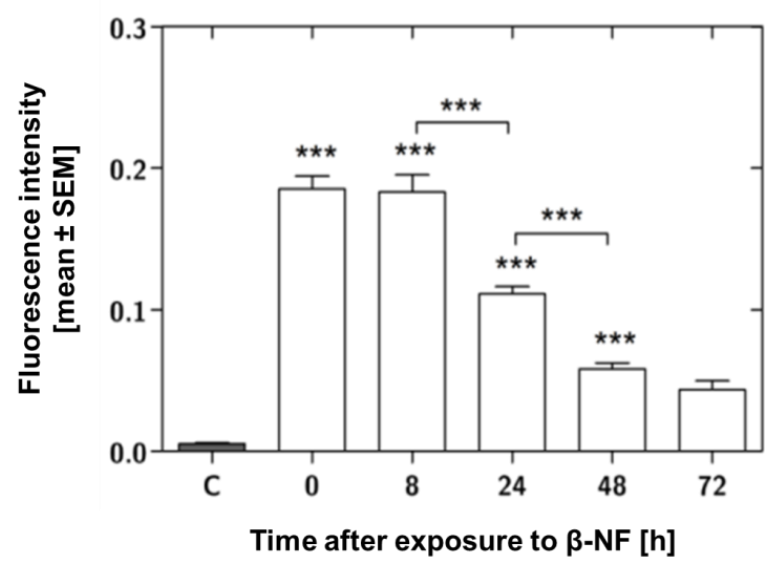

Figure 13. Down-regulation (0-72 h) of cyp-35A3::GFP in transgenic C. elegans after $24 \mathrm{~h}$ exposure to $1 \mathrm{mg} \beta-N F / L$. Negative control $(C)=$ unexposed animals. Significant differences tested against C (if not noted elsewise) as described by Valek (2013)

Biomarkers are furthermore used for assessing environmental samples (table 2). Ecotoxicogenomic studies involving cyp-35As were for instance performed on 
contaminated surface water (Kumar et al. 2015), river sediments (Menzel et al. 2009) and soils (Anbalagan et al. 2012, 2013, Roh et al. 2007). Thereby, different (clusters of) up- or downregulated genes correlated to pollution levels and/or DART. In this thesis, one biologically treated WWTP effluent and corresponding ozonated wastewater (analysed in this thesis) significantly induced cyp-35A3::GFP (table 34 and Abbas et al. 2018). Because the ozonated WWTP effluent induced cyp35A3::GFP to higher extend a possible explanation was given in the context of oxidative TPs (such as corroborated for other aquatic species, da Costa et al. 2014, Stalter et al. 2010, Magdeburg et al. 2012, Giebner et al. 2018). The presumed TPs might thereby either resemble newly generated and/or previously contained cyp$35 A 3$ inducers with higher bioavailability/potency. The causative agents for this effect could not be evaluated, because, except for caffeine, none of the cyp-35A3 inducers cited in table 1 was included in the chemical target monitoring of this thesis. In addition, the MEC of caffeine (see 'Online Resource 3' in Abbas et al. 2018) ranged far below its reported induction concentration (Min et al. 2015). This was also found regarding the concentrations of other wastewater-borne cyp-35A3 inducers in table 1 (e.g., Forsgren 2015). The MECs of chlorpyrifos and triclocarban (< $0.52 \mu \mathrm{g} / \mathrm{L}$, Norberg-King 2001 and $0.17 \mu \mathrm{g} / \mathrm{L}$, Heidler et al. 2006, respectively) were approximately 100 fold lower than their ECs (60-300 $\mu \mathrm{g} / \mathrm{L}$, Roh et al. 2016 and $>170 \mu \mathrm{g} / \mathrm{L}$, Inokuchi et al. 2014, respectively). Therefore, hypotheses on potential cumulative effects of unknown and/or low concentrated cyp-35A3 inducers as well as particle bound (micro)pollutants (such as based on their log Kow values) were discussed (Abbas et al. 2018). These findings further highlighted the need to carefully and case-specifically amend chemical (target) and ecotoxicological analyses (e.g., Chakrabarti et al. 2015, Neale et al. 2018, Tang et al. 2014).

For further establishing CYPs as biomarkers the identification of their inducers and substrate spectra should go in hand with research on their intra- and interspecies relationships. Such information can for instance be obtained from gene ontology studies, by quantitative structure activity relationships (QSAR, e.g., Escher and Hermens 2002, Ristau et al. 2015) and other (experimental) approaches (e.g., Harlow et al. 2018). Due to the evolutionary conservation of CYPs many of their biological functions and toxicological responses (highly) correlate between species. This conservation is apparent at the genetic level. According to a BLAST research 
cyp-35A3 (ceCYP-35A3) shares 100\% DNA sequence homology with CYPs orthologs in three other nematodes (Caenorhabditis brenneri, Caenorhabditis briggsae and Caenorhabditis remanei), but also with one CYP in the flower Camellia japonica (Ensembl 2018, Wormbase 2018). The human ortholog cyp-2C8 (hCYP$2 \mathrm{C} 8$ ) displays $93.7 \%$ sequence homology with ceCYP-35A3, which is higher than for the Drosophila melanogaster ortholog (cyp18a1-PB, 90.7\%). Functional homologies were further ascribed to human CYP-2J2, -2R1 and -2U1 using the 'Protein Analysis Through Evolutionary Relationships Classification System' (Mi et al. 2013). However, the human CYP1 class and CYP1-like metabolism is apparently missing in C. elegans. Nonetheless, C. elegans shares a variety of CYP-based metabolites with humans (Harlow et al. 2018). Similar to other proteins, structures of CYPs have been modelled and reconstructed from crystal structures (Totah and Rettie 2005). From this data in silico methods can generate 3D-models (e.g., figure 14) which are used to compute binding affinities of experimented and probable substrates (Marcus Weber, personal communication). No model of ceCYP-35A3 seemed presently available in the literature.

A

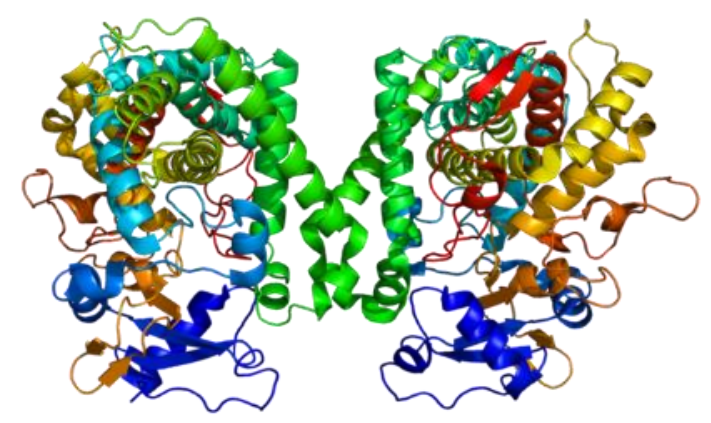

B

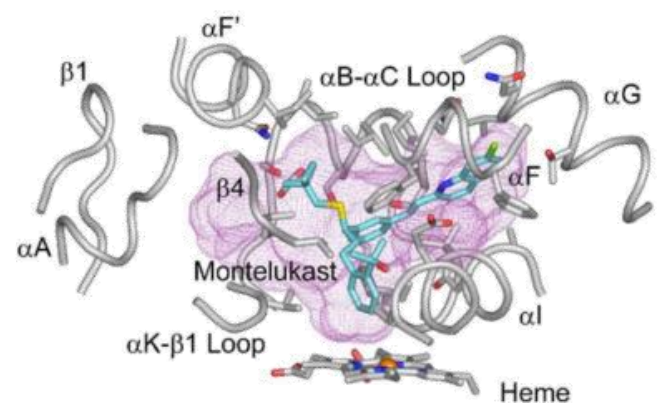

Figure 14. In silico models of hCYP-2C8 (human ceCYP-35A3 ortholog). Ribbon diagram (A) of its asymmetric unit wherein colors represent different domains (Berman et al. 2000). Stereo-image (B) of the active site (Johnson and Stout 2005) including exemplary substrate (center) and heme cofactor (bottom).

Substrate spectra of CYPs can include structurally diverse compounds (e.g., Johnson and Stout 2005, table 1). Markedly, for cyp-35A2 Anbalagan et al. (2012) even reported on an apparent induction by heavy metals. It however remained to be clarified, if this induction was associated with real catalytic, (co-)regulative or unspecific toxicity. Metabolic activities of hCYP-2C8 (ceCYP-35A3 ortholog) have widely been investigated for various drugs and toxicants (e.g., Backman et al. 2016, 
Johnson and Stout 2005, Totah and Rettie 2005). hCYP-2C8 is highly expressed in the liver also representing a main detoxification organ (Backman et al. 2016). Different natural compounds are known hCYP-2C8 substrates/inducers such as certain terpenes, the natural flavone eupatilin (structurally related to $\beta-N F$ ) or ethanol. They also comprise caffeine and lansoprazole (Backman et al. 2016), which have been reported as ceCYP-35A3 inducers (table 1), as well as chlorpyrifos (Abass et al. 2009). No literature was found on $\beta-N F$, fluoranthene or PCB52 as hCYP-2C8 effectors. From the ethoxyresorufin-O-deethylase (EROD) assay it is however known that $\beta-N F$, several other $\mathrm{PAH}$ and dioxin-like toxicants induce hCYP-1A1 in fish (e.g., Kais et al. 2018, Maier et al. 2016, Mohammadi-Bardbori 2014, table 4). $\beta-N F$ is described to be targeted by hCYP-1A2 (Chakrabarti et al. 2008) which also metabolizes caffeine (Omiecinski et al. 1999). The fact that homologous and non-homologous CYPs (such as hCYP-1A1 and ceCYP-35A3) can have overlapping substrate spectra further illustrates their evolutionary relationships and resulting complex metabolic interplay. Future research will further elaborate the usefulness of CYPs as human and environmental biomarkers and their integration into state of the art ecotoxicogenomic investigations (Chakrapani et al. 2008, Fajardo et al. 2020, Harlow et al. 2016, Inokuchi et al. 2014, Min et al. 2015, 2.3.3).

\subsubsection{Functional ecotoxicogenomics, mechanistic ecotoxicology and novel endpoints for (micro)pollutant and (waste)water quality assessment}

A growing number of mechanistic studies are carried out with $C$. elegans deciphering (eco)toxicological responses to anthropogenic and natural chemicals as well as contaminated environmental samples. The complex interplay of these responses are often studied by omic approaches (referring to the genome, proteome and metabolome) to detect and describe the involved physiological processes and toxicological pathways. Genome wide expression screens have been established in ecotoxicogenomics to profile the entirety of up- or down-regulated genes (compare 2.3.2). In general, these screens base on the assumption that chemicals with shared MoA (repeatedly) induce related expression patterns that can be contrasted with homeostatic conditions and other types of environmental stressors. A main aim is to transfer this information to predictive ecotoxicology (e.g., Brooks et al. 2020, Steinberg et al. 2008, Wilson and Khakouli-Duarte 2009). Furthermore, genomic 
and biomarker assessments may provide higher sensitivity and pace than apical and life cycle parameters in reflecting pollutant induced changes. This facilitates more detailed comparisons of acute versus chronic exposures and resulting effects (e.g., Alda Álvarez et al. 2006, Cui et al. 2007, Fajardo et al. 2020, Ruan et al. 2009). Global gene expression patterns upon exposures to wastewater-borne (micro)pollutants were studied in the context of environmental hormones (17ßestradiol and progesterone, Custodia et al. 2001), the PAHs $\beta$-NF and fluoranthene, the herbicide atrazine, the pharmaceutical clofibrate, the EDCs diethylstilbestrol (Reichert and Menzel 2005) and DEHP (Roh et al. 2007), PCB52 (Menzel et al. 2007), the industrial chemical octachlorostyrene (Kim and Choung 2009), the synthetic polycyclic musks 7-acetyl-1,1,3,4,4,6-hexamethyl-1,2,3,4-tetrahydro naphthalene (AHTN) and 1,3,4,6,7,8-hexahydro-4,6,6,7,8,8-hexamethylcyclopentaY-2-benzopyran (HHCB, Mori et al. 2007), heavy metals ( $\mathrm{Cd}, \mathrm{Hg}$ and $\mathrm{Pb}$ with detection thresholds as low as at 48-82 ng/L, Tominaga et al. 2008, Cui et al. 2007), the organophosphate pesticide dichlorvos (Lewis et al. 2013), benzene (Eom et al. 2014, table 2) and caffeine (Min et al. 2015, table 2). Many of these genome wide screens suggested an involvement of CYPs (including the cyp-35A subfamily) in the respective toxicological/metabolic responses/pathways (Custodia et al. 2001, Roh et al. 2007, Schäfer et al. 2009). Kim and Choung (2009) detected transgenerational alterations of gene expression after exposure of the parental generation. Lewis et al. (2009) moreover observed a relatively rapid recovery of overall gene expression $(<26 \mathrm{~h})$ after subacute exposures $(2$ or $8 \mathrm{~h})$ to dichlorvos. When Reichert and Menzel (2005) tested several wastewater-borne pollutants (in the milligram per liter range) the number of induced ( $n \leq 203$ at 2-42 fold higher than the control) or repressed ( $n \leq 153$ at $2-7$ fold lower than the control) genes followed the order fluoranthene $>$ clofibrate $>$ atrazine $>\beta-N F>$ diethylstilbestrol or atrazine $>$ fluoranthene $>$ diethylstilbestrol $>\beta-N F>$ clofibrate respectively. $\beta-N F$ induced 40 genes in total including carboxylesterases, collagen genes, CYPs (with $\beta-N F$ as strongest inducer), cytochrome b5, GST-20, UGTs, 11 genes belonging to different metabolic pathways (such as the metallothionein $m t /-1$ ) and 15 unknown genes. Two $\beta$-NF down-regulated genes were detected including C-type lectin (related to immune defense). Overall, these and earlier experiments (Menzel et al. 2001) supported the described role of these genes in the xenobiotic defense of $C$. elegans. 
Natural compounds have also been examined. Humic substances were for instance investigated using a surface water reverse osmosis isolate (NOM concentration of $6.4 \mathrm{mg} / \mathrm{L}$ ) and the synthetic humic-like substance HS1500 (at $7.6 \mathrm{mg} / \mathrm{L}$ ) wherein CYPs and various other genes were found to be differentially expressed. Albeit potential MoAs of humic substances remained unclear, screening natural compounds is particularly important for investigating environmental samples. Regarding the latter, Menzel et al. (2009) examined surface water sediments that exhibited different contamination degrees (Elbe $>$ Rhine $>$ Danube) with heavy metals, PAHs, PCBs and other POPs (such as octachlorostyrene) resembling the main representatives. All samples induced genes involved in certain metabolic processes, while a few samples also showed DART (using $25-50 \%$ pore water), in vitro genotoxicity and estrogenicity, that in many cases correlated to the respective gene expression profiles. PAH contaminated soils (after remediation) were also screened in a study by Fajardo et al. (2020), who also found growth, reproduction and survival to be significantly impacted.

In general, several methodical aspects have to be considered when performing and evaluating genomic screens (that apply to the entire field of ecotoxicogenomics):

- Robust design (matched with previous data and experimental standards).

- Data normalization (respecting baseline expression and methodical LOQs).

- Inclusion of multivariate statistics (as foundation for gene ontology approaches).

- Functional validation of results (by follow up experiments on downstream effects ruling out unspecific responses / false positives, compare below).

Furthermore, a number of biological/ecotoxicological challenges lie ahead in further establishing genomic screens as robust assessment tools and reliable prediction instruments for (defined and complex/dynamic) environmental stressors such as:

- Detailed comparisons of regular and irregular expression patterns (based on standardized maps/databases such as Davis et al. 2017, EBI 2018, Howe et al. 2017 and Kim et al. 2001). Respective data sets should enable the differentiation between responses such as specific versus unspecific or natural versus (micro)pollutant induced. Accordingly, they may comprise suitable PCs and/or interrogation of relevant ontogenetic/population and environmental factors that could cause variation in expression patterns/levels (e.g., Steinberg et al. 2008, Escher et al. 2017, Roh et al. 2016). 
- Comprehensive involvement of multiple regulation mechanisms on the DNA, transcriptional, RNA, translational or protein level (such as epigenetics or miRNAs, e.g., Camacho et al. 2018, Lundby et al. 2016, Weinhouse et al. 2018, Zhao et al. 2016) that could be impeded by xenobiotic exposure (Reinke et al. 2013, Steinberg et al. 2008, Taki et al. 2014), while escaping detection in regular genomic screens.

- Cross references to aquatic organisms such as Daphnia or fish species (e.g., Colbourne et al. 2015, Harris et al. 2020, Kim et al. 2019c, 2020, Steinberg et al. 2008).

Various "non-genome wide" screens have been carried out focusing on individual or multiple genes in C. elegans, while often applying DNA/RNA sequencing and/or the respective mutant/transgenic strains (e.g., Cong et al. 2020, Mendelski et al. 2019). Due to their reduced methodical effort compared to whole genome assays, these studies generally incorporate an extended set of investigated endpoints (compare table 2 below). The systematic selection of potentially affected endpoints is crucial for ruling out (false) negative results and for examining "downstream" (adverse) effects at higher organizational levels (Harlow et al. 2016). Up to present, mechanistic investigations with $C$. elegans illustrated how biomarker and genomic screens (molecular endpoints) combined with other endpoints (such as biochemical, metabolic, neurological, behavioral or physiological) can be powerful tools to characterize potential MoAs of (micro)pollutants as well as environmental samples. 
Table 2. Selected (non-genome wide) studies on effects and apparent MoAs of environmental chemicals/samples in C. elegans. Studies using multiple endpoints and biomarkers were listed chronologically (latest on top). Certain chemicals were cited by their abbreviation (as given in text) or summarized by pollutant classes (e.g., pesticides). Arrows (“ $\uparrow$ " and “ $\downarrow$ ”) indicate significantly up-/down-regulated genes or stimulation/inhibition of the respective endpoint, while delta symbol (" $\Delta$ ") indicates a relevant/significant alteration. Environmental relevance was estimated regarding selected compartments such as soil, groundwater (GW), surface water (SW), sediments, wastewater including industrial/hospital wastewater (WW), marine waters (MW) or drinking water (DW) as well as specific fields of ecotoxicology (excepting human toxicology).

\begin{tabular}{|c|c|c|c|c|c|}
\hline $\begin{array}{l}\text { Compound / } \\
\text { sample type }\end{array}$ & Investigated endpoints & Toxicity mechanism / MoA & Specific remarks & $\begin{array}{l}\text { Aquatic } \\
\text { relevance }\end{array}$ & $\begin{array}{c}\text { Referen } \\
\text { ce }\end{array}$ \\
\hline \multicolumn{6}{|c|}{ Present study: } \\
\hline$\beta-N F$ & $\begin{array}{l}\text { Apical (brood size } \downarrow \text { ) and molecular } \\
(\text { cyp-35A2 } \uparrow, \text { cyp-35A3 } \uparrow)\end{array}$ & $\begin{array}{l}\text { Involvement of CYPs in the } \\
\text { reproductive toxicity of } \beta-N F\end{array}$ & $\begin{array}{l}\text { Particulates assumed as } \\
\text { main exposure route }\end{array}$ & $\begin{array}{l}\text { WW, TSS, } \\
\text { sediments }\end{array}$ & \multirow{4}{*}{$\begin{array}{l}\text { Abbas et } \\
\text { al. } 2018 \\
\text { and } 2.3\end{array}$} \\
\hline EFF-1 & Apical (brood size $\downarrow$ ) & \multirow{3}{*}{$\begin{array}{l}\text { Effects unexplained by chemical } \\
\text { target analysis. Putative cumulative } \\
\text { effects and potential role of TSS. }\end{array}$} & \multirow{3}{*}{$\begin{array}{l}\text { Chemical target } \\
\text { analyses }(n=111)\end{array}$} & $\begin{array}{l}\text { WW, SW, } \\
\text { TSS }\end{array}$ & \\
\hline EFF-4 & Molecular (cyp-35A3 $\uparrow)$ & & & $\begin{array}{l}\text { WW, SW, } \\
\text { TSS }\end{array}$ & \\
\hline $\mathrm{EFF}+\mathrm{O}_{3}$ & $\begin{array}{l}\text { Apical (growth } \uparrow, \text { insignificant) and } \\
\text { molecular }(c y p-35 A 3 \uparrow)\end{array}$ & & & $\begin{array}{l}\text { Oxidative } \\
\text { TPs, AWWT }\end{array}$ & \\
\hline \multicolumn{6}{|c|}{ (Micro)pollutant studies: } \\
\hline $\begin{array}{l}\text { Chlorpyrifos } \\
\text { (compare below) } \\
\text { and its TPs }\end{array}$ & $\begin{array}{l}\text { Apical (DART } \uparrow) \text {, behavioral } \\
\text { (locomotion } \Delta \text { ), cellular (neuronal } \\
\text { integrity } \downarrow \text { ), biochemical (ROS } \uparrow) \text {, } \\
\text { molecular }(\text { tph-1::GFP } \uparrow \text { ) }\end{array}$ & $\begin{array}{l}\text { Chlorpyrifos }(20-50 \mu \mathrm{g} / \mathrm{L}) \text { and } \\
\text { generated TPs }(0-10 \mathrm{~h}) \text { triggering } \\
\text { multiple (neuro-)toxicity pathways }\end{array}$ & $\begin{array}{l}\text { Higher toxicity of } \\
\text { proposed photolytic TPs } \\
\text { than non-irradiated } \\
\text { standard solution }\end{array}$ & $\begin{array}{l}\text { SW, WW, } \\
\text { TPS }\end{array}$ & $\begin{array}{l}\text { Cao et al. } \\
(2020 b)\end{array}$ \\
\hline Lindane & $\begin{array}{l}\text { Apical (DART } \uparrow) \text {, behavioral } \\
\text { (locomotion } \Delta \text { ), intestinal membrane } \\
\text { permeability } \uparrow \text {, biochemical (ROS } \uparrow) \text {, } \\
\text { molecular ( sod- } 5 \uparrow, \text { isp- } 1 \uparrow, \text { mtm- } 6 \\
\downarrow \text {, opt- } 2 \downarrow \text { ) }\end{array}$ & $\begin{array}{l}\text { Significant correlation of oxidative } \\
\text { stress response and intestinal } \\
\text { membrane permeability with apical } \\
\text { effects }\end{array}$ & LOECs $\geq 1 \mathrm{ng} / \mathrm{L}$ & $\begin{array}{l}\text { Soil, SW, } \\
\text { GW }\end{array}$ & $\begin{array}{l}\text { Yu et al. } \\
(2020)\end{array}$ \\
\hline
\end{tabular}


Table 2 (continued)

\begin{tabular}{|c|c|c|c|c|c|}
\hline \multirow[t]{2}{*}{$\begin{array}{l}\text { Tetrabromo- } \\
\text { BPA }\end{array}$} & \multirow{2}{*}{$\begin{array}{l}\text { Apical (DART } \uparrow) \text {, behavioral } \\
\text { (locomotion } \Delta \text { ), neurotoxicity } \\
\text { (neuronal damage), biochemical } \\
\text { (ROS), molecular ( } 20 \text { genes incl. } \\
\text { cyp-35A2) }\end{array}$} & $\begin{array}{l}\text { Trans-generational neurotoxicity, } \\
\text { whereby gene expression was more } \\
\text { altered at low test concentrations }\end{array}$ & $\begin{array}{l}\text { Locomotion and gene } \\
\text { expression affected in } \\
\mathrm{G}_{1} \text { and } \mathrm{G}_{2}(1-100 \mu \mathrm{g} / \mathrm{L})\end{array}$ & \multirow[t]{2}{*}{ WW, soil } & $\begin{array}{l}\text { Liu et al. } \\
(2020)\end{array}$ \\
\hline & & $\begin{array}{l}\text { Pulsed exposure at environmentally } \\
\text { relevant concentrations triggered } \\
\text { higher toxicity as well as adaption }\end{array}$ & LOECs at $1-10 \mu \mathrm{g} / \mathrm{L}$ & & $\begin{array}{l}\text { Liu et al. } \\
(2019)\end{array}$ \\
\hline $\begin{array}{l}\text { BPA (compare } \\
\text { below) }\end{array}$ & $\begin{array}{l}\text { Apical (DART } \uparrow \text { ), cellular (germline } \\
\text { nuclear loss } \uparrow \text {, apoptosis } \uparrow \text { ), } \\
\text { biochemical (intracellular } \downarrow \text { and } \\
\text { mitochondrial } \downarrow \text { cholesterol level) }\end{array}$ & $\begin{array}{l}\text { Apparent interference of BPA (at } \\
[\mu \mathrm{\mu} / \mathrm{L}]) \text { with mitochondrial cholesterol } \\
\text { transport as mediated by } \\
\text { steroidogenic acute regulatory } \\
\text { protein }\end{array}$ & $\begin{array}{l}\text { Rescue phenotypes by } \\
\text { mutational analysis and } \\
\text { potential endocrine } \\
\text { effects of cholesterol }\end{array}$ & WW, SW & $\begin{array}{l}\text { Chen et } \\
\text { al. }(2019)\end{array}$ \\
\hline $\begin{array}{l}\text { Methampheta- } \\
\text { mine, ketamine }\end{array}$ & $\begin{array}{l}\text { Apical (DART } \uparrow) \text {, behavioral } \Delta \text {, } \\
\text { biochemical (neurotransmitter } \Delta \text { ), } \\
\text { molecular (oxidative stress related } \\
\text { genes } \uparrow \text { ) }\end{array}$ & $\begin{array}{l}\text { Behavioral impairment ( } \geq 50 \mathrm{ng} / \mathrm{L}) \\
\text { reflected in altered neurotransmitter } \\
\text { content and probable association of } \\
\text { oxidative stress response with DART }\end{array}$ & $\begin{array}{l}\text { ECs at MECs incl. risk } \\
\text { estimation }\end{array}$ & WW, SW & $\begin{array}{l}\text { Wang et } \\
\text { al. (2019) }\end{array}$ \\
\hline $\begin{array}{l}\text { Hexabromo- } \\
\text { cyclododecane } \\
\text { (flame retardant) }\end{array}$ & $\begin{array}{l}\text { Apical (DART } \uparrow) \text {, behavioral } \Delta \text {, } \\
\text { biochemical }(\text { ROS } \uparrow) \text {, cellular } \\
\text { (apoptosis } \uparrow) \text {, molecular ( } 16 \text { genes } \Delta \\
\text { incl. cyp-35A2 } \uparrow \text { ) }\end{array}$ & $\begin{array}{l}\text { Apparent protective role of sod-3 and } \\
\text { cep-1 as well as N-acetyl-L-cysteine / } \\
\text { ascorbate against supposedly ROS } \\
\text { derived downstream effects }\end{array}$ & $\begin{array}{l}\text { Detection of altered } \\
\text { gene expression at a } \\
\text { LOEC of } 1.28 \mu \mathrm{g} / \mathrm{L}\end{array}$ & $\begin{array}{l}\text { WW, SW, } \\
\text { soil }\end{array}$ & $\begin{array}{l}\text { Wang et } \\
\text { al. } 2018\end{array}$ \\
\hline \multirow{2}{*}{$\begin{array}{l}\text { Triclosan, } \\
\text { triclocarban } \\
\text { (compare below } \\
\text { and Sreevidya et } \\
\text { al. 2018) }\end{array}$} & $\begin{array}{l}\text { Apical (DART } \uparrow) \text {, behavioral } \\
\text { (locomotion } \Delta \text { ), biochemical (ROS } \uparrow) \\
\text { and molecular (daf-16::GFP } \uparrow)\end{array}$ & $\begin{array}{l}\text { Metabolic shifts in carbohydrates, } \\
\text { amino acids (energy budget) and } \\
\text { tyrosine, serine, polyamines } \\
\text { (neurotransmitter / stress response) }\end{array}$ & $\begin{array}{l}\text { Several phenotypes } \\
\text { could be affected by } \\
\text { triclosan exposure }\end{array}$ & \multirow[t]{2}{*}{ WW, sW } & $\begin{array}{l}\text { Kim et al. } \\
(2019 b)\end{array}$ \\
\hline & $\begin{array}{l}\text { Apical (lethality } \uparrow, \text { DART } \uparrow, \\
\text { lifespan } \downarrow \text { ), biochemical (oxidative } \\
\text { stress) and molecular (daf-16, xol-1) }\end{array}$ & $\begin{array}{l}\text { daf-16::GFP nuclear relocalization } \\
\text { and } x o l-1:: \text { GFP confirming oxidative } \\
\text { stress response and DART } \\
\text { (respectively) }\end{array}$ & $\begin{array}{l}\text { Authors suggest further } \\
\text { research on endocrine } \\
\text { disrupting potential }\end{array}$ & & $\begin{array}{l}\text { Lenz et } \\
\text { al. }(2017)\end{array}$ \\
\hline
\end{tabular}


Table 2 (continued)

\begin{tabular}{|c|c|c|c|c|c|}
\hline Chlorpyrifos & $\begin{array}{l}\text { Molecular (cyp-35A2-3) and } \\
\text { biochemical (AChE } \downarrow \text { ). Study includes } \\
\text { summary on previously investigated } \\
\text { endpoints in the literature. }\end{array}$ & $\begin{array}{l}\text { Metabolisation by CYPs and AChE } \\
\text { inhibition by chlorpyrifos bio-TP. } \\
\text { Internal concentration and time } \\
\text { dependencies }\end{array}$ & $\begin{array}{l}\text { Involves toxicokinetic } \\
\text { parameters and } \\
\text { realistic/low exposure } \\
\text { concentrations }\end{array}$ & $\begin{array}{l}\text { Soil, SW, } \\
\text { GW }\end{array}$ & $\begin{array}{l}\text { Roh et al. } \\
(2016 \\
2014)\end{array}$ \\
\hline 72 pesticides & $\begin{array}{l}\text { Apical (egg viability/hatching } \downarrow \text { ) and } \\
\text { molecular (CYPs } \uparrow \text { ) }\end{array}$ & $\begin{array}{l}\text { DART in relation to humans. } \\
\text { Functional involvement of CYPs }\end{array}$ & $\begin{array}{l}\text { Comparison of } \\
\text { positive/negative } \\
\text { predictive power (incl. } \\
\text { RNAi) }\end{array}$ & $\begin{array}{l}\text { Soil, SW, } \\
\text { GW }\end{array}$ & $\begin{array}{l}\text { Harlow et } \\
\text { al. (2016), } \\
\text { also see } \\
\text { table } 1\end{array}$ \\
\hline BPA & $\begin{array}{l}\text { Apical (growth } \downarrow \text {, reproduction } \downarrow \text { ), } \\
\text { behavioral (locomotion } \Delta \text { ), , cellular } \\
\text { (apoptosis } \uparrow \text { ), biochemical (lipofuscin } \\
\text { accumulation, ROS production), } \\
\text { molecular (incl. cyp-35A2 } \uparrow \text { ) }\end{array}$ & $\begin{array}{l}\text { Cell apoptosis leading to DART and } \\
\text { neurobehavioral toxicities }\end{array}$ & $\begin{array}{l}\text { LOECs in the lower } \\
\text { microgram per liter } \\
\text { range }\end{array}$ & WW, SW & $\begin{array}{l}\text { Zhou et } \\
\text { al. (2016) }\end{array}$ \\
\hline Caffeine & $\begin{array}{l}\text { Apical (brood size } \downarrow \text {, embryonic } \\
\text { lethality } \uparrow \text {, larval arrests } \uparrow \text { ) and } \\
\text { molecular (global gene expression } \\
\uparrow \downarrow \text { ) }\end{array}$ & $\begin{array}{l}\text { Toxicological role of CYPs with focus } \\
\text { on cyp-35As (confirmed by RNAi) }\end{array}$ & $\begin{array}{l}\text { Readily degradable by } \\
\text { biological WWT }\end{array}$ & WW & $\begin{array}{l}\text { Min et al. } \\
(2015) \\
\text { also see } \\
\text { table } 1\end{array}$ \\
\hline Thiabendazole & $\begin{array}{l}\text { Apical (egg number } \downarrow \text { ) and molecular } \\
\text { (CYP activation by NHR-176) }\end{array}$ & $\begin{array}{l}\text { Potential involvement of bio-TPs in } \\
\text { reproductive toxicity }\end{array}$ & $\begin{array}{l}\text { Classification of } \\
\text { transcriptional } \\
\text { regulators }\end{array}$ & $\begin{array}{l}\text { Chemical } \\
\text { analysis } \\
\text { (involving } \\
\text { TPs) }\end{array}$ & $\begin{array}{l}\text { Jones et } \\
\text { al. }(2013, \\
2015) \\
\text { also see } \\
\text { table } 1\end{array}$ \\
\hline $\begin{array}{l}\text { NDMA, } \\
\text { dibromoacetic } \\
\text { acid }\end{array}$ & $\begin{array}{l}\text { Apical (life span } \uparrow, \text { reproduction, body } \\
\text { size } \uparrow, \text { thermal stress resistance), } \\
\text { biochemical (oxidative capacity, lipid } \\
\text { peroxidation) and molecular ( } 17 \\
\text { genes incl. cyp-35A2 } \downarrow \text { ) }\end{array}$ & $\begin{array}{l}\text { Protective stress response or energy } \\
\text { relocation }\end{array}$ & Low dose experiment & WW, SW & $\begin{array}{l}\text { Babersch } \\
\text { ke et al. } \\
(2014)\end{array}$ \\
\hline $\begin{array}{l}\text { Triclosan, } \\
\text { triclocarban } \\
\text { (compare above } \\
\text { and Sreevidya et } \\
\text { al. 2018) }\end{array}$ & $\begin{array}{l}\text { Apical (DART } \uparrow) \text { and molecular ( } 79 \\
\text { CYPs) }\end{array}$ & $\begin{array}{l}19 \text { (triclosan, } 5 \mathrm{mg} / \mathrm{L} \text { ) and } 10 \\
\text { (triclocarban, } 0.17 \mathrm{mg} / \mathrm{L} \text { ) up- } \\
\text { regulated CYPs potentially involved } \\
\text { in the observed DART }\end{array}$ & $\begin{array}{l}\text { Utilization of a DNA } \\
\text { microarray chip } \\
\text { customized for CYPs }\end{array}$ & WW, SW & $\begin{array}{l}\text { Inokuchi } \\
\text { et al. } \\
(2014)\end{array}$ \\
\hline
\end{tabular}


Table 2 (continued)

\begin{tabular}{|c|c|c|c|c|c|}
\hline Benzene & $\begin{array}{l}\text { Apical (survival, reproduction } \downarrow \text { ), } \\
\text { behavioral (locomotion } \Delta \text { ) and } \\
\text { molecular (global gene expression } \\
\uparrow \downarrow \text { incl. cyp-35As) }\end{array}$ & $\begin{array}{l}\text { Distinct roles of cyp-35A2 and cep-1 } \\
\text { in benzene-induced behavioral } \\
\text { responses and reproductive toxicity }\end{array}$ & $\begin{array}{l}\text { Computational } \\
\text { behavior analysis as } \\
\text { integrative evaluation } \\
\text { tool }\end{array}$ & WW, DW & $\begin{array}{l}\text { Eom et al. } \\
(2014), \\
\text { also see } \\
\text { table } 1\end{array}$ \\
\hline $\begin{array}{l}\text { Clenbuterol, } \\
\text { ractopamine }\end{array}$ & $\begin{array}{l}\text { Apical (DART } \uparrow) \text {, behavior } \\
\text { (locomotion } \Delta \text { ), biochemical } \\
\text { (autofluorescence } \uparrow, \text { ROS } \uparrow \text { ), } \\
\text { molecular (gene expression } \uparrow \downarrow \text { ) }\end{array}$ & $\begin{array}{l}\text { Insulin/IGF influenced life span } \\
\text { reduction. Decreased ROS and } \\
\text { DART by sod-2 overexpression }\end{array}$ & $\begin{array}{l}\text { Acute and prolonged } \\
\text { exposure. Low dose } \\
\text { effects (e.g., } 10 \mu \mathrm{g} / \mathrm{L})\end{array}$ & $\begin{array}{l}\text { WW } \\
\text { (depending on } \\
\text { concen-tration) }\end{array}$ & $\begin{array}{l}\text { Zhuang et } \\
\text { al. (2014) }\end{array}$ \\
\hline SMX & $\begin{array}{l}\text { Apical (reproduction } \downarrow \text {, growth } \uparrow \text {, life } \\
\text { span } \uparrow) \text {, behavioral (thermal } \\
\text { resistance, pharynxal pumping } \uparrow \text { ), } \\
\text { biochemical (lipid peroxidation } \uparrow) \text {, } \\
\text { molecular ( } 16 \text { genes } \uparrow \downarrow \text { ) }\end{array}$ & $\begin{array}{l}\text { Lipid peroxidation and up-regulated } \\
\text { hsp-16.1 indicated oxidative stress; } \\
\text { possibly associated with } \\
\text { mitohormesis affecting life span and } \\
\text { pumping frequency affecting growth }\end{array}$ & $\begin{array}{l}\text { Explanation on } \\
\text { bacterivorous } C \text {. } \\
\text { elegans as } r- \\
\text { strategists }\end{array}$ & WW, SW, Soil & $\begin{array}{l}\text { Liu et al. } \\
(2013)\end{array}$ \\
\hline Nicotine & $\begin{array}{l}\text { Behavior (nicotine preference } \uparrow \text {, } \\
\text { locomotion), biochemical (gustatory } \\
\text { plasticity } \downarrow \text { ), molecular (bas-1, cat-2, } \\
\text { lev- } 1 \text {, tph-1 unc-29) }\end{array}$ & $\begin{array}{l}\text { Suggested essential role for } \\
\text { serotonin signaling pathway }\end{array}$ & $\begin{array}{l}\text { Mutant analysis after } \\
\text { acute pre-exposure } \\
\text { and new locomotion } \\
\text { assay }\end{array}$ & $\begin{array}{l}\text { WW } \\
\text { (depending on } \\
\text { concentration) }\end{array}$ & $\begin{array}{l}\text { Matsuura } \\
\text { et al. } \\
(2013)\end{array}$ \\
\hline Fenitrothion & $\begin{array}{l}\text { Apical (DART } \uparrow) \text {, behavioral } \\
\text { (immobility } \uparrow) \text {, biochemical (AChE } \\
\text { activity } \downarrow) \text {, molecular (cyp-35A2 } \uparrow)\end{array}$ & $\begin{array}{l}\text { Role for cyp- } 35 A 2 \text { in the observed } \\
\text { toxic effects suggested by RNAi and } \\
\text { mutant analysis }\end{array}$ & $\begin{array}{l}\text { Quantification of } \\
\text { fenitrothion } \\
\text { degradation in } \\
\text { exposure medium }\end{array}$ & $\begin{array}{l}\text { SW } \\
\text { (depending on } \\
\text { concentration) }\end{array}$ & $\begin{array}{l}\text { Roh and } \\
\text { Choi } \\
\text { (2011) }\end{array}$ \\
\hline 5-fluorouracil & $\begin{array}{l}\text { Apical (DART } \uparrow) \text {, cellular } \\
\text { (apoptosis } \uparrow, \text { cell cycle arrest } \uparrow) \text {, } \\
\text { molecular (lin-29, ung-1) }\end{array}$ & $\begin{array}{l}\text { Cell cycle arrests followed by } \\
\text { germline apoptosis. Repression of } \\
\text { lin- } 29 \text { transcription factor related to } \\
\text { vulval development and egg laying }\end{array}$ & $\begin{array}{l}\text { Partially diminished } \\
\text { effects on embryo } \\
\text { hatching upon } \\
\text { knockdown of ung-1 }\end{array}$ & $\begin{array}{l}\text { WW } \\
\text { (depending on } \\
\text { concentration) }\end{array}$ & $\begin{array}{l}\text { Kumar et } \\
\text { al. }(2010)\end{array}$ \\
\hline Five pesticides & $\begin{array}{l}\text { Apical (DART } \uparrow) \text { and behavioral } \\
\text { (locomotion } \Delta \text { ) }\end{array}$ & $\begin{array}{l}\text { Neurotoxic pesticides affected } \\
\text { locomotion, while pesticides targeting } \\
\text { insect growth diminished C. elegans } \\
\text { reproduction }\end{array}$ & $\begin{array}{l}\text { Comparison of acute } \\
\text { versus chronic } \\
\text { exposure (sensitivity } \\
24 \mathrm{~h}>72 \mathrm{~h} \text { ) }\end{array}$ & Soil, SW, GW & $\begin{array}{l}\text { Ruan et } \\
\text { al. (2009) }\end{array}$ \\
\hline $\begin{array}{l}\text { Levamisole } \\
\text { (pesticide) }\end{array}$ & $\begin{array}{l}\text { Population (males } \Delta \text { ), apical } \\
\text { (survival } \downarrow \uparrow \text {, fecundity } \downarrow \uparrow \text { ), metabolic } \\
\text { (adaption costs) }\end{array}$ & $\begin{array}{l}\text { Multi-generational }(n=20) \\
\text { population/apical effects as potential } \\
\text { result of reduced motility } \\
\text { (encounters } \downarrow \text { thus outcrossing rate } \downarrow \text { ) }\end{array}$ & $\begin{array}{l}\text { Investigation of rapid } \\
\text { adaption and } \\
\text { ecological relevance }\end{array}$ & Soil, SW, GW & $\begin{array}{l}\text { Lopes et } \\
\text { al. (2008) }\end{array}$ \\
\hline
\end{tabular}


Table 2 (continued)

\begin{tabular}{|c|c|c|c|c|c|}
\hline \multicolumn{6}{|c|}{ Environmental studies: } \\
\hline $\begin{array}{l}\text { Sediment } \\
\text { extracts from } \\
\text { superfund site }\end{array}$ & $\begin{array}{l}\text { Apical (DART } \uparrow \text { ), biochemical (ATP } \\
\text { level } \downarrow \text { ), EROD activity (bacteria } \\
\text { and transgenic strain) } \uparrow\end{array}$ & $\begin{array}{l}\text { Basal zebrafish cyp-1A expression, } \\
\text { PAH-biotransformation, downstream } \\
\text { effects in transgenic } C \text {. elegans }\end{array}$ & $\begin{array}{l}\text { Cross-species method } \\
\text { indicating protective role } \\
\text { of } c y p-1 A\end{array}$ & $\begin{array}{l}\text { SW, GW, } \\
\text { sediments }\end{array}$ & $\begin{array}{l}\text { Harris et al. } \\
(2020)\end{array}$ \\
\hline $\begin{array}{l}\text { Acidic and } \\
\text { basic culturing } \\
\text { environment }\end{array}$ & $\begin{array}{l}\text { Apical (DART } \uparrow) \text { and molecular } \\
\text { (transcriptome } \Delta \text { ) }\end{array}$ & $\begin{array}{l}\text { Activation of cuticle synthesis, } \\
\text { structure-related }(\mathrm{pH}=4.33) \text { and } \\
\text { xenobiotic defense }(\mathrm{pH}<4.33) \text { genes }\end{array}$ & $\begin{array}{l}\text { Evaluation of potential } \\
\text { climate change driven } \\
\text { acidification effects }\end{array}$ & $\begin{array}{l}\text { MW, SW, } \\
\text { soils (through } \\
\text { acid rain) }\end{array}$ & $\begin{array}{l}\text { Cong et al. } \\
(2020)\end{array}$ \\
\hline $\begin{array}{l}\text { Micro- } \\
\text { polystyrene } \\
\text { particles }\end{array}$ & $\begin{array}{l}\text { Apical (lifespan } \downarrow \text { ), behavioral } \\
(\text { defecation } \Delta \text { ), cellular (motor } \\
\text { neuron GFP reporter } \Delta \text { ), molecular } \\
\text { (biomarker including skn-1 } \Delta \text { ) }\end{array}$ & $\begin{array}{l}\text { Activation of xenobiotic defense and } \\
\text { defecation pathways (ultimately } \\
\text { impacting AVL and DVB neurons as } \\
\text { well as life span) }\end{array}$ & $\begin{array}{l}\text { Particle size }(1-5 \mu \mathrm{m}) \\
\text { and concentration } \\
\left(\sim 10^{7}-10^{10} \text { particles } / \mathrm{m}^{2}\right) \\
\text { dependency }\end{array}$ & $\begin{array}{l}\text { SW, MW, } \\
\text { WW }\end{array}$ & $\begin{array}{l}\text { Shang et } \\
\text { al. (2020) }\end{array}$ \\
\hline $\begin{array}{l}\text { High-density } \\
\text { polyethylene } \\
\text { particles }\end{array}$ & $\begin{array}{l}\text { Apical (DART } \uparrow \text { ), molecular } \\
\text { (pathway-specific biomarker } \Delta \text { ) }\end{array}$ & $\begin{array}{l}\text { Involvement of nucleotide excision } \\
\text { repair and TGF- } \beta \text { signaling in toxicity } \\
\text { pathway and proposition of two AOPs }\end{array}$ & $\begin{array}{l}\text { Correlation to zebrafish } \\
\text { (experimental) and } \\
\text { humans (in silico) }\end{array}$ & $\begin{array}{l}\text { SW, MW, } \\
\text { WW }\end{array}$ & $\begin{array}{l}\text { Kim et al. } \\
(2020)\end{array}$ \\
\hline $\begin{array}{l}\text { Nano- } \\
\text { polystyrene } \\
\text { particles }\end{array}$ & $\begin{array}{l}\text { Apical (DART } \uparrow) \text {, behavioral } \\
\text { (locomotion } \downarrow) \text {, biochemical } \\
\text { (ROS } \uparrow) \text {, metabolic markers } \Delta\end{array}$ & $\begin{array}{l}\text { Perturbation of energy budget related } \\
\text { metabolites and ROS production } \\
\text { suggests relation to DART/locomotion }\end{array}$ & $\begin{array}{l}\text { Correlation of results } \\
\text { with particle properties } \\
\text { and uptake kinetics }\end{array}$ & $\begin{array}{l}\text { SW, MW, } \\
\text { WW }\end{array}$ & $\begin{array}{l}\text { Kim et al. } \\
(2019 a)\end{array}$ \\
\hline $\begin{array}{l}\text { Model crude oil } \\
\text { from spill event } \\
\text { (water soluble } \\
\text { fraction) }\end{array}$ & $\begin{array}{l}\text { Apical (reproduction } \downarrow \text { ) and } \\
\text { molecular (selected NER pathway } \\
\text { genes } \uparrow \text { ) }\end{array}$ & $\begin{array}{l}\text { Activation of NER pathway and } \\
\text { identification of the PAH C3- } \\
\text { naphthalene as main toxicity } \\
\text { contributor (confirmed by RNAi) }\end{array}$ & $\begin{array}{l}\text { Passive dosing method, } \\
\text { validation of results in } D \text {. } \\
\text { renio and comparison to } \\
\text { human blood biomarker }\end{array}$ & $\begin{array}{l}\text { MW, soil, } \\
\text { GW (e.g., if } \\
\text { similarly } \\
\text { polluted) }\end{array}$ & $\begin{array}{l}\text { Kim et al. } \\
(2019 c)\end{array}$ \\
\hline $\begin{array}{l}\text { Bile acid } \\
\text { metabolites of } \\
\text { synthetic and } \\
\text { natural origin }\end{array}$ & $\begin{array}{l}\text { Apical (DART } \uparrow) \text {, behavioral } \\
\text { (locomotion } \Delta \text { ), molecular } \\
\text { (transcriptome } \Delta, \text { KO-mutants } \Delta \text { ) }\end{array}$ & $\begin{array}{l}\text { Potential endocrine effects of a } \\
\text { bacterial manure-borne bile acid } \\
\text { metabolite could be mediated by } \\
\text { putative androgen receptor NHR- } 69\end{array}$ & $\begin{array}{l}\text { Model system with } \\
\text { Pseudomonas and } \\
\text { metabolisation in } \\
\text { soil/sand microcosm }\end{array}$ & $\begin{array}{l}\text { Soil, SW } \\
\text { (e.g., if } \\
\text { affected by } \\
\text { agricultural } \\
\text { manure) }\end{array}$ & $\begin{array}{l}\text { Mendelski } \\
\text { et al. } \\
(2019)\end{array}$ \\
\hline $\begin{array}{l}\text { Metal } \\
\text { contaminated } \\
\text { soil leachate }\end{array}$ & $\begin{array}{l}\text { Apical (lethality, life span } \downarrow \text { ), } \\
\text { molecular ( } 33 \text { genes incl. cyp- } \\
35 A 2 \uparrow \text { ), metabolism (fatty acid } \\
\text { content } \uparrow \text { ) }\end{array}$ & $\begin{array}{l}\text { Stress response and impacted fat } \\
\text { metabolism with chronic downstream } \\
\text { effects on life span }\end{array}$ & $\begin{array}{l}\text { Metal and PCA to } \\
\text { classify water soluble } \\
\text { and bioavailable fraction }\end{array}$ & Soil, GW & $\begin{array}{l}\text { Rai et al. } \\
(2019)\end{array}$ \\
\hline
\end{tabular}


Table 2 (continued)

\begin{tabular}{|c|c|c|c|c|c|}
\hline $\begin{array}{l}\text { Surface water } \\
\text { upstream of a } \\
\text { large river dam }\end{array}$ & $\begin{array}{l}\text { Apical (lifespan, growth, intestinal } \\
\text { permeability } \uparrow \text { ), behavior (locomotion } \\
\downarrow) \text {, biochemical (ROS } \uparrow \text { ), molecular } \\
(\text { sod- } 2 \uparrow, \text { sod- } 5 \uparrow, \text { clk- } 1 \uparrow, \text { mev- } 1 \downarrow \text { ) }\end{array}$ & $\begin{array}{l}\text { Involvement of sod-2/-5 in ROS } \\
\text { associated (intestinal/behavioral) } \\
\text { toxicity of a backwater sample } \\
\text { (suggested by mutational analysis) }\end{array}$ & $\begin{array}{l}\text { Aqueous and particulate } \\
\text { phase of samples } \\
\text { induced comparable } \\
\text { toxicity }\end{array}$ & $\begin{array}{l}\text { SW, (GW), } \\
\text { DW, TSS }\end{array}$ & $\begin{array}{l}\text { Xiao et al. } \\
(2018)\end{array}$ \\
\hline $\begin{array}{l}\text { River sediments } \\
\text { (aqueous and } \\
\text { solvent } \\
\text { extracts) }\end{array}$ & $\begin{array}{l}\text { Apical (survival } \downarrow \text {, growth } \downarrow \text { ), behavior } \\
\text { (locomotion, body bends } \Delta \text { ), } \\
\text { molecular (cyp-34A9 } \uparrow, \text { gst-1 } \uparrow, \text { gpx-1 } \\
\uparrow, h s p-6 \uparrow, h s p-16.2 \uparrow, h s p-70 \uparrow, \text { mlt-1 } \\
\uparrow, \text { mlt- } 2 \uparrow \text {, sod- } 1 \uparrow, \text { sod-4 } \uparrow \text { ) }\end{array}$ & Toxic profiles for different samples & $\begin{array}{l}\text { Effect correlation to } \\
\text { heavy metal and/or PAH } \\
\text { content (thresholds and } \\
\text { PCA analysis) }\end{array}$ & $\begin{array}{l}\text { Sediments } \\
\text { SW, DW, } \\
\text { WW }\end{array}$ & $\begin{array}{l}\text { Tejeda- } \\
\text { Benitez et } \\
\text { al. }(2016 \mathrm{~b}, \\
2018)\end{array}$ \\
\hline $\begin{array}{l}\text { Soil samples } \\
\text { near wetlands } \\
\text { and farmlands }\end{array}$ & $\begin{array}{l}\text { Community analysis }(\Delta) \text {, apical } \\
\text { (mortality } \uparrow \text {, reproduction } \downarrow \text { ), } \\
\text { molecular }(h s p-70 \downarrow, h s p-90 \uparrow \text { ) }\end{array}$ & $\begin{array}{l}\text { Multi-level disturbances at allocated to } \\
\mathrm{N} \text { - and P-contamination (from non- } \\
\text { point sources such as nearby farms) }\end{array}$ & $\begin{array}{l}\text { Included multivariate } \\
\text { statistics and } 4 \text { soil } \\
\text { parameters }\end{array}$ & Soils, GW & $\begin{array}{l}\text { Wang et al. } \\
(2015)\end{array}$ \\
\hline $\begin{array}{l}\text { Mining-affected } \\
\text { SW }\end{array}$ & $\begin{array}{l}\text { Apical (lethality) and molecular (35 } \\
\text { genes } \uparrow \downarrow \text { incl. cyp-35A2 } \downarrow \text { ) }\end{array}$ & $\begin{array}{l}\text { Deviating MoA for heavy metal. } \\
\text { Reconstituted and native SW. Role of } \\
\text { DOC, metal speciation or undetected } \\
\text { pollutants }\end{array}$ & $\begin{array}{l}\text { No unpolluted reference } \\
\text { site (or spiked SW) } \\
\text { included. Heavy metal } \\
\text { analysis }\end{array}$ & $\begin{array}{l}\text { SW, GW, } \\
\text { (DW) }\end{array}$ & $\begin{array}{l}\text { Kumar et } \\
\text { al. (2015) }\end{array}$ \\
\hline $\begin{array}{l}\text { Dispersed crude } \\
\text { oil (including } \\
\text { cleanup } \\
\text { dispersant) }\end{array}$ & $\begin{array}{l}\text { Cellular (apoptosis } \uparrow \text { ) and molecular } \\
\text { (13 specific genes } \uparrow \downarrow \text { ) }\end{array}$ & $\begin{array}{l}\text { Cep-1 mediated germ cell apoptosis } \\
\text { (e.g., suppressed apoptosis in cep-1 } \\
\text { loss of function mutant) as crucial } \\
\text { driver of the observed DART }\end{array}$ & $\begin{array}{l}\text { Expression patterns } \\
\text { correlated to exposure } \\
\text { levels }\end{array}$ & $\begin{array}{l}\text { MW, soil, } \\
\text { GW (e.g., } \\
\text { if similarly } \\
\text { polluted) }\end{array}$ & $\begin{array}{l}\text { Polli et al. } \\
(2014)\end{array}$ \\
\hline $\begin{array}{l}\text { Mining affected } \\
\text { sediment and } \\
\text { SW samples }\end{array}$ & $\begin{array}{l}\text { Apical (growth } \downarrow \text { ) and molecular (mlt- } \\
1 \text { expression and different } \\
\text { hypersensitive mutants) }\end{array}$ & $\begin{array}{l}\text { SW toxicity was attributed to osmotic } \\
\text { stress, whereas sediment toxicity to } \\
\text { metals/metalloids }\end{array}$ & $\begin{array}{l}\text { Includes chemical and } \\
\text { limnological analysis }\end{array}$ & $\begin{array}{l}\text { SW, } \\
\text { sediments }\end{array}$ & $\begin{array}{l}\text { Turner et } \\
\text { al. (2013) }\end{array}$ \\
\hline Industrial WW & $\begin{array}{l}\text { Apical (life span } \downarrow \text {, dauer formation, } \\
\text { reproduction } \downarrow \text { ), behavior (locomotion } \\
\downarrow \text { ), biochemical (autofluorescence } \uparrow \text { ), } \\
\text { molecular (daf-2) }\end{array}$ & $\begin{array}{l}\text { Toxic effects of different metal } \\
\text { combinations (partially independent of } \\
\text { the insulin-like pathway) }\end{array}$ & $\begin{array}{l}\text { Heavy metals as main } \\
\text { drivers of life span } \\
\text { reduction suggested by } \\
\text { the TIE approach }\end{array}$ & WW, SW & $\begin{array}{l}\text { Wang et al. } \\
(2010 \\
2008\end{array}$ \\
\hline $\begin{array}{l}\text { Landfill } \\
\text { impacted soil } \\
\text { samples }\end{array}$ & $\begin{array}{l}\text { Apical (mortality, growth, } \\
\text { reproduction) and molecular (stress- } \\
\text { related gene expression } \Delta \text { ) }\end{array}$ & $\begin{array}{l}\text { Toxicity and connected stress protein } \\
\text { induction (incl. cyp-35A2) of target } \\
\text { compound DEHP not reflected in } \\
\text { DEHP-contaminated soil samples }\end{array}$ & $\begin{array}{l}\text { Including reference site } \\
\text { and quantification of } \\
\text { DEHP concentrations }\end{array}$ & Soil, GW & $\begin{array}{l}\text { Roh et al. } \\
(2007)\end{array}$ \\
\hline
\end{tabular}


In perspective, the vast amount of information enclosed in these studies may be examined for their integration into recently shaping concepts such as 'multilevel biomarker analysis in environmental toxicology and risk assessment' (Choi 2008), 'adverse outcome pathways' (Angrish et al. 2018, Leist et al. 2017, Kim et al. 2020), 'multi-stressor response profiles' (Prasse et al. 2015), high-throughput toxicology frameworks (e.g., Schroeder et al. 2016), exposomics (Escher et al. 2017) and others (Brooks et al. 2020). Several of these concepts show overlapping features that aim at establishing or underpinning toxicological cause and effect relationships between chemical stressors (including environmental samples) and (adverse) physiological effects (such as approached in figure 15).

In case of the AOP concept these relationships are looked for between 'molecular initiating events'/'key events' and the 'dynamic energy budget' (DEB). Exhaustion of DEB might be followed by associated adverse events (e.g., Connon et al. 2012, Escher et al. 2017, Jager and Ashauer 2018). This approach mainly aims at utilizing the generated quantitative input on the individual level for predictive ecotoxicology on the population level (e.g., OECD 2018). The DEB model was tested as part of modelling approaches such as DEBtox and others. Up to present a number of AOPs have been elaborated for C. elegans (Alda Álvarez et al. 2006, Fueser et al. 2018, Jager et al. 2005, 2014, Jeong et al. 2018, Kim et al. 2020, Margerit et al. 2016, Swain et al. 2010, Wren et al. 2011).

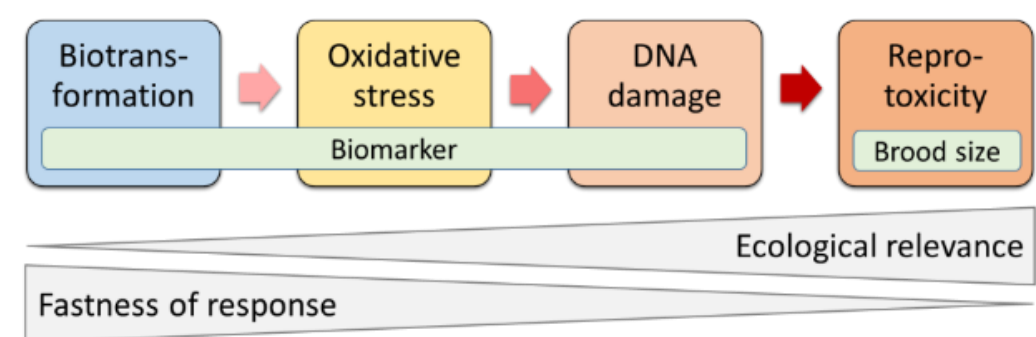

Figure 15. Mechanistic scheme of a hypothetical DART pathway in C. elegans. Therein DART (such as occurring upon exposure to $\beta$-NF) manifests at the cellular/tissue level and propagates to the physiological level through characteristic toxicological events (large boxes). These can be methodically detected via different endpoints/approaches (green boxes) which beholds a decreasing 'fastness of response' and increasing 'ecological relevance' as indicated. 
In general, AOPs further illustrate the complexity of toxicological pathways and (micro)pollutant interactions, whereby (through continuous research and optimisation, compare Lagadic et al. 2020) offering an integrative toolbox for an enhanced understanding and utilization such as in ERA (e.g., Ashauer and Jager 2018, Brooks et al. 2020, Society for Advancement of AOPs 2018). Similar to the field of ecotoxicogenomics important challenges lie ahead regarding mechanistic studies and ecotoxicological concepts with $C$. elegans. Tackling these challenges will further catalyze the establishment of $C$. elegans as relevant and sensitive model organism in chemical and ERA such as referring to urban water cycles. Based on the literature research and experimental results gathered in this thesis (e.g., 1.4, 2.3 and Abbas et al. 2018) its established and prospective usefulness seems mainly reflected in the following arenas:

1) Molecular biomarker-based indication, classification and evaluation of anthropogenic/chemical stressors in complex environmental samples and their toxicological/functional characterisation including genome wide expression "fingerprints". Particular focus is placed on major dysregulated physiological processes/pathways such as development and reproduction while respecting environmental conditions/factors (e.g., Allard et al. 2010, Kumar et al. 2015, table 2). Conducting chemical analyses of water and wastewater samples should be adapted to (known and hypothetical) chemical stressors to C. elegans (Abbas et al. 2018).

2) Enhanced detection of DART and other apical/physiological endpoints with high environmental and human relevance. These evaluations should be optimised by involving mutant and transgenic $C$. elegans strains in combination with HTS to achieve increased statistical power, feasibility and sensitivity (e.g., Allard et al. 2013, Avila et al. 2011, Boyd et al. 2010, Hunt 2016, Lundby et al. 2016, Schroeder et al. 2016, Turner et al. 2013, Xiong et al. 2017, Yu et al. 2020). Mechanistic linkages (compare Anderson and Wild 1994, Angrish et al. 2018, figure 15, table 2 and genome wide screens under 2.3.3) should also facilitate hazard assessment (compare AOP and other cited concepts/studies). Moreover, endpoints/methods (adapted from other fields of research) should continuously be adapted such as under points 2.1-2.5). 
2.1) Molecular and cell biology such as DNA damage/repair detection via the $x o l-$ $1::$ GFP strain (Allard et al. 2013, Lenz et al. 2017, Parodi et al. 2015), the xpa-1 deficient strain (Leung et al. 2010), the hus-1::GFP strain (Hofmann et al. 2002) or via PCR (e.g., Leung et al. 2010, Neher and Stürzenbaum 2006, Zuo et al. 2017). Furthermore, detection of cell cycle arrests (e.g., Cheng et al. 2014, Kumar et al. 2010), mitochondrial integrity (e.g., Behl et al. 2016), epigenetic consequences (e.g., Camacho et al. 2018, Lundby et al. 2016, Weinhouse et al. 2018) or germ line apoptosis via a ced-1::GFP strain (e.g., Allard et al. 2013). In addition, immunostaining and other methods (e.g., Parodi et al. 2015, Chen et al. 2016, 2019) may complement the listed molecular/cellular endpoints.

2.2) Neurotoxicology and behavioral biology (e.g., Aitlhadj and Stürzenbaum 2013, Avila et al. 2011, Bargmann 2006, Boyd et al. 2010, Cao et al. 2020b, Gerhardt et al. 2002, Jones and Candido 1999, Ju et al. 2014, Leung et al. 2008, Liu et al. 2019, Matsuura et al. 2013, Roh and Choi 2011, Tejeda-Benitez and Olivero-Verbel 2016a, Tseng et al. 2013).

2.3) Immunotoxicology. Whereby 'microbial pathogens' (Bruni et al. 2019, Darby 2005, van der Hoeven et al. 2011), 'bacteriological parameters' (Clavijo et al. 2016, Stylianou et al. 2018) and 'immunological markers' (e.g., Lewis et al. 2013, Kumar et al. 2015, Merkx-Jacques et al. 2013, Reichert and Menzel 2005) could play a relevant role in (waste)water assessments. A recent example by Bruni et al. (2019) showed significant effects in $C$. elegans exposed to Micrococcus luteus and Acinetobacter iwoffii isolated from wastewater samples (both strains carrying antibiotic resistances). Further and/or initial activation/weakening of the immune defense could occur through (micro)pollutant co-exposure (compare Stylianou et al. 2018 for C. elegans or Schlüter-Vorberg et al. 2017 for D. magna). In this context WWTP, such as using gravitational systems instead of micro-sieves or UV-based disinfection, may be investigated for potential residual emission of $C$. elegans pathogens (or parasites). Because of the absence of an adaptive immunity in $C$. elegans more research is also needed to correlate these results to other species beholding (more) complex immune systems.

2.4) Biochemistry and metabolomics. Including endpoints/methods such as quantifying ATP levels (e.g., Jones et al. 2011, Lagido et al. 2008, 2009, McLaggan et al. 2012), dauer formation (e.g., Fielenbach and Antebi 2008, Lant and Storey 
2010, Wang et al. 2010), intestinal autofluorescence (e.g., Pincus et al. 2016, Wang et al. 2008b), metabolic activity in connection with different metabolites (e.g., Kim et al. 2019b, Pradhan et al. 2018), oxygen consumption (e.g., Bodhicharla et al. 2014, Han et al. 2018, Schouest et al. 2009, Zuo et al. 2017), oxidative stress (e.g., Liu et al. 2013, Roh et al. 2018, Tseng et al. 2013, Wannous 2011, Xiao et al. 2018, Zhuang et al. 2014) and other biochemical/metabolic parameters.

2.5) Evolutionary developmental biology (e.g., Dutilleul et al. 2014).

3) Comparisons/Correlation of results assembled under point 1-2) to environmental conditions (such as given at multiple sampling sites) and other (nematode) species (Begasse et al. 2015, Boyd and Williams 2003, Haitzer et al. 1999, Höss et al. 2001, 2017, Hägerbäumer et al 2015, Haegerbaeumer et al. 2018, Kim et al. 2020, Maltby et al. 2000, Queirós et al. 2019, Wilson and Khakouli-Duarte 2009) for obtaining a more comprehensive picture about 'ecological realism and relevance' (Escher et al. 2017, Fischer et al. 2013, Jager and Ashauer 2018, Stamm et al. 2016) and for supporting weight-of-evidence frameworks (as far as applicable). 


\subsection{Integrated wastewater quality evaluation - case study of a pilot WWTP equipped with AWWT technologies}

Wastewater-borne (micro)pollutants largely contribute to the pollution of SWs and risks to aquatic ecosystems (1.2). Despite their large number current regulative monitoring focuses on a limited number of (micro)pollutants and physicochemical sum parameters such as TN, $P_{\text {total }}, \mathrm{COD}, \mathrm{BOD}_{5}, \mathrm{DOC}$ and TSS (compare Abbas et al. 2018). The reduction of organic (micro)pollutants is assumed to behave proportionally to a decreasing $\mathrm{COD}$ and $\mathrm{BOD}_{5}$. This holds true for many micropollutants particularly biodegradable and/or TSS-adsorbing compounds. However, a substantial fraction remains unaffected by conventional-biological treatment and becomes emitted into receiving water bodies representing (unpredicted) ecological risks (Link et al. 2017, Loos et al. 2013, Malaj et al. 2014, Pal et al. 2010, Schwarzenbach et al. 2006, Stalter et al. 2013). AWWT technologies have important advantageous in further reducing these emissions (Bui et al. 2016, Prasse et al. 2015, Rizzo et al. 2019, Schwarzenbach et al. 2006, Völker et al. 2019). They are thus currently installed and evaluated at different scales such as within the TransRisk project.

Ecotoxicological methods are essential for evaluating these technologies as the detection of contaminant removal rates by chemical methods alone would be unfeasible respecting their high number, generally low concentrations and potentially associated toxicities (e.g., Brack et al. 2017, Prasse et al. 2015, Reemtsma et al. 2016). In addition, challenges such as the generation of oxidative TPs during ozonation or UV radiation (1.2.2) necessitates bioassays to analyze the removal/generation of toxicity/hazardous compounds/TPs. This is particularly important, because the majority of TPs are presently unknown, difficult to detect (due to the lack of chemical standards / reference substances) and/or hard to predict (due to the complexity and variability of wastewater matrices).

The TransRisk evaluation concept mainly focuses on the reduction of chemical, ecotoxicological and microbial indicators by different AWWT options (Ternes et al. 2017, www.transrisk-projekt.de). The presented case study refers to a full-scale WWTP connected to a pilot scale ozonation and GAC/BF post-filtration systems (figure 16 and Abbas et al. 2018). During the presented case study (April-May 2014) 
an average ozone dose of $1 \mathrm{~g}\left(\mathrm{O}_{3}\right.$,applied $) / \mathrm{g}(\mathrm{DOC})$ and $\mathrm{HRT}$ of $\sim 18 \mathrm{~min}$ were applied. GAC (internal surface 1,200 $\mathrm{m}^{2} / \mathrm{g}$, grain size $1-4.8 \mathrm{~mm}$ ) and BFs (extended clay, grain size 1-5 $\mathrm{mm}$ ) ran in a non-aerated and aerated mode (using ambient air). Further process and WWTP parameters are described in Abbas et al. (2018), Knopp et al. (2016) and Ternes et al. (2017). Effect-based assessments were conducted based on selected in vitro (endocrinicity and mutagenicity) and in vivo (reproductive toxicity) endpoints. According to the change of these detections the efficiency of the respective AWWT option was rated (relative to the conventionally treated WWTP effluent). The $C$. elegans in vivo bioassay (ISO10872) implemented in the present case study was selected from the bioassay battery applied in the TransRisk project.

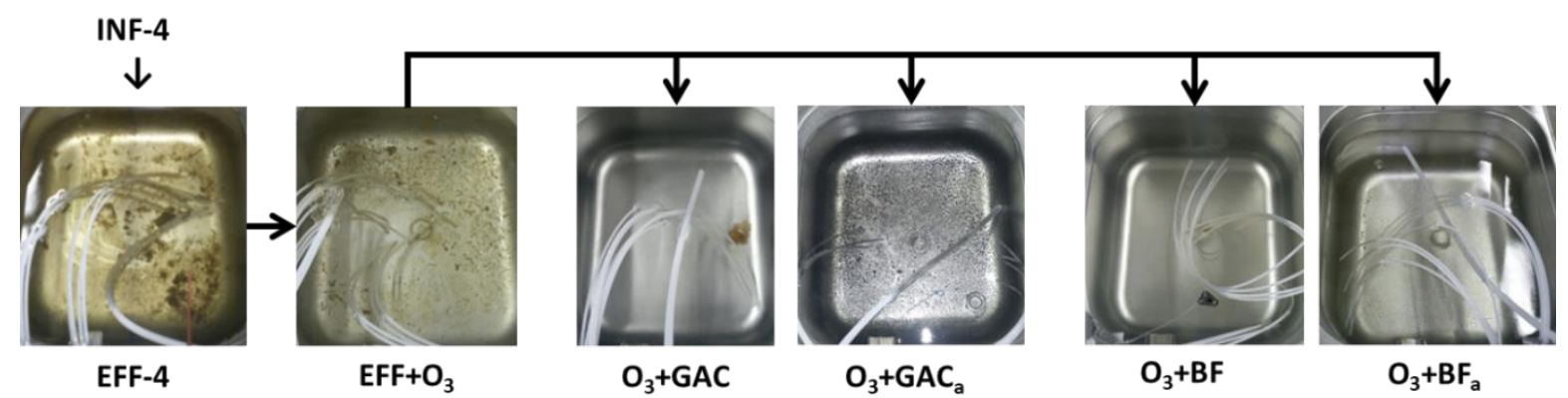

Figure 16. Wastewater collection basins at the pilot WWTP in Hessen, Germany installed during on site testing. Process flow from the WWTP influent (INF-4) to the effluent (EFF-4, activated sludge), ozonated effluent $\left(\mathrm{EFF}+\mathrm{O}_{3}\right)$ and $\mathrm{GAC} / \mathrm{BF}$ post-treatments $\left(\mathrm{O}_{3}+\mathrm{GAC}\right.$, $\mathrm{O}_{3}+\mathrm{GAC}_{\mathrm{a}}, \mathrm{O}_{3}+\mathrm{BF}, \mathrm{O}_{3}+\mathrm{BF}_{\mathrm{a}}$ ) as indicated. Treatment degree was visually reflected by decreasing TSS content and turbidity (from left to right). Sedimented particles in the $\mathrm{O}_{3}+\mathrm{GAC}_{\text {a }}$ were small GAC particles transported via the supply tubes. Samples taken as 24 h composites and prepared on site by SPE. Photographs taken by I. Schneider and A. Abbas.

The latter furthermore included D. magna (OECD 2012), Lemna minor (OECD 2006), the blackworm Lumbriculus variegatus (OECD 2007) and the mud snail Potamopyrgus antipodarum (OECD 2016). These test organisms were investigated in parallel studies by Schneider et al. (2020) and Schlüter-Vorberg et al. (2017) respectively. They proved to be robust sentinel species for characterizing wastewaters (Gartiser et al. 2010, Giebner et al. 2018, Magdeburg et al. 2012, Maltby et al. 2000, Kontana et al. 2009, Rizzo 2011, Stalter et al. 2010). Standardized test systems may however not indicate these properties throughout all wastewater investigations leading to variable bioassay results and compromised 
sensitivities (Berger et al. 2016, Wigh et al. 2018, Völker et al. 2017, 2019). Thus the present aim was to examine an integration of the results obtained with C. elegans into the wastewater evaluation matrix and to deduct its usefulness to wastewater investigations (such as discussed under 2.3). Similar to $P$. antipodarum (endpoint: number of embryos), D. magna (endpoint: number of offspring) and L. variegatus (endpoint: total number of worms), brood size was selected as lifecycle parameter of $C$. elegans and population-relevant endpoint (compare 2.3.1).

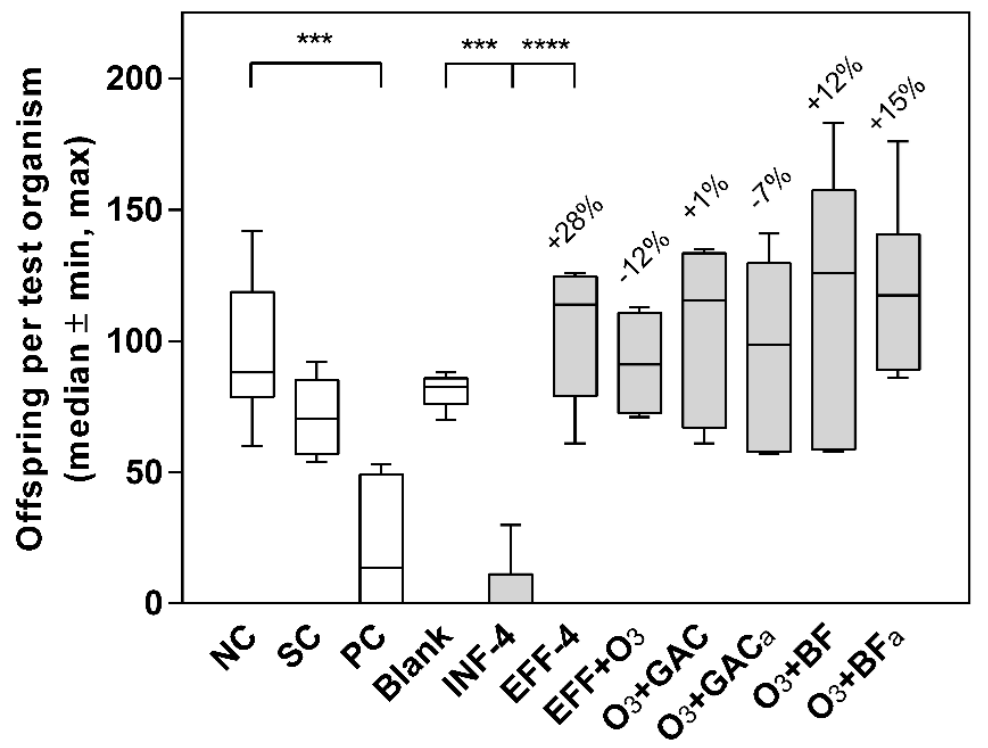

Figure 17. Impacts of WWTP influent (INF-4), effluent (EFF-4), ozonated effluent $\left(E F F+\mathrm{O}_{3}\right)$ and ozone post-filtrations $\left(\mathrm{O}_{3}+\mathrm{GAC}, \mathrm{O}_{3}+\mathrm{GAC}_{\mathrm{a}}, \mathrm{O}_{3}+\mathrm{BF}, \mathrm{O}_{3}+\mathrm{BF}\right.$ ) on the reproduction (brood size) of C. elegans. Samples were taken in April 2014 and analyzed at 10x concentrations. Results pooled from two experiments $(n=60)$. Percent values above bars indicate the percent increase/decrease in brood size relative to the previous treatment stage (except for the EFF-4 which refers to the blank). Significant differences ( ${ }^{* * *} p<0.001,{ }^{* * *} p<0.0001$ ) were tested by one-way ANOVA with Bonferroni's multiple comparison test. $\mathrm{NC}=\mathrm{M9}$ medium. $\mathrm{PC}=\mathrm{BAC}(5 \mathrm{mg} / \mathrm{L}) . \mathrm{SC}=0.2 \%$ DMSO in M9 medium. Blank $=\mathrm{SPE}$ blank prepared from analytically-pure groundwater. Further abbreviations/details given in text.

In the C. elegans analysis seen in figure 17 (from April 2014) the WWTP influent (INF-4) led to a strong reproductive decline, while the brood size of $C$. elegans exposed to the WWTP effluent (EFF-4) returned to a level that was slightly higher than NC and blank. This result indicated an effective removal of reproductive toxicants during activated sludge treatment. Compared to the EFF-4, the brood size in the $\mathrm{EFF}+\mathrm{O}_{3}$ (ozonated effluent) decreased by $12 \%$. Although this decrease was statistically insignificant, it might have been caused by oxidative TPs generated 
during ozonation such as corroborated with other aquatic species (da Costa et al. 2014, Magdeburg et al. 2012, Stalter et al. 2010, Giebner et al. 2018). In the postfiltration stages this reduction diminished $\left(\mathrm{O}_{3}+\mathrm{GAC}, \mathrm{O}_{3}+\mathrm{GAC}_{\mathrm{a}}\right)$ or higher brood sizes compared to the EFF-4 were detected $\left(\mathrm{O}_{3}+\mathrm{BF}, \mathrm{O}_{3}+\mathrm{BF}_{\mathrm{a}}\right)$. The latter result was confirmed at a later sampling date (figure 4A in Abbas et al. 2018) as well as for the endpoint growth (figure 4B in Abbas et al. 2018). Several reasons were considered regarding the enhanced reproduction/growth in the GAC/BF stages. A plausible explanation would be that the quality of the treated wastewater was beneficial (rather than harmful) to $C$. elegans. However, due to the remaining DOC (compare 'Online Resource 2' in Abbas et al. 2018) and residual endocrine activity after advanced treatment (compare figure 2 and 5 in Schneider et al. 2020) the presence of recalcitrant EDCs and/or bioactive natural compounds affecting the growth/reproduction of C. elegans (see Höss et al. 2001, Höss and Weltje 2007 or Vingskes and Spann 2018 for examples) may not be excluded a priori.

The in vitro results incorporated in the present study were obtained from bioassays for (anti)estrogenic (YES and YAES), (anti)androgenic (YAS and YAAS) and mutagenic (Ames fluctuation test) potentials of the wastewater samples (collected during February-April 2014). Similar to the included in vivo test systems these assays/endpoints have been beneficially utilized in related wastewater assessments (Chen et al. 2017, Escher et al. 2009, 2018, Filby et al. 2010, Giebner et al. 2018, Magdeburg et al. 2012, Margot et al. 2013, Schindler-Wildhaber et al. 2015, Stalter et al. 2010, Triebskorn et al. 2017, Välitalo et al. 2017, Völker et al. 2017). Using these assays, the conventional biological effluent indicated characteristic activity/toxicity levels (see Abbas et al. 2019 for further references). Residual estrogenic activity has frequently been observed after activated sludge treatment. Estrogenic activity detected in the EFF-4 was effectively reduced by ozonation, while no further increase occurred in the GAC/BF stages (figure 5A in Schneider et al. 2020). Only minor anti-estrogenic activity was detected in the WWTP effluent. This activity seemed moderately increased by ozonation, similar to previous observations in the course of this thesis (Abbas et al. 2019). It was hypothesized that both phenomena might be the results of shifted ratios of agonistic and antagonistic activities (e.g., Ihara et al. 2014, Rao et al. 2014) and/or anti-estrogenic TPs (e.g., Itzel et al. 2020, Knoop et al. 2018). Albeit GAC filtration reduced the 
antagonistic activity to the level of the EFF-4, it remained elevated in the BFs (figure 5B in Schneider et al. 2020). Plausible causative agents remained to be elucidated.

Table 3. Wastewater quality evaluation matrix based on the TransRisk project. In the present case five in vitro (YES, YAES, YAS, YAAS and Ames, compare Schneider et al. 2020) and one in vivo (ISO10872, figure 17) bioassay were used to estimate the efficacies of the five described AWWT processes (sampled during February-April 2014). Efficacies were scored based on the [\%]-change in biological activity/toxicity relative to the WWTP effluent (note that negative percent values equal to an increase). Scores were weighted according to the precluded environmental relevance of the respective endpoint (compare main text). All samples were analyzed at 10 fold concentration (SPE extracts)

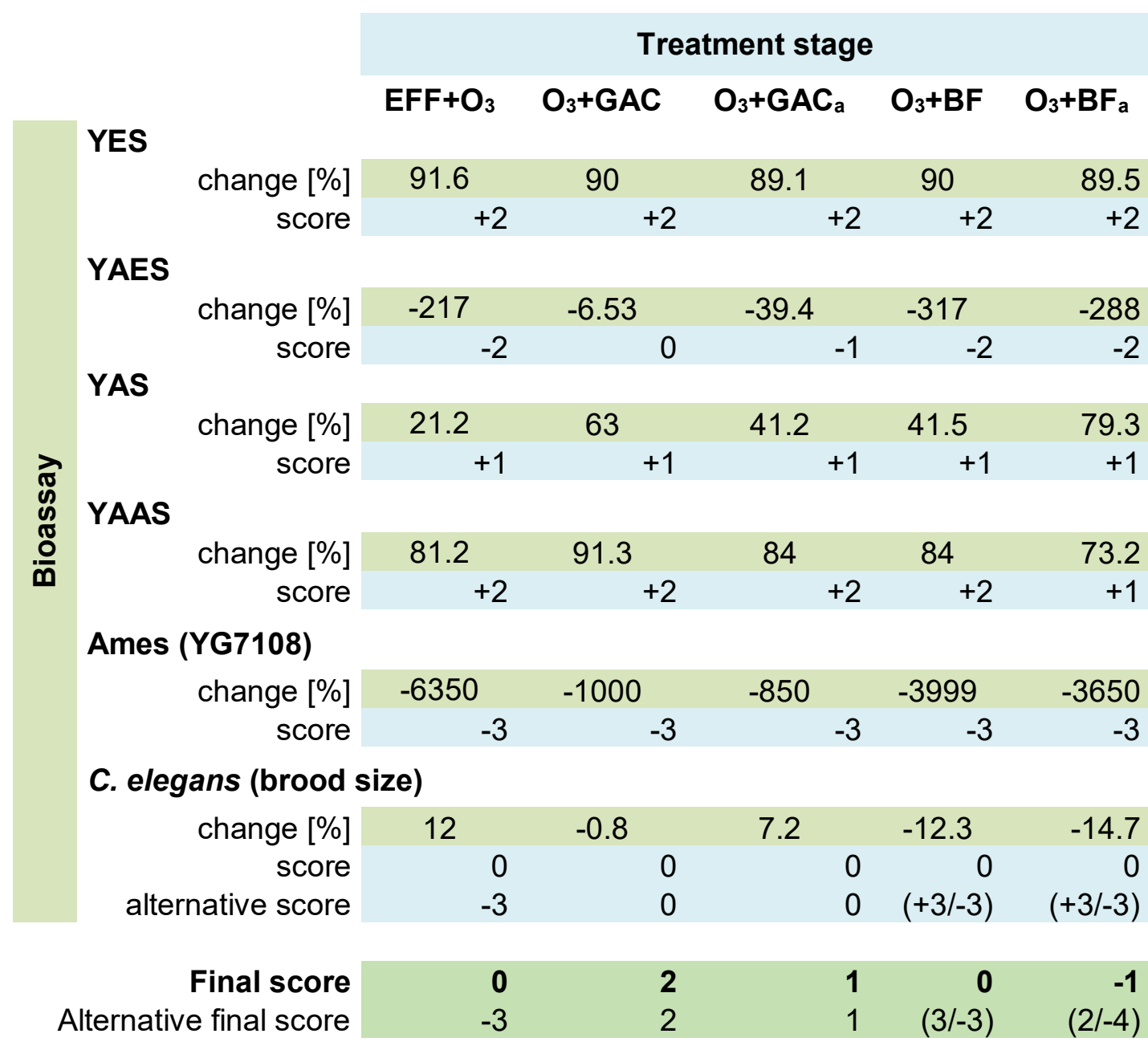

Androgenic and anti-androgenic activities were detected at relatively high levels in the WWTP effluent. Anti-androgenic activities also remained at moderate levels after $\mathrm{O}_{3}$ and in the post-filtration stages (compare Itzel et al. 2020), while androgenic activities were more effectively reduced (figure 5C and 5D in Schneider et al. 2020). Further experiments should thus be performed to confirm the occurrence of 
recalcitrant (anti)androgenic activities in these wastewaters. Moreover, low mutagenicity levels in the WWTP effluent were significantly increased after ozonation. It might be speculated, if and how far this mutagenicity/genotoxicity might have been involved in the reproductive decline observed for this treatment group with $C$. elegans (figure 17). Regarding the post-filtrations systems, GAC filtration more effectively decreased the detected mutagenicity compared to BF (5.4-5.5 fold versus 2.4-2.7 fold respectively), but the residual toxicity was still significant. Due to the high human and environmental relevance of the endpoint mutagenicity, confirmed and reoccurring mutagenicity in WWTP discharges from conventionalbiological as well as advanced treatments should be further investigated.

The described in vivo (figure 17) and in vitro (figure 5 in Schneider et al. 2020) results were taken as an input for the evaluation matrix computed in table 3. Depending on their environmental relevance differently weighted scores (TransRisk workshop in 2015) were ascribed according to the respective detection levels and their changes upon wastewater treatment. In vitro bioassays probing for agonistic (YES/YAS) and antagonistic (YAES/YAAS) endocrine activities were allocated with scores from -2 to +2 depending on their removal degree $(20-80 \%=1$ and $>80 \%=$ $2)$, increase $(-20$ to $-100 \%=-1$ and $<-100 \%=-2)$ or stagnancy $(-20$ to $20 \%=0)$ in activity during the respective wastewater treatment stage. For the Ames fluctuation test these scores ranged from $-3(<-20 \%$ change) to 3 ( $>20 \%$ change), due to the generally higher risk associated with mutagenic compounds. Changes in the brood size of $C$. elegans were assigned the lowest/highest scores ranging from -6 ( $>20 \%$ change) to 6 (<-20\% change) as adverse effects at the whole organism level were ascribed the highest biological/ecological relevance. In addition, an 'alternative score' was examined for the latter endpoint (table 3 ). The respective weighting involved the scores -3 and 3 for changes in brood sizes from 10 to $20 \%$ and -20 to $-10 \%$ respectively. This rating was examined in the light of the discussed sensitivity of C. elegans (2.3) and for its implications on the evaluation matrix (discussed below).

The overall rating ('final score', table 3 ) of the effectivities of the five AWWT options follows the order: $\mathrm{O}_{3}+\mathrm{GAC}>\mathrm{O}_{3}+\mathrm{GAC}_{\mathrm{a}} \sim \mathrm{O}_{3}+\mathrm{BF} \sim \mathrm{EFF}+\mathrm{O}_{3}>\mathrm{O}_{3}+\mathrm{BF}$. This rating indicated a slightly better performance of the GAC filtrations compared to the BFs. It also showed that aeration did not measurably improve the performance of these 
two post filtration systems. The score of the aerated BF was lowest and lower than of ozonation alone. These results reflect in the analytical chemistry part of the TransRisk project (Abbas et al. 2018, Knopp et al. 2016, Ternes et al. 2017). Moreover, comparably high effectivities of activated carbon treatments (GAC, PAC or BAC) as post-filtration systems (but also as independent AWWT) have been documented in the literature (1.2.2). Together with sandfiltration, these technologies have been recommended post-treatments for ozonation (Magdeburg et al. 2014, Knopp et al. 2016, Prasse et al. 2015, Stalter et al. 2010, Reemtsma et al. 2016, 1.2.2).

In the performed case study it may be considered that the presented evaluation matrix allows for a best/worst theoretically-achievable 'final score' of 17/-17 respectively (table 3 ). In this context all five treatment options achieved an intermediate positioning ( -1 to 2$)$ and in this case were to be rated ineffective. To be exact the latter derived from high (e.g., estrogenic and anti-androgenic activities) and moderate (e.g., androgenic activity and mutagenicity) removal rates as well as activities/toxicities that increased (e.g., anti-estrogenicity) or remained stagnant respecting the ascribed thresholds (C. elegans brood size). This result seems expectable due to different reasons:

1) Most estrogenic compounds have been reported to be effectively broken down during ozonation (Abbas et al. 2019, Ma et al. 2005, Maletz et al. 2012, Reungoat et al. 2012). Ozonation was also the main driver of the high removal rates observed for all five AWWT options in the present evaluation.

2) Although anti-estrogens (2.2) were reported to occur in wastewater, not much is known about their concentrations and removal. This means many might not be fully degraded/retained and enter surface waters through WWTPs. This should also be regarded when testing (treated) wastewater for agonistic/antagonistic effects (compare Abbas et al. 2019, Leusch et al. 2017, Rao et al. 2014).

3) Residual androgenic compounds remained speculative as generally higher removal rates have been reported for activated sludge treatment as well as ozonation (Abbas et al. 2019, Rao et al. 2014, Stalter et al. 2011).

4) High removal rates of anti-androgenic activities may however induce changes in agonistic/antagonistic interactions that could lead to higher detections of androgenic activities in the AWWT stages. 
5) Elevated levels of mutagenicity are occasionally observed in connection with ozonation. Different studies suspected this toxicity to derive from generated or potentiated mutagenic/genotoxic TPs (e.g., Jia et al. 2015, table 4). Because of the increased environmental relevance repeated detections of mutagenicity generally call for further research such as on potential causative agents and further optimisation of AWWTs (and other measures, compare 3.).

6) Impacts on the brood size of $C$. elegans ranged within the $\pm 20 \%$ threshold for all five AWWT options (allocating scores of "0"). A hypothetical threshold of $\pm 10 \%$ would lead to an 'alternative final score' of -3 (instead of 0 ) for ozonation, which may better reflect potential adverse effects. The rating of the treatment options $\mathrm{O}_{3}+\mathrm{GAC}$ and $\mathrm{O}_{3}+\mathrm{GAC}_{\mathrm{a}}$ remains unchanged. The $\mathrm{O}_{3}+\mathrm{BF}$ and $\mathrm{O}_{3}+\mathrm{BF}_{\mathrm{a}}$ treatments would be rated as +3 or -3 depending on the exact nature of these effects: positive (wastewater quality higher than in the EFF/NC) or adverse (such as resulting from residual EDCs, compare above discussion of figure 17).

The results of two in vivo on-site studies conducted with D. magna and P. antipodarum at the pilot WWTP (Schlüter-Vorberg et al. 2017, Schneider et al. 2020) well relate to the results gathered with $C$. elegans. In these studies the 'number of embryos' of $P$. antipodarum also slightly increased in the WWTP effluent compared to the NC. For both species a noticeable and in case of $P$. antipodarum significant reproductive decline was detected after ozonation $\left(\mathrm{EFF}+\mathrm{O}_{3}\right)$. This apparent reproductive toxicity was removed in the $\mathrm{O}_{3}+\mathrm{GAC}$ treatment supporting the evaluated effectivity of this AWWT option (table 3 ) and previous observations in related studies (table 4). The $\mathrm{O}_{3}+\mathrm{BF}$ treatment decreased this toxicity (to $P$. antipodarum) to slightly lesser extent, while the $\mathrm{O}_{3}+\mathrm{GAC}_{\mathrm{a}}$ and $\mathrm{O}_{3}+\mathrm{BF}_{\mathrm{a}}$ treatments did not (figure 3 in Schneider et al. 2020). A similar picture was obtained based for the endpoint 'number of offspring' of $D$. magna. However, BF as well as aeration both led to higher offspring numbers than GAC and non-aerated treatments. Regarding the test species L. variegatus, also implemented in TransRisk, the WWTP effluent (EFF-4) adversely affected the reproduction of worms. In the ozonated effluent $\left(\mathrm{EFF}+\mathrm{O}_{3}\right)$ the number of worms was significantly elevated indicating a positive/detoxifying effect of ozonation, while both GAC filtrations performed similarly well and again better than the BFs. 
Table 4. Ecotoxicological effects of corresponding WWTP effluents (EFF), ozonated effluents $\left(E F F+O_{3}\right)$ and three types of post-treatment: $\left.A\right)$ activated carbon $\left.\left(\mathrm{O}_{3}+A C\right), B\right)$ biological treatment $\left(\mathrm{O}_{3}+\mathrm{B}\right)$ and $\left.\mathrm{C}\right) \mathrm{BAC}$ as "hybrid" of $\left.\mathrm{A}\right)$ and $\left.\mathrm{B}\right)\left(\mathrm{O}_{3}+\mathrm{BAC}\right)$ reported in the literature. Arrows " $\downarrow$ " = reduction, “ $\leftrightarrow$ " = stagnancy and " $\uparrow=$ increase in bioactivity/toxicity/expression level compared to the previous treatment stage: in case of the EFF this is given by the WWTP influent or (as the INF often indicated cytotoxicity or mortality respectively) by the NC (indicated with superscript "c"). Red, yellow or green fields indicate high, moderate or low/no residual bioactivity, toxicity or modified expression level respectively. Results may refer to different test systems, exposure conditions (e.g., flowthrough systems, aqueous or extracted samples), process parameters (e.g., ozone doses and implementation scales) and other characteristics as described in the respective references. If a table cell is split into multiple cells this reflects results from different experiments/studies. "Repro" = reproduction. "Histo" = histopathology. "om" = O. mykiss. "dr" = D. rerio. "n.a." = not applicable. "n.d." = not determined.

\begin{tabular}{|c|c|c|c|c|c|c|c|c|c|}
\hline A & Enc & $\begin{array}{l}\text { Treatment stage } \\
\text { point/Species }\end{array}$ & \multicolumn{2}{|c|}{ EFF } & \multicolumn{2}{|c|}{$\mathrm{EFF}+\mathrm{O}_{3}$} & \multicolumn{2}{|c|}{$\mathrm{O}_{3}+\mathrm{AC}$} & Reference \\
\hline \multirow{12}{*}{ 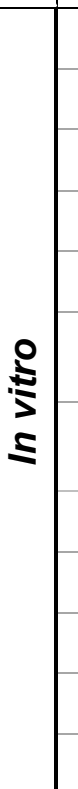 } & & Estrogenicity & $\downarrow$ & $\uparrow^{c}$ & $\downarrow$ & $\leftrightarrow$ & $\leftrightarrow$ & $\downarrow$ & \multirow{3}{*}{$\begin{array}{l}\text { Giebner et al. (2016), } \\
\text { Dopp et al. (2021) }\end{array}$} \\
\hline & & nti-estrogenicity & \multicolumn{2}{|c|}{$\downarrow$} & $\downarrow$ & $\uparrow$ & $\uparrow$ & $\downarrow$ & \\
\hline & & Androgenicity & \multicolumn{2}{|c|}{$\downarrow$} & \multicolumn{2}{|c|}{$\leftrightarrow$} & \multicolumn{2}{|c|}{$\leftrightarrow$} & \\
\hline & & nti-androgenicity & \multicolumn{2}{|c|}{ n.d. } & \multicolumn{2}{|c|}{$\downarrow$} & \multicolumn{2}{|c|}{ n.a. } & Stalter et al. (2011) \\
\hline & & ioxin-like activity & \multicolumn{2}{|c|}{$\downarrow$} & \multicolumn{2}{|c|}{$\downarrow$} & \multicolumn{2}{|c|}{$\downarrow$} & Maier et al. (2016) \\
\hline & & $\begin{array}{l}\text { Mutagenicity, } \\
\text { Genotoxicity }\end{array}$ & $\downarrow$ & n.d. & $\downarrow$ & $\uparrow$ & $\leftrightarrow$ & $\downarrow$ & $\begin{array}{l}\text { Chen et al. (2017), } \\
\text { Giebner et al. (2016) }\end{array}$ \\
\hline & & $\begin{array}{l}\text { Photosynthesis } \\
\text { inhibition }\end{array}$ & \multicolumn{2}{|c|}{$\downarrow$} & \multicolumn{2}{|c|}{$\downarrow$} & \multicolumn{2}{|c|}{$\leftrightarrow$} & \multirow{4}{*}{$\begin{array}{l}\text { Stapf et al. (2017), } \\
\text { Dopp et al. (2021) }\end{array}$} \\
\hline & $\mathrm{Ce}$ & Il growth inhibition & \multicolumn{2}{|c|}{1} & \multicolumn{2}{|c|}{$\downarrow$} & \multicolumn{2}{|c|}{$\leftrightarrow$} & \\
\hline & & AchE inhibition & \multicolumn{2}{|c|}{$\downarrow$} & \multicolumn{2}{|c|}{$\downarrow$} & \multicolumn{2}{|c|}{$\leftrightarrow$} & \\
\hline & & Cytotoxicity & $\downarrow$ & $\downarrow^{c}$ & $\downarrow$ & $\leftrightarrow$ & n.d. & $\uparrow$ & \\
\hline & & Cell morphology & \multicolumn{2}{|c|}{$\uparrow^{c}$} & & \multicolumn{2}{|c|}{$\downarrow$} & lolol ond Dubl (2016) \\
\hline & & ROS & \multicolumn{2}{|c|}{$\uparrow^{c}$} & & & \multicolumn{2}{|c|}{$\downarrow$} & 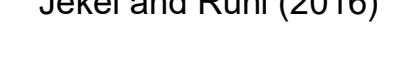 \\
\hline \multirow{7}{*}{$\begin{array}{l}\stackrel{0}{2} \\
\stackrel{2}{2} \\
\Sigma\end{array}$} & & minor growth & \multicolumn{2}{|c|}{$\leftrightarrow^{c}$} & \multicolumn{2}{|c|}{$\leftrightarrow$} & $\leftarrow$ & & $\begin{array}{c}\text { Schlüter-Vorberg et al. } \\
(2017)\end{array}$ \\
\hline & $P$. & antipodarum repro & & & & & & & Giebner et al. (2018) \\
\hline & & mykiss liver-histo & & & & & $\downarrow$ & $\downarrow$ & Triebskorn (2017) \\
\hline & & drVTG & & & & & & & Sun et al. (2017) \\
\hline & 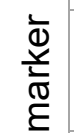 & omVTG & $\uparrow^{c}$ & $\overleftrightarrow{c}$ & $\downarrow$ & n.d. & n.a. & $\uparrow$ & $\begin{array}{c}\text { Magdeburg et al. (2014), } \\
\text { Stalter et al. (2010), } \\
\text { Triebskorn (2017) }\end{array}$ \\
\hline & $\frac{\overline{0}}{\overline{0}}$ & omCYP-1A1 & & & & & 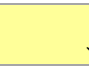 & & Maier et al. (2016) \\
\hline & & ceCYP-35A3 & & & & & $n$ & & Abbas et al. 2018 \\
\hline
\end{tabular}


Table 4. (Continued)

\begin{tabular}{|c|c|c|c|c|c|c|c|c|c|c|}
\hline $\mathbf{B}$ & $\begin{array}{l}\text { Treatment stage } \\
\text { Endpoint/Species }\end{array}$ & \multicolumn{2}{|c|}{ EFF } & \multicolumn{3}{|c|}{$\mathrm{EFF}+\mathrm{O}_{3}$} & \multicolumn{3}{|c|}{$\mathrm{O}_{3}+\mathrm{B}$} & Reference \\
\hline \multirow{7}{*}{$\begin{array}{l}0 \\
\stackrel{2}{s} \\
s \\
s\end{array}$} & Estrogenicity & $\downarrow$ & $\uparrow^{c}$ & $\downarrow$ & $\downarrow \uparrow$ & $\uparrow$ & $\downarrow$ & $\leftrightarrow$ & $\leftrightarrow$ & \multirow{4}{*}{$\begin{array}{l}\text { Itzel et al. (2020), } \\
\text { Dopp et al. (2021) }\end{array}$} \\
\hline & Anti-estrogenicity & \multicolumn{2}{|c|}{$\downarrow$} & \multicolumn{3}{|c|}{$\leftrightarrow$} & \multicolumn{3}{|c|}{$\uparrow$} & \\
\hline & Androgenicity & \multicolumn{2}{|c|}{$\downarrow$} & \multicolumn{3}{|c|}{$\leftrightarrow$} & \multicolumn{3}{|c|}{$\leftrightarrow$} & \\
\hline & Anti-androgenicity & $\downarrow$ & $\uparrow$ & \multicolumn{3}{|c|}{$\uparrow$} & \multicolumn{3}{|c|}{$\leftrightarrow$} & \\
\hline & $\begin{array}{l}\text { Mutagenicity, } \\
\text { Genotoxicity }\end{array}$ & \multicolumn{2}{|c|}{ n.d. } & \multicolumn{3}{|c|}{$\downarrow$} & \multicolumn{3}{|c|}{$\downarrow$} & Wu et al. (2014) \\
\hline & $\begin{array}{l}\text { Bioluminescence } \\
\text { (V. fisheri) }\end{array}$ & n.d. & n.a. & \multicolumn{3}{|c|}{$\downarrow$} & $\uparrow$ & \multicolumn{2}{|c|}{$\uparrow$} & $\begin{array}{c}\text { Li et al. (2015), } \\
\text { Paździor et al. (2017) }\end{array}$ \\
\hline & Cytotoxicity & $\downarrow^{c}$ & $\downarrow^{c}$ & \multicolumn{3}{|c|}{$\downarrow$} & $\leftrightarrow$ & \multicolumn{2}{|c|}{$\downarrow$} & Dopp et al. (2021) \\
\hline$c$ & $\begin{array}{l}\text { Treatment stage } \\
\text { Endpoint/Species }\end{array}$ & \multicolumn{2}{|c|}{ EFF } & \multicolumn{3}{|c|}{$\mathrm{EFF}+\mathrm{O}_{3}$} & \multicolumn{3}{|c|}{$\mathrm{O}_{3}+\mathrm{BAC}$} & Reference \\
\hline \multirow{3}{*}{ 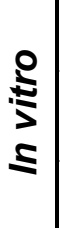 } & Estrogenicity & \multirow{2}{*}{\multicolumn{2}{|c|}{ n.d. }} & $\downarrow$ & $\downarrow$ & $\downarrow$ & $\leftrightarrow$ & $\downarrow$ & $\leftrightarrow$ & \multirow{2}{*}{$\begin{array}{l}\text { Reungoat et al. } \\
\qquad(2012)\end{array}$} \\
\hline & $\begin{array}{l}\text { Bioluminescence } \\
\text { (V. fisheri) }\end{array}$ & & & $\downarrow$ & $\downarrow$ & $\downarrow$ & $\downarrow$ & $\downarrow$ & $\downarrow$ & \\
\hline & Genotoxicity & \multicolumn{2}{|c|}{ n.a. } & \multicolumn{3}{|c|}{$\downarrow$} & \multicolumn{3}{|c|}{$\downarrow$} & Reaume et al. (2014) \\
\hline
\end{tabular}

In summary, for most of the detected toxicities the activated sludge treatment at the inestigateed WWTP constituted an effective treatment barrier. When the activated sludge treatment shows a (very) high performance the (full) capacity of tertiary and AWWT options may be difficult to estimate, since there might be only little residual toxicity to be removed/evaluated (Schlüter-Vorberg et al. 2017, Völker et al. 2017). In addition, it could be argued that an extended selection of robust and sensitive in vitro and in vivo bioassays may have revealed undetected effects, which should be integrated into future wastewater quality evaluations not to overlook significant biological risks (compare Berger et al. 2016, Escher et al. 2014 for DW, Schwarzenbach et al. 2006, Sonne et al. 2018, Stamm et al. 2016, Wigh et al. 2018). However, extensive on-site as well as laboratory-scale bioassay batteries may be unfeasible and costly to perform, thus further bioassay optimisation, prioritisation, complementation and case-specific application has been advised for providing high environmental relevance of the analyses (Berger et al. 2016, Bunzel et al. 2013, Brodin et al. 2014, Rizzo 2011, Stamm et al. 2016). 
In general, residual toxicity in advanced treated wastewater (e.g., Bundschuh et al. 2011a, 2011b, Reungoat et al. 2012, table 4) stresses the identification of respective causative agents, detection of their MEC and and characterisation of their potential ecotoxicity. Chemical target analysis has often been of minor success in elucidating these effects and in computing realistic EQS that are to be transferred to regulation bodies. This inspires to further improve chemical analyses such as by detecting (a higher number of) suspected toxicants that are adapted to environmentally relevant endpoints (Escher et al. 2018, Maier et al. 2016, Sonne et al. 2018, Stamm et al. 2016, Tang et al. 2014, Vasquez and Fatta-Kassinos 2013). In perspective, continued research should be addressed on:

- Best available technologies (BATs, including most effective process combinations).

- Optimal evaluation strategies, ideally involving performance reviews before-andafter technical upgrades and in situ comparisons up-and-downstream of WWTPs (including uncontaminated and contaminated reference sites, Ashauer 2016, Brettschneider et al. 2019, Bundschuh et al. 2011a, 2011b, Henneberg et al. 2014, Hicks et al. 2016, Maier et al. 2016, Triebskorn et al. 2017, Wilhelm et al. 2017).

- Farsighted risk management (acknowledging site specificity and LCA). 


\section{Conclusions and outlook}

Optimising sample preparation for in vitro bioassays (2.1 and Abbas et al. 2019)

- Sample preparation methods such as acidification, filtration and SPE significantly influenced the detected ecotoxicologically relevant in vitro endpoints. These findings hinted on according shifts in the chemical composition of the prepared (waste)water samples.

- Acidified samples mostly showed higher or lower in vitro activities compared to neutral samples that could not be further explained. Sample filtration often led to losses in in vitro activities, but in most cases the investigated in vitro activity remained unaffected. However, in case of antagonistic activities (anti-estrogenic and anti-androgenic) several samples indicated increased activities after filtration, which might have resulted from altered ratios of agonistic/antagonistic compounds in the samples.

- Ecotoxicological optimisation of SPE methods (up to present mainly performed for chemical analyses) proved to be a valuable approach for the effective recovery of biological activity/toxicity from different water and wastewater samples and thus the reduction of false negative results (e.g., undetected in aqueous samples). The commercial SPE column Telos C18/ENV thereby demonstrated higher effectivity than the Oasis HLB and the Supelco ENVICarb+. Moreover, a sample extraction $\mathrm{pH}$ of 7 was favored over a $\mathrm{pH}$ of 2.5 (short-term acidification) as corroborated by multivariate statistics using the Pareto algorithm.

\section{In vitro bioassays as environmental monitoring tools (2.2)}

- MoA-based in vitro bioassays such as integrated and investigated in the longterm environmental monitoring campaign of the water protection model region in Southwest Germany demonstrated to be beneficial tools for the detection and prioritisation of cytotoxicity, genotoxicity, mutagenicity and different endocrine activities in aqueous and extracted (waste)water samples.

- Several endocrine activities were recurrently detected in hospital/raw and to lesser extend in biologically-treated wastewater. The monitoring indicated their effective removal during the activated sludge treatments at the investigated 
WWTPs. Surface water and groundwater samples (used for the production of drinking water) were also found not to exhibit any of the analyzed biological activities/toxicity at significant levels, except for the anti-estrogenicity at one sampling site (compare below).

- Significant anti-estrogenic activity was frequently detected in one out of three groundwater hotspots. These hotspots were previously characterized as part of the long-term chemical monitoring of the model region. Potential anti-estrogenic causative agents, however, remained unknown. Importantly, only one groundwater intake, but none of the other intakes/wells, nor a drinking water supply pipe, showed any significant anti-estrogenicity. This potential hotspot should thus undergo extended chemical and toxicological monitoring.

- Regarding the high anti-estrogenic/-androgenic activity detected in hospital wastewater, several known compounds came into question. Main suspects were pharmaceutical used in anti-cancer therapies. As the sampled hospital connects to one of the investigated WWTPs it would be interesting to clarify, if the reduced activity detected in the WWTP influent resulted from the dilution and/or degradation/adsorption of these compounds in the sewer system and at which concentration they are contained in the WWTP effluent.

\section{Adapted C. elegans bioassay and usefulness for the ecotoxicological characterization of water and wastewater samples (2.3 and Abbas et al. 2018)}

- The sensitivity and ecological relevance of $C$. elegans was exploited for examining the reference PAH $\beta-\mathrm{NF}$ and different (waste)water samples. $\beta-\mathrm{NF}$ exhibited high DART with an $\mathrm{EC}_{50}$ of $114 \mu \mathrm{g} / \mathrm{L}$ (brood size, $96 \mathrm{~h}$ ). One biologically-treated WWTP effluent indicated residual high levels of reproductive toxicity. These investigations were extended for the AWWTs ozonation and GAC/BF-post-filtration, whereby no reproductive toxicity was detected (including the respective WWTP effluent).

- Similar to our filtration results in 2.1 and Abbas et al. 2019 the result obtained with $C$. elegans and unspiked versus $\beta$-NF-spiked (waste)water samples indicated a role for TSS $(>1 \mu \mathrm{m})$ in the toxicity of this PAH (predicted log Kow of 4.7) as well as its putative cumulative effects with other sample constituents.

- The additional developmental endpoint 'larval length' indicated significantly promoted growth after exposure to three extracted WWTP effluents. Although 
plausible causative agents of this effect remained speculative (e.g., if natural or anthropogenic chemicals), it demonstrated the importance of integrating multiple endpoints into wastewater related assessments (compare below).

- A molecular biomarker of exposure was integrated into the $C$. elegans bioassay by the transgenic cyp-35A3::GFP strain. This CYP subfamily is induced by various (micro)pollutants such as $\mathrm{PAH}, \mathrm{PCBs}$ and different pesticides and pharmaceuticals. The present experiments detected a developmental-stage-, tissue- and concentration-dependent induction of cyp-35A3::GFP by $\beta-N F$. High expression levels in adult hermaphrodites were reached after subacute exposures (e.g., EC $\mathrm{E}_{50}$ of $71.5 \mu \mathrm{g} / \mathrm{L}$ after $8 \mathrm{~h}$ ). Furthermore, cyp-35A3::GFP was induced by a conventional-biological and to higher extend by the corresponding ozonated WWTP effluent. It was hypothesized that this could have been caused by oxidative TPs or potentiation of cyp-35A3 inducing compounds. However, the removal of this effect by the GAC/BF-post-filtration-systems remained to be clarified.

- An accompanying chemical analysis quantified several known effectors of the reproduction/growth of $C$. elegans. The conventional-biological and AWWT stages at the pilot WWTP effectively removed the majority of these known effectors as well as most substances included into the target chemical analysis (in most cases below LOQs). Their overall reduction however did not eliminate the reproductive toxicity in one WWTP effluent sample, nor the growth enhancing effect detected in the GAC/BF-post-filtration systems. This and other effects in treated wastewater generally raise the question on the chemical identity of remaining causative agents and calls for a tighter linkage of chemical and ecotoxicological analysis.

- Based on a literature survey, ecotoxicogenomic and mechanistic studies performed with $C$. elegans demonstrate a high potential in characterizing multiple adverse impacts of anthropogenic (micro)pollutants and contaminated environmental samples at different levels of biological organization. Several studies thereby disclosed and describe correlations of molecular/cellular endpoints (e.g., DNA damage or apoptosis) and effects on the physiological level (e.g., DART or survival). The outcome of these studies may thus be beneficially used as input into broader conceptual frameworks such as AOPs and ERA (including high-throughput approaches). In this context it furthermore seems 
promising to assess less frequently examined endpoints (e.g., neurotoxicity or metabolic toxicity) that might affect population/community relevant endpoints (e.g., by exhausting ATP/energy resources or by inducing starvation responses).

\section{Integrated wastewater quality evaluation and perspectives on multifocal reduction of wastewater-borne emissions (2.4)}

- Bioassays have been beneficially implemented for evaluating the effectivity of conventional-biological and advanced WWTP processes. The present innovative evaluation concept followed an integrative approach involving chemical, (eco-)toxicological and microbial assessments for far-sighted and environmentally-relevant risk management.

- In the present effect-based evaluation, pilot scale ozonation combined with (nonaerated) GAC post-filtration $\left(\mathrm{O}_{3}+\mathrm{GAC}\right)$ proved to be the most effective treatment option out of five AWWT processes (namely ozonation, ozonation plus nonaerated or aerated GAC- or BF-post-filtration). This result was obtained using five in vitro and one in vivo test system analysing different MoA-based and environmentally relevant endpoints respectively. Despite the high removal effectivity of the $\mathrm{O}_{3}+\mathrm{GAC}$ process towards most effects, mutagenicity and antiestrogenic activity could not be completely eliminated calling for further investigation.

- The present bioassay battery demonstrated to be useful in evaluating AWWTs. In future studies further endpoints and "non-standard" bioassays should be examined for detecting additional (potentially neglected) effects. As "gold standard" long-term environmental monitoring of key indicator taxa in receiving water bodies before and after WWTP upgrades should be performed (fulfilling both principles 'ecological relevance' and 'environmental context').

- Other conventional, tertiary and advanced wastewater treatment processes as well as non-technical mitigation measures have been researched and evaluated as valuable additions and/or alternative solutions for further reducing WWTP emissions. By interdisciplinary and participatory approaches involving relevant stakeholders (e.g., municipalities, federal agencies, affiliated industries, educators and the general public) discussion and planning of sustainable (waste)water management becomes more realistic and compliant with regulations (e.g., the WFD or Urban Waste Water Directive). 
Therefore the following outlook highlights a few important management paradigms:

- Optimising conventional biological treatment by refining process parameters (e.g., HRTs or sludge ages), tertiary treatments (e.g., mechanical/biological postfiltrations, compare Triebskorn 2017, Völker et al. 2016, 2019) or further improvement of rain/mixed water retention basins.

- Closing smaller/inefficient WWTPs and centralization within larger WWTPs that offer better treatment of (micro)pollutants, toxicity and relevant parameters. Extending the connectivity of remote areas to sewer systems/WWTPs. Sewer systems should be increasingly screened for leakages and combined overflow systems installed where beneficial (Musolff et al. 2010, Phillips et al. 2012).

- Considering cost-benefit analyses and life cycle assessment (LCA) of WWTPs (e.g., Corominas et al. 2013, Papa et al. 2013, Pedrazzani et al. 2018) for creating a broader knowledge and decision base regarding investment and maintenance costs, energy and resource demands, infrastructural requirements, greenhouse gas emissions, ecological footprints and other important sustainability aspects.

- Source control measures such as at households (e.g., through more "ecofriendly" products and controlled discharge of wastes including expired pharmaceuticals), industries/public institutions (e.g., increased on-site pretreatment of hospital/industrial wastewater, Lienert et al. 2011) and agricultural land (e.g., buffer zones against nutrient-/pesticide-rich runoffs, Reichenberger et al. 2007, or transitions towards more 'ecological agriculture')

- In addition to WWTP-related measures for improving surface water quality, the overall drivers and factors of ecosystemic risks (such as hydromorphological and habitat degradation, invasive species and diffuse pollution sources) ought to be evaluated and tackled for sustainable water and wastewater management. This was one of the outcomes of a federal study (BMU 2013) that assessed multiple measures towards their impact on reaching the "good ecological status" of European surface waters. Thereby WWTP upgrades were predicted to contribute to $10 \%$, educational programs (compare above mentioned participatory approach) to $19 \%$, pollution prevention strategies (including source control) to $25 \%$ and river restoration campaigns to $46 \%$ to achieving this status (once performed in a site-specific and context-dependent manner). 


\section{References}

Abass K, Turpeinen M, Pelkonen O (2009) An evaluation of the cytochrome P450 inhibition potential of selected pesticides in human hepatic microsomes. Journal of Environmental Science and Health Part B 44, 553-563

Abdel-Shafy HI, Mansour MS (2016) A review on polycyclic aromatic hydrocarbons: source, environmental impact, effect on human health and remediation. Egyptian Journal of Petroleum 25(1), 107-123

Ahlf W, Hollert H, Neumann-Hensel H, Ricking M (2002) A guidance for the assessment and evaluation of sediment quality a German approach based on ecotoxicological and chemical measurements. Journal of Soils and Sediments 2(1), 37-42

Aitlhadj L, Stürzenbaum SR (2013) The toxicological assessment of two anti-obesity drugs in C. elegans. Toxicology Research 2(2), 145-150

Alberts B, Johnson A, Lewis J, Raff M, Roberts K, Walter P (2002) Caenorhabditis elegans: Development from the perspective of the individual cell. Molecular Biology of the Cell. 4th edition. New York: Garland Science

Allard P, Kleinstreuer NC, Knudsen TB, Colaiácovo MP (2013) A C. elegans screening platform for the rapid assessment of chemical disruption of germline function. Environmental Health Perspectives 121(6), 717-724

Allinson M, Shiraishi F, Salzman SA, Allinson G (2010) In vitro and immunological assessment of the estrogenic activity and concentrations of $17 \beta$-estradiol, estrone, and ethinyl estradiol in treated effluent from 45 wastewater treatment plants in Victoria, Australia. Archives of Environmental Contamination and Toxicology 58(3), 576-586

Allinson M, Shiraishi F, Allinson G (2011) A comparison of recombinant receptor-reporter gene bioassays and a total estrogen enzyme linked immunosorbent assay for the rapid screening of estrogenic activity in natural and waste waters. Bulletin of Environmental Contamination and Toxicology 86(5), 461

Altun ZF, Hall DH (2009) Introduction. In WormAtlas. doi:10.3908/wormatlas.1.1

Alda Álvarez O, Jager T, Colao BN, Kammenga JE (2006) Temporal dynamics of effect concentrations. Environmental Science \& Technology 40(7), 2478-2484

An JH, Blackwell TK (2003) SKN-1 links C. elegans mesendodermal specification to a conserved oxidative stress response. Genes development 17(15), 1882-1893

Anbalagan C, Lafayette I, Antoniou-Kourounioti M, Haque M, King J, Johnsen B, Baillie D, Gutierrez C, Rodriguez Martin JA, De Pomerai D (2012) Transgenic nematodes as biosensors for metal stress in soil pore water samples. Ecotoxicology 21(2), 439-455

Anbalagan C, Lafayette I, Antoniou-Kourounioti M, Gutierrez C, Martin JR, Chowdhuri DK, De Pomerai DI (2013) Use of transgenic GFP reporter strains of the nematode Caenorhabditis elegans to investigate the patterns of stress responses induced by pesticides and by organic extracts from agricultural soils. Ecotoxicology 22, 72-85

Anderson GL, Boyd WA, Williams P (2001) Assessment of sublethal endpoints for toxicity testing with the nematode Caenorhabditis elegans. Environmental Toxicology and Chemistry 20(4), 833-838

Anderson GL, Cole RD, Williams PL (2004) Assessing behavioral toxicity with Caenorhabditis elegans. Environmental Toxicology and Chemistry 23(5), 1235-1240

Anderson SL, Wild GC (1994) Linking genotoxic responses and reproductive success in ecotoxicology. Environmental Health Perspectives 102(Suppl 12), 9 
Angrish MM, Allard P, McCullough SD, Druwe IL, Chadwick LH, Hines E, Chorley BN (2018) Epigenetic applications in adverse outcome pathways and environmental risk evaluation. Environmental Health Perspectives 126(4), 045001

Antebi A (2006) Nuclear hormone receptors in C. elegans. In WormBook. The C. elegans Research Community. http://www.wormbook.org

Ashauer R (2016) Post-ozonation in a municipal wastewater treatment plant improves water quality in the receiving stream. Environmental Sciences Europe 28, 1

Ashauer R, Jager T (2018) Physiological modes of action across species and toxicants: the key to predictive ecotoxicology. Environmental Science: Processes \& Impacts 20(1), 48-57

Avila DS, Adams MR, Chakraborty S, Aschner M (2011) Chapter 16 - Caenorhabditis elegans as a Model to Assess Reproductive and Developmental Toxicity. In Gupta RC, Reproductive and Developmental Toxicology (193-205), Academic Press

Baberschke N, Steinberg CE, Saul N (2015) Low concentrations of dibromoacetic acid and $\mathrm{N}$-nitrosodimethylamine induce several stimulatory effects in the invertebrate model Caenorhabditis elegans. Chemosphere 124, 122-128

Backe WJ, Field JA (2012) Is SPE necessary for environmental analysis? A quantitative comparison of matrix effects from large-volume injection and solidphase extraction based methods. Environmental Science \& Technology 46 (12), 6750-6758

Backhaus T, Brack W, Van den Brink PJ, Deutschmann B, Hollert H, Posthuma L, Segner H, Seiler TB, Teodorovic I, Focks A (2019) Assessing the ecological impact of chemical pollution on aquatic ecosystems requires the systematic exploration and evaluation of four lines of evidence. Environmental Sciences Europe 31(1), 1-9

Backhaus T, Karlsson M (2014) Screening level mixture risk assessment of pharmaceuticals in STP effluents. Water Research 49, 157-165

Backman JT, Filppula AM, Niemi M, Neuvonen PJ (2016) Role of cytochrome P450 2C8 in drug metabolism and interactions. Pharmacological Reviews 68(1), 168-241

Baker DR, Kasprzyk-Hordern B (2011) Critical evaluation of methodology commonly used in sample collection, storage and preparation for the analysis of pharmaceuticals and illicit drugs in surface water and wastewater by solid phase extraction and liquid chromatography-mass spectrometry. Journal of Chromatography A 1218(44), 8036-8059

Baker M (2016) 1,500 scientists lift the lid on reproducibility. Nature 533(7604), 452-454

Barbosa MO, Moreira NF, Ribeiro AR, Pereira MF, Silva AM (2016) Occurrence and removal of organic micropollutants: an overview of the watch list of EU Decision 2015/495. Water Research 94, 257-279

Bargmann Cl (2006) Chemosensation in C. elegans. In WormBook. The C. elegans Research Community. http://www.wormbook.org

Baumstark-Khan C, Cioara K, Rettberg P, Horneck G (2005) Determination of geno- and cytotoxicity of groundwater and sediments using the recombinant SWITCH test. Journal of Environmental Science and Health 40(2), 245-263

Baun A, Jensen SD, Bjerg PL, Christensen TH, Nyholm N (2000) Toxicity of organic chemical pollution in groundwater downgradient of a landfill (Grindsted, Denmark). Environmental Science \& Technology 34(9), 1647-1652

Becker D, Rodriguez-Mozaz S, Insa S, Schoevaart R, Barceló D, de Cazes M, Belleville MP, Sanchez-Marcano J, Misovic A, OehImann J, Wagner M (2017) Removal of endocrine disrupting chemicals in wastewater by enzymatic treatment with fungal laccases. Organic Process Research \& Development 21(4), 480-491 
Begasse M, Leaver M, Vazquez F, Grill S, Hyman A (2015) Temperature dependence of cell division timing accounts for a shift in the thermal limits of $C$. elegans and $C$. briggsae. Cell Reports 10(5), 647-653

Behl M, Rice JR, Smith MV, Co CA, Bridge MF, Hsieh JH, Freedman JH, Boyd WA (2016) Comparative toxicity of organophosphate flame retardants and polybrominated diphenyl ethers to Caenorhabditis elegans. Toxicological Sciences 154(2), 241-252

Benner J, Ternes TA (2009) Ozonation of propranolol: Formation of oxidation products. Environmental Science \& Technology 43(13), 5086-5093

Benotti MJ, Trenholm RA, Vanderford BJ, Holady JC, Stanford BD, Snyder SA (2009) Pharmaceuticals and endocrine disrupting compounds in US drinking water. Environmental Science \& Technology 43(3), 597-603

Berger E, Haase P, Oetken M, Sundermann A (2016) Field data reveal low critical chemical concentrations for river benthic invertebrates. Science of the Total Environment 544, 864873

Bergqvist PA, Augulyte L, Jurjoniene V (2006) PAH and PCB removal efficiencies in Umea (Sweden) and Siauliai (Lithuania) municipal wastewater treatment plants. Water Air and Soil Pollution 175(1-4), 291-303

Berman HM, Westbrook J, Feng Z, Gilliland G, Bhat TN, Weissig H, Shindyalov IN, Bourne PE (2000) The Protein Data Bank. Nucleic Acids Research 28, 235-242

Bistan M, Podgorelec M, Marinšek Logar R, Tišler T (2012) Yeast estrogen screen assay as a tool for detecting estrogenic activity in water bodies. Food Technology and Biotechnology 50(4), 427-433

Bittner M, Saul N, Steinberg CE (2012) Antiandrogenic activity of humic substances. Science of the Total Environment 432, 93-96

Blackledge G (1993) Casodexmechanisms of action and opportunities for usage. Cancer 72(Suppl), 3830-3833

Blanchard M, Teil MJ, Ollivon D, Legenti L, Chevreuil M (2004) Polycyclic aromatic hydrocarbons and polychlorobiphenyls in wastewaters and sewage sludges from the Paris area (France). Environmental Research 95(2), 184-197

Bodhicharla R, Ryde IT, Prasad GL, Meyer JN (2014) The tobacco-specific nitrosamine 4(methylnitrosamino)-1-(3-pyridyl)-1-butanone (NNK) induces mitochondrial and nuclear DNA damage in Caenorhabditis elegans. Environmental and Molecular Mutagenesis 55(1), 43-50

Boehler M, Zwickenpflug B, Hollender J, Ternes T, Joss A, Siegrist H (2012) Removal of micropollutants in municipal wastewater treatment plants by powder-activated carbon. Water Science and Technology 66 (10), 2115-2121

Bollmann AF, Seitz W, Prasse C, Lucke T, Schulz W, Ternes T (2016) Occurrence and fate of amisulpride, sulpiride, and lamotrigine in municipal wastewater treatment plants with biological treatment and ozonation. Journal of Hazardous Materials 320, 204-215

Bougrier C, Battimelli A, Delgenes JP, Carrere H (2007) Combined ozone pretreatment and anaerobic digestion for the reduction of biological sludge production in wastewater treatment. Ozone: Science and Engineering 29(3), 201-206

Boxall ABA, Sinclair CJ, Fenner K, Kolpin D, Maund SJ (2004) When synthetic chemicals degrade in the environment. Environmental Science \& Technology 38, 368A-375A

Boyd WA, Cole RD, Anderson GL, Williams PL (2003) The effects of metals and food availability on the behavior of Caenorhabditis elegans. Environmental Toxicology and Chemistry 22(12), 3049-3055 
Boyd WA, Smith MV, Kissling GE, Freedman JH (2010) Medium-and high-throughput screening of neurotoxicants using C. elegans. Neurotoxicology and Teratology 32(1), 6873

Boyd WA, Williams PL (2003) Comparison of the sensitivity of three nematode species to copper and their utility in aquatic and soil toxicity tests. Environmental Toxicology and Chemistry 22, 2768-2774

Boyd, WA, Smith MV, Freedman JH (2012) Caenorhabditis elegans as a model in developmental toxicology. In Developmental Toxicology (15-24) Humana Press, Totowa, $\mathrm{NJ}$

Brack W, Dulio V, Ågerstrand M, Allan I, Altenburger R, Brinkmann M, Hernández FJ, Burgess RM, Cousins I, Escher BI, Hernández FJ, Hewitt LM, Hilscherová K, Hollender J, Hollert H, Kase R, Klauer B, Lindim C, Herráez Lopez D, Miège C, Munthe J, O'Toole S, Posthuma L, Rüdel H, Schäfer RB, Sengl M, Smedes F, van de Meent D, van den Brink $P$, van Gils J, van Wezel AP, Vethaak AD, Vermeirssen E, von der Ohe PC, Vrana B (2017) Towards the review of the European Union water framework management of chemical contamination in European surface water resources. Science of the Total Environment 576, 720-737

Brazma A, Hingamp P, Quackenbush J, Sherlock G, Spellman P, Stoeckert C, Aach J, Ansorge W, Ball CA, Causton HC, Gaasterland T, Glenisson P, Holstege FC, Kim IF, Markowitz V, Matese JC, Parkinson H, Robinson A, Sarkans U, Schulze-Kremer S, Stewart J, Taylor R, Vilo J, Vingron M (2001) Minimum information about a microarray experiment (MIAME) - toward standards for microarray data. Nature Genetics 29(4):365-371

Brettschneider DJ, Misovic A, Schulte-Oehlmann U, Oetken M, Oehlmann J (2019) Detection of chemically induced ecotoxicological effects in rivers of the Nidda catchment (Hessen, Germany) and development of an ecotoxicological, Water Framework Directivecompliant assessment system. Environmental Sciences Europe 31(1), 7

Brodin T, Piovano S, Fick J, Klaminder J, Heynen M, Jonsson M (2014) Ecological effects of pharmaceuticals in aquatic systems - impacts through behavioural alterations. Philosophical Transactions of the Royal Society B 369(1656), 20130580

Brooks BW, Sabo-Attwood T, Choi K, Kim S, Kostal J, LaLone CA, Langan LM, MargiottaCasaluci L, You J, Zhang X (2020) Toxicology advances for 21st century chemical pollution. One Earth 2(4), 312-316

Brown KH, Schultz IR, Nagler JJ (2007) Reduced embryonic survival in rainbow trout resulting from paternal exposure to the environmental estrogen 17 a-ethynylestradiol during late sexual maturation. Reproduction 134(5), 659-666

Bruni E, Simonetti G, Bovone B, Casagrande C, Castellani F, Riccardi C Pomata D, Di Filippo P, Federici E, Buiarelli F, Uccelletti D (2020) Evaluation of bioaerosol bacterial components of a wastewater treatment plant through an integrate approach and in vivo assessment. International Journal of Environmental Research and Public Health 17, 273

Bui XT, Vo TPT, Ngo HH, Guo WS, Nguyen TT (2016) Multicriteria assessment of advanced treatment technologies for micropollutants removal at large-scale applications. Science of the Total Environment 563, 1050-1067

Bundschuh M, Gessner MO, Fink G, Ternes TA, Sögding C, Schulz R (2011a) Ecotoxicologial evaluation of wastewater ozonation based on detritus-detritivore interactions. Chemosphere 82(3), 355-361

Bundschuh M, Pierstorf R, Schreiber WH, Schulz R (2011b) Positive effects of wastewater ozonation displayed by in situ bioassays in the receiving stream. Environmental Science \& Technology 45(8), 3774-3780 
Bunzel K, Kattwinkel M, Liess M (2013) Effects of organic pollutants from wastewater treatment plants on aquatic invertebrate communities. Water Research 47(2), 597-606

Burgess RM, Ho KT, Brack W, Lamoree M (2013) Effects-directed analysis (EDA) and toxicity identification evaluation (TIE): Complementary but different approaches for diagnosing causes of environmental toxicity. Environmental Toxicology and Chemistry 32(9), 1935-1945

Burns AR, Wallace IM, Wildenhain J, Tyers M, Giaever G, Bader GD, Nislow C, Cutler SR, Roy PJ (2010) A predictive model for drug bioaccumulation and bioactivity in Caenorhabditis elegans. Nature Chemical Biology 6(7), 549-557

Burton GA, Pitt R, Clark S (2000) The role of traditional and novel toxicity test methods in assessing stormwater and sediment contamination. Critical Reviews in Environmental Science and Technology 30(4), 413-447

Byerly L, Cassada RC, Russell RL (1976) The life cycle of the nematode Caenorhabditis elegans. Developmental Biology 51, 23-33

Calisto V, Esteves VI (2009) Psychiatric pharmaceuticals in the environment. Chemosphere $77(10), 1257-1274$

Camacho J, Truong L, Kurt Z, Chen YW, Morselli M, Gutierrez G, Pellegrini M, Yang X, Allard P (2018) The memory of environmental chemical exposure in C. elegans is dependent on the jumonji demethylases jmjd-2 and jmjd-3/utx-1. Cell Reports 23(8), 23922404

Cao X, Yan C, Wu X, Zhou L, Xiu G (2020a) Nonylphenol induced individual and population fluctuation of Caenorhabditis elegans: Disturbances on developmental and reproductive system. Environmental Research 186, 109486

Cao X, Yan C, Yang X, Zhou L, Zou W, Xiu G (2020b) Photolysis-induced neurotoxicity enhancement of chlorpyrifos in aquatic system: A case investigation on Caenorhabditis elegans. Journal of Agricultural and Food Chemistry 68(2), 461-470

Cassada RC, Russell RL (1975) The dauerlarva, a post-embryonic developmental variant of the nematode Caenorhabditis elegans. Developmental Biology 46(2), 326-342

Chakrabarti S, Dicke C, Kalderis D, Kern J (2015) Rice husks and their hydrochars cause unexpected stress response in the nematode Caenorhabditis elegans: reduced transcription of stress-related genes. Environmental Science and Pollution Research 22(16), 12092-12103

Chakrapani BP, Kumar S, Subramaniam JR (2008) Development and evaluation of an in vivo assay in Caenorhabditis elegans for screening of compounds for their effect on cytochrome P 450 expression. Journal of Biosciences 33(2), 269

Chalfie M (2009) GFP: Lighting up life. Proceedings of the National Academy of Sciences 106(25), 10073-10080

Chang HS, Choo KH, Lee B, Choi SJ (2009) The methods of identification, analysis, and removal of endocrine disrupting compounds (EDCs) in water. Journal of Hazardous Materials 172(1), 1-12

Chen J, Caswell-Chen EP (2003) Bagging as a part of the C. elegans life cycle. International Worm Meeting. Citeulike: 3307195

Chen Y, Panter B, Hussain A, Gibbs K, Ferreira D, Allard P (2019) BPA interferes with StAR-mediated mitochondrial cholesterol transport to induce germline dysfunctions. Reproductive Toxicology 90, 24-32 
Chen Y, Shu L, Quu Z, Lee DY, Settle SJ, Hee SQ., Telesca D, Yang X, Allard P (2016) Exposure to the BPA-substitute bisphenol $S$ causes unique alterations of germline function. PLoS Genetics 12(7), e1006223

Chen Z, Li M, Wen Q (2017) Comprehensive evaluation of three sets of advanced wastewater treatment trains for treating secondary effluent: Organic micro-pollutants and bio-toxicity. Chemosphere 189, 426-434

Cheng Z, Tian H, Chu H, Wu J, Li Y, Wang Y (2014) The effect of tributyltin chloride on Caenorhabditis elegans germline is mediated by a conserved DNA damage checkpoint pathway. Toxicology Letters 225(3), 413-421

Chisholm AD, Xu S (2012) The C. elegans epidermis as a model skin. II: differentiation and physiological roles. Wiley Interdisciplinary Reviews. Developmental Biology 1(6), 879-902

Choi JH (2008) Caenorhabditis elegans as a biological model for multilevel biomarker analysis in environmental toxicology and risk assessment. Toxicological Research 24(4), 235-243

Clavijo A, Kronberg MF, Rossen A, Moya A, Calvo D, Salatino SE, Pagano A, Morábito JA, Munarriz ER (2016) The nematode Caenorhabditis elegans as an integrated toxicological tool to assess water quality and pollution. Science of the Total Environment 569, 252-261

Colbourne JK, Singan VR, Gilbert DG (2015) wFleaBase: the Daphnia genome database. BMC Bioinformatics 6(1), 45

Cong Y, Yang H, Zhang P, Xie Y, Cao X, Zhang L (2020) Transcriptome analysis of the nematode Caenorhabditis elegans in acidic stress environments. Frontiers in Physiology 11,1107

Connon RE, Geist J, Werner I (2012) Effect-based tools for monitoring and predicting the ecotoxicological effects of chemicals in the aquatic environment. Sensors 12(9), 1274112771

Coomans AR, Vanderhaeghen (1985) In vitro testing toxicities. In Zuckerman BM, Mai WF, Harrison MB. Plant nematology laboratory manual 29, 173-176. University of Massachusetts

Cordell D, Drangert JO, White S (2009) The story of phosphorus: Global food security and food for thought. Global Environmental Change 19 (2), 292-305

Corish P, Tyler-Smith C (1999) Attenuation of green fluorescent protein half-life in mammalian cells. Protein Engineering 12(12), 1035-1040

Corominas L, Foley J, Guest JS, Hospido A, Larsen HF, Morera S, Shaw A (2013) Life cycle assessment applied to wastewater treatment: state of the art. Water Research 47(15), 5480-5492

Corsi A. K, Wightman, B, Chalfie, M. (2015) A transparent window into biology: a primer on Caenorhabditis elegans. Genetics 200(2), 387-407

Corsi, AK (2006) A biochemist's guide to C. elegans. Analytical Biochemistry 359(1), 1

Cui Y, McBride SJ, Boyd WA, Alper S, Freedman JH (2007) Toxicogenomic analysis of Caenorhabditis elegans reveals novel genes and pathways involved in the resistance to cadmium toxicity. Genome Biology 8, R122

Custodia N, Won SJ, Novillo A, Wieland M, Li C, Callard IP (2001) Caenorhabditis elegans as an environmental monitor using DNA microarray analysis. Annals of the New York Academy of Sciences 948(1), 32-42

Cwiertny DM, Snyder SA, Schlenk D, Kolodziej EP (2014) Environmental designer drugs: when transformation may not eliminate risk. Environmental Science \& Technology 48(20), $11737-11745$ 
Da Costa JB, Rodgher S, Daniel LA, Espíndola ELG (2014) Toxicity on aquatic organisms exposed to secondary effluent disinfected with chlorine, peracetic acid, ozone and UV radiation. Ecotoxicology 23, 1803-1813

Dabrowski JM, Shadung JM, Wepener V (2014) Prioritizing agricultural pesticides used in South Africa based on their environmental mobility and potential human health effects. Environment International 62, 31-40

Dafforn KA, Lewis JA, Johnston EL (2011) Antifouling strategies: history and regulation, ecological impacts and mitigation. Marine Pollution Bulletin 62(3), 453-465

Dagnino S, Gomez E, Picot B, Cavaillès V, Casellas C, Balaguer P, Fenet H (2010) Estrogenic and AhR activities in dissolved phase and suspended solids from wastewater treatment plants. Science of the Total Environment 408(12), 2608-2615

Daniels KD, VanDervort D, Wu S, Leusch FD, van de Merwe, JP, Jia A, Snyder SA (2018) Downstream trends of in vitro bioassay responses in a wastewater effluent-dominated river. Chemosphere 212, 182-192

Darby C (2005) Interactions with microbial pathogens. In WormBook. The C. elegans Research Community. http://www.wormbook.org

Daughton CG (2003) Cradle-to-cradle stewardship of drugs for minimizing their environmental disposition while promoting human health. I. Rationale for and avenues toward a green pharmacy. Environmental Health Perspectives 111(5), 757-774

David HE, Dawe AS, de Pomerai DI, Jones D, Candido EPM, Daniells C (2003) Construction and evaluation of a transgenic hsp16-GFP-lacZ Caenorhabditis elegans strain for environmental monitoring. Environmental Toxicology and Chemistry 22(1), 111-118

Davis AP, Grondin CJ, Johnson RJ, Sciaky D, King BL, McMorran R, Wiegers J, Wiegers TC, Mattingly CJ (2017) The Comparative Toxicogenomics Database: Nucleic Acids Research 45 (D1), D972-D978

De Alda MJL, Barceló D (2001) Use of solid-phase extraction in various of its modalities for sample preparation in the determination of estrogens and progestogens in sediment and water. Journal of Chromatography A 938(1), 145-153

De Baat ML, Van der Oost R, Van der Lee GH, Wieringa N, Hamers T, Verdonschot P, De Voogt P, Kraak M (2020) Advancements in effect-based surface water quality assessment. Water Research 183, 116017

De Bono M (2003) Molecular approaches to aggregation behavior and social attachment. Developmental Neurobiology 54(1), 78-92

De Pomerai D, Anbalagan C, Lafayette I, Rajagopalan D, Loose M, Haque M, King J (2010) High-throughput analysis of multiple stress pathways using GFP reporters in C. elegans. Environmental Toxicology 132, 177-187

Demarche P, Junghanns C, Nair RR, Agathos SN (2012) Harnessing the power of enzymes for environmental stewardship. Biotechnology Advances 30(5), 933-953

Di Benedetto (2009). Ökotoxikologische Charakterisierung mobiler organischer Fremdstoffe aus dem Hessischen Ried (English title: „Ecotoxicological characterization of mobile organic xenobiotics from the Hessian Ried"). PhD thesis. Johann Wolfgang GoetheUniversität, Frankfurt, Germany. URN:nbn:de:hebis:30-74216

Dopp E, Pannekens H, Gottschlich A, Schertzinger G, Gehrmann L, Kasper-Sonnenberg M, Richard J, Joswig M, Grummt T, Schmidt TC, Wilhelm M, Tuerk J (2021) Effect-based evaluation of ozone treatment for removal of micropollutants and their transformation products in waste water. Journal of Toxicology and Environmental Health. Part A 1-22 
Duft M (2004) Ökotoxikologische Sedimentbewertung großer Fließgewässer mit Nematoden und Gastropoden. Vom Biotest zum Freiland (English title "Ecotoxicological sediment assessment of large rivers with nematodes and gastropods. From the bioassay to the field"). Cuvillier Verlag, Göttingen, Germany (Accessed under https://cuvillier.de/de/shop/publications/2984 in February 2018)

Dutilleul M, Bonzom JM, Lecomte C, Goussen B, Daian F, Galas S, Réale D (2014) Rapid evolutionary responses of life history traits to different experimentally-induced pollutions in Caenorhabditis elegans. BMC Evolutionary Biology 14(1), 252

Eggen RI, Behra R, Burkhardt-Holm P, Escher BI, Schweigert N (2004) Challenges in ecotoxicology. Environmental Science \& Technology 38(3), 58A-64A

Ensembl (2018) Ensembl comparative genomics resources. Database query on "Gene: cyp-35A3 (WBGene00019565)". Retrieved under http://www.ensembl.org/Caenorhabditis_elegans/Gene/Summary?g=WBGene00019565;r =V:4022609-4024475;t=K09D9.2 (Accessed data January 2018)

Eom HJ, Kim H, Kim BM, Chon TS, Choi J (2014) Integrative assessment of benzene exposure to Caenorhabditis elegans using computational behavior and toxicogenomic analyses. Environmental Science \& Technology 48(14), 8143-8151

Escher BI, Allinson M, Altenburger R, Bain PA, Balaguer P, Busch W, Crago J, Denslow ND, Dopp E, Hilscherova K, Humpage AR, Kumar A, Grimaldi M, Jayasinghe BS, Jarosova B, Jia A, Makarov S, Maruya KA, Medvedev A, Mehinto AC, Mendez JE, Poulsen A, Prochazka E, Richard J, Schifferli A, Schlenk D, Scholz S, Shiraishi F, Snyder S, Su G, Tang JY, van der Burg B, van der Linden SC, Werner I, Westerheide SD, Wong CK, Yang $\mathrm{M}$, Yeung BH, Zhang X, Leusch FD (2014) Benchmarking organic micropollutants in wastewater, recycled water and drinking water with in vitro bioassays. Environmental Science \& Technology 48(3), 1940-1956

Escher BI, Aït-Aïssa S, Behnisch PA, Brack W, Brion F, Brouwer A, Buchinger S, Crawford SE, Du Pasquier D, Hamers T, Hettwer K, Hilscherová K, Hollert H, Kase R, Kienle C, Tindall AJ, Tuerk J, van der Oost R, Vermeirssen E, Neale PA (2018) Effect-based trigger values for in vitro and in vivo bioassays performed on surface water extracts supporting the environmental quality standards (EQS) of the European Water Framework Directive. Science of the Total Environment 628, 748-765.

Escher BI, Bramaz N, Maurer M, Richter M, Sutter D, von Känel C, Zschokke M (2005) Screening test battery for pharmaceuticals in urine and wastewater. Environmental Toxicology and Chemistry 24(3), 750-758

Escher BI, Bramaz N, Ort C (2009) JEM Spotlight: Monitoring the treatment efficiency of a full scale ozonation on a sewage treatment plant with a mode-of-action based test battery. Journal of Environmental Monitoring 11(10), 1836-1846

Escher BI, Fenner K (2011) Recent advances in environmental risk assessment of transformation products. Environmental Science \& Technology 45(9), 3835-47

Escher BI, Hackermüller J, Polte T, Scholz S, Aigner A, Altenburger R, Böhme A, Bopp SK, Brack W, Busch W, Chadeau-Hyam M, Covaci A, Eisenträger A, Galligan JJ, Garcia-Reyero N, Hartung T, Hein M, Herberth G, Jahnke A, Kleinjans J, Klüver N, Krauss M, Lamoree M,, Lehmann I, Luckenbach T, Miller GW, Müller A, Phillips DH, Reemtsma T, Rolle-Kampczyk U, Schüürmann G, Schwikowski B, Tan YM, Trump S, Walter-Rohde S, Wambaugh JF (2017) From the exposome to mechanistic understanding of chemical-induced adverse effects. Environment International 99, 97-106

Escher BI, Hermens JL (2002) Modes of action in ecotoxicology: their role in body burdens, species sensitivity, QSARs, and mixture effects. Environmental Science \& Technology 36(20), 4201-4217 
Esvelt LA, Kaufman WJ, Selleck RE (1973) Toxicity assessment of treated municipal wastewaters. Journal of the Water Pollution Control Federation 45(7),1558-72

European Bioinformatics Institute (EBI, 2018) Expression Atlas, an open public repository of gene expression pattern data under different biological conditions. https://www.ebi.ac.uk/gxa/home (Accessed on 30.04.2018)

European Commission (2012) Report from the Commission to the European Parliament and the Council on the Implementation of the Water Framework Directive (2000/60/EC) River Basin Management Plans. COM/2012/0670

European Council (1991) Directive of 21 May 1991 concerning urban waste-water treatment (91/271/EEC). Official Journal L 135, 40-52

European Council (1998) Directive of 3 November 1998 on the quality of water intended for human consumption. Official Journal L 330, 32-54

European Parliament and Council (2000) Directive 2000/60/EG of the European Parliament and of the Council of 23 October 2000 establishing a framework for Community action in the field of water policy. Official Journal L 327, 1-73

European Parliament and Council (2008) Directive 2008/105/EC of the European Parliament and of the Council of 16 December 2008 on environmental quality standards in the field of water policy, amending and subsequently repealing Council Directives 82/176/EEC, 83/513/EEC, 84/156/EEC, 84/491/EEC, 86/280/EEC and amending Directive 2000/60/EC of the European Parliament and of the Council. Official Journal L 348, 84-97

European Parliament and Council (2013) Directive 2013/39/EU of the European Parliament and of the Council of 12 August 2013 amending Directives 2000/60/EC and 2008/105/EC as regards priority substances in the field of water policy. Official Journal L 226, 1-17

Fajardo C, Martín M, Nande M, Botías P, García-Cantalejo J, Mengs G, Costa G (2020) Ecotoxicogenomic analysis of stress induced on Caenorhabditis elegans in heavy metal contaminated soil after nZVI treatment. Chemosphere 126909

Feder ME, Walser JC (2005) The biological implications of transcriptomics in elucidating stress and stress responses. Journal of Evolutionary Biology 18(4), 901-910

Federal Ministry for the Environment, Nature Conservation and Nuclear Safety (BMU, 2013)

Die Wasserrahmenrichtlinie. Eine Zwischenbilanz zur Umsetzung der Maßnahmenprogramme 2012 (English title: "The Water Framework Directive. An interim report on the implementation of the programs of measures 2012"). Accessed under: https://www.umweltbundesamt.de/en/publikationen/die-wasserrahmenrichtlinie-eine-

zwischenbilanz-zur

Félix MA, Braedle C (2010) The natural history of Caenorhabditis elegans. Current Biology 20, R965-R969

Fent K, Chew G, Li J, Gomez E (2014) Benzotriazole UV-stabilizers and benzotriazole: antiandrogenic activity in vitro and activation of aryl hydrocarbon receptor pathway in zebrafish eleuthero-embryos. Science of the Total Environment 482, 125-136

Ferreira DW, Chen Y, Allard $P$ (2014) Using the alternative model C. elegans in reproductive and developmental toxicology studies. In Faqi A, Developmental and Reproductive Toxicology. Methods in Pharmacology and Toxicology. Humana Press, New York, NY

Fick J, Lindberg RH, Parkkonen J, Arvidsson B, Tysklind M, Larsson DGJ (2010) Therapeutic levels of levonorgestrel detected in blood plasma of fish: Results from screening rainbow trout exposed to treated sewage effluents. Environmental Science \& Technology 44(7), 2661-2666

Fielenbach N, Antebi A (2008) C. elegans dauer formation and the molecular basis of plasticity. Genes \& Development 22(16), 2149-2165 
Filby AL, Shears JA, Drage BE, Churchley JH, Tyler CR (2010) Effects of advanced treatments of wastewater effluents on estrogenic and reproductive health impacts in fish. Environmental Science \& Technology 44(11), 4348-4354

Fischer BB, Pomati F, Eggen RI (2013) The toxicity of chemical pollutants in dynamic natural systems: the challenge of integrating environmental factors and biological complexity. Science of the Total Environment 449, 253-259

Forsgren AJ (2015) Wastewater Treatment: Occurrence and fate of polycyclic aromatic hydrocarbons (PAHs). CRC Press, Tayler \& Francis, Boca Raton

Freedman JH, Slice LW, Dixon D, Fire A, Rubin CS (1993) The novel metallothionein genes of Caenorhabditis elegans. Structural organization and inducible, cell-specific expression. Journal of Biological Chemistry 268(4), 2554-2564

Fries E, Püttmann W (2004) Monitoring of the antioxidant BHT and its metabolite BHT-CHO in German river water and ground water. Science of the Total Environment 319(1-3), 269282

Fueser $\mathrm{H}$, Majdi N, Haegerbaeumer A, Pilger C, Hachmeister H, Greife P, Huser T, Traunspurger W (2018) Analyzing life-history traits and lipid storage using CARS microscopy for assessing effects of copper on the fitness of Caenorhabditis elegans. Ecotoxicology and Environmental Safety 156, 255-262

Gaillard M (1996) Pharmacodynamics and pharmacokinetics of nilutamide in animal and man. In Antiandrogens in Prostate Cancer, 95-103. ESO Monographs (European School of Oncology). Springer, Berlin, Heidelberg

García-Espiñeira MC, Tejeda-Benítez LP, Olivero-Verbel J (2018) Toxic effects of bisphenol A, propyl paraben, and triclosan on Caenorhabditis elegans. International Journal of Environmental Research and Public Health 15(4), 684

Gartiser S, Hafner C, Hercher C, Kronenberger-Schäfer K, Paschke A (2010) Whole effluent assessment of industrial wastewater for determination of bat compliance. Environmental Science and Pollution Research 17(4), 856-865

Gerhardt A, Schmidt S, Höss S (2002) Measurement of movement patterns of Caenorhabditis elegans (Nematoda) with the Multispecies Freshwater Biomonitor ${ }^{\circledR}$ (MFB) - a potential new method to study a behavioral toxicity parameter of nematodes in sediments. Environmental Pollution 120(3), 513-516

Giebner S, Ostermann S, Straskraba S, Oetken M, Oehlmann J, Wagner M (2018) Effectivity of advanced wastewater treatment: reduction of in vitro endocrine activity and mutagenicity but not of in vivo reproductive toxicity. Environmental Science and Pollution Research 25(5), 3965-3976

Golden JW, Riddle DL (1984) The Caenorhabditis elegans dauer larva: developmental effects of pheromone, food, and temperature. Developmental Biology 102(2), 368-378

Gordon MA, D'Amato NC, Gu H, Babbs B, Wulfkuhle J, Petricoin EF, Gallagher I, Dong T, Torkko K, Liu B, Elias A, Richer JK (2017) Synergy between androgen receptor antagonism and inhibition of mTOR and HER2 in breast cancer. Molecular Cancer Therapy 16(7), 13891400

Gotoh O (1998). Divergent structures of Caenorhabditis elegans cytochrome P450 genes suggest the frequent loss and gain of introns during the evolution of nematodes. Molecular Biology and Evolution 15(11), 1447-1459

Gottschalk C, Libra JA, Saupe A (2009) Ozonation of water and waste water: A practical guide to understanding ozone and its applications. Wiley $\mathrm{VCH}$, Weinheim. John Wiley \& Sons, Hoboken, New Jersey, USA 
Graves AL, Boyd WA, Williams PL (2005) Using transgenic Caenorhabditis elegans in soil toxicity testing. Archives of Environmental Contamination Toxicology 48(4), 490-494

Gros M, Petrovic M, Barcelo D (2006) Multi-residue analytical methods using LC-tandem MS for the determination of pharmaceuticals in environmental and wastewater samples: a review. Analytical and Bioanalytical Chemistry 386(4), 941-952

Grover DP, Balaam J, Pacitto S, Readman JW, White S, Zhou J L (2011) Endocrine disrupting activities in sewage effluent and river water determined by chemical analysis and in vitro assay in the context of granular activated carbon upgrade. Chemosphere 84, $1512-$ 1520

Haegerbaeumer A, Höss S, Heininger P, Traunspurger W (2018) Is Caenorhabditis elegans representative of freshwater nematode species in toxicity testing? Environmental Science and Pollution Research 25(3), 2879-2888

Haegerbaeumer A, Höss S, Traunspurger W (2019) Nematode-Based Effect Assessment in Freshwater Sediments. In: Methods in Pharmacology and Toxicology. Springer Protocolls. Humana Press

Hägerbäumer A, Höss S, Heininger P, Traunspurger W (2015) Experimental studies with nematodes in ecotoxicology: an overview. Journal of Nematology 47(1), 11-27

Haitzer M, Burnison BK, Höss S, Traunspurger W, Steinberg CE (1999) Effects of quantity, quality, and contact time of dissolved organic matter on bioconcentration of benzo[a]pyrene in the nematode Caenorhabditis elegans. Environmental Toxicology and Chemistry 18(3), 459-465

Hall J, Haas KL, Freedman JH (2012) Role of MTL-1, MTL-2, and CDR-1 in mediating cadmium sensitivity in Caenorhabditis elegans. Toxicological Sciences 128(2), 418-426

Han X, Zuo YT, Hu Y, Zhang J, Zhou MX, Chen M, Tang F, Lu WQ, Liu AL (2018) Investigating the performance of three modified activated sludge processes treating municipal wastewater in organic pollutants removal and toxicity reduction. Ecotoxicology and Environmental Safety 148, 729-737

Harju K, Rapinoja ML, Avondet MA, Arnold W, Schär M, Burrell S, LuginbühI W, Vanninen $P$ (2015) Optimization of sample preparation for the identification and quantification of saxitoxin in proficiency test mussel sample using liquid chromatography-tandem mass spectrometry. Toxins 7(12), 4868-4880

Harlow PH, Perry SJ, Stevens AJ, Flemming AJ (2018) Comparative metabolism of xenobiotic chemicals by cytochrome P450s in the nematode Caenorhabditis elegans. Scientific Reports 8(1), 1-8

Harlow PH, Perry SJ, Widdison S, Daniels S, Bondo E, Lamberth C, Currie RA, Flemming AJ (2016) The nematode Caenorhabditis elegans as a tool to predict chemical activity on mammalian development and identify mechanisms influencing toxicological outcome. Scientific Reports 6, 22965

Harris CA, Routledge EJ, Schaffner C, Brian JV, Giger W, Sumpter JP (2007) Benzotriazole is antiestrogenic in vitro but not in vivo. Environmental Toxicology and Chemistry 26(11), 2367-2372

Harris JB, Hartman JH, Luz AL, Wilson JY, Dinyari A, Meyer JN (2020) Zebrafish CYP1A expression in transgenic Caenorhabditis elegans protects from exposures to benzo[a]pyrene and a complex polycyclic aromatic hydrocarbon mixture. Toxicology 440 , 152473

Hartman PS, Herman RK (1982) Radiation-sensitive mutants of Caenorhabditis elegans. Genetics 102, 159-178 
Heberer T (2002) Tracking persistent pharmaceutical residues from municipal sewage to drinking water. Journal of Hydrology 266(3), 175-189

Heidler J, Sapkota A, Halden RU (2006) Partitioning, persistence, and accumulation in digested sludge of the topical antiseptic triclocarban during wastewater treatment. Environmental Science \& Technology 40(11), 3634-3639

Henneberg A, Bender K, Blaha L, Giebner S, Kuch B, Köhler HR, Maier D, Oehlmann J, Richter D, Scheurer M, Schulte-Oehlmann U, Sieratowicz A, Ziebart S, Triebskorn R (2014) Are in vitro methods for the detection of endocrine potentials in the aquatic environment predictive for in vivo effects? Outcomes of the projects SchussenAktiv and SchussenAktivplus in the Lake Constance area, Germany. PloS One 9(6), e98307

Henry ND, Fair PA (2013) Comparison of in vitro cytotoxicity, estrogenicity and antiestrogenicity of triclosan, perfluorooctane sulfonate and perfluorooctanoic acid. Journal of Applied Toxicology 33(4):265-72

Hicks KA, Fuzzen ML, McCann EK, Arlos MJ, Bragg LM, Kleywegt S, Tetreault GR, McMaster ME, Servos MR (2017) Reduction of intersex in a wild fish population in response to major municipal wastewater treatment plant upgrades. Environmental Science \& Technology 51(3), 1811-1819

Hillebrand O, Musallam S, Scherer L, Nodler K, Licha T (2013) The challenge of samplestabilisation in the era of multi-residue analytical methods: a practical guideline for the stabilisation of 46 organic micropollutants in aqueous samples. Science of the Total Environment 454, 289-298

Hirschmann, H (1952) Die Nematoden der Wassergrenze mittelfränkischer Gewässer (Englisch title "The nematodes of the water frontier of Middle-Franconian waters"). Zoologische Jahrbücher Abteilung für Systematik, Ökologie und Geographie der Tiere 81(4), 313-407

Hitchcock DR, Black MC, Williams PL (1997) Investigations into using the nematode Caenorhabditis elegans for municipal and industrial wastewater toxicity testing. Archives of Environmental Contamination and Toxicology 33, 252-260

Hitchcock DR, Law SE, Wu J, Williams PL (1998) Determining toxicity trends in the ozonation of synthetic dye wastewaters using the nematode Caenorhabditis elegans. Archives of Environmental Contamination and Toxicology 34, 259-264

Hodgkin J (1988) Sexual dimorphism and sex determination. In Wood WB, The nematode C. elegans. Cold Spring Harbor Laboratory Press, Cold Spring Harbor, New York, USA

Hofmann ER, Milstein S, Boulton SJ, Ye M, Hofmann JJ, Stergiou L, Gartner A, Vidal M, Hengartner MO (2002) Caenorhabditis elegans HUS-1 is a DNA damage checkpoint protein required for genome stability and EGL-1-mediated apoptosis. Current Biology 12(22), 19081918

Hope IA (1999). C. elegans: A Practical Approach. Oxford University Press, Oxford, UK

Höss S, Ahlf W, Bergtold M, Bluebaum-Gronau E, Brinke M, Donnevert G, Menzel R, Möhlenkamp C, Ratte HT, Traunspurger W, von Danwitz B, Pluta HJ (2012) Interlaboratory comparison of a standardized toxicity test using the nematode Caenorhabditis elegans (ISO 10872). Environmental Toxicololgy and Chemistry 31(7), 1525-35

Höss S, Bergtold M, Haitzer M, Traunspurger W, Steinberg CE (2001) Refractory dissolved organic matter can influence the reproduction of Caenorhabditis elegans (Nematoda). Freshwater Biology 46, 1-10

Höss S, Fritzsche A, Meyer C, Bosch J, Meckenstock RU, Totsche KU (2015) Size- and composition-dependent toxicity of synthetic and soil-derived Fe oxide colloids for the nematode Caenorhabditis elegans. Environment Science Technology 49(1), 544-552 
Höss S, Jänsch S, Moser T, Junker T, Römbke J (2009) Assessing the toxicity of contaminated soils using the nematode $C$. elegans as test organism. Ecotoxicology and Environmental Safety 72(7), 1811-1818

Höss S, Heininger P, Claus E, Möhlenkamp C, Brinke M, Traunspurger W (2017) Validating the NemaSPEAR [\%]-index for assessing sediment quality regarding chemical-induced effects on benthic communities in rivers. Ecological Indicators 73, 52-60

Höss S, Römbke J (2019) Effects of waste materials on Caenorhabditis elegans (Nematoda) using the ISO standard soil toxicity test. Environmental Science and Pollution Research 26, 26304-26312

Höss S, Weltje L (2007) Endocrine disruption in nematodes: effects and mechanisms. Ecotoxicology 16(1), 15-28

Howe KL, Bolt BJ, Shafie M, Kersey P, Berriman M (2017) WormBase ParaSite - a comprehensive resource for helminth genomics. Molecular and Biochemical Parasitology 215, 2-10

Hu XF, Veroni M, de Luise M, Wakeling A, Sutherland R, Watts CKW, Zalcberg JR (1993) Circumvention of tamoxifen resistance by the pure anti-estrogen $\mathrm{ICl} 182,780$. International Journal of Cancer 55, 873-876

Huang GY, Liu YS, Chen XW, Liang YQ, Liu SS, Yang YY, Hu LX, Shi WJ, Tian F, Zhao JL, Chen J, Ying GG (2016) Feminization and masculinization of western mosquitofish (Gambusia affinis) observed in rivers impacted by municipal wastewaters. Scientific Reports 6, 20884

Huggett DB, Foran CM, Brooks BW, Weston J, Peterson B, Marsh KE, La Point TW, Schlenk D (2003) Comparison of in vitro and in vivo bioassays for estrogenicity in effluent from North American municipal wastewater facilities. Toxicological Sciences 72(1), 77-83

Hunt PR (2016) The C. elegans model in toxicity testing. Journal of Applied Toxicology $37(1), 50-59$

Ihara M, Ihara MO, Kumar V, Narumiya M, Hanamoto S, Nakada N, Yamashita N, Miyagawa S, Iguchi T, Tanaka H (2014) Co-occurrence of estrogenic and antiestrogenic activities in wastewater: quantitative evaluation of balance by in vitro ERa reporter gene assay and chemical analysis. Environmental Science \& Technology 48(11), 6366-6373

Inokuchi A, Nihira M, Minakoshi M, Yamamoto R, Ishibashi H, Tominaga N, Arizono $\mathrm{K}$ (2014) Comparative study of the biological effects of antimicrobials, triclosan and trichlocarban, for C. elegans. Journal of Environmental Safety 5(2), 95-98

Inoue H, Hisamoto N, An JH, Oliveira RP, Nishida E, Blackwell TK, Matsumoto K (2005) The $C$. elegans p38 MAPK pathway regulates nuclear localization of the transcription factor SKN-1 in oxidative stress response. Genes development 19(19), 2278-2283

International Organization for Standardization (ISO, 1996) Water Quality - Determination of the Acute Lethal Toxicity of Substances to a Freshwater Fish (Brachydanio rerio Hamilton Buchanan, Teleostei, Cyprinidae) Part 1: Static Method. ISO 7346-1, Geneva, Switzerland

Itzel F, Baetz N, Hohrenk LL, Gehrmann L, Antakyali D, Schmidt TC, Tuerk J (2020) Evaluation of a biological post-treatment after full-scale ozonation at a municipal wastewater treatment plant. Water Research 170, 115316

Itzel F, Gehrmann L, Teutenberg T, Schmidt TC, Tuerk J (2019) Recent developments and concepts of effect-based methods for the detection of endocrine activity and the importance of antagonistic effects. Trends in Analytical Chemistry 118, 699-708

Jackson RB, Carpenter SR, Dahm CN, McKnight, DM, Naiman, RJ, Postel SL, Running SW (2001) Water in a changing world. Ecological Applications 11(4), 1027-1045 
Jager T, Álvarez OA, Kammenga JE, Kooijman SALM (2005) Modelling nematode life cycles using dynamic energy budgets. Functional Ecology 19(1), 136-144

Jager T, Ashauer R (2018) Modelling survival under chemical stress. A comprehensive guide to the GUTS framework. E-book available under https://leanpub.com/guts_book. Acessed in Dezember 2018

Jager T, Gudmundsdóttir EM, Cedergreen N (2014) Dynamic modeling of sublethal mixture toxicity in the nematode Caenorhabditis elegans. Environmental Science \& Technology 48(12), 7026-7033

Janex-Habibi, ML, Huyard A, Esperanza M, Bruchet A (2009) Reduction of endocrine disruptor emissions in the environment: the benefit of wastewater treatment. Water Research 43(6), 1565-1576

Janošek J, Bittner M, Hilscherová K, Bláha L, Giesy JP, Holoubek I (2007) AhR-mediated and antiestrogenic activity of humic substances. Chemosphere 67(6), 1096-1101

Jekel M, Ruhl AS (2016) Anthropogene Spurenstoffe und Krankheitserreger im urbanen Wasserkreislauf, Bewertung, Barrieren und Risikokommunikation, ASKURIS (English title: Anthropogenic organic micro-pollutants and pathogens in the urban water cycle, assessment, barriers and risk communication, ASKURIS). University Press of the Technical University Berlin, Germany

Jeong J, Song T, Chatterjee N, Choi I, Cha YK, Choi J (2018) Developing adverse outcome pathways on silver nanoparticle-induced reproductive toxicity via oxidative stress in the nematode Caenorhabditis elegans using a Bayesian network model. Nanotoxicology 12(10), 1182-1197

Jia A, Escher BI, Leusch FD, Tang JY, Prochazka E, Dong B, Snyder EM, Snyder SA (2015) In vitro bioassays to evaluate complex chemical mixtures in recycled water. Water Research, 80, 1-11

Jobling S, Beresford N, Nolan M, Rodgers-Gray T, Brighty GC, Sumpter JP, Tyler CR (2002) Altered sexual maturation and gamete production in wild roach (Rutilus rutilus) living in rivers that receive treated sewage effluents. Biological Reproduction 66 (2), 272-281

Jobling S, Nolan M, Tyler CR, Brighty G, Sumpter JP (1998) Widespread sexual disruption in wild fish. Environmental Science \& Technology 32 (17), 2498-2506

Johnson EF, Stout CD (2005) Structural diversity of human xenobiotic-metabolizing cytochrome P450 monooxygenases. Biochemical and Biophysical Research Communications 338(1), 331-336

Jones D, Candido EPM (1999) Feeding is inhibited by sublethal concentrations of toxicants and by heat stress in the nematode Caenorhabditis elegans: relationship to the cellular stress response. Journal of Experimental Zoology 284(2), 147-157

Jones LM, Flemming AJ, Urwin PE (2015) NHR-176 regulates cyp-35d1 to control hydroxylation-dependent metabolism of thiabendazole in Caenorhabditis elegans. Biochemical Journal 466(1), 37-44

Jones LM, Rayson SJ, Flemming AJ, Urwin, PE (2013) Adaptive and specialised transcriptional responses to xenobiotic stress in Caenorhabditis elegans are regulated by nuclear hormone receptors. PLoS One 8(7), e69956

Jones, OA, Swain SC, Svendsen C, Griffin JL, Sturzenbaum SR, Spurgeon DJ (2012) Potential new method of mixture effects testing using metabolomics and Caenorhabditis elegans. Journal of Proteome Research 11(2), 1446-1453

Ju J, Lieke T, Saul N, Pu Y, Yin L, Kochan C, Putschew A, Baberschke N, Steinberg CE (2014) Neurotoxic evaluation of two organobromine model compounds and natural AOBr- 
containing surface water samples by a Caenorhabditis elegans test. Ecotoxicology and Environmental Safety 104, 194-201

Jung J, Ishida K, Nishihara T (2004) Anti-estrogenic activity of fifty chemicals evaluated by in vitro assays. Life Sciences 74(25) 3065-3074

Kais B, Ottermanns R, Scheller F, Braunbeck T (2018) Modification and quantification of in vivo EROD live-imaging with zebrafish (Danio rerio) embryos to detect both induction and inhibition of CYP1A. Science of The Total Environment 615, 330-347

Kaiser D, Sieratowicz A, Zielke H, Oetken M, Hollert H, Oehlmann J (2012) Ecotoxicological effect characterisation of widely used organic UV filters. Environmental Pollution 163, 8490

Karlson K, Rosenberg R, Bonsdorff E (2002) Temporal and spatial large-scale effects of eutrophication and oxygen deficiency on benthic fauna in Scandinavian and Baltic waters a review. Oceanography and Marine Biology 40, 427-489

Keiter S, Rastall A, Kosmehl T, Erdinger L, Braunbeck T, Hollert H (2006) Ecotoxicological assessment of sediment, suspended matter and water samples in the upper Danube river. A pilot study in search for the causes for the decline of fish catches. Environmental Science and Pollution Research 13(5), 308-319

Kern S, Fenner K, Singer HP, Schwarzenbach RP, Hollender J (2009) Identification of transformation products of organic contaminants in natural waters by computeraided prediction and high-resolution mass spectrometry. Environmental Science \& Technology 43, 7039-7046

Kidd KA, Blanchfield PJ, Mills KH, Palace VP, Evans RE, Lazorchak JM, Flick RW (2007) Collapse of a fish population after exposure to a synthetic estrogen. Proceedings of the National Academy of Sciences of the United States of America 104(21), 8897-8901

Kim HM, Lee DK, Long NP, Kwon SW, Park JH (2019a) Uptake of nanopolystyrene particles induces distinct metabolic profiles and toxic effects in Caenorhabditis elegans. Environmental Pollution 246, 578-586

Kim HM, Long NP, Yoon SJ, Nguyen HT, Kwon SW (2019b) Metabolomics and phenotype assessment reveal cellular toxicity of triclosan in Caenorhabditis elegans. Chemosphere 236, 124306

Kim S, Thiessen PA, Bolton EE, Chen J, Fu G, Gindulyte A, Han L, He J, He S, Shoemaker BA, Wang J, Yu B, Zhang J, Bryant SH (2016) PubChem substance and compound databases. Nucleic Acids Research 44(D1), 1202-1213

Kim SJ, Choung SY (2009) Whole genomic expression analysis of octachlorostyreneinduced chronic toxicity in Caenorhabditis elegans. Archives of Pharmacal Research 32(11), 1585

Kim SK, Lund J, Kiraly M, Duke K, Jiang M, Stuart JM, Eizinger A, Wylie BN, Davidson, GS (2001) A gene expression map for Caenorhabditis elegans. Science 293(5537), 2087-2092

Kim Y, Jeong J, Chatterjee N, Yim UH, Kwon JH, Park MS, Choi J (2019c) Activation of the nucleotide excision repair pathway by crude oil exposure: A translational study from model organisms to the Hebei Spirit Oil Spill Cohort. Environmental Pollution 254, 112997

Kim Y, Jeong J, Lee S, Choi I, Choi J (2020) Identification of adverse outcome pathway related to high-density polyethylene microplastics exposure: Caenorhabditis elegans transcription factor RNAi screening and zebrafish study. Journal of Hazardous Materials 388,121725

Kim YJ, Nicell JA (2006) Impact of reaction conditions on the laccase-catalyzed conversion of bisphenol A. Bioresource Technology 97, 1431-1442 
Klaassen CD (2007) Casarett and Doull's toxicology: the basic science of poisons. Sevens Edition. McGraw-Hill Medical, New York, USA

Klass MR (1977) Aging in nematode Caenorhabditis elegans - Major biological and environmental factors influencing lifespan. Mechanisms of Ageing and Development 6, 413429

Klavarioti M, Mantzavinos D, Kassinos D (2009) Removal of residual pharmaceuticals from aqueous systems by advanced oxidation processes. Environment International 35(2), $402-$ 417

Knoop O, Itzel F, Tuerk J, Lutze HV, Schmidt TC (2018) Endocrine effects after ozonation of tamoxifen. Science of The Total Environment 622, 71-78

Knopp G, Prasse C, Ternes TA, Cornel P (2016) Elimination of micropollutants and transformation products from a wastewater treatment plant effluent through pilot scale ozonation followed by various activated carbon and biological filters. Water Research 100 , 580-592

Köck-Schulmeyer M, Villagrasa M, de Alda ML, Céspedes-Sánchez R, Ventura F, Barceló D (2013) Occurrence and behavior of pesticides in wastewater treatment plants and their environmental impact. Science of the Total Environment, 458, 466-476

Kontana A, Papadimitriou CA, Samaras P, Zdragas A, Yiangou M (2009) Effectiveness of ozonation and chlorination on municipal wastewater treatment evaluated by a battery of bioassays and biomarkers. Water Science and Technology 60(6), 1497-1505

Kováts N, Refaey M, Varanka B, Reich K, Ferincz Á, Ács A (2012) Comparison of conventional and Vibrio fischeri bioassays for the assessment of municipal wastewater toxicity. Environmental Engineering and Management Journal 11(11), 2073-2076

Kümmerer K (2011) Commentary: Emerging contaminants versus micro-pollutants. Clean - Soil, Air, Water 39 (10), 889-890

Kumar S, Aninat C, Michaux G, Morel F (2010) Anticancer drug 5-fluorouracil induces reproductive and developmental defects in Caenorhabditis elegans. Reproductive Toxicology 29(4), 415-420

Kumar R, Pradhan A, Khan FA, Lindström P, Ragnvaldsson D, Ivarsson P, Olsson PE, Jass $\mathrm{J}$ (2015) Comparative analysis of stress induced gene expression in Caenorhabditis elegans following exposure to environmental and lab reconstituted complex metal mixture. PLOS One 10(7), e0132896

Kusk KO, Krüger T, Long M, Taxvig C, Lykkesfeldt AE, Frederiksen $H$, Andersson AM, Andersen HR, Hansen KM, Nellemann C, Bonefeld-Jørgensen EC (2011) Endocrine potency of wastewater: contents of endocrine disrupting chemicals and effects measured by in vivo and in vitro assays. Environmental Toxicology and Chemistry 30(2), 413-426

Kwon JY, Hong M, Choi MS, Kang S, Duke K, Kim S, Lee S, Lee J (2004) Ethanol-response genes and their regulation analyzed by a microarray and comparative genomic approach in the nematode Caenorhabditis elegans. Genomics 83(4), 600-614

Lagadic L, Wheeler JR, Weltje L (2020) (Mis)Use of the Adverse Outcome Pathway concept for assessing endocrine disruption in nontarget organisms. Integrated Environmental Assessment and Management 16(4), 525-528

Lagido C, McLaggan D, Flett A, Pettitt J, Glover LA (2009) Rapid sublethal toxicity assessment using bioluminescent Caenorhabditis elegans, a novel whole-animal metabolic biosensor. Toxicology Science 109, 88-95

Lagido C, Pettitt J, Flett A, Glover LA (2008) Bridging the phenotypic gap: realtime assessment of mitochondrial function and metabolism of the nematode Caenorhabditis elegans. BMC Physiology 8, 7 
Lant B, Storey KB (2010) An overview of stress response and hypometabolic strategies in Caenorhabditis elegans: conserved and contrasting signals with the mammalian system. International Journal of Biological Sciences 6(1), 9

Laquaz M, Dagot C, Wiest L, Bazin C, Gaschet M, Perrodin Y (2020) Ecotoxicity and antibiotic resistance of wastewater during transport in an urban sewage network. Environmental Science and Pollution Research 27, 19991-19999

Larcher S, Delbès G, Robaire B, Yargeau V (2012) Degradation of 17 a-ethinylestradiol by ozonation - Identification of the by-products and assessment of their estrogenicity and toxicity. Environment International 39, 66-72

Laudet V, Gronemeyer H (2002) The nuclear receptor FactsBook. Academic Press, London, UK

Leist M, Ghallab A, Graepel R, Marchan R, Hassan R, Bennekou SH, Limonciel A, Vinken M, Schildknecht S, Waldmann T, Danen E, van Ravenzwaay B, Kamp H, Gardner I, Godoy P, Bois FY, Braeuning A, Reif R, Oesch F, Drasdo D, Höhme S, Schwarz M, Hartung T, Braunbeck T, Beltman J, Vrieling H, Sanz F, Forsby A, Gadaleta D, Fisher C, Kelm J, Fluri D, Ecker G, Zdrazil B, Terron A, Jennings P, van der Burg B, Dooley S, Meijer AH, Willighagen E, Martens M, Evelo C, Mombelli E, Taboureau O, Mantovani A, Hardy B, Koch B, Escher S, van Thriel C, Cadenas C, Kroese D, van de Water B, Hengstler JG (2017) Adverse outcome pathways: opportunities, limitations and open questions. Archives of Toxicology 91(11), 3477-3505

Lenz KA, Pattison C, Ma H (2017) Triclosan (TCS) and triclocarban (TCC) induce systemic toxic effects in a model organism the nematode Caenorhabditis elegans. Environmental Pollution 231, 462-470

Leung MCK, Goldstone J, Boyd W, Freedman J, Meyer J (2010) Caenorhabditis elegans generates biologically relevant levels of genotoxic metabolites from aflatoxin B1 but not benzo[a]pyrene in vivo. Toxicological Science 118(2), 444-453

Leung MCK, Williams PL, Benedetto A, Au C, Helmcke KJ, Aschner M, Meyer JN (2008) Caenorhabditis elegans: an emerging model in biomedical and environmental toxicology. Toxicological Science 106(1), 5-28

Leusch FD, Khan SJ, Gagnon MM, Quayle P, Trinh T, Coleman H, Rawson C, Chapman HF, Blair P, Nice H, Reitsema T (2014) Assessment of wastewater and recycled water quality: a comparison of lines of evidence from in vitro, in vivo and chemical analyses. Water Research 50, 420-431

Leusch FD, Neale PA, Hebert A, Scheurer M, Schriks MC (2017) Analysis of the sensitivity of in vitro bioassays for androgenic, progestagenic, glucocorticoid, thyroid and estrogenic activity: suitability for drinking and environmental waters. Environment International 99, 120 130

Leusch FDL, Prochazka E, Tan BLL, Carswell S, Neale P, Escher BI (2012) Optimising micropollutants extraction for analysis of water samples: comparison of different solid phase materials and liquid-liquid extraction. Science Forum and Stakeholder Engagement: Building Linkages, Collaboration and Science Quality 191-195

Lewis JA, Gehman EA, Baer CE, Jackson DA (2013) Alterations in gene expression in Caenorhabditis elegans associated with organophosphate pesticide intoxication and recovery. BMC Genomics 14(1), 291

Lewis JA, Szilagyi M, Gehman E, Dennis WE, Jackson DA (2009) Distinct patterns of gene and protein expression elicited by organophosphorus pesticides in Caenorhabditis elegans. BMC Genomics 10, 202

Li J, Chen Y, Zhang G, Ruan W, Shan S, Lai X, Yang D, Yu Z (2020) Integration of behavioural tests and transcriptome sequencing of $C$. elegans reveals how the nematode 
responds to peanut shell biochar amendment. Science of The Total Environment 707, 136024

Li J, Li N, Ma M, Giesy JP, Wang Z (2008) In vitro profiling of the endocrine disrupting potency of organochlorine pesticides. Toxicolology Letters 183, 65

Li J, Wang Z, Ma M, Peng X (2010) Analysis of environmental endocrine disrupting activities using recombinant yeast assay in wastewater treatment plant effluents. Bulletin of Environmental Contamination and Toxicology 84, 529-535

Li Q, Zhang S, Yu Y, Wang L, Guan S, Li P (2012) Toxicity of sodium fluoride to Caenorhabditis elegans. Biomedical Environmental Science 25(2), 216-223

Li X, Shi H, Li K, Zhang L (2015) Combined process of biofiltration and ozone oxidation as an advanced treatment process for wastewater reuse. Frontiers of Environmental Science \& Engineering 9(6), 1076-1083

Li Y, Zhang M, Li S, Lv R, Chen P, Liu R, Liang G, Yin L (2016) The use of the nematode Caenorhabditis elegans to evaluate the adverse effects of epoxiconazole exposure on spermatogenesis. International Journal of Environmental Research and Public Health 13(10), 993

Li Y, Zhu G, Ng WJ, Tan SK (2014) A review on removing pharmaceutical contaminants from wastewater by constructed wetlands: design, performance and mechanism. Science of the Total Environment 468-469, 908-932

Lienert J, Koller M, Konrad J, McArdell CS, Schuwirth N (2011) Multiple-criteria decision analysis reveals high stakeholder preference to remove pharmaceuticals from hospital wastewater. Environmental Science \& Technology 45, 3848-3857

Lindblom TH, Dodd AK (2006) Xenobiotic detoxification in the nematode Caenorhabditis elegans. Journal of Experimental Zoology Part A Comparative Experimental Biology 305(9), $720-30$

Link M, Peter C, Voß K, Schäfer RB (2017) Comparison of dilution factors for German wastewater treatment plant effluents in receiving streams to the fixed dilution factor from chemical risk assessment. Science of the Total Environment 598, 805-813

Liu F, Luo Q, Zhang Y, Huang K, Cao X, Cui C, Lin K, Zhang M (2020) Trans-generational effect of neurotoxicity and related stress response in Caenorhabditis elegans exposed to tetrabromobisphenol A. The Science of The Total Environment 703, 134920

Liu F, Zaman WQ, Peng H, Li C, Cao X, Huang K, Cui C, Zhang W, Lin K, Luo Q (2019) Ecotoxicity of Caenorhabditis elegans following a step and repeated chronic exposure to tetrabromobisphenol A. Ecotoxicology and Environmental Safety 169, 273-281

Liu S, Saul N, Pan B, Menzel R, Steinberg CE (2013) The non-target organism Caenorhabditis elegans withstands the impact of sulfamethoxazole. Chemosphere 10 , 2373-2380

Loos R, Carvalho R, António DC, Comero S, Locoro G, Tavazzi S, Paracchini B, Ghiani M, Lettieri T, Blaha L, Jarosova B, Voorspoels S, Servaes K, Haglund P, Fick J, Lindberg RH, Schwesig D, Gawlik BM (2013) EU wide monitoring survey on waste water treatment plant effluents. Water Research 47(17), 6475-87

Lopes PC, Sucena E, Santos ME, Magalhaes S (2008) Rapid experimental evolution of pesticide resistance in $C$. elegans entails no costs and affects the mating system. PLoS One 3(11), e3741

Lundby Z, Camacho J, Allard P (2016) Fast functional germline and epigenetic assays in the nematode Caenorhabditis elegans. In Zhu H, Xia M. High-Throughput Screening Assays in Toxicology. Methods in Molecular Biology 1473. Humana Press, New York, USA 
Ma D, Wei J, Zhang H, Zhou Y, Shen J, Wang L, Zhang P (2019). Acute toxicity evolution during ozonation of mono-chlorophenols and initial identification of highly toxic intermediates. Environmental Science. Processes \& Impacts 21(9), 1509-1518

Ma M, Li J, Wang Z (2005) Assessing the detoxication efficiencies of wastewater treatment processes using a battery of bioassays/biomarkers. Archives of Environmental Contamination and Toxicology 49(4), 480-487

Macova M, Escher BI, Reungoat J, Carswell S, Chue KL, Keller J, Mueller JF (2010) Monitoring the biological activity of micropollutants during advanced wastewater treatment with ozonation and activated carbon filtration. Water Research 44(2), 477-492

Macova M, Toze S, Hodgers L, Mueller JF, Bartkow M, Escher BI (2011) Bioanalytical tools for the evaluation of organic micropollutants during sewage treatment, water recycling and drinking water generation. Water Research 45(14), 4238-4247

Magdeburg A, Stalter D, Oehlmann J (2012) Whole effluent toxicity assessment at a wastewater treatment plant upgraded with a full-scale post-ozonation using aquatic key species. Chemosphere 88, 1008-1014

Magdeburg A, Stalter D, Schlüsener M, Ternes T, Oehlmann J (2014) Evaluating the efficiency of advanced wastewater treatment: target analysis of organic contaminants and (geno-)toxicity assessment tell a different story. Water Research 50, 35-47

Maier D, Blaha L, Giesy JP, Henneberg A, Köhler HR, Kuch B, Osterauer R, Peschke K, Richter D, Scheurer M, Triebskorn R (2015) Biological plausibility as a tool to associate analytical data for micropollutants and effect potentials in wastewater, surface water, and sediments with effects in fishes. Water Research 72, 127-144

Mailler R, Gasperi J, Coquet Y, Deshayes S, Zedek S, Cren-Olivé C, Cartiser N, Eudes V, Bressy A, Caupos E, Moilleron R, Chebbo G, Rocher V (2015) Study of a large scale powdered activated carbon pilot: removals of a wide range of emerging and priority micropollutants from wastewater treatment plant effluents. Water Research 72, 315-330

Malaj E, von der Ohe PC, Grote M, Kühne R, Mondy CP, Usseglio-Polatera P, Brack W, Schafer RB (2014) Organic chemicals jeopardize the health of freshwater ecosystems on the continental scale. Proceedings of the National Academy of Sciences 111 (26), 95499554

Maletz S, Floehr T, Beier S, Klümper C, Brouwer A, Behnisch P, Higley E, Giesy JP, Hecker M, Gebhardt W, Linnemann V, Pinnekamp J, Hollert H (2013) In vitro characterization of the effectiveness of enhanced sewage treatment processes to eliminate endocrine activity of hospital effluents. Water Research 47(4), 1545-1557

Maltby L, Clayton SA, Yu H, McLoughlin N, Wood RM, Yin D (2000) Using single-species toxicity tests, community-level responses, and toxicity identification evaluations to investigate effluent impacts. Environmental toxicology and chemistry 19(1), 151-157

Margerit A, Gomez E, Gilbin R (2016) Dynamic energy-based modeling of uranium and cadmium joint toxicity to Caenorhabditis elegans. Chemosphere 146, 405-412

Margot J, Maillard J, Rossi L, Barry DA, Holliger C (2013) Influence of treatment conditions on the oxidation of micropollutants by Trametes versicolor laccase. New Biotechnology 30(6), 803-813

Martin MT, Dix DJ, Judson RS, Kavlock RJ, Reif DM, Richard AM, Rotroff DM, Romanov S, Medvedev A, Poltoratskaya N, Gambarian M, Moeser M, Makarov SS, Houck KA (2010) Impact of environmental chemicals on key transcription regulators and correlation to toxicity end points within EPA's ToxCast program. Chemical Research in Toxicology 23 (3), 578590 
Marttinen SK, Kettunen RH, Rintala JA (2003) Occurrence and removal of organic pollutants in sewages and landfill leachates. Science of The Total Environment 301, 1-12

Maruya KA, Dodder NG, Mehinto AC, Denslow ND, Schlenk D, Snyder SA, Weisberg SB (2016) A tiered, integrated biological and chemical monitoring framework for contaminants of emerging concern in aquatic ecosystems. Integrated Environmental Assessment and Management 12(3), 540-547

Matsumoto T, Kobayashi M, Moriwaki T, Kawai S, Watabe S (2004) Survey of estrogenic activity in fish feed by yeast estrogen-screen assay. Comparative Biochemistry \& Physiology 139, 147-152

Matsuura T, Miura H, Nishino A (2013) Inhibition of gustatory plasticity due to acute nicotine exposure in the nematode Caenorhabditis elegans. Neuroscience Research 77(3), 155-161

Matthai A (2009). Zur Bedeutung der Schadstoffexposition auf die Genexpression und Reproduktion von Caenorhabditis elegans (English title: „Role of pollutant exposure for gene expression and reproduction of Caenorhabditis elegans"). PhD thesis. Technische Universität Hamburg-Harburg, Hamburg, Germany

Maupas E (1899) La mue et l'enkystement chez les nématodes (English title: „Molting and encystment in nematodes“). Archives de Zoologie Expérimentale et Générale (3. Série), 7, 563-628

McLaggan D, Amezaga MR, Petra E, Frost A, Duff El, Rhind SM, Fowler PA, Glover LA, Lagido C (2012) Impact of sublethal levels of environmental pollutants found in sewage sludge on a novel Caenorhabditis elegans model biosensor. PLoS One 7(10), e46503

Mendelski MN, Dölling R, Feller FM, Hoffmann D, Ramos Fangmeier L, Ludwig KC, Yücel O, Mährlein A, Paul RJ, Philipp B (2019) Steroids originating from bacterial bile acid degradation affect Caenorhabditis elegans and indicate potential risks for the fauna of manured soils. Scientific Reports 9, 11120

Menzel R, Swain SC, Höss S, Claus E, Menzel S, Steinberg CE, Reifferscheid G, Stürzenbaum SR (2009) Gene expression profiling to characterize sediment toxicity - a pilot study using Caenorhabditis elegans whole genome microarrays. BMC Genomics 10, $160-$ 174

Menzel R, Bogaert T, Achazi R (2001) A systematic gene expression screen of Caenorhabditis elegans cytochrome P450 genes reveals CYP35 as strongly xenobiotic inducible. Archives of Biochemistry and Biophysics 395, 158-168

Menzel R, Reichert K, Achazi R (2002) Nutzung der induzierbaren Genexpression des Nematoden Caenorhabditis elegans als Biomonitor. Umweltwissenschaften und Schadstoff-Forschung 14(1), 18-23

Menzel R, Rodel M, Kulas J, Steinberg CE (2005a) CYP35: xenobiotically induced gene expression in the nematode Caenorhabditis elegans. Archives of Biochemistry and Biophysics 438, 93-102

Menzel R, Stürzenbaum S, Bärenwaldt A, Kulas J, Steinberg CEW (2005b) Humic material induces behavioral and global transcriptional responses in the nematode Caenorhabditis elegans. Environmental Science \& Technology 39(21), 8324-8332

Menzel R, Yeo HL, Rienau S, Li S, Steinberg CE, Sturzenbaum SR (2007) Cytochrome P450s and short-chain dehydrogenases mediate the toxicogenomic response of PCB52 in the nematode Caenorhabditis elegans. Journal of Molecular Biology 370, 1-13

Mersha MD, Patel BM, Patel D, Richardson BN, Dhillon HS (2015) Effects of BPA and BPS exposure limited to early embryogenesis persist to impair non-associative learning in adults. Behavioral and Brain Functions 11(1), 27 
Merkx-Jacques A, Coors A, Brousseau R, Masson L, Mazza A, Tien YC, Topp E (2013) Evaluating the pathogenic potential of environmental Escherichia coli using the Caenorhabditis elegans infection model. Applied and Environmental Microbiology 79(7), 2435-2445

Mi H, Muruganujan A, Casagrande JT, Thomas PD (2013) Large-scale gene function analysis with the PANTHER classification system. Nature Protocols 8(8), 1551

Min H, Kawasaki I, Gong J, Shim YH (2015) Caffeine induces high expression of cyp-35A family genes and inhibits the early larval development in Caenorhabditis elegans. Molecular Cells 38(3), 236-242

Misik M, Knasmueller S, Ferk F, Cichna-Markl M, Grummt T, Schaar H, Kreuzinger N (2011) Impact of ozonation on the genotoxic activity of tertiary treated municipal wastewater. Water Research 45(12), 3681-3691

Mohammadi-Bardbori A (2014) Assay for quantitative determination of CYP1A1 enzyme activity using 7-Ethoxyresorufin as standard substrate (EROD assay). Nature Protocol Exchange. https://protocolexchange.researchsquare.com/article/nprot-3473/v1

Mori T, Inokuchi A, Nihira M, Yamamoto R, Ishibashi H, Kohra S, Tominaga N, Arizono K (2007) Eco-toxicological effect of polycyclic musks for C. elegans. Japanese Society for Alternatives to Animal Experiments 14, 569-573

Morimoto RI (1998) Regulation of the heat shock transcriptional response: cross talk between a family of heat shock factors, molecular chaperones, and negative regulators. Genes development 12(24), 3788-3796

Morrissey CA, Mineau P, Devries JH, Sanchez-Bayo F, Liess M, Cavallaro MC, Liber K (2015) Neonicotinoid contamination of global surface waters and associated risk to aquatic invertebrates: a review. Environment International 74, 291-303

Mosser T, Matic I, Leroy M (2011) Bacterium-induced internal egg hatching frequency is predictive of life span in Caenorhabditis elegans populations. Applied and Environmental Microbiology 77(22), 8189-8192

Mueller MT, Fueser H, Höss S, Traunspurger W (2020a) Species-specific effects of longterm microplastic exposure on the population growth of nematodes, with a focus on microplastic ingestion. Ecological Indicators 118, 106698

Mueller MT, Fueser H, Trac LN, Mayer P, Traunspurger W, Höss S (2020b) Surface-related toxicity of polystyrene beads to nematodes and the role of food availability. Environmental Science \& Technology 54(3), 1790-1798

Munz NA, Fu Q, Stamm C, Hollender J (2018) Internal concentrations in gammarids reveal increased risk of organic micropollutants in wastewater-impacted streams. Environmental Science \& Technology 52(18), 10347-10358

Münze R, Hannemann C, Orlinskiy P, Gunold R, Paschke A, Foit K, Becker J, Kaske O, Paulson E, Peterson M, Jernstedt H, Kreuger J, Schüürmann G, Liess M (2017) Pesticides from wastewater treatment plant effluents affect invertebrate communities. Science of The Total Environment 599, 387-399

Muschket M, Di Paolo C, Tindall AJ, Touak G, Phan A, Krauss M, Kirchner K, Seiler TB, Hollert H, Brack W (2017) Identification of unknown antiandrogenic compounds in surface waters by effect-directed analysis (EDA) using a parallel fractionation approach. Environmental Science \& Technology 52(1), 288-297

Musolff A, Leschik S, Reinstorf F, Strauch G, Schirmer M (2010) Micropollutant loads in the urban water cycle. Environmental Science \& Technology 44, 4877-4883 
Nam S, An Y (2010) Assessing the ecotoxicity of vinyl chloride using green alga $P$. subcapitata, nematode $C$. elegans, and the SOS chromotest in a closed system without headspace. Science of The Total Environment 408(16), 3148-5

Neale PA, Brack W, Aït-Aïssa S, Busch W, Hollender J, Krauss M, Maillot-Maréchal E, Munz NA, Schlichting R, Schulze T, Vogler B, Escher BI (2018) Solid-phase extraction as sample preparation of water samples for cell-based and other in vitro bioassays. Environmental Science Process Impacts 20(3), 493-504

Neale PA, Escher BI, Leusch FD (2015) Understanding the implications of dissolved organic carbon when assessing antagonism in vitro: an example with an estrogen receptor assay. Chemosphere 135, 341-346

Neale PA, Munz NA, Aït-Aïssa S, Altenburger R, Brion F, Busch W, Escher BI, Hilscherová K, Kienle C, Novák J, Seiler TB, Shao Y, Stamm C, Hollender J (2017) Integrating chemical analysis and bioanalysis to evaluate the contribution of wastewater effluent on the micropollutant burden in small streams. Science of the Total Environment 576, 785-795

Neher DA, Stürzenbaum SR (2006) Extra-long PCR, an identifier of DNA adducts in single nematodes (Caenorhabditis elegans). Comparative Biochemistry and Physiology Part C: Toxicology Pharmacology 144(3), 279-285

Nelson DR, Koymans L, Kamataki T, Stegeman JJ, Feyereisen R, Waxman DJ, Waterman, MR, Gotoh O, Coon MJ, Estabrook RW, Gunsalus IC, Nebert DW (1996) P450 superfamily: update on new sequences, gene mapping, accession numbers and nomenclature. Pharmacogenetics 6, 1-42

$\mathrm{Ng} \mathrm{CK}$, Cao B (2015) What exactly are you filtering out? Environmental Science \& Technology 49, 5259-5260

Nigon V (1949) Les modalités de la reproduction et le déterminisme du sexe chez quelques nematodes libres (English title "Reproductive modalities and sex determinism in certain free nematodes"). Annales des Sciences Naturelles. Zoologie et Biologie animale 11, 1-132

Norberg-King TJ (2001) Toxicity reduction and toxicity identification evaluations for effluents, ambient waters, and other aqueous media (Vol. 1) SETAC special symposium publication. Society of Environmental Toxicology and Chemistry, Pensacola, USA

Norberg-King TJ, Embry MR, Belanger SE, Braunbeck T, Butler JD, Dorn PB, Farr B, Guiney PD, Hughes SA, Jeffries M, Journel R, Lèonard M, McMaster M, Oris JT, Ryder K, Segner H, Senac T, Van Der Kraak G, Whale G, Wilson P (2018) An international perspective on the tools and concepts for effluent toxicity assessments in the context of animal alternatives: reduction in vertebrate use. Environmental Toxicology and Chemistry 37(11), 2745-2757

OECD (1992). Test no. 210. Fish Early Life Stage Toxicity Test. OECD guidelines for the testing of chemicals. OECD Publishing, Paris, France

OECD (2006) Test no. 221: Lemna sp. Growth inhibition test. OECD Publishing, Paris, France

OECD (2007) Test no. 225: Sediment-water Lumbriculus toxicity test using spiked sediment. OECD Publishing, Paris, France

OECD (2012) Test no. 211: Daphnia magna reproduction test. OECD Publishing, Paris, France

OECD (2016) Test no. 242: Potamopyrgus antipodarum reproduction test. OECD Publishing, Paris, France

OECD (2018) Users' Handbook supplement to the guidance document for developing and assessing Adverse Outcome Pathways". OECD Series on Adverse Outcome Pathways 1. OECD Publishing, Paris, France 
Offermann K, Matthäi A, Ahlf W (2009) Assessing the importance of dietborne cadmium and particle characteristics on bioavailability and bioaccumulation in the nematode Caenorhabditis elegans. Environmental Toxicology and Chemistry 28(6), 1149-1158

Ohba K, Ishibashi N (1984) A nematode, Caenorhabditis elegans, as test organism for nematicide evaluation. Journal of Pesticide Science 9(1), 91-96

Omiecinski CJ, Remmel RP, Hosagrahara VP (1999) Concise review of the cytochrome P450s and their roles in toxicology. Toxicological Sciences 48(2), 151-156

Oulton RL, Kohn T, Cwiertny DM (2010) Pharmaceuticals and personal care products in effluent matrices: a survey of transformation and removal during wastewater treatment and implications for wastewater management. Journal of Environmental Monitoring 12 (11), 1956-1978

Pal A, Gin KYH, Lin AYC, Reinhard M (2010) Impacts of emerging organic contaminants on freshwater resources: review of recent occurrences, sources, fate and effects. Science of The Total Environment 408(24), 6062-6069

Pan ST, Xue D, Li ZL, Zhou ZW, He ZX, Yang Y, Yang T, Qiu JX, Zhou SF (2016) Computational identification of the paralogs and orthologs of human cytochrome P450 superfamily and the implication in drug discovery. International Journal of Molecular Sciences 17(7), 1020

Papa M, Pedrazzani R, Bertanza G (2013) How green are environmental technologies? A new approach for a global evaluation: The case of WWTP effluents ozonation. Water Research 47(11) 3679-3687

Park KJ, Müller CT, Markman S, Swinscow-Hall O, Pascoe D, Buchanan KL (2009) Detection of endocrine disrupting chemicals in aerial invertebrates at sewage treatment works. Chemosphere 77(11), 1459-1464

Parodi DA, Damoiseaux R, Allard P (2015) Comprehensive assessment of germline chemical toxicity using the nematode Caenorhabditis elegans. Journal of Visual Experiments 96, e52445

Parrott JL, Blunt BR (2005) Life-cycle exposure of fathead minnows (Pimephales promelas) to an ethinylestradiol concentration below $1 \mathrm{ng} / \mathrm{L}$ reduces egg fertilization success and demasculinizes males. Environmental Toxicology 20(2), 131-141

Paździor K, Wrębiak J, Klepacz-Smółka A, Gmurek M, Bilińska L, Kos L, Sójka-Ledakowicz J, Ledakowicz S (2017) Influence of ozonation and biodegradation on toxicity of industrial textile wastewater. Journal of Environmental Management 195(2), 166-173

Peakall DB (1994) The role of biomarkers in environmental assessment (1) Introduction. Ecotoxicology 3(3), 157-160

Pedrazzani R, Cavallotti I, Bollati E, Ferreri M, Bertanza G (2018) The role of bioassays in the evaluation of ecotoxicological aspects within the PEF/OEF protocols: The case of WWTPs. Ecotoxicology and Environmental Safety 147, 742-748

Peredney CL, Williams PL (2000) Utility of Caenorhabditis elegans for assessing heavy metal contamination in artificial soil. Archives of Environmental Contamination and Toxicology 39(1), 113-118

Pérez-González A, Urtiaga AM, Ibáñez R, Ortiz I (2012) State of the art and review on the treatment technologies of water reverse osmosis concentrates. Water Research 46(2), 267283

Peter E, Candido M, Jones D (1996) Transgenic Caenorhabditis elegans strains as biosensors. Trends in Biotechnoly 14(4), 125-129 
Philip R, Anton A, Loftus AC (2011) Strategic planning - preparing for the future. SWITCH training kit module 1. Integrated urban water management in the city of the future. ICLEI European Secretariat GmbH, Freiburg, Germany

Phillips PJ, Chalmers AT, Gray JL, Kolpin DW, Foreman WT, Wall GR (2012) Combined sewer overflows: An environmental source of hormones and wastewater micropollutants. Environmental Science \& Technology 46, 5336-5343

Pietrogrande MC, Basaglia G (2007) GC-MS analytical methods for the determination of personal-care products in water matrices. Trends in Analytical Chemistry 26(11), 1086-1094

Pihl L, Baden SP, Diaz RJ (1991) Effects of periodic hypoxia on distribution of demersal fish and crustaceans. Marine Biology 108(3), 349-360

Pincus Z, Mazer TC, Slack FJ (2016) Autofluorescence as a measure of senescence in $C$. elegans: look to red, not blue or green. Aging 8(5), 889

Polli JR, Zhang Y, Pan X (2014) Dispersed crude oil amplifies germ cell apoptosis in Caenorhabditis elegans, followed a CEP-1-dependent pathway. Archives of Toxicology 88(3), 543-551

Polo M, Llompart M, Garcia-Jares C, Cela R (2005) Multivariate optimization of a solidphase microextraction method for the analysis of phthalate esters in environmental waters. Journal of Chromatography A 1072(1), 63-72

Popham JD, Webster JM (1979) Cadmium toxicity in the free-living nematode, Caenorhabditis elegans. Environmental Research 20(1), 183-91

Powell-Coffman JA, Bradfield CA, Wood WB (1998) Caenorhabditis elegans orthologs of the aryl hydrocarbon receptor and its heterodimerization partner the aryl hydrocarbon receptor nuclear translocator. Proceedings of the National Academy of Sciences 95(6), 2844-2849

Pradhan A, Olsson PE, Jass J (2018) Di(2-ethylhexyl)phthalate and diethylphthalate disrupt lipid metabolism, reduce fecundity and shortens lifespan of Caenorhabditis elegans. Chemosphere 190, 375-382

Prasse C, Stalter D, Schulte-Oehlmann U, Oehlmann J, Ternes TA (2015) Spoilt for choice: a critical review on the chemical and biological assessment of current wastewater treatment technologies. Water Research 87, 237-270

Prieto-Rodríguez L, Oller I, Klamerth N, Agüera A, Rodríguez EM, Malato S (2013) Application of solar AOPs and ozonation for elimination of micropollutants in municipal wastewater treatment plant effluents. Water Research 47(4), 1521-1528

Quednow K, Püttmann W (2008) Endocrine disruptors in freshwater streams of Hesse, Germany: Changes in concentration levels in the time span from 2003 to 2005. Environmental Pollution 152(2), 476-483

Queirós L, Pereira JL, Gonçalves FJM, Pacheco M, Aschner M, Pereira P (2019) Caenorhabditis elegans as a tool for environmental risk assessment: emerging and promising applications for a "nobelized worm". Critical Reviews in Toxicology 49(5), 411 429

Racz PI, Wildwater M, Rooseboom M, Kerkhof E, Pieters R, Yebra-Pimentel ES, Dirks RP, Spaink HP, Smulders C, Whale GF (2017) Application of Caenorhabditis elegans (nematode) and Danio rerio embryo (zebrafish) as model systems to screen for developmental and reproductive toxicity of piperazine compounds. Toxicology In Vitro 44, 11-16

Rai N, Sjöberg V, Forsberg G, Karlsson S, Olsson PE, Jass J (2019) Metal contaminated soil leachates from an art glass factory elicit stress response, alter fatty acid metabolism 
and reduce lifespan in Caenorhabditis elegans. Science of The Total Environment 651, 2218-2227

Ramos S, Homem V, Alves A, Santos L (2015) Advances in analytical methods and occurrence of organic UV-filters in the environment. Science of The Total Environment 526, 278-311

Rankin CH (2002) From gene to identified neuron to behaviour in Caenorhabditis elegans. Nature Reviews Genetics 3(8) 622-630

Rao K, Li N, Ma M, Wang Z (2014) In vitro agonistic and antagonistic endocrine disrupting effects of organic extracts from waste water of different treatment processes. Frontiers of Environmental Science \& Engineering 8(1), 69-78

Reaume MJ, Seth R, McPhedran KN, da Silva EF, Porter LA (2015) Effect of media on biofilter performance following ozonation of secondary treated municipal wastewater effluent: sand vs. GAC. Ozone: Science \& Engineering 37(2), 143-153

Rechavi OL, Houri-Ze'evi S, Anava WS, Goh SY, Kerk SY, Hannon GJ, Hobert O (2014) Starvation-induced transgenerational inheritance of small RNAs in C. elegans. Cell 158, 277-287

Reemtsma T, Berger U, Arp HPH, Gallard H, Knepper TP, Neumann M, Quintana PM, Voogt PD (2016) Mind the gap: persistent and mobile organic compounds - water contaminants that slip through. Environmental Science \& Technology 50, 10308-10315

Rehberger K, Kropf C, Segner H (2018) In vitro or not in vitro: a short journey through a long history. Environmental Sciences Europe 30(1), 1-12

Reichenberger S, Bach M, Skitschak A, Frede HG (2007) Mitigation strategies to reduce pesticide inputs into ground- and surface water and their effectiveness, a review. Science of The Total Environment 384, 1-35

Reichert K, Menzel R (2005) Expression profiling of five different xenobiotics using a Caenorhabditis elegans whole genome microarray. Chemosphere 61, 229-237

Reinke V, Kraus M, Okkema $\mathrm{P}$ (2013) Transcriptional regulation of gene expression in C. elegans. In WormBook. The C. elegans Research Community. http://www.wormbook.org

Rempel MA, Schlenk D (2008) Effects of environmental estrogens and antiandrogens on endocrine function, gene regulation, and health in fish. International Reviews of Cell and Molecualr Biology 267, 207-52

Reungoat J, Escher BI, Macova M, Argaud FX, Gernjak W, Keller J (2012) Ozonation and biological activated carbon filtration of wastewater treatment plant effluents. Water Research 46(3), 863-872

Ribeiro AR, Nunes OC, Pereira MFR, Silva AMT (2015) An overview on the advanced oxidation processes applied for the treatment of water pollutants defined in the recently launched Directive 2013/39/EU. Environment International 75, 33-51

Richardson SD (2009) Water analysis: emerging contaminants and current issues. Analytical Chemistry 81(12), 4645-467

Riddle DL, Blumenthal T, Meyer BJ, Priess JR (1997) Section III, Life History and Evolution. In C. elegans II, Cold Spring Harbor Laboratory Press, NY, USA

Riss TL, Moravec RA (2004) Use of multiple assay endpoints to investigate the effects of incubation time, dose of toxin, and plating density in cell-based cytotoxicity assays. Assay and Drug Develpment Technologies 2(1), 51-62

Ristau K, Akgül Y, Bartel AS, Fremming J, Müller MT, Reiher L, Stapela F, Splett JP, Spann $N$ (2015) Toxicity in relation to mode of action for the nematode Caenorhabditis elegans: 
acute-to-chronic ratios and quantitative structure-activity relationships. Environmental Toxicology and Chemistry 34, 2347-2353

Rizzo L (2011) Bioassays as a tool for evaluating advanced oxidation processes in water and wastewater treatment. Water Research 45(15), 4311-4340

Rizzo L, Manaia C, Merlin C, Schwartz T, Dagot C, Ploy MC, Michael I, Fatta-Kassinos D (2013) Urban wastewater treatment plants as hotspots for antibiotic resistant bacteria and genes spread into the environment: a review. Science of the Total Environment 447, 345360

Rockström J, Steffen W, Noone K, Persson Å, Chapin III FS, Lambin EF, Lenton TM, Scheffer M, Folke C, Schellnhuber HJ, Nykvist B, de Wit CA, Hughes T, van der Leeuw S, Rodhe H, Sörlin S, Snyder PK, Costanza R, Svedin U, Falkenmark M, Karlberg L, Corell RW, Fabry VJ, Hansen J, Walker B, Liverman D, Richardson K, Crutzen P, Foley JA (2009) A safe operating space for humanity. Nature 461, 472-475

Rodriguez M, Snoek LB, De Bono M, Kammenga JE (2013) Worms under stress: C. elegans stress response and its relevance to complex human disease and aging. Trends in Genetics 29(6), 367-374

Roh JY, Choi J (2008) Ecotoxicological evaluation of chlorpyrifos exposure on the nematode Caenorhabditis elegans. Ecotoxicology and Environmental Safety 71(2), 483489

Roh JY, Choi J (2011) Cyp35a2 gene expression is involved in toxicity of fenitrothion in the soil nematode Caenorhabditis elegans. Chemosphere 84(10), 1356-1361

Roh JY, Jung IH, Lee JY, Choi J (2007) Toxic effects of di(2-ethylhexyl)phthalate on mortality, growth, reproduction and stress-related gene expression in the soil nematode Caenorhabditis elegans. Toxicology 237(1-3), 126-133

Roh JY, Kim PG, Kwon JH (2018) Comparative study of oxidative stress caused by anthracene and alkyl-anthracenes in Caenorhabditis elegans. Environmental Health and Toxicology 33(1), e2018006

Roh JY, Lee H, Kwon JH (2014) Changes in the expression of cyp35a family genes in the soil nematode Caenorhabditis elegans under controlled exposure to chlorpyrifos using passive dosing. Environmental Science \& Technology 48(17), 10475-10481

Roh JY, Lee HJ, Kwon JH (2016) Internal concentration and time are important modifiers of toxicity: the case of chlorpyrifos on Caenorhabditis elegans. Environmental Science \& Technology 50(17), 9689-9696

Roh JY, Sim SJ, Yi J, Park K, Chung KH, Ryu DY, Choi J (2009) Ecotoxicity of silver nanoparticles on the soil nematode Caenorhabditis elegans using functional ecotoxicogenomics. Environmental Science \& Technology 43(10), 3933-3940

Routledge EJ, Sumpter JP (1996) Estrogenic activity of surfactants and some of their degradation products assessed using a recombinant yeast screen. Environmental Toxicology and Chemistry 15, 241-248

Routledge EJ (2003) Identifying the causative agents: the use of combined chemical and biological strategies in monitoring programs. Pure and Applied Chemistry 75(11-12), 24612466

Ruan QL, Ju JJ, Li YH, Liu R, Pu YP, Yin LH, Wang DY (2009) Evaluation of pesticide toxicities with differing mechanisms using Caenorhabditis elegans. Journal of Toxicology and Environmental Health A 72, 746-51

Ruan QL, Ju JJ, Li YH, Li XB, Liu R, Liang GY, Zhang J, Pu YP, Wang DY, Yin LH (2012) Chlorpyrifos exposure reduces reproductive capacity owing to a damaging effect on 
gametogenesis in the nematode Caenorhabditis elegans. Journal of Applied Toxicology 32(7), 527-535

Sadaria AM, Supowit SD, Halden RU (2016) Mass balance assessment for six neonicotinoid insecticides during conventional wastewater and wetland treatment: nationwide reconnaissance in United States wastewater. Environmental Science \& Technology 50(12), 6199-6206

Sambongi Y, Nagae T, Liu Y, Yoshimizu T, Takeda K, Wada Y, Futai M (1999) Sensing of cadmium and copper ions by externally exposed ADL, ASE, and ASH neurons elicits avoidance response in Caenorhabditis elegans. Neuroreport 10(4), 753-757

Sanchez-Bayo F, Hyne RV (2014) Detection and analysis of neonicotinoids in river waters - development of a passive sampler for three commonly used insecticides. Chemosphere 99, 143-151

Sawada K, Inoue D, Wada Y, Sei K, Nakanishi T, Ike M (2012) Detection of retinoic acid receptor agonistic activity and identification of causative compounds in municipal wastewater treatment plants in Japan. Environmental Toxicology and Chemistry 31(2), 307315

Schäfer P, Müller M, Krüger A, Steinberg CE, Menzel R (2009) Cytochrome P450dependent metabolism of PCB52 in the nematode Caenorhabditis elegans. Archives of Biochemistry and Biophysics 488(1), 60-68

Schertzinger G, Zimmermann S, Grabner D, Sures B (2017) Assessment of sublethal endpoints after chronic exposure of the nematode Caenorhabditis elegans to palladium, platinum and rhodium. Environmental Pollution 230, 31-39

Schindler-Wildhaber Y, Mestankova H, Schärer M, Schirmer K, Salhi E, Von Gunten U (2015) Novel test procedure to evaluate the treatability of wastewater with ozone. Water Research 75, 324-335

Schlüter-Vorberg L, Knopp G, Cornel P, Ternes T, Coors A (2017) Survival, reproduction, growth, and parasite resistance of aquatic organisms exposed on-site to wastewater treated by advanced treatment processes. Aquatic Toxicology 186, 171-179

Schlüter-Vorberg L, Prasse C, Ternes TA, Mückter H, Coors A (2015) Toxification by transformation in conventional and advanced wastewater treatment: the antiviral drug acyclovir. Environmental Science \& Technology Letters 2(12), 342-346

Schroeder AL, Ankley GT, Houck KA, Villeneuve DL (2016) Environmental surveillance and monitoring - the next frontiers for high-throughput toxicology. Environmental Toxicology \& Chemistry 35(3), 513-525

Schulenburg H, Félix MA (2017) The natural biotic environment of Caenorhabditis elegans. Genetics 206(1), 55-86

Schulze T, Ahel M, Ahlheim J, Aït-Aïssa S, Brion F, Di Paolo C, Froment J, Hidasi AO, Hollender J, Hollert H, Hu M, Kloß A, Koprivica S, Krauss M, Muz M, Oswald P, Petre M, Schollée JE, Seiler TB, Shao Y, Slobodnik J, Sonavane M, Suter MJ, Tollefsen KE, Tousova Z, Walz KH, Brack W (2017) Assessment of a novel device for onsite integrative largevolume solid phase extraction of water samples to enable a comprehensive chemical and effect-based analysis. Science of The Total Environment 581, 350-358

Schwarzenbach RP, Escher BI, Fenner K, Hofstetter TB, Johnson CA, von Gunten U, Wehrli B (2006) The challenge of micropollutants in aquatic systems. Science 313(5790), 1072-1077

Seeland A, Oetken M, Kiss A, Fries E, Oehlmann J (2012) Acute and chronic toxicity of benzotriazoles to aquatic organisms. Environmental Science and Pollution Research 19(5), $1781-1790$ 
Seitz W, Winzenbacher R (2017) A survey on trace organic chemicals in a German water protection area and the proposal of relevant indicators for anthropogenic influences. Environmental Monitoring and Assessment 189(6), 244

Sese BT, Grant A, Reid BJ (2009) Toxicity of polycyclic aromatic hydrocarbons to the nematode Caenorhabditis elegans. Journal of Toxicology and Environmental Health Part A 72(19), 1168-1180

Shang X, Lu J, Feng C, Ying Y, He Y, Fang S, lin Y, Dahlgren R, Ju J (2020) Microplastic (1 and $5 \mu \mathrm{m}$ ) exposure disturbs lifespan and intestine function in the nematode Caenorhabditis elegans. Science of The Total Environment 705, 135837

Shieh BH, Louie A, Law FC (2016) Factors Affecting distribution of estrogenicity in the influents, effluents, and biosolids of Canadian wastewater treatment plants. Archives of Environmental Contamination and Toxicology 70(4), 682-691

Silva E, Rajapakse N, Kortenkamp A (2002) Something from "nothing" - eight weak estrogenic chemicals combined at concentrations below NOECs produce significant mixture effects. Environmental Science \& Technology 36(8), 1751-1756

Smith M, Zhang Y, Polli J, Wu H, Zhang B, Xiao P, Farwell M, Pan X (2013) Impacts of chronic low-level nicotine exposure on Caenorhabditis elegans reproduction: identification of novel gene targets. Reproductive Toxicology 40, 69-75

Soares A, Guieysse B, Jefferson B, Cartmell E, Lester JN (2008) Nonylphenol in the environment: a critical review on occurrence, fate, toxicity and treatment in wastewaters. Environment International 34(7), 1033-1049

Society for Advancement of AOPs (2018) AOP-Wiki. Available from http://aopwiki.org (Last accessed: October 2020)

Sohoni P, Sumpter JP (1998) Several environmental oestrogens are also anti-androgens. Journal of Endocrinology 158, 327-339

Sonne AT, Rasmussen JJ, Höss S, Traunspurger W, Bjerg PL, McKnight US (2018) Linking ecological health to co-occurring organic and inorganic chemical stressors in a groundwater-fed stream system. Science of The Total Environment 642, 1153-1162

Spann N, Goedkoop W, Traunspurger W (2015) Phenanthrene bioaccumulation in the nematode Caenorhabditis elegans. Environmental Science \& Technology 49(3), 1842-1850

Sreevidya VS, Lenz KA, Svoboda KR, Ma H (2018) Benzalkonium chloride, benzethonium chloride, and chloroxylenol - three replacement antimicrobials are more toxic than triclosan and triclocarban in two model organisms. Environmental Pollution 235, 814-824

Stalter D (2010) Ecotoxicological assessment of advanced wastewater treatment technologies. PhD thesis. Johann Wolfgang Goethe University, Frankfurt, Germany. URN:nbn:de:hebis:30-95029

Stalter D, Magdeburg A, Quednow K, Botzat A, Oehlmann J (2013) Do contaminants originating from state-of-the-art treated wastewater impact the ecological quality of surface waters? PloS One 8(4), e60616

Stalter D, Peters LI, O'Malley E, Tang JYM, Revalor M, Farré MJ, Watson K, von Gunten U, Escher BI (2016) Sample enrichment for bioanalytical assessment of disinfected drinking water: concentrating the polar, the volatiles, and the unknowns. Environmental Science \& Technology 50(12), 6495-6505.

Stalter D, Magdeburg A, Wagner M, Oehlmann J (2011) Ozonation and activated carbon treatment of sewage effluents: Removal of endocrine activity and cytotoxicity. Water Research 45(3), 1015-1024 
Stamm C, Räsänen K, Burdon FJ, Altermatt F, Jokela J, Joss A, Ackermann M, Eggen RIL (2016) Chapter four - unravelling the impacts of micropollutants in aquatic ecosystems: interdisciplinary studies at the interface of large-scale ecology. Advances in Ecological Research 55, 183-223

Stapf M, Schumann P, Völker J, Miehe U (2017) Studie über Effekte und Nebeneffekte bei der Behandlung von kommunalem Abwasser mit Ozon (English title: „Study on effects and side effects in the treatment of urban wastewater with ozone"). Kompetenzzentrum Wasser Berlin, Germany. Accessed under: http://www.kompetenz-wasser.de/wpcontent/uploads/2017/10/senbao_abschlussbericht_final.pdf

Stylianou M, Björnsdotter MK, Olsson PE, Jogsten IE, Jass J (2019) Distinct transcriptional response of Caenorhabditis elegans to different exposure routes of perfluorooctane sulfonic acid. Environmental Research 168, 406-413

Sulston JE, Horvitz HR (1977) Post-embryonic cell lineages of the nematode, Caenorhabditis elegans. Developmental Biology 56(1), 110-156

Sumpter JP (2005) Endocrine disrupters in the aquatic environment: an overview. Clean Soil, Air, Water 33(1), 9-16

Swain SC, Keusekotten K, Baumeister R, Sturzenbaum SR (2004) C. elegans metallothioneins: new insights into the phenotypic effects of cadmium toxicosis. Journal of Molecular Biology 341, 951-959

Taki F, Pan X, Zhang B (2014) Chronic nicotine exposure systemically alters microRNA expression profiles during post-embryonic stages in Caenorhabditis elegans. Journal of Cell Physiology 229(1), 79-89

Tang JY, Busetti F, Charrois JW, Escher BI (2014) Which chemicals drive biological effects in wastewater and recycled water? Water Research 60, 289-299

Taubert S, Ward JD, Yamamoto KR (2011) Nuclear hormone receptors in nematodes: evolution and function. Molecular and Cellular Endocrinology 334(1), 49-55

Tchobanoglous G, Burton FL (1991) Wastewater engineering: treatment, disposal and reuse. Metcalf \& Eddy. McGraw-Hill, New York

Tejeda-Benitez L, Flegal R, Odigie K, Olivero-Verbel J (2016a) Pollution by metals and toxicity assessment using Caenorhabditis elegans in sediments from the Magdalena River, Colombia. Environmental Pollution 212, 238-250

Tejeda-Benitez L, Olivero-Verbel, J (2016b) Caenorhabditis elegans, a biological model for research in toxicology. In de Voogt W, Reviews of Environmental Contamination and Toxicology Volume 237 (1-35). Springer, Cham. Switzerland

Tejeda-Benitez L, Noguera-Oviedo K, Aga DS Olivero-Verbel J (2018) Toxicity profile of organic extracts from Magdalena River sediments. Environmental Science and Pollution Research 25, 1519-1532

Ternes TA, Prasse C, Eversloh CL, Knopp G, Cornel P, Schulte-Oehlmann U, Schwartz T, Alexander J, Seitz W, Coors A, Oehlmann J (2016) Integrated evaluation concept to assess the efficacy of advanced wastewater treatment processes for the elimination of micropollutants and pathogens. Environmental Science \& Technology 51(1), 308-319

Ternes TA, Stumpf M, Mueller J, Haberer K, Wilken RD, Servos M (1999) Behavior and occurrence of estrogens in municipal sewage treatment plants - I. Investigations in Germany, Canada and Brazil. Science of the Total Environment 225(1-2), 81-90

Tetreault GR, Bennett CJ, Shires K, Knight B, Servos MR, McMaster ME (2011) Intersex and reproductive impairment of wild fish exposed to multiple municipal wastewater discharges. Aquatic Toxicology 104(3-4), 278-290 
Thrupp TJ, Runnalls TJ, Scholze M, Kugathas S, Kortenkamp A, Sumpter JP (2018) The consequences of exposure to mixtures of chemicals: Something from 'nothing' and 'a lot from a little' when fish are exposed to steroid hormones. Science of The Total Environment $619,1482-1492$

Tillmann M (2003) Sedimenttoxikologische Untersuchungen mit Gastropoden und Insekten unter besonderer Berücksichtigung endokrin-wirksamer Substanzen (English title „Sediment toxicological studies with gastropods and insects with special consideration of endocrine disrupting substances"). PhD thesis, Johann Wolfgang Goethe-Universität, Frankfurt am Main, Germany

Tominaga N, Matsuno T, Kohra S, Arizono K (2008) Sensing of heavy metals using Caenorhabditis elegans DNA microarray. In Murakami Y, Nakayama K, Kitamura SI, Iwata $\mathrm{H}$, Tanabe S. Interdisciplinary Studies on Environmental Chemistry-Biological Responses to Chemical Pollutants (155-161). Terrapub, Tokyo, Japan.

Totah RA, Rettie AE (2005) Cytochrome P450 2C8: substrates, inhibitors, pharmacogenetics, and clinical relevance. Clinical Pharmacology \& Therapeutics 77, 341352

Tran DQ, Ide CF, Mclachlan JA, Arnold SF (1996) The anti-estrogenic activity of selected polynuclear aromatic hydrocarbons in yeast expressing human estrogen receptor. Biochemical and Biophysical Research Communications 229(1), 102-108

Traunspurger W (1997) Bathymetric, seasonal and vertical distribution of feeding types of nematodes in an oligotrophic lake. Vie et Milieu 47, 1-7

Triebskorn R, Braunbeck T, Grummt T, Hanslik L, Huppertsberg S, Jekel M, Knepper TP, Krais S, Müller YK, Pittross M, Ruhl AS, Schmieg H, Schür C, Strobel C, Wagner M, Zumbülte N, Kohler HR (2019) Relevance of nano-and microplastics for freshwater ecosystems: a critical review. Trends in Analytical Chemistry 110, 375-392

Triebskorn R, Casper H, Scheil V, Schwaiger J (2007) Ultrastructural effects of pharmaceuticals (carbamazepine, clofibric acid, metoprolol, diclofenac) in rainbow trout (Oncorhynchus mykiss) and common carp (Cyprinus carpio). Analytical and Bioanalytical Chemistry 387(4), 1405-1416

Triebskorn R (2017) Weitergehende Abwasserreinigung: ein wirksames und bezahlbares Instrument zur Verminderung von Spurenstoffen und Keimen im Wasserkreislauf: gemeinsamer Schlussbericht der vom Bundesministerium für Bildung und Forschung sowie vom Ministerium für Umwelt, Klima und Energiewirtschaft Baden-Württemberg geförderten Projekte SchussenAktiv, SchussenAktivplus und SchussenAktivplus (English title: "Advanced wastewater treatment. An effective and affordable instrument for the reduction of trace substances and pathogens in the water cycle: joint final report of the projects SchussenAktiv, SchussenAktivplus and SchussenAktivplus funded by the Federal Ministry of Education and Research and the Ministry of Environment, Climate and Energy, BadenWürttemberg"). University of Tübingen, Germany. Accessed under http://dx.doi.org/10.15496/publikation-15721

Tseng IL, Yang YF, Yu CW, Li WH, Liao VHC (2013) Phthalates induce neurotoxicity affecting locomotor and thermotactic behaviors and AFD neurons through oxidative stress in Caenorhabditis elegans. PLoS One 8(12), e82657

Turner E, Kroeger G, Arnold M, Thornton B, Di Giulio R, Meyer J (2013) Assessing different mechanisms of toxicity in mountaintop removal/valley fill coal mining-affected watershed samples using Caenorhabditis elegans. PLoS One 8(9), e75329

United Nations Environmental Program (UNEP, 2005). Ridding the world of POPs: A guide to the Stockholm Convention on Persistent Organic Pollutants. The Secretariat of the Stockholm Convention and UNEP's Information Unit for Conventions, Geneva, Switzerland 
United States Environmental Protection Agency (US EPA, 1972). Clean water act 33 USC $\S 1251$ et seq. United States Congress, Washington, USA

Ura K, Kai T, Sakata S, Iguchi T, Arizono K (2002) Aquatic acute toxicity testing using the nematode Caenorhabditis elegans. Journal of Health Science 48(6), 583-586

Urbatzka R, van Cauwenberge A, Maggioni S, Viganò L, Mandich A, Benfenati E, Lutz I, Kloas W (2007) Androgenic and antiandrogenic activities in water and sediment samples from the river Lambro, Italy, detected by yeast androgen screen and chemical analyses. Chemosphere 67(6), 1080-1087

US EPA (2002) National pollutant discharge elimination system: secondary treatment standards. Accessed under http://cfpub.epa.gov/npdes/techbasedpermitting/sectreat.cfm

US EPA (2011) UN PIC U.S. PIC-nominated pesticides list. http://www.epa.gov/oppfead1/international/us-unlist.htm. Retrieved on December 2017

Utgikar R, Riddick DS (2017) Downregulation of cytochrome P450 2C8 by 3methylcholanthrene in human hepatocellular carcinoma cell lines. Canadian Journal of physiology and Pharmacology 95(6), 768-771

Valek L (2013) CYP35A3::GFP induction and reproductive toxicity of $\beta$-Naphthoflavone and environmental water samples in Caenorhabditis elegans. Master thesis. Johann Wolfgang Goethe University, Frankfurt am Main, Germany

Välitalo P, Massei R, Heiskanen I, Behnisch P, Brack W, Tindall AJ, Du Pasquier D, Küster E, Mikola A, Schulze T, Sillanpää M (2017) Effect-based assessment of toxicity removal during wastewater treatment. Water Research 126, 153-163

van der Hoeven R, McCallum KC, Cruz MR, Garsin DA (2011) Ce-Duox1/BLI-3 generated reactive oxygen species trigger protective SKN-1 activity via p38 MAPK signaling during infection in C. elegans. PLoS pathogens 7(12), e1002453

Van Hege K, Dewettinck T, Claeys T, de Smedt G, Verstraete W (2002) Reclamation of treated domestic wastewater using biological membrane assisted carbon filtration (BIOMAC). Environmental Technology 23(9), 971-980

Van Kessel WHM, Zaalberg RB, Seinen W (1989) Testing environmental pollutants on soil organisms: a simple assay to investigate the toxicity of environmental pollutants on soil organisms, using $\mathrm{CdCl} 2$ and nematodes. Ecotoxicology and Environmental Safety, 18(2), 181-190

Vanderford BJ, Mawhinney DB, Trenholm RA, Zeigler-Holady JC, Snyder SA (2011) Assessment of sample preservation techniques for pharmaceuticals, personal care products, and steroids in surface and drinking water. Analytical and Bioanalytical Chemistry 399(6), 2227-2234

Vasquez MI, Fatta-Kassinos D (2013) Is the evaluation of "traditional" physicochemical parameters sufficient to explain the potential toxicity of the treated wastewater at sewage treatment plants? Environmental Science and Pollution Research 20, 3516-3528

Vingskes A K, Spann N (2018) The toxicity of a mixture of two antiseptics, triclosan and triclocarban, on reproduction and growth of the nematode Caenorhabditis elegans. Ecotoxicology 27(4), 420-429

Völker J, Castronovo S, Wick A, Ternes TA, Joss A, Oehlmann J, Wagner M (2016) Advancing biological wastewater treatment: extended anaerobic conditions enhance the removal of endocrine and dioxin-like activities. Environmental Science \& Technology 50(19) 10606-10615

Völker J, Stapf M, Miehe U, Wagner M (2019) Systematic review of toxicity removal by advanced wastewater treatment technologies via ozonation and activated carbon. Environmental Science \& Technology 53(13), 7215-7233 
Völker J, Vogt T, Castronovo S, Wick A, Ternes TA, Joss A, Oehlmann J, Wagner M (2017) Extended anaerobic conditions in the biological wastewater treatment: Higher reduction of toxicity compared to target organic micropollutants. Water Research 116, 220-230

Wagner M, Schlüsener MP, Ternes TA, Oehlmann J (2013) Identification of putative steroid receptor antagonists in bottled water: combining bioassays and high-resolution mass spectrometry. PLoS One 8(8), e72472

Wagner M, Kienle C, Vermeirssen EL, Oehlmann J (2017) Endocrine disruption and in vitro ecotoxicology: recent advances and approaches. Advances in Biochemical Engineering/Biotechnology 157, 1-58

Wagner M, Oehlmann J (2011) Endocrine disruptors in bottled mineral water: estrogenic activity in the E-Screen. The Journal of Steroid Biochemistry and Molecular Biology 127(1), 128-135

Wagner M, Vermeirssen ELM, Buchinger S, Behr M, Magdeburg A, Oehlmann J (2013) Deriving bio-equivalents from in vitro bioassays: assessment of existing uncertainties and strategies to improve accuracy and reporting. Environmental Toxicology and Chemistry 32 (8), 1-12

Walker CH (1998) Biomarker strategies to evaluate the environmental effects of chemicals. Environmental Health Perspectives 106(Suppl 2), 613

Wang D, Wang Y, Shen L (2010) Confirmation of combinational effects of calcium with other metals in a paper recycling mill effluent on nematode lifespan with toxicity identification evaluation method. Journal of Environmental Sciences 22(5), 731-737

Wang S, Tang M, Pei B, Xiao X, Wang J, Hang H, Wu L (2008a) Cadmium induced germline apoptosis in Caenorhabditis elegans: the roles of HUS1, p53, and MAPK signaling pathways. Toxicological Science 102, 345-351

Wang X, Shen L, Yu H, Wang D (2008b) Toxicity evaluation in a paper recycling mill effluent by coupling bioindicator of aging with the toxicity identification evaluation method in nematode Caenorhabditis elegans. Journal of Environmental Sciences 20(11), 1373-1380

Wang X, Yang J, Li H, Guo S, Tariq M, Chen H, Wang C, Liu Y (2018) Chronic toxicity of hexabromocyclododecane (HBCD) induced by oxidative stress and cell apoptosis on nematode Caenorhabditis elegans. Chemosphere 208, 31-39

Wang Y, Qiao J, He C, Wang Z, Luo W, Sheng L (2015) Towards multi-level biomonitoring of nematodes to assess risk of nitrogen and phosphorus pollution in Jinchuan Wetland of Northeast China. Ecotoxicology 24(10), 2190-2199

Wang Z, Xu Z, Li X (2019) Impacts of methamphetamine and ketamine on C. elegans's physiological functions at environmentally relevant concentrations and eco-risk assessment in surface waters. Journal of Hazardous Materials 363, 268-276

Wannous G (2011) Caenorhabditis elegans - Testsysteme für Freiland und Labor (English title "Caenorhabditis elegans - Test systems for the field and laboratory"). PhD thesis. Universität Trier, Trier, Germany

Web of Science (2017) Topic/Title "wastewater toxicity". Clarivate Analytics, USA. Retrieved under http://apps.webofknowledge.com. proxy.ub.uni-frankfurt.de on January 2018

Weinhouse C, Truong L, Meyer JN, Allard P (2018) Caenorhabditis elegans as an emerging model system in environmental epigenetics. Environmental and Molecular Mutagenesis 59(7), 560-575

Wernersson AS, Carere M, Maggi C, Tusil P, Soldan P, James A, Sanchez W, Dulio V, Broeg K, Reifferscheid G, Buchinger S, Maas H, Van Der Grinten E, O'Toole S, Ausili A, Manfra L, Marziali L, Polesello S, Lacchetti I, Mancini L, Lilja K, Linderoth M, Lundeberg T, Fjällborg B, Porsbring T, Larsson J, Bengtsson-Palme J, Förlin L, Kienle C, Kunz P, 
Vermeirssen E, Werner I, Robinson CD, Lyons B, Katsiadaki I, Whalley C, den Haan K, Messiaen M, Clayton H, Lettieri T, Negrão Carvalho R, Gawlik BM, Hollert H, Di Paolo C, Brack W, Kammann U, Kase R (2015) The European technical report on aquatic effectbased monitoring tools under the water framework directive. Environmental Science Europe 27(1), 1-11

Wigh A, Aït-Aïssa S, Creusot N, Terrisse H, Delignette-Muller ML, Bergé A, Vulliet E, Domenjoud B, Gonzalez-Ospina A, Brosselin V, Devaux A, Bony S (2018) Assessment of ozone or not-treated wastewater ecotoxicity using mechanism-based and zebrafish embryo bioassays. Journal of Environmental Protection 9(4), 325-346

Wigh A, Devaux A, Brosselin V, Gonzalez-Ospina A, Domenjoud B, Aït-Aïssa S, Creusot N, Gosset A, Bazin C, Bony S (2016) Proposal to optimize ecotoxicological evaluation of wastewater treated by conventional biological and ozonation processes. Environmental Science and Pollution Research 23(4), 3008-3017

Wigh A, Geffard O, Abbaci K, Francois A, Noury P, Bergé A, Vulliet E, Domenjoud B, Gonzalez-Ospina A, Bony S, Devaux A (2017) Gammarus fossarum as a sensitive tool to reveal residual toxicity of treated wastewater effluents. Science of the Total Environment $584,1012-1021$

Wilhelm S, Henneberg A, Köhler HR, Rault M, Richter D, Scheurer M, Suchail S, Triebskorn $\mathrm{R}$ (2017) Does wastewater treatment plant upgrading with activated carbon result in an improvement of fish health? Aquatic Toxicology 192, 184-197

Williams DC, Bailey DC, Fitsanakis VA (2017) Chapter 17 - Caenorhabditis elegans as a Model to Assess Reproductive and Developmental Toxicity. In Gupta RC, Reproductive and Developmental Toxicology, Academic Press, USA

Williams PL, Dusenbery DB (1990) Aquatic toxicity testing using the nematode, Caenorhabditis elegans. Environmental Toxicology and Chemistry 9(10), 1285-1290

Wilson MJ, Khakouli-Duarte T. (2009) Nematodes as environmental indicators. CABI, Wallingford, UK

Winder C, Azzi R, Wagner D (2005) The development of the globally harmonized system (GHS) of classification and labelling of hazardous chemicals. Journal of Hazardous Materials 125(1), 29-44

Wittkowski P, Marx-Stoelting P, Violet N, Fetz V, Schwarz F, Oelgeschläger M, Schönfelder G, Vogl S (2019) Caenorhabditis elegans as a promising alternative model for environmental chemical mixture effect assessment-a comparative study. Environmental Science \& Technology 53(21), 12725-12733

World Health Organization (2002) Integrated risk assessment: nonylphenol case study. WHO/IPCS/IRA/12/04. World Health Organization, Geneva, Switzerland

World Health Organization (2016) Global report on urban health: equitable, healthier cities for sustainable development. Accessed under https://apps.who.int/iris/handle/10665/204715 on January 2018

WormBase (2018) Database entry for species: C. elegans (Genome assembly: WBcel235) and gene: cyp-35A3. Version WS266. Accessed under http://dev.wormbase.org:9004/species/c_elegans/gene/cyp-35A3 on February 2018

Wren JF, Kille P, Spurgeon DJ, Swain S, Sturzenbaum SR, Jager T (2011) Application of physiologically based modelling and transcriptomics to probe the systems toxicology of aldicarb for Caenorhabditis elegans (Maupas 1900). Ecotoxicology 20(2), 397-408

Wu C, Gao Z, Zhou Y, Liu M, Song J, Yu Y (2015) Treatment of secondary effluent from a petrochemical wastewater treatment plant by ozonation-biological aerated filter. Journal of Chemical Technology \& Biotechnology 90(3), 543-549 
Wu QY, Zhou YT, Li W, Zhang X, Du Y, Hu HY (2019) Underestimated risk from ozonation of wastewater containing bromide: Both organic byproducts and bromate contributed to the toxicity increase. Water Research 162, 43-52

Xiao G, Zhao L, Huang Q, Yang J, Du H, Guo D, Xia M, Li G, Chen Z, Wang D (2018) Toxicity evaluation of Wanzhou watershed of Yangtze Three Gorges reservior in the flood season in Caenorhabditis elegans. Scientific Reports 8, 6734

Xiao Y, De Araujo C, Sze CC, Stuckey DC (2015) Toxicity measurement in biological wastewater treatment processes: a review. Journal of Hazardous Materials 286, 15-29

Xiong H, Pears C, Woollard A (2017) An enhanced C. elegans based platform for toxicity assessment. Scientific Reports 7(1), 9839

Yang K, Yu J, Guo Q, Wang C, Yang M, Zhang Y, Xia P, Zhang D, Yu Z (2017) Comparison of micropollutants' removal performance between pre-ozonation and post-ozonation using a pilot study. Water Research 111, 147-153

Yang XL, Xia MQ, Chen M, Shen DQ, Fu DF, Song HL (2014) Optimization of solid-phase extraction for pretreatment of selected estrogens in sewage by response surface methodology. Polish Journal of Environmental Studies 23(6)

Yeargers E (1981) Effect of gamma-radiation on dauer larvae of Caenorhabditis elegans. Journal of Nematology 13(2), 235-237

Yeates GW, Bongers T, De Goede RGM, Freckman DW, Geogieva SS (1993) Feeding habits in soil nematode families and genera - An outline for soil ecologists. Journal of Nematology 25, 315-331

Yeom IT, Lee KR, Lee YH, Ahn KH, Lee SH (2002) Effects of ozone treatment on the biodegradability of sludge from municipal wastewater treatment plants. Water Science and Technology 46(4-5), 421-425

Yu Y, Hua X, Chen H, Li Z, Han Y, Xiang M (2020) Toxicity of lindane induced by oxidative stress and intestinal damage in Caenorhabditis elegans. Environmental Pollution 114731

Yu Z, Jiang L, Yin D (2011) Behavior toxicity to Caenorhabditis elegans transferred to the progeny after exposure to sulfamethoxazole at environmentally relevant concentrations. Journal of Environmental Sciences 23(2), 294-300

Zhang D, Gersberg RM, Ng WJ, Tan SK (2014) Removal of pharmaceuticals and personal care products in aquatic plant-based systems: a review. Environmental Pollution 184, 620639

Zhang Q, Lu M, Dong X, Wang C, Zhang C, Liu W, Zhao M (2014) Potential estrogenic effects of phosphorus-containing flame retardants. Environmental Science \& Technology 48(12), 6995-7001

Zhao JL, Chen XW, Yan B, Wei C, Jiang YX, Ying GG (2015) Estrogenic activity and identification of potential xenoestrogens in a coking wastewater treatment plant. Ecotoxicology and Environmental Safety 112, 238-246

Zhao JL, Ying GG, Yang B, Liu S, Zhou LJ, Chen ZF, Lai HJ (2011) Screening of multiple hormonal activities in surface water and sediment from the Pearl river system, South China, using effect-directed in vitro bioassays. Environmental Toxicology and Chemistry 30(10), 2208-2215

Zhao Y, Wu Q, Wang D (2016) An epigenetic signal encoded protection mechanism is activated by graphene oxide to inhibit its induced reproductive toxicity in Caenorhabditis elegans. Biomaterials 79, 15-24

Zhou D, Yang J, Li H, Lu Q, Lin KF (2016) Ecotoxicological evaluation of low-concentration bisphenol A exposure on the soil nematode Caenorhabditis elegans and intrinsic 
mechanisms of stress response in vivo. Environmental Toxicology and Chemistry 35(8), 2041-2047

Zhou JL, Zhang, ZL, Banks E, Grover D, Jiang JQ (2009) Pharmaceutical residues in wastewater treatment works effluents and their impact on receiving river water. Journal of Hazardous Materials 166(2-3), 655-661

Zhuang Z, Zhao Y, Wu Q, Li M, Liu H, Sun L, Gao W, Wang D (2014) Adverse effects from clenbuterol and ractopamine on nematode Caenorhabditis elegans and the underlying mechanism. PLoS One 9(1), e85482

Zuo YT, Hu Y, Lu WW, Cao JJ, Wang F, Han X, Lu WQ, Liu AL (2017) Toxicity of 2, 6 dichloro-1, 4-benzoquinone and five regulated drinking water disinfection by-products for the Caenorhabditis elegans nematode. Journal of Hazardous Materials 321, 456-463

Zwart N, Nio SL, Houtman CJ, de Boer J, Kool J, Hamers T, Lamoree MH (2018) Highthroughput effect-directed analysis using downscaled in vitro reporter gene assays to identify endocrine disruptors in surface water. Environmental Science \& Technology 52(7), 4367-4377 
Annex 


\section{A.1 What you extract is what you see: Optimising the preparation of water and wastewater samples for in vitro bioassays}

Aennes Abbas*, llona Schneider*, Anna Bollmann, Jan Funke, Jörg Oehlmann, Carsten Prasse, Ulrike Schulte-Oehlmann, Wolfram Seitz, Thomas Ternes, Marcus Weber, Henning Wesley, Martin Wagner

Water Research 125, 47-60

(First Online: 02 Jan 2019)

*shared first-authorship (see next page for further details) 


\section{Declaration (paper A.1)}

Author contributions to the publication: "What you extract is what you see: Optimising the preparation of water and wastewater samples for in vitro bioassays"

Status: accepted \& printed Journal: Water Research

Contributing authors (names and distinct initials):

- Aennes Abbas (AA)

- Ilona Schneider (IS)

- Anna Bollmann (AB)

- Jan Funke (JF)

- Jörg Oehlmann (JO)

- Carsten Prasse (CP)

- Ulrike Schulte-Oehlmann (US)

- Wolfram Seitz (WS)

- Thomas Ternes (TT)

- Marcus Weber (MC)

- Henning Wesley (HW)

- Martin Wagner (MW)

Individual contributions of the first authors and co-authors:

\section{Study conception and design:}

Doctoral candidate (AA): $40 \%$

Shared first author (IS): $40 \%$

Coauthors (AB, CP, JF, JO, US, TT, MW): $20 \%$

\section{Performance of experiments and assays}

Doctoral candidate (AA): $45 \%$

Shared first author (IS): $45 \%$

Coauthors (AB, JF, HW, CP): 10\%

\section{Compilation of data sets and tables/figures:}

Doctoral candidate (AA): $40 \%$

Shared first author (IS): $50 \%$

Coauthors (MC, MW): 10\%

\section{Data analyses and interpretation:}

Doctoral candidate (AA): $37,5 \%$

Shared first author (IS): $37,5 \%$

Coauthors (MC, MW): 15\%

Coauthors (JO, CP): 10\%

\section{Drafting of the Manuscript:}

Doctoral candidate (AA): $40 \%$

Shared first author (IS): $40 \%$

Coauthor (MW): 15\%

Coauthors (CP, JO, US): $5 \%$ 


\title{
Author accepted manuscript (paper A.1)
}

\author{
Accepted Manuscript
}

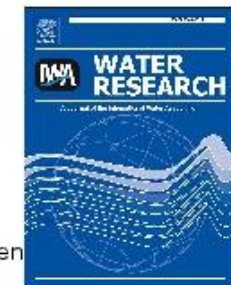

Prasse, Ulrike Schulte-Oehlmann, Wolfram Seitz, Thomas Ternes, Marcus Weber, Henning Wesely, Martin Wagner

PII: S0043-1354(18)31067-4

DOI: https://doi. org/10.1016/j.watres.2018.12.049

Reference: WR 14345

To appear in: Water Research

Received Date: 26 August 2018

Revised Date: 19 December 2018

Accepted Date: 21 December 2018

Please cite this article as: Abbas, A., Schneider, I., Bollmann, A., Funke, J., Oehlmann, J., Prasse, C., Schulte-Oehlmann, U., Seitz, W., Ternes, T. Weber, M. Wesely, H., Wagner, M. What you extract is what you see: Optimising the preparation of water and wastewater samples for in vitro bioassays, Water Research, https://doi.org/10.1016/j.watres.2018.12.049.

This is a PDF file of an unedited manuscript that has been accepted for publication. As a service to our customers we are providing this early version of the manuscript. The manuscript will undergo copyediting, typesetting, and review of the resulting proof before it is published in its final form. Please note that during the production process errors may be discovered which could affect the content, and all legal disclaimers that apply to the journal pertain.

This manuscript version is made available under the CC-BY-NC-ND 4.0 license https://creativecommons.org/licenses/by-nc-nd/4.0/ 


\section{WR46063 \\ Graphical abstract - revised version}

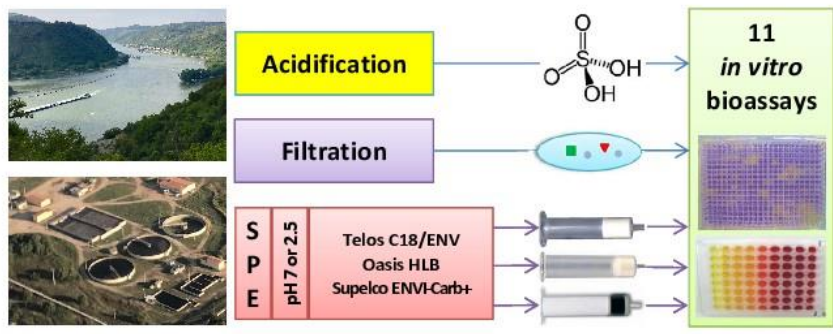


1 What you extract is what you see: Optimising the preparation of water and

2 wastewater samples for in vitro bioassays

3 Aennes Abbas ${ }^{1, *}$ \& Ilona Schneider ${ }^{1, *}$, Anna Bollmann ${ }^{2}$, Jan Funke ${ }^{3}$, Jörg Oehlmann ${ }^{1}$, Carsten

4 Prasse $^{4}$, Ulrike Schulte-Oehlmann ${ }^{1}$, Wolfram Seitz ${ }^{2}$, Thomas Ternes ${ }^{5}$, Marcus Weber ${ }^{6}$,

5 Henning Wesely ${ }^{5}$, Martin Wagner ${ }^{1,7}$

$6{ }^{1}$ Department Aquatic Ecotoxicology, Goethe University Frankfurt am Main, Max-von-Laue-

7 Str. 13, D-60438 Frankfurt, Germany

$8{ }^{2}$ Zweckverband Landeswasserversorgung, Am Spitzigen Berg 1, D-89129 Langenau,

9 Germany

$10{ }^{3}$ IWW Rheinisch-Westfälisches Institut für Wasser Beratungs- und Entwicklungsgesellschaft

$11 \mathrm{mbH}$, Moritzstraße 26, D-45476 Muelheim an der Ruhr, Germany

$12{ }^{4}$ Department of Environmental Health and Engineering, Johns Hopkins University,

13 Baltimore, MD, 21218, USA

$14 \quad{ }^{5}$ Federal Institute of Hydrology (BfG), Am Mainzer Tor 1, D-56068 Koblenz, Germany

$15{ }^{6}$ Department of Numerical Analysis and Modelling, Konrad-Zuse-Zentrum für

16 Informationstechnik Berlin (ZIB), Takustraße 7, D-14195 Berlin, Germany

$17{ }^{7}$ Department of Biology, Norwegian University of Science and Technology, NO-7491

18 Trondheim, Norway

19

20 "These authors contributed equally to the work.

21 Email addresses of corresponding authors: abbas@bio.uni-frankfurt.de, i.schneider@bio.uni-

22 frankfurt.de 


\section{Abstract}

24 The assessment of water quality is crucial for safeguarding drinking water resources and

25 ecosystem integrity. To this end, sample preparation and extraction is critically important,

26 especially when investigating emerging contaminants and the toxicity of water samples. As

27 extraction methods are rarely optimised for bioassays but rather adopted from chemical

28 analysis, this may result in a misrepresentation of the actual toxicity.

29 In this study, surface water, groundwater, hospital and municipal wastewater were used to

30 characterise the impacts of common sample preparation techniques (acidification, filtration

31 and solid phase extraction (SPE)) on the outcomes of eleven in vitro bioassays. The latter

32 covered endocrine activity (reporter gene assays for estrogen, androgen, aryl-hydrocarbon,

33 retinoic acid, retinoid $\mathrm{X}$, vitamin $\mathrm{D}$, thyroid receptor), mutagenicity (Ames fluctuation test),

34 genotoxicity (umu test) and cytotoxicity. Water samples extracted using different SPE

35 sorbents (Oasis HLB, Supelco ENVI-Carb+, Telos C18/ENV) at acidic and neutral pH were

36 compared for their performance in recovering biological effects.

37 Acidification, commonly used for stabilisation, significantly altered the endocrine activity and toxicity of most (waste)water samples. Sample filtration did not affect the majority of

39 endpoints but in certain cases affected the (anti-)estrogenic and dioxin-like activities. SPE

40 extracts (10.4× final concentration), including WWTP effluents, induced significant endocrine

41 effects that were not detected in aqueous samples ( $0.63 \times$ final concentration $)$, such as

42 estrogenic, (anti-)androgenic and dioxin-like activities. When ranking the SPE methods using

43 multivariate Pareto optimisation an extraction with Telos C18/ENV at $\mathrm{pH} 7$ was most

44 effective in recovering toxicity. At the same time, these extracts were highly cytotoxic

45 masking the endpoint under investigation. Compared to that, extraction at $\mathrm{pH} 2.5$ enriched

46 less cytotoxicity,

47 In summary, our study demonstrates that sample preparation and extraction critically affect

48 the outcome of bioassays when assessing the toxicity of water samples. Depending on the 
49 water matrix and the bioassay, these methods need to be optimised to accurately assess water

50 quality.

51

52 Keywords

53 Activated carbon, advanced treatment, endocrine disrupting chemicals, micropollutants,

54 ozonation, transformation products, tertiary treatment

55

56 Abbreviations

\begin{tabular}{|ll|}
\hline 9-cis-RA & 9-cis retinoic acid \\
4-NOPD & 4-nitro-o-phenylenediamine \\
4-NQO & 4-nitroquinoline N-oxide \\
AhR & aryl-hydrocarbon receptor \\
Ames & bacterial reverse mutation test \\
ANOVA & analysis of variance \\
at-RA & all-trans retinoic acid \\
CAS & Chemical Abstracts Service \\
CPRG & chlorophenol red- $\beta$-D-galactopyranoside \\
DIN & German Institute of Standardisation (Deutsches Institut für Normung) \\
DMSO & dimethyl sulfoxide \\
DNA & deoxyribonucleic acid \\
DOC & dissolved organic carbon \\
E $_{2}$ & 17 $\beta$-estradiol \\
EC & European Commission \\
EC SO & Median effect concentration \\
EDCs & endocrine disrupting chemicals \\
EFF & effluent \\
FB & filtration basin \\
Flu & flutamide \\
GW & groundwater \\
hAR & human androgen receptor \\
hER $\alpha$ & \\
&
\end{tabular}




\begin{tabular}{|c|c|}
\hline HOS & hospital \\
\hline IB & infiltration basin \\
\hline INF & influent \\
\hline IR & induction rate \\
\hline ISO & International Standard Organisation \\
\hline $\operatorname{lac} Z$ & bacterial gene coding $\beta$-galactosidase \\
\hline LOQ & limit of quantification \\
\hline MS & microsieve \\
\hline n.a. & not analysed \\
\hline NF & nitrofurantoin \\
\hline$\beta-N F$ & $\beta$-naphthoflavone \\
\hline n.s. & not significant \\
\hline OD & optical density \\
\hline OHT & 4-hydroxytamoxifen \\
\hline ONPG & $o$-nitrophenyl $\beta$-D-galactopyranoside \\
\hline PTFE & polytetrafluorethylene \\
\hline $\operatorname{RAR} \alpha$ & retinoic acid receptor $\alpha$ \\
\hline $\mathrm{RXR} \alpha$ & retinoid $X$ receptor $\alpha$ \\
\hline SOS & inducible bacterial DNA repair system \\
\hline SPE & solid phase extraction \\
\hline SW & surface water \\
\hline $\mathrm{T}$ & testosterone \\
\hline $\mathrm{T}_{3}$ & 3,3',5-triiod-L-thyronine \\
\hline TA100 & recombinant strain of Salmonella typhimurium \\
\hline TA98 & recombinant strain of Salmonella typhimurium \\
\hline $\operatorname{TR} \alpha$ & thyroid receptor $\alpha$ \\
\hline TSS & total suspended solids \\
\hline umu & bacterial test for the determination of genotoxicity \\
\hline итис & bacterial ultra violet mutagenesis gene $\mathrm{C}$ \\
\hline US EPA & United States Environmental Protection Agency \\
\hline$u v r B$ & gene of a bacterial DNA repair system \\
\hline VDR & vitamin $\mathrm{D}$ receptor \\
\hline WWTP & wastewater treatment plant \\
\hline YAAS & yeast anti-androgen screen \\
\hline
\end{tabular}


5

YAES yeast anti-estrogen screen

YAS yeast androgen screen

YDS yeast dioxin screen

YES yeast estrogen screen

57 


\section{$58 \quad 1$ Introduction}

59 Anthropogenic micropollutants typically occur at nanogram to microgram per litre

60 concentrations in urban water cycles. Micropollutants may pose a risk to ecosystems as they

61 have been associated with negative impacts on aquatic biota (Malaj et al. 2014, Prasse et al.

62 2015). Micropollutants are found amongst pharmaceuticals, personal care products, industrial

63 chemicals, pesticides and biocides (Kümmerer 2011) that are emitted from different

64 anthropogenic sources. These sources can be diffuse, such as agricultural runoffs, or point

65 sources, such as wastewater treatment plant (WWTP) discharges. Several studies have

66 demonstrated an incomplete removal of micropollutants and relevant toxicity after

67 conventional wastewater treatment using activated sludge (Prasse et al. 2015). Therefore,

68 advanced wastewater treatment technologies utilising chemical oxidation or adsorption are

69 being developed to increase the removal of micropollutants and toxicity (Miklos et al. 2018,

70 Rizzo 2011). In vitro bioassays play a crucial role for the ecotoxicological assessment of

71 water and wastewater quality because they determine the joint toxicity caused by complex

72 samples, often regarding a specific mode of action (Escher et al. 2014, 2018, Leusch et al.

73 2017). Bioassays are routinely used in monitoring campaigns and sufficiently advanced to be

74 integrated into water and wastewater regulations (Brack et al. 2017, Escher et al. 2018).

75 Environmental water and wastewater samples represent complex mixtures of known and

76 unknown chemicals (Schwarzenbach et al. 2006) and are characterised by a variable

77 composition with respect to matrix parameters (e.g., suspended solids or dissolved organic

78 carbon (DOC)). The toxicity of the samples is mainly determined by the type and

79 concentration of the active, anthropogenic or natural compound(s) and their cumulative

80 effects. However, the sample matrix can also affect the outcome of a bioassay (Janošek et al.

81 2007, Neale et al. 2015). In addition, samples can undergo physicochemical and biological

82 processes that can transform or degrade the active compounds and may, therefore, modulate

83 the biological effects under investigation. 
84 Because of their ability to reduce matrix effects, to preserve and to concentrate dissolved organic chemicals in aqueous samples, different extraction methods, such as solid phase extraction (SPE), are used in chemical and ecotoxicological studies (Prasse et al. 2015). While

87 sample preparation and extraction methods are commonly optimised for chemical analysis,

88 i.e., to maximise the recovery of specific target compounds, this is rarely done in bioassay

89 studies (Bistan et al. 2012, Neale et al. 2018, Schulze et al. 2017) because the "true" toxicity

90 to recover remains unknown. Thus, standard extraction procedures adapted from chemical

91 analysis are mainly used. Comparative studies have indicated that such chemical "standard"

92 methods can be ineffective in extracting unknown, active compounds from water samples

93 (Hendriks et al. 1994, Wagner and Oehlmann 2011). Because this can lead to an

94 underestimation or false negative results, optimising sample preparation and extraction to

95 recover a maximum of toxicity should be imperative for bioassay studies.

96 The aim of our study was to assess the impacts of common samples preparation methods on

97 the detection of environmentally-relevant endocrine activities, genotoxicity and cytotoxicity

98 in water and wastewater samples. These samples consisted of surface water, groundwater,

99 hospital wastewater, raw (untreated), conventionally-treated and ozonated wastewater. These

100 samples consisted of grab as well as composite samples with low to high contamination

101 degrees to allow for an optimal comparison of SPE methods. The toxicity of untreated

102 aqueous samples and samples that were acidified $(24 \mathrm{~h}$ at $\mathrm{pH} 2.0)$ or filtered $(1 \mu \mathrm{m}$ pore size $)$

103 was compared in eleven in vitro bioassays. Furthermore, the effectiveness of six SPE methods

104 was compared by extracting samples with three SPE sorbents at acidic and neutral sample pH

105 (2.5 and 7 right before loading). Aqueous and extracted samples were analysed using

106 bioassays for nine human hormone receptors, the umu test and the Ames fluctuation test. The

107 outcome of these bioassays was evaluated by a multivariate Pareto optimisation to identify the most effective sample extraction method. 
1092 Material and methods

\section{$110 \quad$ 2.1 Characterisation of sampling sites}

111 Sampling locations were selected according to their relevance and representativeness

112 regarding the water cycle in a model region in Baden-Württemberg (Germany, Table 1,

113 samples 1-14, see Seitz and Winzenbacher 2017 for details). Samples comprised influents

114 and effluents of three municipal WWTPs (WWTP 1-3) with activated sludge treatment, two

115 hospital wastewaters, three rivers (surface water), influent and effluent of a filtration basin,

116 two storm water sedimentation tanks, one storm water overflow tank (with infiltration basin),

117 and three groundwater monitoring wells (hotspots). Additional wastewater samples were taken from a pilot WWTP (WWTP 4) in Hessen, Germany (Knopp et al. 2016), equipped with advanced treatment technologies, including a full-scale ozonation of conventionally treated effluent (activated sludge) filtered using a microsieve (MS, filtration at mesh size: $10 \mu \mathrm{m}$ ) to reduce total suspended solids (TSS, Table 1, samples 15-19). The ozonation was

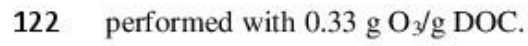

\section{$124 \quad 2.2$ Collection of water and wastewater samples}

125 Wastewater samples (influent and effluent) from the municipal WWTPs in Baden-

126 Württemberg (sampling period: April (B), July (C, D) and December (E) 2012) and the pilot

127 WWTP in Hessen (sampling period: March (A), April (B), July 2012 (C, D) and December (E) 2012, January (F) 2013) were collected as grab (samples 1, 6, 8-14, 18) or $24 \mathrm{~h}$ composite

129 samples (samples 2-5, 7, 15-17, Table 1). The results of corresponding samples (e.g.,

130 influents or effluents) were compared to each other, only, with exception of the event-driven

131 sampling of samples 6 and 7 (FB-IN and FB-OUT, Table 1). For the collection of $24 \mathrm{~h}$

132 composite samples, wastewater was continuously pumped through polytetrafluoroethylene

133 (PTFE) tubes into $5 \mathrm{~L}$ glass bottles. Bottles were kept at $4^{\circ} \mathrm{C}$ in darkness during sampling.

134 Hospital effluents, surface waters, samples from storm water sedimentation and an overflow 
135 tank (with infiltration basin) as well as groundwater hotspots were grab samples (sampling period: April (B), July (C, D) and December (E) 2012). All samples were stored at $4^{\circ} \mathrm{C}$ in precleaned, amber glass bottles with PTFE lids and analysed (aqueous samples for acidification and filtration experiments) or further processed (comparison of SPE methods) within $48 \mathrm{~h}$ after sampling.

\subsection{Sample preparation}

\subsubsection{Acidification for testing aqueous samples}

One aliquot $(40 \mathrm{~mL}$ ) of the aqueous (waste)water sample was kept at the original $\mathrm{pH}$, another aliquot $(40 \mathrm{~mL})$ was acidified with sulphuric acid ( $5 \mathrm{~mol} / \mathrm{L}$, purity "pro analysi") to $\mathrm{pH} 2.0$ directly after sampling. After storage for $24 \mathrm{~h}$ at $4^{\circ} \mathrm{C}$ in the dark, acidified samples were neutralised with sodium hydroxide ( $1 \mathrm{~mol} / \mathrm{L}$, purity "pro analysi") to $\mathrm{pH} 7$ prior to analysing the aqueous samples in the bioassays (in contrast to short-term acidification for SPE, 2.3.3).

\subsubsection{Filtration for testing aqueous samples}

One aliquot of the (waste)water sample remained unfiltered while another aliquot was filtered using glass fibre filters (Whatman GF6, pore size $1 \mu \mathrm{m}$ ) to reduce TSS. Selected filtered and unfiltered aqueous samples were tested as aqueous samples (not SPE extracts) in the in vitro assays (2.4). The glass fibre filters containing the retentate were suspended in ultrapure water (10 $\mathrm{min}$ in an ultrasonic bath) and the obtained aqueous suspensions were analysed for endocrine activity retained on the filters. A filter control was run and analysed in parallel: ultra-pure water was filtered and an empty glass fibre filter was suspended as well. Additionally, the influence of a microsieve (mesh size: $10 \mu \mathrm{m}$ ) on endocrine and genotoxic activity of conventionally treated effluent after final sedimentation at WWTP 4 was investigated by taking wastewater samples before and after the microsieve. A microsieve control was analysed as well (data not shown): fragments of the microsieve were incubated in 
10

161 ultra-pure water and in methanol for $70 \mathrm{~d}$ and the resulting suspensions were tested in the in

162

163

164

165

166

167

168

169

170

171

172

173

174

175

176

177

178

179

180

181

182

vitro bioassays.

\subsubsection{Solid phase extraction}

Three commonly used types of SPE sorbents were tested for the recovery of endocrine, genotoxic, and mutagenic activities: Oasis HLB (200 mg), Kinesis Telos C18/ENV (500 mg C18, $200 \mathrm{mg} \mathrm{ENV})$ and Supelco ENVI-Carb+ $(200 \mathrm{mg})$. Prior to sample loading, the cartridges were conditioned as follows: Oasis HLB and Telos C18/ENV were conditioned consecutively with $1 \times 2 \mathrm{~mL}$ heptane, $1 \times 2 \mathrm{~mL}$ acetone, $3 \times 2 \mathrm{~mL}$ methanol (LC-MS Optigrade) and $4 \times 2 \mathrm{~mL}$ ultrapure water. Supelco ENVI-Carb+ cartridges were turned (top to bottom) before they were conditioned with $1 \times 2 \mathrm{~mL}$ acetone and $1 \times 2 \mathrm{~mL}$ methanol. Afterwards, the columns were turned again (loading direction) and conditioned with $1 \times 2 \mathrm{~mL}$ acetone, $3 \times 2 \mathrm{~mL}$ methanol and $4 \times 2 \mathrm{~mL}$ ultrapure water. For each sample, $500 \mathrm{~mL}$ sample was extracted at two $\mathrm{pH}$ values, neutral $(\mathrm{pH} 7)$ and acidified with sulphuric acid $(3.5 \mathrm{~mol} / \mathrm{L})$ to $\mathrm{pH} 2.5$.

SPE was performed within $48 \mathrm{~h}$ after collection and directly after acidification. The columns were dried under a stream of nitrogen and stored at $-20^{\circ} \mathrm{C}$. Samples extracted at neutral $\mathrm{pH}$ were eluted with $5 \times 2 \mathrm{~mL}$ acidified methanol and $5 \times 2 \mathrm{~mL}$ acetone, each containing $0.2 \%$ formic acid. Acidified samples were consecutively eluted with $5 \times 2 \mathrm{~mL}$ methanol and $5 \mathrm{x}$ $2 \mathrm{~mL}$ acetone at neutral $\mathrm{pH}$. After adding $100 \mu \mathrm{L}$ dimethyl sulfoxide (DMSO), the combined methanol-acetone extract was concentrated to $100 \mu \mathrm{L}$ final volume under a gentle nitrogen stream. The extracts (5000-fold concentrated compared to the aqueous sample) were stored at $-20^{\circ} \mathrm{C}$ until testing. A SPE blank was prepared in parallel to each sampling campaign to control for contamination by loading each column type with ultrapure water and extracting them with neutral and acidified methanol and acetone, respectively. 
$187 \quad 2.4 \mathrm{In}$ vitro bioassays

188

\subsubsection{Recombinant yeast screens for endocrine activities}

In this study, nine recombinant yeast-based reporter-gene assays were used to detect endocrine activities: Yeast Estrogen Screen (YES, human estrogen receptor $\alpha(\mathrm{hER} \alpha)$ ), Yeast Anti-Estrogen Screen (YAES), Yeast Androgen Screen (YAS, human androgen receptor (hAR)), Yeast Anti-Androgen Screen (YAAS) first described by Routledge and Sumpter (1996) and Sohoni and Sumpter (1998), Yeast Dioxin Screen (YDS, aryl-hydrocarbon receptor (AhR, Miller 1997)), as well as yeast two-hybrid assays for retinoic acid receptor $\alpha$ (RAR $\alpha$ ), retinoid X receptor $\alpha(\mathrm{RXR} \alpha)$, vitamin $\mathrm{D}$ receptor (VDR) and thyroid receptor $\alpha$ (TR $\alpha$ ) introduced by Inoue et al. (2009). We used yeast-based assays rather than mammalian cell lines because they are robust in terms of cytotoxicity, because they have been validated by ISO (ISO 19040-1:2018) and to compare the results to our previous work.

All bioassays have the same principle: The activation of the respective receptor by chemicals present in the sample triggers the expression of $\beta$-galactosidase, which cleaves the chromogenic substance chlorophenol red- $\beta$-D-galactopyranoside (CPRG; CAS 99792-79-7, Sigma-Aldrich, Germany). The intensity of the colour change (yellow to red) is proportional to the agonistic activity of the sample and is measured with a photometer (Multiskan Ascent,

Thermo Fisher Scientific, Braunschweig, Germany) at a wavelength of $540 \mathrm{~nm}\left(\mathrm{OD}_{540}\right)$. To screen for antagonistic activities (YAES and YAAS), a known agonist is added. Thus, antagonistic compounds reduced the reporter gene activity induced by the agonist.

7 All bioassays were conducted in 96-well microtiter plates (f-form, VWR Darmstadt, Germany) as described previously (Völker et al. 2016, Wagner et al. 2013, Stalter et al. 2011,

9 Wagner and Oehlmann 2009). In brief, aqueous samples were analysed in eight replicates with a dilution factor of 1.6 (i.e., 0.625 -fold final sample concentration). SPE extracts were 1 diluted 480 -fold resulting in a 10.4-fold final sample concentration $(0.2 \% \mathrm{v} / \mathrm{v}$ solvent 
212 concentration, eight replicates). This enrichment factor was used for all SPE extracts

213 (compare 2.2 and Table 1). After 18-22 $\mathrm{h}$ incubation (depending on the assay) at $30^{\circ} \mathrm{C}$ and

$2141200 \mathrm{rpm}$, cell number (absorbance at $595 \mathrm{~nm}, \mathrm{OD}_{595}$, to detect cytotoxic effects) and

215 reporter-gene activity $\left(\mathrm{OD}_{540}\right)$ were determined photometrically. In each assay and

216 experiment, concentration-response curves for the appropriate reference compound were

217 generated (see Table S1 and Figures S1-S5 for details).

218 The $\mathrm{OD}_{540}$ was corrected for the respective cell density $\left(\mathrm{OD}_{595}\right)$. If $>20 \%$ cytotoxicity

219 occurred (see 2.5) results were not used. The corrected absorbance was normalised to the

220 negative/solvent controls $(0 \%)$ and the maximum activity of the reference compound $(100 \%)$

221 to calculate relative activities (\%). For the antagonist assays, a control without agonist was

222 used to represent $100 \%$ receptor inhibition.

223 The limit of quantification (LOQ) was calculated for each bioassay and experiment using the

224 mean activity of the negative control and adding threefold it's standard deviation. As the

225 LOQs varied between bioassays and experiments, they were not shown for the sake of clarity.

226 However, in general only results above the LOQs were considered. In a few cases, such as

227 estrogenic activity, lower activities were shown because of their ecotoxicological relevance

228 (low effect threshold) and for comparing WWTP effectivities.

$230 \quad$ 2.4.2 Genotoxicity assay (umu test)

231 Genotoxic effects were assessed using the umu test (ISO 13829) with the genetically modified

232 Salmonella typhimurium strain TA1535 (pSK1002). The umu test detects primary reversible

233 or irreversible DNA damages that induce the expression of the DNA SOS-repair system

234 associated with the UV mutagenesis gene C (umuC gene). Genotoxic substances in the

235 samples lead to an expression of $\beta$-galactosidase from the umuC-lac $Z$ construct. The reporter-

236 gene activity is determined by the cleavage of the chromogenic substance $o$-nitrophenyl $\beta$-D-

237 galactopyranoside (ONPG, CAS 369-07-3, Sigma-Aldrich, Germany). The umu test was 
238 conducted as described by Magdeburg et al. (2014). In brief, aqueous samples were analysed

239 after sterile filtration (injection filter with PTFE membrane: pore size $0.2 \mu \mathrm{m}$, neoLab,

240 Germany) with a dilution factor of 1.7 and SPE extracts in a 20 -fold final sample

241 concentration $(0.4 \% \mathrm{v} / \mathrm{v}$ solvent $)$ in eight replicates. Ten concentrations between $5-2000 \mu \mathrm{g} / \mathrm{L}$

242 final concentration in the well of 4-nitroquinoline N-oxide (4-NQO; CAS 56-57-5, Sigma-

243 Aldrich, Germany) were used as genotoxic reference compound (Table S1). Cytotoxicity

244

245

246 $\left(\mathrm{OD}_{595}\right)$ and genotoxicity $\left(\mathrm{OD}_{414}\right)$ were determined photometrically. The $\mathrm{OD}_{414}$ was corrected for the respective cell density $\left(\mathrm{OD}_{595}\right)$ if no cytotoxicity occurred (see 2.5). A linear regression line was generated using the corrected $\mathrm{OD}_{414}$ of the reference compound (Figure S6). The induction rate (IR) was calculated using the corrected $\mathrm{OD}_{414}$ of the samples. An IR $\geq 1.5$ is considered potentially genotoxic.

\subsubsection{Mutagenicity assay (Ames fluctuation test)}

Mutagenic effects (i.e., irreversible DNA damage) were analysed using the Ames fluctuation test (ISO/DIN 11350) with two genetically modified strains of Salmonella typhimurium (TA98 and TA100). The assay detects the induction of point mutations in special marker genes coding for enzymes involved in histidine biosynthesis as frameshift mutations (TA98) and base pair substitutions (TA100). To increase sensitivity, the strains TA98 and TA100 have a mutation in the $\operatorname{uvr} B$ DNA repair gene. In the absence of mutagens, the strains do not grow in histidine-free medium and a reverse mutation in the marker genes enables histidine synthesis and thus growth. This leads to a $\mathrm{pH}$ change in the assay medium that is determined photometrically at a wavelength of $414 \mathrm{~nm}$.

The Ames test was conducted as described by Magdeburg et al. (2014). In brief, aqueous samples were tested after sterile filtration (see 2.3.2) with a dilution factor of 1.25 and SPE extracts in a 10 -fold final sample concentration $(0.2 \% \mathrm{v} / \mathrm{v}$ solvent). Mutagenic reference compounds were used as positive controls (TA98: $10 \mathrm{mg} / \mathrm{L}$ final concentration in the well 4 - 
265 TA100: $0.25 \mathrm{mg} / \mathrm{L}$ final concentration in the well nitrofurantoin (NF; CAS 67-20-9, Sigma

266 Aldrich, Germany, Table S1). The mutagenic activity of the sample was determined photometrically with a cut-off value at a wavelength of $414 \mathrm{~nm}$ by counting the number of wells that shifted from purple (negative) to yellow (positive).

\subsection{Data analysis}

In this study, cytotoxicity was defined as a cell number in the sample of $\leq 80 \%$ compared to

2 the negative control (solvent control) analysed in parallel in each experiment.

3 Statistical analyses were performed using GraphPad Prism (version 5.03, GraphPad Software Inc., San Diego, California, USA). Datasets were analysed using the D'Agostino and Pearson omnibus normality test for Gaussian distribution and the Bartlett's test for homogeneity of variances. In case of a normal distribution and equal variances significant differences between the datasets were determined using a one-way ANOVA with Dunnett's post-test. If the datasets were not normally distributed, the nonparametric Kruskal-Wallis test with Dunn's post-test was used. An unpaired t-test was used to determine significant differences between neutral and acidified samples and unfiltered and filtered samples. A p-value $\leq 0.05$ was considered significant.

2 The mathematical part of the methodological optimisation was carried out using a Pareto strategy (Ehrgott 2000) further adapted for the multivariate optimisation, similar to the use of colour coding in in silico toxicology (Durmaz et al. 2015). The main optimisation criterion was to assess sample preparation methodologies that achieved the highest measured biological activity in six different parameters. Pareto thereby classified a preparation method as nonoptimal, if another preparation method exists that delivers "better" values regarding all parameters (YES, YAS, etc.) and all tested samples. Non-optimal preparation methods are 
289 excluded from the list leading to a ranked set of Pareto-optimal sample preparation methods.

290 The applied strategy also tackled scenarios with missing data 


\section{$291 \quad 3$ Results and discussion}

\section{3.1 Sample acidification for testing aqueous samples}

293 Analytical chemists use acid as a standard method to stabilise aqueous samples and prevent the biodegradation of (micro)pollutants (Prasse et al. 2015). Stabilisation is thought to occur by deactivating microorganisms (Baker and Kasprzyk-Hordern 2011, US EPA 2010) that may use target analytes as substrates. Therefore, the procedure is often adopted in ecotoxicology for conserving the toxicity of samples but often without studying its effectiveness.

The present results show that sample acidification and storage over $24 \mathrm{~h}$ significantly affected the endocrine activities and mutagenicity of aqueous samples compared to the samples kept at neutral $\mathrm{pH}$ (Figure 1, full data sets in Table S2). Focusing on a change of the endocrine activities or mutagenicity of $\geq 10 \%$, untreated wastewater was most affected by acidification (Table S3) whereby $50 \%$ of the assays $(n=22)$ showed decreased activities between -13 and $94 \%$. In case of the influent and effluent of the filtration basin $32 \%$ of the bioassays $(n=22)$ indicated altered activities between $-13 \%$ and $-37 \%$. Groundwater $(9 \%, \mathrm{n}=33)$, ozonated wastewater $(9 \%, \mathrm{n}=11)$ and surface water $(3 \%, \mathrm{n}=33)$ were least affected (Table S3). Regarding the different bioassays, the activities in the YAES, RXR and Ames TA100 assays were most affected by acidification (Table S4). 65\% of the YAES experiments showed decreased $(-13$ to $-32 \%)$ or increased $(+15$ to $+34 \%)$ activities (Figure 1A). The Ames TA100 was affected in $24 \%$ of the experiments with decreasing (-13 to $-77 \%)$ as well as increasing mutagenicity $(+17 \%)$ compared to neutral samples (Figure 1C, Table S4). Acidification

311 caused the highest decrease of mutagenicity in the Ames TA98 with $-94 \%$ followed by the

312 RAR assay with $-88 \%$ (Figure 1B). In the remaining bioassays, low endocrine or genotoxic

313 activities were detected. Thus, no conclusion of the influence of acidification on these endpoints was possible (Figure S7, Table S2).

315 In summary, sample acidification led to a decrease (-13 to $-94 \%)$ of activity in $81 \%$ and to an 316 increase $(+10$ to $+34 \%)$ of activity in $19 \%$ of the cases $(n=32)$. This indicates that sample 
317 acidification significantly affects the outcomes of bioassays. Two hypotheses may explain the changes in toxicity: 1) In acidified samples, acids may interfere with active chemicals or 2) in neutral samples, microbial activity may degrade or transform the active chemicals Basically, the key question is whether the neutral (hypothesis 1) or the acidified sample (hypothesis 2) represent the "true" toxicity. For chemical analysis, there is consensus that acidification stabilises most compounds and prevents microbial degradation (Baker and Kasprzyk-Hordern 2011, Vanderford et al. 2011, US EPA 2010). However, our data implies that besides few exceptions the in vitro activity is lower at acidic compared to neutral $\mathrm{pH}$ (Figure 1, Table S2). Accordingly, samples at a neutral $\mathrm{pH}$ may better represent the actual toxicity. If this hypothesis holds true, an acidification of samples would either reduce the concentration of active chemicals by increasing adsorption to suspended matter (Baker and Kasprzyk-Hordern 2011) or by increasing hydrolysis (Prasse et al. 2015). Alternatively, it can be assumed that the higher activity in neutral samples is an artefact caused by a change in sample composition. Here, continuous microbial activity may deconjugate compounds resulting in a higher biological activity. This occurs during biological wastewater treatment (Andersen et al. 2003, Koh et al. 2008, Wu et al. 2017). However, an on-going microbial degradation of active compounds would counteract this process (Giebner et al. 2018).

335 In reality, the toxicity of an aqueous sample may change at either neutral or acidic $\mathrm{pH}$. As this depends on the chemical and biological composition of a sample, it is difficult to generalise which condition best represents the actual toxicity. Based on the present data, we argue that a neutral $\mathrm{pH}$ comes closest to reality, as the sample is minimally processed. In addition, a higher biological activity will result in a more protective water quality assessment if one accepts that the risks of false-positives outweighs the risk of false-negatives. 
343 Sample filtration is beneficial to stabilise compounds (Baker and Kasprzyk-Hordern 2011), to

344 avoid clogging of SPE cartridges, to remove TSS (Janex-Habibi et al. 2009) and to sterilise

345 samples (Gehrmann et al. 2018). In the present study, unfiltered and corresponding glass fibre

346 filtered (pore size $1 \mu \mathrm{m}$ ) aqueous samples as well as aqueous suspension of the filter

347 retentates were compared to investigate the impacts of filtration on the toxicity. These

348 comparisons further included a microsieve (pore size $10 \mu \mathrm{m}$ ) installed at one WWTP, which

349 had a minimal effect on the toxicity (full data set in Table S5).

350 Focusing on a change of the different endocrine activities or mutagenicity of $\geq 10 \%$ again, the

351 untreated wastewater was affected at most by filtration (Tables S5 and S6). Here, the toxicity

352 was decreased by -20 and $-54 \%$ and increased by +28 and $+61 \%$ in $22 \%$ of the bioassays

$353(n=18$, Figure 2A, 2B). For surface water, activities were altered in $14 \%(n=7)$ of the

354 bioassays with one affected endpoint (Figure S8). Conventionally treated wastewater and

355 groundwater were less or not affected by filtration (Figures 2C and S8, Table S6).

356 Filtration had the strongest impact on the YAES (50\% of the assays, $n=8$; Table S7)

357 followed by the YES and YAAS $(25 \%, \mathrm{n}=8$ each) and YDS $(13 \%, \mathrm{n}=8)$. The effects

358 observed in the other bioassays were too low to evaluate the influence of filtration on these

359 endpoints (Figures 2 and S8, Table S5).

360 The aqueous suspension of the filter retentates also showed relevant changes in endocrine

361 activities $\geq 10 \%$ in $19 \%(\mathrm{n}=36)$ of the yeast-based assays. The retentates were anti-

362 estrogenic $(57 \%, \mathrm{n}=7)$ and anti-androgenic $(43 \%, \mathrm{n}=7)$ with activities from $21-80 \%$

363 (YAES) and 30-45\% (YAAS, Table S5). In two samples, the endocrine activity in the filtered

364 sample was significantly $(\mathrm{p} \leq 0.001)$ lower than in the unfiltered sample. As the retentate was

365 also active, the activity was retained by filtration. In two cases, significantly higher

$366(\mathrm{p} \leq 0.001)$ activities were detected in the filtered compared to the unfiltered samples. Here,

367 the retentate was active as well. In two YAES experiments, the endocrine activities in the

368 filtered and unfiltered samples were on a comparable high level (84-91\%) and the retentate 
369 was active as well (46 and $80 \%$ ). One sample was not anti-androgenic as filtered and unfiltered water, but as filter retentate (45\%, Figure S8, Table S5).

371 In summary, sample filtration led to a decrease (-18 to $-54 \%)$ of activity in $33 \%$ and to an increase $(+13$ to $+61 \%)$ of activity in $67 \%$ of the cases $(n=9)$ and, thus, has a significant impact on the bioassay results. The retention of particle-associated hormones and endocrine disrupting chemicals (EDCs) may explain this observation. This is supported by the detection of significant endocrine activities in the filter retentates and previous observations (Dagnino et al. 2010, Routledge 2003, Shieh et al. 2016).

377 Interestingly, few filtered samples had significantly higher endocrine activities than the corresponding unfiltered samples. For the WWTP effluent filtered by a microsieve we detected an approximately 2 -fold increase in anti-estrogenic activity (Table S5). This may be the result of an altered ratio of agonistic and antagonistic activities (Ihara et al. 2014, Rao et al. 2014) or the leaching of "new" compounds by the filter materials (filter controls confirmed this was not the case). In the present case, dissimilar affinities towards filter materials and/or suspended solids ( $\mathrm{Ng}$ and Cao 2015, Wangmo et al. 2018) could have resulted in a retention of antagonistic and thus increased agonistic activities in the filtrate and vice versa. In conclusion, the application of sample filtration should be well-adjusted to the aims of a study, the characteristics of investigated (waste)water samples and bioassay specificities, as

387 this is crucial to avoid misestimating the in vitro toxicity (Dagnino et al. 2010, EC 2003). In

388 the present study, this was amongst others observed when evaluating the removal of (anti)estrogenic and dioxin-like activities at WWTP 1 (Figure 2). Depending on whether the either increases or decreases the toxicity.

\subsection{Comparison of aqueous and extracted samples}


394 Comparing the toxicity of aqueous samples and corresponding SPE extracts is rarely done but

395 has a number of advantages, such as the possibility to calculate recovery rates and evaluate

396 the environmental relevance of obtained results (Giebner et al. 2018, Muschket et al. 2017,

397 Tousova et al. 2017, Wangmo et al. 2018).

398 In the present case, most aqueous samples induced minimal estrogenic, anti-androgenic and

399 retinoic acid-like activities (Figure 3, Tables S8, S9, S10). However, anti-estrogenic activities

400 between 21 and $91 \%$ were detected in all aqueous samples (Figure 3B). The activities were

$401<19 \%$ in the other bioassays (Figures 3D and S9, Table S8). In extracted samples, the

402 estrogenic activity $(\leq 8 \%, \mathrm{n}=35)$ was generally as low as in the corresponding aqueous

403 samples $(\leq 13 \%, \mathrm{n}=8$; Figures 3 and 4 , Table S9). The minor estrogenic activity detected in

404 most samples in this study is in line with other studies on biological (Jalova et al. 2013, Keiter

405 et al. 2006, Metcalfe et al. 2013) and advanced wastewater treatment (Ma et al. 2005, Maletz

406 et al 2013).

407 The anti-estrogenic activity of the extracts was variable and, depending on the SPE method, in parts very high $(13-89 \%, \mathrm{n}=35)$ and comparable to the corresponding aqueous samples (Figures 3B and 4). This indicated that the causative compounds were either only partially

410 recovered or that the anti-estrogenicity of the aqueous samples is caused by the matrix (Neale

411 et al. 2015). Interestingly, the high anti-estrogenic activities in the extracts point towards

412 potential masking effects, whereby receptor antagonists reduce the detection of agonistic

413 activity in water sample. This phenomenon has also been discussed by other authors (Giebner

414 et al. 2018, Gehrmann et al. 2018, Thara et al. 2014, Rao et al. 2014, Stalter et al. 2011). In

415 addition, groundwater was significantly anti-estrogenic (Figure 3B, Table S8 and S9). This

416 calls for further clarification regarding the presence of EDCs in groundwater.

417 In contrast, the anti-androgenic activity was low in most aqueous samples $(\leq 5 \%, n=7)$ but

418 higher in the extracts (9-89\%, $\mathrm{n}=30$, Figures $3 \mathrm{C}$ and 4 , Table S9) indicating a successful

extraction. Except for hospital wastewater, which may contain anti-androgenic 
420 pharmaceuticals (Sohoni and Sumpter 1998, Stalter et al. 2011), the majority of aqueous

421 samples exhibited only low androgenic and anti-androgenic activities (Figures 3C and S9,

422 Table S8). The androgenic activities remained low in the corresponding extracts, whereas

423 anti-androgenic activities were detected at moderate to high levels. As in case of the anti-

424 estrogenic activity, androgen receptor antagonists may mask the androgenic activity. Such

425 interactions were described for WWTP effluents (Leusch et al. 2017, Rao et al. 2014) and

426 ozonated hospital wastewater (Gehrmann et al. 2018). The high removal of these activities

427 reported for activated sludge treatment (Rao et al. 2014) and ozonation (Stalter et al. 2011)

428 were not observed in this study.

429 The highest RAR activity was detected in aqueous hospital and untreated wastewater (HOS:

$43093 \%$, INF-1: $23 \%$ ) and corresponding extracts, depending on the SPE-method (HOS: 14

$43191 \%$, INF-1: 0-54\%; Figures 3E and 4, Table S9). This implies that the active compounds

432 were only partially extracted. Only hospital and untreated wastewater induced RAR activities,

433 which was removed in the effluent (Figure 3E, Tables S8 and S9). RXR activities were

434 detected in extracted WWTP effluent and ozonated effluent (Figure S9, Table S8). So far,

435 only few studies reported RAR and RXR activities in water (Inoue et al. 2009) and

436 wastewater (Allinson et al. 2011, Inoue et al. 2011). In the experiments by Sawada et al.

437 (2012) and Cao et al. (2009) these activities readily degraded during activated sludge

438 treatment and lab-scale ozonation, respectively. Likewise, only a few studies exist on VDR-

439 and TR-like activities in (waste)water samples (Escher et al. 2014, Inoue et al. 2011, Kusk et

440 al. 2011, Leusch et al. 2017). In any case, activity levels in the present aqueous/extracted

441 samples were negligible.

442 Moderate dioxin-like activities were detected in a number of extracted but none of the

443 aqueous samples (Table S8). Highest activities were observed in raw, treated and hospital

444 wastewater. Lowest activities were observed for ozonated wastewater and groundwater. Its

removal during biological and advanced wastewater treatment has been observed in several 
446 (Allinson et al. 2011, Loos et al. 2012, Stalter et al. 2011) but not all studies (Jia et al. 2015,

447 Rao et al. 2014, Reungoat et al. 2010) supporting its detection in the present WWTP effluents.

448 While none of the aqueous samples $(n=6)$ was active in the umu assay, $33 \%(n=27)$ of the

\section{3.4 Identifying the optimal SPE method} ozonation (Cao et al. 2009, Misik et al. 2011). extracts were potentially genotoxic (Figure 3F, Tables S8 and S9). Low to moderate genotoxicity was detected in extracted hospital, raw and treated wastewater but in none of the other samples. Other studies observed genotoxicity in extracted WWTP effluents (Macova et al. 2011, Keiter et al. 2006, Escher et al. 2014). These potentials generally decreased upon

Similar to analytical chemistry (Baker and Kasprzyk-Hordern 2011, Maruya et al. 2016, Polo et al. 2005), SPE of (waste)water samples is advantageous for in vitro bioassays. Extraction prevents the microbial degradation of untreated samples and improves the detection of toxicological effects caused by low (micro)pollutant concentrations (Escher et al. 2005, Janošek et al. 2007, Macova et al. 2011, Neale et al. 2015, 2018). SPE can also minimise matrix interferences by reducing natural organic matter and excluding ions, nutrients or acids (Neale and Escher 2014, Prasse et al. 2015, Escher et al. 2018).

In contrast to chemical analysis of target compounds, the recovery of toxicity by SPE cannot be evaluated because the causative chemicals and mixture effects remain unknown. Thus, this study aimed at maximising the extraction of toxicity by comparing two mixed-mode hydrophilic/hydrophobic (Oasis HLB and Supelco ENVI-Carb+) and one composite (Telos C18/ENV) SPE sorbents. These SPE sorbents enrich a broad and heterogeneous spectrum of chemicals (Köke et al. 2018, Leusch et al. 2012, Neale et al. 2018). Extracting both neutral and acidified samples, six different SPE methods were evaluated by a semi-quantitative (3.4.1-3.4.4) approach followed by multivariate statistics (3.4.5). 
473 In parallel to the extraction of the samples, a SPE blank was prepared to control for potential

474 contaminants in reference waters and used materials (Kolkman et al. 2013, Neale et al. 2018,

475 Schulze et al. 2017). Each cartridge type was loaded with ultrapure water and extracted as

476 described in 2.3.3. The extracts of the 60 SPE blanks were negative in all bioassays except in

477 two cases (3\%): Supelco ENVI-Carb+ at pH 7 and pH 2.5 in the YAAS. Here, the activities

478 were $2 \%$ and $3 \%$ higher than the limit of quantification. In addition, a DMSO sample was

479 included in parallel to the SPE extracts in each in vitro bioassay as a solvent control. These

480 solvent controls did not induce an effect in the bioassays.

481

\section{$482 \quad 3.4 .2$ Cytotoxicity}

483 Cytotoxicity is often used as indicator of the reactive toxicity of environmental samples and their overall (micro)pollutant load. It, thus, represents an important endpoint which is integrated into several water quality assessments (Escher et al. 2014, 2018, Leusch et al. 2014, Välitalo et al. 2017). However, depending on the investigated endpoint, cytotoxicity can also prevent or mask the detection of specific toxicity (see 4).

In the present study, none of the aqueous samples induced cytotoxic effects (Figure 4, Tables S8 and S9). Cytotoxicity was, however, frequently detected in SPE extracts (Figure 4). Untreated wastewater induced cytotoxicity in 50\% (HOS) and 38\% (INF-1) of sample extracts $(n=60$, each) tested in ten in vitro bioassays (Table 2). For conventionally treated wastewater (EFF-1, EFF-4, EFF-4-MS, $n=54-60$ ) cytotoxicity was observed in $\leq 25 \%$ of extracts (Table 2). The occurrence of cytotoxicity in extracted ozonated wastewater (sample EFF-4-MS- ${ }_{3}, \mathrm{n}=54$ ) and groundwater (sample GW-1, $\mathrm{n}=60$ ) was 35 and $2 \%$, respectively (Table 2),

The choice of the SPE method had a substantial influence on the detection of cytotoxicity: the extracts of the Oasis HLB and the Telos C18/ENV (neutral $\mathrm{pH}$ ) were cytotoxic in $32 \%$ and 
$49850 \%$ of the bioassays $(n=78$ each, Table 2$)$. At acidified $\mathrm{pH}$, these extracts induced similar

499 cytotoxicity with 15 and $13 \%$, respectively $(n=78$ each, Table 2$)$. Samples extracted with the

500 Supelco ENVI-Carb+ at neutral $\mathrm{pH}$ were more cytotoxic (12\%) compared to the

501 corresponding samples that were extracted at acidified $\mathrm{pH}$ (not cytotoxic effects, $\mathrm{n}=78$ each,

502 Table 2).

503 In general, samples extracted at neutral $\mathrm{pH}$ induced higher cytotoxicity than acidified samples

504 (Figure 4) and Telos C18/ENV extracts were more cytotoxic than those of Oasis HLB and

505 Supelco ENVI-Carb+. Thus, extraction at neutral $\mathrm{pH}$ with Telos C18/ENV was the method

506 where the highest cytotoxicity was detected (Figure 4). Escher et al. (2005) found an

507 extraction at $\mathrm{pH} 3$ (using the Oasis HLB) to be more effective than $\mathrm{pH} 7$ and $\mathrm{pH} 11$ in a study

508 on spiked urine samples. Stalter et al. (2011) observed this for acidified biologically-treated

509 and ozonated wastewater. Both studies suggest that compounds with acidic moieties to be

510 responsible for the recovered cytotoxicity. This is in contrast to the present results, which

511 suggest that the cytotoxicity in a broad range of bioassays is extracted more effectively at

512 neutral $\mathrm{pH}$.

513 In a recent study by Stalter et al. (2016) the Telos ENV (without C18 sorbent) followed by the

514 Oasis HLB recovered most cytotoxicity amongst nine other SPE sorbents from disinfected

515 drinking water (acidified before extraction). Polar compounds adsorbed by the ENV as well

516 as the HLB sorbent material were suspected as main causative agents. Although Stalter et al.

517 (2016) did not compare an extraction at neutral $\mathrm{pH}$ the results support the effectivity of the

518 Telos C18/ENV and Oasis HLB observed in the present study. Along the same line, a

519 multilayer SPE based on Oasis HLB induced more cytotoxicity than a single sorbent method

520 in a study by Neale et al. (2018).

521 Conventional wastewater treatment decreased the occurrence of cytotoxicity from $38 \%$ of the

522 extracts to $7 \%$ in case of WWTP 1 (Table 2). In contrast, ozonation increased the number of

cytotoxic extracts from 24 to $35 \%$ (Table 2). This observation supports earlier hypotheses on 
525 Lundström et al. 2010, Magdeburg et al. 2014). In contrast to the WWTP samples, only $2 \%$ of

526 groundwater extracts were cytotoxic. This is in agreement with the high water quality

527 monitored at GW sampling sites 1-3 (Seitz and Winzenbacher 2017) as well as the rare

528 detection of cytotoxicity in groundwater, unless influenced by landfill leachates, industrial or

529 other contaminated sites (Baumstark-Khan et al. 2005, Baun et al. 2000).

530

\section{3.4.3 Endocrine endpoints}

532 Pooling the results according to water sample type, the highest mean estrogenic activity was

533 found in conventionally treated wastewater (EFF-1, EFF-4, EFF-4-MS) extracted with Telos

$534 \mathrm{C} 18 / \mathrm{ENV}(\mathrm{pH} 2.5)$ with $5 \%(\mathrm{n}=4)$ relative activity and Oasis HLB $(\mathrm{pH} 2.5)$ with $5 \%(\mathrm{n}=4)$

535 relative activity (Table S11, Figure S10). Samples extracted at neutral $\mathrm{pH}$ with the same SPE

536 sorbents induced lower estrogenic activities $(3 \%, n=2 ; 2 \%, n=3)$. Extracts produced with

537 Supelco ENVI-Carb+ showed low estrogenic activity regardless of the adjusted $\mathrm{pH}$.

538 With regard to the anti-estrogenic activity of conventionally treated (EFF) and ozonated

$539\left(\mathrm{EFF}^{-\mathrm{O}_{3}}\right)$ wastewater as well as groundwater (GW) both sorbents, Oasis HLB and Telos

$540 \mathrm{C} 18 / \mathrm{ENV}$ showed similar effectivity when samples were extracted at $\mathrm{pH} 2.5$ (Figures 4 and

541 S10, Tables S8 and S11). For conventionally treated wastewater (EFF) and groundwater

$542(\mathrm{GW})$ extracted at neutral $\mathrm{pH}$ with the same sorbents the mean anti-estrogenic activity was

543 higher. The highest mean anti-estrogenic activity was found in samples extracted with

544 Supelco ENVI-Carb+ at neutral $\mathrm{pH}(62-87 \%, \mathrm{n}=1-2)$.

545 In case of the anti-androgenic activity of all sample types, acidified samples extracted with

546 Oasis HLB and Telos C18/ENV produced similar results again (Figures 4 and S11). Because

547 of high cytotoxicity, the activities of neutrally extracted samples could not be analysed.

548 Treated wastewater and groundwater extracted with Supelco ENVI-Carb+ at both $\mathrm{pH}$ values induced lower anti-androgenic activities than the other SPE methods. As the activity in the 
550 other bioassays was minor, no comparison of the SPE methods on these endpoints was possible (Figures S11-S14).

552 Based on the above results the Telos C18/ENV sorbent followed by the Oasis HLB recovered

553 highest endocrine activities from the majority of (waste)water samples. However, the Supelco

554 ENVI-Carb+ sorbent was more effective in recovering androgenic activities. This is in part

555 reflected in previous studies. In a study on bottled mineral water, a C18 material recovered

556 higher estrogenic activity compared to the Oasis HLB and Supelco ENVI-Carb+ (Wagner and

557 Oehlmann 2011). The authors argue that non-polar chemicals are responsible for this effect.

558 In the present study, most estrogenicity was recovered by the Telos C18/ENV (involving a

559 similar C18 material), while Oasis HLB achieved comparable levels.

560 Except for estrogenicity, endocrine activities were more effectively recovered at $\mathrm{pH} 2.5$.

561 However, the more frequent detection of cytotoxicity in $\mathrm{pH} 7$ extracts might have masked the

562 respective activities. Despite the effective extraction of endocrine activities, it remained insufficient from some (waste)waters and endpoints (Figures 3 and S9, Table S8). This includes the anti-estrogenicity, which was enriched from several but not all samples. The difficulty in extracting anti-estrogenic activity has been observed and discussed in previous studies (Giebner et al. 2018).

567

\section{$568 \quad$ 3.4.4 Genotoxicity and mutagenicity}

569 The highest genotoxicity (IR 4.37) was detected in the Telos C18/ENV pH 2.5 extract of 570 untreated hospital wastewater (HOS, Tables S8 and S9, Figure S14). Seven extracts (100\%)

571 of the Oasis HLB and Telos C18/ENV sorbents at both $\mathrm{pH} 7$ and 2.5 of the conventionally

572 treated wastewater of the pilot WWTP 4 (EFF-4 and EFF-4-MS) were genotoxic with 573 induction rates between 1.50 and 1.87. The extracts of a WWTP 1 (INF-1 and EFF-1), except

574 one extract produced with Oasis HLB, $\mathrm{pH} 2.5$, and groundwater (GW-1) did not induce 
575 genotoxicity. All extracts produced with Supelco ENVI-Carb+ $(\mathrm{pH} 7$ and $\mathrm{pH} 2.5)$ were not

576 active, either.

577 Genotoxicity was enriched from four out of six sampling sites (Figure S14, Tables S8 and S9)

578 but IRs remained only moderately increased compared to the corresponding aqueous samples

579 (except for hospital wastewater). One reason for this could be that genotoxicity of

580 (waste)water samples is generally detected at higher sample enrichment factors (e.g., 100-

581 fold, Keiter et al. 2006, Schulze et al. 2017, Stalter et al. 2016) or at contamination hotspots

582 (Baumstark-Khan et al. 2005, Baun et al. 2000).

583 In line with the efficiency of the Telos C18/ENV pH 2.5 method, Magdeburg et al. (2014)

584 extracted genotoxicity and mutagenicity from wastewater (biological and advanced treatment)

585 using the Oasis HLB at $\mathrm{pH} 2$. Although the authors did not compare different SPE methods,

586 their results seem in agreement with the present results. Mutagenicity and cytotoxicity were

587 also higher in biologically-treated and ozonated wastewater extracted at $\mathrm{pH} 2$ (instead of $\mathrm{pH}$

588 7) using a C18 sorbent (Misik et al. 2011). For the other investigated in vitro endpoints, no

589 SPE optimisation study was found in the literature.

590

591 3.4.5 What is the best SPE method?

592 Regarding the results of five types of water samples tested with five in vitro bioassays the

593 most effective SPE method for the extraction of endocrine activities was Telos C18/ENV pH

5947 (7x), followed by Telos C18/ENV pH 2.5 and Supelco ENVI-Carb+ pH 7 (each 5x), Oasis

595 HLB pH 7 (4x), Oasis HLB pH 2.5 (2x) and Supelco ENVI-Carb+ pH 2.5 (1x, Table 3). To

596 statistically distinguish between optimal (and non-optimal) SPE methods a multivariate

597 optimisation based on Pareto was implemented (Durmaz et al. 2015, Ehrgott 2000). Pareto

598 computed sample type and bioassay specific "Pareto optimal" methods.

599 The Pareto results are exemplified for conventionally treated wastewater (EFF-4) in five in

600 vitro bioassays, whereby Pareto is based on the activity percentiles (Table S12) for ranking 
601 the SPE methods (Table S13). The best extraction methods ("Pareto best") were Telos

$602 \mathrm{C} 18 / \mathrm{ENV} \mathrm{pH} 7$ followed by Oasis HLB pH 7 and Telos C18/ENV pH 2.5 (see Table S13 for

603 detailed results). The ranking of these methods was computed as follow: Instead of looking at

604 the "best" extraction results within a certain matrix, the "worst" results were classified as

605 "false negative responders". The Supelco ENVI-Carb+ method at pH 2.5 was three times

606 "Pareto-worst" as it extracted the lowest activity in a maximal number of bioassays. All other

607 methods performed better. When an extract was cytotoxic, the result was marked with the

608 label "cytotoxic" instead of providing a value. The Pareto algorithm is capable of evaluating

609 data sets with a limited number of such results. In case of an excessive degree of cytotoxicity

610 (HOS and INF-1), the corresponding SPE method was, however, not listed in the respective

611 ranking matrix and the level of relevance decreases for this parameter. This means that the

612 ranking for this parameter is not reaching the "worst" class anymore. This evaluation

613 procedure was performed for all data sets referring to the different samples, SPE methods and

614 in vitro bioassays to obtain the following overall ranking of "Pareto optimal" SPE methods:

615 Regarding the five sample types, the method Telos C18/ENV at $\mathrm{pH} 7$ was four times "Pareto

616 best", followed by Oasis HLB pH 7 and pH 2.5 (each $2 x$, Tables 3 and S14). In terms of the

617 five bioassays, the methods Telos C18/ENV at $\mathrm{pH} 2.5$ and Supelco ENVI-Carb+ at $\mathrm{pH} 7$ were

618 two times "Pareto best", respectively (details in Table S14).

619 Accordingly, the method Telos C18/ENV at pH 7 was "Pareto best" regarding the effectivity

620 in extracting different types of water and wastewater samples with respect to the highest

621 endocrine activities (Table 3). Higher recoveries at neutral $\mathrm{pH}$ (over acidic and basic $\mathrm{pH}$ )

622 were also observed by Tousova et al. (2017) for several endpoints also investigated in this

623 study. The authors, however, used other sorbents for large volume SPE of surface waters.

624 Summing up the results of the in vitro bioassays and Pareto optimisation, the methods Telos

$625 \mathrm{C} 18 / \mathrm{ENV} \mathrm{pH} 7$ and Oasis HLB pH 7 were optimal to enrich endocrine activities but also the

626 highest cytotoxicity (Table 2). The corresponding methods at $\mathrm{pH} 2.5$ showed good results as 
627 well as lower cytotoxicity (Tables 2 and S14). The final recommendation for most effective

628 recovery of in vitro toxicity from diverse (waste)waters is, thus, to use the Telos C18/ENV

629 method at a sample $\mathrm{pH}$ of 7. 
6304 Challenges in optimising sample preparation for bioassays

631 Despite the advantages of optimising the sample preparation for bioassay analyses (Muschket

632 et al. 2017, Neale et al. 2018, Ternes et al. 2017), a number of important challenges remain.

633 The first challenge is that the "true" toxicity of a sample (at a given sampling site and time)

634 remains unknown. The reason for this is that for complex environmental samples, the

635 causative compounds, potential mixture effects and confounding factors (e.g., matrix effects)

636 are largely unspecified. Accordingly, each step of sampling and sample preparation and

637 storage may change the chemical composition of a sample and its toxicity. Active compounds

638 may be added (via contaminated materials) or removed (via adsorption to materials) during

639 sampling, added or removed during transport and storage (via microbial activity) and added or

640 removed during sample preparation.

641 Second, the differentiation between toxicity caused by anthropogenic pollutants and naturally

642 occurring compounds, often referred to a matrix effects, remains challenging. For instance,

643 our approach in maximising the recovery of toxicities may come at the costs of also

644 maximising matrix effects. One such example is the co-extraction of DOC that may induce

645 artefacts in bioassays for receptor antagonism (Neale and Escher 2014). Several confounding

646 factors resulting in false-positive or negative result need to be considered when interpreting

647 bioassay data (discussed in Giebner et al. 2018). However, sample preparation may not be the

648 appropriate tool to address these. Instead, post-extraction analysis (such as effect-directed

649 analysis) can be a way to separate the toxicity caused by anthropogenic and natural

650 compounds

651 The third challenge is the selectivity of sample extraction: While SPE methods with broad

652 selectivity exist, an extraction of chemicals is always selective, resulting in a loss of

653 compounds with low affinity to the sorbent (Köke et al. 2018, Neale et al. 2018, Niss et al.

6542018 , Stalter et al. 2016). Accordingly, the toxicity of an extract will never fully represent the

655 toxicity of the extracted sample. Thus, the question is rather how much loss in toxicity during 
656 extraction is acceptable. One way of addressing this is to compare the toxicity of extracts to

657 aqueous samples (Dagnino et al. 2010, EC 2003). Another way is to optimise the recovery of

658 toxicity. Both strategies were adopted in this study to identify the best extraction method.

659 The forth challenge arises from cytotoxicity masking the effect under investigation, which is

660 often the case at high concentration factors. While cytotoxicity can be considered an

661 important toxicological endpoint by itself outweighing the specific effect is masks, it is most

662 commonly rather regarded an obstacle that needs to be removed. This can be achieved by

663 diluting a sample to a non-cytotoxic concentration (Inoue et al. 2009, 2011, Leusch et al.

664 2017, Neale et al. 2018, Välitalo et al. 2017). However, this also dilutes the effect of interest.

665 Alternative approaches, such as minimising the dilution of aqueous samples (Niss et al. 2018)

666 or reducing exposure times in the bioassay as well as cleaning up the cytotoxicity (e.g., by

667 fractionation), have so far not been widely adopted.

668 These challenges are connected to a range of SPE parameters. Thus, the sorbent (Chang et al.

669 2009, Escher et al. 2005, Stalter et al. 2016), sample volumes (Macova et al. 2011, Schulze et

670 al. 2017), eluting solvents (Lu et al. 2010, Välitalo et al. 2017, Yang et al. 2014), fractionation

671 steps (Leusch et al. 2017, Välitalo et al. 2017) and operating modes such as large volume or

672 multilayer SPE (Köke et al. 2018, Schulze et al. 2017) can be optimised.

673 Acknowledging that it is impractical to perform an optimisation for every sample and every

674 bioassay, a range of case studies for different matrices can be used to evaluate whether

675 specific sample preparation methods perform generally better than others. We have taken such

676 approach in the present study and conclude that the Telos C18/ENV method at neutral sample

$677 \mathrm{pH}$ performs best in recovering multiple endocrine activities and cytotoxicity from aqueous

678 samples. 
1. Acidification of aqueous (waste)water samples significantly alters a range of in vitro toxicities, including anti-estrogenic, anti-androgenic and retinoic acid-like activities as well as mutagenicity. Sample filtration has a minor impact on the samples' toxicity.

683

2. Compared to aqueous samples, solid phase extraction enriches most in vitro toxicities. However, some activities (e.g., anti-estrogenicity) remain poorly extractable.

3. When comparing six SPE methods, the choice of the optimal method depends on the matrix as well as the in vitro endpoint.

687 4. In general, an extraction using Telos $\mathrm{C} 18 / \mathrm{ENV}$ at a sample $\mathrm{pH}$ of 7 was most effective in recovering in vitro toxicity from (waste)water samples. However, these methods also coextract a high cytotoxicity masking other endpoints. Using the same method at a sample $\mathrm{pH}$ of 2.5 reduced the extraction of cytotoxicity.

691 5. Sample preparation needs to be optimised when analysing the toxicity of water samples.

692 While this is a resource-consuming task involving multiple methodological parameters, water quality can only be accurately assessed when the recovery of the toxicity of a sample is maximal. 
6956 Acknowledgements

696 This study was funded by the German Federal Ministry of Education and Research (BMBF)

697 Project TransRisk "Charakterisierung, Kommunikation und Minimierung von Risiken durch

698 neue Schadstoffe und Krankheitserreger im Wasserkreislauf" (funding number:

699 02WRS1275B, contract period: $11 / 2011$ to 04/2015) within the funding measure "Risk

700 Management of Emerging Compounds and Pathogens in the Water Cycle (RiSKWa)" which

701 is gratefully appreciated. The authors cordially thank all TransRisk project partners for fruitful

702 discussions and extensive collaboration that helped to compile and improve this manuscript as

703 well as Johannes Völker and Andrea Misovic for their scientific and technical support.

704 Furthermore, the authors would like to thank Prof. Dr. P. Cornel and Gregor Knopp and the

705 employees at the investigated WWTP and water works for supporting this study such as by

706 provision of samples.

707 
Allinson, M., Shiraishi, F., Allinson, G., 2011. A comparison of recombinant receptorreporter gene bioassays and a total estrogen enzyme linked immunosorbent assay for the rapid screening of estrogenic activity in natural and waste waters. Bulletin of Environmental Contamination and Toxicology, $86(5), 461$.

Andersen, H., Siegrist, H., Halling-Sørensen, B., Ternes, T. A., 2003. Fate of estrogens in a municipal sewage treatment plant. Environmental Science \& Technology, 37 (18), 40214026.

Baker, D. R., Kasprzyk-Hordern, B., 2011. Critical evaluation of methodology commonly used in sample collection, storage and preparation for the analysis of pharmaceuticals and illicit drugs in surface water and wastewater by solid phase extraction and liquid chromatography-mass spectrometry. Journal of Chromatography A, 1218 (44), 8036-8059.

Baumstark-Khan, C., Cioara, K., Rettberg, P., Horneck, G., 2005. Determination of geno- and cytotoxicity of groundwater and sediments using the recombinant SWITCH test. Journal of Environmental Science and Health, 40 (2), 245-263.

Baun, A., Jensen, S. D., Bjerg, P. L., Christensen, T. H., Nyholm, N., 2000. Toxicity of organic chemical pollution in groundwater downgradient of a landfill (Grindsted, Denmark). Environmental Science \& Technology, 34 (9), 1647-1652.

Bistan, M., Podgorelec, M., Marinšek Logar, R., Tišler, T., 2012. Yeast estrogen screen assay as a tool for detecting estrogenic activity in water bodies. Food Technology and

728 Biotechnology, 50 (4), 427-433.

729 Brack, W., Dulio, V., Ågerstrand, M., Allan, I., Altenburger, R., Brinkmann, M., Bunke, D., 730 Burgess, R. M., Cousins, I., Escher, B. I., Hernández, F. J., Hewitt, L. M., Hilscherová, K., 731 Hollender, J., Hollert, H., Kase, R., Klauer, B., Lindim, C., Herráez, D. L., Miège, C., 732 Munthe, J., OToole, S., Posthuma, L., Rüdel, H., Schäfer, R. B., Sengl, M., Smedes, F., van 733 de Meent, D., van den Brink, P. J., van Gils, J., van Wezel, A. P., Vethaak, A. D., Vermeirssen, E., von der Ohe, P. C., Vrana, B., 2017. Towards the review of the European Union Water Framework Directive: Recommendations for more efficient assessment and management of chemical contamination in European surface water resources. Science of The Total Environment (576), 720-737. 2009. Evaluation of wastewater reclamation technologies based on in vitro and in vivo bioassays. Science of the Total Environment, 407 (5), 1588-1597.

Chang, H. S., Choo, K. H., Lee, B., Choi, S. J., 2009. The methods of identification, analysis, and removal of endocrine disrupting compounds (EDCs) in water. Journal of Hazardous Materials 172(1), 1-12.

Dagnino, S., Gomez, E., Picot, B., Cavaillès, V., Casellas, C., Balaguer, P., Fenet, H., 2010. Estrogenic and AhR activities in dissolved phase and suspended solids from wastewater treatment plants. Science of the Total Environment, 408 (12), 2608-2615. 
747 Durmaz, V., Weber, M., Meyer, J., Mückter, H., 2015. Computergestützte Simulationen zur 748 Abschätzung gesundheitlicher Risiken durch anthropogene Spurenstoffe in der Wassermatrix. 749 KA Korrespondenz Abwasser, Abfall, 3/15, 264-267.

750 Ehrgott, M., 2000. Approximation algorithms for combinatorial multicriteria optimization 751 problems. International Transactions in Operational Research, 7 (1), 5-31.

Escher, B. I., Ait-Aissa, S., Behnisch, P. A., Brack, W., Brion, F., Brouwer, A., Buchinger, S., Crawford, S. E., Du Pasquier, D., Hamers, T., Hettwer, K., Hilscherová, K., Hollert, H., Kase, R., Kienle, C., Tindall, A. J., Tuerk, J., van der Oost, R., Vermeirssen, E., Neale P. A., 2018. Effect-based trigger values for in vitro and in vivo bioassays performed on surface water extracts supporting the environmental quality standards (EQS) of the European Water Framework Directive. Science of the Total Environment, 628, 748-765.

758 Escher, B. I., Bramaz, N., Maurer, M., Richter, M., Sutter, D., von Känel, C., Zschokke, M., 2005. Screening test battery for pharmaceuticals in urine and wastewater. Environmental

760 Toxicology and Chemistry, 24 (3), 750-758.

761 Escher, B. I., Allinson, M., Altenburger, R., Bain, P. A., Balaguer, P., Busch, W., Crago, J., 762 Denslow, N. D., Dopp, E., Hilscherova, K., Humpage, A. R., Kumar, A., Grimaldi, M., 763 Jayasinghe, B. S., Jarosova, B., Jia, A., Makarov, S., Maruya, K. A., Medvedev, A., Mehinto, 764 A. C., Mendez, J. E., Poulsen, A., Prochazka, E., Richard, J., Schifferli, A., Schlenk, D., 765 Scholz, S., Shiraishi, F., Snyder, S, Su, G., Tang, J. Y., van der Burg, B., van der Linden, S. 766 C., Werner, I., Westerheide, S. D., Wong, C. K., Yang, M., Yeung, B. H., Zhang, X., Leusch, 767 F. D., 2014. Benchmarking organic micropollutants in wastewater, recycled water and 768 drinking water with in vitro bioassays. Environmental Science \& Technology, 48 (3), 19407691956.

Gehrmann, L., Bielak, H., Behr, M., Itzel, F., Lyko, S., Simon, A., Kunze, G., Dopp, E., 771 Wagner, M., Tuerk, J., 2018. (Anti-) estrogenic and (anti-) androgenic effects in wastewater during advanced treatment: comparison of three in vitro bioassays. Environmental Science and Pollution Research, 25 (5), 4094-4104.

Giebner, S., Ostermann, S., Straskraba, S., Oetken, M., Oehlmann, J., Wagner, M., 2018. Effectivity of advanced wastewater treatment: reduction of in vitro endocrine activity and mutagenicity but not of in vivo reproductive toxicity. Environmental Science and Pollution

777 Research, 25 (5), 3965-3976.

778 Hendriks, A. J., Maas-Diepeveen, J. L., Noordsij, A., Van der Gaag, M. A., 1994. Monitoring response of XAD-concentrated water in the Rhine delta: a major part of the toxic compounds remains unidentified. Water Research, 28 (3), 581-598.

Ihara, M., Ihara, M. O., Kumar, V., Narumiya, M., Hanamoto, S., Nakada, N., Yamashita N., Miyagawa S., Iguchi T., Tanaka, H., 2014. Co-occurrence of estrogenic and antiestrogenic activities in wastewater: quantitative evaluation of balance by in vitro $\mathrm{ER} \alpha$. reporter gene assay and chemical analysis. Environmental Science \& Technology, 48 (11), 6366-6373. assay. Bulletin of Environmental Contamination and Toxicology, 82, 399-404. 
Inoue, D., Nakama, K., Sawada, K., Watanabe, T., Matsui, H., Sei, K., Nakanishi T., Ike, M.,

789 2011. Screening of agonistic activities against four nuclear receptors in wastewater treatment plants in Japan using a yeast two-hybrid assay. Journal of Environmental Sciences, 23 (1), 125-132.

792 International Standard Organisation (ISO)/Deutsches Institut für Normung (DIN), 2012.

793 ISO/DIN 11350: Water quality - Determination of the genotoxicity of water and waste water -

794 Salmonella/microsome fluctuation test (Ames fluctuation test). Geneva, Switzerland.

795 International Standard Organisation (ISO), 2002. ISO13829: Water quality-determination of

796 the genotoxicity of water and waste water using the umu test. Geneva, Switzerland.

797 International Standard Organisation (ISO), 2018. ISO 19040-1: Water quality -798 Determination of the estrogenic potential of water and waste water -- Part 1: Yeast estrogen screen (Saccharomyces cerevisiae). Geneva, Switzerland.

800 Jalova, V., Jarošová, B., Blaha, L., Giesy, J. P., Ocelka, T., Grabic, R., Jurčíková J., Vrana B., 801 Hilscherova, K., 2013. Estrogen-, androgen-and aryl hydrocarbon receptor mediated activities

802

826 Kolkman, A., Schriks, M., Brand, W., Bäuerlein, P. S., van der Kooi, M. M., van Doorn, R. 827 H., Emke, E., Reus, A. A., van der Linden, S. C., de Voogt, P., Heringa, M. B., 2013. Sample 
preparation for combined chemical analysis and in vitro bioassay application in water quality assessment. Environmental Toxicology and Pharmacology, 36 (3), 1291-1303.

Kümmerer, K., 2011. Commentary: Emerging contaminants versus micro-pollutants. Clean -

831 Soil, Air, Water, 39 (10), 889-890.

832 Kusk, K. O., Krüger, T., Long, M., Taxvig, C., Lykkesfeldt, A. E., Frederiksen, H., Emke E.,

833 Reus A. A., van der Linden S. C., de Voogt P., Bonefeld-Jørgensen, E. C., 2011. Endocrine 834 potency of wastewater: contents of endocrine disrupting chemicals and effects measured by in 835 vivo and in vitro assays. Environmental Toxicology and Chemistry, 30 (2), 413-426.

Leusch, F. D. L., Prochazka, E., Tan, B. L. L., Carswell, S., Neale, P., Escher, B. I., 2012. Optimising micropollutants extraction for analysis of water samples: comparison of different solid phase materials and liquid-liquid extraction. Sci. Forum Stakehold. Engagem. Build. Link. Collab. Sci. Qual., Brisbane, Queensland (pp. 191-195).

Leusch, F. D., Khan, S. J., Gagnon, M. M., Quayle, P., Trinh, T., Coleman, H., Rawson C., Chapman H. F., Blair P., Nice H., Reitsema, T., 2014. Assessment of wastewater and recycled water quality: a comparison of lines of evidence from in vitro, in vivo and chemical analyses. Water Research, 50, 420-431.

Leusch, F. D., Neale, P. A., Hebert, A., Scheurer, M., Schriks, M. C., 2017. Analysis of the sensitivity of in vitro bioassays for androgenic, progestagenic, glucocorticoid, thyroid and estrogenic activity: Suitability for drinking and environmental waters. Environment International, 99, 120-130.

Loos, R., Carvalho R., António D. C., Comero S., Locoro G., Tavazzi S., Paracchini B., Ghiani M., Lettieri T., Blaha L., Jarosova B., Voorspoels S., Servaes K., Haglund P., Fick J., Lindberg R. H., Schwesig D., Gawlik B. M., 2012. EU wide monitoring survey on waste water treatment plant effluents. JRC Scientific and Policy Report, JRC 76400.

Lu, G., Zhang, H., Wang, C., 2010. Assessment of estrogenic activity conducted by combining bioassay and chemical analyses of the effluent from wastewater treatment plants in Nanjing, China. Environmental Toxicology and Chemistry, 29 (6), 1279-1286.

Lundström, E., Adolfsson-Erici, M., Alsberg, T., Björlenius, B., Eklund, B., Lavén, M., Breitholtz, M., 2010. Characterization of additional sewage treatment technologies: Ecotoxicological effects and levels of selected pharmaceuticals, hormones and endocrine disruptors. Ecotoxicology and Environmental Safety, 73 (7), 1612-1619.

Ma, M., Li, J., Wang, Z., 2005. Assessing the detoxication efficiencies of wastewater treatment processes using a battery of bioassays/biomarkers. Archives of Environmental Contamination and Toxicology, 49 (4), 480-487.

Macova, M., Toze, S., Hodgers, L., Mueller, J. F., Bartkow, M., Escher, B. I., 2011. Bioanalytical tools for the evaluation of organic micropollutants during sewage treatment, water recycling and drinking water generation. Water Research, 45 (14), 4238-4247.

Magdeburg, A., Stalter, D., Schlüsener, M., Ternes, T., Oehlmann, J., 2014. Evaluating the efficiency of advanced wastewater treatment: target analysis of organic contaminants and (geno-) toxicity assessment tell a different story. Water research, 50, 35-47. 
Malaj, E., von der Ohe, P. C., Grote, M., Kühne, R., Mondy, C. P., Usseglio-Polatera, P.,

869 Brack, W., Schäfer, R. B., 2014. Organic chemicals jeopardize the health of freshwater

870 ecosystems on the continental scale. Proceedings of the National Academy of Sciences of the

871 United States of America, 111 (26), 9549-9554.

872 Maletz, S., Floehr, T., Beier, S., Klümper, C., Brouwer, A., Behnisch, P., Higley E., Giesy J.

873 P., Hecker M., Gebhardt W., Linnemann V., Pinnekamp J., Hollert H., 2013. In vitro characterization of the effectiveness of enhanced sewage treatment processes to eliminate endocrine activity of hospital effluents. Water Research, 47 (4), 1545-1557.

876 Maruya, K. A., Dodder, N. G., Mehinto, A. C., Denslow, N. D., Schlenk, D., Snyder, S. A., Weisberg, S. B., 2016. A tiered, integrated biological and chemical monitoring framework for contaminants of emerging concern in aquatic ecosystems. Integrated Environmental Assessment and Management, 12 (3), 540-547.

Metcalfe, C. D., Kleywegt, S., Letcher, R. J., Topp, E., Wagh, P., Trudeau, V. L., Moon, T. W., 2013. A multi-assay screening approach for assessment of endocrine-active contaminants in wastewater effluent samples. Science of the Total Environment, 454, 132-140.

Miklos, D. B., Remy, C., Jekel, M., Linden, K., G., Drewes, J. E., Hübner U., 2018. Evaluation of advanced oxidation processes for water and wastewater treatment - A critical review. Water Research (139), 118-131.

Miller, C. A., 1997. Expression of the human aryl hydrocarbon receptor complex in yeast activation of transcription by indole compounds. Journal of Biological Chemistry 272 (52), 32824-32829.

Misik, M., Knasmueller, S., Ferk, F., Cichna-Mark1, M., Grummt, T., Schaar, H., Kreuzinger, N., 2011. Impact of ozonation on the genotoxic activity of tertiary treated municipal wastewater. Water Research, 45 (12), 3681-3691.

Muschket, M., Di Paolo, C., Tindall, A. J., Touak, G., Phan, A., Krauss, M., Kirchner K., Seiler T. B., Hollert H., Brack, W., 2018. Identification of unknown antiandrogenic compounds in surface waters by effect-directed analysis (EDA) using a parallel fractionation approach. Environmental Science \& Technology, 52 (1), 288-297.

Neale, P. A., Escher, B. I., 2014. Does co-extracted dissolved organic carbon cause artefacts in cell-based bioassays? Chemosphere, 108, 281-288.

Neale, P. A., Escher, B. I., Leusch, F. D., 2015. Understanding the implications of dissolved organic carbon when assessing antagonism in vitro: an example with an estrogen receptor assay. Chemosphere, 135, 341-346.

Neale, P. A., Brack, W., Aït-Aïssa, S., Busch, W., Hollender, J., Krauss, M., MaillotMaréchal, E., Munz, N. A., Schlichting, R., Schulze, T., Vogler, B., Escher, B. I, 2018. Solidphase extraction as sample preparation of water samples for cell-based and other in vitro bioassays. Environmental Science: Processes \& Impacts, 20 (3), 493-504.

Niss, F., Rosenmai, A. K., Mandava, G., Örn, S., Oskarsson, A., Lundqvist, J., 2018. Toxicity bioassays with concentrated cell culture media - a methodology to overcome the chemical 
loss by conventional preparation of water samples. Environmental Science and Pollution

908 Research, 25(12), 12183-12188.

Ng, C. K., Cao, B., 2015. What exactly are you filtering out? Environmental Science and

910 Technology, 49, 5259-5260.

911 Polo, M., Llompart, M., Garcia-Jares, C., Cela, R., 2005. Multivariate optimization of a solidphase microextraction method for the analysis of phthalate esters in environmental waters.

913 Journal of Chromatography A, 1072 (1), 63-72.

914 Prasse, C., Stalter, D., Schulte-Oehlmann, U., Oehlmann, J., Ternes, T., 2015. Spoilt for 915 choice: A critical review on chemical and biological evaluation of current wastewater 916 treatment technologies. Water Research, 87, 237-270.

917 Rao, K., Li, N., Ma, M., Wang, Z., 2014. In vitro agonistic and antagonistic endocrine 918 disrupting effects of organic extracts from waste water of different treatment processes. 919 Frontiers of Environmental Science \& Engineering, 8(1), 69-78.

920 Reungoat, J., Macova, M., Escher, B. I., Carswell, S., Mueller, J. F., Keller, J., 2010. Removal

921 of micropollutants and reduction of biological activity in a full scale reclamation plant using 922 ozonation and activated carbon filtration. Water Research, 44 (2), 625-637.

923 Rizzo, L., 2011. Bioassays as a tool for evaluating advanced oxidation processes in water and 924 wastewater treatment. Water Research, 45 (15), 4311-4340.

925 Routledge, E. J., 2003. Identifying the causative agents: the use of combined chemical and 926 biological strategies in monitoring programs. Pure and Applied Chemistry, 75 (11-12), 2461 9272466.

928 Routledge, E. J., Sumpter, J. P., 1996. Estrogenic activity of surfactants and some of their 929 degradation products assessed using a recombinant yeast screen. Environmental Toxicology 930 and Chemistry, 15, 241-248.

931 Sawada, K., Inoue, D., Wada, Y., Sei, K., Nakanishi, T., Ike, M., 2012. Detection of retinoic acid receptor agonistic activity and identification of causative compounds in municipal wastewater treatment plants in Japan. Environmental Toxicology and Chemistry, 31 (2), 307 934315.

935 Schulze, T., Ahel, M., Ahlheim, J., Aït-Aïssa, S., Brion, F., Di Paolo, C., Froment, J., Hidasi, 936 A. O., Hollender, J., Hollert, H., Hu, M., Kloß, A., Koprivica, S., Krauss, M., Muz, M., 937 Oswald, P., Petre, M., Schollée, J. E., Seiler, T. B., Shao, Y., Slobodnik, J., Sonavane, M., 938 Suter, M. J., Tollefsen, K. E., Tousova, Z., Walz, K. H., Brack, W., 2017. Assessment of a novel device for onsite integrative large-volume solid phase extraction of water samples to enable a comprehensive chemical and effect-based analysis. Science of the Total

941 Environment, 581, 350-358.

942 Schwarzenbach, R. P., Escher, B. I., Fenner, K., Hofstetter, T. B., Johnson, C. A., von 943 Gunten, U., Wehrli, B., 2006. The challenge of micropollutants in aquatic systems. Science 944 313(5790), 1072-1077 
946 protection area and the proposal of relevant indicators for anthropogenic influences.

947 Environmental Monitoring and Assessment, 189 (6), 244.

948 Shieh, B. H., Louie, A., Law, F. C., 2016. Factors affecting distribution of estrogenicity in the 949 influents, effluents, and biosolids of Canadian wastewater treatment plants. Archives of 950 Environmental Contamination and Toxicology, 70 (4), 682-691.

951 Sohoni P., Sumpter J. P., 1998. Several environmental oestrogens are also antiandrogens.

952 Journal of Endocrinology, 158, 327-339.

Stalter, D., Peters, L. I., O'Malley, E., Tang, J. Y., Revalor, M., Farré, M. J., Watson, K., von

954 Gunten, U., Escher, B. I., 2016. Sample enrichment for bioanalytical assessment of

955 disinfected drinking water: Concentrating the polar, the volatiles, and the unknowns.

956 Environmental Science and Technology, 50 (12), 6495-6505.

957 Stalter, D., Magdeburg, A., Wagner, M., Oehlmann, J., 2011. Ozonation and activated carbon 958 treatment of sewage effluents: Removal of endocrine activity and cytotoxicity. Water 959 Research, $45(3), 1015-1024$.

960 Ternes, T. A., Prasse, C., Eversloh, C. L., Knopp, G., Comel, P., Schulte-Oehlmann, U., 961 Schwartz, T., Alexander, J., Seitz, W., Coors, A., Oehlmann, J., 2017. Integrated evaluation 962 concept to assess the efficacy of advanced wastewater treatment processes for the elimination 963 of micropollutants and pathogens. Environmental Science \& Technology, 51 (1), 308-319.

964 Tousova, Z., Oswald, P., Slobodnik, J., Blaha, L., Muz, M., Hu, M., Brack, W., Krauss, M., 965 Di Paolo, C., Tarcai, Z., Seiler, T. B., Hollert, H., Koprivica, S., Ahel, M., Schollée, J. E., 966 Hollender, J., Suter, M. J., Hidasi, A. O., Schirmer, K., Sonavane, M., Ait-Aissa, S., Creusot, 967 N., Brion, F., Froment, J., Almeida, A. C., Thomas, K., Tollefsen. K. E., Tufi, S., Ouyang, X., 968 Leonards, P., Lamoree, M., Torrens, V. O., Kolkman, A., Schriks, M., Spirhanzlova, P., 969 Tindall, A., Schulze, T., 2017. European demonstration program on the effect-based and chemical identification and monitoring of organic pollutants in European surface waters. Science of the Total Environment, 601, 1849-1868.

972 US EPA, 2010. Stability of Pharmaceuticals, Personal Care Products, Steroids, and Hormones 973 in Aqueous Samples, POTW Effluents, and Biosolids. U.S. Environmental Protection 974 Agency, Office of Water. p. 1-38. EPA-820-R-10-008.

975 US EPA, 2002. Short-term method for estimating the chronic toxicity of effluents and receiving waters to freshwater organisms. In assessments of effluents. Fourth Edition, October. Office of Water, U.S. Environmental Protection Agency Washington, DC. D., Küster, E., Mikola, A., Schulze, T., Sillanpää, M., 2017. Effect-based assessment of toxicity removal during wastewater treatment. Water Research, 126, 153-163.

981 Vanderford, B. J., Mawhinney, D. B., Trenholm, R. A., Zeigler-Holady, J. C., Snyder, S. A., 982 2011. Assessment of sample preservation techniques for pharmaceuticals, personal care 983 products, and steroids in surface and drinking water. Analytical and Bioanalytical Chemistry, $984399(6), 2227-2234$. 
Völker, J., Castronovo, S., Wick, A., Ternes, T. A., Joss, A., Oehlmann, J., Wagner, M., 2016.

986 Advancing biological wastewater treatment: extended anaerobic conditions enhance the removal of endocrine and dioxin-like activities. Environmental Science and Technology, 50, 10606-10615

989 Wagner, M., Vermeirssen, E. L. M., Buchinger, S., Behr, M., Magdeburg, A., Oehlmann, J., 2013. Deriving bio-equivalents from in vitro bioassays: assessment of existing uncertainties and strategies to improve accuracy and reporting. Environmental Toxicology and Chemistry, $32(8), 1-12$.

Wagner, M., Oehlmann, J., 2011. Endocrine disruptors in bottled mineral water: estrogenic activity in the E-Screen. Journal of Steroid Biochemistry and Molecular Biology, 127 (1), 128-135.

Wagner, M., Oehlmann, J, 2009. Endocrine disruptors in bottled mineral water: total estrogenic burden and migration from plastic bottles. Environmental Science and Pollution Research, 16, 278-286.

Wangmo, C., Jarque, S., Hilscherová, K., Bláha, L., Bittner, M., 2018. In vitro assessment of sex steroids and related compounds in water and sediments - a critical review. Environmental

1001 Science: Processes \& Impacts, 20 (2), 270-287.

1002 Wu, Q., Lam, J. C., Kwok, K. Y., Tsui, M. M., Lam, P. K., 2017. Occurrence and fate of endogenous steroid hormones, alkylphenol ethoxylates, bisphenol $\mathrm{A}$ and phthalates in municipal sewage treatment systems. Journal of Environmental Sciences, 61, 49-58. Yang, X. L., Xia, M. Q., Chen, M., Shen, D. Q., Fu, D. F., Song, H. L., 2014. Optimization of solid-phase extraction for pretreatment of selected estrogens in sewage by response surface methodology. Polish Journal of Environmental Studies, 23 (6), 2287-2294. 
1010 Table 1: Overview of the investigated samples; WWTP: wastewater treatment plant. Details

1011 on samples 1-14 can be found in Seitz and Winzenbacher (2017).

\begin{tabular}{|c|c|c|c|}
\hline $\begin{array}{l}\text { Sample } \\
\text { No. }\end{array}$ & Type of sample & Sample acronym & $\begin{array}{c}\text { Sampling } \\
\text { mode }\end{array}$ \\
\hline 1 & untreated wastewater (hospital effluent) & HOS & grab \\
\hline 2 & $\begin{array}{l}\text { untreated wastewater } \\
\text { (WWTP } 1 \text { influent) }\end{array}$ & INF-1 & composite \\
\hline 3 & $\begin{array}{l}\text { conventionally treated wastewater } \\
\text { (WWTP } 1 \text { effluent) }\end{array}$ & EFF-1 & composite \\
\hline 4 & $\begin{array}{l}\text { conventionally treated wastewater } \\
\text { (WWTP } 2 \text { effluent) }\end{array}$ & EFF-2 & composite \\
\hline 5 & $\begin{array}{l}\text { conventionally treated wastewater } \\
\text { (WWTP } 3 \text { effluent) }\end{array}$ & EFF-3 & composite \\
\hline 6 & $\begin{array}{l}\text { conventionally treated wastewater (WTTP } 4 \\
\text { influent of a filtration basin) }\end{array}$ & FB-IN & grab \\
\hline 7 & $\begin{array}{l}\text { conventionally treated wastewater (WTTP } 4 \\
\text { effluent of a filtration basin) }\end{array}$ & FB-OUT & composite \\
\hline 8 & surface water of an infiltration basin & IB (SW) & grab \\
\hline 9 & surface water 1 (river) & SW-1 & grab \\
\hline 10 & surface water 2 (river) & SW-2 & grab \\
\hline 11 & surface water 3 (river) & SW-3 & grab \\
\hline 12 & groundwater 1 (hotspot) & GW-1 & grab \\
\hline 13 & groundwater 2 (hotspot) & GW-2 & grab \\
\hline 14 & groundwater 3 (hotspot) & GW-3 & grab \\
\hline 15 & $\begin{array}{l}\text { conventionally treated wastewater (pilot } \\
\text { WWTP) }\end{array}$ & EFF-4 & composite \\
\hline 16 & $\begin{array}{l}\text { ozonated conventionally treated wastewater } \\
\text { (before microsieve, pilot WWTP) }\end{array}$ & $\mathrm{EFF}-4-\mathrm{O}_{3}$ & composite \\
\hline 17 & $\begin{array}{l}\text { conventionally treated wastewater (after } \\
\text { microsieve, pilot WWTP) }\end{array}$ & EFF-4-MS & composite \\
\hline 18 & $\begin{array}{l}\text { ozonated microfiltered conventionally-treated } \\
\text { wastewater (pilot WWTP) }\end{array}$ & EFF-4-MS-O 3 & composite \\
\hline 19 & tap water (pilot WWTP) & TAP & grab \\
\hline
\end{tabular}


43

1013 Table 2: Occurrence of cytotoxicity (\%) during the analysis of all sample extracts in ten in

1014 vitro bioassays (except EFF-4-MS (F) and EFF-4-MS-O $\mathrm{O}_{3}(\mathrm{~F}): \mathrm{n}=9$ ) pooled according to SPE

1015 method. Corresponding samples were taken on the same sampling dates in July (D) 2012 and

1016 in January (F) 2013.

\begin{tabular}{cccccccc}
\hline sample & \multicolumn{2}{c}{ Oasis HLB } & \multicolumn{2}{c}{ Telos C18/ENV } & \multicolumn{2}{c}{ Supelco ENVI-Carb+ } & Sample \\
& pH 7 & pH 2.5 & pH 7 & pH 2.5 & pH 7 & pH 2.5 & mean \\
\hline HOS & 80 & 70 & 100 & 50 & 0 & 0 & $50(\mathrm{n}=60)$ \\
INF-1 & 60 & 50 & 70 & 50 & 0 & 0 & $38(\mathrm{n}=60)$ \\
EFF-1 & 0 & 0 & 30 & 0 & 10 & 0 & $7(\mathrm{n}=60)$ \\
EFF-4 & 0 & 0 & 0 & 0 & 0 & 0 & $0(\mathrm{n}=60)$ \\
EFF-4-MS (D) & 0 & 0 & 50 & 0 & 0 & 0 & $8(\mathrm{n}=60)$ \\
EFF-4-MS (F) & 44 & 0 & 56 & 0 & 44 & 0 & $24(\mathrm{n}=54)$ \\
EFF-4-MS-O 3 (F) & 78 & 0 & 100 & 0 & 33 & 0 & $35(\mathrm{n}=54)$ \\
GW-1 & 0 & 0 & 0 & 0 & 10 & 0 & $2(\mathrm{n}=60)$ \\
\hline \multirow{2}{*}{ Method mean } & 32 & 15 & 50 & 13 & 12 & 0 & \\
\hline
\end{tabular}

1017 
1018 Table 3: Most effective SPE methods for the extraction of estrogenic (YES), anti-estrogenic

1019 (YAES), androgenic (YAS), anti-androgenic (YAAS) and dioxin-like (YDS) activity from

1020 water and wastewater samples (inner table, based on Table S8). In addition, "Pareto best"

1021 methods for each bioassay and sample type were computed. Double/triple listings represent

1022 equally effective methods. Hospital wastewater (HOS) and one WWTP influent (INF-1) were

1023 not analysed due to excessive cytotoxicity. Brackets: activity $\leq 10 \%$; "“-": no endocrine

1024 activity/cytotoxicity

\begin{tabular}{|c|c|c|c|c|c|c|}
\hline Sample type & YES & YAES & YAS & YAAS & YDS & $\begin{array}{l}\text { Pareto best: } \\
\text { sample type }\end{array}$ \\
\hline EFF-1 & (Oasis 2.5) & Supelco 7 & & Oasis 2.5 & Telos 7 & $\begin{array}{c}\text { Oasis } 2.5 \\
\text { Telos } 7\end{array}$ \\
\hline EFF-4 & (Telos 2.5) & Telos 7 & (Oasis 7) & Telos 7 & Telos 7 & $\begin{array}{c}\text { Oasis } 7 \\
\text { Telos } 7 \\
\text { Telos } 2.5\end{array}$ \\
\hline EFF-4-MS & (Telos 2.5) & Oasis 7 & (Supelco 7) & Oasis 7 & Telos 7 & Telos 7 \\
\hline EFF-4-MS-O ${ }_{3}$ & - & Supelco 7 & (Supelco 2.5) & Telos 2.5 & (Telos 2.5) & Supelco 7 \\
\hline GW-1 & (Telos 7) & Telos 7 & (Supelco 7) & Telos 2.5 & (Supelco 7) & $\begin{array}{c}\text { Oasis } 7 \\
\text { Oasis } 2.5 \\
\text { Telos } 7\end{array}$ \\
\hline $\begin{array}{l}\text { Pareto best: } \\
\text { bioassay }\end{array}$ & Telos 2.5 & Supelco 7 & Supelco 7 & $\begin{array}{c}\text { Telos } 2.5 \\
\text { Supelco } 2.5\end{array}$ & Telos 7 & Telos 7 \\
\hline
\end{tabular}

1025 


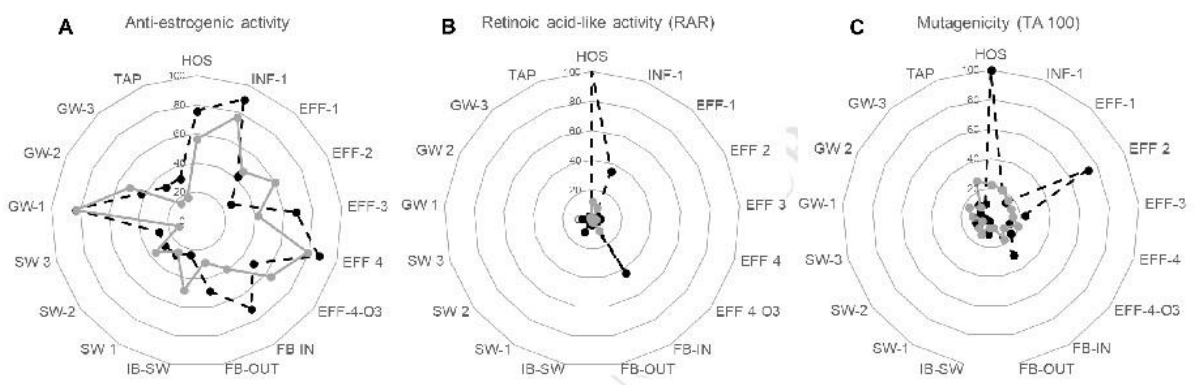

Figure 1: Impact of acidification. Anti-estrogenic activity (A), retinoic acid-like activity (RAR, B) and mutagenicity (Ames TA 100, C) of neutral (black) and acidified (grey) aqueous water and wastewater samples (mean in \%). Corresponding samples (INF-1/EFF-1, EFF-4/EFF-4-O 3 and FBIN/FB-OUT) were taken on the same sampling date in March 2012 and April 2012, respectively.
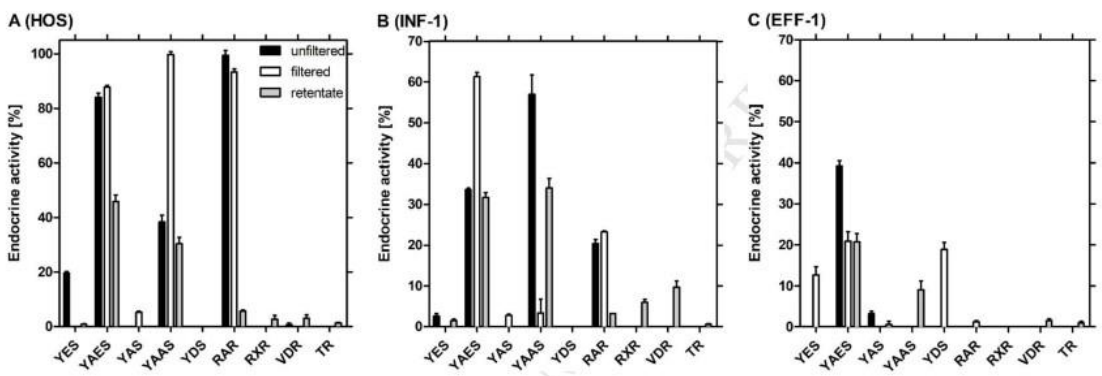

Figure 2: Impact of filtration. Endocrine activity ( $\%$, mean \pm SEM) of unfiltered (black bars) and filtered (white bars) wastewater samples and the aqueous suspensions of the filter retentate (grey bars). A: untreated hospital wastewater (HOS), B: untreated municipal wastewater of WWTP 1 (INF-1), C: conventionally treated effluent of WWTP 1 (EFF-1). YES: estrogenic, YAES: anti-estrogenic, YAS: androgenic, YAAS: antiandrogenic, YDS: dioxin-like, RAR: retinoic acid-like, RXR: retinoid-X-like, VDR: vitamin D-like, TR: thyronine-like. Corresponding samples (INF-1/EFF-1) taken on the same sampling date in July 2012. 

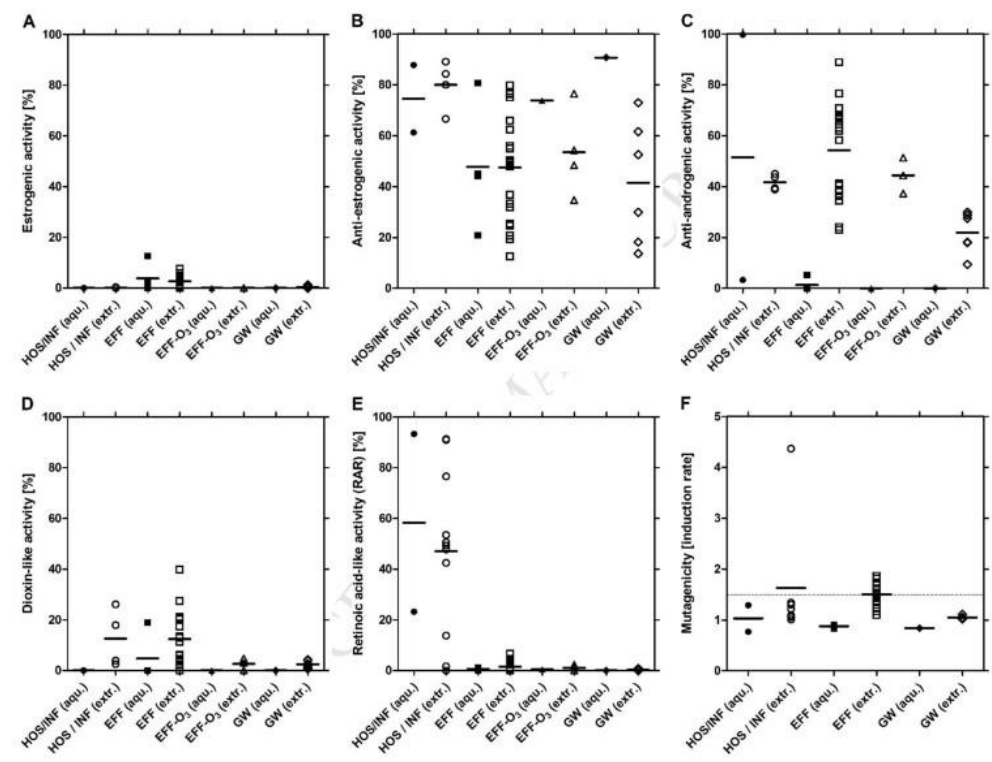

Figure 3: Comparison of aqueous and extracted samples. Estrogenic (A), anti-estrogenic (B), anti-androgenic (C), dioxin-like (D) and retinoic acid-like (RAR, E) activity in \% and genotoxicity as induction rate (umu, F) of the pooled data of aqueous (aqu.) water and wastewater samples (0.63-fold final concentration) and of the corresponding 10.4-fold concentrated SPE extracts (extr.). Symbols: mean activity of the individual sample, line: mean of all samples of one sample type, filled symbol: aqueous sample, clear symbol: SPE extract, HOS: untreated hospital wastewater, INF: untreated influent, $\mathrm{EFF}$ : conventionally treated effluent, $\mathrm{EFF}-\mathrm{O}_{3}$ : ozonated conventionally treated wastewater, GW: groundwater. Corresponding samples were taken within the same sampling period in July 2012 and January 2013. 
WTPID MANIISCRIPT
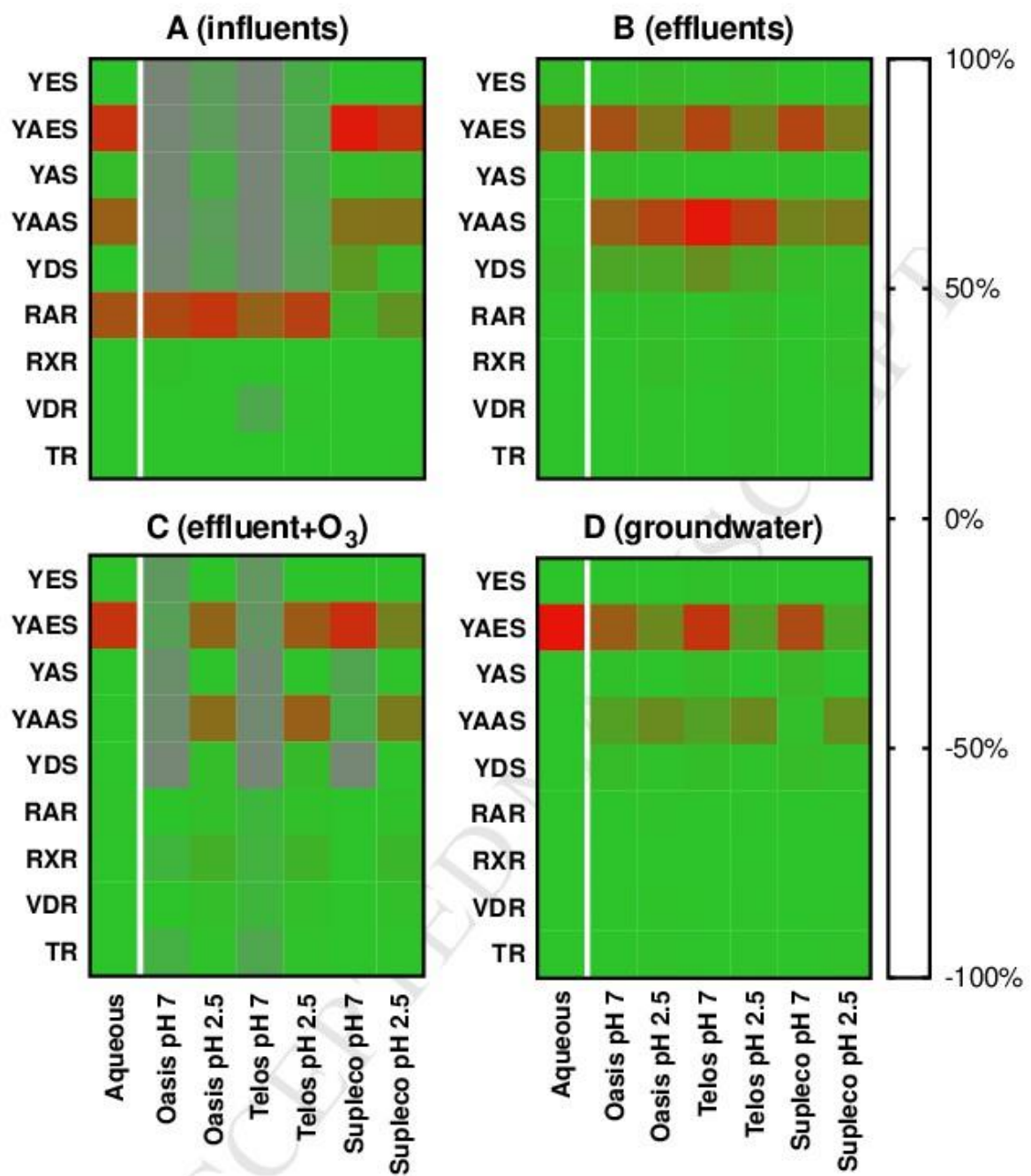

Figure 4: Comparison of the six SPE methods. Endocrine activity (0\% to 100\%) and cytotoxicity ( $0 \%$ to $-100 \%)$ of aqueous samples and the corresponding SPE extracts $(0.63$ and 10.4-fold final concentration, respectively) of wastewater treatment plant influents (A), effluents (B), ozonated effluent (C) and groundwater (D). Six SPE methods were compared: Oasis HLB, Telos C18/ENV and Supelco ENVI-Carb+ extraction at $\mathrm{pH} 7$ and $\mathrm{pH} 2.5$. The results were pooled from the different samples according to water type. Green: $0.0 \%$ endocrine activity/cytotoxicity, red: $100 \%$ endocrine activity, grey: $100 \%$ cytotoxicity. 
What you extract is what you see: Optimising the preparation of water and wastewater samples for in vitro bioassays

Aennes Abbas \& Ilona Schneider, Anna Bollmann, Jan Funke, Jörg Oehlmann, Carsten Prasse, Ulrike Schulte-Oehlmann, Wolfram Seitz, Thomas Ternes, Marcus Weber, Henning Wesely, Martin Wagner

\section{HIGHLIGHTS}

- Acidification of (waste)water samples significantly affects their in vitro toxicity

- Filtration does not affect the toxicity of most (waste)water samples

- All six SPE methods recovered in vitro toxicity, depending on endpoints/matrices

- Best SPE methods were identified for each matrix and endpoint

- Multivariate optimisation identified Telos C18/ENV $(\mathrm{pH} 7)$ as overall best SPE method 


\section{Supplementary information (paper A.1)}

\section{Material and methods}

Table S1: Overview of the bioassays used in this study, including endpoints (in brackets), concentration range $\left[\mathrm{mol} \mathrm{L}^{-1}\right]$ of the respective reference compound (positive control), background agonists and $\mathrm{EC}_{50}$ values.

\begin{tabular}{|c|c|c|c|}
\hline In vitro bioassay & Positive control & $\begin{array}{c}\text { Concentration range } \\
{\left[\mathrm{mol} \mathrm{L}^{-1}\right]}\end{array}$ & $\mathrm{EC}_{50}$-values \\
\hline $\begin{array}{l}\text { YES } \\
\text { (estrogenicity) }\end{array}$ & $\begin{array}{l}17 \beta \text {-estradiol }\left(\mathrm{E}_{2}\right) \\
\text { (CAS: } 50-28-2)\end{array}$ & $1.0 \times 10^{-12}-1.0 \times 10^{-08}$ & $1.23 \times 10^{-10}$ \\
\hline $\begin{array}{l}\text { YAES } \\
\text { (anti- } \\
\text { estrogenicity) }\end{array}$ & $\begin{array}{l}\text { 4-Hydroxytamoxifen } \\
\text { (OHT, CAS: } 68392-35-8) \\
\text { background agonist: } \\
0.1 \text { nmol/L } 17 \beta \text {-estradiol }\left(\mathrm{E}_{2}\right)\end{array}$ & $1.25 \times 10^{-06}-8.0 \times 10^{-05}$ & $6.53 \times 10^{-06}$ \\
\hline $\begin{array}{l}\text { YAS } \\
\text { (androgenicity) }\end{array}$ & $\begin{array}{l}\text { Testosterone } \\
(\mathrm{T}, \mathrm{CAS}: 58-22-0)\end{array}$ & $3 \times 10^{-11}-1.0 \times 10^{-07}$ & $4.36 \times 10^{-09}$ \\
\hline $\begin{array}{l}\text { YAAS } \\
\text { (anti- } \\
\text { androgenicity) }\end{array}$ & $\begin{array}{l}\text { Flutamide } \\
\text { (Flu, CAS: 13311-84-7) } \\
\text { background agonist: } \\
3 \text { nmol/L testosterone }\end{array}$ & $7.81 \times 10^{-07}-5.0 \times 10^{-05}$ & $3.13 \times 10^{-06}$ \\
\hline $\begin{array}{l}\text { YDS } \\
\text { (dioxin-like) }\end{array}$ & $\begin{array}{l}\beta \text {-Naphthoflavone } \\
(\beta-N F, \text { CAS: } 6051-87-2)\end{array}$ & $1.0 \times 10^{-09}-1.0 \times 10^{-05}$ & $1.19 \times 10^{-07}$ \\
\hline $\begin{array}{l}\text { RAR } \\
\text { (vitamin A-like) }\end{array}$ & $\begin{array}{l}\text { All-trans retinoic acid } \\
\text { (at-RA, CAS: } 302-79-4 \text { ) }\end{array}$ & $1.0 \times 10^{-09}-3.0 \times 10^{-06}$ & $3.14 \times 10^{-08}$ \\
\hline $\begin{array}{l}\text { RXR } \\
\text { (vitamin A-like) }\end{array}$ & $\begin{array}{l}\text { 9-cis retinoic acid } \\
\text { (9-cis-RA, CAS: 5300-03-8) }\end{array}$ & $1.0 \times 10^{-08}-1.0 \times 10^{-05}$ & $4.50 \times 10^{-07}$ \\
\hline $\begin{array}{l}\text { VDR } \\
\text { (vitamin D-like) }\end{array}$ & $\begin{array}{l}\text { 1 } \alpha, 25 \text {-Dihydroxyvitamin D3 } \\
\text { (Calcitriol, CAS: } 322222-06-3 \text { ) }\end{array}$ & $1.0 \times 10^{-10}-3.0 \times 10^{-07}$ & $5.28 \times 10^{-08}$ \\
\hline $\begin{array}{l}\text { TR } \\
\text { (thyronine-like) }\end{array}$ & $\begin{array}{l}\text { 3,3',5-Triiod-L-thyronine } \\
\left(\mathrm{T}_{3}, \mathrm{CAS}: 6893-02-3\right)\end{array}$ & $1.0 \times 10^{-09}-3.0 \times 10^{-06}$ & $2.23 \times 10^{-07}$ \\
\hline $\begin{array}{l}\text { Ames } \\
\text { (TA98) }\end{array}$ & $\begin{array}{l}\text { 4-Nitro-o-phenylenediamine } \\
\text { (4-NOPD, CAS: 99-56-9) }\end{array}$ & $10 \mathrm{mg} / \mathrm{L}$ & - \\
\hline $\begin{array}{l}\text { Ames } \\
(\mathrm{TA1} 100)\end{array}$ & $\begin{array}{l}\text { Nitrofurantoin } \\
\text { (NF, CAS: 67-20-9) }\end{array}$ & $0.25 \mathrm{mg} / \mathrm{L}$ & - \\
\hline $\begin{array}{l}\text { Umu-test } \\
\text { (genotoxicity) }\end{array}$ & $\begin{array}{l}\text { 4-Nitroquinoline N-oxide } \\
\text { (4-NQO, CAS 56-57-5) }\end{array}$ & $5.0-2000 \mu \mathrm{g} / \mathrm{L}$ & - \\
\hline
\end{tabular}




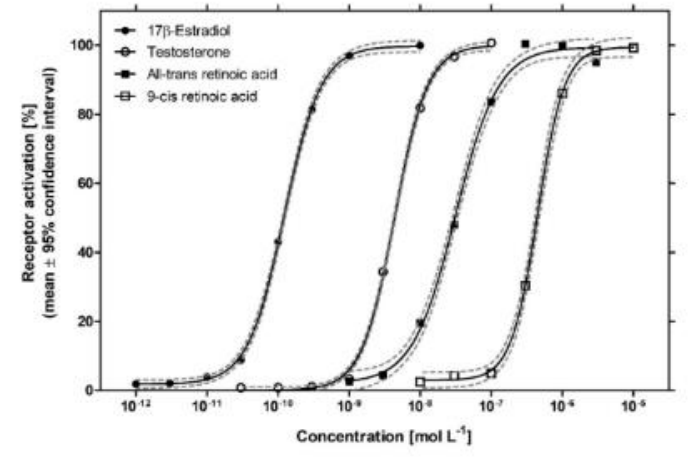

Figure S1: Receptor activation (\%, mean $\pm 95 \%$ confidence interval) as concentration-response relationships of six (RAR, RXR) and seven (YES, YAS) experiments at the human estrogen (left), androgen (second left), retinoic acid receptor (third left) and retinoid $X$ (right) receptor (YES: 17p-estradiol; YAS: testosterone; RAR: all-trans retinoic acid; RXR: 9-cis retinoic acid).

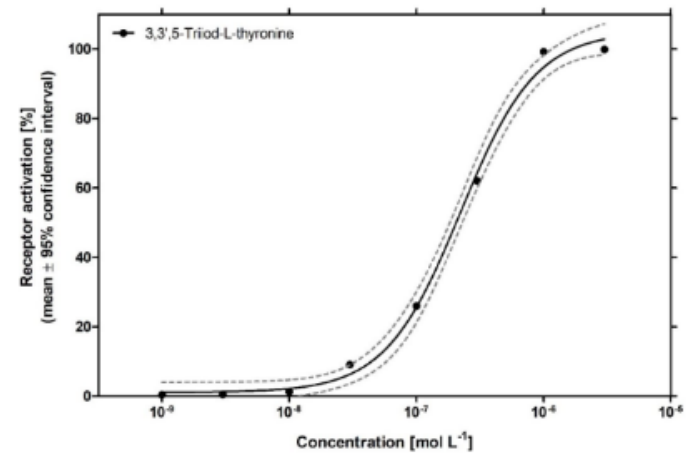

Figure S3: Receptor activation (\%, mean $\pm 95 \%$ confidence interval) as concentration-response relationship of six experiments at the human thyronine receptor (TR: 3,3',5-triiod-L-thyronine).

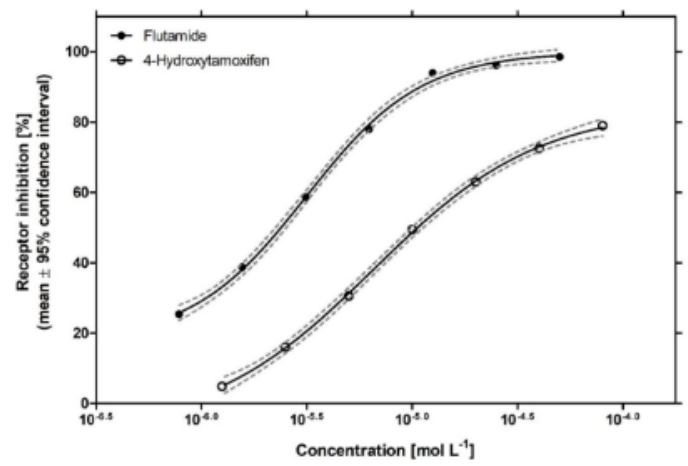

Figure S5: Receptor inhibition (\%, mean $\pm 95 \%$ confidence interval) as concentration-response relationships of seven experiments, each, at the human androgen (left) and estrogen (right) receptor (YAAS: flutamide; YAES: 4-hydroxytamoxifen)).

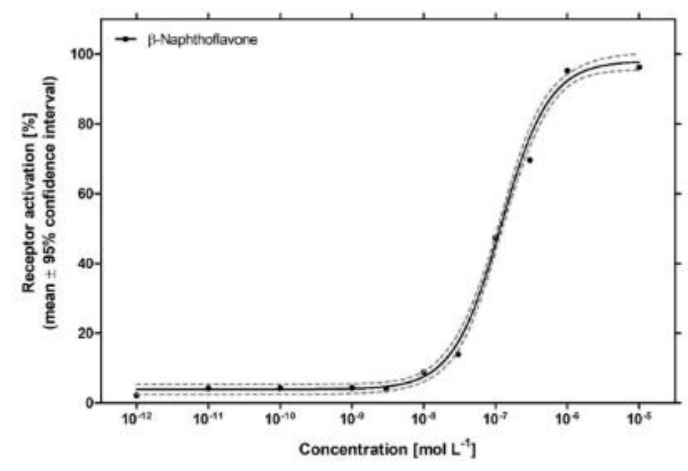

Figure S2: Receptor activation ( $\%$, mean $\pm 95 \%$ confidence interval) as concentration-response relationship of seven experiments at the human aryl-hydrocarbon receptor (YDS: $\beta$ Naphthoflavone).

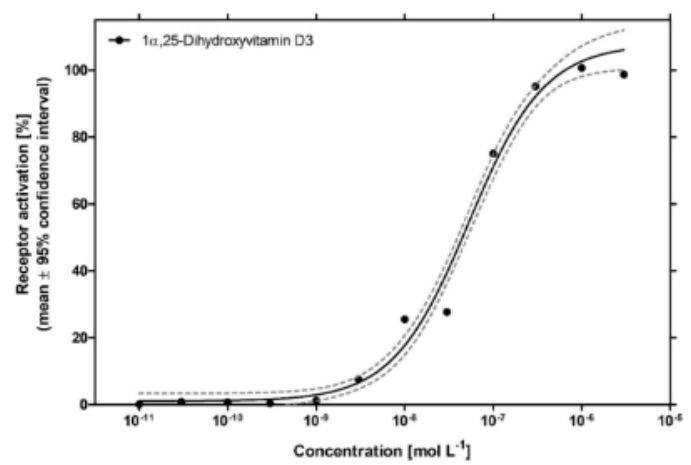

Figure S4: Receptor activation ( $\%$, mean $\pm 95 \%$ confidence interval) as concentration-response relationship of six experiments at the human vitamin D receptor (VDR: $1 \alpha, 25$ dihydroxyvitamin D3).

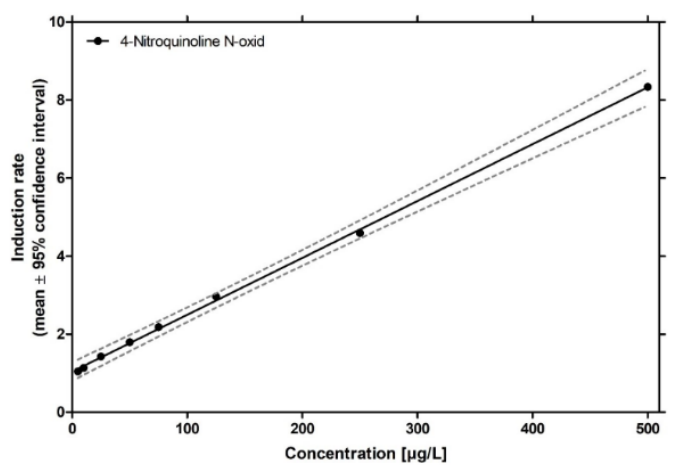

Figure S6: Induction rate (mean $\pm 95 \%$ confidence interval) of the positive control as linear regression of seven experiments of the umu test (4-nitroquinoline $\mathrm{N}$-oxid) 
2 Results and discussion

2.1 Sample acidification

Table S2: Estrogenic (YES), anti-estrogenic (YAES) and androgenic (YAS) activity (\%; mean \pm SEM) of neutral and acidified (pH 2) aqueous samples. Significant differences between neutral and acidified samples are marked with asterisks: $\star p \leq 0.05, \star \star p \leq 0.01, \star \star \star p \leq 0.001$ (unpaired t-test), n.s.: not significant. Corresponding samples were taken on the same sampling dates in March (A) and April (B) 2012.

\begin{tabular}{|c|c|c|c|c|c|c|c|c|c|}
\hline \multirow[t]{3}{*}{ sample } & \multicolumn{9}{|c|}{ in vitro bioassay } \\
\hline & \multicolumn{2}{|c|}{ YES } & \multirow{2}{*}{$\begin{array}{l}\text { significance } \\
\text { neutral/pH } 2\end{array}$} & \multicolumn{2}{|c|}{ YAES } & \multirow{2}{*}{$\begin{array}{l}\text { significance } \\
\text { neutral/pH } 2\end{array}$} & \multicolumn{2}{|c|}{ YAS } & \multirow{2}{*}{$\begin{array}{l}\text { significance } \\
\text { neutral } / \mathrm{pH} 2\end{array}$} \\
\hline & neutral & pH 2 & & neutral & $\mathrm{pH} 2$ & & neutral & pH 2 & \\
\hline HOS (B) & $1.38 \pm 0.22$ & $1.80 \pm 0.96$ & n.s. & $75.8 \pm 1.88$ & $56.5 \pm 1.30$ & $\star \star \star$ & $25.7 \pm 0.52$ & $9.61 \pm 0.52$ & $\star \star \star$ \\
\hline INF-1 (B) & 0.0 & 0.0 & n.s. & $89.8 \pm 0.63$ & $76.9 \pm 1.08$ & $\star \star \star$ & $19.4 \pm 0.82$ & $16.5 \pm 0.50$ & $\star \star$ \\
\hline EFF-1 (B) & 0.0 & 0.0 & n.s. & $41.8 \pm 1.89$ & $46.5 \pm 1.82$ & n.s. & 0.0 & $0.84 \pm 0.24$ & $\star \star \star$ \\
\hline EFF-2 (B) & 0.0 & 0.0 & n.s. & $26.1 \pm 2.66$ & $60.0 \pm 0.80$ & $\star \star \star$ & 0.0 & $0.35 \pm 0.32$ & $\star \star$ \\
\hline EFF-4-O ${ }_{3}(\mathrm{~A})$ & $0.08 \pm 1.10$ & $0.39 \pm 1.08$ & n.s. & $48.3 \pm 1.28$ & $63.5 \pm 0.71$ & $\star \star \star$ & 0.0 & $1.47 \pm 0.12$ & $\star \star \star$ \\
\hline FB-IN (B) & $1.41 \pm 0.24$ & $1.08 \pm 0.12$ & n.s. & $71.0 \pm 2.19$ & $38.6 \pm 0.99$ & $\star \star \star$ & $30.3 \pm 1.54$ & $15.7 \pm 1.46$ & $\star \star \star$ \\
\hline FB-OUT (B) & 0.0 & 0.0 & n.s. & $48.9 \pm 1.49$ & $28.8 \pm 1.22$ & $\star \star \star$ & $0.19 \pm 0.07$ & $0.16 \pm 0.21$ & n.s. \\
\hline IB (SW) (B) & 0.0 & 0.0 & n.s. & $23.6 \pm 1.07$ & $48.3 \pm 1.49$ & $\star \star \star$ & $0.12 \pm 0.05$ & $0.55 \pm 0.08$ & $\star \star \star$ \\
\hline SW-1 (B) & 0.0 & 0.0 & n.s. & $27.1 \pm 0.61$ & $24.9 \pm 1.49$ & n.s. & 0.0 & 0.0 & n.s. \\
\hline GW-2 (B) & 0.0 & 0.0 & n.s. & $42.9 \pm 2.32$ & $51.5 \pm 1.73$ & $\star \star$ & 0.0 & 0.0 & n.s. \\
\hline GW-3 (B) & 0.0 & 0.0 & n.s. & $31.7 \pm 1.69$ & $16.4 \pm 0.31$ & $\star \star \star$ & 0.0 & $0.61 \pm 0.13$ & $\star \star \star$ \\
\hline TAP (A) & $2.21 \pm 0.63$ & $0.52 \pm 0.43$ & $\star$ & $31.5 \pm 3.96$ & $17.5 \pm 4.65$ & $\star$ & 0.0 & $1.14 \pm 0.18$ & $\star \star \star$ \\
\hline
\end{tabular}

Table S2 continued: Anti-androgenic (YAAS), dioxin-like (YDS) and retinoic acid-like (RAR) activity (\%; mean \pm SEM) of neutral and acidified ( $\mathrm{pH}$ 2) aqueous samples. Significant differences between neutral and acidified samples are marked with asterisks: $\star \mathrm{p} \leq 0.05, \star \star \mathrm{p} \leq 0.01$, $\star$ $\mathrm{p} \leq 0.001$ (unpaired t-test), n.s.: not significant. Corresponding samples were taken on the same sampling dates in March (A) and April (B) 2012 .

\begin{tabular}{|c|c|c|c|c|c|c|c|c|c|}
\hline \multirow[t]{3}{*}{ sample } & \multicolumn{9}{|c|}{ in vitro bioassay } \\
\hline & \multicolumn{2}{|c|}{ YAAS } & \multirow{2}{*}{$\begin{array}{l}\text { significance } \\
\text { neutral/pH } 2\end{array}$} & \multicolumn{2}{|c|}{ YDS } & \multirow{2}{*}{$\begin{array}{l}\text { significance } \\
\text { neutral/pH } 2\end{array}$} & \multicolumn{2}{|c|}{ RAR } & \multirow{2}{*}{$\begin{array}{l}\text { significance } \\
\text { neutral/pH2 }\end{array}$} \\
\hline & neutral & $\mathrm{pH} 2$ & & neutral & $\mathrm{pH} 2$ & & neutral & $\mathrm{pH} 2$ & \\
\hline INF-1 (B) & 0.0 & 0.0 & n.s. & $16.3 \pm 2.77$ & $1.92 \pm 0.67$ & $\star \star \star$ & $35.1 \pm 0.95$ & $8.68 \pm 1.36$ & $\star \star \star$ \\
\hline EFF-1 (B) & 0.0 & 0.0 & n.s. & 0.0 & 0.0 & n.s. & $3.92 \pm 1.10$ & $1.22 \pm 0.44$ & $\star$ \\
\hline EFF-2 (B) & $5.38 \pm 4.70$ & 0.0 & n.s. & 0.0 & 0.0 & n.s. & $4.16 \pm 0.35$ & $1.68 \pm 0.72$ & $\star \star$ \\
\hline EFF-4 (A) & $21.3 \pm 1.38$ & $17.0 \pm 3.82$ & n.s. & 0.0 & 0.0 & n.s. & $3.61 \pm 0.31$ & $0.71 \pm 0.33$ & $\star \star \star$ \\
\hline $\mathrm{EFF}-4-\mathrm{O}_{3}(\mathrm{~A})$ & 0.0 & 0.0 & n.s. & 0.0 & 0.0 & n.s. & $1.58 \pm 0.86$ & $0.68 \pm 0.51$ & n.s. \\
\hline FB-IN (B) & 0.0 & 0.0 & n.s. & $37.0 \pm 1.08$ & $4.96 \pm 1.00$ & $\star \star \star$ & $42.7 \pm 2.35$ & $8.49 \pm 0.44$ & $\star \star \star$ \\
\hline FB-OUT (B) & $0.67 \pm 1.42$ & 0.0 & $\star \star \star$ & 0.0 & 0.0 & n.s. & $2.60 \pm 0.32$ & $1.67 \pm 0.47$ & n.s. \\
\hline IB (SW) (B) & 0.0 & 0.0 & n.s. & 0.0 & 0.0 & n.s. & $3.59 \pm 0.28$ & $1.72 \pm 0.35$ & $\star \star \star$ \\
\hline GW-2 (B) & 0.0 & 0.0 & n.s. & 0.0 & 0.0 & n.s. & $2.74 \pm 0.30$ & $0.78 \pm 0.50$ & $\star \star$ \\
\hline GW-3 (B) & 0.0 & 0.0 & n.s. & 0.0 & 0.0 & n.s. & $3.50 \pm 0.84$ & $2.62 \pm 0.73$ & n.s. \\
\hline TAP (A) & $0.91 \pm 4.81$ & $7.19 \pm 4.80$ & n.s. & 0.0 & 0.0 & n.s. & $1.71 \pm 0.14$ & 0.0 & $\star \star \star$ \\
\hline
\end{tabular}


Table S2 continued: Retinoid X-like (RXR), vitamin D-like (VDR) and thyronin-like (TR) activity ( $\%$; mean \pm SEM) of neutral and acidified (pH 2) aqueous samples. Significant differences between neutral and acidified samples are marked with asterisks: $\star p \leq 0.05, \star \star p \leq 0.01$, $\mathrm{p} \leq 0.001$ (unpaired t-test), n.s.: not significant. Corresponding samples were taken on the same sampling dates in March (A) and April (B) 2012 .

\begin{tabular}{|c|c|c|c|c|c|c|c|c|c|}
\hline \multirow[t]{3}{*}{ sample } & \multicolumn{9}{|c|}{ in vitro bioassay } \\
\hline & \multicolumn{2}{|c|}{ RXR } & \multirow{2}{*}{$\begin{array}{l}\text { significance } \\
\text { neutral/pH } 2\end{array}$} & \multicolumn{2}{|c|}{ VDR } & \multirow{2}{*}{$\begin{array}{l}\text { significance } \\
\text { neutral/pH } 2\end{array}$} & \multicolumn{2}{|c|}{ TR } & \multirow{2}{*}{$\begin{array}{l}\text { significance } \\
\text { neutral } / \mathrm{pH} 2\end{array}$} \\
\hline & neutral & $\mathrm{pH} 2$ & & neutral & $\mathrm{pH} 2$ & & neutral & $\mathrm{pH} 2$ & \\
\hline HOS (B) & $25.0 \pm 7.01$ & 0.0 & $\star \star \star$ & $4.19 \pm 1.10$ & 0.0 & $\star \star \star$ & $0.38 \pm 0.28$ & $0.47 \pm 0.28$ & n.s. \\
\hline INF-1 (B) & $32.2 \pm 4.50$ & 0.0 & $\star \star \star$ & $1.99 \pm 1.36$ & 0.0 & $\star \star$ & $0.86 \pm 0.24$ & 0.0 & $\star \star$ \\
\hline EFF-1 (B) & 0.0 & 0.0 & n.s. & 0.0 & 0.0 & n.s. & 0.0 & 0.0 & n.s. \\
\hline EFF-2 (B) & 0.0 & 0.0 & n.s. & 0.0 & 0.0 & n.s. & 0.0 & 0.0 & n.s. \\
\hline EFF-3 (B) & 0.0 & $10.2 \pm 3.53$ & n.s. & 0.0 & 0.0 & n.s. & 0.0 & 0.0 & n.s. \\
\hline EFF-4 (A) & $0.46 \pm 0.15$ & $0.88 \pm 0.32$ & n.s. & $0.36 \pm 0.19$ & $0.21 \pm 0.13$ & n.s. & 0.0 & 0.0 & n.s. \\
\hline $\mathrm{EFF}-4-\mathrm{O}_{3}(\mathrm{~A})$ & 0.0 & $9.74 \pm 4.36$ & $\star \star$ & 0.0 & 0.0 & n.s. & 0.0 & $0.09 \pm 0.21$ & n.s. \\
\hline FB-IN (B) & $36.8 \pm 7.32$ & 0.0 & $\star \star \star$ & $0.94 \pm 1.18$ & 0.0 & $\star$ & $0.08 \pm 0.27$ & 0.0 & $\star \star \star$ \\
\hline FB-OUT (B) & 0.0 & 0.0 & n.s. & 0.0 & 0.0 & n.s. & $0.22 \pm 0.24$ & 0.0 & $\star$ \\
\hline IB (SW) (B) & $13.1 \pm 8.06$ & 0.0 & n.s. & $0.45 \pm 0.43$ & 0.0 & $\star$ & $0.50 \pm 0.27$ & 0.0 & $\star \star$ \\
\hline SW-1 (B) & 0.0 & 0.0 & n.s. & 0.0 & 0.0 & n.s. & 0.0 & 0.0 & n.s. \\
\hline SW-2 (B) & 0.0 & 0.0 & n.s. & 0.0 & 0.0 & n.s. & 0.0 & 0.0 & n.s. \\
\hline SW-3 (B) & 0.0 & $9.74 \pm 4.07$ & $\star$ & 0.0 & $4.63 \pm 1.50$ & $\star \star \star$ & 0.0 & 0.0 & n.s. \\
\hline GW-1 (B) & $50.5 \pm 7.02$ & $32.7 \pm 2.18$ & $\star$ & $0.94 \pm 0.53$ & $0.49 \pm 0.27$ & n.s. & $1.43 \pm 0.15$ & 0.0 & $\star \star \star$ \\
\hline GW-2 (B) & 0.0 & 0.0 & n.s. & 0.0 & 0.0 & n.s. & 0.0 & 0.0 & n.s. \\
\hline GW-3 (B) & 0.0 & $18.7 \pm 3.05$ & $\star \star \star$ & 0.0 & $0.50 \pm 0.25$ & $\star \star \star$ & 0.0 & $0.14 \pm 0.27$ & $\star \star$ \\
\hline TAP (A) & $0.25 \pm 0.27$ & $0.25 \pm 0.27$ & n.s. & $0.19 \pm 0.11$ & $0.22 \pm 0.11$ & n.s. & 0.0 & 0.0 & n.s. \\
\hline
\end{tabular}

Table S2 continued: Genotoxicity (umu: induction rate as mean $\pm \mathrm{SEM}]$ ) and mutagenicity (Ames, \% as mean]) of neutral and acidified (pH 2) aqueous samples; umu: potential genotoxicity if induction rate is $\geq 1.5$, Ames: potential mutagenicity if mean is $\geq 20.8 \%$. Significant differences between neutral and acidified samples are marked with asterisks: $\star \mathrm{p} \leq 0.05, \star \star \mathrm{p} \leq 0.01, \star \star \star \mathrm{p} \leq 0.001$ (umu: unpaired t-test, Ames: Fisher's exact test), n.s.: not significant. Corresponding samples were taken on the same sampling dates in March (A) and April (B) 2012.

\begin{tabular}{|c|c|c|c|c|c|c|c|c|c|}
\hline \multirow[t]{3}{*}{ sample } & \multicolumn{9}{|c|}{ in vitro bioassay } \\
\hline & \multicolumn{2}{|c|}{ Umu } & \multirow{2}{*}{$\begin{array}{l}\text { significance } \\
\text { neutral/pH } 2\end{array}$} & \multicolumn{2}{|c|}{ Ames TA98 } & \multirow{2}{*}{$\begin{array}{l}\text { significance } \\
\text { neutral/pH } 2\end{array}$} & \multicolumn{2}{|c|}{ Ames TA100 } & \multirow{2}{*}{$\begin{array}{l}\text { significance } \\
\text { neutral/pH } 2\end{array}$} \\
\hline & neutral & $\mathrm{pH} 2$ & & neutral & $\mathrm{pH} 2$ & & neutral & $\mathrm{pH} 2$ & \\
\hline HOS (B) & $0.96 \pm 0.02$ & $0.92 \pm 0.04$ & n.s. & 100 & 6.25 & $\star \star \star$ & 100 & 22.9 & $\star \star \star$ \\
\hline INF-I (B) & $0.92 \pm 0.03$ & $0.98 \pm 0.04$ & n.s. & 6.25 & 4.17 & n.s. & 20.8 & 20.8 & n.s. \\
\hline EFF-1 (B) & $0.85 \pm 0.03$ & $0.74 \pm 0.02$ & $\star \star$ & 0.0 & 8.33 & n.s. & 14.6 & 16.7 & n.s. \\
\hline EFF-2 (B) & $0.95 \pm 0.06$ & $0.94 \pm 0.05$ & n.s. & 66.7 & 0.0 & $\star \star \star$ & 72.9 & 14.6 & $\star \star \star$ \\
\hline EFF-3 (B) & $0.87 \pm 0.04$ & $0.87 \pm 0.03$ & n.s. & 6.25 & 4.17 & n.s. & 22.9 & 14.6 & n.s. \\
\hline $\mathrm{EFF}-4$ (A) & $0.80 \pm 0.02$ & $1.11 \pm 0.01$ & $\star \star \star$ & 4.17 & 6.25 & n.s. & 12.5 & 18.8 & n.s. \\
\hline $\mathrm{EFF}-4-\mathrm{O}_{3}(\mathrm{~A})$ & $0.86 \pm 0.03$ & $0.89 \pm 0.04$ & n.s. & 6.25 & 8.33 & n.s. & 16.7 & 10.4 & n.s. \\
\hline FB-IN (B) & $0.83 \pm 0.02$ & $0.94 \pm 0.03$ & $\star \star$ & 6.25 & 6.25 & n.s. & 29.2 & 16.7 & n.s. \\
\hline FB-OUT (B) & $1.21 \pm 0.03$ & $0.87 \pm 0.03$ & $\star \star \star$ & 4.17 & 4.17 & n.s. & 6.25 & 6.25 & n.s. \\
\hline IB (SW) (B) & $0.98 \pm 0.02$ & $1.20 \pm 0.05$ & $\star \star \star$ & 0.0 & 10.4 & n.s. & 10.4 & 6.25 & n.s. \\
\hline SW-1 (B) & $1.01 \pm 0.07$ & $0.95 \pm 0.02$ & n.s. & 2.08 & 10.4 & n.s. & 2.08 & 12.5 & n.s. \\
\hline SW-2 (B) & $1.15 \pm 0.05$ & $0.63 \pm 0.03$ & $\star \star \star$ & 6.25 & 4.17 & n.s. & 10.4 & 10.4 & n.s. \\
\hline SW-3 (B) & $0.91 \pm 0.05$ & $0.99 \pm 0.06$ & n.s. & 4.17 & 2.08 & n.s. & 10.4 & 6.25 & n.s. \\
\hline GW-1 (B) & $0.96 \pm 0.04$ & $0.98 \pm 0.05$ & n.s. & 4.17 & 4.17 & n.s. & 4.17 & 12.5 & n.s. \\
\hline GW-2 (B) & $1.21 \pm 0.04$ & $0.61 \pm 0.03$ & $\star \star \star$ & 2.08 & 2.08 & n.s. & 8.33 & 16.7 & n.s. \\
\hline GW-3 (B) & $0.87 \pm 0.02$ & $0.85 \pm 0.02$ & n.s. & 8.33 & 0.0 & n.s. & 12.5 & 10.4 & n.s. \\
\hline TAP (A) & $0.86 \pm 0.02$ & $1.86 \pm 0.08$ & $\star \star \star$ & 2.08 & 4.17 & n.s. & 10.4 & 27.1 & n.s. \\
\hline
\end{tabular}


Table S3: Percentage of in vitro bioassays $(\mathrm{n}=11$ per sample, umu test excluded $)$ in which acidification caused a change in activity of $\geq 10 \%$ within one sample type. Corresponding samples were taken on the same sampling dates in March (A) and April (B) 2012.

\begin{tabular}{lcc}
\hline Type of sample & {$[\%]$} & \\
\hline untreated wastewater (HOS (B), INF-1 (B)) & 50.0 & $\mathrm{n}=22$ \\
influent and effluent of a filtration basin (FB-IN (B), FB-OUT (B)) & 31.8 & $\mathrm{n}=22$ \\
surface water of an infiltration basin (IB (SW) (B)) & 18.2 & $\mathrm{n}=11$ \\
tap water (TAP (A)) & 18.2 & $\mathrm{n}=11$ \\
conventionally treated wastewater (EFF-1 (B), EFF-2 (B), EFF-3 (B), EFF-4 (A)) & 11.4 & $\mathrm{n}=44$ \\
groundwater (hotspots; GW-1 (B), GW-2 (B), GW-3 (B)) & 9.1 & $\mathrm{n}=33$ \\
ozone-treated wastewater (EFF-4-O3 (A)) & 9.1 & $\mathrm{n}=11$ \\
surface water (SW-1 (B), SW-2 (B), SW-3 (B)) & 3.0 & $\mathrm{n}=33$ \\
\hline
\end{tabular}

Table S4: Percentage of the number of analysed samples ( $n=17$ per bioassay) in which acidification caused a change in the activity of $\geq 10 \%$ in one in vitro bioassay.

\begin{tabular}{lc}
\hline Type of in vitro bioassay & {$[\%]$} \\
\hline YAES & 64.7 \\
RXR & 41.2 \\
Ames TA100 & 23.5 \\
RAR & 17.6 \\
YDS & 17.6 \\
YAS & 11.8 \\
Ames TA98 & 11.8 \\
YES & 0.0 \\
YAAS & 0.0 \\
VDR & 0.0 \\
TR & 0.0 \\
\hline
\end{tabular}



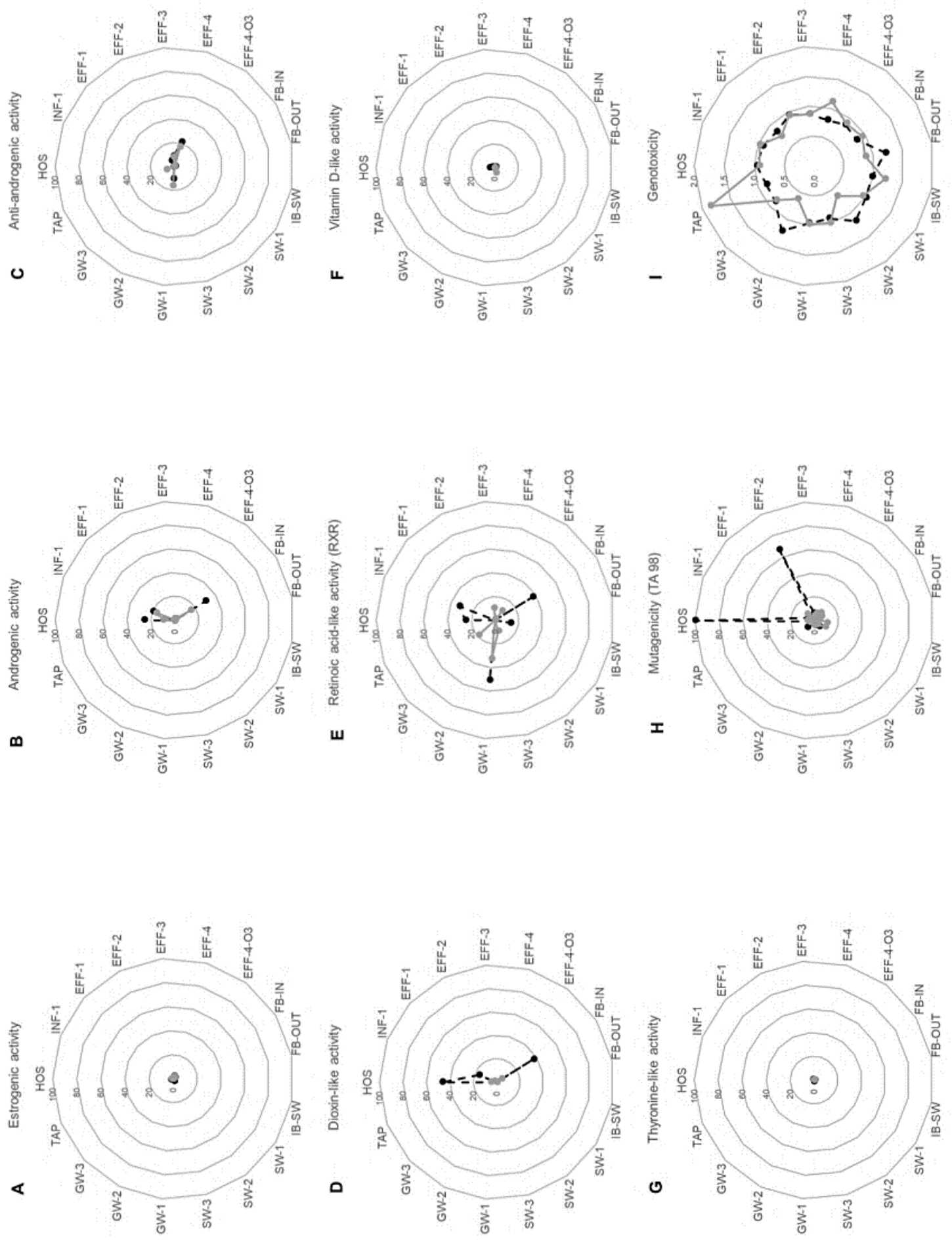

Figure S7: Estrogenic (A), androgenic (B), anti-androgenic (C), dioxin-like (D), retinoic acid-like (RXR, E), vitamin D-like (F) and thyronine-like (G) activity, mutagenicity (TA98, H) and genotoxicity (I) in \% of neutral (black) and acidified (grey) aqueous water and wastewater samples. Corresponding samples were taken on the same sampling dates in March and April 2012, respectively. 


\subsection{Sample filtration}

Table S5: Endocrine activity (\%; mean \pm SEM), genotoxicity (umu: induction rate [mean \pm SEM]) and mutagenicity (Ames [\%; mean]) of unfiltered and filtered samples (Whatman GF6) and aqueous suspensions of the filter retentate; n.a.: not analysed. Umu: genotoxic if induction rate is $\geq 1.5$; Ames: mutagenic if mean is $\geq 20.8 \%$. Significant differences between unfiltered and filtered samples are marked with asterisks: $\star \mathrm{p} \leq 0.05, \star \star \mathrm{p} \leq 0.01, \star \star \star \mathrm{p} \leq 0.001$ (endocrine activity and umu: unpaired t-test, Ames: Fisher's exact test), n.s.: not significant. Samples taken in March (A), middle of July (C), end of July (D) 2012 and December (E) 2012.

\begin{tabular}{|c|c|c|c|c|c|}
\hline sample & $\begin{array}{l}\text { in vitro } \\
\text { bioassay }\end{array}$ & unfiltered & filtered & $\begin{array}{c}\text { aqueous } \\
\text { suspension }\end{array}$ & $\begin{array}{c}\text { significance } \\
\text { unfiltered/filtered }\end{array}$ \\
\hline \multirow[t]{10}{*}{$\operatorname{HOS}(\mathrm{C})$} & YES & $19.6 \pm 0.61$ & 0.0 & $0.75 \pm 0.24$ & $\star \star \star$ \\
\hline & YAES & $84.1 \pm 1.47$ & $87.8 \pm 0.68$ & $45.9 \pm 2.33$ & $\star$ \\
\hline & YAS & 0.0 & $5.28 \pm 0.37$ & 0.0 & $\star \star \star$ \\
\hline & YAAS & $38.3 \pm 2.49$ & $99.7 \pm 1.07$ & $30.3 \pm 2.34$ & $\star \star \star$ \\
\hline & YDS & 0.0 & 0.0 & 0.0 & n.s. \\
\hline & RAR & $99.4 \pm 1.78$ & $93.3 \pm 1.18$ & $5.66 \pm 0.33$ & $\star$ \\
\hline & RXR & 0.0 & 0.0 & $2.70 \pm 1.31$ & n.s. \\
\hline & VDR & $0.64 \pm 0.64$ & 0.0 & $3.11 \pm 1.22$ & $\star \star \star$ \\
\hline & $\mathrm{TR}$ & 0.0 & 0.0 & $1.32 \pm 0.22$ & n.s. \\
\hline & Umu & $1.10 \pm 0.11$ & $1.29 \pm 0.12$ & $0.85 \pm 0.05$ & n.s. \\
\hline \multirow[t]{10}{*}{ INF-1 (C) } & YES & $2.56 \pm 0.67$ & 0.0 & $1.48 \pm 0.36$ & $\star \star \star$ \\
\hline & YAES & $33.6 \pm 0.35$ & $61.3 \pm 1.03$ & $31.7 \pm 1.15$ & $\star \star \star$ \\
\hline & YAS & 0.0 & $2.74 \pm 0.33$ & 0.0 & $\star \star \star$ \\
\hline & YAAS & $57.0 \pm 4.70$ & $3.31 \pm 3.46$ & $34.0 \pm 2.32$ & $\star \star \star$ \\
\hline & YDS & 0.0 & 0.0 & 0.0 & n.s. \\
\hline & RAR & $20.4 \pm 0.97$ & $23.2 \pm 0.27$ & $3.21 \pm 0.07$ & $\star$ \\
\hline & RXR & 0.0 & 0.0 & $5.93 \pm 0.75$ & n.s. \\
\hline & VDR & 0.0 & 0.0 & $9.69 \pm 1.50$ & n.s. \\
\hline & $\mathrm{TR}$ & 0.0 & 0.0 & $0.53 \pm 0.21$ & n.s. \\
\hline & Umu & $0.77 \pm 0.04$ & $0.77 \pm 0.04$ & $0.79 \pm 0.05$ & n.s. \\
\hline
\end{tabular}


Table S5 continued.

\begin{tabular}{|c|c|c|c|c|c|}
\hline sample & $\begin{array}{l}\text { in vitro } \\
\text { bioassay }\end{array}$ & unfiltered & filtered & $\begin{array}{c}\text { aqueous } \\
\text { suspension }\end{array}$ & $\begin{array}{c}\text { significance } \\
\text { unfiltered/filtered }\end{array}$ \\
\hline \multirow[t]{10}{*}{ EFF-1 (C) } & YES & 0.0 & $12.7 \pm 1.98$ & 0.0 & $\star \star \star$ \\
\hline & YAES & $39.2 \pm 1.32$ & $20.9 \pm 2.32$ & $20.7 \pm 2.06$ & $\star \star \star$ \\
\hline & YAS & $3.21 \pm 0.58$ & 0.0 & $0.56 \pm 0.78$ & $\star \star$ \\
\hline & YAAS & 0.0 & 0.0 & $9.04 \pm 2.12$ & n.s. \\
\hline & YDS & 0.0 & $18.9 \pm 1.69$ & 0.0 & $\star \star \star$ \\
\hline & RAR & 0.0 & $1.23 \pm 0.27$ & 0.0 & $\star \star \star$ \\
\hline & RXR & 0.0 & 0.0 & 0.0 & n.s. \\
\hline & VDR & 0.0 & 0.0 & $1.51 \pm 0.51$ & n.s. \\
\hline & TR & 0.0 & 0.0 & $1.01 \pm 0.33$ & n.s. \\
\hline & Umu & $0.75 \pm 0.03$ & $0.83 \pm 0.06$ & $0.73 \pm 0.04$ & n.s. \\
\hline \multirow[t]{10}{*}{ EFF-1 (E) } & YES & 0.0 & 0.0 & n.a. & n.s. \\
\hline & YAES & $29.0 \pm 2.40$ & $22.6 \pm 1.43$ & n.a. & $\star$ \\
\hline & YAS & 0.0 & $1.20 \pm 0.49$ & n.a. & $\star \star \star$ \\
\hline & YAAS & 0.0 & 0.0 & n.a. & n.s. \\
\hline & YDS & 0.0 & 0.0 & n.a. & n.s. \\
\hline & RAR & 0.0 & 0.0 & n.a. & n.s. \\
\hline & RXR & n.a. & n.a. & n.a. & n.a. \\
\hline & VDR & n.a. & n.a. & n.a. & n.a. \\
\hline & TR & $12.1 \pm 0.41$ & $9.74 \pm 1.53$ & n.a. & n.s. \\
\hline & Umu & n.a. & n.a. & n.a. & n.a. \\
\hline \multirow[t]{12}{*}{ EFF-4 (A) } & YES & 0.0 & 0.0 & n.a. & n.s. \\
\hline & YAES & $60.6 \pm 1.91$ & $59.2 \pm 1.23$ & n.a. & n.s. \\
\hline & YAS & 0.0 & 0.0 & n.a. & n.s. \\
\hline & YAAS & 0.0 & 0.0 & n.a. & n.s. \\
\hline & YDS & 0.0 & 0.0 & n.a. & n.s. \\
\hline & RAR & $2.74 \pm 0.30$ & $9.16 \pm 4.01$ & n.a. & n.s. \\
\hline & RXR & 0.0 & 0.0 & n.a. & n.s. \\
\hline & VDR & 0.0 & $1.64 \pm 2.11$ & n.a. & n.s. \\
\hline & $\mathrm{TR}$ & 0.0 & $0.15 \pm 0.11$ & n.a. & n.s. \\
\hline & Umu & $1.05 \pm 0.04$ & $0.87 \pm 0.03$ & n.a. & $\star \star$ \\
\hline & Ames TA98 & 4.17 & 0.0 & n.a. & n.s. \\
\hline & Ames TA100 & 14.6 & 18.8 & n.a. & n.s. \\
\hline
\end{tabular}


Table S5 continued.

\begin{tabular}{|c|c|c|c|c|c|}
\hline sample & $\begin{array}{l}\text { in vitro } \\
\text { bioassay }\end{array}$ & unfiltered & filtered & $\begin{array}{c}\text { aqueous } \\
\text { suspension }\end{array}$ & $\begin{array}{c}\text { significance } \\
\text { unfiltered/filtered }\end{array}$ \\
\hline \multirow[t]{10}{*}{ SW-1 (E) } & YES & 0.0 & 0.0 & n.a. & n.s. \\
\hline & YAES & $17.7 \pm 0.97$ & $31.0 \pm 2.11$ & n.a. & $\star \star \star$ \\
\hline & YAS & 0.0 & $0.12 \pm 0.58$ & n.a. & n.s. \\
\hline & YAAS & 0.0 & 0.0 & n.a. & n.s. \\
\hline & YDS & 0.0 & 0.0 & n.a. & n.s. \\
\hline & RAR & 0.0 & 0.0 & n.a. & n.s. \\
\hline & RXR & n.a. & n.a. & n.a. & n.a. \\
\hline & VDR & n.a. & n.a. & n.a. & n.a. \\
\hline & TR & $9.81 \pm 0.91$ & $9.07 \pm 0.81$ & n.a. & n.s. \\
\hline & Umu & n.a. & n.a. & n.a. & n.a. \\
\hline \multirow[t]{10}{*}{ GW-1 (C) } & YES & 0.0 & 0.0 & $2.17 \pm 1.36$ & n.s. \\
\hline & YAES & $85.6 \pm 0.47$ & $90.7 \pm 0.52$ & $79.5 \pm 0.57$ & $\star \star \star$ \\
\hline & YAS & $3.00 \pm 0.63$ & $0.43 \pm 1.44$ & 0.0 & n.s. \\
\hline & YAAS & 0.0 & 0.0 & $45.2 \pm 9.34$ & n.s. \\
\hline & YDS & 0.0 & 0.0 & 0.0 & n.s. \\
\hline & RAR & $0.17 \pm 0.21$ & 0.0 & $1.33 \pm 0.19$ & $\star$ \\
\hline & RXR & 0.0 & 0.0 & $5.25 \pm 0.92$ & n.s. \\
\hline & VDR & 0.0 & 0.0 & $2.95 \pm 0.96$ & n.s. \\
\hline & TR & 0.0 & 0.0 & $1.99 \pm 0.69$ & n.s. \\
\hline & Umu & $0.82 \pm 0.04$ & $0.84 \pm 0.04$ & $0.85 \pm 0.05$ & n.s. \\
\hline \multirow{10}{*}{$\begin{array}{l}\text { EFF-4 (D)/ } \\
\text { EFF-4-MS } \\
\text { (D) }\end{array}$} & YES & 0.0 & 0.0 & n.a. & n.s. \\
\hline & YAES & $45.1 \pm 0.81$ & $80.8 \pm 0.53$ & n.a. & $\star \star \star$ \\
\hline & YAS & $0.05 \pm 0.69$ & 0.0 & n.a. & n.s. \\
\hline & YAAS & 0.0 & $5.24 \pm 4.52$ & n.a. & n.s. \\
\hline & YDS & 0.0 & 0.0 & n.a. & n.s. \\
\hline & RAR & $0.40 \pm 0.16$ & $0.65 \pm 0.14$ & n.a. & n.s. \\
\hline & RXR & 0.0 & 0.0 & n.a. & n.s. \\
\hline & VDR & $0.64 \pm 0.75$ & 0.0 & n.a. & n.s. \\
\hline & TR & $0.96 \pm 0.34$ & 0.0 & n.a. & n.s. \\
\hline & Umu & $0.89 \pm 0.04$ & $0.91 \pm 0.05$ & n.a. & n.s. \\
\hline
\end{tabular}

Table S6: Percentage of in vitro bioassays (except umu test) in which filtration caused a change in activity of $\geq 10 \%$ within one sample type. Corresponding samples were taken on the same sampling dates in March (A), in the middle of July (C), at the end of July (D) and in December 2012 .

\begin{tabular}{lcc}
\hline Type of sample & {$[\%]$} & \\
\hline untreated wastewater (HOS (C), INF-1 (C)) & 22.2 & $\mathrm{n}=18$ \\
surface water (SW-1 (E)) & 14.3 & $\mathrm{n}=7$ \\
conventionally treated wastewater (EFF-1 (C), EFF-1 (E), EFF-4 (A), EFF-4 (D)) & 11.1 & $\mathrm{n}=36$ \\
groundwater (hotspot; GW-1 (C)) & 0.0 & $\mathrm{n}=9$ \\
\hline
\end{tabular}



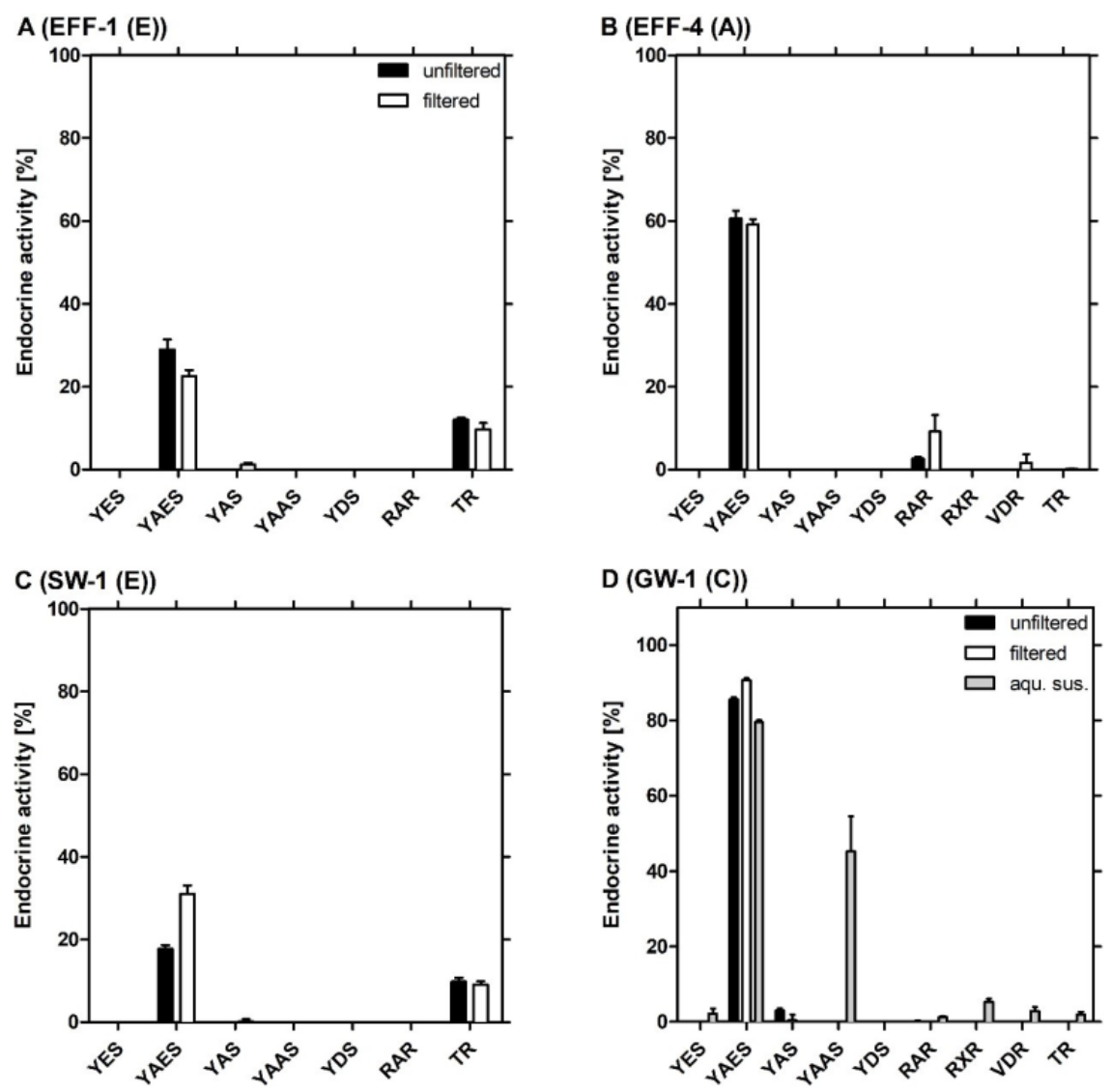

Figure S8: Endocrine activity (\%, mean \pm SEM) of unfiltered (black bars) and filtered (white bars) water and wastewater samples and the aqueous suspensions of the filter retentate (grey bars) of conventionally treated wastewater (A and B: effluents of two WWTPs (EFF-1 and EFF4)), surface water (C: SW-1) and groundwater (D: GW-1). YES: estrogenic; YAES: antiestrogenic; YAS: androgenic; YAAS: anti-androgenic; YDS: dioxin-like, RAR: retinoic acidlike, RXR: retinoid X-like, VDR: vitamin D-like, TR: thyronine-like. No aqueous suspension of the filter retentate was analysed in A, B and C. No RXR and VDR assays were performed in A and C. Corresponding samples were taken on the same sampling dates in March (A), July (C) and December (E) 2012. 
Table S7: Percentage of the number of analysed samples in which filtration caused a change in the activity of $\geq 10 \%$ within one type of in vitro bioassay.

\begin{tabular}{lcc}
\hline Type of in vitro bioassay & {$[\%]$} & \\
\hline YAES & 50.0 & $\mathrm{n}=8$ \\
YES & 25.0 & $\mathrm{n}=8$ \\
YAAS & 25.0 & $\mathrm{n}=8$ \\
YDS & 12.5 & $\mathrm{n}=8$ \\
YAS & 0.0 & $\mathrm{n}=8$ \\
RAR & 0.0 & $\mathrm{n}=8$ \\
RXR & 0.0 & $\mathrm{n}=6$ \\
VDR & 0.0 & $\mathrm{n}=6$ \\
TR & 0.0 & $\mathrm{n}=6$ \\
Ames TA98 & 0.0 & $\mathrm{n}=1$ \\
Ames TA100 & 0.0 & $\mathrm{n}=1$ \\
\hline
\end{tabular}

\subsection{Solid Phase Extraction}

Table S8: Endocrine activity (\%, mean \pm SEM) and genotoxicity (umu: induction rate, mean \pm SEM) of aqueous samples and 10 -fold concentrated SPE extracts, $2:$ cytotoxic. Umu: potential genotoxicity if induction rate is $\geq 1.5$. Corresponding samples were taken on the same sampling date in the middle of July (C) 2012, the end of July (D) 2012 and in January (F) 2013

\begin{tabular}{|c|c|c|c|c|c|c|c|c|}
\hline \multirow[t]{2}{*}{ sample } & \multirow{2}{*}{$\begin{array}{c}\text { in vitro } \\
\text { bioassay }\end{array}$} & \multirow[t]{2}{*}{ aqueous } & \multicolumn{2}{|c|}{ Oasis HLB } & \multicolumn{2}{|c|}{ Telos C18/ENV } & \multicolumn{2}{|c|}{ Supelco ENVI-Carb+ } \\
\hline & & & pH 7 & pH 2.5 & pH 7 & pH 2.5 & pH 7 & $\mathrm{pH} 2.5$ \\
\hline \multirow[t]{10}{*}{$\operatorname{HOS}(\mathrm{C})$} & YES & 0.0 & 2 & 2 & 2 & 2 & 0.0 & $0.35 \pm 0.26$ \\
\hline & YAES & $87.8 \pm 0.68$ & 2 & 2 & 2 & 2 & $89.1 \pm 0.74$ & $66.6 \pm 1.41$ \\
\hline & YAS & $5.28 \pm 0.37$ & $\approx$ & $\approx$ & $\stackrel{2}{*}$ & $\approx$ & 0.0 & $6.67 \pm 0.83$ \\
\hline & YAAS & $99.7 \pm 1.07$ & 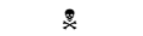 & 2 & 2 & 2 & $38.9 \pm 5.40$ & $39.4 \pm 4.95$ \\
\hline & YDS & 0.0 & $\approx$ & $\approx$ & $\approx$ & $\approx$ & $26.1 \pm 0.67$ & $2.58 \pm 0.22$ \\
\hline & RAR & $93.3 \pm 1.18$ & $76.6 \pm 1.22$ & $91.3 \pm 1.39$ & 2 & $91.0 \pm 2.12$ & $13.8 \pm 0.65$ & $47.8 \pm 0.98$ \\
\hline & RXR & 0.0 & $2.75 \pm 0.63$ & $\approx$ & 2 & 0.0 & 0.0 & 0.0 \\
\hline & VDR & 0.0 & 2 & $0.77 \pm 0.26$ & 2 & 0.0 & 0.0 & 0.0 \\
\hline & TR & 0.0 & 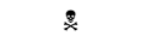 & 0.0 & 2 & 0.0 & 0.0 & 0.0 \\
\hline & Umu & $1.29 \pm 0.12$ & $\approx$ & 2 & 2 & $4.37 \pm 0.19$ & $1.22 \pm 0.04$ & $1.01 \pm 0.04$ \\
\hline \multirow[t]{10}{*}{ INF-1 (C) } & YES & 0.0 & 2 & 2 & 2 & 2 & 0.0 & 0.0 \\
\hline & YAES & $61.3 \pm 1.03$ & 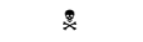 & 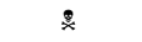 & 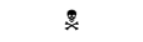 & 2 & $84.3 \pm 2.75$ & $80.0 \pm 2.44$ \\
\hline & YAS & $2.74 \pm 0.33$ & 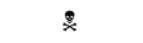 & 2 & 2 & 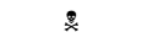 & $5.33 \pm 0.70$ & $3.47 \pm 0.40$ \\
\hline & YAAS & $3.31 \pm 3.46$ & $\approx$ & 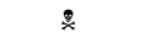 & 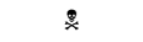 & 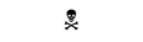 & $43.7 \pm 4.31$ & $45.0 \pm 2.18$ \\
\hline & YDS & 0.0 & $\approx$ & 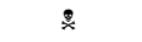 & $\approx$ & $\approx$ & $17.9 \pm 0.23$ & $3.95 \pm 0.14$ \\
\hline & RAR & $23.2 \pm 0.27$ & $49.3 \pm 1.01$ & $53.5 \pm 0.97$ & $50.6 \pm 1.19$ & $42.5 \pm 0.34$ & 0.0 & $1.67 \pm 0.27$ \\
\hline & RXR & 0.0 & 0.0 & 0.0 & 0.0 & 0.0 & 0.0 & 0.0 \\
\hline & VDR & 0.0 & 0.0 & 0.0 & 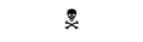 & $1.20 \pm 0.60$ & 0.0 & 0.0 \\
\hline & TR & 0.0 & 0.0 & 0.0 & 0.0 & 0.0 & 0.0 & $0.72 \pm 0.26$ \\
\hline & Umu & $0.77 \pm 0.04$ & $\dot{2}$ & $1.34 \pm 1.34$ & $\stackrel{2}{x}$ & $1.06 \pm 0.02$ & $1.30 \pm 0.06$ & $1.09 \pm 0.03$ \\
\hline
\end{tabular}


Table $\mathbf{S 8}$ continued.

\begin{tabular}{|c|c|c|c|c|c|c|c|c|}
\hline \multirow[t]{2}{*}{ sample } & \multirow{2}{*}{$\begin{array}{c}\text { in vitro } \\
\text { bioassay }\end{array}$} & \multirow[t]{2}{*}{ aqueous } & \multicolumn{2}{|c|}{ Oasis HLB } & \multicolumn{2}{|c|}{ Telos C18/ENV } & \multicolumn{2}{|c|}{ Supelco ENVI-Carb+ } \\
\hline & & & $\mathrm{pH} 7$ & $\mathrm{pH} 2.5$ & $\mathrm{pH} 7$ & $\mathrm{pH} 2.5$ & $\mathrm{pH} 7$ & $\mathrm{pH} 2.5$ \\
\hline \multirow[t]{7}{*}{ EFF-1 (C) } & YES & $12.7 \pm 1.98$ & 0 & $7.60 \pm 0.41$ & $3.93 \pm 0.58$ & $5.04 \pm 0.47$ & $0.08 \pm 0.19$ & 0.0 \\
\hline & YAS & 0.0 & $1.55 \pm 0.45$ & $0.81 \pm 0.55$ & $\approx$ & 0.0 & $1.43 \pm 0.46$ & 0.0 \\
\hline & YAAS & 0.0 & $24.1 \pm 2.84$ & $70.8 \pm 1.37$ & $\approx$ & $66.6 \pm 2.14$ & $36.3 \pm 4.65$ & $41.2 \pm 2.30$ \\
\hline & RXR & 0.0 & 0.0 & 0.0 & $2.07 \pm 2.71$ & $0.12 \pm 0.71$ & 0.0 & 0.0 \\
\hline & VDR & 0.0 & 0.0 & 0.0 & 0.0 & $0.91 \pm 0.15$ & 0.0 & $0.25 \pm 0.19$ \\
\hline & TR & 0.0 & 0.0 & 0.0 & 0.0 & $0.42 \pm 0.14$ & 0.0 & $0.44 \pm 0.13$ \\
\hline & Umu & $0.83 \pm 0.06$ & $1.10 \pm 0.04$ & $1.62 \pm 0.07$ & $*$ & $1.36 \pm 0.03$ & $\approx$ & $1.17 \pm 0.04$ \\
\hline \multirow[t]{7}{*}{ EFF-4 (D) } & YES & 0.0 & $3.05 \pm 0.26$ & $5.08 \pm 0.38$ & $2.07 \pm 0.49$ & $5.91 \pm 0.38$ & $0.66 \pm 0.29$ & 0.0 \\
\hline & YDS & 0.0 & $20.3 \pm 0.78$ & $13.6 \pm 0.64$ & $21.2 \pm 1.54$ & $13.0 \pm 1.10$ & $4.56 \pm 1.43$ & $1.12 \pm 1.32$ \\
\hline & RAR & $0.40 \pm 0.16$ & 0.0 & $2.86 \pm 0.53$ & $0.47 \pm 0.68$ & $2.74 \pm 0.42$ & 0.0 & $2.44 \pm 0.65$ \\
\hline & RXR & 0.0 & 0.0 & $3.61 \pm 2.85$ & 0.0 & $1.87 \pm 0.65$ & 0.0 & $1.21 \pm 0.82$ \\
\hline & VDR & $0.64 \pm 0.75$ & 0.0 & $0.97 \pm 0.12$ & 0.0 & $1.92 \pm 0.31$ & 0.0 & $1.60 \pm 0.28$ \\
\hline & $\mathrm{TR}$ & $0.96 \pm 0.34$ & 0.0 & $1.86 \pm 0.14$ & $0.01 \pm 0.58$ & $1.96 \pm 0.23$ & 0.0 & 0.0 \\
\hline & Umu & $0.89 \pm 0.04$ & $1.54 \pm 0.03$ & $1.82 \pm 0.02$ & $1.50 \pm 0.08$ & $1.87 \pm 0.03$ & $1.43 \pm 0.09$ & $1.25 \pm 0.07$ \\
\hline
\end{tabular}

Table S8 continued.

\begin{tabular}{|c|c|c|c|c|c|c|c|c|}
\hline \multirow[t]{2}{*}{ sample } & \multirow{2}{*}{$\begin{array}{c}\text { in vitro } \\
\text { bioassay }\end{array}$} & \multirow[t]{2}{*}{ aqueous } & \multicolumn{2}{|c|}{ Oasis HLB } & \multicolumn{2}{|c|}{ Telos C18/ENV } & \multicolumn{2}{|c|}{ Supelco ENVI-Carb+ } \\
\hline & & & $\mathrm{pH} 7$ & $\mathrm{pH} 2.5$ & $\mathrm{pH} 7$ & $\mathrm{pH} 2.5$ & $\mathrm{pH} 7$ & $\mathrm{pH} 2.5$ \\
\hline \multirow[t]{10}{*}{ EFF-4-MS (D) } & YES & 0.0 & $3.09 \pm 0.60$ & $3.20 \pm 0.38$ & 2 & $4.77 \pm 0.37$ & $1.24 \pm 0.17$ & $0.78 \pm 0.33$ \\
\hline & YAES & $80.8 \pm 0.53$ & $76.3 \pm 1.46$ & $33.3 \pm 1.85$ & 2 & $48.0 \pm 2.33$ & $62.4 \pm 1.37$ & $31.9 \pm 2.34$ \\
\hline & YAS & 0.0 & $0.43 \pm 0.56$ & $0.29 \pm 0.36$ & 2 & $0.71 \pm 0.71$ & $2.44 \pm 1.14$ & 0.0 \\
\hline & YAAS & $5.24 \pm 4.52$ & $68.0 \pm 2.80$ & $63.1 \pm 2.89$ & 2 & $67.3 \pm 2.39$ & $23.0 \pm 1.56$ & $34.5 \pm 1.56$ \\
\hline & YDS & 0.0 & $20.6 \pm 0.27$ & $6.21 \pm 1.21$ & $39.8 \pm 2.09$ & $11.4 \pm 1.66$ & $5.85 \pm 0.16$ & $4.26 \pm 1.46$ \\
\hline & RAR & $0.65 \pm 0.14$ & $0.25 \pm 0.16$ & $2.72 \pm 0.35$ & 0.0 & $3.59 \pm 0.50$ & 0.0 & $0.94 \pm 0.24$ \\
\hline & RXR & 0.0 & $2.06 \pm 0.28$ & $0.59 \pm 0.44$ & 0.0 & 0.0 & 0.0 & 0.0 \\
\hline & VDR & 0.0 & $0.22 \pm 0.14$ & $1.74 \pm 0.29$ & 0.0 & $1.88 \pm 0.35$ & 0.0 & $0.87 \pm 0.47$ \\
\hline & TR & 0.0 & 0.0 & 0.0 & 0.0 & $0.55 \pm 0.24$ & 0.0 & 0.0 \\
\hline & Umu & $0.91 \pm 0.05$ & $1.61 \pm 0.08$ & $1.72 \pm 0.11$ & 2 & $1.73 \pm 0.07$ & $1.45 \pm 0.06$ & $1.35 \pm 0.04$ \\
\hline \multirow[t]{9}{*}{ EFF-4-MS (F) } & YES & $2.39 \pm 1.16$ & $\stackrel{2}{*}$ & $4.49 \pm 0.11$ & 2 & $4.77 \pm 0.12$ & 0.0 & $0.83 \pm 0.25$ \\
\hline & YAES & $44.2 \pm 4.45$ & $75.1 \pm 1.17$ & $20.8 \pm 0.83$ & 2 & $24.7 \pm 0.69$ & $\approx$ & $36.8 \pm 1.66$ \\
\hline & YAS & 0.0 & $\approx$ & $0.44 \pm 0.13$ & 2 & $0.22 \pm 0.08$ & $\approx$ & $0.71 \pm 0.15$ \\
\hline & YAAS & 0.0 & $\approx$ & $58.3 \pm 0.81$ & $\approx$ & $64.6 \pm 0.69$ & $\approx$ & $40.4 \pm 2.21$ \\
\hline & YDS & 0.0 & $*$ & $20.4 \pm 0.38$ & 2 & $27.5 \pm 0.65$ & $\approx$ & $2.44 \pm 0.09$ \\
\hline & RAR & 0.0 & $4.06 \pm 0.33$ & $4.67 \pm 0.12$ & $3.22 \pm 0.19$ & $6.71 \pm 0.12$ & 0.0 & $0.90 \pm 0.18$ \\
\hline & RXR & 0.0 & 0.0 & $9.68 \pm 1.12$ & 0.0 & $9.88 \pm 1.91$ & 0.0 & $8.36 \pm 0.64$ \\
\hline & VDR & 0.0 & 0.0 & $0.92 \pm 0.19$ & 0.0 & $0.95 \pm 0.24$ & 0.0 & $1.23 \pm 0.19$ \\
\hline & TR & 0.0 & 0.0 & $1.02 \pm 0.08$ & 0.0 & $1.51 \pm 0.04$ & 0.0 & $0.27 \pm 0.09$ \\
\hline
\end{tabular}


Table S8 continued.

\begin{tabular}{|c|c|c|c|c|c|c|c|c|}
\hline \multirow[t]{2}{*}{ sample } & \multirow{2}{*}{$\begin{array}{l}\text { in vitro } \\
\text { bioassay }\end{array}$} & \multirow[t]{2}{*}{ aqueous } & \multicolumn{2}{|c|}{ Oasis HLB } & \multicolumn{2}{|c|}{ Telos C18/ENV } & \multicolumn{2}{|c|}{ Supelco ENVI-Carb+ } \\
\hline & & & pH 7 & $\mathrm{pH} 2.5$ & $\mathrm{pH} 7$ & $\mathrm{pH} 2.5$ & $\mathrm{pH} 7$ & $\mathrm{pH} 2.5$ \\
\hline \multirow[t]{9}{*}{ EFF-4-MS-O ${ }_{3}(\mathrm{~F})$} & YES & $0.04 \pm 0.35$ & 2 & 0.0 & 2 & 0.0 & 0.0 & 0.0 \\
\hline & YAES & $73.9 \pm 1.13$ & 2 & $48.4 \pm 0.48$ & 2 & $54.3 \pm 0.53$ & $76.5 \pm 0.86$ & $34.7 \pm 0.60$ \\
\hline & YAS & 0.0 & $*$ & $0.08 \pm 0.09$ & 2 & $0.01 \pm 0.08$ & $\approx$ & $0.27 \pm 0.12$ \\
\hline & YAAS & 0.0 & 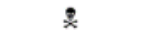 & $44.4 \pm 1.32$ & $\approx$ & $51.4 \pm 1.05$ & $\approx$ & $37.3 \pm 2.62$ \\
\hline & YDS & 0.0 & 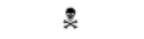 & $3.03 \pm 0.17$ & 2 & $4.76 \pm 0.18$ & $*$ & $0.12 \pm 0.05$ \\
\hline & RAR & $0.46 \pm 0.31$ & 0.0 & $2.26 \pm 0.25$ & 2 & $1.57 \pm 0.22$ & 0.0 & $1.23 \pm 0.17$ \\
\hline & RXR & 0.0 & 2 & $10.5 \pm 0.85$ & 2 & $8.69 \pm 0.64$ & 0.0 & $6.74 \pm 0.79$ \\
\hline & VDR & 0.0 & 0.0 & $2.22 \pm 0.15$ & 2 & $1.70 \pm 0.24$ & 0.0 & $1.73 \pm 0.16$ \\
\hline & TR & 0.0 & $\approx$ & $0.72 \pm 0.10$ & 2 & $0.78 \pm 0.10$ & 0.0 & $0.31 \pm 0.06$ \\
\hline \multirow[t]{10}{*}{ GW-1 (C) } & YES & 0.0 & 0.0 & 0.0 & $1.17 \pm 0.35$ & $0.03 \pm 0.20$ & $0.40 \pm 0.22$ & $0.05 \pm 0.46$ \\
\hline & YAES & $90.7 \pm 0.52$ & $52.6 \pm 0.97$ & $29.9 \pm 0.96$ & $73.0 \pm 0.43$ & $18.2 \pm 1.75$ & $61.6 \pm 1.65$ & $13.7 \pm 2.07$ \\
\hline & YAS & $0.43 \pm 1.44$ & $1.28 \pm 0.57$ & 0.0 & $3.45 \pm 0.69$ & 0.0 & $6.15 \pm 0.46$ & 0.0 \\
\hline & YAAS & 0.0 & $18.2 \pm 1.84$ & $28.8 \pm 2.09$ & $17.9 \pm 0.86$ & $29.9 \pm 1.24$ & $9.40 \pm 2.61$ & $27.5 \pm 2.51$ \\
\hline & YDS & 0.0 & $4.09 \pm 0.91$ & $0.91 \pm 0.69$ & $2.75 \pm 0.30$ & $0.72 \pm 1.08$ & $4.22 \pm 0.65$ & $1.83 \pm 0.23$ \\
\hline & RAR & 0.0 & 0.0 & $0.83 \pm 0.20$ & 0.0 & $0.25 \pm 0.16$ & 0.0 & $0.46 \pm 0.23$ \\
\hline & RXR & 0.0 & 0.0 & 0.0 & 0.0 & 0.0 & 0.0 & 0.0 \\
\hline & VDR & 0.0 & 0.0 & $0.49 \pm 0.12$ & 0.0 & $0.37 \pm 0.06$ & 0.0 & $0.34 \pm 0.21$ \\
\hline & TR & 0.0 & 0.0 & $0.87 \pm 0.18$ & 0.0 & $0.47 \pm 0.11$ & 0.0 & $0.82 \pm 0.14$ \\
\hline & Umu & $0.84 \pm 0.04$ & $1.03 \pm 0.02$ & $1.11 \pm 0.03$ & $1.05 \pm 0.01$ & $1.04 \pm 0.02$ & $\approx$ & $1.02 \pm 0.01$ \\
\hline
\end{tabular}

Table S9: Minimum and maximum of endocrine activity ( $\%$; mean \pm SEM) and genotoxicity (umu: induction rate, mean \pm SEM) of selected in vitro bioassays of aqueous samples and 10-fold concentrated SPE extracts, n.a.: not analysed. Umu: potential genotoxicity if induction rate is $\geq 1.5$. Corresponding samples were taken on the same sampling dates in the middle of July (C) and at the end of July (D) 2012 and in January (F) 2013.

\begin{tabular}{|c|c|c|c|c|c|c|c|c|c|c|}
\hline \multirow{3}{*}{ sample } & \multicolumn{10}{|c|}{ in vitro bioassay } \\
\hline & \multicolumn{2}{|c|}{ YES } & \multicolumn{2}{|c|}{ YAES } & \multicolumn{2}{|c|}{ YAAS } & \multicolumn{2}{|c|}{ RAR } & \multicolumn{2}{|c|}{ Umu } \\
\hline & aqueous & extract & aqueous & extract & aqueous & extract & aqueous & extract & aqueous & extract \\
\hline $\operatorname{HOS}(\mathrm{C})$ & 0.0 & $\begin{array}{c}0.0- \\
0.35 \pm 0.26 \\
(\mathrm{n}=2)\end{array}$ & $\begin{array}{c}87.8 \pm \\
0.68\end{array}$ & $\begin{array}{c}66.6 \pm 1.41- \\
89.1 \pm 0.74 \\
(\mathrm{n}=2)\end{array}$ & $\begin{array}{c}99.7 \pm \\
1.07\end{array}$ & $\begin{array}{c}38.9 \pm 5.40- \\
39.4 \pm 4.95 \\
(\mathrm{n}=2)\end{array}$ & $\begin{array}{c}93.3 \pm \\
1.18\end{array}$ & $\begin{array}{c}13.8 \pm 0.65- \\
91.3 \pm 1.39 \\
(\mathrm{n}=5)\end{array}$ & $1.29 \pm 0.12$ & $\begin{array}{c}1.01 \pm 0.04- \\
4.37 \pm 0.19 \\
(\mathrm{n}=3)\end{array}$ \\
\hline INF-1 (C) & 0.0 & $\begin{array}{c}0.0 \\
(\mathrm{n}=2)\end{array}$ & $\begin{array}{c}61.3 \pm \\
1.03\end{array}$ & $\begin{array}{c}80.0 \pm 2.44- \\
84.3 \pm 2.75 \\
(\mathrm{n}=2)\end{array}$ & $\begin{array}{c}3.31 \pm \\
3.46\end{array}$ & $\begin{array}{c}43.7 \pm 4.31- \\
45.0 \pm 2.18 \\
(\mathrm{n}=2)\end{array}$ & $\begin{array}{c}23.2 \pm \\
0.27\end{array}$ & $\begin{array}{c}0.0- \\
53.5 \pm 0.97 \\
(\mathrm{n}=6)\end{array}$ & $0.77 \pm 0.04$ & $\begin{array}{c}1.06 \pm 0.02- \\
1.34 \pm 1.34 \\
(\mathrm{n}=4)\end{array}$ \\
\hline EFF-1 (C) & $12.7 \pm 1.98$ & $\begin{array}{c}0.0- \\
7.60 \pm 0.41 \\
(\mathrm{n}=6)\end{array}$ & $\begin{array}{c}20.9 \pm \\
2.32\end{array}$ & $\begin{array}{c}12.6 \pm 3.22- \\
55.0 \pm 0.49 \\
(\mathrm{n}=6)\end{array}$ & 0.0 & $\begin{array}{c}24.1 \pm 2.84- \\
70.8 \pm 1.37 \\
(\mathrm{n}=5)\end{array}$ & $\begin{array}{c}1.23 \pm \\
0.27\end{array}$ & $\begin{array}{c}0.0- \\
0.83 \pm 0.20 \\
(\mathrm{n}=6)\end{array}$ & $0.83 \pm 0.06$ & $\begin{array}{c}1.10 \pm 0.04- \\
1.62 \pm 0.07 \\
(\mathrm{n}=4)\end{array}$ \\
\hline EFF-4 (D) & 0.0 & $\begin{array}{c}0.0- \\
5.91 \pm 0.38 \\
(\mathrm{n}=6)\end{array}$ & $\begin{array}{c}45.1 \pm \\
0.81\end{array}$ & $\begin{array}{c}48.6 \pm 1.83- \\
79.8 \pm 0.77 \\
(\mathrm{n}=6)\end{array}$ & 0.0 & $\begin{array}{c}38.2 \pm 2.69- \\
88.9 \pm 1.25 \\
(\mathrm{n}=6)\end{array}$ & $\begin{array}{c}0.40 \pm \\
0.16\end{array}$ & $\begin{array}{c}0.0- \\
2.86 \pm 0.53 \\
(\mathrm{n}=6)\end{array}$ & $0.89 \pm 0.04$ & $\begin{array}{c}1.25 \pm 0.07- \\
1.87 \pm 0.03 \\
(\mathrm{n}=6)\end{array}$ \\
\hline $\begin{array}{l}\text { EFF-4-MS } \\
\text { (D) }\end{array}$ & 0.0 & $\begin{array}{c}0.78 \pm 0.33- \\
4.77 \pm 0.37 \\
(\mathrm{n}=5)\end{array}$ & $\begin{array}{l}80.8 \pm \\
0.53\end{array}$ & $\begin{array}{c}31.9 \pm 2.34- \\
76.3 \pm 1.46 \\
(\mathrm{n}=5)\end{array}$ & $\begin{array}{c}5.24 \pm \\
4.52\end{array}$ & $\begin{array}{c}23.0 \pm 1.56- \\
68.0 \pm 2.80 \\
(\mathrm{n}=5)\end{array}$ & $\begin{array}{c}0.65 \pm \\
0.14\end{array}$ & $\begin{array}{c}0.0- \\
3.59 \pm 0.50 \\
(\mathrm{n}=6)\end{array}$ & $0.91 \pm 0.05$ & $\begin{array}{c}1.35 \pm 0.04- \\
1.73 \pm 0.07 \\
(\mathrm{n}=5)\end{array}$ \\
\hline $\begin{array}{l}\text { EFF-4-MS } \\
\text { (F) }\end{array}$ & $2.39 \pm 1.16$ & $\begin{array}{c}0.0- \\
4.77 \pm 0.12 \\
(\mathrm{n}=4)\end{array}$ & $\begin{array}{l}44.2 \pm \\
4.45\end{array}$ & $\begin{array}{c}20.8 \pm 0.83- \\
75.1 \pm 1.17 \\
(\mathrm{n}=4)\end{array}$ & 0.0 & $\begin{array}{c}40.4 \pm 2.21- \\
64.6 \pm 0.69 \\
(n=3)\end{array}$ & 0.0 & $\begin{array}{c}0.0- \\
6.71 \pm 0.12 \\
(\mathrm{n}=6)\end{array}$ & n.a. & n.a. \\
\hline $\begin{array}{c}\text { EFF-4-MS- } \\
\mathrm{O}_{3}(\mathrm{~F})\end{array}$ & $0.04 \pm 0.35$ & $\begin{array}{c}0.0 \\
(n=4)\end{array}$ & $\begin{array}{l}73.9 \pm \\
1.13\end{array}$ & $\begin{array}{c}34.7 \pm 0.60- \\
76.5 \pm 0.86 \\
(\mathrm{n}=4)\end{array}$ & 0.0 & $\begin{array}{c}37.3 \pm 2.62- \\
51.4 \pm 1.05 \\
(\mathrm{n}=3)\end{array}$ & $\begin{array}{c}0.46 \pm \\
0.31\end{array}$ & $\begin{array}{c}0.0- \\
2.26 \pm 0.25 \\
(\mathrm{n}=5)\end{array}$ & n.a. & n.a. \\
\hline GW-1 (C) & 0.0 & $\begin{array}{c}0.0- \\
1.17 \pm 0.35 \\
(\mathrm{n}=6)\end{array}$ & $\begin{array}{l}90.7 \pm \\
0.52\end{array}$ & $\begin{array}{c}13.7 \pm 2.07- \\
73.0 \pm 0.43 \\
(\mathrm{n}=6)\end{array}$ & 0.0 & $\begin{array}{c}9.40 \pm 2.61- \\
29.9 \pm 1.24 \\
(\mathrm{n}=6)\end{array}$ & 0.0 & $\begin{array}{c}0.0- \\
0.83 \pm 0.20 \\
(\mathrm{n}=6)\end{array}$ & $0.84 \pm 0.04$ & $\begin{array}{c}1.02 \pm 0.01- \\
1.11 \pm 0.03 \\
(\mathrm{n}=5)\end{array}$ \\
\hline
\end{tabular}


Table S10: Pooled data ((waste)water type and SPE-extracts) of endocrine activity ( $\%$; mean \pm SEM) and genotoxicity (umu: induction rate, mean \pm SEM) of aqueous samples and 10-fold concentrated SPE extracts, n.a.: not analysed. Umu: potential genotoxicity if induction rate is $\geq 1.5$. Corresponding samples were taken on the same sampling dates in the middle of July (C) and at the end of July (D) 2012 and in January (F) 2013.

\begin{tabular}{|c|c|c|c|c|c|c|c|c|c|c|}
\hline \multirow{3}{*}{ sample } & \multicolumn{10}{|c|}{ in vitro bioassay } \\
\hline & \multicolumn{2}{|c|}{ YES } & \multicolumn{2}{|c|}{ YAES } & \multicolumn{2}{|c|}{ YAS } & \multicolumn{2}{|c|}{ YAAS } & \multicolumn{2}{|c|}{ YDS } \\
\hline & aqueous & extract & aqueous & extract & aqueous & extract & aqueous & extract & aqueous & extract \\
\hline $\begin{array}{l}\operatorname{HOS}(\mathrm{C}) / \\
\text { INF-1 (C) }\end{array}$ & $\begin{array}{c}0.0 \\
(\mathrm{n}=2)\end{array}$ & $\begin{array}{c}0.09 \pm 0.09 \\
(\mathrm{n}=4)\end{array}$ & $\begin{array}{c}74.6 \pm 13.3 \\
(\mathrm{n}=2)\end{array}$ & $\begin{array}{c}80.0 \pm 4.84 \\
(\mathrm{n}=4)\end{array}$ & $\begin{array}{c}4.01 \pm 1.27 \\
(\mathrm{n}=2)\end{array}$ & $\begin{array}{c}3.87 \pm 1.45 \\
(\mathrm{n}=4)\end{array}$ & $\begin{array}{c}51.5 \pm 48.2 \\
(\mathrm{n}=2)\end{array}$ & $\begin{array}{c}41.8 \pm 1.53 \\
(\mathrm{n}=4)\end{array}$ & $\begin{array}{c}0.0 \\
(n=2)\end{array}$ & $\begin{array}{c}12.6 \pm 5.67 \\
(n=4)\end{array}$ \\
\hline $\begin{array}{c}\text { EFF-1 (C) / } \\
\text { EFF-4 (D) / } \\
\text { EFF-4-MS } \\
\text { (D and F) }\end{array}$ & $\begin{aligned} 3.77 & \pm 3.03 \\
(n & =4)\end{aligned}$ & $\begin{array}{c}2.70 \pm 0.51 \\
(\mathrm{n}=21)\end{array}$ & $\begin{aligned} & 47.8 \pm 12.4 \\
&(n=4)\end{aligned}$ & $\begin{array}{c}47.6 \pm 4.50 \\
(\mathrm{n}=21)\end{array}$ & $\begin{array}{c}0.01 \pm 0.01 \\
(\mathrm{n}=4)\end{array}$ & $\begin{array}{c}0.79 \pm 0.26 \\
(\mathrm{n}=19)\end{array}$ & $\begin{aligned} & 1.31 \pm 1.31 \\
&(\mathrm{n}=4)\end{aligned}$ & $\begin{array}{c}54.3 \pm 4.29 \\
(\mathrm{n}=19)\end{array}$ & $\begin{array}{c}4.73 \pm 4.73 \\
(n=4)\end{array}$ & $\begin{array}{c}12.4 \pm 2.28 \\
(\mathrm{n}=21)\end{array}$ \\
\hline $\begin{array}{c}\text { EFF-4-MS- } \\
\mathrm{O}_{3}(\mathrm{~F})\end{array}$ & $\begin{array}{c}0.04 \\
(n=1)\end{array}$ & $\begin{array}{c}0.0 \\
(n=4)\end{array}$ & $\begin{array}{c}73.9 \\
(\mathrm{n}=1)\end{array}$ & $\begin{aligned} 53.5 & \pm 8.70 \\
(\mathrm{n} & =4)\end{aligned}$ & $\begin{array}{c}0.0 \\
(\mathrm{n}=1)\end{array}$ & $\begin{array}{c}0.12 \pm 0.08 \\
(\mathrm{n}=3)\end{array}$ & $\begin{array}{c}0.0 \\
(\mathrm{n}=1)\end{array}$ & $\begin{aligned} & 44.4 \pm 4.07 \\
&(\mathrm{n}=3)\end{aligned}$ & $\begin{array}{c}0.0 \\
(\mathrm{n}=1)\end{array}$ & $\begin{aligned} & 2.64 \pm 1.35 \\
&(\mathrm{n}=3)\end{aligned}$ \\
\hline \multirow[t]{3}{*}{ GW-1 (C) } & $\begin{array}{c}0.0 \\
(\mathrm{n}=1)\end{array}$ & $\begin{array}{c}0.28 \pm 0.19 \\
(\mathrm{n}=6)\end{array}$ & $\begin{array}{c}90.7 \\
(n=1)\end{array}$ & $\begin{aligned} 41.5 & \pm 9.95 \\
(\mathrm{n} & =6)\end{aligned}$ & $\begin{array}{c}0.43 \\
(\mathrm{n}=1)\end{array}$ & $\begin{array}{c}1.81 \pm 1.03 \\
(\mathrm{n}=6)\end{array}$ & $\begin{array}{c}0.0 \\
(\mathrm{n}=1)\end{array}$ & $\begin{aligned} & 22.0 \pm 3.31 \\
&(\mathrm{n}=6)\end{aligned}$ & $\begin{array}{c}0.0 \\
(\mathrm{n}=1)\end{array}$ & $\begin{aligned} & 2.42 \pm 0.62 \\
&(\mathrm{n}=6)\end{aligned}$ \\
\hline & \multicolumn{2}{|c|}{ RAR } & \multicolumn{2}{|c|}{ RXR } & \multicolumn{2}{|c|}{ VDR } & \multicolumn{2}{|c|}{ TR } & \multicolumn{2}{|c|}{ Umu } \\
\hline & aqueous & extract & aqueous & extract & aqueous & extract & aqueous & extract & aqueous & extract \\
\hline $\begin{array}{l}\operatorname{HOS}(\mathrm{C}) / \\
\text { INF-1 (C) }\end{array}$ & $\begin{aligned} 58.3 & \pm 35.1 \\
(\mathrm{n} & =2)\end{aligned}$ & $\begin{array}{c}47.1 \pm 9.64 \\
(\mathrm{n}=11)\end{array}$ & $\begin{array}{c}0.0 \\
(\mathrm{n}=2)\end{array}$ & $\begin{array}{c}0.28 \pm 0.28 \\
(\mathrm{n}=10)\end{array}$ & $\begin{array}{c}0.0 \\
(\mathrm{n}=2)\end{array}$ & $\begin{aligned} 0.22 & \pm 0.15 \\
(\mathrm{n} & =9)\end{aligned}$ & $\begin{array}{c}0.0 \\
(\mathrm{n}=2)\end{array}$ & $\begin{array}{c}0.07 \pm 0.07 \\
(\mathrm{n}=10)\end{array}$ & $\begin{array}{c}1.03 \pm 0.26 \\
(\mathrm{n}=2)\end{array}$ & $\begin{aligned} 1.63 & \pm 0.46 \\
(\mathrm{n} & =7)\end{aligned}$ \\
\hline $\begin{array}{l}\text { EFF-1 (C) / } \\
\text { EFF-4 (D) / } \\
\text { EFF-4-MS } \\
(\mathrm{D} \text { and F) }\end{array}$ & $\begin{aligned} 0.57 & \pm 0.26 \\
(\mathrm{n} & =4)\end{aligned}$ & $\begin{array}{c}1.54 \pm 0.38 \\
(\mathrm{n}=24)\end{array}$ & $\begin{array}{c}0.0 \\
(n=4)\end{array}$ & $\begin{array}{c}1.64 \pm 0.64 \\
(\mathrm{n}=24)\end{array}$ & $\begin{aligned} & 0.16 \pm 0.16 \\
&(\mathrm{n}=4)\end{aligned}$ & $\begin{array}{c}0.56 \pm 0.14 \\
(\mathrm{n}=24)\end{array}$ & $\begin{aligned} 0.24 & \pm 0.24 \\
(\mathrm{n} & =4)\end{aligned}$ & $\begin{array}{c}0.34 \pm 0.13 \\
(\mathrm{n}=24)\end{array}$ & $\begin{array}{c}0.88 \pm 0.02 \\
(\mathrm{n}=3)\end{array}$ & $\begin{array}{c}1.50 \pm 0.06 \\
(\mathrm{n}=15)\end{array}$ \\
\hline $\begin{array}{c}\text { EFF-4-MS- } \\
\mathrm{O}_{3}(\mathrm{~F})\end{array}$ & $\begin{array}{c}0.46 \\
(\mathrm{n}=1)\end{array}$ & $\begin{array}{c}1.01 \pm 0.45 \\
(\mathrm{n}=5)\end{array}$ & $\begin{array}{c}0.0 \\
(\mathrm{n}=1)\end{array}$ & $\begin{array}{c}6.48 \pm 2.29 \\
(\mathrm{n}=4)\end{array}$ & $\begin{array}{c}0.0 \\
(\mathrm{n}=1)\end{array}$ & $\begin{array}{c}1.13 \pm 0.47 \\
(\mathrm{n}=5)\end{array}$ & $\begin{array}{c}0.0 \\
(\mathrm{n}=1)\end{array}$ & $\begin{aligned} 0.45 & \pm 0.18 \\
(\mathrm{n} & =4)\end{aligned}$ & n.a. & n.a. \\
\hline GW-1 (C) & $\begin{array}{c}0.0 \\
(n=1)\end{array}$ & $\begin{array}{c}0.26 \pm 0.14 \\
(\mathrm{n}=6)\end{array}$ & $\begin{array}{c}0.0 \\
(n=1)\end{array}$ & $\begin{array}{c}0.0 \\
(\mathrm{n}=6)\end{array}$ & $\begin{array}{c}0.0 \\
(\mathrm{n}=1)\end{array}$ & $\begin{array}{c}0.2 \pm 0.09 \\
(\mathrm{n}=6)\end{array}$ & $\begin{array}{c}0.0 \\
(n=1)\end{array}$ & $\begin{array}{c}0.36 \pm 0.17 \\
(\mathrm{n}=6)\end{array}$ & $\begin{array}{c}0.84 \\
(\mathrm{n}=1)\end{array}$ & $\begin{array}{c}1.05 \pm 0.02 \\
(\mathrm{n}=5)\end{array}$ \\
\hline
\end{tabular}



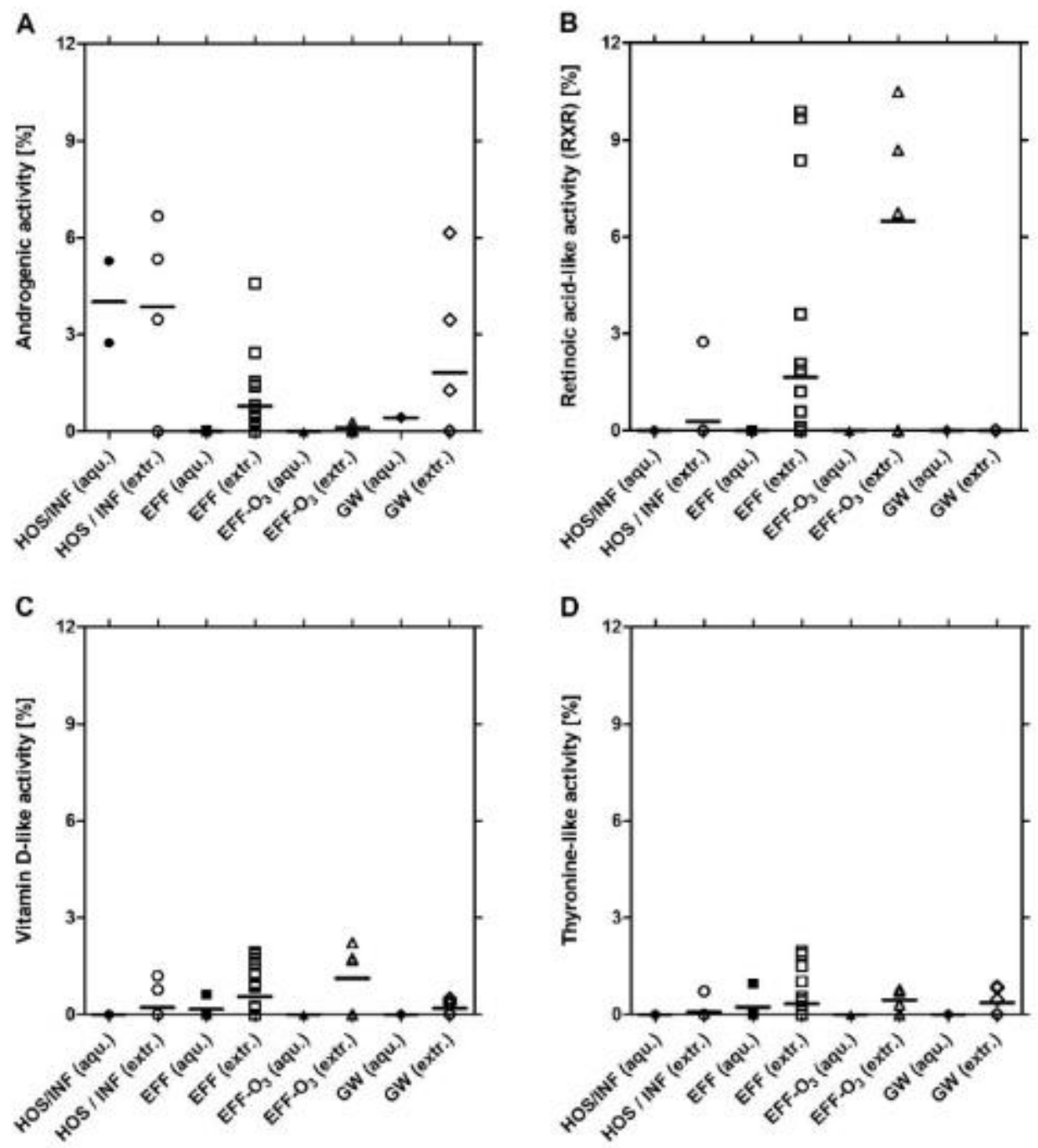

Figure S9: Androgenic (A), retinoid X-like (RXR, B), vitamin D-like (C) and thyronine-like (D) activity in $\%$ of the pooled data of aqueous (aqu.) water and wastewater samples and of the corresponding pooled 10-fold SPE extracts (extr). Symbols: activity of the individual sample, line: mean of all samples of one water type, filled symbol: aqueous sample, clear symbol: SPE extract, HOS: hospital effluent (untreated wastewater), INF: influent (untreated wastewater), EFF: effluent (conventionally treated wastewater), $\mathrm{EFF}-\mathrm{O}_{3}$ : ozonated conventionally treated wastewater, $\mathrm{GW}$ : groundwater. The corresponding samples were taken on the same sampling dates in July 2012 and January 2013. 
Table S11: Pooled data ((waste)water type) of endocrine activity (\%; mean \pm SEM) and genotoxicity (umu: induction rate, mean \pm SEM) of aqueous samples and 10-fold concentrated SPE extracts, \&: cytotoxic. Umu: potential genotoxicity if induction rate is $\geq 1.5$. Corresponding samples were taken on the same sampling date in the middle of July (C) 2012, the end of July (D) 2012 and in January (F) 2013

\begin{tabular}{|c|c|c|c|c|c|c|c|c|}
\hline \multirow[t]{2}{*}{ sample } & \multirow{2}{*}{$\begin{array}{l}\text { in vitro } \\
\text { bioassay }\end{array}$} & \multirow[t]{2}{*}{ aqueous } & \multicolumn{2}{|c|}{ Oasis HLB } & \multicolumn{2}{|c|}{ Telos C18/ENV } & \multicolumn{2}{|c|}{ Supelco ENVI-Carb+ } \\
\hline & & & $\mathrm{pH} 7$ & $\mathrm{pH} 2.5$ & $\mathrm{pH} 7$ & $\mathrm{pH} 2.5$ & $\mathrm{pH} 7$ & $\mathrm{pH} 2.5$ \\
\hline \multirow[t]{10}{*}{$\begin{array}{l}\operatorname{HOS}(\mathrm{C}) / \\
\text { INF-1 (C) }\end{array}$} & YES & $\begin{array}{c}0.0 \\
(\mathrm{n}=2)\end{array}$ & 2 & 2 & 2 & $\approx$ & $\begin{array}{c}0.0 \\
(\mathrm{n}=2)\end{array}$ & $\begin{array}{c}0.18 \pm 0.18 \\
(\mathrm{n}=2)\end{array}$ \\
\hline & YAES & $\begin{array}{c}74.6 \pm 13.3 \\
(\mathrm{n}=2)\end{array}$ & $\approx$ & $\approx$ & 2 & 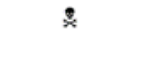 & $\begin{aligned} 86.7 & \pm 2.40 \\
(\mathrm{n} & =2)\end{aligned}$ & $\begin{aligned} 73.3 & \pm 6.70 \\
(\mathrm{n} & =2)\end{aligned}$ \\
\hline & YAS & $\begin{array}{c}4.01 \pm 1.27 \\
(n=2)\end{array}$ & $\approx$ & $\approx$ & $\approx$ & $\approx$ & $\begin{array}{c}2.67 \pm 2.67 \\
(\mathrm{n}=2)\end{array}$ & $\begin{array}{c}5.07 \pm 1.60 \\
(\mathrm{n}=2)\end{array}$ \\
\hline & YAAS & $\begin{array}{c}51.5 \pm 48.2 \\
(\mathrm{n}=2)\end{array}$ & 2 & 2 & $\approx$ & $\approx$ & $\begin{aligned} 41.3 & \pm 2.40 \\
(\mathrm{n} & =2)\end{aligned}$ & $\begin{aligned} 42.2 & \pm 2.80 \\
(\mathrm{n} & =2)\end{aligned}$ \\
\hline & YDS & $\begin{array}{c}0.0 \\
(n=2)\end{array}$ & 2 & 2 & 2 & 2 & $\begin{array}{c}22.0 \pm 4.10 \\
(\mathrm{n}=2)\end{array}$ & $\begin{array}{c}3.27 \pm 0.69 \\
(\mathrm{n}=2)\end{array}$ \\
\hline & RAR & $\begin{array}{c}58.3 \pm 35.1 \\
(\mathrm{n}=2)\end{array}$ & $\begin{array}{c}63.0 \pm 13.7 \\
(n=2)\end{array}$ & $\begin{array}{c}72.4 \pm 18.9 \\
(\mathrm{n}=2)\end{array}$ & $\begin{array}{c}50.6 \\
(n=1)\end{array}$ & $\begin{array}{c}66.8 \pm 24.3 \\
(\mathrm{n}=2)\end{array}$ & $\begin{array}{c}6.90 \pm 6.90 \\
(\mathrm{n}=2)\end{array}$ & $\begin{aligned} 24.7 & \pm 23.1 \\
(\mathrm{n} & =2)\end{aligned}$ \\
\hline & RXR & $\begin{array}{c}0.0 \\
(n=2)\end{array}$ & $\begin{array}{c}1.38 \pm 1.38 \\
(n=2)\end{array}$ & $\begin{array}{c}0.0 \\
(\mathrm{n}=1)\end{array}$ & $\begin{array}{c}0.0 \\
(n=1)\end{array}$ & $\begin{array}{c}0.0 \\
(\mathrm{n}=2)\end{array}$ & $\begin{array}{c}0.0 \\
(\mathrm{n}=2)\end{array}$ & $\begin{array}{c}0.0 \\
(\mathrm{n}=2)\end{array}$ \\
\hline & VDR & $\begin{array}{c}0.0 \\
(\mathrm{n}=2)\end{array}$ & $\begin{array}{c}0.0 \\
(\mathrm{n}=1)\end{array}$ & $\begin{array}{c}0.39 \pm 0.39 \\
(\mathrm{n}=2)\end{array}$ & 2 & $\begin{aligned} 0.60 & \pm 0.60 \\
(\mathrm{n} & =2)\end{aligned}$ & $\begin{array}{c}0.0 \\
(\mathrm{n}=2)\end{array}$ & $\begin{array}{c}0.0 \\
(\mathrm{n}=2)\end{array}$ \\
\hline & TR & $\begin{array}{c}0.0 \\
(\mathrm{n}=2)\end{array}$ & $\begin{array}{c}0.0 \\
(\mathrm{n}=1)\end{array}$ & $\begin{array}{c}0.0 \\
(\mathrm{n}=2)\end{array}$ & $\begin{array}{c}0.0 \\
(n=1)\end{array}$ & $\begin{array}{c}0.0 \\
(\mathrm{n}=2)\end{array}$ & $\begin{array}{c}0.0 \\
(\mathrm{n}=2)\end{array}$ & $\begin{aligned} & 0.36 \pm 0.36 \\
&(\mathrm{n}=2)\end{aligned}$ \\
\hline & Umu & $\begin{array}{c}1.03 \pm 0.26 \\
(\mathrm{n}=2)\end{array}$ & 2 & $\begin{array}{c}1.34 \\
(\mathrm{n}=1)\end{array}$ & 2 & $\begin{array}{c}2.72 \pm 1.66 \\
(\mathrm{n}=2)\end{array}$ & $\begin{array}{c}1.26 \pm 0.04 \\
(\mathrm{n}=2)\end{array}$ & $\begin{array}{c}1.05 \pm 0.04 \\
(\mathrm{n}=2)\end{array}$ \\
\hline
\end{tabular}

Table S11 continued.

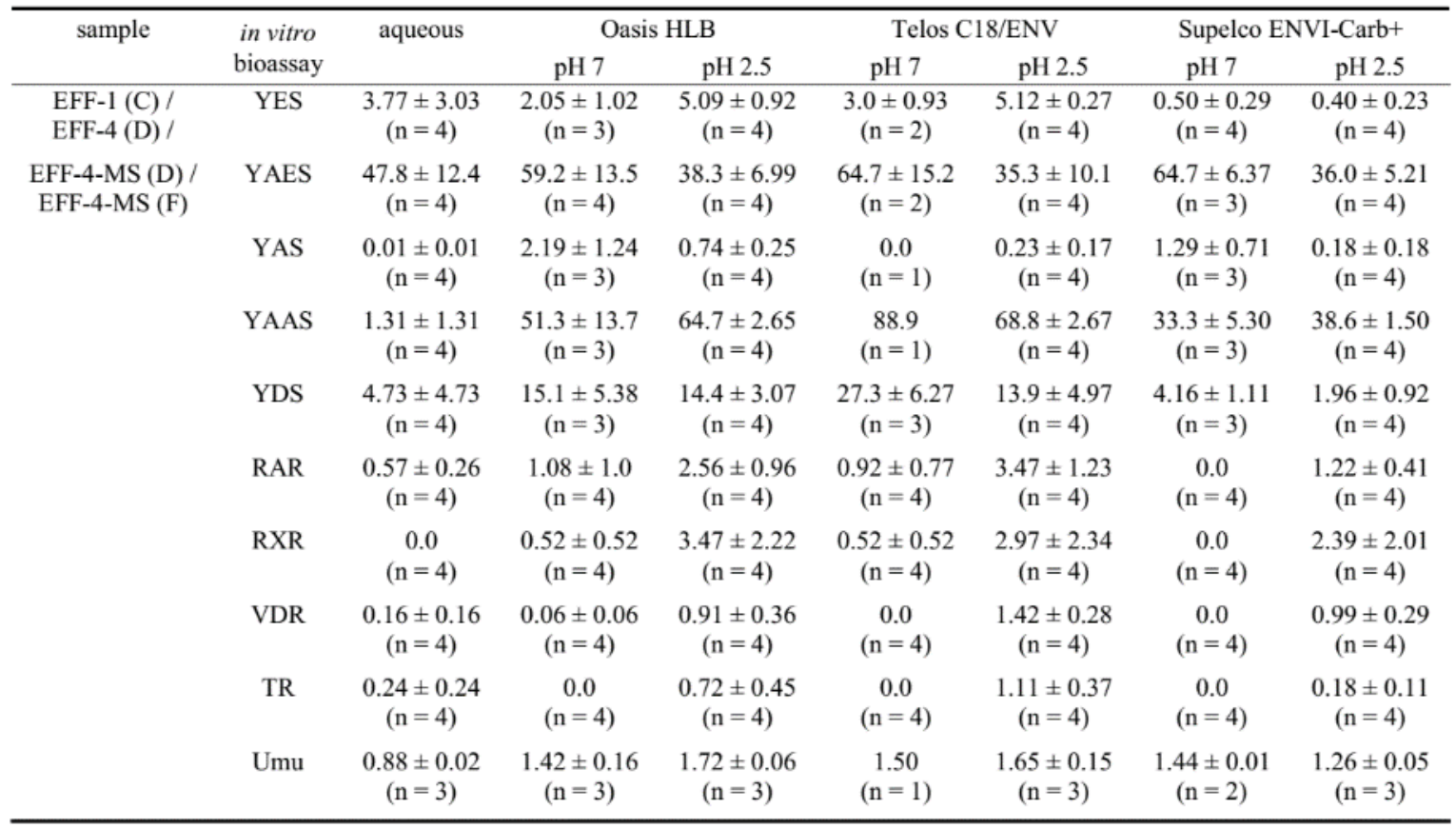



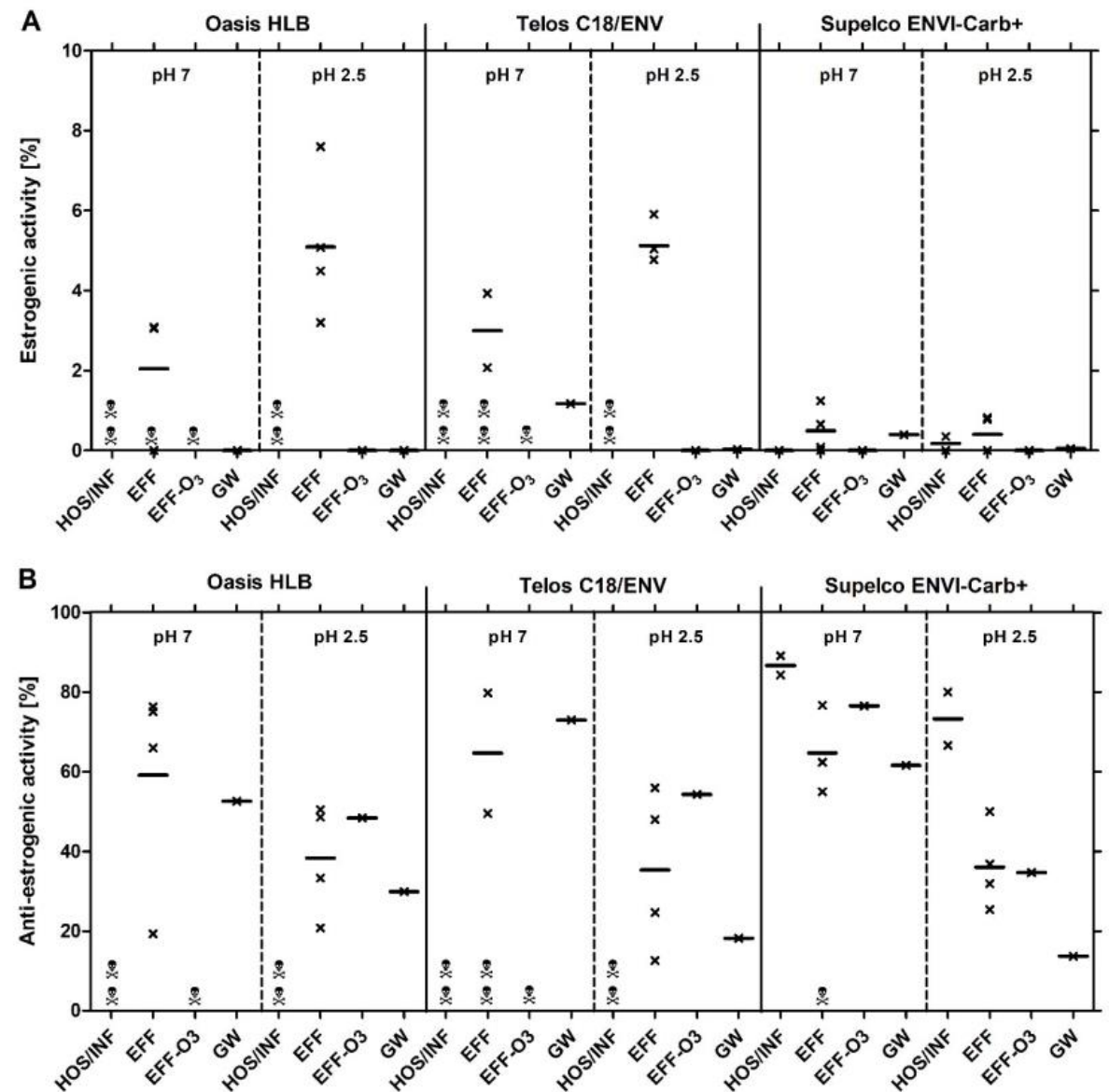

Figure S10: Estrogenic (A) and anti-estrogenic (B) activity in \% of SPE extracts of water and wastewater samples using three different SPE columns and two different $\mathrm{pH}$ values. The results were pooled from the different samples according to water type. Symbols: activity of the individual sample, line: mean of all samples of one water type, $\stackrel{\gtrless}{*}$ cytotoxic, HOS: hospital effluent (untreated wastewater), INF: influent (untreated wastewater), EFF: effluent (conventionally treated wastewater), $\mathrm{EFF}-\mathrm{O}_{3}$ : ozonated conventionally treated wastewater, $\mathrm{GW}$ : groundwater. 

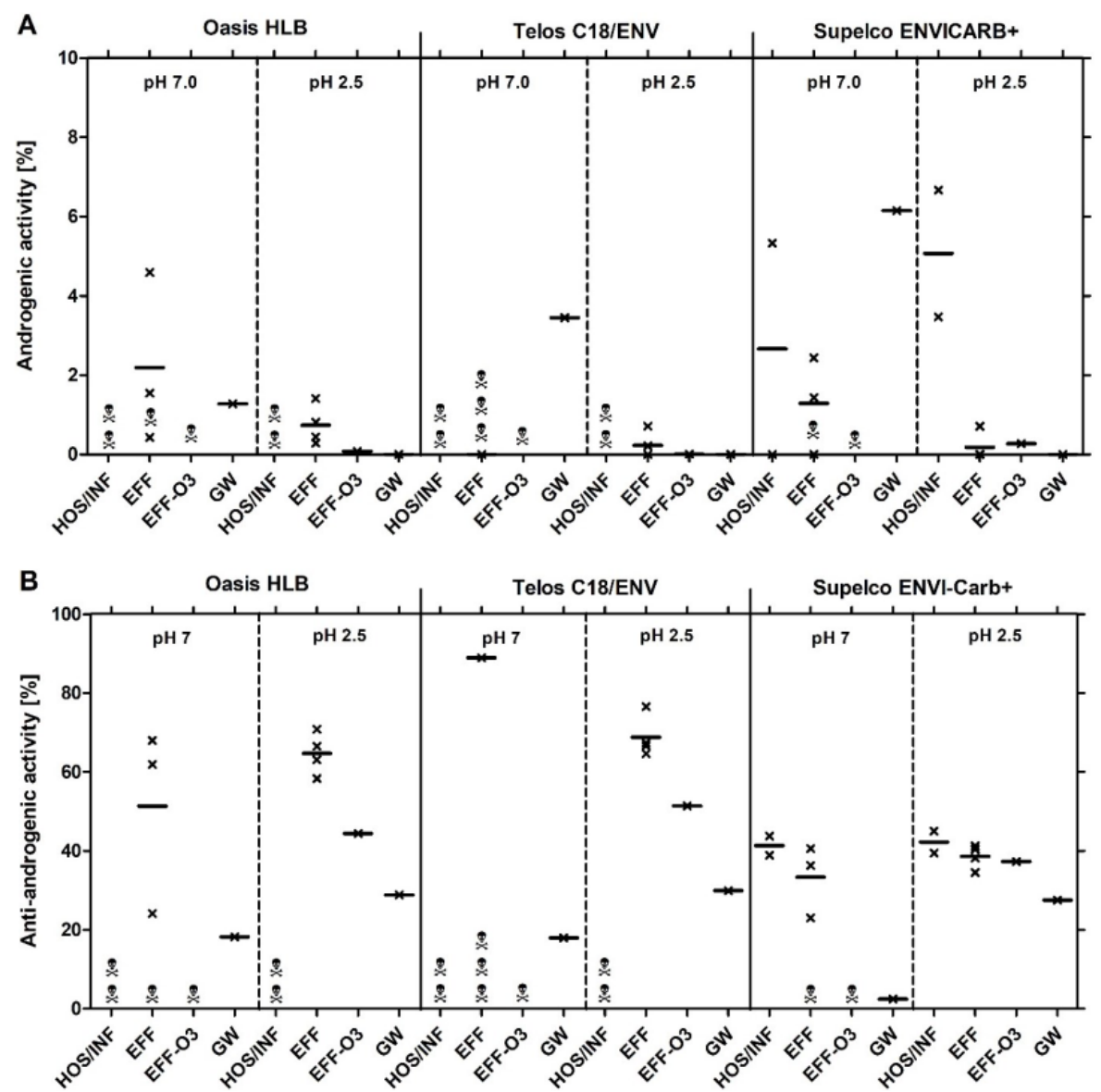

Figure S11: Androgenic (A) and anti-androgenic (B) activity in \% of SPE extracts of water and wastewater samples using three different SPE columns and two different $\mathrm{pH}$ values. The results were pooled from the different samples according to water type. Symbols: activity of the individual sample, line: mean of all samples of one water type, $\$:$ cytotoxic, HOS: hospital effluent (untreated wastewater), INF: influent (untreated wastewater), EFF: effluent (conventionally treated wastewater), $\mathrm{EFF}-\mathrm{O}_{3}$ : ozonated conventionally treated wastewater, $\mathrm{GW}$ : groundwater. 

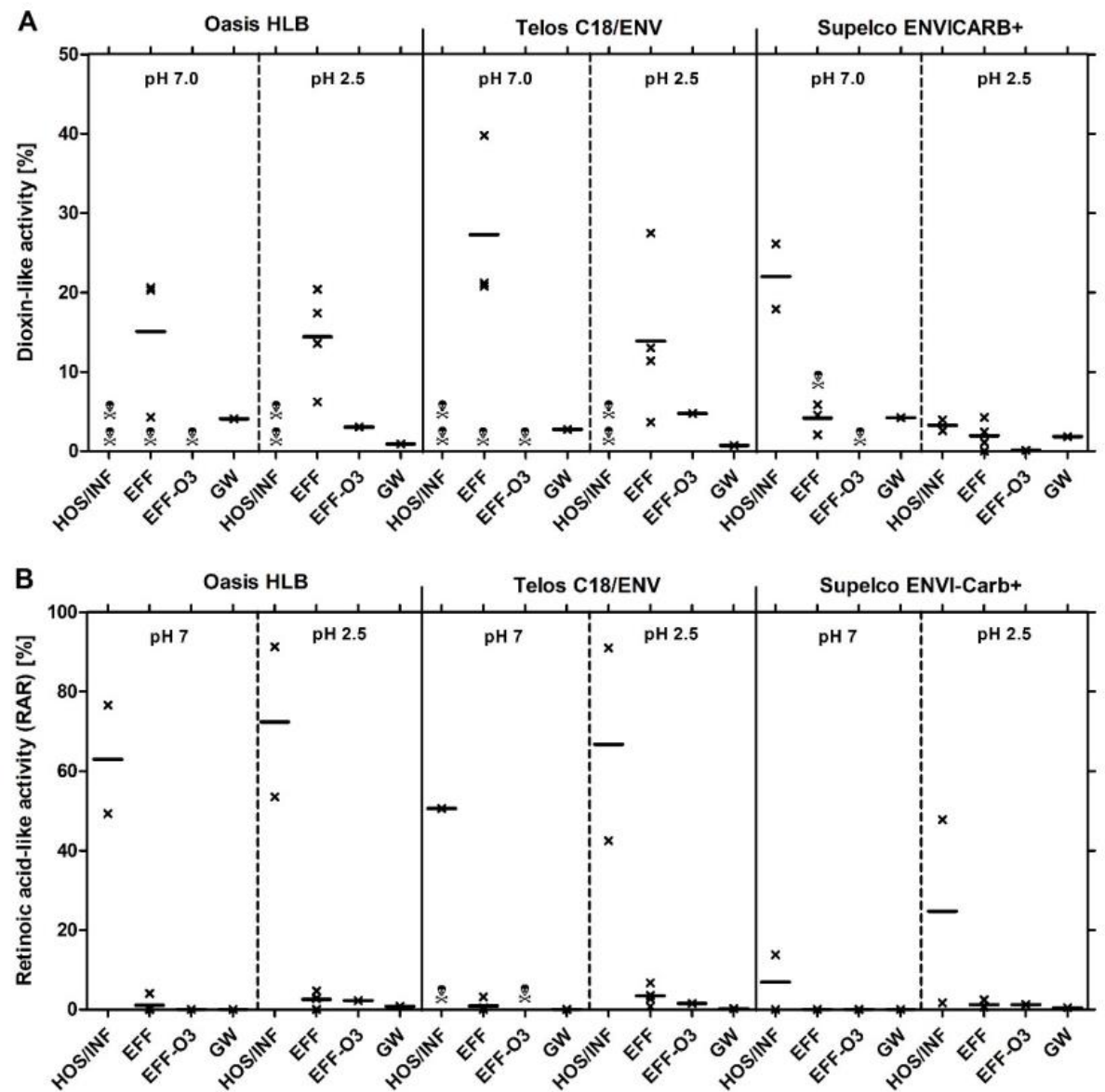

Figure S12: Dioxin-like (A) and retinoic acid-like (RAR, B) activity in \% of SPE extracts of water and wastewater samples using three different SPE columns and two different $\mathrm{pH}$ values. The results were pooled from the different samples according to water type. Symbols: activity of the individual sample, line: mean of all samples of one water type, $\$$ : cytotoxic, HOS: hospital effluent (untreated wastewater), INF: influent (untreated wastewater), EFF: effluent (conventionally treated wastewater), $\mathrm{EFF}-\mathrm{O}_{3}$ : ozonated conventionally treated wastewater, $\mathrm{GW}$ : groundwater. 

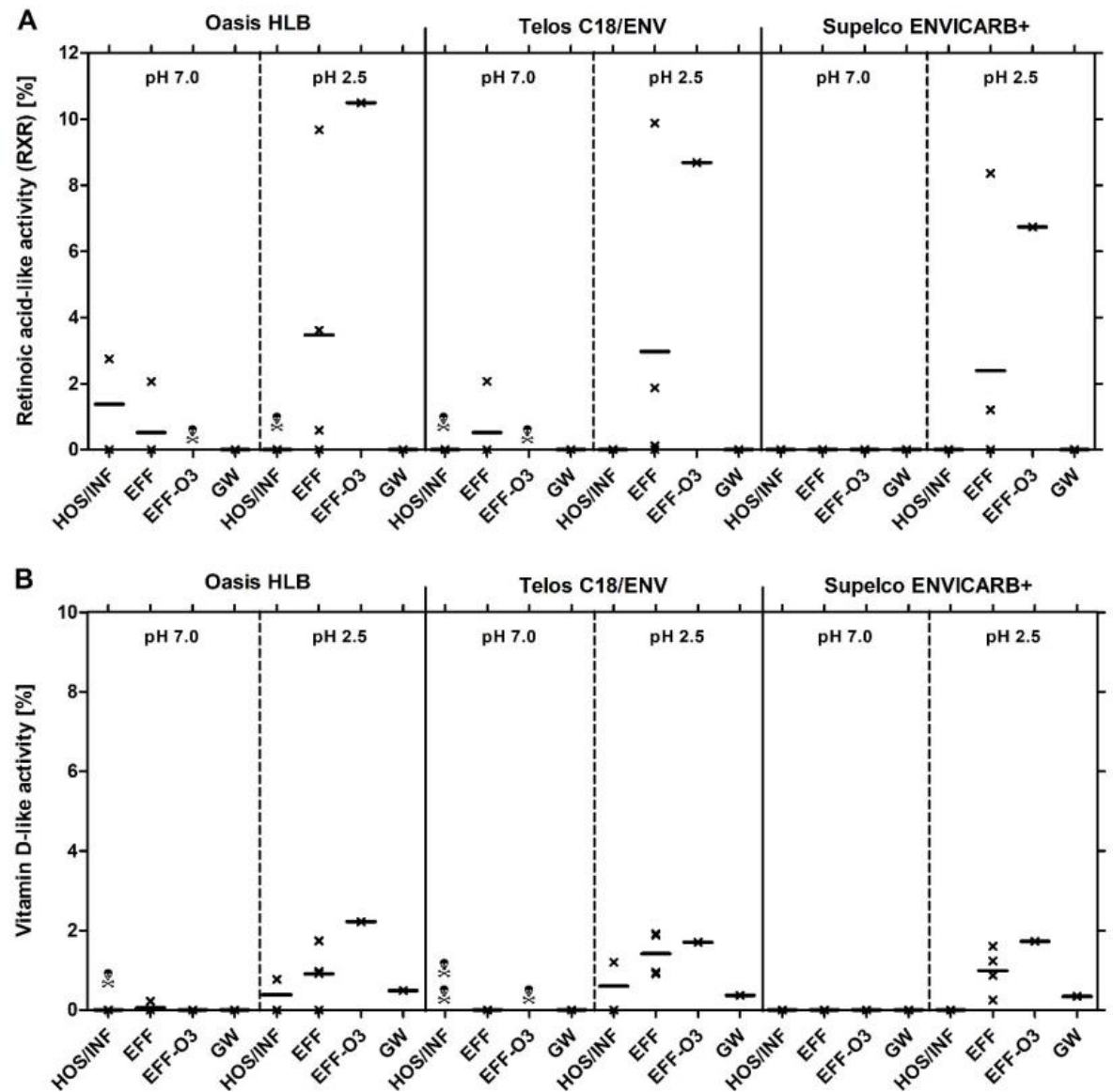

Figure S13: Retinoid X-like (RXR, A) and vitamin D-like (B) activity in \% of SPE extracts of water and wastewater samples using three different SPE columns and two different $\mathrm{pH}$ values. The results were pooled from the different samples according to water type. Symbols: activity of the individual sample, line: mean of all samples of one water type, $\$$ : cytotoxic, HOS: hospital effluent (untreated wastewater), INF: influent (untreated wastewater), EFF: effluent (conventionally treated wastewater), EFF-O ${ }_{3}$ : ozonated conventionally treated wastewater, GW: groundwater. 

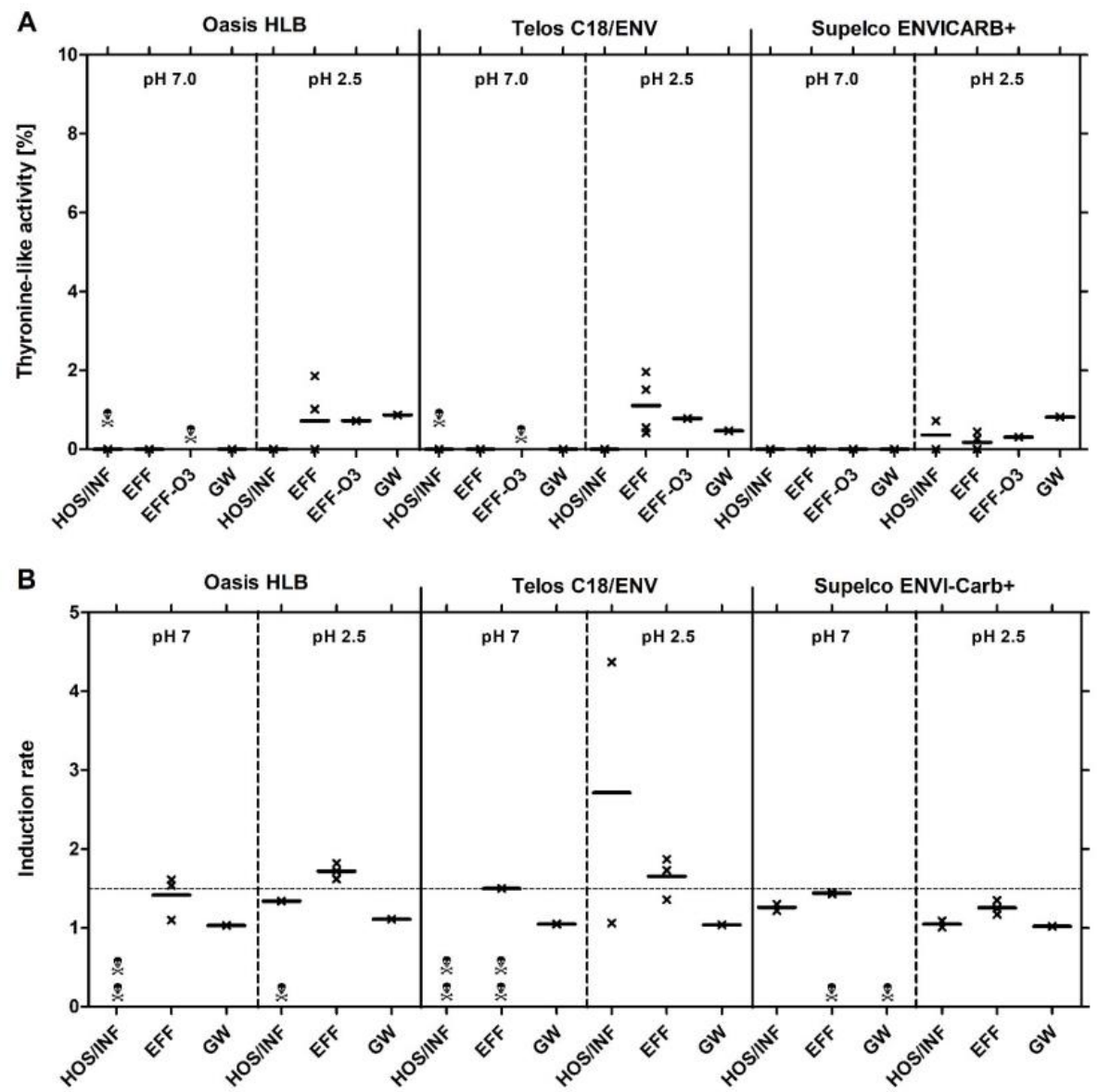

Figure S14: Thyronine-like (A) activity in \% and induction rate (umu, B) of SPE extracts of water and wastewater samples using three different SPE columns and two different $\mathrm{pH}$ values. The results were pooled from the different samples according to water type. Symbols: activity of the individual sample, line: mean of all samples of one water type, $\$$ : cytotoxic, umu: potential genotoxicity if induction rate is $\geq 1.5$, HOS: hospital effluent (untreated wastewater), INF: influent (untreated wastewater), EFF: effluent (conventionally treated wastewater), EFF-O ${ }_{3}$ : ozonated conventionally treated wastewater, GW: groundwater. 


\subsection{Pareto optimisation and ranking}

Table S12: Endocrine activity (\%) of conventionally treated wastewater (sample EFF-4 (D)) as aqueous sample and 10-fold concentrated SPE extracts from six different methods: three solid phase extraction (SPE) columns (Oasis HLB, Telos C18/ENV and Supelco ENVI-Carb+) were used at two $\mathrm{pH}$ values $(\mathrm{pH} 7$ and $\mathrm{pH} 2.5)$ and tested in five recombinant yeast screens (YES, YAES, YAS, YAAS and YDS).

\begin{tabular}{ccccccc|c}
\hline \multirow{2}{*}{ method } & \multicolumn{2}{c}{ Oasis HLB } & \multicolumn{2}{c}{ Telos C18/ENV } & \multicolumn{2}{c|}{ Supelco ENVI-Carb+ } & aqueous \\
bioassay & $\mathrm{pH} \mathrm{7.0}$ & $\mathrm{pH} \mathrm{2.5}$ & $\mathrm{pH} \mathrm{7.0}$ & $\mathrm{pH} \mathrm{2.5}$ & $\mathrm{pH} \mathrm{7.0}$ & $\mathrm{pH} \mathrm{2.5}$ & sample \\
\hline YES & 3.05 & 5.08 & 2.07 & 5.91 & 0.66 & 0.0 & 0.0 \\
YAES & 66.0 & 48.6 & 79.8 & 56.0 & 76.7 & 50.0 & 45.1 \\
YAS & 4.59 & 1.41 & 0.0 & 0.0 & 0.0 & 0.01 & 0.05 \\
YAAS & 61.9 & 66.5 & 88.9 & 76.6 & 40.6 & 38.2 & 0.0 \\
YDS & 20.3 & 13.6 & 21.2 & 13.0 & 4.56 & 1.12 & 0.0 \\
\hline
\end{tabular}

Table S13: Pareto ranking ( $1^{\text {st }}$ rank = "best", $6^{\text {th }}$ rank = "worst") of the six different SPE methods according to their effectivity in extracting different endocrine activities from conventionally treated wastewater (sample EFF-4 (D), Table S12). Oasis: Oasis HLB, Telos: Telos C18/ENV, Supelco: Supelco ENVI-Carb+, 7: $\mathrm{pH} 7,2.5: \mathrm{pH} 2.5$. In case of the YAS no $5^{\text {th }}$ and $6^{\text {th }}$ rank existed.

\begin{tabular}{lcccccc}
\hline & best & $2^{\text {nd }}$ & $3^{\text {rd }}$ & $4^{\text {th }}$ & $5^{\text {th }}$ & worst \\
bioassay & & & & & & \\
\hline YES & Telos 2.5 & Oasis 2.5 & Oasis 7 & Telos 7 & Supelco 7 & Supelco 2.5 \\
YAES & Telos 7 & Supelco 7 & Oasis 7 & Telos 2.5 & Supelco 2.5 & Oasis 2.5 \\
YAS & Oasis 7 & Oasis 2.5 & Supelco 2.5 & Telos 7 & - & - \\
& & & & Telos 2.5 & & \\
YAAS & Telos 7 & Telos 2.5 & Oasis 2.5 & Oasis 7 & Supelco 7 & Supelco 2.5 \\
YDS & Telos 7 & Oasis 7 & Oasis 2.5 & Telos 2.5 & Supelco 7 & Supelco 2.5 \\
\hline
\end{tabular}


Table S14: Pareto ranking (best, $2^{\text {nd }}-4^{\text {th }}$ best) of SPE methods of all water types according to their effectivity in extracting different types of water and wastewater samples with respect to the highest endocrine activities. Hospital wastewater (HOS) and WWTP influent (INF-1) was not ranked due to excessive cytotoxicity. Oasis: Oasis HLB, Telos: Telos C18/ENV, Supelco: Supelco ENVICarb+, 7: $\mathrm{pH} 7,2.5$ : $\mathrm{pH} 2.5$. Corresponding samples were taken on the same sampling dates in the middle of July (C) and at the end of July (D) 2012 and in January (F) 2013.

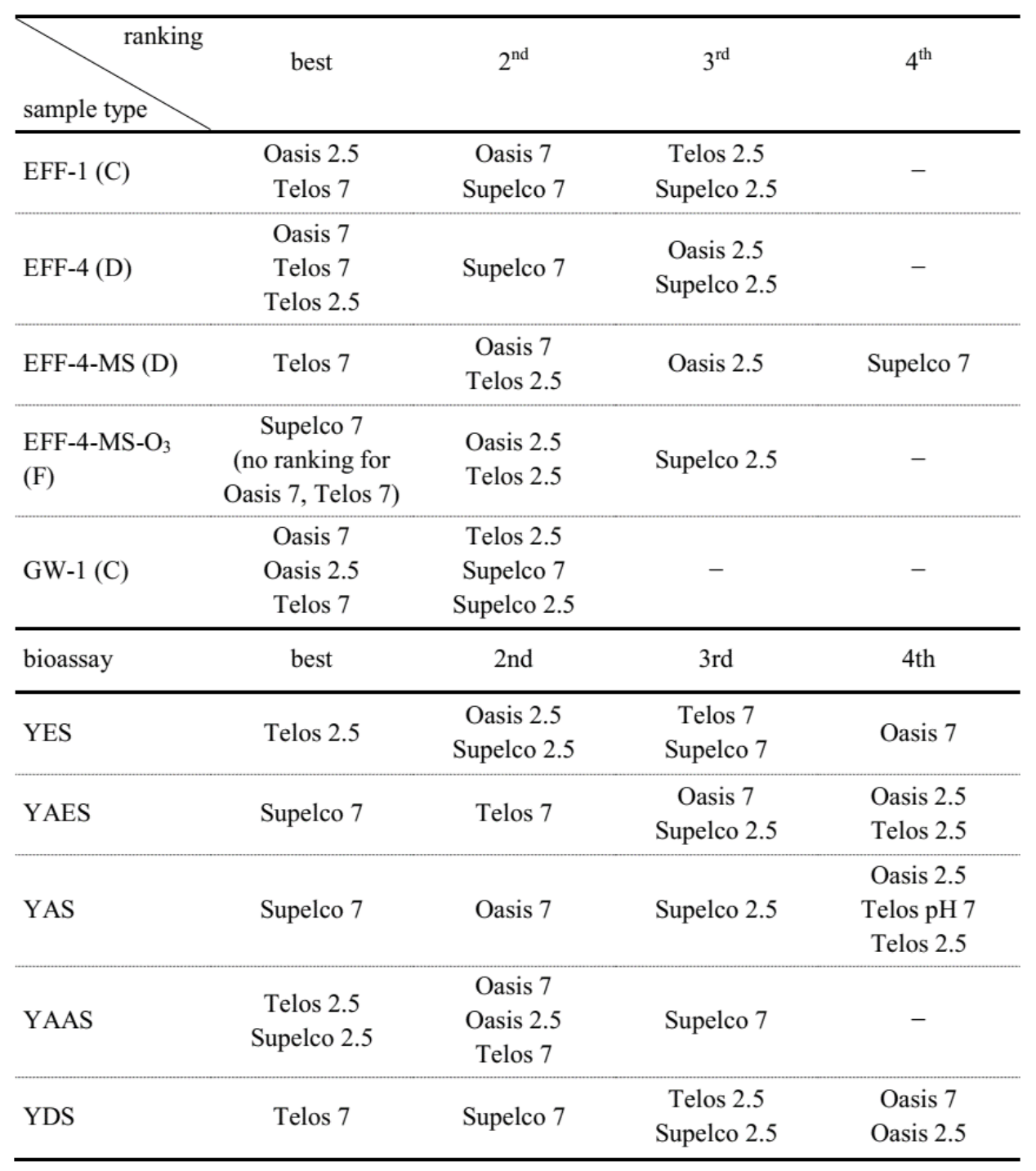


A.2 Ecotoxicological impacts of surface water and wastewater from conventional and advanced treatment technologies on brood size, larval length and cytochrome P450 (35A3) expression in Caenorhabditis elegans

Aennes Abbas, Lucie Valek, Ilona Schneider, Anna Bollmann, Gregor Knopp, Wolfram Seitz, Ulrike Schulte-Oehlmann, Jörg Oehlmann, Martin Wagner

Environmental Science and Pollution Research 25, 13868-13880

(First Online: 06 March 2018) 


\section{Declaration (paper A.2)}

Author contributions to the publication: "Ecotoxicological impacts of surface water and wastewater from conventional and advanced treatment technologies on brood size, larval length and cytochrome P450 (35A3) expression in Caenorhabditis elegans"

Status: accepted \& printed

Journal: Environmental Science and Pollution Research

Contributing authors (names and distinct initials):

- Aennes Abbas (AA)

- Lucie Valek (LV)

- Ilona Schneider (IS)

- Anna Bollmann (AB)

- Gregor Knopp (GK)

- Wolfram Seitz (WS)

- Ulrike Schulte-Oehlmann (US)

- Jörg Oehlmann (JO)

- Martin Wagner (MW)

Individual contributions of the first author and co-authors:

\section{Study conception and design:}

Doctoral candidate (AA): $85 \%$

Coauthors (LV, JO): $10 \%$

Coauthors (MW, US): $5 \%$

\section{Performance of experiments and assays}

Doctoral candidate (AA): $50 \%$

Coauthor (LV): $40 \%$

Coauthor (IS): $5 \%$

Coauthors (AB, WS): $5 \%$

\section{Compilation of data sets and tables/figures:}

Doctoral candidate (AA): $65 \%$

Coauthor (LV): $30 \%$

Coauthors (GK, AB, WS): $5 \%$

\section{Data analyses and interpretation:}

Doctoral candidate (AA): $75 \%$

Coauthors (LV, MW): $20 \%$

Coauthors (JO, IS): $5 \%$

\section{Drafting of the Manuscript:}

Doctoral candidate (AA): $80 \%$

Coauthors (MW, LV): 15\%

Coauthor (IS): $5 \%$ 


\section{Author accepted manuscript (paper A.2)}

This version of the article has been accepted for publication, after peer review (when applicable) and is subject to Springer Nature's AM terms of use (https://www.springernature.com/gp/open-research/policies/accepted-manuscriptterms), but is not the Version of Record and does not reflect post-acceptance improvements, or any corrections. The Version of Record is available online at: https://doi.org/10.1007/s11356-018-1605-2.

Ecotoxicological impacts of surface water and wastewater from conventional and advanced treatment technologies on brood size, larval length and cytochrome P450 (35A3) expression in Caenorhabditis elegans

Aennes Abbas ${ }^{1, *}$, Lucie Valek ${ }^{2}$, Ilona Schneider ${ }^{1}$, Anna Bollmann $^{3}$, Gregor Knopp ${ }^{4}$, Wolfram Seitz ${ }^{3}$, Ulrike Schulte-Oehlmann ${ }^{1}$, Jörg Oehlmann ${ }^{1}$, Martin Wagner ${ }^{1,5}$

${ }^{1}$ Department of Aquatic Ecotoxicology, Goethe Universität Frankfurt, Max-von-Laue-

Str. 13, D-60438 Frankfurt, Germany

2Department of Clinical Pharmacology, Goethe-University Hospital, Theodor Stern Kai

7, D-60590 Frankfurt, Germany

${ }^{3}$ Zweckverband Landeswasserversorgung, Spitziger Berg 1, 89129 Langenau,

Germany

${ }^{4}$ Department of Wastewater Technology and Water Reuse, Technische Universität

Darmstadt, Franziska-Braun-Str. 7, D-64287 Darmstadt, Germany

${ }^{5}$ Department of Biology, Norwegian University of Science and Technology,

Høgskoleringen 5, Realfagbygget, Trondheim, Norway

*corresponding author: abbas@bio.uni-frankfurt.de, +49 1776257026 (phone), +49 69

79842141 (fax)

Keywords: municipal effluents, contaminants of emerging concern, CYP biomarker, persistent organic pollutants (POPs), toxic effects, transformation products, in vivo bioassay, ozonation

Acknowledgments: This work was partly supported by the German Federal Ministry of Education and Research (BMBF) within the project TransRisk [02WRS1275A] which is gratefully appreciated. The authors further thank Ralph Menzel (Humboldt University Berlin), Wolfgang Ahlf (Technical University Hamburg-Harburg) and all TransRisk project partners for fruitful discussions and collaboration that greatly helped to improve this manuscript. We also thank the Caenorhabditis Genetics Center, funded by the National Institutes of Health Office of Research Infrastructure Programs [P40 OD010440] (USA), for supplying the Caenorhabditis elegans N2 and Escherichia coli OP50 strain. 


\section{Abstract}

32 Anthropogenic micropollutants and transformation products (TPs) negatively affect

33 aquatic ecosystems and water resources. Wastewater treatment plants (WWTP)

34 represent major point sources for (micro)pollutants and TP in urban water cycles.

35 The aim of the current study was to assess the removal of micropollutants and toxicity

36 during conventional and advanced wastewater treatment. Using wild type and transgenic

37 Caenorhabditis elegans the endpoints reproduction, growth and cytochrome P450 (CYP)

38 35A3 induction (via cyp-35A3::GFP) were assessed. Samples were collected at four

39 WWTPs and a receiving surface water. One WWTP included the advanced treatments:

40 ozonation followed by granular activated carbon (GAC) or biological filtration (BF),

41 respectively. Relevant micropollutants and WWTP parameters $(n=111)$ were included.

42 Significant reproductive toxicity was detected for one WWTP effluent (31-83\% reduced

43 brood size). Three of four effluents significantly promoted the growth of $C$. elegans larvae

44 (49-55\% increased lengths). This effect was also observed for the GAC (34-41\%) and

45 BF $(30 \%)$ post-treatments. Markedly, significant cyp-35A3::GFP induction was detected for one effluent before and after ozonation, being more pronounced for the ozonated

47 samples (5 and 7.4 fold above controls).

48 While the advanced treatments decreased the concentrations of most micropollutants, the observed effects may be attributed to effects of residual target compounds and/or compounds not included in the target chemical analysis. This highlights the need for an integrated assessment of (advanced) wastewater treatment covering both, biological and chemical parameters. 
54 The nematode Caenorhabditis elegans is one of the main model organisms in biology.

55 C. elegans has a versatile and well characterized physiology, with several biochemical

56 pathways conserved to those in humans (Leung et al. 2008). In addition, C. elegans

57 implies a short lifespan $(12-20 \mathrm{~d})$, fast reproductive cycle $\left(3 \mathrm{~d}\right.$ at $\left.20{ }^{\circ} \mathrm{C}\right)$ and facile

58 cultivation. Based on its ecological relevance (Félix and Braendle 2010), the widespread

59 particle feeder is increasingly used in ecotoxicology (Hägerbäumer et al. 2015; Leung et

60 al. 2008), comprising a wide range of methodologies as well as molecular, apical and

61 community endpoints (Wilson and Khakouli-Duarte 2009). Since the late 1990s mutant

62 and transgenic strains, which became readily available for $C$. elegans, have been utilized

63 in ecotoxicology (e.g., Peter et al. 1996). These strains contain gene knockouts, artificial

64 mutations such as causing hypersensitivity to certain xenobiotics and/or recombinant

65 reporter genes, such as green fluorescent protein (GFP), coupled to target genes of

66 ecotoxicological interest (e.g., Wilson and Khakouli-Duarte 2009; Xiong et al. 2017). The

67 cytochrome P450 (CYP) gene family counts more than 80 candidates in C. elegans.

68 CYPs fulfill essential cellular functions, such as phase I detoxification (Lindblom and Dodd

69 2006). In ecotoxicogenomics, gene expression profiling of CYPs thus became an

70 established biomarker (Reichert and Menzel 2005; Wilson and Khakouli-Duarte 2009).

71 Menzel et al. $(2001,2007)$ showed that exposure to xenobiotics induced the expression

72 of specific sets of CYPs. cyp-35A3 (human CYP2-like) investigated in this study is

73 induced by the polycyclic aromatic hydrocarbons (PAH) $\beta$-naphthoflavone ( $\beta$-NF), and

74 fluoranthene, the polychlorinated biphenyl (PCB) 2,2',5,5'-tetrachlorobiphenyl (PCB52),

75 the pharmaceuticals primaquine and lansoprazol (Menzel et al. 2001), benzene (Eom et 
al. 2014), the insecticides chlorpyrifos, diazinon (Roh et al. 2014) and imidacloprid, the

77 anthelmintic thiabendazole (Jones et al. 2013), the antimicrobials triclosan and trichlocarban (Inokuchi et al. 2014), as well as caffeine (Min et al. 2015). The rationale for selecting cyp-35A3 in this study was that several of these compounds induced cyp-35A3

80 at higher levels than most other CYPs. This responsiveness seems to be a common

81 feature of all members of the cyp-35A subfamily (Menzel et al. 2001; Min et al. 2015).

82 Because several cyp-35A inducers represent known environmental pollutants, members

83 of this gene subfamily have been integrated into ecotoxicogenomics studies on

84 environmental samples, such as contaminated soil (Anbalagan et al. 2013) and river

85 sediments (Menzel et al. 2009). In general, its fully sequenced genome renders

86 C. elegans an ideal model for (eco)toxicogenomics studies (Reichert and Menzel 2005)

87 that is applied for the testing of chemicals, technical materials and in environmental risk

88 assessment (ERA; Hägerbäumer et al. 2015; Leung et al. 2008; Wilson and Khakouli-

89 Duarte 2009).

90 WWTPs represent major point sources for (micro)pollutants in aquatic ecosystems (e.g.,

91 Loos et al. 2013). Discharges from conventionally treated wastewater (activated sludge

92 treatment) are associated with multiple adverse effects on sensitive aquatic species

93 (Prasse et al. 2015), including C. elegans (Hitchcock et al. 1997). These discharges

94 contain complex mixtures of various pollutant classes, such as PAHs. PAHs belong to the

95 group of persistent organic pollutants (POP) that despite their reduced emission in the

96 last few decades are regularly detected in WWTP effluents, surface water and river

97 sediments (Forsgren 2015). As a consequence, fluoranthene was listed as priority

98 pollutant by the US EPA and in the EU water framework directive (WFD) representing 
other hazardous PAHs (European Commission 2000). PAHs are known for their genotoxicity in various species. Unlike other PAHs, $\beta-N F$ is not carcinogenic and seemed not to cause DNA damage to $C$. elegans (Leung et al. 2010). Nonetheless, $\beta$-NF caused significant reproduction toxicity and growth inhibitions (Leung et al. 2010; Menzel et al. 2001).

With the improvement of analytical methods novel anthropogenic chemicals, including pharmaceuticals, biocides, nutrient related or industrial chemicals, have been detected in WWTP effluents and receiving water bodies. Despite a growing knowledge base, the majority of natural and anthropogenic chemicals in wastewater remain presently unknown (Petrie et al. 2015). Moreover, a significant fraction of these substances, including micropollutants, are not or only incompletely removed during conventional wastewater treatment (Loos et al. 2013). To tackle this, advanced treatment technologies have been developed and implemented, including oxidative treatment technologies (e.g., ozonation or UV $+\mathrm{H}_{2} \mathrm{O}_{2}$ ), adsorptive technologies (e.g., granulated or powdered activated carbon (GAC, PAC)) and biotechnology (e.g., immobilized enzymes). Different technologies (and their combinations) effectively increase the removal of residual (micro)pollutants and toxicity. However, they also indicated negative side effects (Prasse et al. 2015). Adsorptive treatment technologies do not remove highly polar chemicals. Oxidative and enzymatic treatments do not fully mineralize a large set of substances. Oxidative treatments thereby generate unknown transformation products (TP) (Magdeburg et al. 2012) that can be more toxic than their parental compounds (Sinclair and Boxall 2003). Because of this they require additional post-treatment, such as by sandfiltration (e.g., Magdeburg et al. 2012). From the research on wastewater treatment processes it also 
became apparent that the removal of target compounds does not necessarily result in a removal of toxicity.

The present study aimed at extending on this knowledge by assessing the removal of (micro)pollutants and toxicity (xenobiotic metabolism) by conventional and advanced wastewater treatment. Samples were collected at four WWTPs of different size classes (small, medium and large) equipped with conventional activated sludge and different advanced treatments. The latter were installed at one WWTP and comprised of an ozonation of the WWTP effluent and sequential GAC filtration or biofiltration (BF). In addition, surface water was sampled downstream of one of the investigated WWTPs. For the analysis of these samples an established C. elegans bioassay was adapted from the International Organization for Standardization (ISO) guideline 10872 (Höss et al. 2012). Lab-scale in vivo bioassays such as ISO 10872 are valuable tools in assessing the toxicity and biological activity of environmental samples. Their outcome thereby provides valuable indications on the quality of (waste)water and can serve as proxy of potential biological impacts of chemicals. This standardized bioassay has also been used to examine the impacts of various chemicals with different modes of action in other studies (e.g., Ristau et al. 2015; Haegerbaeumer et al. 2018). The guideline comprises the apical endpoints reproduction and growth that respond sensitively to testing environmental samples (Wilson and Khakouli-Duarte 2009). A main objective of this study was to integrate molecular endpoints for xenobiotic metabolism into the assay, which may be more sensitive. Cyp-35A3::GFP (Menzel et al. 2007) was selected as biomarker for CYP35A3 related xenobiotic metabolism in transgenic C. elegans (e.g., Min et al. 2015; Roh et al. 2014). Using the PAH and potent cyp-35A3 inducer $\beta-N F$, proof of principle 
145 experiments were carried out on surface water and wastewater prepared by different 146 techniques. These experiments aimed at determining the assay sensitivity and 147 characterizing the impact of the sample matrix, such as from total suspended solids (TSS) 148 content or background (micro)pollutant concentrations. Based on these results, 15 149 relevant sampling points, representative for the urban water cycle, were analyzed. Special 150 focus was put on the comparison of conventional and advanced treatments, the 151 respective micropollutant removal efficacies and the occurrence of residual 152 micropollutants and/or toxicity in WWTP discharges and receiving surface water. Two 153 main hypotheses were tested: 1) Advanced wastewater treatment is more effective in 154 removing (micro)pollutants and toxicity. 2) The removal of target compounds does not per 155 se translate to a removal of toxicity. For quantification of (micro)pollutants and TPs the 156 concentrations of 92 chemical indicator substances (Seitz and Winzenbacher 2017) and

15719 WWTP parameters (Knopp et al. 2016) were determined. 


\subsection{Conventional wastewater treatment plants}

Three WWTPs and one surface water were sampled in the state Baden-Württemberg, Germany, in December 2012, October 2013 and February 2014. The considered region comprises a water protection area of $513 \mathrm{~km}^{2}$ that provides drinking water for approximately 3.5 million inhabitants. WWTP-1 (440,000 population equivalents, PE) is located near this area $(3.5 \mathrm{~km}), 12 \mathrm{~km}$ upstream of the SW sampling site. WWTP-2 (16,000 PE) and WWTP-3 (16,600 PE) are situated within the water protected area. The SW was sampled from the Danube (near Leipheim), one of the largest rivers in Germany. At the sampling point, a wastewater fraction of approximate $6 \%$ was measured (Seitz and Winzenbacher 2017). WWTP-4 (50,000 PE) is located in the state of Hessen, Germany. Samples were taken in March and April 2015. All WWTPs (1-4) use conventional treatment based on activated sludge, but differ in their catchment areas, corresponding wastewater quality, receiving surface waters and other specifications (Online Resource 1; Knopp et al. 2016; Seitz and Winzenbacher 2017). Samples were collected at WWTP influents (INF 1-4) and effluents (EFF 1-4) according to 2.3.

\subsection{Pilot wastewater treatment plant equipped with advanced treatment} technologies

The pilot WWTP was fed by the conventionally treated wastewater of WWTP-4 and included an ozonation $\left(\mathrm{O}_{3}\right)$ coupled to GAC or BF (Fig. 1; Knopp et al. 2016). The WWTP effluent was filtered by a $10 \mu \mathrm{m}$ microscreen to reduce suspended solids prior to $\mathrm{O}_{3}$. Samples were taken according to 2.3 from the influent (INF-4), after activated sludge 
treatment $(\mathrm{EFF}-4)$, after the ozonation $\left(\mathrm{EFF}+\mathrm{O}_{3}\right), \mathrm{GAC}\left(\mathrm{O}_{3}+\mathrm{GAC}\right)$ and $\mathrm{BF}\left(\mathrm{O}_{3}+\mathrm{BF}\right) . \mathrm{GAC}$ and $\mathrm{BF}$ were operated in parallel in an unaerated $\left(\mathrm{O}_{3}+\mathrm{GAC}\right.$ and $\left.\mathrm{O}_{3}+\mathrm{BF}\right)$ and aerated $\left(\mathrm{O}_{3}+\mathrm{GAC}_{\mathrm{a}}\right.$ and $\left.\mathrm{O}_{3}+\mathrm{BF}_{\mathrm{a}}\right)$ mode using compressed ambient air. Details on process parameters can be found in Online Resource 2.

\subsection{Sampling and sample preparation}

Wastewater samples (1-5 L) were collected as $24 \mathrm{~h}$ composite samples. Surface water samples were collected as $1 \mathrm{~L}$ grab samples. Aqueous samples were kept in amber glass bottles at $4^{\circ} \mathrm{C}$ until testing (max. $3 \mathrm{~d}$ after sampling) or extracted on site directly after sampling by an optimized solid phase extraction (SPE) method (Abbas et al., in prep.). The procedure in brief: Prior to SPE, $500 \mathrm{~mL}$ of each sample were filtered through Whatman GF6 filters (pore size $<1 \mu \mathrm{m})$, acidified with sulfuric acid $(3.5 \mathrm{M}$, picograde) to pH 2.5 and extracted using Telos C18/ENV columns (Kinesis). A SPE blank was included by applying the same procedure to an analytically pure groundwater (GW) sample. SPE columns were eluted with $5 \times 2 \mathrm{~mL}$ methanol (Carl Roth, Rotisolv, Ultra LC-MS) and $5 \times 2 \mathrm{~mL}$ acetone (Carl Roth, Rotisolv, GC Ultra). $100 \mu \mathrm{L}$ dimethyl sulfoxide (DMSO, Sigma-Aldrich, 99.5\%) was added to each extract. The methanol/acetone was evaporated under a gentle nitrogen stream. This resulted in a 5000 fold increase in solute concentration (5000x). SPE extracts were kept at $-20^{\circ} \mathrm{C}$ until bioassay analysis.

\subsection{Spiking of samples with $\beta$-naphthoflavone}

Aqueous SW and EFF-1 from December 2012 were spiked to $1 \mathrm{mg} / \mathrm{L} \beta-\mathrm{NF}$ (CAS 605187-2, Alfa Aesar, >98\%). Ultrapure water (UPW) was used as blank sample (TKA GenPure, Thermo Fisher Scientific). $\beta$-NF was selected as a reference compound for 
reproductive toxicity, growth inhibition (Leung et al. 2010) and cyp-35A3 expression (Menzel et al. 2001, 2007). For spiking $1 \mu \mathrm{L}$ of a $1 \mathrm{mg} / \mathrm{mL}$ stock solution in DMSO was added to $1 \mathrm{~L}$ of the respective sample (0.1\% DMSO final). Aqueous and spiked samples were analyzed as 1:2 dilution, resulting in a final $\beta-N F$ concentration of $0.5 \mathrm{mg} / \mathrm{L}$ for the spiked samples. In addition, aqueous (UPW, SW and EFF-1) and spiked (UPW's, SW and $\mathrm{EFF}-1^{\mathrm{s}}$ ) samples were subjected to SPE (according to 2.3).

\subsection{C. elegans strains and maintenance}

The C. elegans N2 strain, variety Bristol was obtained from the Caenorhabditis Genetic Center (CGC, Minneapolis, USA). The transgenic strain expressing the cyp-35A3::GFP construct was kindly provided by Dr. Ralph Menzel (Humboldt Universität zu Berlin, Germany). C. elegans were maintained on agar plates containing nematode growth medium (NGM). The Escherichia coli OP50 strain (uracil-deficient, obtained from the CGC) was used as food source. C. elegans stock plates (prepared according to ISO 10872) were kept at $20 \pm 1{ }^{\circ} \mathrm{C}$ in the dark. Fresh stock plates were prepared $3-5 \mathrm{~d}$ prior to bioassay analysis.

\subsection{Adapted C. elegans bioassay}

ISO 10872 was adapted as follows: For the endpoints brood size and larval length synchronized L1 larvae were transferred into 24 well microtiter plates $(n=5-10$ per replicate, compare 2.6.1 and 2.6.2). Each well contained $0.8 \mathrm{~mL}$ M9 medium. After transfer of L1 larvae 400 or $401-402.5 \mu \mathrm{L}$ M9 were removed for testing aqueous samples or SPE extracts respectively. $100 \mu \mathrm{L}$ of an OP50 suspension (500 FAU, final concentration) in M9 including cholesterol (CAS 57-88-5, Sigma-Aldrich, > 92.5\% GC, 
$0.1 \%$ final concentration) was supplemented to all wells. The resulting bacterial suspension was used as negative control (NC). For testing SPE extracts: depending on the final concentration factor, $10 x$ or $25 x$, an extract volume of $1 \mu \mathrm{L}(1: 500)$ or $2.5 \mu \mathrm{L}$ (1:200) of the 5000x SPE extracts (2.3) was added respectively. For testing aqueous samples: $0.5 \mathrm{~mL}$ of sample was added (1:2). Addition of samples/extracts represented the starting point $\left(\mathrm{t}_{0}\right)$ of the bioassays. Microtiter plates were incubated at $20^{\circ} \mathrm{C}$ in the dark for 1-96 $\mathrm{h}$ depending on the endpoint (2.6.1 and 2.6.2). Highest final SPE enrichment factor tested $(25 \mathrm{x})$ represented a DMSO concentration of $0.5 \%(\mathrm{v} / \mathrm{v})$. At this solvent concentration no adverse effects on C. elegans were reported (Boyd et al. 2010). In prescreening experiments $10 x$ concentrated samples were tested (3.1). For samples from WWTP 1-3 a 25x concentration factor was applied (3.2). However, for these samples mortality occurred in the INF 1-3 (data not shown) thus 1:2 dilutions were prepared. Accordingly, WWTPs 1-3 were tested in 12.5x concentrations. Samples from WWTP-4 were tested in $25 x$ concentrations (3.3).

\subsubsection{Endpoint brood size and larval length}

Benzylcetyldimethylammonium chloride (BAC-C16, 5 mg/L, CAS 122-18-9, Alfa Aesar, $95 \%$ ) was used as additional positive control (PC) for reprotoxicity and inhibition of growth (Höss et al. 2012). The duration of the respective bioassays was $96 \mathrm{~h}$. At their termination ( $t_{\text {end }}=96 \mathrm{~h}$ ) adult and larval nematodes were sacrificed by heat shock $\left(15 \mathrm{~min}\right.$ at $\left.80^{\circ} \mathrm{C}\right)$ and stained with rose bengal (CAS 632-69-9, AppliChem) for microscopic evaluation (30x). For the endpoint brood size (reproduction) 10 individuals were exposed in 3 replicates each per experiment. Total $\mathrm{n}$ per treatment group are indicated in figure captions. For a comparative analysis in selected experiments, 5 individuals in 5 replicates 
were used (adapted from ISO 10872). The offspring of each replicate was counted after $96 \mathrm{~h}$ and presented as mean number of offspring per adult hermaphrodite. For determining larval lengths (endpoint growth), 20 randomly picked larvae from each replicate were measured. Data of the replicates were pooled if no statistical difference occurred.

\subsubsection{Endpoint cyp-35A3::GFP expression}

$\beta-N F$ served as reference substance for the expression of cyp-35A3::GFP in transgenic C. elegans (Menzel et al. 2007). For the exposure to $\beta-N F$, wastewater samples and SPE extracts adult specimens were used. The procedure was analogous to the endpoints in 2.6.1 except shorter exposure times (1-48 h). cyp-35A3::GFP expression levels were evaluated for a minimum of 10 adults per treatment group using fluorescence microscopy. Individuals were mounted onto microscopy slides and immobilized by a drop of sodium azide (Sigma-Aldrich, $10 \mathrm{mM}$ ). GFP localization and fluorescence intensities were determined using an Olympus BX50 microscope at 100x magnification, an excitation wavelength of $470-490 \mathrm{~nm}$ and emission wavelength of $515 \mathrm{~nm}$. Images were taken with a digital imaging system (Discus software) and processed with ImageJ (National Institute of Health, USA). Background fluorescence was subtracted based on the average GFP signal of unexposed (NC) organisms.

\subsection{Chemical analysis and WWTP parameters}

Water and wastewater samples were analyzed for selected WWTP parameters and micropollutants (Online Resource 2-3). Quantification of micropollutants was performed by HPLC (Thermo Dionex UltiMate 3000 RSLC) and electrospray MS/MS detection 
270 were determined according to regulatory standards (as described by Knopp et al. 2016).

271 A defined set of process parameters $(n=7)$ was documented for the advanced

272 wastewater treatment technologies (Online Resource 2).

$273 \quad 2.8$ Statistical analysis

274 Statistical analysis was performed using GraphPad Prism, version 5.0-7.0 (GraphPad

275 Software, San Diego, USA) and Microsoft Excel 2010 (Microsoft, Redmond, USA).

276 Statistically significant differences between treatments were analyzed as indicated in

277 figure captions. $\beta$-NF concentration response curves were computed based on the 278 reprotoxicity and cyp-35A3::GFP expression levels of $0.01,0.1,1$ and $5 \mathrm{mg} / \mathrm{L} \beta-\mathrm{NF}$ after $27996 \mathrm{~h}$ and $1-48 \mathrm{~h}$ of exposure, respectively. Logistic regression models were used to 280 derive the median effective concentrations $\mathrm{EC}_{50}$ (Online Resource 5-6).

281 


\section{Results}

\subsection{Aqueous and $\beta$-naphthoflavone spiked surface water and wastewater}

In previous studies, $\beta$-NF affected the reproduction and growth of $C$. elegans at exposure concentrations of > $273 \mu \mathrm{g} \beta-\mathrm{NF} / \mathrm{L}$ (Leung et al. 2010; Reichert and Menzel 2005). In the present experiments $\beta-\mathrm{NF}$ caused a concentration-dependent decrease in brood size with the lowest observed effect concentration (LOEC) of $100 \mu \mathrm{g} / \mathrm{L}$ and an $\mathrm{EC}_{50}$ of $140 \mu \mathrm{g} / \mathrm{L}$ (Online Resource 5). Based on this proof of principle experiments were conducted using the reference compound $\beta-\mathrm{NF}$ as well as aqueous surface water (SW) and WWTP effluent (EFF-1). Aqueous samples, including an ultrapure water control (UPW), were spiked to $1 \mathrm{mg} / \mathrm{L} \beta-\mathrm{NF}$ and tested as 1:2 dilutions. Average offspring numbers were $98.6 \pm$ 8.1 juveniles per adult in the UPW control. The SW did not induce reprotoxicity, but slightly increased the reproduction by $10 \%$ compared to the UPW (Fig. 2). The same was true for the 10x concentrated SW extract. In contrast, the aqueous WWTP effluent (EFF-1) significantly reduced reproduction by $83 \%$ compared to the control. The $10 x$ concentrated extract of EFF-1 induced a $31 \%$ reduction in brood size compared to the extracted ultrapure water. This reprotoxicity was however not as pronounced as for the aqueous sample. As expected, the presence of $0.5 \mathrm{mg} / \mathrm{L} \beta-\mathrm{NF}$ in spiked samples significantly reduced brood sizes. For the spiked ultrapure water $\left(\mathrm{UPW}^{\mathrm{s}}\right)$ reproduction was $46 \%$ lower than in the unspiked reference. Along that line, exposure to spiked surface water (SW $\left.{ }^{\text {s }}\right)$ resulted in a $40 \%$ smaller brood size compared to SW. The spiked WWTP effluent induced more than $90 \%$ mortality thus reproduction was not assessed. The extracts of spiked UPW and SW significantly reduced the reproduction to levels comparable to the 
aqueous spiked samples. Despite a 10x concentration factor, the spiked WWTP effluent sample induced lower reprotoxicity than the aqueous EFF-1 ${ }^{\text {s }}$.

\subsection{Conventional wastewater treatment}

The impacts of influent and effluent samples from three WWTPs applying conventional activated sludge treatment on the brood size and larval length of $C$. elegans were investigated. Samples were analyzed in $12.5 x$ concentrations. Regarding the endpoint brood size (Fig. 3A), a high variability in the influent samples was observed. Mean offspring numbers for INF-1, INF-2 and INF-3 were 19, 11 and $14 \%$ lower than in the GW control (85.6 \pm 7.9 juveniles per adult), respectively. For the effluent samples variability was lower and for EFF 1-2 comparable to those of NC and GW. Here, the mean offspring numbers in EFF-1, EFF-2 and EFF-3 were increased by 40,45 and $80 \%$ respectively compared to GW. Larval lengths were quantified to detect possible impacts on C. elegans growth (Fig. 3B). Larvae of NC and GW had grown to a mean length of $391 \pm 14.2 \mu \mathrm{m}$ and $336 \pm 11.9 \mu \mathrm{m}$, respectively. Length distributions of EFF-1, EFF-2 and EFF-3 were broader than for GW and larvae were observed to be significantly longer (mean lengths of $515 \pm 21.5 \mu \mathrm{m}, 495 \pm 16.5 \mu \mathrm{m}$ and $517 \pm 17.4 \mu \mathrm{m}$, respectively). Larval growth was not determined for the influent samples.

\subsection{Advanced wastewater treatment technologies}

The samples from the conventional and subsequent advanced wastewater treatments at WWTP-4 were analyzed for their effects on brood size and larval lengths. These samples were tested as $25 \mathrm{x}$ concentrated extracts as no significant mortality occurred (compare 2.6). A high reprotoxicity was induced by the INF-4 sample with an average offspring 
number $98 \%$ lower than in the GW control $(68.2 \pm 9.8$, Fig. $4 A)$. The samples from the subsequent treatments $\mathrm{EFF}-4$ and $\mathrm{EFF}+\mathrm{O}_{3}$ were not reprotoxic but increased the average offspring number by $11.6 \%$ and $19.4 \%$ compared to GW, respectively ( $p>0.05$ ). For $\mathrm{O}_{3}+\mathrm{GAC}, \mathrm{O}_{3}+\mathrm{GAC}_{\mathrm{a}}, \mathrm{O}_{3}+\mathrm{BF}$ and $\mathrm{O}_{3}+\mathrm{BF}_{\mathrm{a}}$ an increase of average offspring numbers was observed (17.8, 26.9, 30.6 and $42 \%$ compared to GW, respectively), which was not significant. Similarly, the larvae length tends to increase (Fig. 4B). Here, larvae exposed to the conventionally treated effluent (EFF-4) had an average length of (389 $\pm 17.4 \mu \mathrm{m})$ that was slightly but not significantly higher than in the NC (345 $\pm 15 \mu \mathrm{m})$ and GW $(350 \pm$ $15.7 \mu \mathrm{m})$. For $\mathrm{EFF}+\mathrm{O}_{3}(422 \pm 23.8 \mu \mathrm{m})$ a further non-significant increase was observed. In the $\mathrm{O}_{3}+\mathrm{GAC}(494 \pm 26.5 \mu \mathrm{m}), \mathrm{O}_{3}+\mathrm{GAC}_{\mathrm{a}}(469 \pm 25.4 \mu \mathrm{m})$ and $\mathrm{O}_{3}+\mathrm{BF}_{\mathrm{a}}(456 \pm 23 \mu \mathrm{m})$ treatments larvae were significantly larger compared to NC and GW. The length of larvae exposed to $\mathrm{O}_{3}+\mathrm{BF}(347 \pm 16.4 \mu \mathrm{m})$ was at the level of $\mathrm{GW}$. These results were qualitatively confirmed throughout multiple experiments $(n=6)$.

\section{4 cyp-35A3::GFP induction in transgenic C. elegans}

To evaluate potential impacts of water and wastewater samples on the xenobiotic metabolism of C. elegans the Pcyp-35A3::GFP transgenic strain was used (Menzel et al. 2007). CYP-35A3 served as biomarker for the exposure to PAH, PCB and other cyp-35A3 inducing compounds. First, it was investigated whether the reference compound $\beta$-NF induces cyp-35A3::GFP expression. A concentration- and time-dependent increase (0.01-5 mg $\beta-N F / L, 1-48$ h) in GFP signal was observed (Online Resource 6). EC Fo $_{50}$ values of 71.5 and $78.6 \mu \mathrm{g} / \mathrm{L}$ were reached after 8 and $24 \mathrm{~h}$ respectively. The highest expression levels ( 21.3 and 24 fold above the control) were reached after $8 \mathrm{~h}$ of exposure 
to 1 and $5 \mathrm{mg} / \mathrm{L} \beta-\mathrm{NF}$, respectively. cyp-35A3::GFP expression responded fast to an exposure to $5 \mathrm{mg} / \mathrm{L} \beta-\mathrm{NF}$ (after $1 \mathrm{~h}$ ). From $4 \mathrm{~h}$ onwards, the LOEC was $0.1 \mathrm{mg} / \mathrm{L} \beta-\mathrm{NF}$. Based on these results the sensitivity of cyp-35A3::GFP expression towards different aqueous, spiked and enriched water and wastewater samples was compared (Fig. 5 and Online Resource 7). None of the aqueous samples (UPW, SW, EFF-1) significantly induced cyp-35A3::GFP. Similar to their aqueous equivalents, exposure to $10 \mathrm{x}$ concentrated extracts of these samples did not significantly induce cyp-35A3::GFP at any exposure time. In contrast, the $\beta-N F$-spiked aqueous samples (UPW ${ }^{\mathrm{s}}, \mathrm{SW}^{\mathrm{s}}$ and $\mathrm{EFF}-1^{\mathrm{s}}$ ) significantly induced the expression. Similar to $\beta-\mathrm{NF}$, this increase was time-dependent $(1-48 \mathrm{~h})$ and maximal expression levels were reached after 24-48 h. The earliest significantly increased expression was detected after $1 \mathrm{~h}$ of exposure to EFF-15. The exposure to the extracted spiked samples UPW's and SW's led to slightly higher CYP35A3::GFP levels compared to the aqueous spiked samples. Interestingly, cyp-35A3 expression induced by EFF-1 ${ }^{\text {s }}$ extracts was significantly lower than for the aqueous EFF$1^{\text {s }}$ sample (Fig. $5 B$ ).

With regard to advanced wastewater treatment technologies, the effluents of conventional WWTPs (EFF-1, EFF-4) were compared to ozonation (EFF+O ${ }_{3}$, Fig. $\left.5 \mathrm{C}\right)$. Samples were analyzed as $10 x$ extracts for multiple exposure times (4-48 h, Online Resource 8). Again, EFF-1 did not cause any significant cyp-35A3::GFP induction. In contrast, EFF-4 and its subsequent treatment by ozonation $\left(\mathrm{EFF}+\mathrm{O}_{3}\right)$ significantly increased cyp-35A3::GFP expression. The induction by $\mathrm{EFF}+\mathrm{O}_{3}(7.4$ fold above the control level, at $24 \mathrm{~h}$ ) was significantly higher than by EFF-4 (5 fold above the control level, at $24 \mathrm{~h}$ ). 


\subsection{Chemical analysis and WWTP parameters}

The experiments with C. elegans were accompanied by a detailed chemical analysis of (micro)pollutants and WWTP parameters (Online Resource 2-4). Focusing on WWTP-4, DOC, conductivity, $\mathrm{UV}_{254}, \mathrm{NH}_{4}{ }^{+}$and $\mathrm{P}_{\text {total }}$ were removed with rates characteristic for conventional biological and advanced wastewater treatment (Knopp et al. 2016). For instance, the advanced technologies (EFF-4 vs. EFF $+\mathrm{O}_{3}$ and $\mathrm{EFF}+\mathrm{O}_{3}$ vs. $\mathrm{O}_{3}+\mathrm{GAC} / \mathrm{GAC}$, $\mathrm{O}_{3}+\mathrm{BF} / \mathrm{BF}_{\mathrm{a}}$ ) demonstrated additional removal rates in terms of these parameters although to a different extent. The DOC was reduced by only $9 \%$ from EEF-4 to EFF $+\mathrm{O}_{3}$, but further $32,37,21$ and $26 \%$ by $\mathrm{O}_{3}+\mathrm{GAC}, \mathrm{O}_{3}+\mathrm{GAC}_{\mathrm{a}}, \mathrm{O}_{3}+\mathrm{BF}, \mathrm{O}_{3}+\mathrm{BF}_{\mathrm{a}}$, respectively.

Out of the 92 target compounds, 57 substances and TPs were detected above the LOQ in the INF-4 and 50 in the EFF-4. The concentrations of 14 of these compounds were reduced by $>90 \%$, of 10 by $50-90 \%$ and of 14 by $<50 \%$. Further 19 compounds occurred at higher concentrations in the effluent than in the influent, whereby the concentration of 13 was increased by $>25 \%$. Carboxy-acyclovir (main TP of acyclovir), acesulfame, sucralose, 4-formylaminoantipyrin (TP of phenazone) and benzotriazole occurred at the highest concentration in the effluent $(20,13,10,9.8,8.4 \mu \mathrm{g} / \mathrm{L}$, respectively). Ozonation effectively reduced the concentration of the majority of substances. From 50 substances above the LOQ in the EFF-4 only 20 were detected in the EFF+O 3 . The concentrations of only 5 substances decreased by less than $50 \%$, including diatrizoic acid, acesulfame, sucralose, melamine and iomeprol (Online Resource 3). The four post-treatments resulted in a low (BFs) to moderate (GAC filtrations) additional removal. An average removal rate of $36,39,11$ and $18 \%\left(\mathrm{O}_{3}+\mathrm{GAC}, \mathrm{O}_{3}+\mathrm{GAC}_{\mathrm{a}}, \mathrm{O}_{3}+\mathrm{BF}, \mathrm{O}_{3}+\mathrm{BF}_{\mathrm{a}}\right.$ compared to 
$392 \mathrm{EFF}+\mathrm{O}_{3}$ ) was determined. Diatrizoate had the highest concentrations after post-treatment

$3935.6-6.1 \mu \mathrm{g} / \mathrm{L}$, followed by acesulfame (4.1-5.1 and sucralose $(2-4.4 \mu \mathrm{g} / \mathrm{L})$. 


\section{Discussion}

\section{1 $\beta$-naphthoflavone and spiked environmental samples}

The detected reprotoxicity of the reference substance $\beta$-NF (3.1) was higher than reported in the literature (Leung et al. 2010; Reichert and Menzel 2005). Regarding the biomarker CYP-35A3 an intestinal expression of cyp-35A3::GFP (Online Resource 9 and Menzel et al. 2007) was confirmed for all $\beta-N F$ ECs $(0.1-5 \mathrm{mg} / \mathrm{L})$. The intestine of $C$. elegans is known as its detoxification organ, which may hint on the physiological role of CYP-35A3 and/or mode of action of $\beta-N F$. EC 50 values of 71.5 and $78.6 \mu \mathrm{g} / \mathrm{L}$ for the 8 and $24 \mathrm{~h}$ time point respectively were recorded (Online Resource 6). These ECs indicated a slightly higher sensitivity of the biomarker compared to the endpoint reproduction $\left(\mathrm{EC}_{50}=140\right.$ $\mu \mathrm{g} / \mathrm{L}, 96 \mathrm{~h})$. Markedly, $\beta-N F$ strongly induced all cyp-35A subfamily members and several other CYPs (Menzel et al. 2001). Menzel et al. (2005) knocked down cyp-35A subfamily members, which decreased the reproductive toxicity of PCB52 and fluoranthene. Inokuchi et al. (2014) suggested a role for CYPs (including CYP-35A3) in the tolerance against triclosan and trichlocarban. Roh et al. (2014) supposed an involvement of CYP-35A3 in the metabolic toxicity of chlorpyrifos. Accordingly, the reprotoxicity of $\beta-\mathrm{NF}$ (and its potential metabolites) may be mediated via CYP-35As.

The potential impact of the sample matrix on the $\beta-N F$ effects was examined by spiking surface water and wastewater samples. Spiked surface water induced a high reprotoxicity similar to the spiked ultrapure water control. For the unspiked surface water sample no reprotoxicity was detected. This indicated that no reprotoxicity is present and that the surface water matrix does not interfere with the $\beta-N F$ toxicity. This is further supported by 
the detected low micropollutant concentrations (Online Resource 3; Seitz and Winzenbacher 2017). The effluent of WWTP-1 decreased the brood size by $83 \%$ and spiking further increased this effect to $100 \%$ (Fig. 2). This suggests a joint effect of $\beta$-NF and other reprotoxic wastewater constituents including natural factors that may affect these toxicities. Mixture toxicity was previously suggested for wastewater contaminants in C. elegans (Hitchcock et al. 1997). The fact that there was no difference in the reprotoxicity induced by the spiked aqueous and extracted ultrapure water and surface water (Fig. 2) suggested a low recovery rate towards $\beta-N F$, which may not effectively elute from the SPE sorbent due to its hydrophobicity. In contrast, the extracted effluent sample (EFF-1) induced toxicity indicating that other reprotoxic compounds than $\beta-N F$ were extractable. However, the reprotoxicity in the extracted EFF-1 and EFF-1 ${ }^{\mathrm{S}}$ was lower than in their aqueous equivalents, which may attribute to particle associated reprotoxicity filtered out during SPE pre-filtration (compare below) and/or the absence of nonextractable natural factors (compare above).

Unspiked surface water and effluent of WWTP-1 did not cause any significant cyp35A3::GFP induction (Fig. 5 and Online Resource 7). Spiking with $\beta-N F$, however, resulted in an effective induction, which was higher in the aqueous effluent compared to the surface water sample. This is in accordance with the results observed for reproduction and might be explained by joint effects caused by low concentrations of multiple CYPinducers in the effluent, which do not induce expression without $\beta$-NF and/or natural factors affecting the latter. Another factor might have contributed: $\beta-N F$ has a log Kow of 4.7 (estimated using US EPA's EPISuite) and will adsorb to particles, such as from TSS in wastewater. Higher TSS can thus partition more bioavailable $\beta-N F$ into the particulate 
phase of wastewater compared to surface water. As ingestion of contaminated food particles is the main exposure route for several pollutants in C. elegans (Offermann et al. 2009), the interaction of $\beta-N F$ and wastewater-borne particles may thus explain the higher toxicity observed in the aqueous sample. In addition, this was not the case for extracted samples in which particulate matter larger than $1 \mu \mathrm{m}$ and sample impurities were generally removed prior to or during extraction respectively. These results underline the importance to consider contaminated suspended solids in ecotoxicological evaluations of WWTP discharges (Burton et al. 2000) for which particle-feeding species such as C. elegans may offer several advantages.

\subsection{Conventional wastewater treatment}

Hitchcock et al. (1997) observed high levels of mortality when exposing C. elegans to WWTP effluent samples from conventional activated sludge treatment. In the present study mortality occurred in most of the 25x WWTP influent, but not effluent samples of WWTPs 1-3 (data not shown). However, aqueous and extracted effluent samples of WWTP-1 (from December 2012) exhibited a respective 31-83\% decrease in brood size (Fig. 2). Similar (repro)toxicity has been reported for other species exposed to conventionally treated WWTP effluents (e.g., Giebner et al. 2016; Magdeburg et al. 2012). In contrast, none of the extracted effluent samples of WWTPs 1-3 from October 2013 and February 2014 induced significant (repro)toxicity (Fig. 3). The corresponding influent samples however exhibited moderate to high levels of reprotoxicity. Growth was selected as additional endpoint (Höss et al. 2012). C. elegans larvae exposed to the effluents from WWTPs 1-3 were significantly longer compared to the NC and GW control. The lengths of the majority of these larvae herby corresponded to the L3 instead of the L1 stage, which 
suggests that the samples strongly promoted the growth of $C$. elegans. Such effects have been observed for other conventionally treated effluents and model invertebrates as well (e.g., Völker et al. 2017) where they were caused by residual nutrients (compare 4.3). The extracted effluent from WWTP-1 did apparently not induce cyp-35A3 to any significant extend. In contrast, the extracted effluent from WWTP-4 caused a significantly elevated expression, implying this WWTP emits CYP inducers. Generally, known cyp$35 A 3$ inducing (micro)pollutants, such as $\beta-N F$, fluoranthene, PCB52, chlorpyrifos or thiabendazole, have been detected in treated wastewaters in the microgram per liter range (e.g., Quevauviller et al. 2006; Peris-Vicente et al. 2016). Diazinon, imidacloprid and lansoprazol ranged at the nanogram per liter scale (e.g., Loos et al. 2013). Caffeine is the only known cyp-35A3 inducer analyzed in this study (3.4) and was detected in the EFF-4 and EFF $+\mathrm{O}_{3}$ below the LOQ $(<0.05 \mu \mathrm{g} / \mathrm{L})$. For cyp-35A3 expression experiments most of these compounds were tested in the lower milligram per liter range, thus far above their reported wastewater concentrations. However, hydrophobic cyp-35A3 inducing compounds, such as triclosan and trichlocarban, benzene and the mentioned PCBs and PAHs, readily adsorb to sludge (McLaggan et al. 2012; Chalew and Halden 2010). This indicated that the particulate phase of environmental samples should be considered when estimating realistic exposure concentrations of these compounds.

\subsection{Advanced wastewater treatment technologies}

An early ecotoxicological contribution to the research on advanced wastewater treatment technologies was performed with C. elegans (Hitchcock et al. 1998). The authors observed that the toxicity of an acid-based dye wastewater increased along the duration 
of ozonation. The effect was attributed to the generation of toxic TPs during ozonation. This hypothesis has been corroborated using several aquatic species exposed to ozonated wastewater (Magdeburg et al. 2012; Giebner et al. 2016). In contrast to these studies neither conventionally nor advanced treated wastewater at WWTP-4 negatively affected the reproduction of $C$. elegans (Fig. 4A). Accordingly, the removal of toxicity by the post-treatments (such as postulated in hypothesis 1 ) in the Introduction) could not be assessed. This is in accordance with other model species, which were not sufficiently sensitive for the evaluation of advanced wastewater treatment (Völker et al. 2017). Mutant and transgenic of $C$. elegans strains, such as the mentioned hypersensitive mutant (e.g., Xiong et al. 2017), may thus represent promising alternative tools for assessing the toxicity of (highly) treated wastewaters and micropollutant effects at (very) low concentrations. Another explanation for the observation at WWTP-4 might be the general variability of the wastewater matrix. (Micro)pollutants and natural compounds in WWTP influents and effluents can vary significantly depending on the catchment area and WWTP characteristics respectively (e.g., WWTP-1 and WWTP-4, Online Resource 1). Moreover, toxic oxidation products amongst (highly) polar compounds may be lost during SPE of ozonated (waste)water samples (Stalter et al. 2016).

In comparison, the endpoint larval growth was affected by the advanced wastewater treatment stages with a significantly increased larvae length in the activated charcoal treatments and the aerated biofilter (Fig. 4B). The largest increase was observed for the $\mathrm{O}_{3}+$ GAC. Different anthropogenic compounds (Höss and Weltje 2007) and natural organic matter (NOM) constituents (Höss et al. 2001) demonstrated to affect C. elegans reproduction and/or growth. As most of these compounds are effectively removed during 
activated sludge treatments (e.g., nonylphenol) or hardly enriched by the applied SPE method (e.g., inorganic trace nutrients or macromolecular NOM) the causes of the observed effect remain speculative.

A significant impact of the advanced wastewater treatment ozonation was detected utilizing cyp-35A3::GFP. The extracted effluent from WWTP-4 (EFF-4) led to significant inductions of $c y p-35 A 3:: G F P$. Markedly, the induction levels of EFF-4 were higher after ozonation (Fig. 5C, Online Resource 8). As observed for other species (Magdeburg et al. 2012), this increased CYP expression may have been the result of toxic/bioactive TPs generated by the oxidative treatment. This result further speaks for the usefulness of $C$. elegans mutant/transgenic strains in wastewater quality assessments. Unfortunately, we did not investigate the fate of this biological activity in the post-treatments and it remains to be determined whether the CYP induction is removed here.

\subsection{Micropollutant removal}

The concentrations of most target compounds, DOC and other relevant wastewater parameters decreased in the conventional biological and the advanced treatment stages (3.5). This confirmed the additional reduction capacity of ozonation and the GAC/BF posttreatments such as postulated in hypothesis 1) in the Introduction. The causes of the observed effects of the respective wastewater samples on C. elegans (3.1-3.4) however remain to be clarified.

Chemical indicators analyzed in this study (Online Resource 3-4) for which toxicological data was available in the $C$. elegans literature mainly ranged amongst pharmaceuticals, which may attribute to its growing application in biomedical research (Leung et al. 2008). 
Certain of the chemical indicators indicated (repro)toxicity, including 1-adamantylamine (Kao et al. 2016), 2-(thiocyanomethylthio)-benzothiazol (Allard et al. 2013), caffeine (Boyd et al. 2010), carbamazepine (Olga Kolychalow, personal communication), DEET (Hartman and Freedman 2005), as well as depressed fertility, such as saccharin (Sofia Allison, personal communication) or growth promotion, such as sulfamethoxazole (Liu et al. 2013). Nonetheless, none of these compounds seemed individually responsible for the effects observed in this study, because their concentrations (Online Resource 3) were lower than their reported ECs. A few chemical indicators were tested positively for biochemical or molecular endpoints in C. elegans which occurred in the microgram per liter range in the wastewater samples from conventional treatment, such as diclofenac or sotalol (Petersen et al. 2004) as well as the advanced wastewater treatment stages, such as acesulfame or gabapentin (Caylor et al. 2013). It should also be considered that the concentrations of chemical indicators measured in this and most of the cited studies referred to the aqueous phase of the respective wastewater samples. In contrast, their accumulation to sludge particles (Chalew and Halden 2010; McLaggan et al. 2012) and potential mixture toxicity effects (e.g., additive or synergistic) have rarely been compared. However, it is also likely, that the chemical analysis of target micropollutants did not cover the toxicologically relevant compounds (e.g., Tang et al. 2014), supporting hypothesis 2) postulated in the Introduction. This further highlights the need to combine biological and chemical methods to assess the effectiveness of (advanced) wastewater treatment. 
551 The technical removal of anthropogenic micropollutants and transformation products from

552 WWTP discharges is pivotal for improving water quality and mitigating potential ecological risks (European Commission 2000). Assessing the effectiveness of wastewater treatment in removing chemicals and toxicity is a pre-requisite to the success of this measure. For this, efficient and sensitive methods have been developed and implemented (e.g., Wernersson et al. 2015). Along that line, this study aimed at adapting a well-established C. elegans bioassay for combining apical (growth and reproduction) and molecular (CYP35A3 related xenobiotic metabolism) endpoints.

The bioassay was validated using $\beta-N F$ as reference compound and different sample matrices. $\beta$-NF dose-dependently induced reproductive toxicity and cyp-35A3 expression at concentrations $>100 \mu \mathrm{g} / \mathrm{L}$. The matrix wastewater effluent was discussed to have modulated the $\beta$-NF effects either because of sorption to suspended solids or the presence of other toxic compounds as well as natural factors affecting the latter. Furthermore, a comparison of aqueous and extracted samples demonstrated that cyp35A3-inducing compounds were not completely extractable. These results support earlier scientific consent about case-specific sample preparation in wastewater quality assessments.

In this study, wastewater from four conventional WWTPs was assessed to investigate efficiencies of the activated sludge treatments in removing (micro)pollutants and toxicity. One effluent significantly inhibited the reproduction of $C$. elegans indicating the presence of residual toxicity. Three effluents significantly promoted larval growth due to unknown 
causes. The forth effluent significantly induced the biomarker cyp-35A3::GFP. The variety of effects observed in the different WWTPs demonstrates the importance of integrating multiple biological endpoints and chemical analysis when assessing their removal capacities.

This approach is even more relevant when evaluating advanced wastewater treatment technologies. At WWTP-4 they consisted of a pilot scale ozonation and ozonation followed by granular activated carbon filtration or biofiltration. Because the conventionally treated effluent did not affect the reproduction of $C$. elegans, it was not possible to evaluate the performance of the post-treatments in removing reprotoxicity. However, the post-treatment with granular activated carbon filtration and aerated biofiltration significantly promoted larval growth. The conventionally treated effluent significantly induced cyp-35A3::GFP expression, which was further increased by ozonation. As reported by previous studies, this might be the cause of toxic transformation products generated during oxidative treatment. It however remained to be investigated whether this effect persisted in the post-treatments (GAC/BF). Because the advanced treatments decreased the concentrations of most chemical indicators below the LOQs, the observed effects might be attributed to effects of chemical indicators that were not (fully) eliminated and/or compounds not covered by the target chemical analysis. This highlights the need for an integrated assessment of (advanced) wastewater treatment covering both, biological and chemical parameters. 


\section{Figure captions}

Fig. 1 Process scheme of WWTP-4. The first part of the WWTP (left) operates a conventional biological treatment process. The second part (right) is a pilot WWTP with advanced wastewater treatment technologies: Ozonation connected to aerated and nonaerated granular activated carbon (GAC) filtration or biofiltration. Grey dots indicate sampling points (24 h composites)

Fig. 2 Impacts of aqueous and extracted ultrapure water (UPW), surface water (SW) and wastewater treatment plant effluent (EFF-1) on the brood size of C. elegans. Aqueous (white bars) and extracted (grey bars) samples were analyzed in $0.5 \mathrm{x}$ and $10 \mathrm{x}$ concentrations, respectively. Spiked aqueous samples (marked by superscript s) contained $0.5 \mathrm{mg} / \mathrm{L} \beta$-naphthoflavone. Results pooled from two experiments $(n=40-120$ per treatment). Significant differences $\left({ }^{* \star} p<0.01,{ }^{\star \star \star} p<0.001,{ }^{\star \star \star *} p<0.0001\right)$ tested unspiked against spiked samples (if not noted elsewise) by one-way ANOVA with Tukey's post-hoc analysis. $₹>90 \%$ mortality

Fig. 3 Impacts of extracted groundwater (GW, SPE blank), wastewater treatment plant influent (INF 1-3) and effluent (EFF 1-3) on the brood size (A) and length of larvae (B) of C. elegans. Samples (grey bars) were analyzed in $12.5 x$ concentrations. Results pooled from three experiments for brood size ( $n=45$ per treatment group) and two experiments for larval lengths $\left(n=120-125\right.$ per treatment group). Significant differences $\left({ }^{* *} p<0.01\right.$, $\left.{ }^{\star \star \star} \mathrm{p}<0.001,{ }^{\star \star \star \star} \mathrm{p}<0.0001\right)$ were tested against NC and GW (A, B) as well as INFs against EFFs (A) by Kruskal-Wallis test with Dunn's post-test. NC (white bar) $=$ M9 medium. $\mathrm{PC}$ (white bar) = BAC $(5 \mathrm{mg} / \mathrm{L}) . \mathrm{ns}=$ not significant 
Fig. 4 Impacts of extracted groundwater (GW, SPE blank), wastewater treatment plant influent (INF-4), effluent (EFF-4) and advanced treatments on the brood size (A) and length of larvae (B) of C. elegans. Advanced treatments comprised of ozonation $\left(\mathrm{EFF}+\mathrm{O}_{3}\right)$ and ozonation followed by aerated and non-aerated granular activated carbon filtration $\left(\mathrm{O}_{3}+\mathrm{GAC}, \mathrm{O}_{3}+\mathrm{GAC}_{\mathrm{a}}\right)$ or biofiltration $\left(\mathrm{O}_{3}+\mathrm{BF}, \mathrm{O}_{3}+\mathrm{BF}\right.$ ). Samples (grey bars) were analyzed in $25 x$ concentrations. Results pooled from four experiments for brood size ( $n=$ 95 per treatment group) and one experiments for larval length $(n=60$ per treatment group). Significant differences $\left({ }^{\star} p<0.05,{ }^{\star \star} p<0.01,{ }^{\star \star \star} p<0.001\right)$ were tested against NC and GW by Kruskal-Wallis test with Dunn's post-test. NC (white bar) = M9 medium. $\mathrm{PC}($ white bar $)=\mathrm{BAC}(5 \mathrm{mg} / \mathrm{L})$

Fig. 5 A) cyp-35A3::GFP expression in transgenic C. elegans after $8 \mathrm{~h}$ exposure to $1 \mathrm{mg} / \mathrm{L}$ $\beta$-naphthoflavone ( $\beta$-NF). Exposed adult hermaphrodites showed a strong GFP signal along their intestine, as detected by fluorescence microscopy (100x). Images (NC, $\beta-N F)$ show an overlay of differential interference contrast microscopy (DIC) and GFP channel. $\mathrm{NC}=\mathrm{M9}$ medium. Bar $=200 \mu \mathrm{m} . \mathrm{B}$ and $\mathrm{C}$ ) Impacts of aqueous and extracted ultrapure water (UPW), surface water (SW), wastewater treatment plant effluent (EFF-1, EFF-4) and ozonated effluent $\left(\mathrm{EFF}+\mathrm{O}_{3}\right)$ on cyp-35A3::GFP expression. Aqueous (white bars) and extracted (grey bars) samples were analyzed in $0.5 \mathrm{x}$ and $10 \mathrm{x}$ concentrations respectively after $24 \mathrm{~h}$ exposure. Spiked aqueous samples (marked by superscript s) contained $0.5 \mathrm{mg} / \mathrm{L} \beta-\mathrm{NF}$. Results pooled from two experiments $(\mathrm{n}=10$ per treatment group, respectively). Significant differences $\left({ }^{\star} p<0.05,{ }^{\star \star} p<0.01,{ }^{\star \star \star} p<0.001,{ }^{\star \star \star \star} p<\right.$ $0.0001)$ tested unspiked against spiked samples $(B)$ and against controls $(B, C)$ by oneway ANOVA with Tukey's post-hoc analysis. Dashed lines = limit of quantification. C) 
$638 \mathrm{NC}$ (white bar) $=\mathrm{M} 9$ medium. Solvent control $(\mathrm{SC}$, white bar $)=0.2 \%$ DMSO in M9 639 medium. Fluorescence intensity of $\mathrm{PC}(1 \mathrm{mg} / \mathrm{L} \beta-\mathrm{NF})=0.185$ (result not shown).

640 


\section{References}

Allard P, Kleinstreuer NC, Knudsen TB, Colaiácovo MP (2013) A C. elegans screening platform for the rapid assessment of chemical disruption of germline function. Environ Health Perspect 121(6): 717-724

Anbalagan C, Lafayette I, Antoniou-Kourounioti M, Gutierrez C, Martin JR, Chowdhuri DK, De Pomerai DI (2013) Use of transgenic GFP reporter strains of the nematode Caenorhabditis elegans to investigate the patterns of stress responses induced by pesticides and by organic extracts from agricultural soils. Ecotoxicology 22:72-85 Boyd WA, McBride SJ, Rice JR, Snyder DW, Freedman JH (2010) A high throughput method for assessing chemical toxicity using a Caenorhabditis elegans reproduction assay. Toxicol Appl Pharmacol 245(2): 153-159 Burton GA, Pitt R, Clark S (2000) The role of traditional and novel toxicity test methods in assessing stormwater and sediment contamination. Crit Rev Environ Sci Technol $30(4), 413-447$

Peter E, Candido M, Jones D (1996) Transgenic Caenorhabditis elegans strains as biosensors. Trends Biotechnol 14(4), 125-129

Caylor RC, Jin Y, Ackley BD (2013) The Caenorhabditis elegans voltage-gated calcium channel subunits UNC-2 and UNC-36 and the calcium-dependent kinase UNC43/CaMKII regulate neuromuscular junction morphology. Neural Dev 8:10 Eom HJ, Kim H, Kim BM, Chon TS, Choi J (2014) Integrative assessment of benzene exposure to Caenorhabditis elegans using computational behavior and toxicogenomic analyses. Environ Sci Technol 48(14), 8143-8151 
European Commission (EC) (2000) Directive 2000/60/EC of the European Parliament and of the Council of 23 October 2000 establishing a framework for community action in the field of water policy. EU Water Framework Directive. Official Journal of the European Union (OJ) L 327, pp. 1-73

Félix MA, Braendle C (2010) The natural history of Caenorhabditis elegans. Curr Bio $20,22,965-969$

Forsgren AJ (2015) Wastewater Treatment: Occurrence and Fate of Polycyclic Aromatic Hydrocarbons (PAHs). CRC Press, Boca Raton

Giebner S, Ostermann S, Straskraba S, Oetken M, Oehlmann J, Wagner M (2016)

Effectivity of advanced wastewater treatment: reduction of in vitro endocrine activity and mutagenicity but not of in vivo reproductive toxicity. Environ Sci Pollut Res Int. [Epub ahead of print]. DOI: 10.1007/s11356-016-7540-1

Jones LM, Rayson SJ, Flemming AJ, Urwin, PE (2013) Adaptive and specialised transcriptional responses to xenobiotic stress in Caenorhabditis elegans are regulated by nuclear hormone receptors. PLoS one 8(7), e69956 Hartman PS, Freedman M (2005) Getting Off on DEET: C. elegans as a model system to study an insect repellent. Conference-Abstract. International Worm Meeting, 2005 Hägerbäumer A, Höss S, Heininger P, Traunspurger W (2015) Experimental studies with nematodes in ecotoxicology: an overview. J Nematol 47(1):11-27 Haegerbaeumer A, Höss S, Heininger P, Traunspurger W (2018) Is Caenorhabditis elegans representative of freshwater nematode species in toxicity testing? Environ Sci Pollut Res 25(3), 2879-2888 
685

686

687

688

689

690

691

692

693

694

695

696

697

698

699

700

701

702

703

704

705

Hitchcock DR, Black MC, Williams PL (1997) Investigations into using the nematode Caenorhabditis elegans for municipal and industrial wastewater toxicity testing. Arch Environ Contam Toxicol 33, 252-260

Hitchcock DR, Law SE, Wu J, Williams PL (1998) Determining toxicity trends in the ozonation of synthetic dye wastewaters using the nematode Caenorhabditis elegans. Arch Environ Contam Toxicol 34, 259-264

Höss S, Ahlf W, Bergtold M, Bluebaum-Gronau E, Brinke M, Donnevert G, Menzel R, Möhlenkamp C, Ratte HT, Traunspurger W, von Danwitz B, Pluta HJ (2012) Interlaboratory comparison of a standardized toxicity test using the nematode Caenorhabditis elegans (ISO 10872). Environ Toxicol Chem 31(7):1525-35

Höss S, Bergtold M, Haitzer M, Traunspurger W, Steinberg CE (2001) Refractory dissolved organic matter can influence the reproduction of Caenorhabditis elegans (Nematoda). Freshwater Biology 46, 1-10 Höss S, Weltje L (2007) Endocrine disruption in nematodes: effects and mechanisms. Ecotoxicology 16(1):15-28 Inokuchi A, Nihira M, Minakoshi M, Yamamoto R, Ishibashi H, Tominaga N, Arizono K (2014) Comparative study of the biological effects of antimicrobials, triclosan and trichlocarban, for C. elegans. J Environ Safety 5(2), 95-98 Kao A, Liu R, Gupta D, Feng Z (2016) Amantadine alleviates the motor and dopamine receptor changes in a levodopa-induced dyskinesia (LID) model of C. elegans (P5.373). Neurology 86(16 Supplement), P5.373 
Knopp G, Prasse C, Ternes TA, Cornel P (2016) Elimination of micropollutants and transformation products from a wastewater treatment plant effluent through pilot scale ozonation followed by various activated carbon and biological filters. Water Res 100 , $580-592$

Leung MCK, Williams PL, Benedetto A, Au C, Helmcke KJ, Aschner M, Meyer JN (2008) Caenorhabditis elegans: an emerging model in biomedical and environmental toxicology. Toxicol Sci 106(1), 5-28

Leung MCK, Goldstone J, Boyd W, Freedman J, Meyer J (2010) Caenorhabditis elegans generates biologically relevant levels of genotoxic metabolites from aflatoxin B1 but not benzo[a]pyrene in vivo. Toxicol Sci 118(2):444-453 Lindblom TH, Dodd AK (2006) Xenobiotic detoxification in the nematode Caenorhabditis elegans. J Exp Zool A Comp Exp Biol 305(9):720-30. DOI: 10.1002/jez.a.324 Liu S, Saul N, Pan B, Menzel R, Steinberg CE (2013) The non-target organism Caenorhabditis elegans withstands the impact of sulfamethoxazole. Chemosphere 10, $2373-2380$

Loos R, Carvalho R, António DC, Comero S, Locoro G, Tavazzi S, Paracchini B, Ghiani M, Lettieri T, Blaha L, Jarosova B, Voorspoels S, Servaes K, Haglund P, Fick J, Lindberg RH, Schwesig D, Gawlik BM (2013) EU wide monitoring survey on waste water treatment plant effluents. Water Res 47(17), 6475-87 Magdeburg A, Stalter D, Oehlmann J (2012) Whole effluent toxicity assessment at a wastewater treatment plant upgraded with a full-scale post-ozonation using aquatic key species. Chemosphere 88, 1008-1014 
McLaggan D, Amezaga MR, Petra E, Frost A, Duff El, Rhind SM, Fowler PA, Glover LA, Lagido C (2012) Impact of sublethal levels of environmental pollutants found in sewage sludge on a novel Caenorhabditis elegans model biosensor. PLoS ONE 7(10), e46503 Menzel R, Bogaert T, Achazi R (2001) A systematic gene expression screen of Caenorhabditis elegans cytochrome P450 genes reveals CYP35 as strongly xenobiotic inducible. Arch Biochem Biophys 395, 158-168

Menzel R, Rodel M, Kulas J, Steinberg CE (2005) CYP35: xenobiotically induced gene expression in the nematode Caenorhabditis elegans. Arch Biochem Biophys 438, 93102

Menzel R, Swain SC, Höss S, Claus E, Menzel S, Steinberg CE, Reifferscheid G, Stürzenbaum SR (2009) Gene expression profiling to characterize sediment toxicity - a pilot study using Caenorhabditis elegans whole genome microarrays. BMC Genomics $10,160-174$

Menzel R, Yeo HL, Rienau S, Li S, Steinberg CE, Sturzenbaum SR (2007) Cytochrome P450s and short-chain dehydrogenases mediate the toxicogenomic response of PCB52 in the nematode Caenorhabditis elegans. J Mol Biol 370, 1-13 Min H, Kawasaki I, Gong J, Shim YH (2015) Caffeine induces high expression of cyp$35 \mathrm{~A}$ family genes and inhibits the early larval development in Caenorhabditis elegans. Mol Cells 38(3), 236-242

Offermann K, Matthäi A, Ahlf W (2009) Assessing the importance of dietborne cadmium and particle characteristics on bioavailability and bioaccumulation in the nematode Caenorhabditis elegans. Environ Toxicol Chem 28(6): 1149-1158 
Peris-Vicente J, Roca-Genovés P, Tayeb-Cherif K, Esteve-Romero J (2016)

Development and validation of a method to determine thiabendazole and ophenylphenol in wastewater using micellar liquid chromatography-fluorescence detection. Electrophoresis 37(19), 2517-2521

Petersen Cl, McFarland TR, Stepanovic SZ, Yang P, Reiner DJ, Hayashi K, George AL, Roden DM, Thomas JH, Balser JR (2004) In vivo identification of genes that modify ether-a-go-go-related gene activity in Caenorhabditis elegans may also affect human cardiac arrhythmia. Proc Natl Acad Sci 101(32): 11773-11778

Petrie B, Barden R, Kasprzyk-Hordern B (2015) A review on emerging contaminants in wastewaters and the environment: current knowledge, understudied areas and recommendations for future monitoring. Water $\operatorname{Res} 72,3-27$

Prasse C, Stalter D, Schulte-Oehlmann U, Oehlmann J, Ternes TA (2015) Spoilt for choice: a critical review on the chemical and biological assessment of current wastewater treatment technologies. Water Res 87:237-270

Quevauviller P, Thomas O, Van Der Beken A (2007). Wastewater quality monitoring and treatment (Vol. 13). John Wiley \& Sons, Hoboken

Reichert K, Menzel R (2005) Expression profiling of five different xenobiotics using a Caenorhabditis elegans whole genome microarray. Chemosphere 61, 229-237

Ristau K, Akgül Y, Bartel AS, Fremming J, Müller MT, Reiher L, Stapela F, Splett JP, Spann N (2015) Toxicity in relation to mode of action for the nematode Caenorhabditis elegans: acute-to-chronic ratios and quantitative structure-activity relationships. Environ Toxicol Chem, 34(10): 2347-2353 
Roh J, Lee H, Kwon J (2014) Changes in the expression of cyp35a family genes in the soil nematode Caenorhabditis elegans under controlled exposure to chlorpyrifos using passive dosing. Environ Sci Technol 48(17):10475-10481 Seitz W, Winzenbacher R (2017) A survey on trace organic chemicals in a German water protection area and the proposal of relevant indicators for anthropogenic influences. Environ Monit Assess 189(6), 244

Sinclair CJ, Boxall AB (2003) Assessing the ecotoxicity of pesticide transformation products. Environ Sci Technol 37(20), 4617-4625

Stalter D, Peters LI, O'Malley E, Tang JY, Revalor M, Farré MJ, Watson K, von Gunten U, Escher BI (2016) Sample enrichment for bioanalytical assessment of disinfected drinking water: concentrating the polar, the volatiles, and the unknowns. Environ Sci Technol 50(12):6495-505 Tang JY, Busetti F, Charrois JW, Escher BI (2014) Which chemicals drive biological effects in wastewater and recycled water? Water Res 60, 289-299 Völker J, Vogt T, Castronovo S, Wick A, Ternes TA, Joss A, Oehlmann J, Wagner M (2017) Extended anaerobic conditions in the biological wastewater treatment: Higher reduction of toxicity compared to target organic micropollutants. Water Res 116, 220230

Wernersson AS, Carere M, Maggi C, Tusil P, Soldan P, James A, Sanchez W, Dulio V, Broeg K, Reifferscheid G, Buchinger S, Maas H, Van Der Grinten E, O'Toole S, Ausili A, Manfra L, Marziali L, Polesello S, Lacchetti I, Mancini L, Lilja K, Linderoth M, Lundeberg T, Fjällborg B, Porsbring T, Larsson J, Bengtsson-Palme J, Förlin L, Kienle C, Kunz P, 
794 Vermeirssen E, Werner I, Robinson CD, Lyons B, Katsiadaki I, Whalley C, den Haan K,

795 Messiaen M, Clayton H, Lettieri T, Negrão Carvalho R, Gawlik BM, Hollert H, Di Paolo

796 C, Brack W, Kammann U, Kase R (2015) The European technical report on aquatic

797 effect-based monitoring tools under the water framework directive. Environ Sci Eur

$798 \quad 27(1), 1-11$

799 Wilson MJ, Khakouli-Duarte T (2009) Nematodes as environmental indicators. CABI,

800 Wallingford 


\section{Supplementary information (paper A.2)}

Online Resource 1: Tab. Characteristics of wastewater treatment plants (WWTPs) 1-4, including population equivalents (PE), connected PE (in brackets), WWTP capacity and amount of treated wastewater (WW), hydraulic retention time (HRT) in the conventional-biological stage, average sludge age in the activated sludge treatment, approximate wastewater fraction in receiving water body and further specifications. "n.d." = not detected

\begin{tabular}{|c|c|c|c|c|c|c|}
\hline WWTP & PE & $\begin{array}{c}\text { Treated } \\
W W\left[\mathrm{~m}^{3} / \mathrm{a}\right]\end{array}$ & $\begin{array}{l}\text { HRT } \\
{[\mathrm{h}]}\end{array}$ & $\begin{array}{l}\text { Sludge } \\
\text { age [d] }\end{array}$ & $\begin{array}{l}\text { Receiving } \\
\text { water body }\end{array}$ & Further specifications \\
\hline 1 & $\begin{array}{l}440,000 \\
\text { (n.d.) }\end{array}$ & $38.8 \times 10^{6}$ & 19 & 14 & $\begin{array}{c}\sim 6 \% \\
\text { wastewater }\end{array}$ & $\begin{array}{l}\text { Catchment area in metropolitan } \\
\text { area, including commercial } \\
\text { districts and hospitals. Receiving } \\
\text { surface water used as drinking } \\
\text { water resource. }\end{array}$ \\
\hline 2 & $\begin{array}{c}16,000 \\
(10,000)\end{array}$ & $1.5 \times 10^{6}$ & $9-16$ & 14 & $\begin{array}{c}\sim 50 \% \\
\text { wastewater }\end{array}$ & $\begin{array}{l}\text { Receiving water body with high } \\
\text { groundwater infiltration. }\end{array}$ \\
\hline 3 & $\begin{array}{c}16,600 \\
(14,000)\end{array}$ & $2.5 \times 10^{6}$ & $9-15$ & 11 & $\begin{array}{c}\sim 18 \% \\
\text { wastewater }\end{array}$ & $\begin{array}{l}\text { Periodically high proportion of } \\
\text { external WW (from a hospital). }\end{array}$ \\
\hline 4 & $\begin{array}{l}50,000 \\
(42,000)\end{array}$ & $2.3 \times 10^{6}$ & 45 & $12-18$ & n.d. & $\begin{array}{l}\text { WWTP connected to a pilot } \\
\text { WWTP with advanced WW } \\
\text { treatment. Catchment area with } \\
\text { commercial and hospital } \\
\text { wastewater discharges. }\end{array}$ \\
\hline
\end{tabular}


Online Resource 2: Tab. Selected wastewater and process parameters of WWTP-4. Parameters included: $\mathrm{pH}$, electric conductivity, spectral absorption coefficient at $254 \mathrm{~nm}\left(\mathrm{UV}_{254}\right)$, dissolved organic carbon (DOC), chemical oxygen demand (COD), filtered chemical oxygen demand $\left(\mathrm{COD}_{0.45 \mu \mathrm{m}}\right)$, ammonium $\left(\mathrm{NH}_{4}{ }^{+}\right)$, nitrate $\left(\mathrm{NO}_{3}{ }^{-}\right)$, nitrite $\left(\mathrm{NO}_{2}{ }^{-}\right)$, total phosphorous $\left(\mathrm{P}_{\text {total }}\right)$, hydraulic retention time of ozone $\left(\mathrm{O}_{3}-\mathrm{HRT}\right)$, ozone dose $(\mathrm{D})$, specific ozone dose (d), ozone consumption ( $\mathrm{Z}$ ), specific ozone consumption ( $\mathrm{z}$ ), filtration speed $\left(\mathrm{V}_{\mathrm{F}}\right)$ and empty bed contact time (EBCT). Parameters were determined in $24 \mathrm{~h}$ composite (CS) or grab samples (GS) from April 14 ${ }^{\text {th }}, 2015$. A few grab samples were taken on April $16^{\text {th }}, 2015$. The average of both samples is reported $\left(\mathrm{GS}_{2}\right)$. "n.a." = not applicable. "n.d." = not detected. LOQ are indicated with "<"

\begin{tabular}{|c|c|c|c|c|c|c|c|c|}
\hline \multirow{2}{*}{$\begin{array}{c}\text { Sample } \\
\text { type }\end{array}$} & \multirow{2}{*}{ Parameter } & \multicolumn{7}{|c|}{ Sampling point } \\
\hline & & INF-4 & EFF-4 & $\mathrm{EFF}+\mathrm{O}_{3}$ & $\mathrm{O}_{3}+\mathrm{GAC}$ & $\mathrm{O}_{3}+\mathrm{GAC}_{\mathrm{a}}$ & $\mathrm{O}_{3}+\mathrm{BF}$ & $\mathrm{O}_{3}+\mathrm{BF}_{\mathrm{a}}$ \\
\hline $\mathrm{GS}_{2}$ & $\mathrm{pH}$ & 7.59 & 7.33 & n.d. & n.d. & n.d. & n.d. & n.d. \\
\hline $\mathrm{GS}_{2}$ & $\begin{array}{l}\text { Conductivity } \\
{[\mu \mathrm{S} / \mathrm{cm}]}\end{array}$ & 1601 & 1213 & n.d. & n.d. & n.d. & n.d. & n.d. \\
\hline $\mathrm{CS}$ & $\mathrm{SAC}_{254}$ & 0.66 & 0.22 & 0.09 & 0.06 & 0.05 & 0.08 & 0.07 \\
\hline $\mathrm{GS}_{2}$ & $U_{254}$ & 0.67 & 0.22 & 0.08 & 0.25 & 0.05 & 0.06 & 0.06 \\
\hline CS & DOC [mg/L] & 96.5 & 13.4 & 12.2 & 8.3 & 7.6 & 9.7 & 9.0 \\
\hline $\mathrm{GS}_{2}$ & DOC [mg/L] & 105 & 13.5 & 13.1 & 8.1 & 7.9 & 9.2 & 8.9 \\
\hline CS & $\begin{array}{c}\mathrm{COD} / \mathrm{COD}_{0,45 \mu \mathrm{m}} \\
{[\mathrm{mg} / \mathrm{L}]}\end{array}$ & $938 / 309$ & 32.4 & 27.7 & 18.1 & 16.8 & 21.9 & 19.7 \\
\hline $\mathrm{GS}_{2}$ & $\begin{array}{c}\mathrm{COD} / \mathrm{COD}_{0,45 \mu \mathrm{m}} \\
{[\mathrm{mg} / \mathrm{L}]}\end{array}$ & $935 / 325$ & 33.1 & 27.8 & 17.7 & 17.2 & 20.3 & 19.9 \\
\hline GS & $P_{\text {total }}[\mathrm{mg} / \mathrm{L}]$ & 13.0 & 0.65 & 0.64 & 0.66 & 0.76 & 0.63 & 0.81 \\
\hline GS & $\mathrm{NH}_{4}{ }^{+}[\mathrm{mg} / \mathrm{L}]$ & 67.5 & 0.12 & 0.17 & $<0.015$ & $<0.015$ & $<0.015$ & $<0.015$ \\
\hline GS & $\mathrm{NO}_{2}-[\mathrm{mg} / \mathrm{L}]$ & 0.04 & 0.15 & $<0.015$ & $<0.015$ & $<0.015$ & $<0.015$ & $<0.015$ \\
\hline GS & $\mathrm{NO}_{3}{ }^{-}[\mathrm{mg} / \mathrm{L}]$ & 0.5 & 1.64 & 1.89 & 2.32 & 2.44 & 2.25 & 2.36 \\
\hline GS & $\mathrm{O}_{3}-\mathrm{HRT}$ [min] & n.a. & n.a. & 16.7 & n.a. & n.a. & n.a. & n.a. \\
\hline GS & $D\left[g / m^{3}\right]$ & n.a. & n.a. & 13.8 & n.a. & n.a. & n.a. & n.a. \\
\hline GS & $\mathrm{d}\left[\mathrm{g}\left(\mathrm{O}_{3}\right) / \mathrm{g}(\mathrm{DOC})\right]$ & n.a. & n.a. & 1.0 & n.a. & n.a. & n.a. & n.a. \\
\hline GS & $\mathrm{Z}\left[\mathrm{g} / \mathrm{m}^{3}\right]$ & n.a. & n.a. & 13.5 & n.a. & n.a. & n.a. & n.a. \\
\hline GS & $z\left[g\left(\mathrm{O}_{3}\right) / g(\mathrm{DOC})\right]$ & n.a. & n.a. & 1.0 & n.a. & n.a. & n.a. & n.a. \\
\hline GS & $V_{F}[m / h]$ & n.a. & n.a. & n.a. & 4.8 & 5.5 & 4.7 & 5.5 \\
\hline GS & EBCT [min.] & n.a. & n.a. & n.a. & 25.3 & 23.7 & 29.2 & 25.8 \\
\hline
\end{tabular}




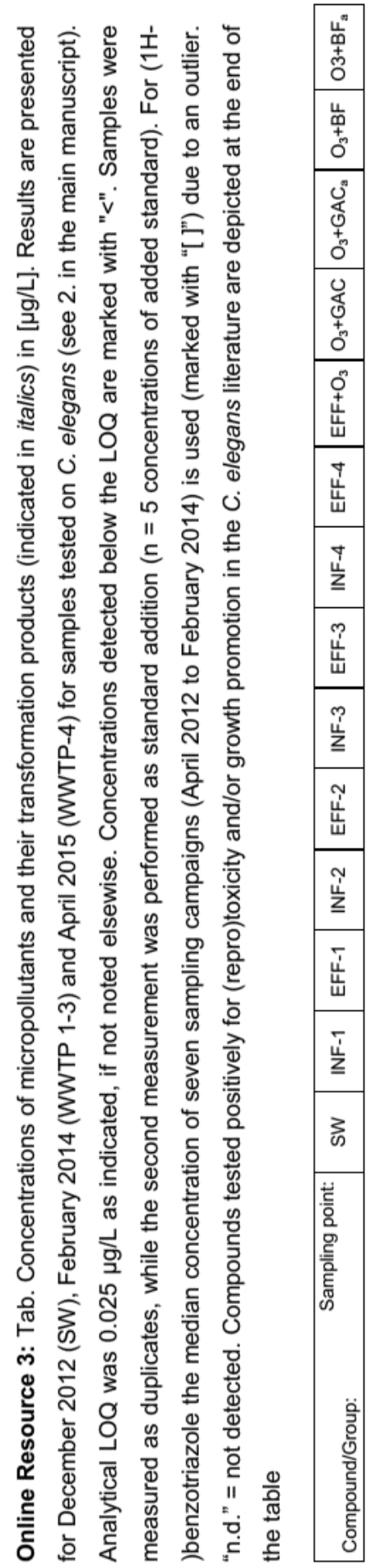

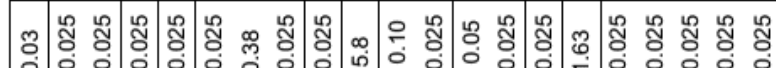

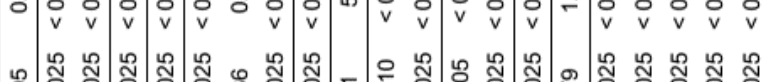

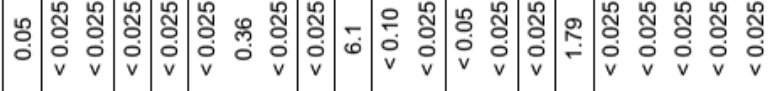

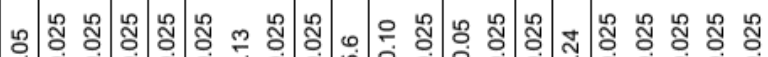

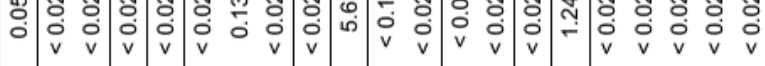

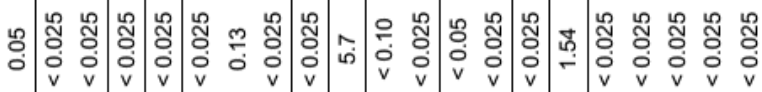

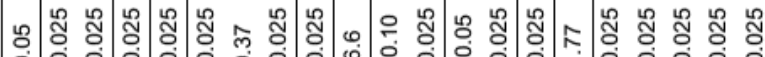

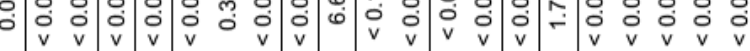

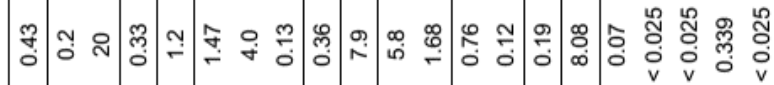

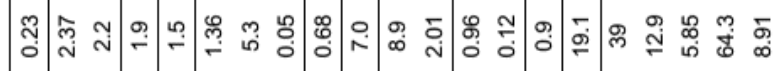
万人 产号员

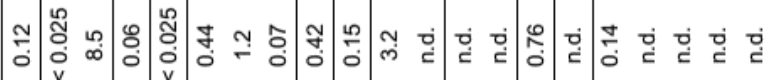

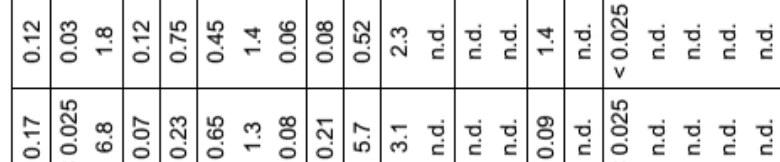
望望管

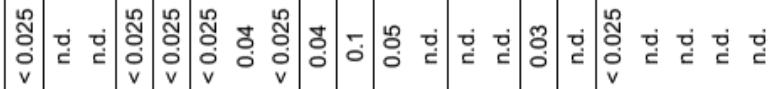

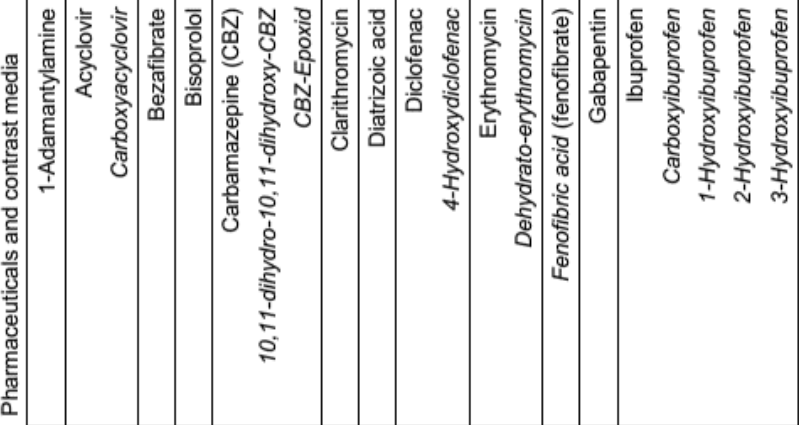




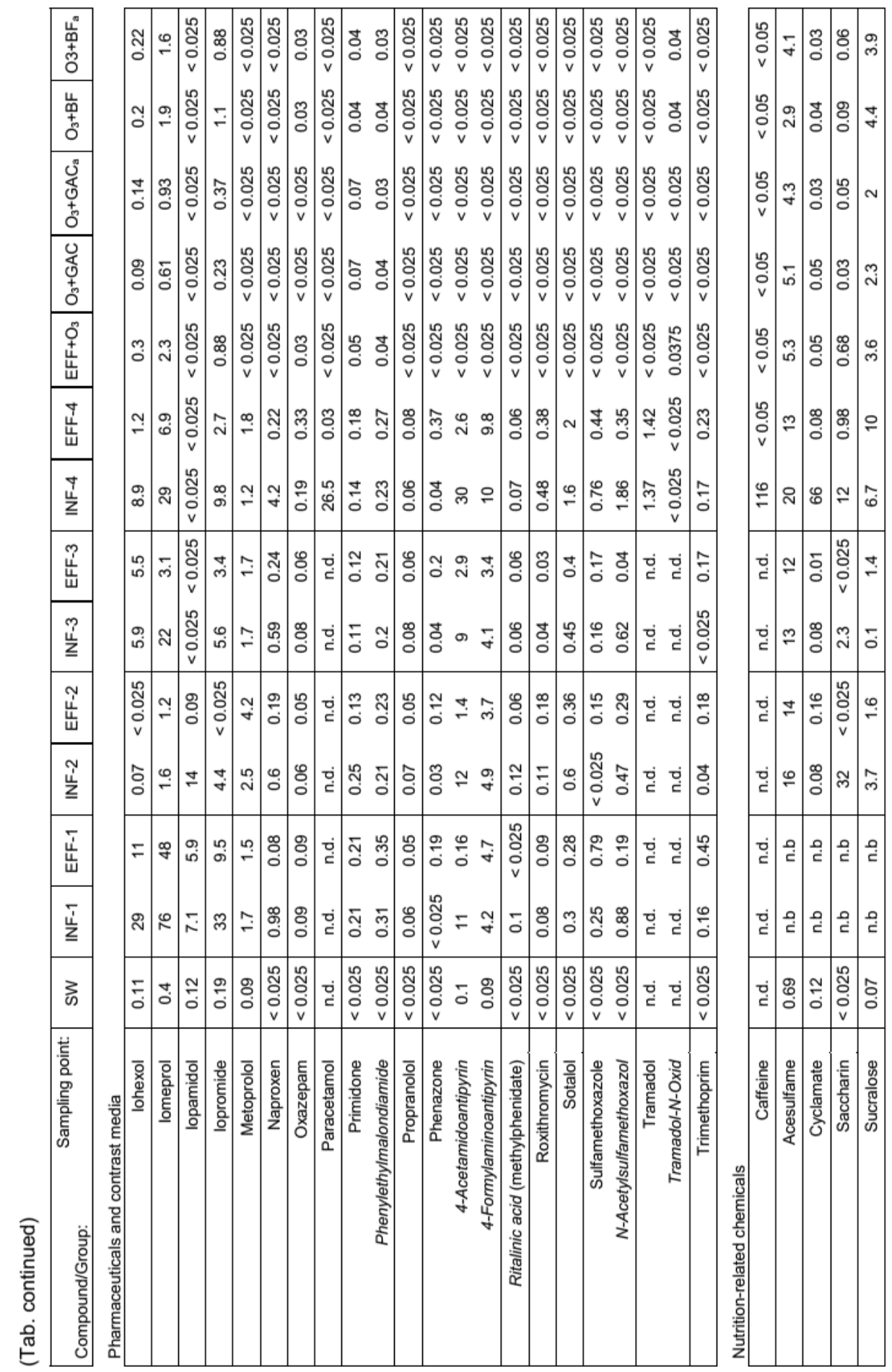




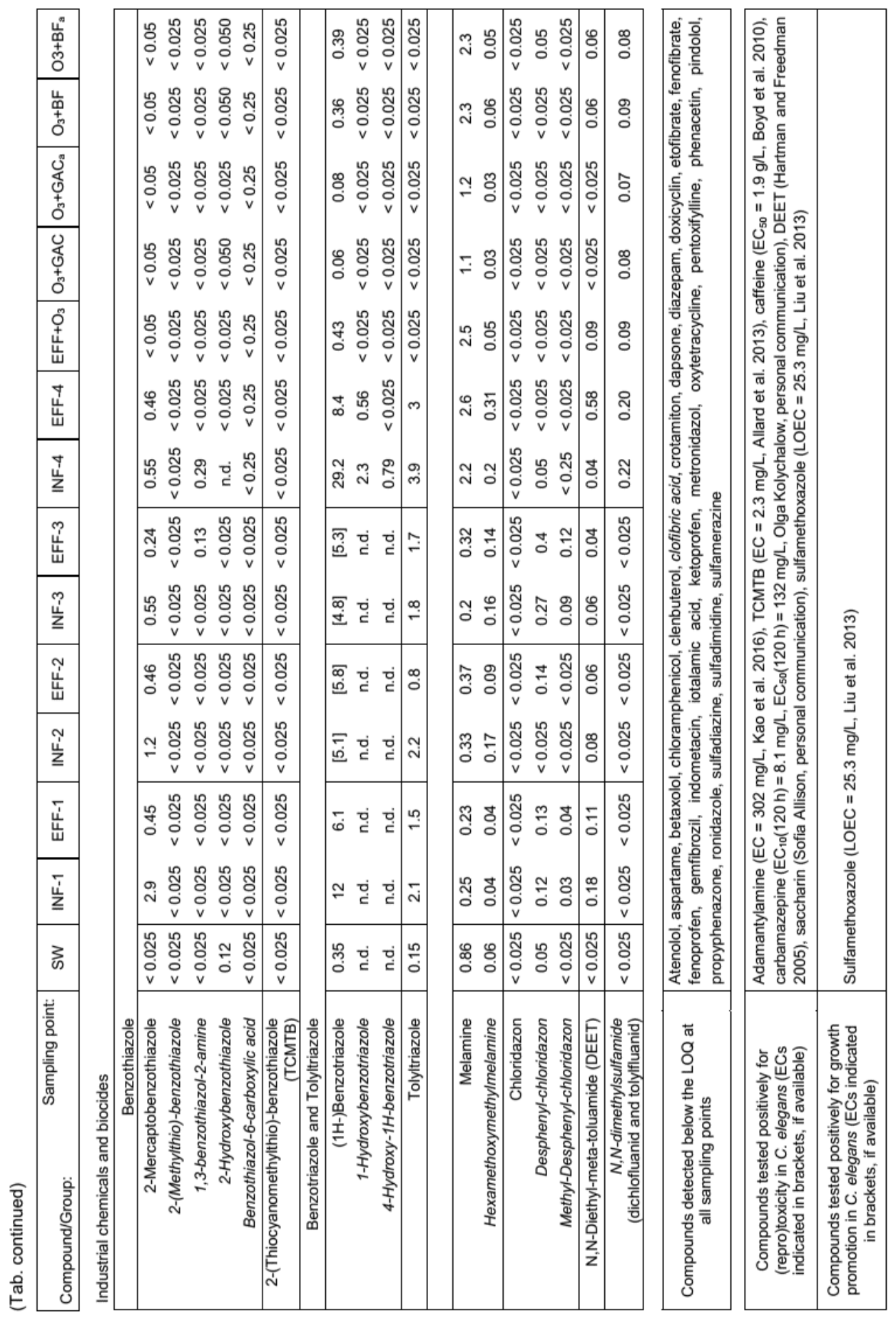




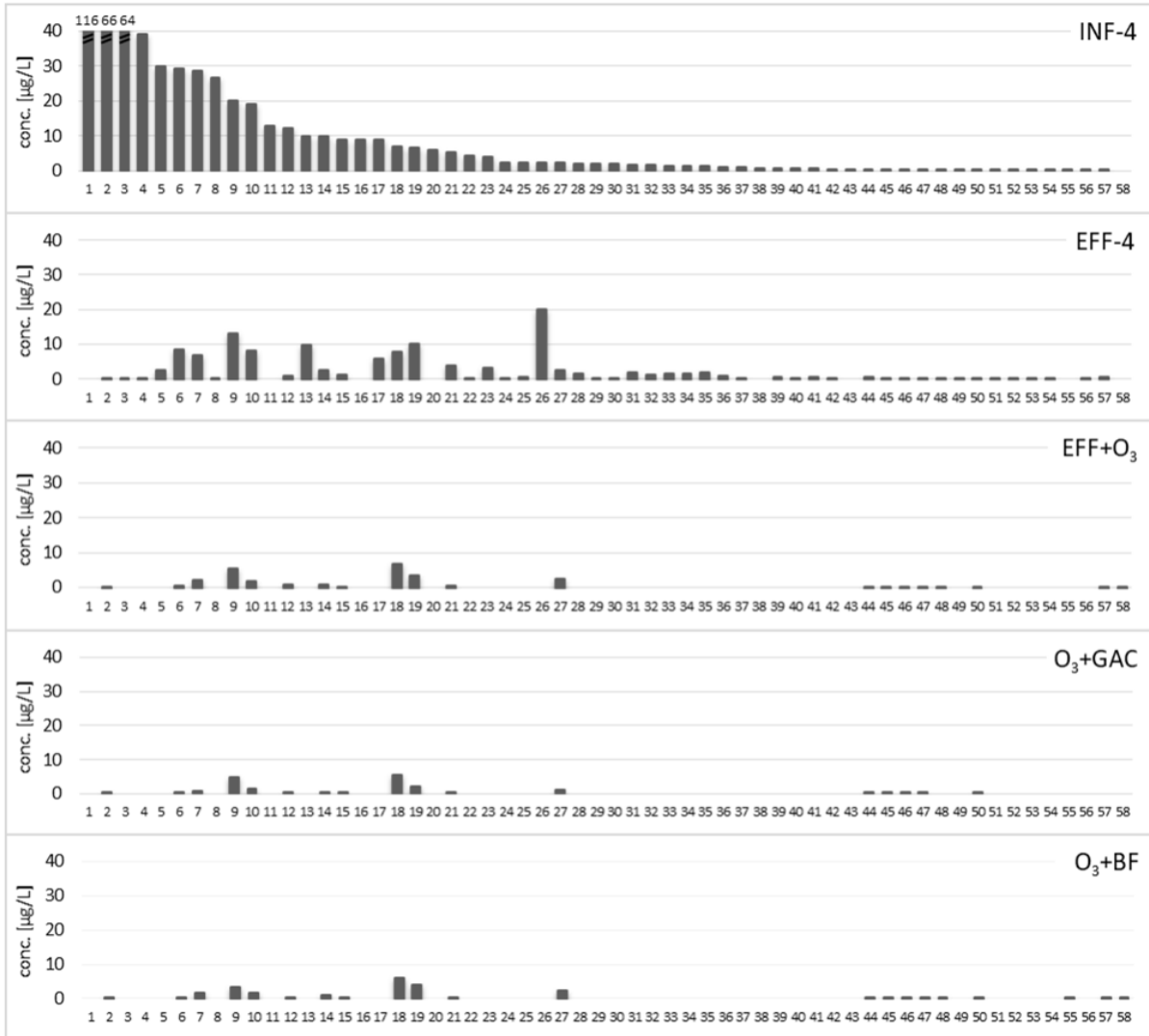

Online Resource 4: Fig. Micropollutant removal during conventional and advanced wastewater treatment at WWTP-4. Concentrations (conc., in [ $\mu \mathrm{g} / \mathrm{L}], \mathrm{y}$-axes) of micropollutants and transformation products $(\mathrm{n}=$ 58) were quantified for samples from April 2015. WWTP stages are WWTP influent (INF-4), WWTP effluent (EFF-4), ozonated effluent $\left(\mathrm{EFF}+\mathrm{O}_{3}\right)$, granulated activated carbon filtration or biofiltration of ozonated WWTP effluent $\left(\mathrm{O}_{3}+\mathrm{GAC}, \mathrm{O}_{3}+\mathrm{BF}\right)$. Post-filtrations by $\mathrm{GAC}$ and $\mathrm{BF}$ are given as mean of the aerated and non-aerated systems due to equivalent removal efficiencies (compare 3.5 and 4.4 in the main manuscript). Compound annotations (Transformation products indicated in italics): caffeine (1), cyclamate (2), 2hydroxyibuprofen (3), ibuprofen (4), 4-acetamidoantipyrin (5), (1H-)benzotriazole (6), iomeprol (7), paracetamol (8), acesulfame (9), gabapentin (10), carboxyibuprofen (11), saccharin (12), 4formylaminoantipyrin (13), iopromide (14), iohexol (15), 3-hydroxyibuprofen (16), diclofenac (17), diatrizoic acid (18), sucralose (19), 1-hydroxyibuprofen (20), 10,11-dihydro-10,11-dihydroxycarbamazepin (21), naproxen (22), tolyltriazole (23), acyclovir (24), 1-hydroxybenzotriazol (25), carboxyacyclovir (26), melamine (27), 4-hydroxydiclofenac (28), bezafibrate (29), N-acetylsulfamethoxazol (30), sotalol (31), bisoprolol (32), tramadol (33), carbamazepine (34), metoprolol (35), erythromycin (36), fenofibric acid (37), 4-hydroxy-1H-benzotriazol (38), sulfamethoxazole (39), clarithromycin (40), 2-(methylthio)-benzothiazole (41), roxithromycin (42), 2-hydroxybenzothiazol (43), 1-adamantylamine (44), primidone (45), N,Ndimethylsulfamide (46), hexamethoxymethylmelamine (47), oxazepam (48), trimethoprim (49), PEMA (phenylethylmalondiamide) (50), dehydrato-erythromycin (51), ritalinic acid (52), propranolol (53), carbamazepine-epoxid (54), desphenyl-chloridazon (55), phenazone (56), N,N-diethyl-meta-toluamide (DEET) (57), tramadol-N-oxid (58) 


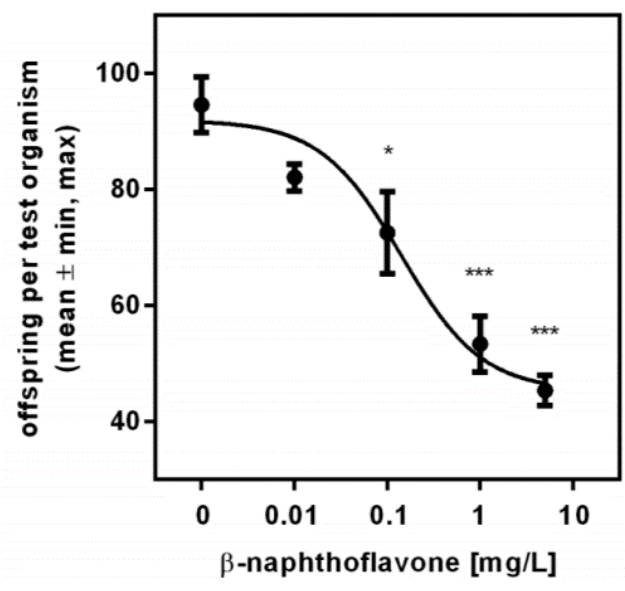

Online Resource 5: Fig. Concentration-response-curve of $\beta$-naphthoflavone for the endpoint brood size of C. elegans. The mean number of offspring per adult was quantified after $96 \mathrm{~h}$ of exposure to $\beta$-NF $(0.01-$ $5.0 \mathrm{mg} / \mathrm{L})$ commencing at the L1 stage. Significant differences $\left({ }^{*}=p<0.05,{ }^{* * *}=p<0.001\right)$ were tested against the control (M9 media, indicated as $0 \mathrm{mg} / \mathrm{L} \beta$-naphthoflavone) by one-way ANOVA with Tukey's post-hoc analysis. The median effective concentration $\mathrm{EC}_{50}(0.14 \mathrm{mg} / \mathrm{L})$ was derived using a logistic regression model $\left(y=45.3+46.6 /\left(1+10^{\lg (x)+0.85}\right), R^{2}=0.74\right)$ 


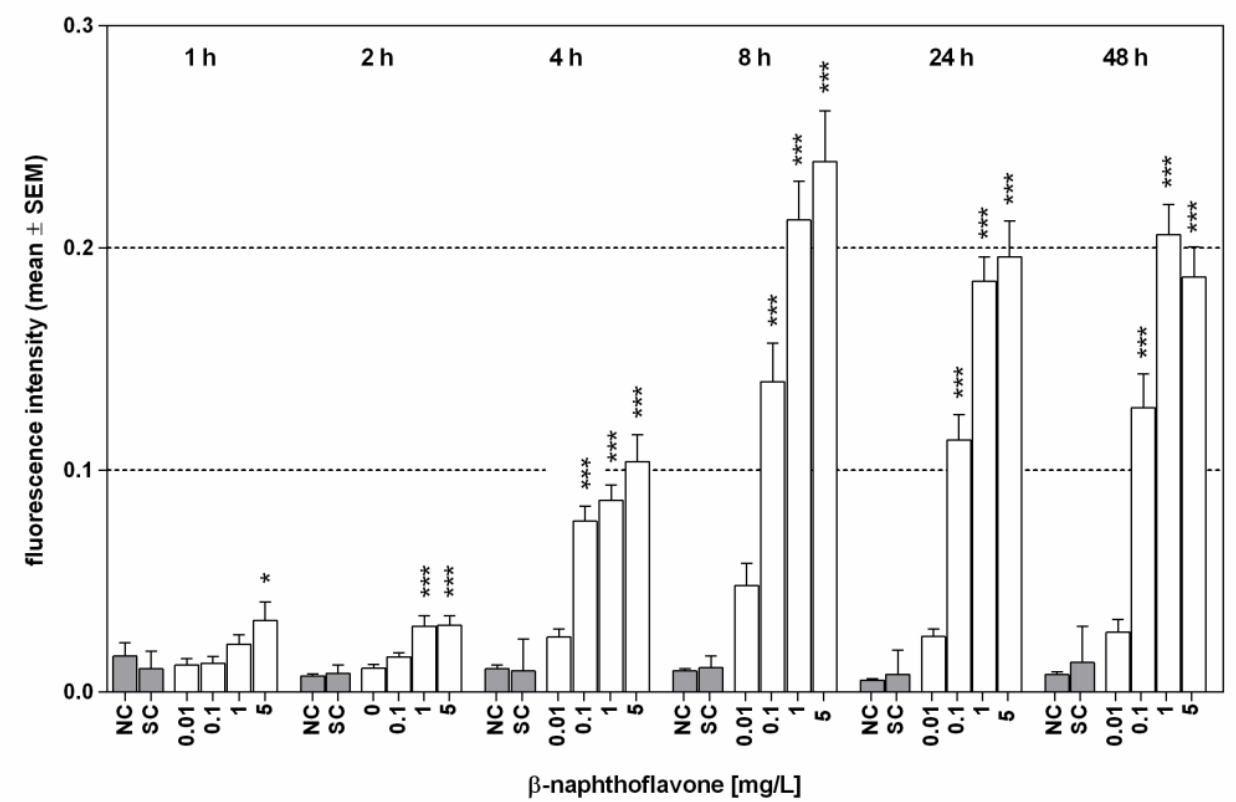

Online Resource 6: Fig. Impacts of $\beta$-naphthoflavone on cyp-35A3::GFP expression in transgenic C. elegans. Samples were analyzed after $1-48 \mathrm{~h}$ exposure of adult hermaphrodites to $0.01-5.0 \mathrm{mg} / \mathrm{L} \beta$ naphthoflavone. Results pooled from two experiments ( $n=25$ per treatment). Significant differences ( ${ }^{*} p<$ 0.05 , ${ }^{* *} p<0.001$ ) were tested against the controls of each time point by one-way ANOVA followed by Dunett's comparison test. NC $=$ M9 medium. Solvent control $(\mathrm{SC})=0.2 \%$ DMSO in M9 medium. Median effective concentrations $\mathrm{EC}_{50}$ for the $8 \mathrm{~h}(71.5 \mu \mathrm{g} / \mathrm{L})$ and $24 \mathrm{~h}(78.6 \mu \mathrm{g} / \mathrm{L})$ time point were derived using logistic regression models $\left(8 \mathrm{~h}: \mathrm{y}=-0.0039+0.26 /\left(1+10^{(-1.15-\lg (\mathrm{x}))^{*} 0.67}\right), \mathrm{R}^{2}=0.68\right.$ and $24 \mathrm{~h}: \mathrm{y}=0.0046+$ $0.19 /\left(1+10^{\left.\left.(-1.11-\lg (x))^{*} 1.03\right), R^{2}=0.69\right)}\right.$ 


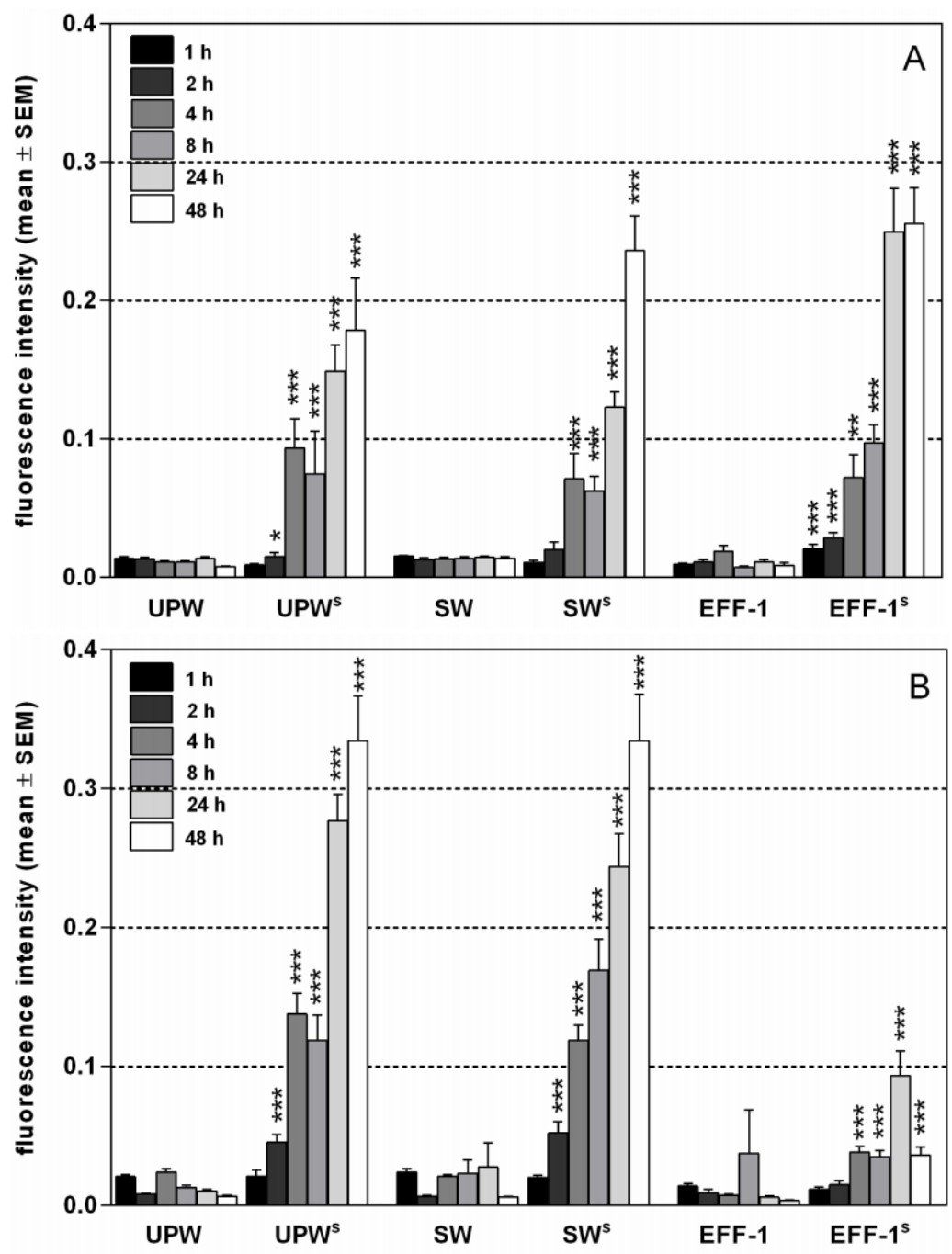

Online Resource 7: Fig. Impacts of aqueous (A) and extracted (B) ultrapure water (UPW), surface water (SW) and wastewater treatment plant effluent (EFF-1) on cyp-35A3::GFP expression in transgenic C. elegans. Aqueous and extracted samples were analyzed in 0.5 and 10-fold concentrations (respectively) after 1-48 $\mathrm{h}$ of exposure of adult hermaphrodites. Spiked aqueous samples (marked by superscript s) contained $0.5 \mathrm{mg} / \mathrm{L} \beta$-naphthoflavone. Results pooled from two experiments ( $\mathrm{n}=15$ per treatment group). Significant differences $\left({ }^{*} p<0.05,{ }^{* *} p<0.01,{ }^{* * *} p<0.001\right)$ were tested aqueous against spiked samples of each time point $(A, B)$ by unpaired t-test 


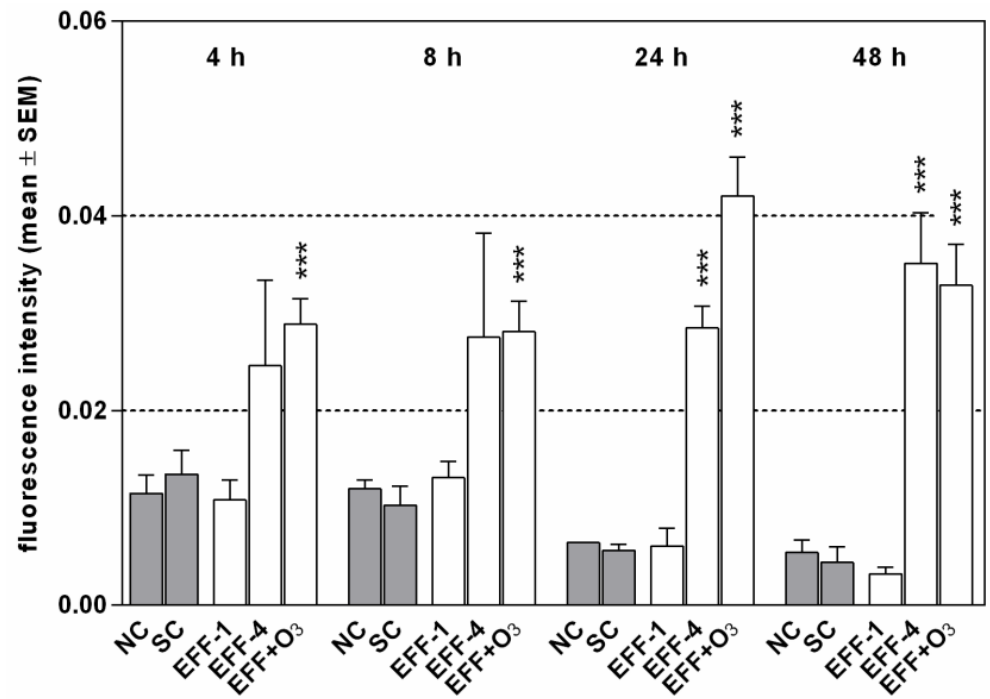

Online Resource 8: Fig. Impacts of extracted wastewater treatment plant effluents (EFF-1, EFF-4) and ozonated effluent $\left(\mathrm{EFF}+\mathrm{O}_{3}\right)$ on cyp-35A3::GFP expression in transgenic $\mathrm{C}$. elegans. Extracted samples (white bars) were analyzed in 10-fold concentrations after 4-48 $\mathrm{h}$ of exposure of adult hermaphrodites. Results pooled from two experiments $\left(n=10\right.$ per treatment group). Significant differences $\left({ }^{\star \star \star} p<0.001\right)$ tested against controls of each time point by one-way ANOVA followed by Dunett's comparison test. $\mathrm{NC}=\mathrm{M} 9$ medium. Solvent control $(\mathrm{SC})=0.2 \% \mathrm{DMSO}$ in M9 medium 


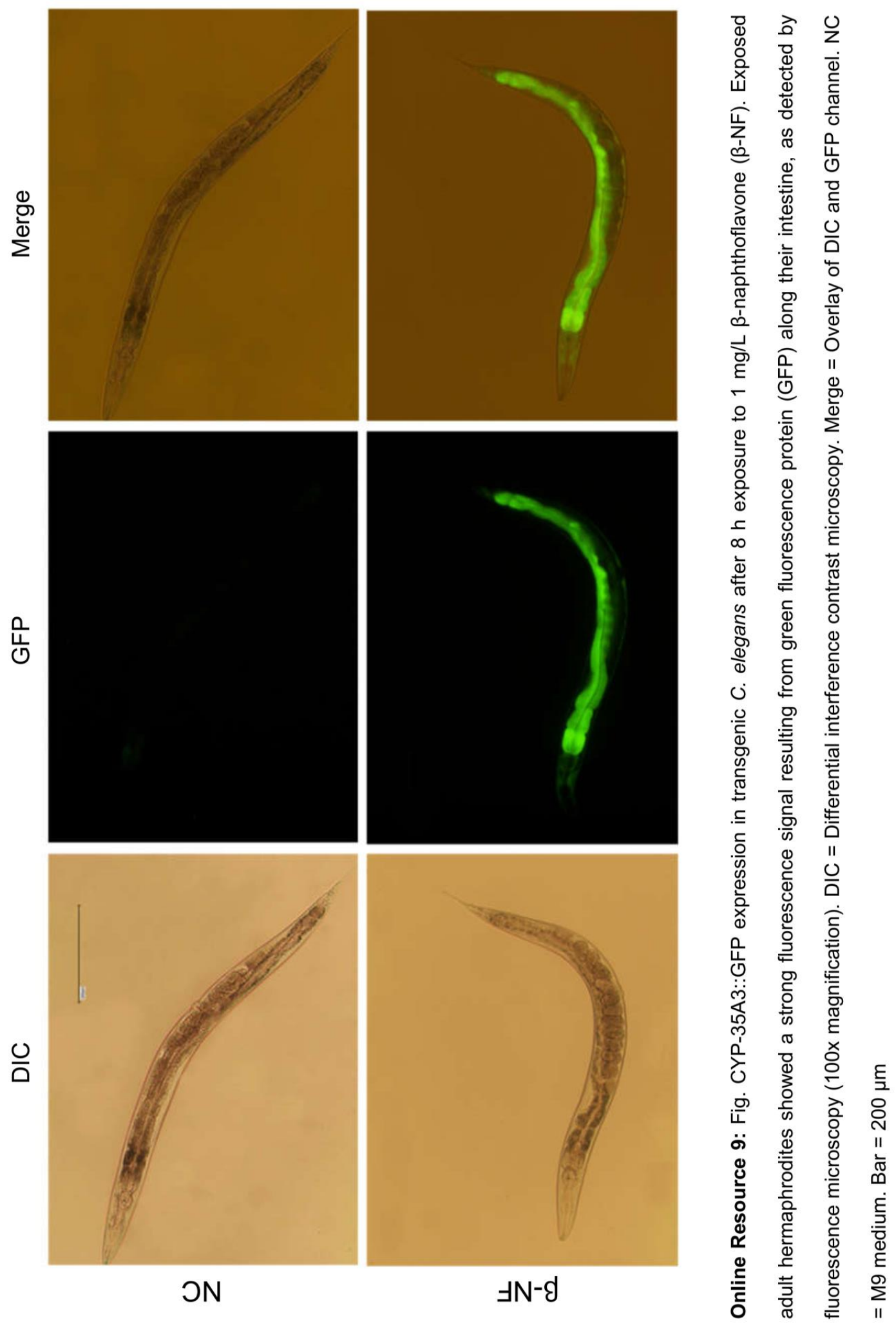




\section{A.3 Post-treatment of ozonated wastewater with activated carbon and biofiltration compared to membrane bioreactors: Toxicity removal in vitro and in Potamopyrgus antipodarum}

Ilona Schneider, Aennes Abbas, Anna Bollmann, Andrea Dombrowski, Gregor Knopp, Ulrike Schulte-OehImann, Wolfram Seitz, Martin Wagner, Jörg OehImann

Water Research 185, 116104

(First Online: 06 Aug 2020) 


\section{Declaration (paper A.3)}

Author contributions to the publication: "Post-treatment of ozonated wastewater with activated carbon, biofiltration and membrane bioreactors: Toxicity removal in vitro and in Potamopyrgus antipodarum"

Status: accepted \& online

Journal: Water Research

Contributing authors (names and distinct initials):

- Ilona Schneider (IS)

- Aennes Abbas (AA)

- Anna Bollmann (AB)

- Gregor Knopp (GK)

- Andrea Dombrowski (AD)

- Wolfram Seitz (WS)

- Ulrike Schulte-Oehlmann (US)

- Martin Wagner (MW)

- Jörg Oehlmann (JO)

Individual contributions of the first author and co-authors:

\section{Study conception and design:}

First author (IS): $75 \%$

Doctoral candidate (AA): $10 \%$

Coauthors (JO, US): $10 \%$

Coauthors (GK, MW): $5 \%$

\section{Performance of experiments and assays}

First author (IS): $75 \%$

Doctoral candidate (AA): $20 \%$

Other coauthors (AB, AD, WS): $5 \%$

\section{Compilation of data sets and tables/figures:}

First author (IS): $90 \%$

Doctoral candidate (AA): $5 \%$

Other coauthors (MW, AB, WS): $5 \%$

\section{Data analyses and interpretation:}

First author (IS): $80 \%$

Doctoral candidate (AA): $10 \%$

Other coauthors (MW, JO): 10\%

\section{Drafting of the Manuscript:}

First author (IS): $70 \%$

Doctoral candidate (AA): $20 \%$

Other coauthors (MW, JO): 10\% 


\title{
Author accepted manuscript (paper A.3)
}

\author{
Journal Pre-proof

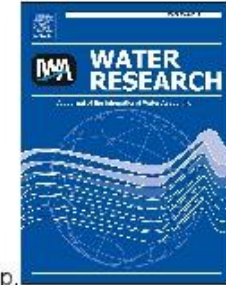 \\ Post-treatment of ozonated wastewater with activated carbon and biofiltration \\ compared to membrane bioreactors: Toxicity removal in vitro and in Potamopyrgus \\ antipodarum \\ Ilona Schneider, Aennes Abbas, Anna Bollmann, Andrea Dombrowski, Gregor Knopp, \\ Ulrike Schulte-Oehlmann, Wolfram Seitz, Martin Wagner, Jörg Oehlmann \\ PII: \\ S0043-1354(20)30641-2 \\ DOl: $\quad$ https://doi. org/10.1016/j.watres.2020.116104 \\ Reference: WR 116104 \\ To appear in: Water Research \\ Received Date: 9 April 2020 \\ Revised Date: 7 June 2020 \\ Accepted Date: 22 June 2020
}

\begin{abstract}
Please cite this article as: Schneider, I., Abbas, A., Bollmann, A., Dombrowski, A., Knopp, G., SchulteOehlmann, U., Seitz, W. Wagner, M., Oehlmann, Jö., Post-treatment of ozonated wastewater with activated carbon and biofiltration compared to membrane bioreactors: Toxicity removal in vitro and in Potamopyrgus antipodarum, Water Research (2020), doi: https://doi.org/10.1016/j. watres.2020.116104

This is a PDF file of an article that has undergone enhancements after acceptance, such as the addition of a cover page and metadata, and formatting for readability, but it is not yet the definitive version of record. This version will undergo additional copyediting, typesetting and review before it is published in its final form, but we are providing this version to give early visibility of the article. Please note that, during the production process, errors may be discovered which could affect the content, and all legal disclaimers that apply to the joumal pertain.

(c) 2020 Published by Elsevier Ltd.
\end{abstract}

Author Accepted Manuscript of Schneider et al. 2020: " Post-treatment of ozonated wastewater with activated carbon and biofiltration compared to membrane bioreactors: Toxicity removal in vitro and in Potamopyrgus antipodarum", Water Research 185, 116104, https://doi.org/10.1016/j.watres.2020.116104

Published under a Creative Commons Attribution - NonCommercial - NoDerivatives (CC-BY-NC-ND) 4.0 License https://creativecommons.org/licenses/by-nc-nd/4.0 


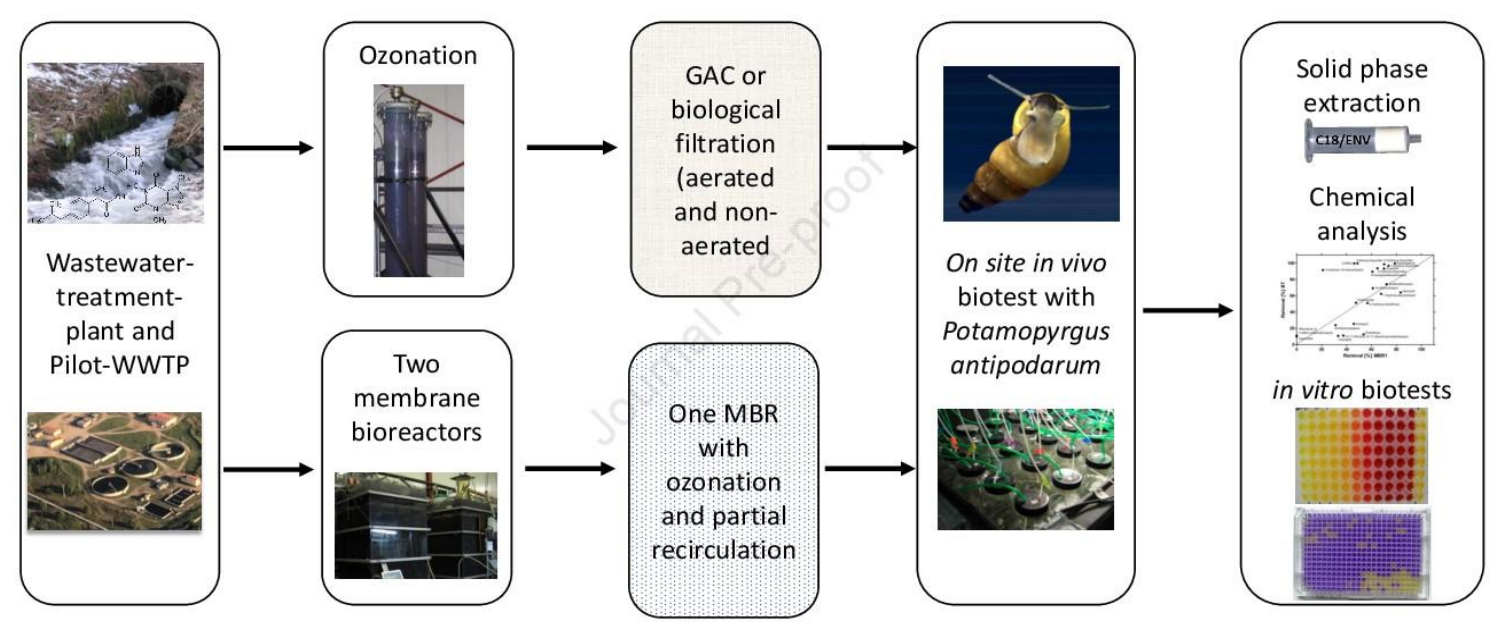


compared to membrane bioreactors: Toxicity removal in vitro and in 3 Potamopyrgus antipodarum

5 Ilona Schneider ${ }^{\mathrm{a},}$, Aennes Abbas ${ }^{\mathrm{a}}$, Anna Bollmann ${ }^{\mathrm{b}}$, Andrea Dombrowski ${ }^{\mathrm{a}}$, Gregor Knopp ${ }^{\mathrm{c}, 1}$,

6 Ulrike Schulte-Oehlmann ${ }^{\mathrm{a}}$, Wolfram Seitz ${ }^{\mathrm{b}}$, Martin Wagner ${ }^{\mathrm{a}, 2}$, Jörg Oehlmann ${ }^{\mathrm{a}}$

8 " Department Aquatic Ecotoxicology, Goethe University Frankfurt am Main, Max-von Laue-Straße

9 13, D-60438 Frankfurt am Main, Germany

10 b Zweckverband Landeswasserversorgung, Am Spitzigen Berg 1, D-89129 Langenau, Germany

11 ' Department of Wastewater Technology and Water Reuse, Technische Universität Darmstadt,

12 Franziska-Braun-Str. 7, D-64287 Darmstadt, Germany

$13 \quad{ }^{1}$ Unger Ingenieure, Julius-Reiber-Straße 19, D-64293 Darmstadt, Germany

$14{ }^{2}$ Department of Biology, Norwegian University of Science and Technology, Høgskoleringen 5,

15 Realfagbygget, NO-7491, Trondheim, Norway

$17 *$ corresponding author. E-mail address: I.Schneider@ bio.uni-frankfurt.de 
19 Wastewater treatment plants are major point sources of (micro)pollutant emissions and advanced

20 wastewater treatment technologies can improve their removal capacity. While abundant data on

21 individual advanced treatment technologies is available, there is limited knowledge regarding the

22 removal performance of ozonation combined with multiple post-treatments and stand-alone

23 membrane bioreactors. This is especially true for the removal of in vitro and in vivo toxicity.

24 Therefore, we investigated the removal of 40 micropollutants and toxicity by a pilot-scale ozonation

25 with four post-treatments: non-aerated and aerated granular activated carbon and biological

26 filtration. In addition, two stand-alone membrane bioreactors fed with untreated wastewater and one

27 MBR operating with ozonated partial flow recirculation were analysed. Aqueous and extracted

28 samples were analysed in vitro for (anti)estrogenic, (anti)androgenic and mutagenic effects. To

29 assess in vivo effects, the mudsnail Potamopyrgus antipodarum was exposed in an on-site flow-

30 through system.

31 Multiple in vitro effects were detected in conventionally treated wastewater including estrogenic

32 and anti-androgenic activity. Ozonation largely removed these effects, while anti-estrogenic and

33 mutagenic effects increased suggesting the formation of toxic transformation products. These

34 effects were significantly reduced by granular activated carbon being more effective than biological

35 filtration. The membrane bioreactor performed similarly to the conventional treatment while the

36 membrane bioreactor with ozonation had a comparable removal performance like ozonation.

37 Conventionally treated wastewater increased the growth of $P$. antipodarum. Ozonation reduced the

38 reproduction indicating a potential formation of toxic transformation products. In the post-

39 treatments, these effects were compensated or remained unaffected. The effluents of the membrane

40 bioreactors induced reproductive toxicity

41 Our results show that ozonation is effective in further reducing toxicity and micropollutan

42 concentrations. However, the formation of toxicity requires a post-treatment. Here, ozonation

43 coupled to granular activated carbon filtration seemed the most promising treatment process. 
Kawands Journal Pre-proot

45 reporter-gene assays, endocrine disrupting chemicals, sewage, advanced wastewater treatment, on-

46 site testing, transformation product 


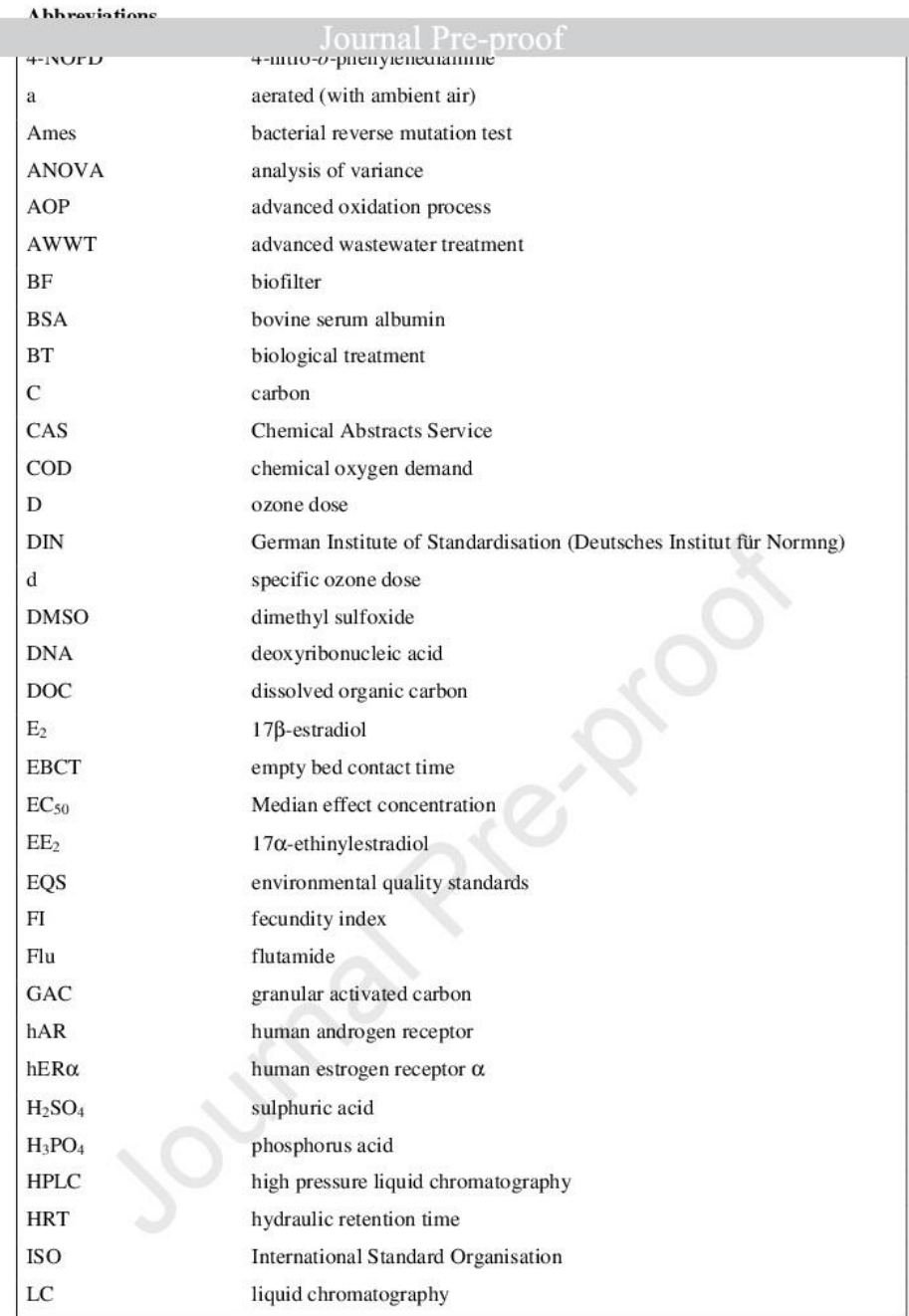




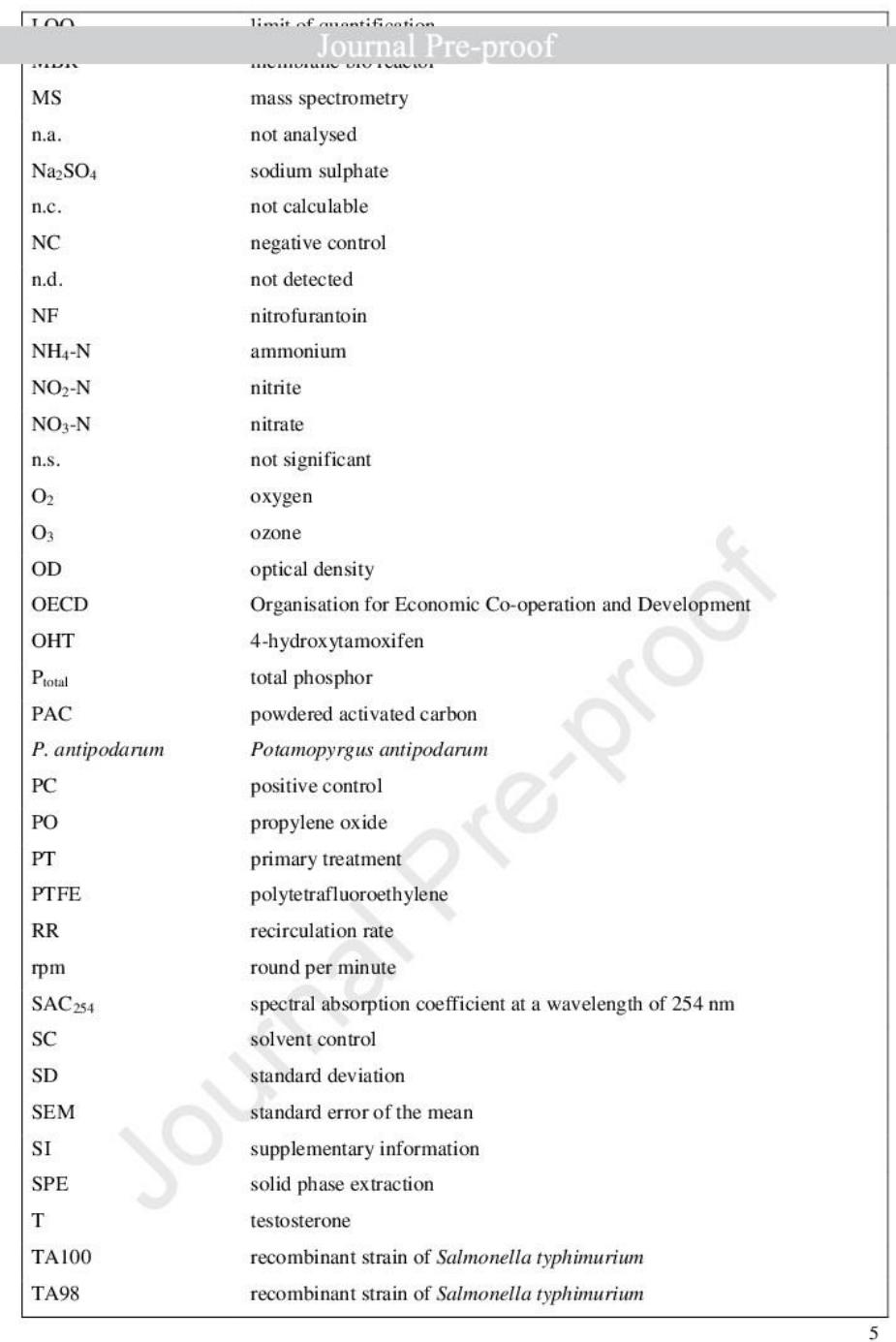




\begin{tabular}{|ll|}
\hline TD & \multicolumn{1}{|l|}{ Journal Pre-proof } \\
$\mathrm{V}_{\mathrm{F}}$ & filter velocity \\
WWTP & without \\
YAAS & wastewater treatment plant \\
YAES & Yeast anti-androgen screen \\
YAS & Yeast anti-estrogen screen \\
YES & Yeast androgen screen \\
YG7108 & Yeast estrogen screen \\
$\mathrm{Z}$ & recombinant strain of Salmonella typhimurium \\
$\mathrm{Z}$ & ozone consumption \\
\hline
\end{tabular}

48 
51 chemicals to aquatic ecosystems, including pollutants of emerging concern (Loos et al. 2013) and

52 micropollutants (Schwarzenbach et al. 2006). WWTPs are known to incompletely remove different

53 micropollutants during conventional, biological wastewater treatment, such as using activated

54 sludge. Reasons for this are low biodegradability and/or high polarity of chemicals (Knopp et al.

55 2016). Certain micropollutants have been detected throughout the water cycle including nanogram

56 per liter concentrations in drinking water (Benotti et al. 2009) and have been characterised as

57 relevant risk to ecosystem integrity and drinking water resources (Malaj et al. 2014). Chemical

58 contamination resulted in the establishment of environmental quality standards (EQS) in many

59 countries, including their integration into different (waste)water policies (e.g., European Parliament

60 and Council 2008, 2013) and the implementation of technical mitigation measures.

61 One major measure is the development and implementation of advanced wastewater treatment

62 (AWWT) technologies (Bui et al. 2016). Key AWWT include advanced oxidation processes

63 (AOPs, e.g., ozonation in combination with UV radiation), activated carbon treatments (e.g.,

64 granular activated carbon (GAC) or powdered activated carbon (PAC)) or pressure-driven

65 membranes (e.g., reverse osmosis). These technologies demonstrated additional removal of

66 (micro)pollutants from biologically treated wastewater. However, each technology has certain

67 weaknesses such as the formation of potentially toxic transformation products (TPs) during AOP or

68 an insufficient sorption of polar chemicals to activated carbon (Rizzo 2011). Accordingly, the

69 addition of a post-treatment (i.e., filtration after ozonation) and optimised parameter settings (e.g.,

70 ozone $\left(\mathrm{O}_{3}\right)$ doses and hydraulic retention times (HRTs)) have been recommended (Völker et al.

71 2019). The present study investigates an innovative process combination for the further reduction of

72 relevant (micro)pollutants and toxicity. The focus was the upgrade of a municipal WWTP with

73 activated sludge treatment in Hesse, Germany with a pilot-scale ozonation in combination with

74 subsequent non-aerated and aerated GAC/biofilter (BF) (Figure 1). Ozonation was chosen because

75 the chemical oxidation induces a transformation of (micro)pollutants in the wastewater and, thus, 


\section{6 incrascas the amoccihility to and docradation in the hinloni
Journal Pre-proof}

77 processes and the resulting TPs can result in the formation of in vitro and in vivo toxicity (Völker et

78 al. 2019). Therefore, ozonation was combined with GAC and biofilter as adsorptive techniques to

79 reduce these effects. This is novel because commonly GAC filtration is used as a post-treatment

80 technology for activated sludge treatments but not in combination with other AWWT technologies.

81 Membrane bioreactors (MBRs) present a stand-alone technology to treat raw wastewater, such as

82 hospital wastewater (Bui et al. 2016, Skouteris et al.2012, Verlicchi et al. 2010). The benefits of

83 using MBRs are amongst others that a final sedimentation is not needed and that a higher solid

84 content in the MBR results in smaller construction volumes and higher sludge ages that may

85 positively affect micropollutant removal. Again, little is known regarding their performance in

86 reducing toxicity (Gehrmann et al. 2018, Maletzt et al. 2013, Snyder et al. 2007). Thus, two MBRs

87 fed with untreated wastewater, one incorporating a partial flow recirculation of ozonated

88 wastewater, were examined (Figure 1) focusing on the combination of oxidation and biological

89 treatment. The aim was to test whether higher removal rates can be achieved with the lowest ozone

90 concentration. Such an approach has not yet been investigated. Another benefit of the

91 implementation of the recirculation concept was that it does not require an expansion of existing

92 activated sludge treatment and, thus, lowers the operating costs.

93 As multiple AWWT technologies and combinations thereof are available, it is important to compare

94 their performance in removing chemicals and toxicity. So far, most previous studies investigated

95 only a single AWWT technology, often alone or less frequently in combination with one post-

96 treatment (e.g., ozonation combined with sand filtration). In addition, most studies are performed at

97 different WWTPs complicating the comparison of technological performance and efficiency of

98 multiple technologies. Studies comparing multiple process combinations at the same plant are rather

99 scarce (Stalter et al. 2010, Völker et al. 2016). However, such studies are needed to assess the

100 benefits of conventional and AWWT technologies. 
102 complementary because the former allows for determining the removal of priority compounds while

103 the latter enables the assessment of toxicity removal caused by an overall mixture of chemicals

104 (Cao et al. 2009). This combination is particularly important because the removal of target

105 compounds does not per se correlate to toxicity removal (Magdeburg et al. 2014). Case-specific

106 combinations of bioassays and chemical analyses were thus rated as 'gold standard' (Ternes et al.

107 2017),

108 In the current study, we used multiple in vitro bioassays and one in vivo bioassay with the New

109 Zealand mudsnail Potamopyrgus antipodarum and quantified 28 representative micropollutants and

110 twelve standard wastewater parameters. The performance of a full scale conventional biological

111 wastewater treatment (BT) combined with a subsequent pilot scale ozonation $\left(\mathrm{BT}+\mathrm{O}_{3}\right)$ followed by

112 GAC filtration or BF as well as two stand-alone MBRs, one MBR with partial flow ozonation

113 (MBR1, MBR1+O ${ }_{3}$ and MBR2, respectively) were investigated. The evaluation focused on the

114 removal of target chemicals and toxicity compared to the activated sludge treatment $\left(\mathrm{O}_{3}, \mathrm{GAC}, \mathrm{BF}\right)$

115 or raw wastewater (MBRs). In this context, three hypotheses were tested: 1) Increasing the ozone

116 dose and HRT increases the removal of micropollutants and in vitro toxicities; 2) Ozonation

117 generates toxic TPs that adversely affect different in vitro and/or in vivo endpoints while a post-

118 treatment reduces these effects; 3 ) The MBRs remove chemicals and toxicity with a performance

119 comparable to an activated sludge treatment with a partial flow ozonation further increasing the

120 performance. The aim of this work was to compare the toxicity and micropollutant removal of the

121 multiple combinations of AWWT technologies implemented at the same WWTP and provide

122 recommendations on which technologies perform best. 


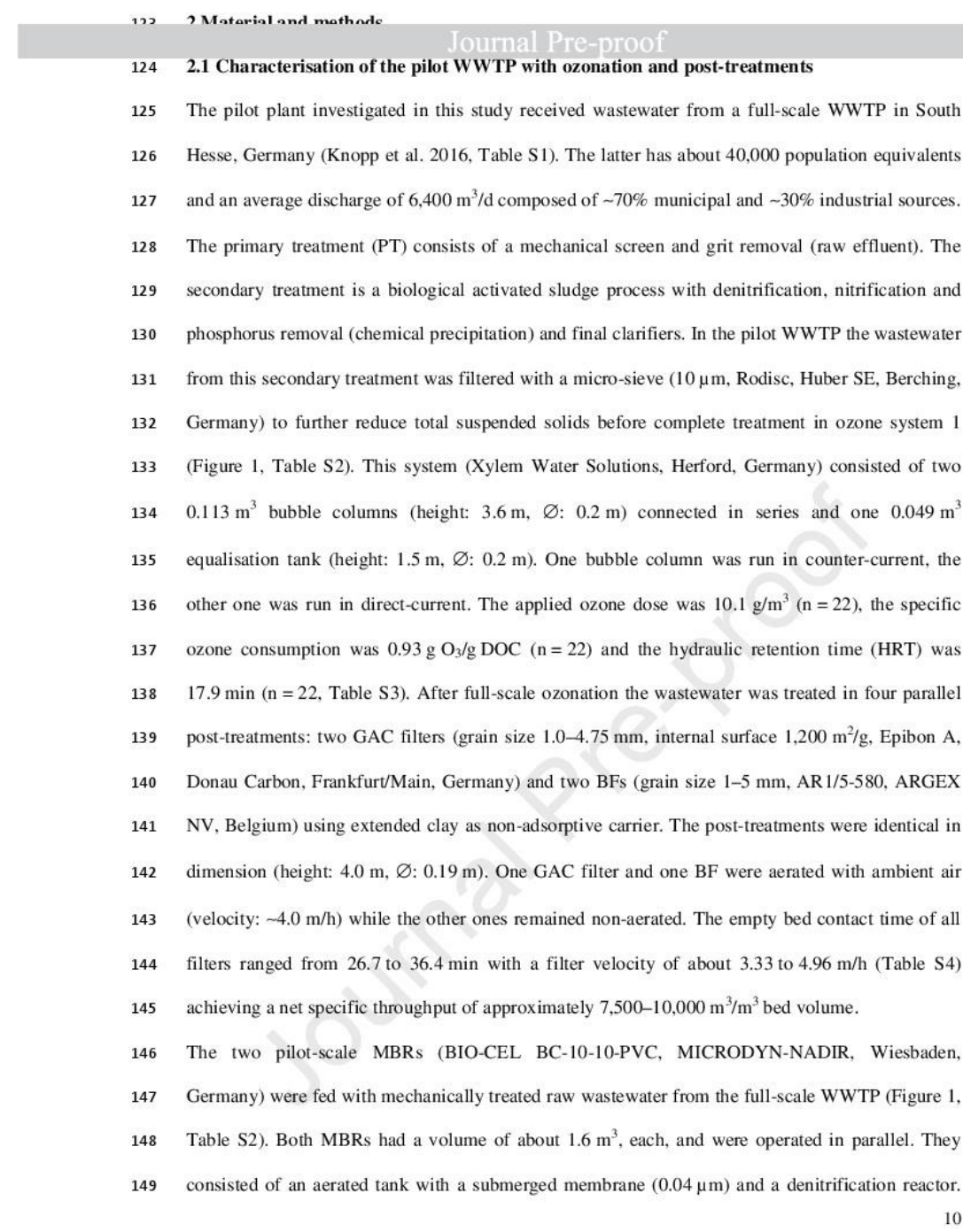


152 equalisation tank (height: $0.9 \mathrm{~m}, \varnothing: 0.2 \mathrm{~m}$, volume: $0.03 \mathrm{~m}^{3}$ ). The applied ozone dose was

$1536.78 \mathrm{~g} / \mathrm{m}^{3}(\mathrm{n}=5)$, the specific ozone consumption was $0.96 \mathrm{~g} \mathrm{O}_{3} / \mathrm{g} \mathrm{DOC}(\mathrm{n}=5)$ and the HRT was

$15426.1 \mathrm{~min}(\mathrm{n}=5$, Table S3). A defined fraction of the ozonated wastewater was recirculated into

155 MBR1 with a recirculation rate of $2.02(\mathrm{n}=5)$. The sludge retention time was 55 days. MBR2

156 served as reference and its wastewater was neither ozonated nor recirculated. Further technical

157 details and process parameters are described in the supplementary information (Tables S 1-S4).

158

$159 \quad 2.2$ Optimal ozone dose and hydraulic retention time

160 Prior to the on-site experiment with P. antipodarum (2.3), an experiment to determine the optimal

161 ozone dose and HRT was performed. Conventionally treated wastewater from the municipal

162 WWTP was ozonated using four increasing ozone doses $\left(0.18-0.51 \mathrm{~g} \mathrm{O}_{3 \text {, applied }} / \mathrm{g}\right.$ DOC) at a

163 constant HRT of $12.6 \mathrm{~min}$ as well as a constant ozone dose of $0.53 \mathrm{~g} \mathrm{O}_{3}$ applied $/ \mathrm{g}$ DOC using five

164 HRTs (4.6-15.1 min). Three $24 \mathrm{~h}$ composite samples were taken from each adjusted ozone dose and

165 HRT. These wastewater samples were extracted (2.4) and analysed in five in vitro bioassays (2.5).

166

$167 \quad 2.3$ On-site in vivo experiment with Potamopyrgus antipodarum

168 P. antipodarum was collected in the stream Lumda in Hesse, Germany (50 $38^{\prime} 52.64^{\prime \prime} \mathrm{N}$,

$1698^{\circ} 5349.28^{\prime \prime}$ E) and acclimatised in the laboratory to culture medium at $16.0^{\circ} \mathrm{C}$ and a light-dark-

170 regime of $16: 8 \mathrm{~h}$ for four weeks. Animals with shell heights between 3.4 and $4.0 \mathrm{~mm}$ were used for

171 the experiment (mean \pm SD: $3.66 \pm 0.16 \mathrm{~mm}, \mathrm{n}=50$ ). The endpoints reproduction (number of

172 embryos), growth (shell height) and biomarkers for energy reserves (protein, lipid and glycogen

173 content) were analysed.

174 The on-site experiment was carried out in a continuous flow-through system directly at the pilot

175 WWTP based on OECD guideline 242 (OECD 2016). Wastewater from nine points representing

176 different treatment stages and degrees were tested (Figure 1): after conventional BT, after ozone 
179 The PT was not investigated because other studies reported on high mortality upon exposure to raw

180 wastewater (Giebner et al. 2018, Smital et al. 2011).

181 Peristaltic pumps (Otto Huber, Böttingen, Germany) constantly pumped the undiluted wastewater

182 through polytetrafluoroethylene (PTFE) tubes from the nine treatment stages to $10 \mathrm{~L}$ high-grade

183 stainless-steel reservoirs allowing residual ozone to gas out. From these reservoirs, smaller

184 peristaltic pumps (IPC 24, Ismatec, Wertheim-Mondfeld, Germany) pumped the wastewater

185 constantly through PTFE tubes into the exposure vessels containing the test organism. The exposure

186 vessels were placed in random order in a tank filled with water nearly up to the passive overflows of

187 the exposure vessels. Water temperature was adjusted to $16^{\circ} \mathrm{C}$ using four heating elements and an

188 external cooling unit (Julabo, Seelbach, Germany). A negative control group (NC) with culture

189 medium (OECD 2016) and a positive control group (PC) with culture medium containing $25.0 \mathrm{ng} / \mathrm{L}$

$19017 \alpha$-ethinylestradiol $\left(\mathrm{EE}_{2}\right)$ ran in parallel to the wastewater treatments in a flow-through system as

191 well. Fresh culture medium of the NC and PC was prepared regularly (Table S5). Each test vessel

192 (1 L) was filled with $600 \mathrm{~mL}$ medium or wastewater and had a 6-fold volume water exchange rate

193 per day. All vessels were aerated with ambient air filtered with a $0.2 \mu \mathrm{m}$ laboratory injection filter.

194 Twenty-five mudsnails were exposed in each replicate (four replicates per treatment group) and fed

195 every third day with $0.25 \mathrm{mg}$ fine powdered fish feed (Tetra Phyll) per snail and day. After 28 days

196 of exposure under a light:dark regime of $16: 8 \mathrm{~h}$, the mudsnails were frozen in liquid nitrogen and

197 stored at $-80^{\circ} \mathrm{C}$ until analysis. For the analyses, the mudsnails were defrosted, shell height was

198 measured to the nearest $0.1 \mathrm{~mm}$ and shells were cracked and carefully removed to determine the

199 total number of embryos in the brood pouch. In addition, aqueous grab samples of the NC and the

$200 \mathrm{PC}$ medium and aqueous $24 \mathrm{~h}$ composite samples and 5000 -fold enriched samples of the different

201 wastewaters were tested in vitro (see 2.4-2.5, Table S5). Protein, glycogen and lipid content as

202 biomarkers for energy reserves were determined as described in the Supplementary Information

203 (S1.3, Figures S1-S3, Tables S6-S8). In brief, each mudsnail was weighed (accuracy $\pm 0.01 \mathrm{mg}$ ) 
205 turns per second using a grinding ball and a swing mill (MM 400, Retsch GmbH, Haan, Germany).

The protein content was determined as described in Bradford (1976). Glycogens and lipids were

207 separated as described by van Handel (1965) and determined using hot anthrone and vanillin

208 reactions (van Handel 1985a, b). The protein, glycogen and lipid content of the samples was

calculated in $\mu \mathrm{g} / \mathrm{mg}$ mudsnail and then converted to an energy content of the lipid reserve in $\mathrm{J} / \mathrm{mg}$ mudsnail using the specific calorific value (Berg et al. 2007).

211

2122.4 Wastewater sample preparation: Solid phase extraction (SPE)

213 The SPE column Telos C18/ENV, $500 \mathrm{mg}+200 \mathrm{mg} / 6 \mathrm{~mL}$ (Kinesis Ltd., St. Neots, Great Britain)

214 was used for extracting the wastewater samples because they were optimal for the enrichment of 215 endocrine activity and mutagenicity from wastewater (Abbas et al. 2019). The SPE columns were

216 conditioned consecutively with $1 \times 2.0 \mathrm{~mL}$ heptane, $1 \times 2.0 \mathrm{~mL}$ acetone, $3 \times 2.0 \mathrm{~mL}$ methanol and

$2174 \times 2.0 \mathrm{~mL}$ ultra-pure water. SPE was performed within $48 \mathrm{~h}$ after sample collection. Each

218 wastewater sample was collected as $24 \mathrm{~h}$ composite sample (Table S5). After filtration with GF 6

219 filters (Whatman, GE Healthcare Life Sciences, Chalfont St. Giles, England), $500 \mathrm{~mL}$ of each

220 sample were acidified to $\mathrm{pH} 2.5$ with sulphuric acid $(3.5 \mathrm{~mol} / \mathrm{L})$ directly before enrichment and

221 extracted. The columns were dried under $\mathrm{N}_{2}$ and eluted with methanol and acetone at neutral

222 conditions $(5 \times 2.0 \mathrm{~mL}$, respectively). After adding $100 \mu \mathrm{L}$ dimethyl sulphoxide (DMSO) each

223 methanol-acetone extract was concentrated to $100 \mu \mathrm{L}$ final volume under a gentle $\mathrm{N}_{2}$ stream. All

224 DMSO extracts (5,000-fold concentrated compared to the aqueous sample) were stored at $-20^{\circ} \mathrm{C}$

225 until testing. A SPE blank (solvent control, SC) was prepared by extracting $500 \mathrm{~mL}$ ultra-pure

226 water. SPE blanks were identically prepared in parallel to the enrichment of samples from each

sampling campaign.

228

2292.5 In vitro bioassays for endocrine activities and mutagenicity

$230 \quad$ 2.5.1 Recombinant yeast screens for endocrine activities 
233 Estrogen Screen (YAES), Yeast Androgen Screen (YAS, human androgen receptor (hAR)) and

234 Yeast Anti-Androgen Screen (YAAS) as first described by Routledge and Sumpter (1996) and

235 Sohoni and Sumpter (1998). The YES and YAS are used to study compounds activating the hER $\alpha$

and hAR (receptor agonists) while the YAES and YAAS detect chemicals blocking the respective

238 Darmstadt, Germany) as previously described by Völker et al. (2016). In brief, aqueous samples

239 were analysed in a 0.63 -fold final sample concentration (1.6-fold dilution). SPE extracts were were analysed in each experiment (see Figures S4 and S5 and Table S9 for details). The incubation times at $30^{\circ} \mathrm{C}$ and $1200 \mathrm{rpm}$ depended on the bioassay and were between 18 and $22 \mathrm{~h}$. Results were not used if $>20 \%$ cytotoxicity compared to the NC/SC occurred. Relative endocrine activities were calculated by normalising the reported gene activity to the $\mathrm{NC} / \mathrm{SC}(0 \%)$ and the maximum activity of the reference compound (100\%). A control without agonist was used for the antagonistic assays to represent $100 \%$ receptor inhibition. Selected SPE extracts, particularly those that were cytotoxic,

249 were tested with dilution factors of 1:2 to 1:16 to generate concentration-response-relationships

250 (Figure S6). 251

253 The Ames fluctuation test (ISO DIN 11350, 2012) was used to identify mutagenic activity (i.e., 254 irreversible DNA damages) with three genetically-modified strains of the bacterium Salmonella 255 typhimurium (TA98, TA 100 and YG7108) as described by Magdeburg et al. (2014). In brief, SPE 256 extracts were tested in a 10.4-fold final sample concentration $(0.2 \% \mathrm{v} / \mathrm{v}$ solvent). Mutagenic 257 reference compounds were used as PC (Table S9). A SC (DMSO) ran in parallel to the extracts in 
259 counting the number of wells that shifted from purple (negative) to yellow (positive) the mutagenic

260 activity of the sample was determined photometrically.

261

262 2.6 Chemical analysis

263 Chemical analysis of wastewater samples was carried out once per week (four times) during the

26428 days on-site experiment (2.3). The selection criteria of the 28 micropollutants were amongst

265 others their high polarity and no/low reduction by conventional and/or AWWT technologies, the

266 formation of stable TPs, their ecotoxicological relevance, their detection frequency in aqueou

267 environments and their use as wastewater tracer. Thus, an analysis of these micropollutants and

268 their corresponding TPs was conducted by high performance liquid chromatography (HPLC;

269 Thermo Dionex UltiMate 3000 RSLC, Thermo Fisher Scientific Inc., Waltham, USA) coupled via

270 an electrospray interface with a mass spectrometry (MS) system (MS/MS; Sciex Qtrap 5500, AB

271 Sciex, Framingham, USA) without sample enrichment (Seitz and Winzenbacher 2017). The

272 injection volume was $100 \mu 1$. Ultrapure water (Purelab Ultra, Elga, Celle, Germany) was used for

273 dilution or as eluent. Furthermore, the LC/MS grade formic acid (Fluka, MS grade, 98\%),

274 ammonium formate (Sigma-Aldrich, > 99.995\%) and acetonitrile (Carl Roth, LC-MS grade, >

$27599.95 \%)$ were used. Separation was achieved on a Kinetex $2.6 \mu \mathrm{m} \mathrm{C} 18$ column $(100 \times 4.6 \mathrm{~mm}$,

276 Phenomenex Inc., Torrance, USA) at a flow rate of $0.6 \mathrm{~mL} / \mathrm{min}$ with a pre-column (Security Guard

277 KIT KJO-4282, Phenomenex, Torrance, USA). Mass spectrometry was carried out in

278 positive/negative polarity switching electrospray ionization mode. The limit of quantification

279 (LOQ) was $0.025 \mu \mathrm{g} / \mathrm{L}$. The chemical analysis was done using the following standard methods DIN

$280 \quad 38407-36$ (2014) and DIN 38407-47 (2015).

281

2.7 Measurement of physical-chemical wastewater parameters

283 The following water parameters were determined directly at the pilot WWTP using standardised

284 cuvette tests (Hach Lange, Düsseldorf, Germany): chemical oxygen demand (COD), dissolved 
286 and spectral absorption coefficient at $254 \mathrm{~nm}\left(\mathrm{SAC}_{254}\right.$ ) (Table S 10). In addition, the following wate

287 parameters were measured directly in the exposure vessels as requested by OECD (2016): $\mathrm{pH}$

288 conductivity, oxygen saturation and oxygen concentration using potentiometric electrodes (Multi

$289340 \mathrm{i} / \mathrm{SET}$, WTW Weilheim, Germany), nitrite $\left(\mathrm{NO}_{2}-\mathrm{N}\right)$, nitrate $\left(\mathrm{NO}_{3}-\mathrm{N}\right)$, ammonium $\left(\mathrm{NH}_{4}-\mathrm{N}\right)$ and

290 total hardness using rapid test kits (Aquamerck, Merck, Darmstadt, Germany, Table S11)

291 Temperature was measured in the tank with two data loggers that recorded the temperature every 15

$292 \min$

293

$294 \quad 2.8$ Statistical analysis

295 Statistical analyses were performed using GraphPad Prism (version 5.03, GraphPad Software, San

296 Diego, California, USA). Mortality data were analysed using Fisher's exact test. Gaussian

297 distribution was tested with the D'Agostino and Pearson omnibus normality test and homogeneity

298 of variances with the Bartlett's test. In case of a normal distribution and equal variances, significant

299 differences between the datasets were analysed using a one-way ANOVA with Bonferroni's post-

300 test (glycogen and total energy content). If the datasets were not normally distributed, the

301 nonparametric Kruskal-Wallis test with Dunn's post-test was used (shell height, total number of

embryos and energy contents as protein and lipid). Significant differences between treatments were

303

marked with asterisks: $\mathrm{p}<0.05: \star, \mathrm{p}<0.01: \star \star, \mathrm{p}<0.001: \star \star \star$. 
307 The mean estrogenic and anti-estrogenic activity of the BT was $7.31 \pm 0.21 \%$ and $61.7 \pm 0.55 \%$,

308 respectively. With increasing ozone dose, the estrogenic activity decreased by $94.0 \%$ to

$3090.44 \pm 0.07 \%$ at the highest ozone dose whereas the anti-estrogenic activity increased by $29.1 \%$ to

$31079.6 \pm 1.37 \%$ (Figure 2A, Table S12). No androgenic activity was detected in the BT and at al

311 ozone doses (Figure 2B). In contrast, the anti-androgenic activity in the BT was $76.1 \pm 0.72 \%$. With

312 increasing ozone dose, the anti-androgenic activity decreased by $35.1 \%$ to $49.3 \pm 0.73 \%$ at the

313 highest ozone dose (Figure 2B, Table S12).

314 None of the treatments was mutagenic in the Ames TA98 strain (Figure 2C). However, the Ames

315 TA100 strain indicated a potential mutagenicity in the BT $(21.2 \pm 2.59 \%)$ which increased by

$67.1 \%$ with increasing ozone dose to maximal $35.4 \pm 2.10 \%$ (Table S12).

317

$318 \quad 3.1 .2$ Optimal hydraulic retention time

319 The mean estrogenic activity of the BT was $3.58 \pm 0.12 \%$. Ozonation reduced the estrogenic 320 activity by 81.3 to $95.7 \%$ independent of the HRT (Figure 2D). The mean anti-estrogenic activity of 321 the BT was $71.0 \pm 0.45 \%$ and decreased by $12.9 \%$ at the lowest HRT to $61.9 \pm 0.91 \%$. With

322 increasing HRTs the anti-estrogenic activity first increased before it remained constant within the 323 same range like the BT (Table S13). Again, no androgenic activity was detected in the BT and at all 324 tested HRTs (Figure 2E). However, the anti-androgenic activity of the BT was $70.9 \pm 0.80 \%$ and 325 decreased by $43.6 \%$ to $39.9 \pm 2.21 \%$ at the lowest HRT. With increasing HRTs, the anti-androgenic 326 activity first increased to $60.7 \pm 0.88 \%$ before it decreased to $40.7 \pm 0.93 \%$ at highest HRT $(-42.6 \%$ 327 compared to the BT, Table S13).

328 Again, none of the treatments was mutagenic in the Ames TA98 strain (Figure 2F). In contrast, the 329 Ames TA100 indicated potential mutagenicity in the BT $(21.5 \pm 1.64 \%)$. This effect increased by $33093.5 \%$ at higher HRTs to maximal $41.7 \pm 3.18 \%$ (Table S13). 


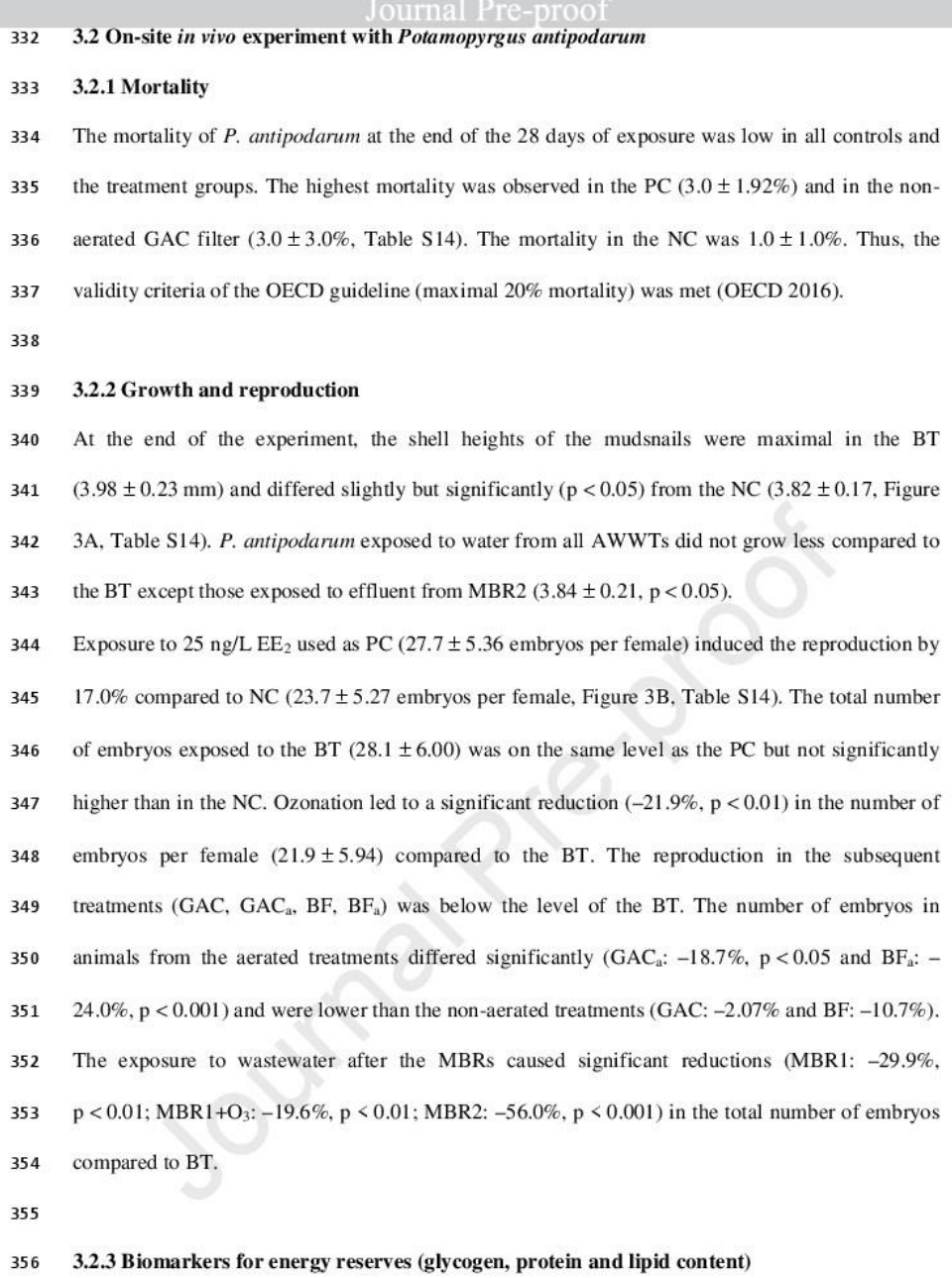


359 found in the MBR2 $(0.23 \pm 0.08 \mathrm{~J} / \mathrm{mg})$. However, no significant differences were detected.

360 The glycogen content was highest $(+29.2 \%, \mathrm{p}<0.05$, Figure $4 \mathrm{~B}$, Table $\mathrm{S} 15)$ in animals from the non-aerated GAC filter $(0.24 \pm 0.08 \mathrm{~J} / \mathrm{mg})$ and significantly higher compared to the BT $(0.19 \pm 0.04$ $\mathrm{J} / \mathrm{mg})$ and lowest in P. antipodarum from the MBR1 $(0.15 \pm 0.05 \mathrm{~J} / \mathrm{mg})$.

363 The lipid contents of the mudsnails in the PC $(0.96 \pm 0.42 \mathrm{~J} / \mathrm{mg})$ and BT $(0.95 \pm 0.73 \mathrm{~J} / \mathrm{mg})$ were significantly lower $(-39.8 \%, \mathrm{p}<0.01$ and $-40.1 \%, \mathrm{p}<0.05)$ compared to the NC $(1.59 \pm 0.54 \mathrm{~J} / \mathrm{mg}$, Figure 4C, Table S15). The highest lipid content was determined in animals from the non-aerated $\mathrm{BF}(2.05 \pm 0.31 \mathrm{~J} / \mathrm{mg})$ and differed together with aerated GAC filter treatment $(1.52 \pm 0.51 \mathrm{~J} / \mathrm{mg})$ significantly from the BT $(+115 \%, \mathrm{p}<0.001$ and $+59.7 \%, \mathrm{p}<0.05$, respectively). The total energy content in mudsnails from the PC $(1.44 \pm 0.43 \mathrm{~J} / \mathrm{mg})$ and the BT $(1.38 \pm 0.77$

$369 \mathrm{~J} / \mathrm{mg})$ were lowest with significant differences $(-30.6 \%, \mathrm{p}<0.001$ and $-33.2 \%, \mathrm{p}<0.001)$

370 compared to the NC $(2.07 \pm 0.56 \mathrm{~J} / \mathrm{mg}$, Figure 4D, Table S16). The total energy content of the 371 mudsnails exposed to water from the AWWT were higher than in the BT with significant 372 differences in the $\mathrm{GAC}_{\mathrm{a}}(1.94 \pm 0.36 \mathrm{~J} / \mathrm{mg},+40.2 \%, \mathrm{p}<0.01)$, the $\mathrm{BF}(2.54 \pm 0.35 \mathrm{~J} / \mathrm{mg},+83.7 \%$, $373 \mathrm{p}<0.001)$ and $\mathrm{BF}_{\mathrm{a}}(1.87 \pm 0.47 \mathrm{~J} / \mathrm{mg},+35.2 \%, \mathrm{p}<0.05)$. 374 375 3.2.4 In vitro bioassays for endocrine and mutagenic activity

379 The aqueous samples of the PC spiked with $25 \mathrm{ng} / \mathrm{L} \mathrm{EE}_{2}$ had a mean estrogenic activity of 28.2 $380 \pm 0.47 \mathrm{ng}$ ethinylestradiol-equivalents/L that corresponds to a receptor activation of $26.1 \pm 0.78 \%$.

381 The aqueous PT samples were neither estrogenic $(1.60 \pm 0.27 \%)$ nor anti-androgenic $382(1.03 \pm 0.41 \%)$ but induced a high anti-estrogenic $(95.0 \pm 0.71 \%)$ and androgenic $(38.2 \pm 2.30 \%)$ 


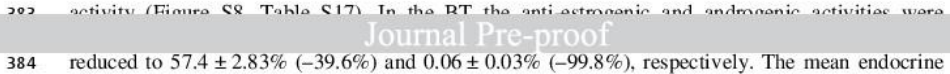

385 activities in all $\mathrm{AWWT}\left(\mathrm{BT}+\mathrm{O}_{3}, \mathrm{GAC}, \mathrm{GAC}_{\mathrm{a}}, \mathrm{BF}\right.$ and $\left.\mathrm{BF}_{\mathrm{a}}\right)$ and $\mathrm{MBR}$ systems $\left(\mathrm{MBR} 1, \mathrm{MBR} 1+\mathrm{O}_{3}\right.$

386 and MBR2) were on a comparable level to BT.

387 The SPE extracts of the BT indicated a mean estrogen activity of $16.9 \pm 1.60 \%$ (Figure 5A, Table

388 S18). Ozonation reduced the estrogenic activity by $96.5 \%$ to $0.59 \pm 0.11 \%$. The following GAC

389 filter and BF showed a reduction of the estrogen activity compared to the BT by 95.1 to $95.9 \%$ as

390 well. For the MBR systems this reduction ranged between $81.7 \%$ in MBR2 and $97.4 \%$ in

$391 \mathrm{MBR} 1+\mathrm{O}_{3}$.

392 Ozonation of the BT increased the anti-estrogenic activity of the extracts by $163 \%$ from

$39314.1 \pm 1.53 \%$ to $37.2 \pm 1.43 \%$ (Figure 5B, Table S18). Post-filtration reduced this anti-estrogenic

394 activity by 5.03 to $49.9 \%$ but the activity was still higher compared to the BT ( $+31.8 \%$ (GAC),

$395+65.7 \%\left(\mathrm{GAC}_{4}\right),+150 \%(\mathrm{BF})$ and $\left.+144 \%\left(\mathrm{BF}_{\mathrm{a}}\right)\right)$. The wastewater of the MBR1, MBR $1+\mathrm{O}_{3}$ and

396 MBR2 indicated a higher anti-estrogen activity compared to the BT with an increase by $162,93.3$

397 and $201 \%$, respectively and a maximal activity of $42.6 \pm 2.95 \%$ in MBR2.

398 The mean androgenic activity (Figure 5C, Table S18) of the BT extracts was $1.76 \pm 0.31 \%$ and was

399 reduced by 10.1 to $84.0 \%$ in all $\mathrm{AWWT}\left(\mathrm{BT}+\mathrm{O}_{3}, \mathrm{GAC}, \mathrm{GAC}_{\mathrm{a}}, \mathrm{BF}\right.$ and $\left.\mathrm{BF}_{\mathrm{a}}\right)$ and $\mathrm{MBR}$ systems

400 (MBR1, MBR1+O 3 and MBR2).

401 A mean anti-androgenic activity (Figure 5D, Table S18) of $72.1 \pm 2.05 \%$ was determined in the

402 SPE extracts of the BT. Compared to this treatment the AWWT $\left(B T+\mathrm{O}_{3}, \mathrm{GAC}, \mathrm{GAC}_{\mathrm{a}}, \mathrm{BF}\right.$ and $\left.\mathrm{BF}_{\mathrm{a}}\right)$

403 and MBR systems (MBR1, MBR1+O ${ }_{3}$ and MBR2) reduced the anti-androgenic activity by 7.68 to

$40472.6 \%$.

405

406 Ames fluctuation test for mutagenicity

407 No mutagenic activity was detectable in the BT in the Ames strain YG7108 (Figure 6, Table S18).

408 Ozonation of the BT induced a high mutagenicity of $93.2 \pm 1.29 \%$. Water treated with GAC and

$409 \mathrm{GAC}_{\mathrm{a}}$ was not mutagenic in contrast to the $\mathrm{BF}$ and $\mathrm{BF}_{\mathrm{a}}$ with $50.8 \pm 2.29 \%$ and $52.9 \pm 4.87 \%$, 
11 mutagenicity of $67.5 \pm 4.62 \%$.

412

$413 \quad 3.3$ Chemical analysis

414 The chemical analysis was conducted in parallel to the ecotoxicological investigations and included

41528 micropollutants mainly belonging to the group of pharmaceuticals such as radio-opaque

416 substances, anticonvulsants, antibiotics (including metabolites such as of carbamazepine, diclofenac

417 or ibuprofen) as well as nutrition-related chemicals (caffeine), herbicides (mecoprop) and industria

418 chemicals (benzotriazole and tolyltriazole). In the PT, caffeine was detected at the highest

419 concentration of $162 \pm 23.2 \mu \mathrm{g} / \mathrm{L}$ followed by carboxy-ibuprofen $(74.7 \pm 6.27 \mu \mathrm{g} / \mathrm{L}), 2$-hydroxy-

420 ibuprofen $(47.3 \pm 4.97 \mu \mathrm{g} / \mathrm{L})$ and $1 \mathrm{H}$-benzotriazole $(25.0 \pm 0.71 \mu \mathrm{g} / \mathrm{L})$. The concentrations of the

421 other substances were between 0.025 and $14.4 \mu \mathrm{g} / \mathrm{L}$ (Table S19). The BT reduced the

422 concentrations of 15 out of 28 chemicals by more than $50 \%$ (highest reduction, $-99.8 \%$ for caffeine

423 and carboxy-ibuprofen). For nine chemicals, the reduction was low $(<-25 \%)$. For carbamazepine

424 and carboxy-acyclovir a concentration increase was detected.

425 Ozonation led to a further reduction of 21 substances ranging from $-11.1 \%$ (iopamidol) and $-99.1 \%$

426 (carboxy-acyclovir)) compared to the BT (Figure 7A, Table S19). The concentrations of 18

427 substances decreased by more than $50 \%$. For another three compounds, the concentrations

428 decreased by between 10 and $50 \%$. Two TPs (3-hydroxy-ibuprofen and tramadol-N-oxide)

429 indicated higher concentrations in the $\mathrm{BT}+\mathrm{O}_{3}$ than in the $\mathrm{BT}$.

430 The post-treatments further reduced the concentrations of most target substances (Figures S9 and

431 S10, Tables S19 and S20). For certain compounds for which ozonation did not achieve a complete

432 removal (e.g., 3-hydroxy-ibuprofen, diclofenac, sulfamethoxazole), a post filtration led to an overall

433 removal of 75.0 to $90.7 \%$ compared to the $\mathrm{BT}+\mathrm{O}_{3}$. For a small set of compounds (2-hydroxy-

434 ibuprofen, 4-hydroxy-1H-benzotriazole, carboxy-acyclovir, paracetamol), a moderate additional

435 removal between 31.1 and $42.9 \%$ occurred in the GAC filters and BFs compared to the $\mathrm{BT}+\mathrm{O}_{3}$.

436 GAC filters showed a higher removal rate for seven compounds including 1H-benzotrialzole, 
438 Certain compounds such as caffeine or mecoprop could however not be further removed by the

$439 \mathrm{GAC}$ filters and $\mathrm{BFs}$ compared to the $\mathrm{BT}+\mathrm{O}_{3}$.

440 MBR1 and MBR2 had slightly lower removal efficacies regarding the 28 chemicals than the BT

441 (Figures 7B and S11, Tables S20-S21). The ozonation increased the removal in the MBR1 with

442 efficiencies comparable to the $\mathrm{BT}+\mathrm{O}_{3}$. However, the concentration of carboxy-acyclovir increased

443 in the BT $(+367 \%), \mathrm{MBR} 1$ and MBR2 $\left(+146\right.$ and $+343 \%$, respectively) as well as MBR $1+\mathrm{O}_{3}$

$444(+39.3 \%)$.

445 The results for the water parameters can be found in the Supplementary Information (S2.4, Tables

446 S22-S29). 


\section{$448 \quad 4.1$ Optimal ozone dose and hydraulic retention time}

449 4.1.1 Optimal ozone dose

450 In line with previous research, an additional ozonation of conventionally treated wastewater

451 efficiently reduced the estrogenic activity (Völker et al. 2019). The removal of estrogenicity

452 increased with ozone dose and doses $\geq 0.44 \mathrm{~g} \mathrm{O}_{3} / \mathrm{g}$ DOC were most effective (Figure 2A, supports

453 hypothesis 1). Interestingly, we observed a marked increase of the anti-estrogenic activity with

454 higher ozone dosage (falsifies hypothesis 1), a phenomenon that has been reported previously

455 (Giebner et al. 2018, Gehrmann et al. 2018, Itzel et al. 2020, Stalter et al. 2011). One potential

456 reason is the removal of estrogens masking the anti-estrogenicity (Thara et al. 2014, Leusch et al.

457 2017, Ma et al. 2005, Rao et al. 2014) or the formation of anti-estrogenic TPs during ozonation

458 (compare hypothesis 2, Knoop et al. 2018).

459 In contrast to previous studies that reported an effective removal of anti-androgenic activity in

460 biologically treated (Rao et al. 2014) and ozonated (Stalter et al. 2011) wastewater, we detected a

461 high anti-androgenicity in the $\mathrm{BT}$ as well as the $\mathrm{BT}+\mathrm{O}_{3}$ samples (Figure $2 \mathrm{~B}$ ) that was not fully

462 removed by the applied ozone doses. Treatment with the highest dose $\left(0.51 \mathrm{~g} \mathrm{O}_{3}\right.$ applied $/ \mathrm{g}$ DOC) led

463 to a $35.1 \%$ reduction. This indicated the presence of relatively stable anti-androgenic substances

464 (Itzel et al. 2020).

465 The Ames TA100 was more suitable for detecting mutagenicity than the Ames TA98 (Figure 2C).

466 Again, this is in line with previous research (Völker et al. 2019). The mutagenicity (TA100)

467 increased at higher ozone doses indicating the formation of mutagenic TPs. Higher mutagenicity in

468 ozonated wastewater was previously reported (Chen et al. 2017, Giebner et al. 2018, Jia et al. 2015,

469 Magdeburg et al. 2014). These findings underline the importance of implementing ozonation post-

470 treatments (4.4).

471 With regards to determining the optimal ozone dose, it becomes obvious that a balance needs to be

472 found between the removal of estrogenic and anti-androgenic compounds on the one, and the 


\section{$476 \quad$ 4.1.2 Optimal hydraulic retention time}

477 The experiment with a high ozone dose and different HRTs supports the results of the previous

478 experiment: The mean estrogenic activity was reduced in ozonated wastewater compared to the BT

479 for all HRTs (Figure 2D). The anti-estrogenic activity decreased at the lowest HRT but remained at

480 the level of BT at higher HRTs. The results support the idea of a generation of anti-estrogenic TPs

481 during ozonation because the estrogenic activity was on a comparable low level at all HRTs.

482 Again, the anti-androgenic activity was high in BT (Figure 2E) and was reduced most at the shortest

483 and longest HRT. The lower removal in the intermediate HRTs might be explained by anti-

484 androgenic TPs (hypothesis 2). The mutagenicity detected in the Ames TA100 in the BT increased

485 at particular longer HRTs (Figure 2F). This observation further substantiates the formation of

486 mutagenic TPs during ozonation (hypothesis 2).

487

$488 \quad 4.2$ In vivo effects in Potamopyrgus antipodarum

$489 \quad$ 4.2.1 Growth and reproduction

490 P. antipodarum were larger when exposed to water from BT compared to the NC (Figure 3A)

491 which may be the result of a better nutrient supply in the BT containing additional organic matter.

492 Furthermore, a significantly lower shell height was detected in the MBR2 compared to the BT

493 which may indicate a lower removal of general toxicity in MBR2.

494 The reproduction of $P$. antipodarum was increased in the BT and the PC (Figure 3B) compared to

495 the NC. One reason for this could be a better nutrition (compare above). Here, several studies

496 showed that gastropods with a better nutrient supply produced a higher number of eggs (Augusto et

497 al. 2012, Keas \& Esch 1997, Ter Maat et al. 2007). Another reason might be the presence of

498 residual endocrine disrupting substances (Duft et al. 2007, Stalter et al. 2011, Stange et al. 2012) in 
503 each individual. The FI of the PC and BT were not significantly higher compared to the NC (Figure

504 S7, Table S14) which illustrates that the mudsnails carried a normal number of embryos according

505 to their size. Hence, the higher number of embryos in the BT and the PT could not definitely be the post-treatments $\mathrm{GAC}_{\mathrm{a}}, \mathrm{BF}_{\mathrm{a}}$ as well as from MBR1, MBR1+O $\mathrm{O}_{3}$ and MBR2. Here, the significantly decreased FI indicated a reproductive toxicity compared to the BT (Figure S7, Table

510 S14). The reproductive toxicity could be induced by unspecific toxicity of the ozonated wastewater

511 and/or toxic TPs (Völker et al. 2019). In a study by Giebner et al. (2018) the total number of treatment. The authors assumed that the decreased reproduction was caused by a general toxicity of the wastewater. Interestingly, the reproductive toxicity in snails exposed to water from MBR2

515 implies that it does not remove toxicity as good as a conventional BT (falsifies hypothesis 3).

516

517 4.2.2 Biomarkers for energy reserves (glycogen, protein and lipid content)

518 Glycogen, protein and lipid content have not been previously analysed in P. antipodarum exposed

519 to wastewater. They are of interest because the energy content has an influence on reproduction of

520 gastropods (Gust et al. 2011). In the present study, differences in biomarker sensitivity were

521 observed in the order of lipid > glycogen > protein content after the exposure to the different

522 wastewaters (Figure 4). Gust et al (2011) reported that glycogen was the preferred energy invested

523 in the reproduction of $P$. antipodarum followed by lipids. In this study, exposure to differently

524 treated wastewater did not affect the protein content but the glycogen content of the mudsnails 
529 result in a higher reproduction. The total energy content mirrors that picture because lipids are the 530 dominant energy storage in $P$. antipodarum.

\section{$532 \quad 4.2 .3$ In vitro endocrine activity and mutagenicity}

533 The aqueous samples taken in parallel to the in vivo experiment did not induce any relevant 534 estrogenic and anti-androgenic activities in any sample (Figure S8, Table S17). Accordingly, the 535 removal capacity could not be evaluated for these two parameters. In contrast, high anti-estrogenic and androgenic activities were detected in PT. The androgenic activity was almost completely removed in the BT whereas the anti-estrogenic activity was substantially reduced but remained on a relatively high level throughout all AWWT technologies (Figure S8, Table S17). Hence, the

539 cleaning capacity of the BT seemed not sufficient in removing the latter, which has been suggested 540 in earlier studies on the present (Abbas et al. 2019) and on other activated sludge treatments (Harth 541 et al. 2018, Ihara et al. 2014, Rao et al. 2014, Tang et al. 2014)

542 Regarding the 10.4-fold concentrated extracts, the estrogenic activity in the BT was almost 543 completely removed by ozonation (Figures 6, Table S18). Accordingly, an additional removal by 544 the post-treatments could not be assessed. In contrast, the anti-estrogenic activity increased 545 markedly in $\mathrm{BT}+\mathrm{O}_{3}$. The $\mathrm{BF}$ and $\mathrm{BF}_{\mathrm{a}}$ did not reduce the anti-estrogenic activity whereas GAC and $546 \mathrm{GAC}_{\mathrm{a}}$ were more effective. One explanation might be that the activated carbon is better in

547 adsorbing more polar ozonation TPs than the more non-polar BF

548 Ozonation led to reduction of the anti-androgenic activity but it remained on a relatively high level 549 compared to previous reports (Gehrmann et al. 2018, Itzel et al. 2020) indicating an incomplete 
555 An almost total reduction of estrogenic activity and simultaneous increase of anti-estrogenic activity

556 in the MBR $1+\mathrm{O}_{3}$ is consistent with the observation for the $\mathrm{BT}+\mathrm{O}_{3}$ (compare above) indicating an

557 incomplete removal of substances with anti-estrogenic activity.

558 The results of the Ames test with the strain YG7108 (Figure 6) support previous hypotheses on

559 mutagenic TPs generated during ozonation $\left(\mathrm{BT}+\mathrm{O}_{3}\right.$ and $\left.\mathrm{MBR} 1+\mathrm{O}_{3}\right)$. Interestingly, water treated

560 with $\mathrm{BF}$ was also mutagenic. Here, the causes remain unknown. Again, the GAC treatments did not

561 generate mutagenic activity. These results again indicated a higher performance of the GAC filters

562 compared to the BFs.

563

$564 \quad 4.3$ Removal of micropollutants

Twenty-eight micropollutants and twelve wastewater parameters were analysed in parallel to the on-site experiment with P. antipodarum to evaluate the performance of the AWWT technologies.

567 The BT effectively reduced the COD, DOC, $\mathrm{NH}_{4}-\mathrm{N}, \mathrm{P}_{\text {total }}$ and $\mathrm{SAC}_{254}$. These parameters were only

568 minimally affected by ozonation, except for the $\mathrm{SAC}_{254}$. GAC and $\mathrm{BF}$ achieved an additional 569 reduction of the COD, DOC and $\mathrm{SAC}_{254}$ whereby GAC was more effective than BF (Tables S22-

570 S29)

571 The MBR systems decreased most of these parameters, except for $\mathrm{NO}_{3}-\mathrm{N}, \mathrm{NH}_{4}-\mathrm{N}$ and $\mathrm{P}_{\text {total }}$ at

572 comparable or higher effectivity than the BT. MBR1 had a slightly higher effectivity than MBR2,

573 which may have been the result of the recirculated ozonated wastewater from the MBR $1+\mathrm{O}_{3}$.

574 Generally, the $\mathrm{MBR} 1+\mathrm{O}_{3}$ only showed a comparable $\left(\mathrm{SAC}_{254}\right)$ or better $\left(\mathrm{COD}, \mathrm{DOC}, \mathrm{NO}_{2}-\mathrm{N}\right)$

575 removal than the $\mathrm{BT}+\mathrm{O}_{3}$ (hypothesis 3). 
579 et al. 2012). Ozonation decreases the concentration of carboxy-acyclovir with an additional removal

580 in the subsequent post-treatments. In general, ozonation resulted in an additional removal of target

581 compounds compared to the conventional treatment (Figure 7) with the exception of 3-hydroxy-

582 ibuprofen, 4-hydroxy-1H-benzotriazol, 4-nitro-sulfmethoxazole, carboxy-ibuprofen, caffeine,

583 paracetamol and mecoprop. This is in line with a multitude of previous studies demonstrating the

584 performance of ozone treatments in further reducing micropollutants (Prasse et al. 2015).

585 A post-treatment with GAC further reduced the concentrations of compounds detected after

586 ozonation (Table S19). In most cases, this reduction was to levels below the LOQ for both, non-

587 aerated and aerated GAC filtration. This demonstrates that a combination of ozonation and activated

588 carbon post-treatments is very effective in removing micropollutants. The two BF systems also

589 reduced the concentrations of micropollutants further with no marked difference between non-

590 aerated and aerated BF. They were, however, less effective in removing some compounds (e.g.,

591 iopromide) than the GAC systems (Table S20).

592 The MBR systems had a very similar performance in removing target chemicals like the

593 conventional activated sludge treatment (Figure 7). This is in line with previous studies (Bertanza et

594 al. 2017, Maletz et al. 2013). The combination of an MBR with ozonation further improved the

reduction of recalcitrant chemicals (Table S20). Accordingly, MBRs can be a suitable alternative

598 4.4 What is the optimal wastewater treatment from an ecotoxicological point of view?

599 Residual ecotoxicological effects and micropollutants were detected in the present full-scale

600 WWTP using an activated sludge treatment. This highlights the need for alternative and/or AWWT

601 treatment options and/or optimisation of the activated sludge treatment. Here, ozonation was 


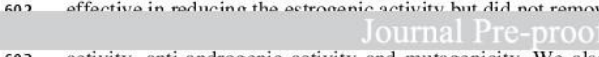

603 activity, anti-androgenic activity and mutagenicity. We also observed a reduction in growth and

604 reproduction of $P$. antipodarum exposed on-site to ozonated wastewater. These findings support the

605 idea that ozonation is effective in removing some specific toxicities while it generates toxic TPs that

606 induce other adverse effects (Völker et al. 2019, hypothesis 2). Accordingly, a post-treatment is

607 needed to reduce these effects. Here, GAC filtration was more effective than the BFs in reducing

608 the residual/generated in vitro toxicity. The same was true for some micropollutants. No specific

609 differences were observed for aerated versus non-aerated systems. As all post-treatments were fed

610 with the same wastewater, we conclude that a GAC post-treatment is preferable to BF when

611 improving the toxicity/chemical removal of ozonated wastewater. However, other considerations

612 (e.g., energy demand, available space, carbon footprint) need to be taken into account when

613 deciding on a suitable post-treatment.

614 MBR systems can be a promising alternative to conventional activated sludge processes (Bui et al

615 2016). In the present study, MBR1 but not MBR2 had a similar removal performance for toxicity

616 and micropollutants like the BT (hypothesis 3). Raw wastewater treated in MBR2 induced a marked

617 reproductive toxicity in $P$. antipodarum. Thus, a combination with ozonation (MBR1) might be

618 preferable. However, the latter treatment generated a high mutagenicity which was removed by

619 recirculating the ozonated water in the MBR. Accordingly, a combination of MBR and ozonation

620 technologies might represent a promising option for specific situations, such as little available space

621 for WWTP in urban settings.

622 
624 - To determine optimal ozone doses and HRTs, maximum removal rates and generation of in

625 vitro toxicity have to be balanced. An ozone dose of $0.33 \mathrm{~g} \mathrm{O}_{3} / \mathrm{g}$ DOC and an HRT of $12.6 \mathrm{~min}$

626 seemed optimal.

627 - While ozonation was effective in further reducing toxicity and micropollutants it also formed

628 toxic TPs. Thus, post-treatment is needed. Activated carbon and biological post-filtration

629 (further) reduced most of the effect with GAC being more effective than BF.

630 - MBR systems as alternatives to an activated sludge treatment were similarly effective like the

631 BT and even performed better (e.g., removal of estrogenicity). $M B R+\mathrm{O}_{3}$ improved the removal

632 performance but also generated mutagenicity. The latter was reduced by recirculation to the

633 MBR which might represent a promising option

634 - A significant anti-estrogenic activity remained in all AWWTs which should be further

635 investigated.

636 - Conventionally treated wastewater affected growth and reproduction of $P$. antipodarum (better 637 nutrient supply or exposure of estrogenic chemicals). Ozonation reduced the reproduction 638 indicating a potential formation of toxic TPs. In the post-treatments these effects were 639 compensated or remained unaffected. All MBR treatments induced reproductive toxicity.

640 - Ozonation of conventionally treated wastewater reduced micropollutants and improved 641 wastewater parameters. Post-treatment with GAC/BF resulted in an additional reduction. MBRs 642 were comparable to $\mathrm{BT}$ while $\mathrm{MBR}+\mathrm{O}_{3}$ was similarly effective like $\mathrm{BT}+\mathrm{O}_{3}$.

643 - For an optimised effect-based assessment of wastewater quality of conventional and AWWT 644 sensitive and environmentally relevant in vitro and in vivo endpoints as well as an adapted 645 chemical analysis are needed. In addition, further parameters (e.g., energy demand, carbon 646 emission), alternative technical options (e.g., optimising activated sludge treatments) and 647 socioeconomic factors (i.e., source control) have to be considered. 
649 This study was funded by the German Federal Ministry of Education and Research (BMBF) Project

650 TransRisk "Charakterisierung, Kommunikation und Minimierung von Risiken durch neue

651 Schadstoffe und Krankheitserreger im Wasserkreislauf" (funding number: 02WRS1275B, contract

652 period: 11/2011 to 04/2015) within the funding measure "Risk Management of Emerging

653 Compounds and Pathogens in the Water Cycle (RiSKWa)".

654 The authors would like to thank Lisa Schlüter-Vorberg from ECT Oekotoxikologie GmbH in

655 Flörsheim for her support during the on-site experiments as well as Prof. Dr. P. Cornel from

656 Technische Universität Darmstadt and the employees at the investigated WWTP for their technical

657 assistance and providing the wastewater samples. 


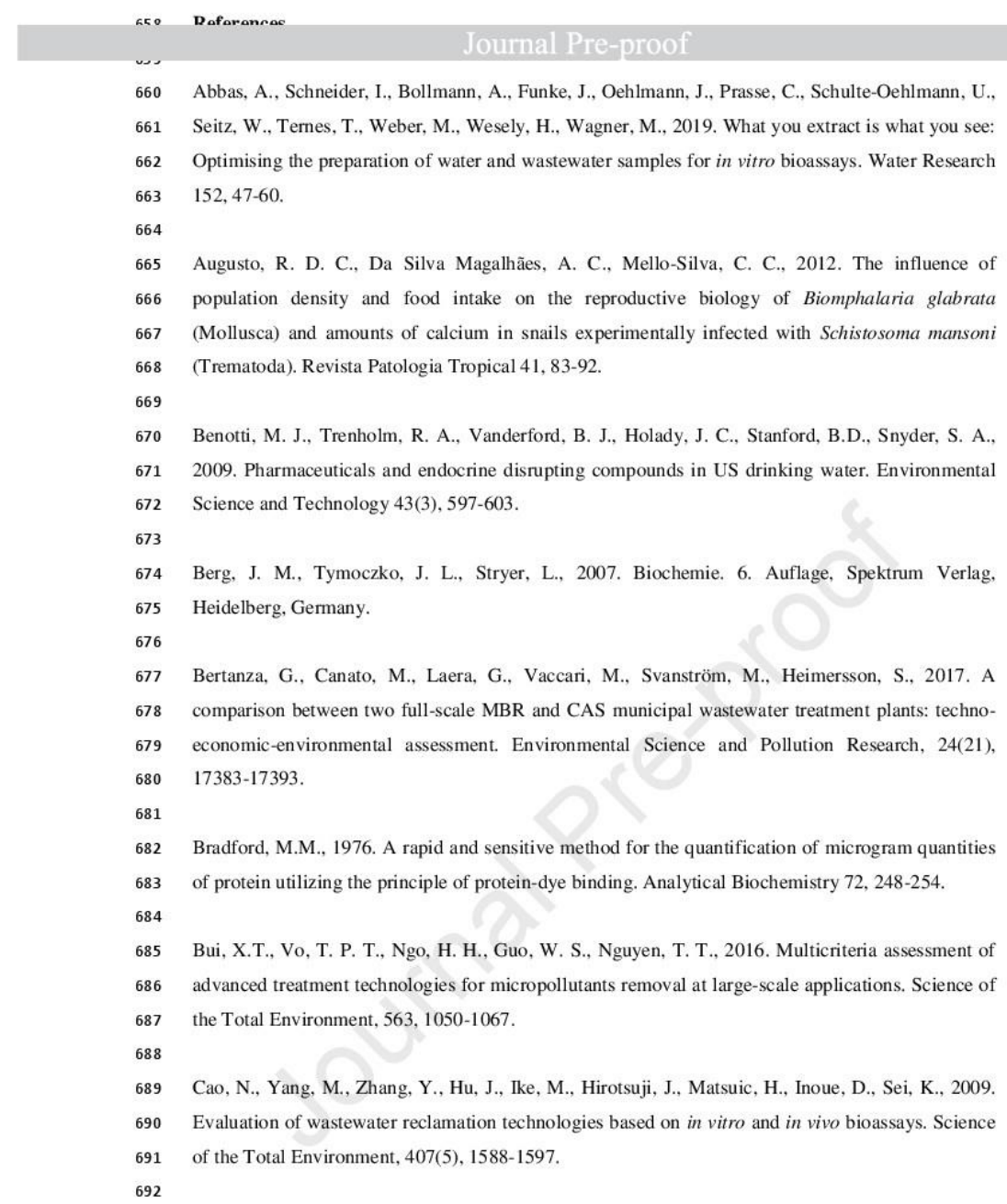




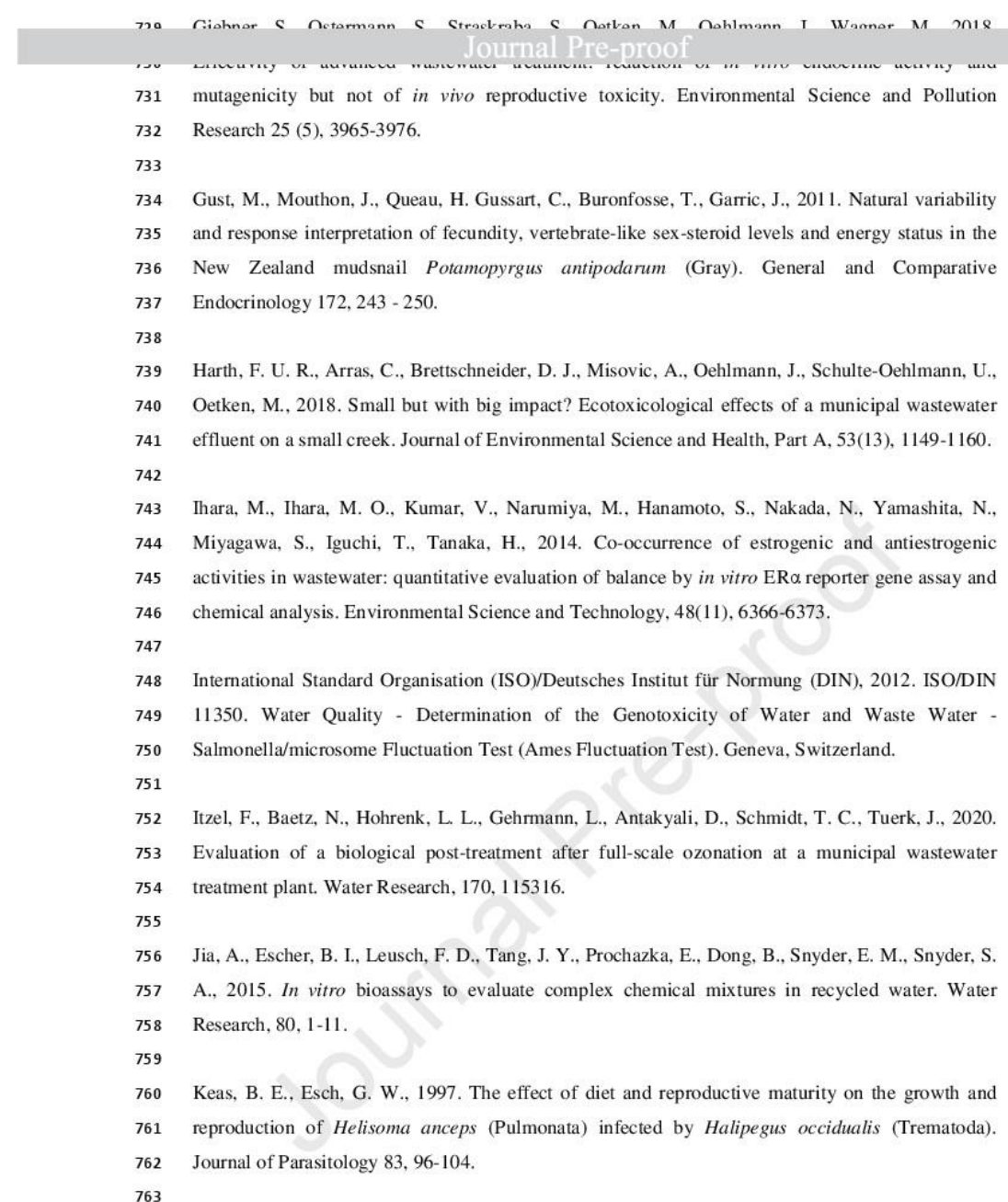




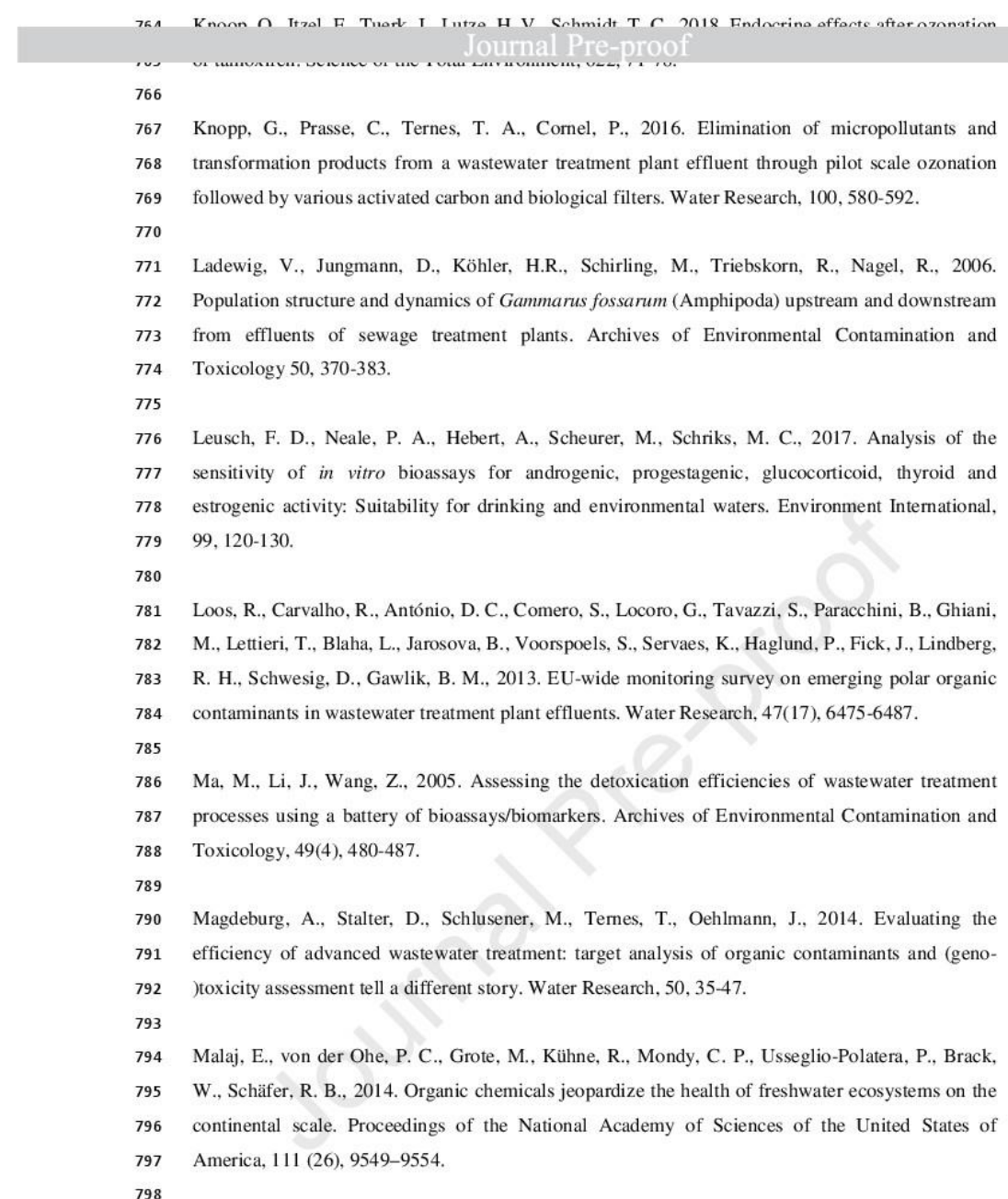




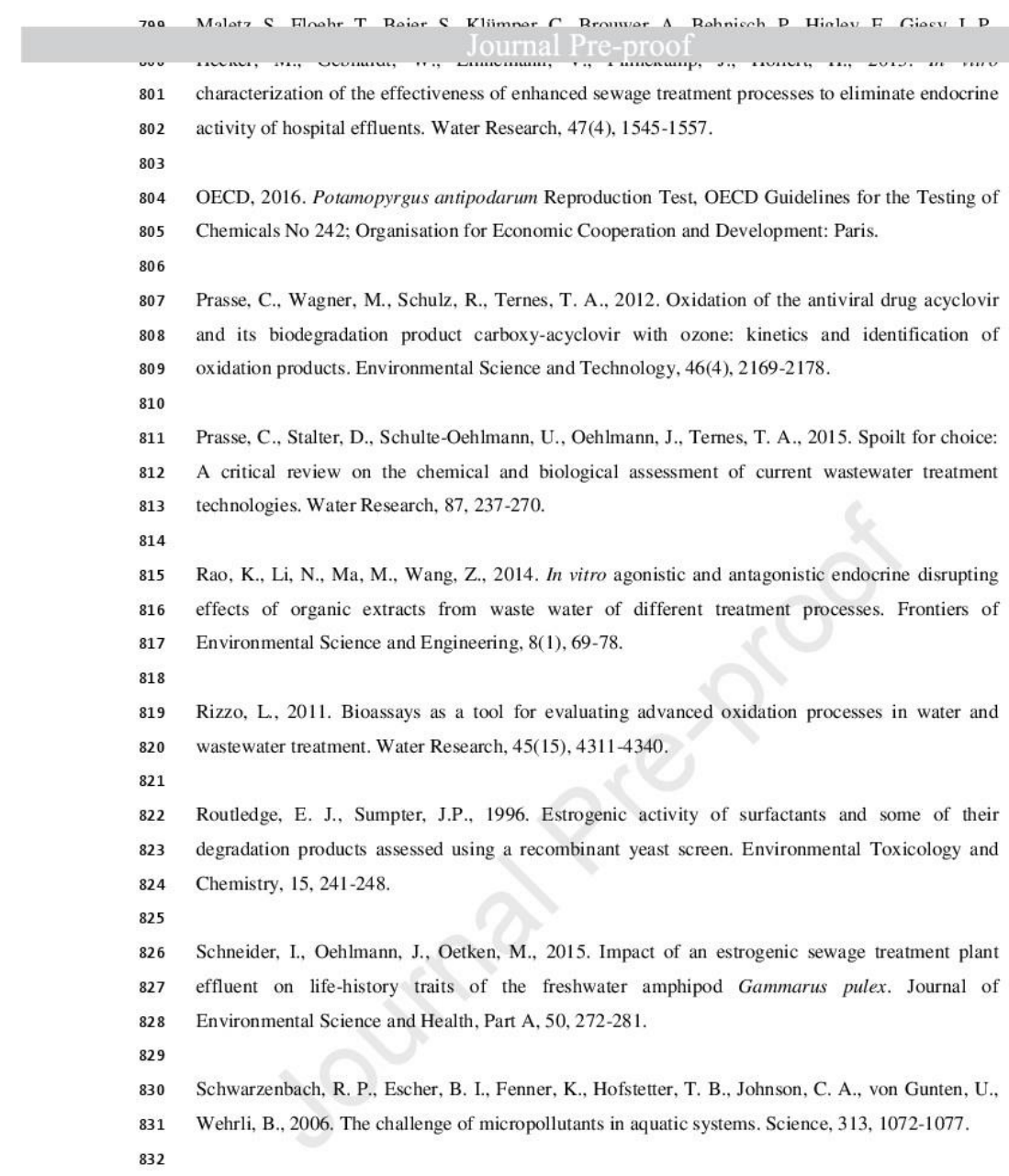




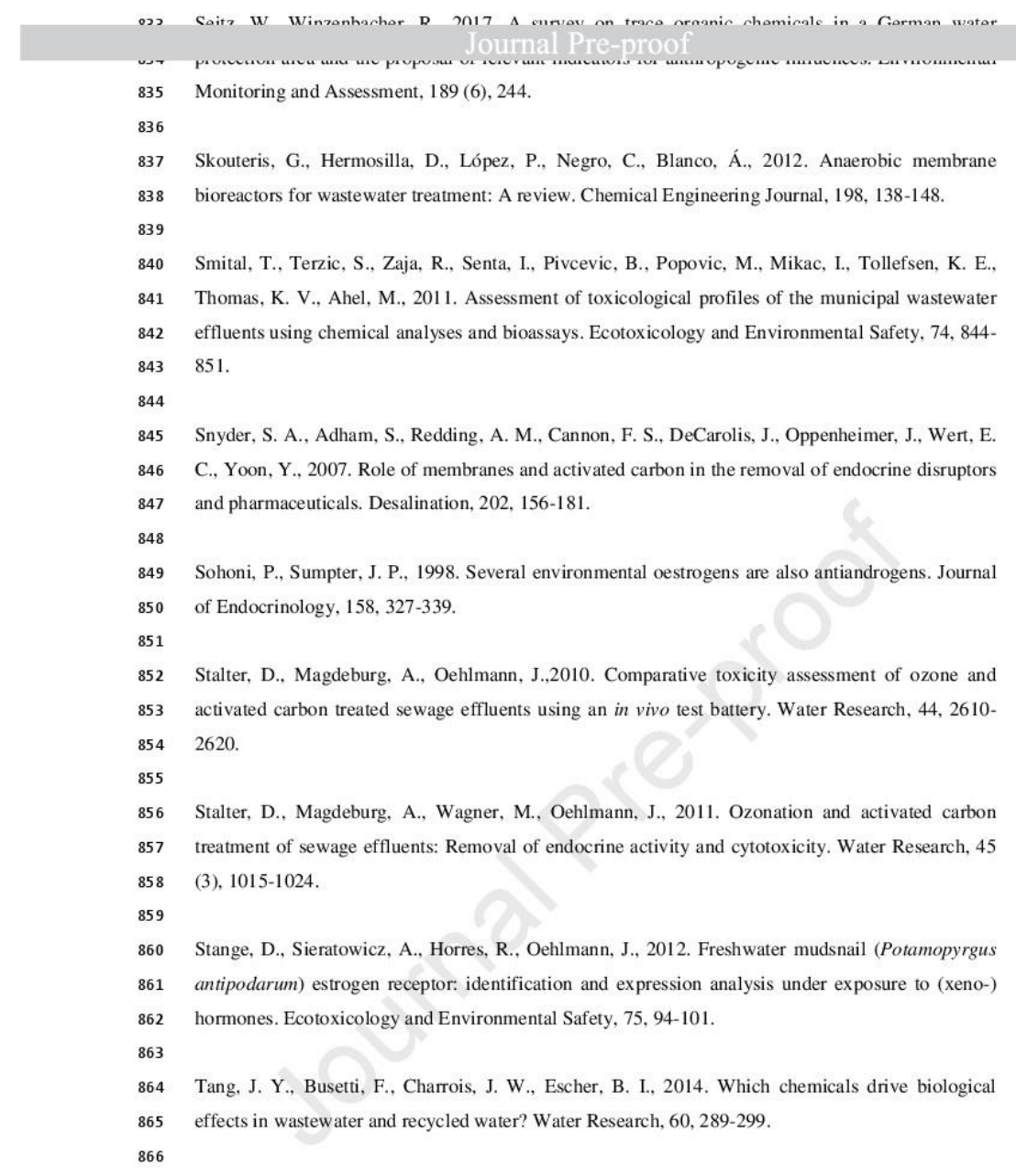




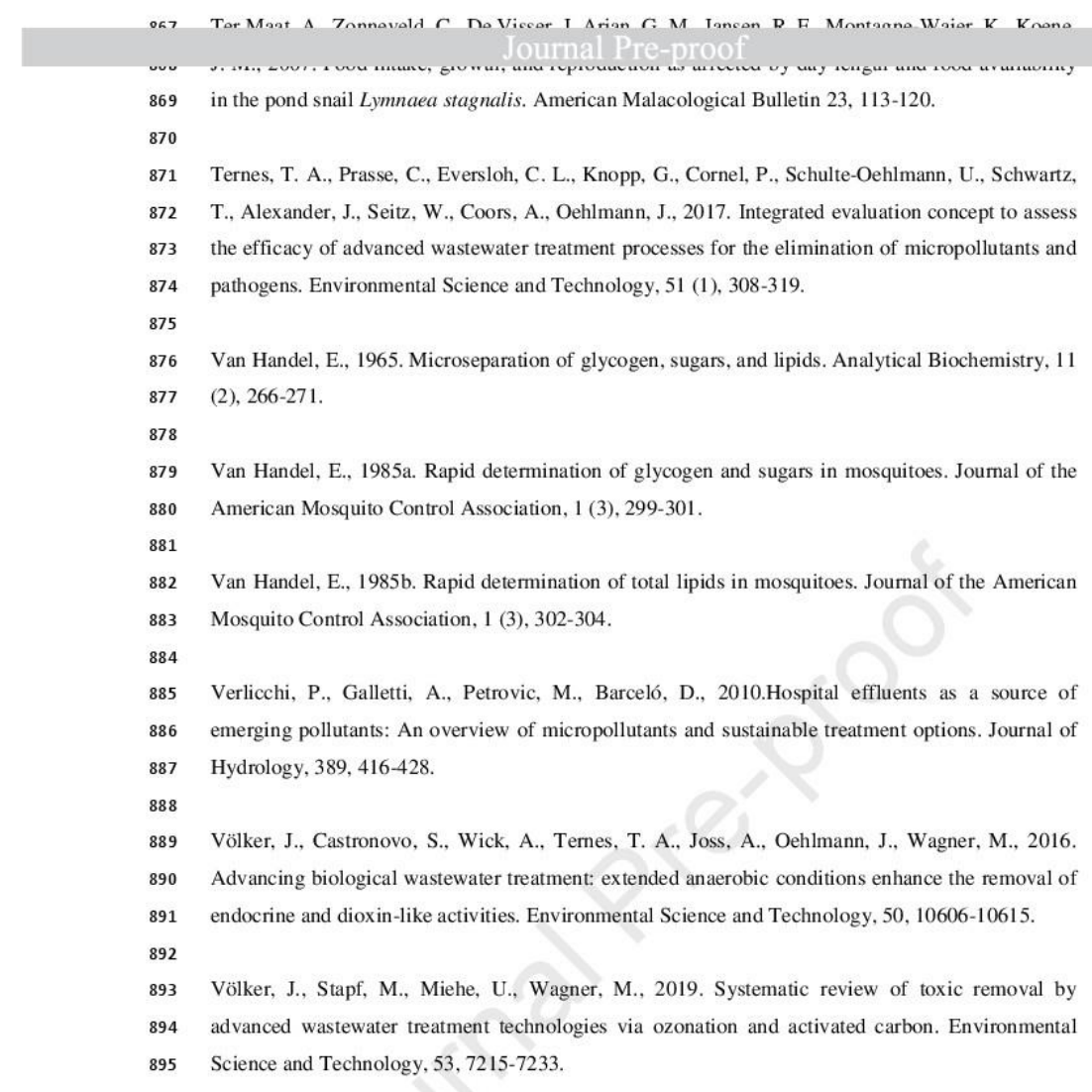




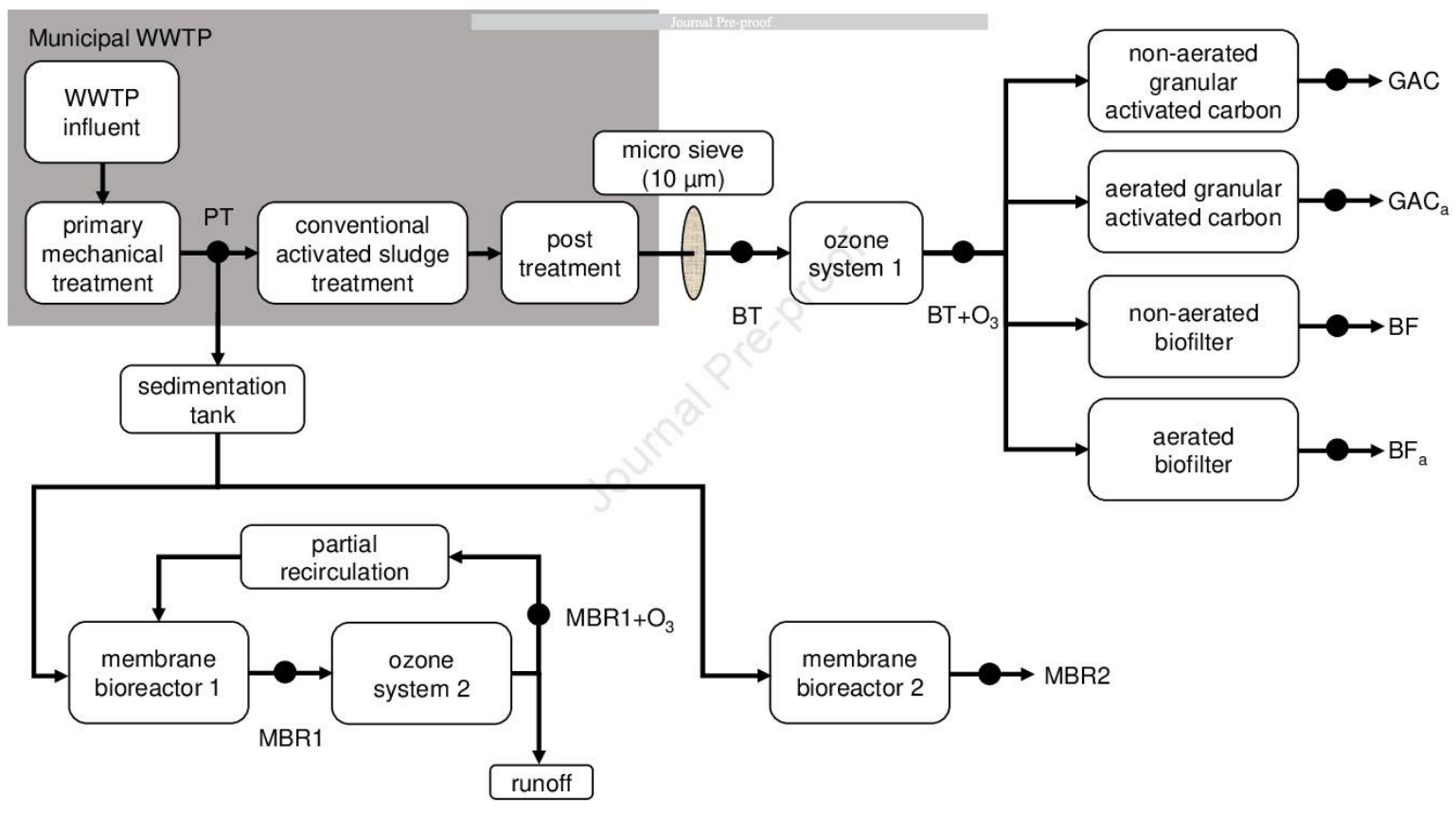




\section{Journal Pre-proof}

Figure 1: Process design of the WWTP and AWWT. Process design of the municipal wastewater treatment plant (WWTP) and the pilot-scale advanced wastewater treatment technologies (AWWT). Sampling points are marked with black dots. PT: after primary treatment, BT: after conventional biological treatment, $\mathrm{BT}+\mathrm{O}_{3}$ : biological treatment after ozonation, GAC: non-aerated granular activated carbon, GAC $\mathrm{a}$ : granular activated carbon aerated with ambient air, BF: non-aerated biofilter, $\mathrm{BF}_{\mathrm{a}}$ : biofilter aerated with ambient air, MBR1/2: membrane bioreactor 1/2, MBR1+O $\mathrm{O}_{3}$ : membrane bioreactor 1 after ozonation. 

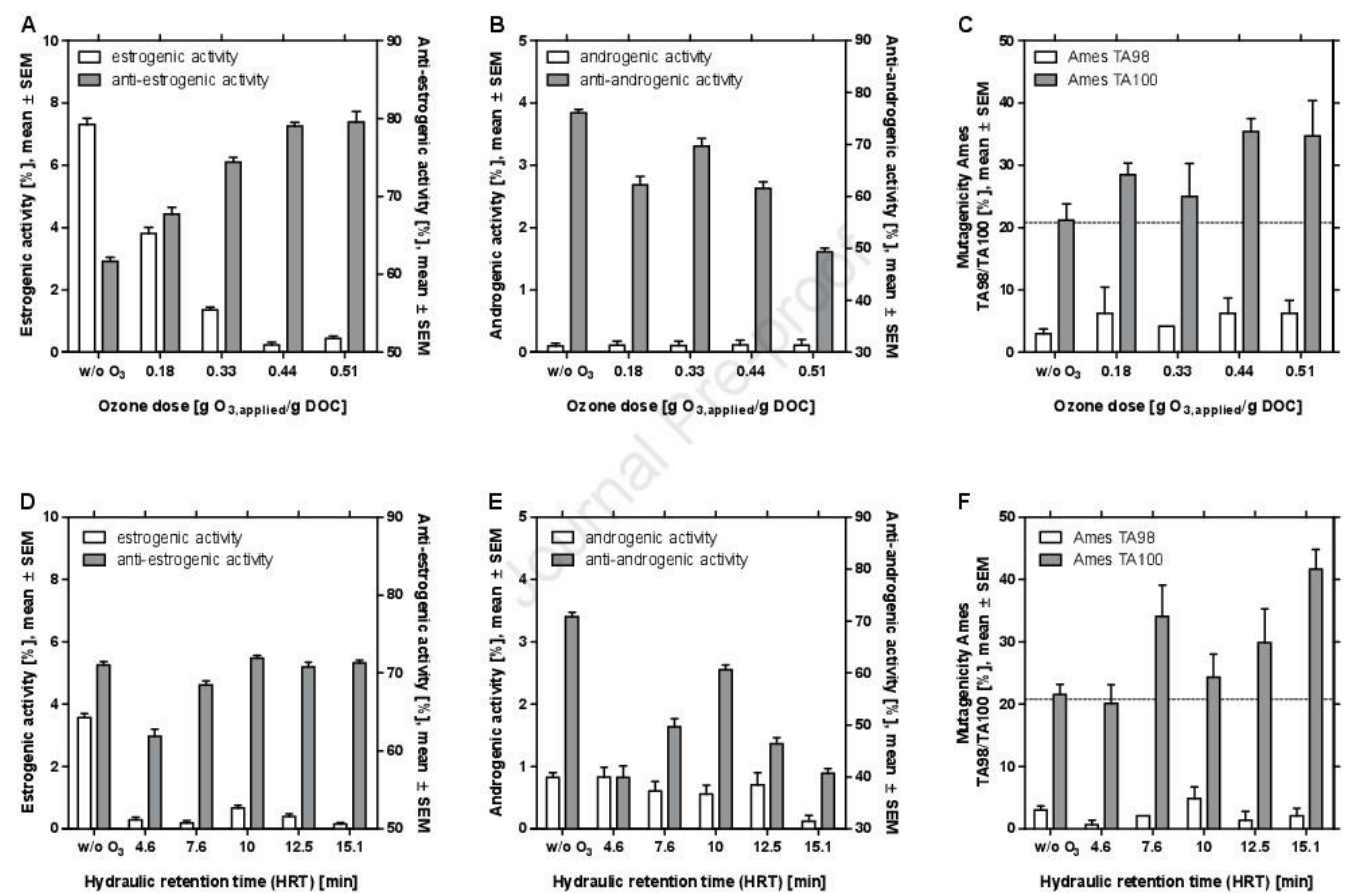


\section{Journal Pre-proo}

Figure 2: Optimal ozone dose and hydraulic retention time. Estrogenic and anti-estrogenic activity (A, D), androgenic and anti-androgenic activity (B, E) and mutagenicity (C, F) in \% (mean $\pm \mathrm{SEM}$ ) of conventional biological treated wastewater (without ozone; A, B: $n=93-$ 96; D, E: $n=117-120 ; C, F: n=12-15$ ) and ozonated wastewater (three SPE extracts per ozone dose (A, B: $n=16-24, C: n=3)$ and hydraulic retention time (D, E: $n=21-24 ; F$ : $\mathrm{n}=3)$ ). A, B, C: multiple ozone dose $\left(0.18-0.51 \mathrm{~g} \mathrm{O}_{3}\right.$, applied $\left./ \mathrm{g} \mathrm{DOC}\right)$ at a constant hydraulic retention time of $12.6 \mathrm{~min}$; D, E, F: multiple hydraulic retention times $(4.6-15.1 \mathrm{~min})$ at a constant ozone dose of $0.53 \mathrm{~g} \mathrm{O}_{3}$, applied $/ \mathrm{g}$ DOC. w/o $\mathrm{O}_{3}$ : without ozone. 

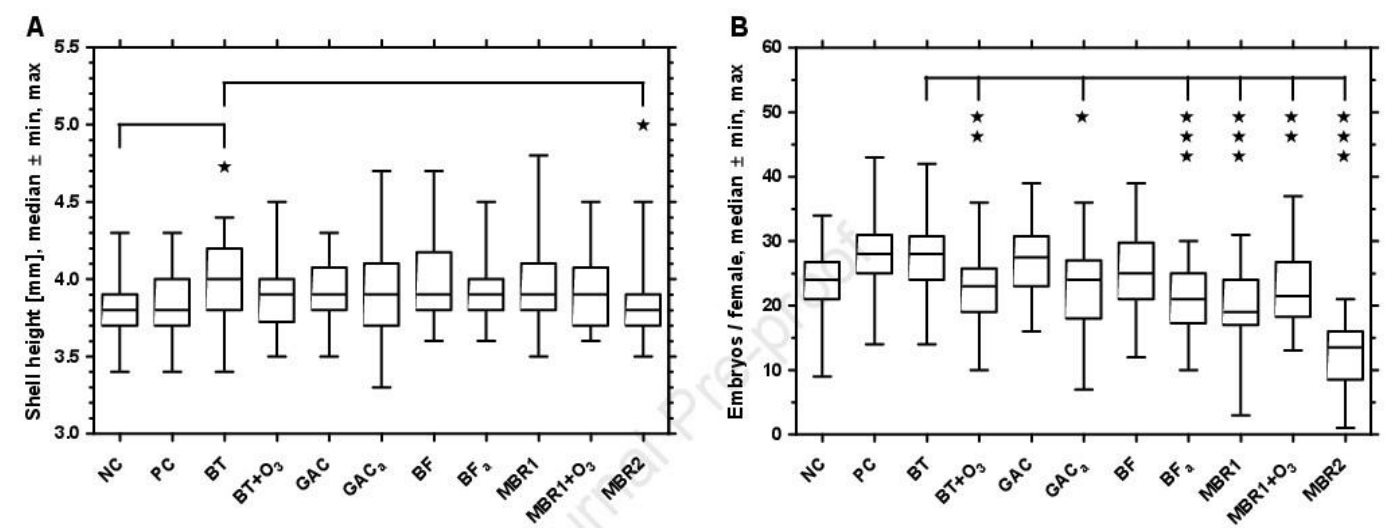


\section{Journal Pre-proof}

Figure 3: Growth and reproduction. Size (A) and reproduction (B) of Potamopyrgus antipodarum after 28 days of exposure to the negative control (NC), the positive control (PC), the conventional biological treatment (BT) and the eight advanced treatment technologies. $\mathrm{BT}+\mathrm{O}_{3}$ : after ozone system 1, GAC: after non-aerated granular activated carbon treatment, $\mathrm{GAC}_{\mathrm{a}}$ : after aerated granular activated carbon treatment, BF: after non-aerated biofilter treatment, $\mathrm{BF}_{\mathrm{a}}$ : after aerated biofilter treatment, MBR1/2: after membrane bioreactor $1 / 2$, MBR1 $+\mathrm{O}_{3}$ : after ozone system 2. Significant differences to BT are indicated with asterisks: $\mathrm{p}<0.05, \star \star \mathrm{p}<0.01, \star \star \star \mathrm{p}<0.001$ (Kruskal-Wallis with Dunn's post-test), $\mathrm{n}=35-40$. 

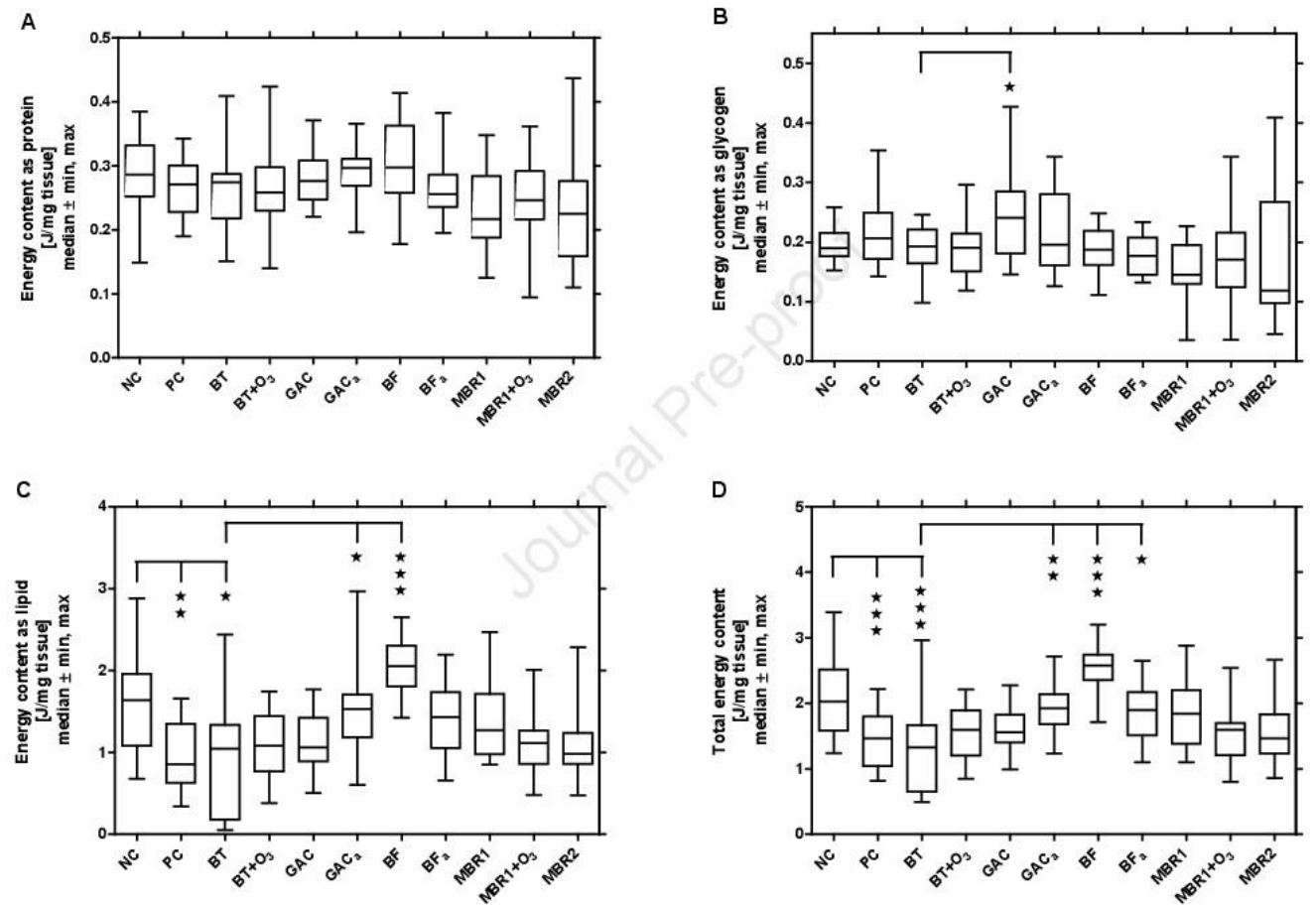


\section{Iournal Pre-proo}

Figure 4: Biomarkers for energy reserves. Energy content as protein (A), glycogen (B), lipid (C) and total energy content (D) in $\mathrm{J} / \mathrm{mg}$ tissue of Potamopyrgus antipodarum after 28 days exposure to water from the negative control (NC), the positive control (PC), the conventional biological treatment (BT) and the eight advanced treatment technologies in an on-site flow-through system. $\mathrm{BT}+\mathrm{O}_{3}$ : after ozone system 1, GAC: after non-aerated activated granular carbon treatment, $\mathrm{GAC}_{\mathrm{a}}$ : after aerated activated granular carbon treatment, $\mathrm{BF}$ : after non-aerated biofilter treatment, $\mathrm{BF}_{\mathrm{a}}$ : after aerated biofilter treatment, $\mathrm{MBR} 1 / 2$ : after membrane bioreactor $1 / 2, M B R 1+\mathrm{O}_{3}$ : after ozone system 2 . Significant differences to NC and $\mathrm{BT}$, are indicated with asterisks: $\star \mathrm{p}<0.05, \star \star \mathrm{p}<0.01, \star \star \star \mathrm{p}<0.001$ (One-way ANOVA with Bonferroni's post-test (B, D) or Kruskal-Wallis with Dunn's post-test (A, C)), $\mathrm{n}=17-20$. 

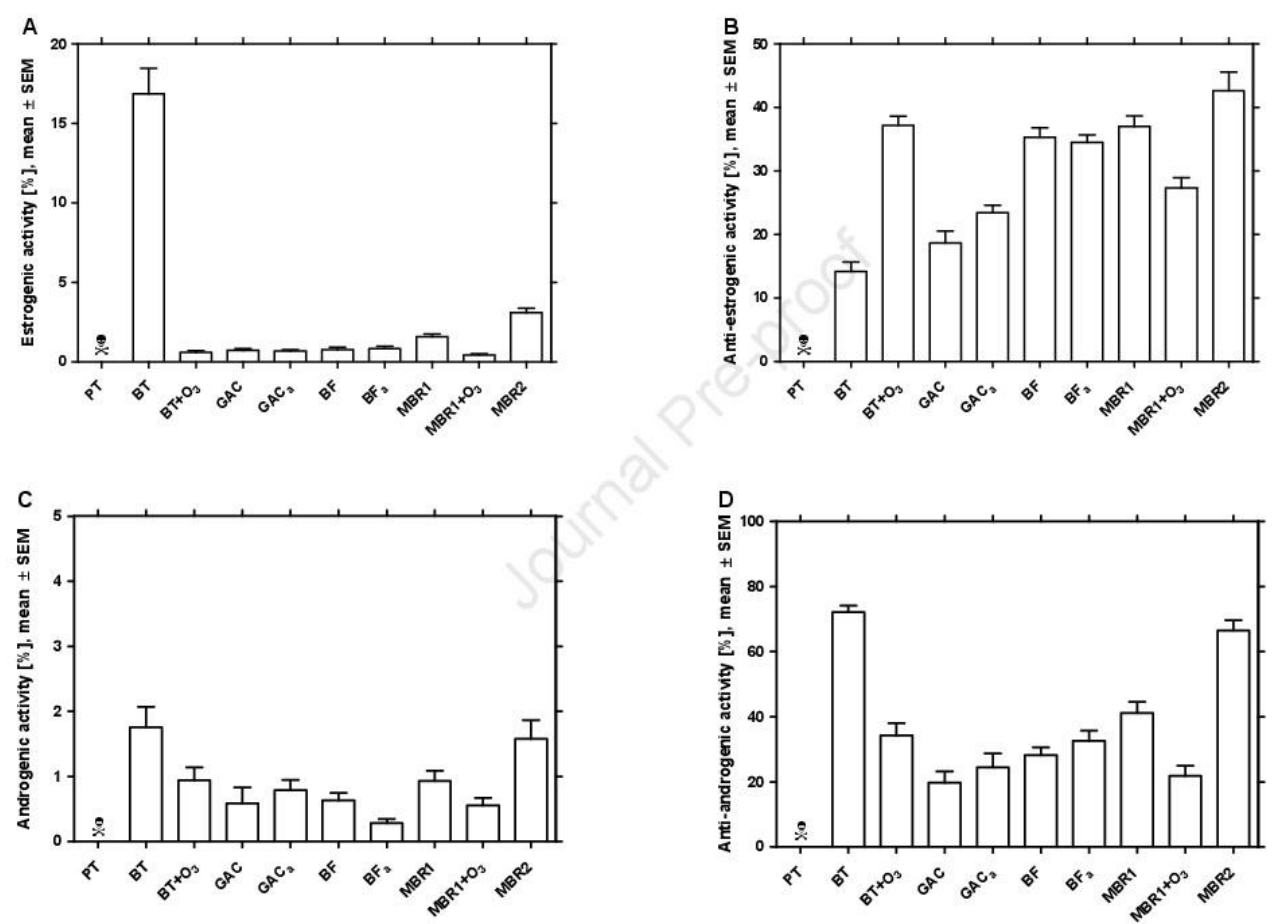


\section{Journal Pre-proof}

Figure 5: Endocrine activities of the on-site biotest. Estrogenic (A), anti-estrogenic (B), androgenic (C) and anti-androgenic activity (D) in SPE extracts produced from $24 \mathrm{~h}$ composite samples taken in parallel to the in vivo experiment. PT: after primary treatment, BT: after conventional biological treatment, $\mathrm{BT}+\mathrm{O}_{3}$ : after ozone system 1, GAC: after non-aerated granular activated carbon treatment, $\mathrm{GAC}_{\mathrm{a}}$ : after aerated granular activated carbon treatment, $\mathrm{BF}$ : after non-aerated biofilter treatment, $\mathrm{BF}_{\mathrm{a}}$ : after aerated biofilter treatment, MBR1/2: after membrane bioreactor $1 / 2$, MBR $1+\mathrm{O}_{3}$ : after ozone system $2, \stackrel{2}{*}$ cytotoxic, $\mathrm{n}=32$. 


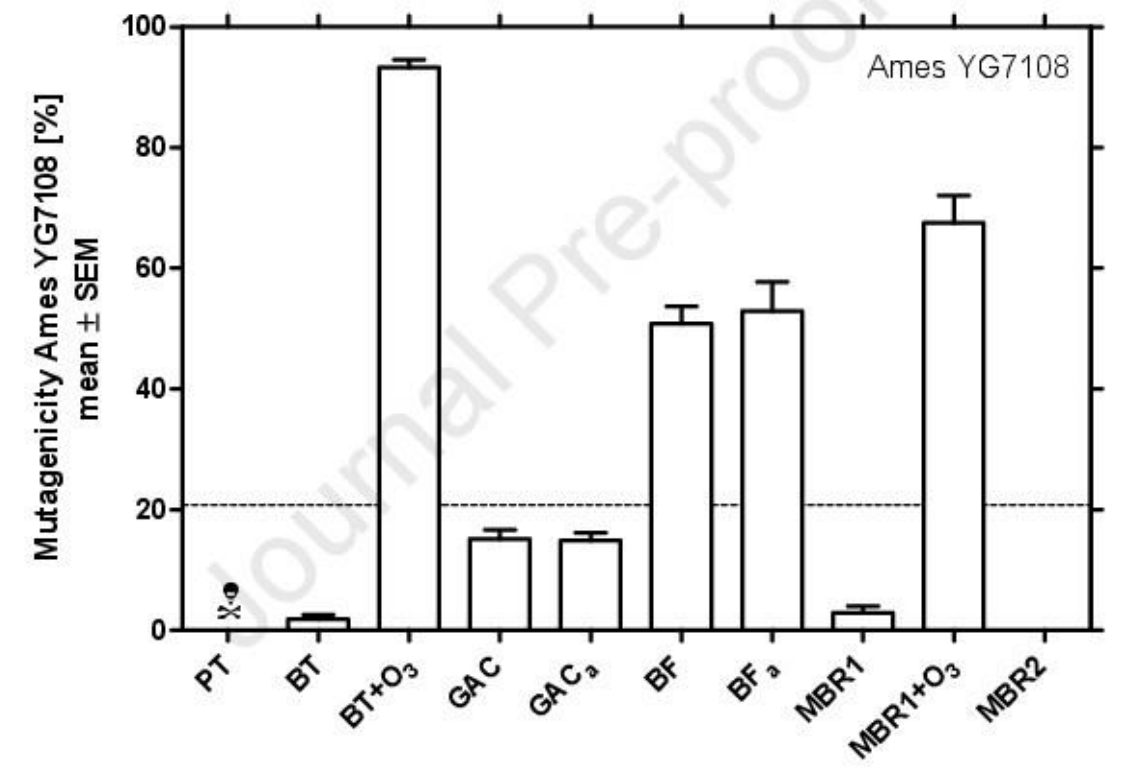




\section{Iournal Pre-proof}

Figure 6: Mutagenicity of the on-site biotest. Mutagenicity in the Ames strain YG7108 in SPE extracts produced from $24 \mathrm{~h}$ composite samples taken in parallel to the in vivo experiment. PT: after primary treatment, BT: after conventional biological treatment, $\mathrm{BT}+\mathrm{O}_{3}$ : after ozone system 1, GAC: after non-aerated granular activated carbon treatment, GACa: after aerated granular activated carbon treatment, BF: after non-aerated biofilter treatment, $\mathrm{BF}_{\mathrm{a}}$ : after aerated biofilter treatment, MBR1/2: after membrane bioreactor $1 / 2, \mathrm{MBR} 1+\mathrm{O}_{3}$ : after ozone system $2, \gtrless$ : cytotoxic, $\mathrm{n}=8$. 


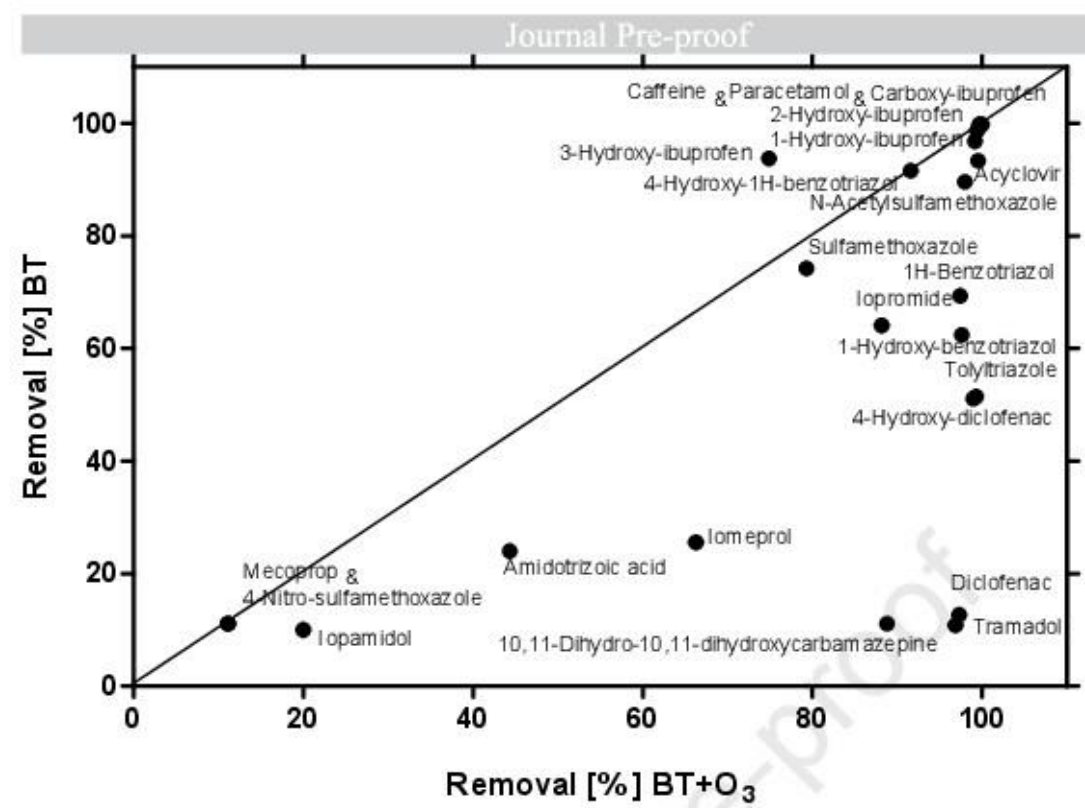

B

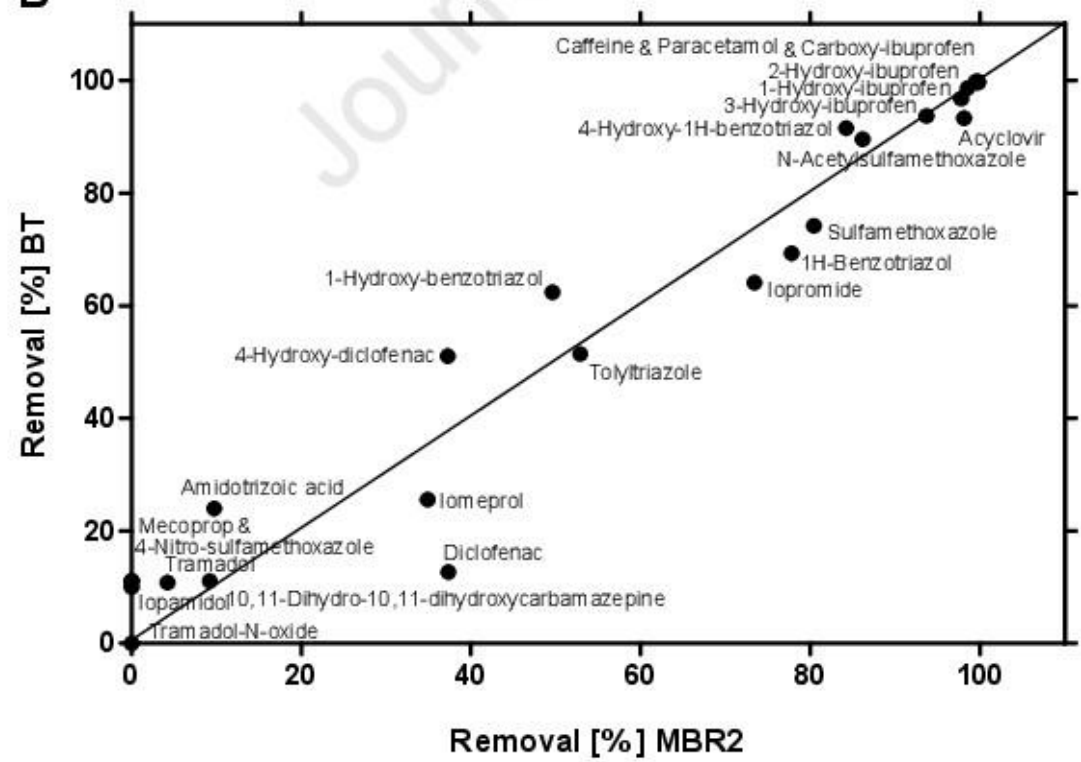


Figure 7: Chemical analysis. Removal of micropollutants by the conventional biological treatment (BT), by the ozonation $\left(\mathrm{BT}+\mathrm{O}_{3}, \mathrm{~A}\right)$ and by the membrane bioreactor $2(\mathrm{MBR} 2, \mathrm{~B})$ compared to the primary treatment. $n=1-4$. 
Post-treatment of ozonated wastewater with activated carbon and biofiltration compared to membrane bioreactors: Toxicity removal in vitro and in Potamopyrgus antipodarum

Ilona Schneider, Aennes Abbas, Anna Bollmann, Andrea Dombrowski, Gregor Knopp, Ulrike Schulte-OehImann, Wolfram Seitz, Martin Wagner, Jörg Oehlmann

\section{HIGHLIGHTS}

- Comprehensive comparison of toxicity and micropollutant removal by advanced wastewater treatment

- Ozonation reduces estrogenicity and micropollutants but forms anti-estrogenicity and mutagenicity

- Post-treatment with granular activated carbon is more effective than biofilters

- Membrane bioreactors are as effective as conventional biological wastewater treatment

- Effluents of ozonation and membrane bioreactors induce reproductive toxicity in $P$. antipodarum 


\title{
Supplementary information (paper A.3)
}

\author{
S1 Material and methods
}

S1.1 Technical parameters of the municipal and the pilot wastewater treatment plant

Table S1: Technical parameters of the municipal wastewater treatment plant.

\begin{tabular}{ll}
\hline conventional activated sludge: & $11,750 \mathrm{~m}^{3}$ \\
volume of the pipelines: & $100 \mathrm{~m}^{3}$ \\
average dry weather flow rate: & $3750 \mathrm{~m}^{3} / \mathrm{d}$ \\
hydraulic retention time: & $75.8 \mathrm{~h}(3.16 \mathrm{~d})$ \\
\hline
\end{tabular}

Table S2: Technical parameters of the pilot wastewater treatment plant.

\begin{tabular}{|c|c|c|c|}
\hline & volume $\left[\mathrm{m}^{3}\right]$ & flow rate $\left[\mathrm{m}^{3} / \mathrm{h}\right]$ & retention time [h]; [d] \\
\hline Micro sieve & 5 & 2.32 & $2.15 ; 0.090$ \\
\hline Ozone system 1 & 0.212 & 0.709 & $0.299 ; 0.012$ \\
\hline Surge tank 1 (ozone system 1) & 0.150 & 0.709 & $0.212 ; 0.009$ \\
\hline Surge tank 2 (ozone system 1 ) & 0.150 & 0.650 & $0.231 ; 0.010$ \\
\hline $\begin{array}{l}\text { Granular activated carbon filter } \\
\text { (non-aerated) }\end{array}$ & 0.110 & 0.131 & $0.840 ; 0.035$ \\
\hline $\begin{array}{l}\text { Granular activated carbon filter } \\
\text { (aerated) }\end{array}$ & 0.110 & 0.085 & $1.30 ; 0.054$ \\
\hline Biofilter (non-aerated) & 0.110 & 0.124 & $0.886 ; 0.037$ \\
\hline Biofilter (aerated) & 0.110 & 0.128 & $0.858 ; 0.036$ \\
\hline $\begin{array}{l}\text { Reservoir with wastewater of } \\
\text { the primary treatment }\end{array}$ & 3.86 & $7.74\left(186 \mathrm{~m}^{3} / \mathrm{d}\right)$ & $0.499 ; 0.021$ \\
\hline Previous tank before MBR & 2.0 & $0.684\left(16.4 \mathrm{~m}^{3} / \mathrm{d}\right)$ & $2.92 ; 0.122$ \\
\hline MBR1 & 1.64 & 0.122 & $13.5 ; 0.561$ \\
\hline MBR2 & 1.64 & 0.043 & $38.4 ; 1.60$ \\
\hline Surge tank 1 (ozone system 2 ) & 0.100 & $0.117\left(2.81 \mathrm{~m}^{3} / \mathrm{d}\right)$ & $0.853 ; 0.036$ \\
\hline Ozone system 2 & 0.049 & $0.117\left(2.81 \mathrm{~m}^{3} / \mathrm{d}\right)$ & $0.416 ; 0.017$ \\
\hline
\end{tabular}


Table S3: Process parameters (mean \pm SD) of ozone system 1 and 2 during the test period of 28 days. D: ozone dose, d: specific ozone dose, DOC: dissolved organic carbon, HRT: hydraulic retention time, $\mathrm{O}_{3}$ : ozone, $\mathrm{RR}$ : recirculation rate, $\mathrm{Z}$ : ozone consumption, $\mathrm{z}$ specific ozone consumption.

\begin{tabular}{ccccccc}
\hline & HRT [min] & $\mathbf{D}\left[\mathbf{g} / \mathbf{m}^{3}\right]$ & $\mathbf{d}\left[\mathbf{g ~ O}_{3} / \mathbf{g ~ D O C}\right]$ & $\mathbf{Z}\left[\mathbf{g} / \mathbf{m}^{3}\right]$ & $\mathbf{z}\left[g_{3} / \mathbf{g ~ D O C}\right]$ & $\mathbf{R R}$ \\
\hline $\begin{array}{c}\text { Ozone } \\
\begin{array}{c}\text { system } \\
1\end{array}\end{array}$ & $\begin{array}{c}17.9 \pm 0.38 \\
(\mathrm{n}=22)\end{array}$ & $\begin{array}{c}10.1 \pm 1.35 \\
(\mathrm{n}=22)\end{array}$ & $\begin{array}{c}0.95 \pm 0.21 \\
(\mathrm{n}=22)\end{array}$ & $\begin{array}{c}9.86 \pm 1.35 \\
(\mathrm{n}=22)\end{array}$ & $\begin{array}{c}0.93 \pm 0.20 \\
(\mathrm{n}=22)\end{array}$ & - \\
$\begin{array}{c}\text { Ozone } \\
\text { system }\end{array}$ & $\begin{array}{c}26.1 \pm 1.36 \\
(\mathrm{n}=5)\end{array}$ & $\begin{array}{c}6.78 \pm 0.35 \\
(\mathrm{n}=5)\end{array}$ & - & & $0.96 \pm 0.08$ & $2.02 \pm 0.10$ \\
2 & & & & - & $(\mathrm{n}=5)$ & \\
\hline
\end{tabular}

A recirculation rate of 2.0 means that for example $100 \mathrm{~L}$ wastewater from ozone system 2 was recirculated to $50 \mathrm{~L}$ wastewater of the primary treatment that in the end the ozone system 2 was fed with $150 \mathrm{~L}$ of a mixture of both wastewaters.

Table S4: Filter velocity $\left(\mathrm{V}_{\mathrm{F}}\right)$ and empty bed contact time (EBCT) (mean \pm SD, respectively) of the advanced treatment processes after ozone system 1 during the test period of 28 days. GAC: nonaerated granular activated carbon treatment, $\mathrm{GAC}_{\mathrm{a}}$ : aerated granular activated carbon treatment, BF: non-aerated biofilter treatment, $\mathrm{BF}_{\mathrm{a}}$ : aerated biofilter treatment.

\begin{tabular}{ccccc}
\hline & GAC & GAC $_{a}$ & BF & BF $_{a}$ \\
\hline$V_{F}[\mathrm{~m} / \mathrm{h}]$ & $4.92 \pm 0.08(n=31)$ & $3.33 \pm 0.64(n=8)$ & $4.96 \pm 0.14(n=10)$ & $4.94 \pm 0.06(n=11)$ \\
EBCT $[\mathrm{min}]$ & $28.3 \pm 0.57(n=31)$ & $36.4 \pm 5.66(n=8)$ & $27.4 \pm 0.99(n=10)$ & $26.7 \pm 0.28(n=11)$ \\
\hline
\end{tabular}

Table S5: Overview of the sampling dates of the medium of the negative control (NC) and the positive control (PC) and the wastewater treatments. BT: after conventional biological treatment, $\mathrm{BT}+\mathrm{O}_{3}$ : after ozone system 1, GAC: after non-aerated granular activated carbon treatment, $\mathrm{GAC}_{\mathrm{a}}$ : after aerated granular activated carbon treatment, BF: after non-aerated biofilter treatment, BF $F_{\mathrm{a}}$ after aerated biofilter treatment, MBR1/2: after membrane bioreactor 1/2, MBR1+O ${ }_{3}$ : after ozone system $2,{ }^{*}$ : fresh medium was prepared.

\begin{tabular}{|c|c|c|}
\hline $\begin{array}{l}\text { Sample } \\
\text { acronym }\end{array}$ & Sampling date in 2014 & Sampling mode \\
\hline$\overline{\mathrm{NC}}$ & 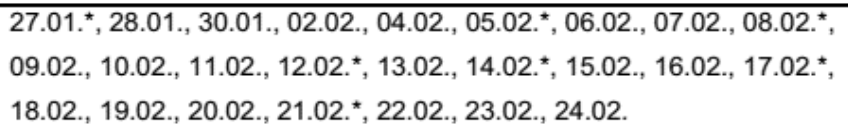 & grab \\
\hline $\mathrm{PC}$ & 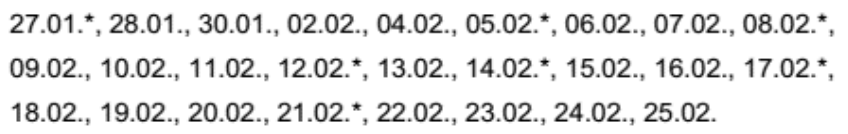 & grab \\
\hline BT & 28./29.01., 04./05.02., 11./12.02., 18./19.02. & $24 \mathrm{~h}$ composite \\
\hline $\mathrm{BT}+\mathrm{O}_{3}$ & 28./29.01., 04./05.02., 11./12.02., 18./19.02. & 24 h composite \\
\hline GAC & 28./29.01., 04./05.02., 11./12.02., 18./19.02. & 24 h composite \\
\hline $\mathrm{GAC}_{\mathrm{a}}$ & 28./29.01., 04./05.02., 11./12.02., 18./19.02. & 24 h composite \\
\hline BF & 28./29.01., 04./05.02., 11./12.02., 18./19.02. & 24 h composite \\
\hline $\mathrm{BF}_{\mathrm{a}}$ & 28./29.01., 04./05.02., 11./12.02., 18./19.02. & 24 h composite \\
\hline PT & 29./30.01., 04./05.02., 11./12.02., 18./19.02. & 24 h composite \\
\hline MBR1 & 29./30.01., 04./05.02., 11./12.02., 18./19.02. & 24 h composite \\
\hline $\mathrm{MBR} 1+\mathrm{O}_{3}$ & 29./30.01., 04./05.02., 11./12.02., 18./19.02. & 24 h composite \\
\hline MBR2 & 29./30.01., 04./05.02., 11./12.02., 18./19.02. & 24 h composite \\
\hline
\end{tabular}




\section{S1.3 Measurement of biomarkers for energy reserves (protein, glycogen and lipid content)}

The measurement of the protein content was done according to Bradford (1976). $50.0 \mu \mathrm{L}$ of the homogenate were mixed with $1.5 \mathrm{~mL}$ Bradford reagent (AppliChem $\mathrm{GmbH}$, Darmstadt, Germany) using a vortex and incubated at room temperature for five minutes. Five increasing concentrations of a bovine serum albumin solution (BSA; $0.1 \%$ ) mixed with Bradford reagent and incubated as well served as a linear standard calibration curve (Tables S6 and S7, Figure S1). The absorption was measured at a wavelength of $595 \mathrm{~nm}$ using a photometer (BioSpectrometer, Eppendorf, Hamburg, Germany). The protein content of the samples was calculated in $\mu \mathrm{g} / \mathrm{mg}$ tissue and then converted to an energy content of the protein reserve in $\mathrm{J} / \mathrm{mg}$ tissue using the specific calorific value of $17.0 \mathrm{~kJ} / \mathrm{g}$ (Berg et al. 2007).

The separation of the glycogen and lipids was done using the micro-separation method as described in van Handel (1965). $100 \mu \mathrm{L}$ of the homogenate were mixed with $1.6 \mathrm{~mL}$ of a chloroform-methanol solution (1:1) and centrifuged (centrifuge 5702, Eppendorf, Hamburg, Germany) for two minutes at $3,000 \mathrm{rpm}$. The generated pellet at the ground contained the glycogen. The separated chloroformmethanol supernatant containing the lipids was mixed with $0.6 \mathrm{~mL}$ demineralised water and centrifugalised for two minutes at 3,000 rpm. The upper water-methanol fraction was discharged, the lower chloroform fraction contained the lipids.

The glycogen content was measured using hot anthrone reaction (van Handel 1965, 1985a). The glycogen pellets were mixed with $5.0 \mathrm{~mL}$ anthrone reagent and incubated in a water bath (Grand Instruments, Cambridge, England) at $95.0^{\circ} \mathrm{C}$ for 17 minutes. Six increasing concentrations of a glucose solution $(0.1 \%)$, mixed with anthrone reagent and incubated as well, served as a linear standard calibration curve (Tables S6 and S8, Figure S2). The absorption was measured at a wavelength of $625 \mathrm{~nm}$ using a photometer (BioSpectrometer, Eppendorf, Hamburg, Germany). The glycogen content of the samples was calculated in $\mu \mathrm{g} / \mathrm{mg}$ tissue and converted to an energy content of the glycogen reserve in $\mathrm{J} / \mathrm{mg}$ tissue using the specific calorific value of $17.0 \mathrm{~kJ} / \mathrm{g}$ (Berg et al. 2007). The lipid content was measured using the vanillin reaction (van Handel 1965, 1985b). After evaporation of the chloroform in a water bath $\left(95.0^{\circ} \mathrm{C}\right)$ lipids were mixed with $200 \mu \mathrm{L}$ sulphuric acid $\left(\mathrm{H}_{2} \mathrm{SO}_{4} ; 95 \%\right)$ and incubated at $95.0^{\circ} \mathrm{C}$ for 10 minutes. After adding $5.0 \mathrm{~mL}$ of the vanillin reagent the samples were mixed with a vortex and incubated for five minutes at room temperature. Five increasing concentrations of a colza solution $(0.1 \%)$ were treated like the samples and served as a linear standard calibration curve (Tables S6 and S8, Figure S3). The absorption was measured at a wavelength of $625 \mathrm{~nm}$ using a photometer (BioSpectrometer, Eppendorf, Hamburg, Germany). The lipid content of the samples was calculated in $\mu \mathrm{g} / \mathrm{mg}$ tissue and then converted to an energy content of the lipid reserve in $\mathrm{J} / \mathrm{mg}$ tissue using the specific calorific value of $37.0 \mathrm{~kJ} / \mathrm{g}$ (Berg et al. 2007). 
Table S6: Solvents needed for the determination of the protein, glycogen and lipid content. BSA: bovine serum albumin, $\mathrm{H}_{2} \mathrm{SO}_{4}$ : sulphuric acid, $\mathrm{H}_{3} \mathrm{PO}_{4}$ : phosphorus acid, $\mathrm{Na}_{2} \mathrm{SO}_{4}$ : sodium sulphate solution.

\begin{tabular}{ll}
\hline Sodium sulphate solution (2.0\%) & $2.0 \mathrm{~g} \mathrm{Na}_{2} \mathrm{SO}_{4}+100 \mathrm{~mL}$ demineralised water \\
Bovine serum albumin solution (0.1\%) & $100 \mathrm{mg} \mathrm{BSA}+100 \mathrm{~mL} \mathrm{Na} \mathrm{SO}_{4}$ solution \\
Chloroform-methanol solution & mix $1: 1$ \\
Anthrone reagent & $150 \mathrm{~mL}$ cooled demineralised water \\
& add $385 \mathrm{~mL}$ sulphuric acid $\left(\mathrm{H}_{2} \mathrm{SO}_{4}\right)$ stepwise \\
& dissolve $750 \mathrm{mg}$ anthrone \\
& storage in the refrigerator \\
& $100 \mathrm{~mL}$ heated demineralised water \\
Vanillin reagent & dissolve $600 \mathrm{mg}$ vanillin \\
& add $400 \mathrm{~mL}$ phosphorus acid $\left(\mathrm{H}_{3} \mathrm{PO}_{4}\right)$ \\
& storage in brown glass bottles in the dark \\
\hline
\end{tabular}

Table S7: Determination of the protein content: pipette scheme for the standard calibration curve with the five increasing concentrations of a bovine serum albumin solution (BSA; $0.1 \%$ ) and sodium sulphate solution $\left(\mathrm{Na}_{2} \mathrm{SO}_{4}\right)$.

\begin{tabular}{ccc}
\hline No. & BSA solution $(\mathbf{0 . 1 \% )}[\mu \mathrm{L}]$ & $\mathrm{Na}_{2} \mathrm{SO}_{4}$ Solution $(\mathbf{2 . 0} \%)[\mu \mathrm{L}]$ \\
\hline 1 & 0.0 & 50.0 \\
2 & 12.5 & 37.5 \\
3 & 25.0 & 25.0 \\
4 & 37.5 & 12.5 \\
5 & 50.0 & 0.0 \\
\hline
\end{tabular}

Table S8: Determination of the glycogen and lipid content: volumes $[\mu \mathrm{L}]$ of the glucose and colza solutions needed for the five, respectively six, increasing concentrations for the standard calibration curves.

\begin{tabular}{ccc}
\hline No. & Glucose solution $(\mathbf{0 . 1 \% )}[\mu \mathrm{L}]$ & Colza solution $(0.1 \%)[\mu \mathrm{L}]$ \\
\hline 1 & 0.0 & 0.0 \\
2 & 25.0 & 50.0 \\
3 & 50.0 & 100 \\
4 & 100 & 200 \\
5 & 150 & 400 \\
6 & 200 & - \\
\hline
\end{tabular}




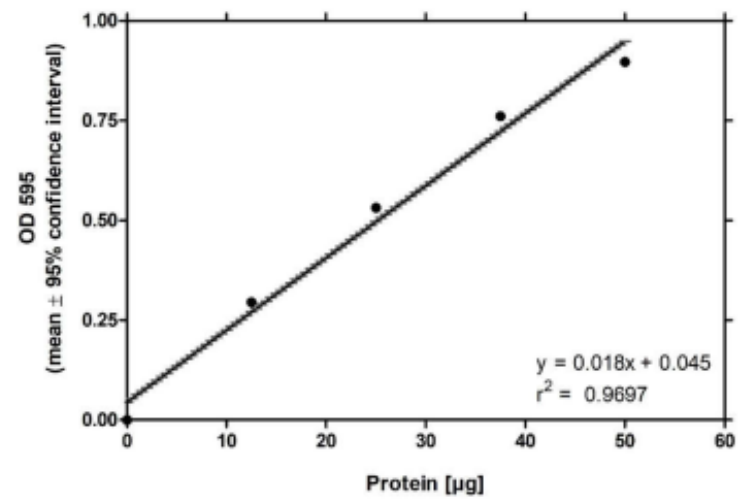

Figure S1: Optical density at a wavelength of $595 \mathrm{~nm}$ (OD 595) of five protein concentrations [ $\mu \mathrm{g}]$ as linear regression (mean $\pm 95 \%$ confidence interval) of seven experiments.

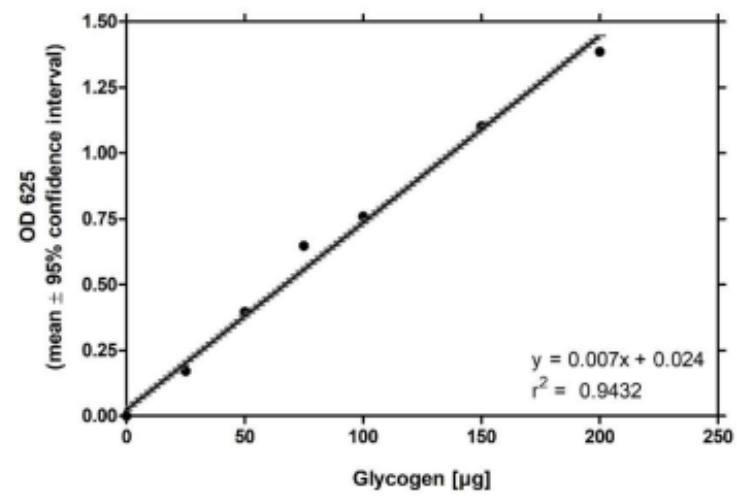

Figure S2: Optical density at a wavelength of $625 \mathrm{~nm}(\mathrm{OD} 625)$ of seven glycogen concentrations $[\mu \mathrm{g}]$ as linear regression (mean $\pm 95 \%$ confidence interval) of seven experiments.

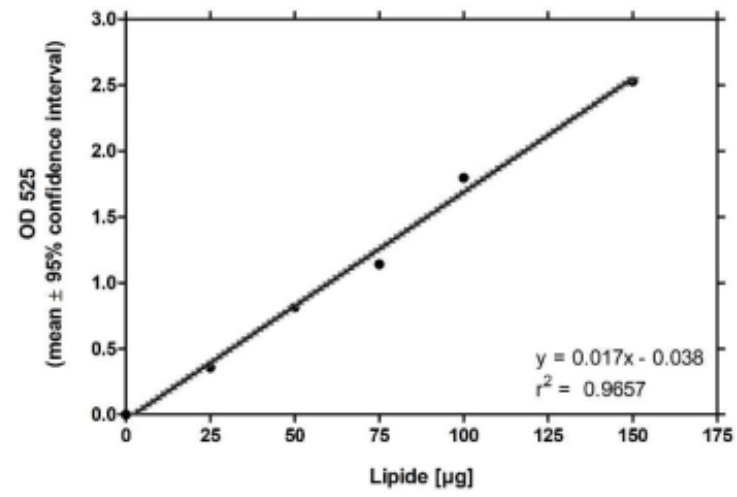

Figure S3: Optical density at a wavelength of $525 \mathrm{~nm}$ (OD 525) of six lipid concentrations [ $\mu \mathrm{g}]$ as linear regression (mean $\pm 95 \%$ confidence interval) of seven experiments. 


\section{S1.4 In vitro bioassays for endocrine and mutagenic activity}

Table S9: Overview of the bioassays used in this study, including endpoints, concentration range of the respective reference compound (positive control), background agonists and $\mathrm{EC}_{50}$ values.

\begin{tabular}{|c|c|c|c|}
\hline $\begin{array}{l}\text { In vitro } \\
\text { bioassay }\end{array}$ & Positive control & $\begin{array}{l}\text { Concentration range } \\
{\left[\mathrm{mol} \mathrm{L}^{-1}\right]}\end{array}$ & $\begin{array}{l}\mathrm{EC}_{50} \\
{\left[\mathrm{~mol} \mathrm{~L}^{-1}\right]}\end{array}$ \\
\hline $\begin{array}{l}\text { YES } \\
\text { (estrogenicity) }\end{array}$ & $\begin{array}{l}\text { 17 } \beta \text {-estradiol } \\
\left(\mathrm{E}_{2}, \mathrm{CAS}: 50-28-2\right)\end{array}$ & $1.0 \times 10^{-12}-1.0 \times 10^{-08}$ & $1.25 \times 10^{-10}$ \\
\hline $\begin{array}{l}\text { YES } \\
\text { (estrogenicity) }\end{array}$ & $\begin{array}{l}17 \alpha \text {-ethinylestradiol } \\
\left(\mathrm{EE}_{2}, \mathrm{CAS}: 57-63-6\right)\end{array}$ & $1.0 \times 10^{-12}-1.0 \times 10^{-08}$ & $1.32 \times 10^{-10}$ \\
\hline $\begin{array}{l}\text { YAES } \\
\text { (anti- } \\
\text { estrogenicity) }\end{array}$ & $\begin{array}{l}\text { 4-hydroxytamoxifen } \\
(\mathrm{OHT}, \mathrm{CAS}: 68392-35-8) \\
\text { background agonist: } \\
0.1 \mathrm{nmol} / \mathrm{L} 17 \beta \text {-estradiol }\left(\mathrm{E}_{2}\right)\end{array}$ & $1.25 \times 10^{-06}-8.0 \times 10^{-05}$ & $1.09 \times 10^{-05}$ \\
\hline $\begin{array}{l}\text { YAS } \\
\text { (androgenicity) }\end{array}$ & $\begin{array}{l}\text { testosterone } \\
(\mathrm{T}, \mathrm{CAS}: 58-22-0)\end{array}$ & $3.0 \times 10^{-11}-1.0 \times 10^{-07}$ & $4.54 \times 10^{-09}$ \\
\hline $\begin{array}{l}\text { YAAS } \\
\text { (anti- } \\
\text { androgenicity) }\end{array}$ & $\begin{array}{l}\text { flutamide } \\
\text { (Flu, CAS: 13311-84-7) } \\
\text { background agonist: } \\
3 \mathrm{nmol} / \mathrm{L} \text { testosterone }\end{array}$ & $7.81 \times 10^{-07}-5.0 \times 10^{-05}$ & $3.37 \times 10^{-06}$ \\
\hline $\begin{array}{l}\text { Ames TA98 } \\
\text { (mutagenicity) }\end{array}$ & $\begin{array}{l}\text { 4-nitro-o-phenylenediamine } \\
\text { (4-NOPD, CAS: 99-56-9) }\end{array}$ & $10 \mathrm{mg} / \mathrm{L}$ & - \\
\hline $\begin{array}{l}\text { Ames TA100 } \\
\text { (mutagenicity) }\end{array}$ & $\begin{array}{l}\text { nitrofurantoin } \\
\text { (NF, CAS: 67-20-9) }\end{array}$ & $0.25 \mathrm{mg} / \mathrm{L}$ & - \\
\hline $\begin{array}{l}\text { Ames YG7108 } \\
\text { (mutagenicity) }\end{array}$ & $\begin{array}{l}\text { propylene oxide } \\
\text { (PO, CAS 75-56-9) }\end{array}$ & $0.2 \%$ & - \\
\hline
\end{tabular}

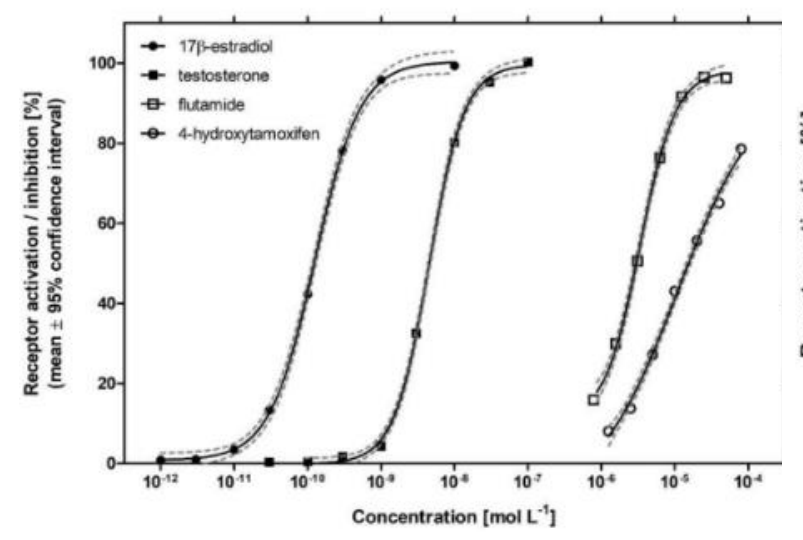

Figure S4: Receptor activation (YES, YAS) and inhibition (YAES, YAAS) as concentration-response relationships of six (YAES, YAAS) and seven (YES, YAS) experiments at the human estrogen and androgen receptor (YES: 17ß-estradiol; YAS: testosterone; YAAS: flutamide; YAES: 4hydroxytamoxifen)

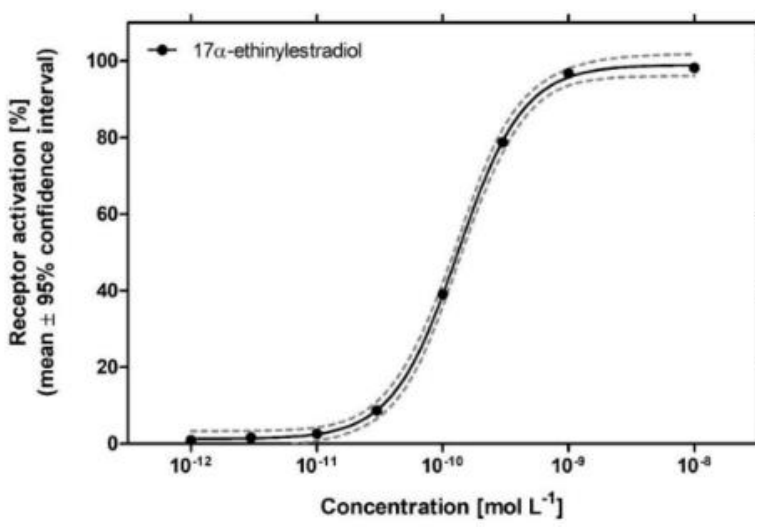

Figure S5: Concentration response relation-ships of $17 \alpha$-ethinylestradiol in four YES experiments 
Table S10: Methods and measurement ranges of the water parameters measured directly in the effluents of the nine wastewater treatment reactors.

\begin{tabular}{|c|c|c|}
\hline physical-chemical parameter & method & range \\
\hline chemical oxygen demand (COD) & $\begin{array}{l}\text { DIN ISO 15705-H45 } \\
\text { HACH-LANGE cuvette test LCK414 and LCK514 }\end{array}$ & $\begin{array}{l}5-60 \mathrm{mg} \mathrm{O}_{2} / \mathrm{L} \text { and } \\
100-2000 \mathrm{mg} \mathrm{O}_{2} / \mathrm{L}\end{array}$ \\
\hline dissolved organic carbon (DOC) & $\mathrm{HACH}$-Lange cuvette test LCK385 & $3-30 \mathrm{mg} \mathrm{C} / \mathrm{L}$ \\
\hline dissolved organic carbon (DOC) & DIN 1484 & $0.5-100 \mathrm{mg} \mathrm{C} / \mathrm{L})$ \\
\hline nitrite $\left(\mathrm{NO}_{2}-\mathrm{N}\right)$ & $\begin{array}{l}\text { cuvette test corresponding to EN ISO } 26777 \text {, DIN } \\
38405 \text { D10 } \\
\text { HACH-Lange cuvette test LCK341 }\end{array}$ & $\begin{array}{l}0.015-0.6 \mathrm{mg} \mathrm{NH}_{4}- \\
\mathrm{N} / \mathrm{L}\end{array}$ \\
\hline nitrate $\left(\mathrm{NO}_{3}-\mathrm{N}\right)$ & $\begin{array}{l}\text { cuvette test corresponding to ISO } 7890-1-2-1986 \text {, } \\
\text { DIN } 38405 \text { D9-2 } \\
\text { HACH-Lange cuvette test LCK339 and LCK340 }\end{array}$ & $\begin{array}{l}0.23-13.5 \mathrm{mg} \mathrm{NO}_{3}- \\
\mathrm{N} / \mathrm{L} \text { and } 5-35 \mathrm{mg} \\
\mathrm{NO}_{3}-\mathrm{N} / \mathrm{L}\end{array}$ \\
\hline ammonium $\left(\mathrm{NH}_{4}-\mathrm{N}\right)$ & $\begin{array}{l}\text { cuvette test corresponding to ISO } 7150-1 \text {, } \\
\text { DIN } 38406 \text { E5-1 } \\
\text { HACH-LANGE cuvette test LCK303 and LCK } 304\end{array}$ & $\begin{array}{l}2.0-47 \mathrm{mg} \mathrm{NH}{ }_{4}-\mathrm{N} / \mathrm{L} \\
\text { and } 0.015-2 \mathrm{mg} \\
\mathrm{NH}_{4}-\mathrm{N} / \mathrm{L}\end{array}$ \\
\hline total phosphor & $\begin{array}{l}\text { cuvette test corresponding to ISO } 6878-1-1986 \text {, } \\
\text { DIN } 38405 \text { D11-4 } \\
\text { HACH-Lange cuvette test LCK339 and LCK340 }\end{array}$ & $\begin{array}{l}0.23-13.5 \mathrm{mg} \mathrm{NO} 3- \\
\mathrm{N} / \mathrm{L} \text { and } 5-35 \mathrm{mg} \\
\mathrm{NO}-\mathrm{N} / \mathrm{L}\end{array}$ \\
\hline $\begin{array}{l}\text { Spectral absorption coefficient at } \\
254 \mathrm{~nm}\left(\mathrm{SAC}_{254}\right)\end{array}$ & $\begin{array}{l}\text { Determination of the decrease of light of a filtered } \\
\text { sample at a wavelength of } 254 \mathrm{~nm} \text { following the } \\
\text { principle of Beer-Lambert law }\end{array}$ & \\
\hline
\end{tabular}

Table S11: Methods and measurement ranges of the water parameters measured directly in the exposure vessels.

\begin{tabular}{lll}
\hline \multicolumn{1}{c}{$\begin{array}{c}\text { physical-chemical } \\
\text { parameter }\end{array}$} & \multicolumn{1}{c}{ method } & \multicolumn{1}{c}{ range } \\
\hline temperature $\left[{ }^{\circ} \mathrm{C}\right]$ & TetraCon-325, Multi 340i / SET, WTW Weilheim & - \\
$\mathrm{pH}$ & SenTix 41, Multi 340i / SET, WTW Weilheim & - \\
conductivity $[\mu \mathrm{S} / \mathrm{cm}]$ & TetraCon-325, Multi 340i / SET, WTW Weilheim & -- \\
oxygen content [mg/L] & OxiCal-SL, Multi 340i / SET, WTW Weilheim & - \\
oxygen saturation [\%] & OxiCal-SL, Multi 340i / SET, WTW Weilheim & - \\
nitrite [mg/L] & nitrite-test, Aquamerck, MERCK Darmstadt & $0.025-0.5 \mathrm{mg} / \mathrm{L}$ \\
nitrate [mg/L] & nitrate-test, Aquamerck, MERCK Darmstadt & $10-150 \mathrm{mg} / \mathrm{L}$ \\
ammonium [mg/L] & ammonium-test, Aquamerck, MERCK Darmstadt & $0.5-10 \mathrm{mg} / \mathrm{L}$ \\
total hardness [ $\left.{ }^{\circ} \mathrm{d}\right]$ & $\begin{array}{l}\text { total hardness-test Merckoquant, MERCK } \\
\text { Darmstadt }\end{array}$ & $<3->21^{\circ} \mathrm{d}$ \\
\hline
\end{tabular}




\section{S2 Results and discussion}

\section{S2.1 Optimal ozone dose and hydraulic retention time}

Table S12: Estrogenic (YES), anti-estrogenic (YAES), androgenic (YAS) and anti-androgenic (YAAS) activity and mutagenicity (Ames TA98, Ames TA100) in \% (mean \pm SEM) from three SPE-extracts each produced from $24 \mathrm{~h}$ composite samples of conventionally treated wastewater (BT) and ozonated wastewater with ozone dose of $0.18-0.51 \mathrm{~g} \mathrm{O}_{3}$, applied $/ \mathrm{g} \mathrm{DOC}$ at a constant hydraulic retention time (HRT) of $12.6 \mathrm{~min}$. The change of endocrine activity and mutagenicity compared to the conventional biological treatment $\left(\Delta \mathrm{w} / \mathrm{o} \mathrm{O}_{3}\right)$ is given in \%.

\begin{tabular}{|c|c|c|c|c|c|c|c|c|c|}
\hline & \multirow[b]{2}{*}{$\mathrm{w} / \mathrm{oO}_{3}$} & \multicolumn{8}{|c|}{ ozone dose $\left[\mathrm{g} \mathrm{O}_{3, \text { applied }} / \mathrm{g} \mathrm{DOC}\right]$} \\
\hline & & 0.18 & $\begin{array}{c}\Delta \mathrm{w} / \mathrm{o}_{3} \\
{[\%]}\end{array}$ & 0.33 & $\begin{array}{c}\Delta \mathrm{w} / \mathrm{o} \mathrm{O} \mathrm{O}_{3} \\
{[\%]}\end{array}$ & 0.44 & $\begin{array}{c}\Delta \mathrm{w} / \mathrm{o} \mathrm{O}_{3} \\
{[\%]}\end{array}$ & 0.51 & $\begin{array}{c}\Delta \mathrm{w} / \mathrm{o} \mathrm{O}_{3} \\
{[\%]}\end{array}$ \\
\hline YES & $\begin{array}{c}7.31 \pm 0.21 \\
(n=96)\end{array}$ & $\begin{array}{c}3.81 \pm 0.19 \\
(n=24)\end{array}$ & -47.8 & $\begin{array}{c}1.35 \pm 0.08 \\
(n=24)\end{array}$ & -81.5 & $\begin{array}{c}0.23 \pm 0.09 \\
(n=16)\end{array}$ & -96.9 & $\begin{array}{c}0.44 \pm 0.07 \\
(n=24)\end{array}$ & -94.0 \\
\hline YAES & $\begin{array}{c}61.7 \pm 0.55 \\
(n=93)\end{array}$ & $\begin{array}{c}67.7 \pm 0.87 \\
(n=23)\end{array}$ & +9.85 & $\begin{array}{c}74.4 \pm 0.60 \\
(n=22)\end{array}$ & +20.7 & $\begin{array}{c}79.1 \pm 0.45 \\
(n=21)\end{array}$ & +28.2 & $\begin{array}{c}79.6 \pm 1.37 \\
(n=20)\end{array}$ & +29.1 \\
\hline YAS & $\begin{array}{c}0.10 \pm 0.04 \\
(n=96)\end{array}$ & $\begin{array}{c}0.11 \pm 0.07 \\
(n=24)\end{array}$ & +9.28 & $\begin{array}{c}0.11 \pm 0.07 \\
(n=24)\end{array}$ & +6.39 & $\begin{array}{c}0.12 \pm 0.07 \\
(n=24)\end{array}$ & +18.7 & $\begin{array}{c}0.11 \pm 0.09 \\
(n=24)\end{array}$ & +10.0 \\
\hline YAAS & $\begin{array}{c}76.1 \pm 0.72 \\
(n=95)\end{array}$ & $\begin{array}{c}62.2 \pm 1.64 \\
(n=24)\end{array}$ & -18.2 & $\begin{array}{c}69.7 \pm 1.45 \\
(n=24)\end{array}$ & -8.39 & $\begin{array}{c}61.6 \pm 1.28 \\
(n=24)\end{array}$ & -19.0 & $\begin{array}{c}49.3 \pm 0.73 \\
(n=24)\end{array}$ & -35.1 \\
\hline Ames TA98 & $\begin{array}{c}2.95 \pm 0.79 \\
(n=12)\end{array}$ & $\begin{array}{c}6.25 \pm 4.17 \\
(n=3)\end{array}$ & - & $\begin{array}{c}4.17 \pm 0.00 \\
(n=3)\end{array}$ & - & $\begin{aligned} 6.25 & \pm 2.41 \\
(n & =3)\end{aligned}$ & - & $\begin{array}{c}6.25 \pm 2.08 \\
(n=3)\end{array}$ & - \\
\hline Ames TA100 & $\begin{array}{c}21.2 \pm 2.59 \\
(n=12)\end{array}$ & $\begin{aligned} 28.5 & \pm 1.85 \\
(n & =3)\end{aligned}$ & +34.5 & $\begin{aligned} 25.0 & \pm 5.25 \\
(n & =3)\end{aligned}$ & +18.1 & $\begin{aligned} 35.4 & \pm 2.10 \\
(n & =3)\end{aligned}$ & +67.1 & $\begin{aligned} 34.7 & \pm 5.67 \\
(n & =3)\end{aligned}$ & +63.9 \\
\hline
\end{tabular}

Table S13: Estrogenic (YES), anti-estrogenic (YAES), androgenic (YAS) and anti-androgenic (YAAS) activity and mutagenicity (Ames TA98, Ames TA100) in \% (mean \pm SEM) from three SPE-extracts each produced from $24 \mathrm{~h}$ composite samples of conventionally treated wastewater (BT) and ozonated wastewater with hydraulic retention times (HRTs) of 4.6-15.1 min at a constant ozone dose of $0.53 \mathrm{~g} \mathrm{O}$, appled $/ \mathrm{g} \mathrm{DOC}$. The change of endocrine activity and mutagenicity compared to the conventional biological treatment $\left(\Delta \mathrm{w} / \mathrm{o} \mathrm{O}_{3}\right)$ is given in $\%$.

\begin{tabular}{|c|c|c|c|c|c|c|c|c|c|c|c|}
\hline & \multirow[b]{2}{*}{ w/o $\mathrm{O}_{3}$} & \multicolumn{10}{|c|}{ hydraulic retention time [min] } \\
\hline & & 4.6 & $\begin{array}{c}\Delta \mathrm{w} / \mathrm{o} \mathrm{O}_{3} \\
{[\%]}\end{array}$ & 7.6 & $\begin{array}{c}\Delta \mathrm{w} / \mathrm{O}_{3} \\
{[\%]}\end{array}$ & 10.0 & $\begin{array}{c}\Delta \mathrm{w} / \mathrm{o} \mathrm{O}_{3} \\
{[\%]}\end{array}$ & 12.5 & $\begin{array}{c}\Delta w^{\prime} / \mathrm{O}_{3} \\
{[\%]}\end{array}$ & 15.1 & $\begin{array}{c}\Delta \mathrm{w} / \mathrm{o}_{3} \\
{[\%]}\end{array}$ \\
\hline YES & $\begin{array}{c}3.58 \pm 0.12 \\
(n=119)\end{array}$ & $\begin{array}{c}0.29 \pm 0.07 \\
(n=24)\end{array}$ & -91.8 & $\begin{array}{c}0.19 \pm 0.07 \\
(n=21)\end{array}$ & -94.6 & $\begin{array}{c}0.67 \pm 0.10 \\
(n=24)\end{array}$ & -81.3 & $\begin{array}{c}0.40 \pm 0.09 \\
(n=23)\end{array}$ & -88.8 & $\begin{array}{c}0.16 \pm 0.04 \\
(n=22)\end{array}$ & -95.7 \\
\hline YAES & $\begin{array}{c}71.0 \pm 0.45 \\
(n=117)\end{array}$ & $\begin{array}{c}61.9 \pm 0.91 \\
(n=23)\end{array}$ & -12.9 & $\begin{array}{c}68.5 \pm 0.55 \\
(n=23)\end{array}$ & -3.65 & $\begin{array}{c}71.9 \pm 0.38 \\
(n=23)\end{array}$ & +1.21 & $\begin{array}{c}70.8 \pm 0.62 \\
(n=21)\end{array}$ & -0.32 & $\begin{array}{c}71.3 \pm 0.34 \\
(n=23)\end{array}$ & +0.35 \\
\hline YAS & $\begin{array}{c}0.82 \pm 0.08 \\
(n=120)\end{array}$ & $\begin{array}{c}0.83 \pm 0.16 \\
(n=24)\end{array}$ & +0.96 & $\begin{array}{c}0.60 \pm 0.15 \\
(n=23)\end{array}$ & -26.5 & $\begin{array}{c}0.56 \pm 0.14 \\
(n=24)\end{array}$ & -32.0 & $\begin{array}{c}0.71 \pm 0.20 \\
(n=24)\end{array}$ & -14.1 & $\begin{array}{c}0.12 \pm 0.09 \\
(n=24)\end{array}$ & -85.2 \\
\hline YAAS & $\begin{array}{c}70.9 \pm 0.80 \\
(n=117)\end{array}$ & $\begin{array}{c}39.9 \pm 2.21 \\
(n=24)\end{array}$ & -43.6 & $\begin{array}{c}49.7 \pm 1.58 \\
(n=24)\end{array}$ & -29.9 & $\begin{array}{c}60.7 \pm 0.88 \\
(n=24)\end{array}$ & -14.4 & $\begin{array}{c}46.4 \pm 1.15 \\
(n=23)\end{array}$ & -34.6 & $\begin{array}{c}40.7 \pm 0.93 \\
(n=24)\end{array}$ & -42.6 \\
\hline $\begin{array}{l}\text { Ames } \\
\text { TA98 }\end{array}$ & $\begin{array}{c}3.05 \pm 0.67 \\
(n=15)\end{array}$ & $\begin{array}{c}0.69 \pm 0.69 \\
(n=3)\end{array}$ & - & $\begin{array}{c}2.08 \pm 0.00 \\
(n=3)\end{array}$ & - & $\begin{array}{c}4.86 \pm 1.84 \\
(n=3)\end{array}$ & - & $\begin{array}{c}1.39 \pm 1.39 \\
(n=3)\end{array}$ & - & $\begin{array}{c}2.08 \pm 1.20 \\
(n=3)\end{array}$ & - \\
\hline $\begin{array}{l}\text { Ames } \\
\text { TA100 }\end{array}$ & $\begin{array}{c}21.5 \pm 1.64 \\
(n=15)\end{array}$ & $\begin{array}{c}20.1 \pm 3.02 \\
(n=3)\end{array}$ & -6.55 & $\begin{array}{c}34.1 \pm 5.02 \\
(n=3)\end{array}$ & +58.2 & $\begin{array}{c}24.3 \pm 3.68 \\
(n=3)\end{array}$ & +13.0 & $\begin{aligned} 29.9 & \pm 5.44 \\
(n & =3)\end{aligned}$ & +38.7 & $\begin{aligned} 41.7 & \pm 3.18 \\
(n=3) & =3\end{aligned}$ & +93.5 \\
\hline
\end{tabular}




\section{S2.2.1 Mortality, growth and reproduction}

Table S14: Mortality in \% (mean \pm SEM), shell height in $\mathrm{mm}$ (mean \pm SD), total number of embryos (mean \pm SD) and fecundity index (mean \pm SD) of Potamopyrgus antipodarum after 28 days of exposure to the negative control (NC), the positive control (PC), the conventional biological treatment (BT) and the eight advanced treatment technologies. $\mathrm{BT}+\mathrm{O}_{3}$ : after ozone system 1, GAC: after non-aerated granular activated carbon filter treatment, $\mathrm{GAC}_{\mathrm{a}}$ : after aerated granular activated carbon filter treatment, $\mathrm{BF}$ : after non-aerated biofilter treatment, $\mathrm{BF}$ : after aerated biofilter treatment, MBR1/2: after membrane bioreactor $1 / 2, M B R 1+\mathrm{O}_{3}$ : after ozone system 2 . The change of the shell height and the total number of embryos compared to the negative control $(\triangle \mathrm{NC})$ or the conventional biological treatment $(\Delta \mathrm{BT})$ is given in \%. Significant differences compared to $\Delta N C$ and $\Delta \mathrm{BT}$ are marked with asterisks: $\star p \leq 0.05, \star \star p \leq 0.01, \star \star \star p \leq 0.001$ (Kruskal-Wallis with Dunn's post-test), n.s.: not significant.

\begin{tabular}{|c|c|c|c|c|c|c|c|}
\hline treatment & mortality [\%] & shell height [mm] & $\Delta[\%]$ & total number of embryos & $\Delta[\%]$ & fecundity index & $\Delta[\%]$ \\
\hline $\mathrm{NC}$ & $\begin{array}{c}1.00 \pm 1.00 \\
(n=100)\end{array}$ & $\begin{array}{c}3.82 \pm 0.17 \\
(n=40)\end{array}$ & - & $\begin{array}{c}23.7 \pm 5.27 \\
(n=40)\end{array}$ & - & $\begin{array}{c}6.17 \pm 1.21 \\
(n=40)\end{array}$ & - \\
\hline PC & $\begin{array}{c}3.00 \pm 1.92 \\
(n=100)\end{array}$ & $\begin{array}{c}3.87 \pm 0.21 \\
(n=37)\end{array}$ & $\begin{array}{c}\Delta \mathrm{NC}+1.31 \\
\text { (n.s.) }\end{array}$ & $\begin{array}{c}27.7 \pm 5.36 \\
(n=40)\end{array}$ & $\begin{array}{c}\Delta \mathrm{NC}+17.0 \\
\text { (n.s.) }\end{array}$ & $\begin{array}{c}7.07 \pm 1.23 \\
(\mathrm{n}=37)\end{array}$ & $\begin{array}{c}\Delta \mathrm{NC}+14.6 \\
\text { (n.s.) }\end{array}$ \\
\hline BT & $\begin{array}{c}2.00 \pm 1.16 \\
(n=100)\end{array}$ & $\begin{array}{c}3.98 \pm 0.23 \\
(\mathrm{n}=39)\end{array}$ & $\begin{array}{c}\Delta \mathrm{NC}+4.30 \\
(\star)\end{array}$ & $\begin{array}{c}28.1 \pm 6.00 \\
(n=40)\end{array}$ & $\begin{array}{c}\Delta \mathrm{NC}+18.7 \\
\text { (n.s.) }\end{array}$ & $\begin{array}{c}7.02 \pm 1.25 \\
(n=39)\end{array}$ & $\begin{array}{c}\Delta \mathrm{NC}+13.8 \\
\text { (n.s.) }\end{array}$ \\
\hline $\mathrm{BT}+\mathrm{O}_{3}$ & $\begin{array}{c}1.00 \pm 1.00 \\
(n=100)\end{array}$ & $\begin{array}{c}3.90 \pm 0.21 \\
(n=40)\end{array}$ & $\begin{array}{l}\Delta \mathrm{BT}-1.98 \\
\text { (n.s.) }\end{array}$ & $\begin{array}{c}21.9 \pm 5.94 \\
(n=40)\end{array}$ & $\begin{array}{c}\Delta \mathrm{BT}-21.9 \\
(\star \star)\end{array}$ & $\begin{array}{c}5.61 \pm 1.43 \\
(n=40)\end{array}$ & $\begin{array}{c}\Delta \mathrm{BT}-20.1 \\
(\star \star \star)\end{array}$ \\
\hline GAC & $\begin{array}{c}3.00 \pm 3.00 \\
(n=100)\end{array}$ & $\begin{array}{c}3.90 \pm 0.20 \\
(n=40)\end{array}$ & $\begin{array}{l}\Delta \mathrm{BT}-1.98 \\
\text { (n.s.) }\end{array}$ & $\begin{array}{c}27.5 \pm 5.30 \\
(n=40)\end{array}$ & $\begin{array}{l}\Delta \mathrm{BT}-2.07 \\
\text { (n.s.) }\end{array}$ & $\begin{array}{c}7.01 \pm 1.10 \\
(n=40)\end{array}$ & $\begin{array}{c}\Delta \mathrm{BT}-0.11 \\
\text { (n.s.) }\end{array}$ \\
\hline $\mathrm{GAC}_{\mathrm{a}}$ & $\begin{array}{c}1.33 \pm 1.33 \\
(n=100)\end{array}$ & $\begin{array}{c}3.92 \pm 0.28 \\
(n=35)\end{array}$ & $\begin{array}{c}\Delta \mathrm{BT}-1.56 \\
\text { (n.s.) }\end{array}$ & $\begin{array}{c}22.8 \pm 6.19 \\
(n=35)\end{array}$ & $\begin{array}{c}\Delta \mathrm{BT}-18.7 \\
(\star)\end{array}$ & $\begin{array}{c}5.79 \pm 1.36 \\
(n=35)\end{array}$ & $\begin{array}{c}\Delta \mathrm{BT}-17.5 \\
(\star \star)\end{array}$ \\
\hline BF & $\begin{array}{c}2.00 \pm 1.16 \\
(n=100)\end{array}$ & $\begin{array}{c}3.94 \pm 0.25 \\
(n=40)\end{array}$ & $\begin{array}{c}\Delta \mathrm{BT}-1.05 \\
\text { (n.s.) }\end{array}$ & $\begin{array}{c}25.1 \pm 6.25 \\
(n=40)\end{array}$ & $\begin{array}{c}\Delta \mathrm{BTT}-10.7 \\
\text { (n.s.) }\end{array}$ & $\begin{array}{c}6.34 \pm 1.44 \\
(n=40)\end{array}$ & $\begin{array}{c}\Delta \mathrm{BT}-9.63 \\
\text { (n.s.) }\end{array}$ \\
\hline $\mathrm{BF}_{\mathrm{a}}$ & $\begin{array}{c}1.00 \pm 1.00 \\
(n=100)\end{array}$ & $\begin{array}{c}3.93 \pm 0.19 \\
(n=40)\end{array}$ & $\begin{array}{l}\Delta \mathrm{BT}-1.43 \\
\text { (n.s.) }\end{array}$ & $\begin{array}{c}21.3 \pm 5.04 \\
(n=40)\end{array}$ & $\begin{array}{c}\Delta \mathrm{BT}-24.0 \\
(\star \star \star)\end{array}$ & $\begin{array}{c}5.43 \pm 1.24 \\
(n=40)\end{array}$ & $\begin{array}{c}\Delta \mathrm{BT}-22.6 \\
(\star \star \star)\end{array}$ \\
\hline MBR1 & $\begin{array}{c}0.00 \pm 0.00 \\
(n=100)\end{array}$ & $\begin{array}{c}3.93 \pm 0.27 \\
(n=40)\end{array}$ & $\begin{array}{l}\Delta \mathrm{BT}-1.36 \\
\text { (n.s.) }\end{array}$ & $\begin{array}{c}19.7 \pm 5.98 \\
(n=40)\end{array}$ & $\begin{array}{c}\Delta \mathrm{BT}-29.9 \\
(\star \star \star)\end{array}$ & $\begin{array}{c}5.01 \pm 1.48 \\
(n=40)\end{array}$ & $\begin{array}{c}\Delta \mathrm{BT}-28.7 \\
(\star \star \star)\end{array}$ \\
\hline $\mathrm{MBR} 1+\mathrm{O}_{3}$ & $\begin{array}{c}1.00 \pm 1.00 \\
(n=100)\end{array}$ & $\begin{array}{c}3.92 \pm 0.24 \\
(n=40)\end{array}$ & $\begin{array}{l}\Delta \mathrm{BT}-1.68 \\
\text { (n.s.) }\end{array}$ & $\begin{array}{c}22.6 \pm 6.20 \\
(n=40)\end{array}$ & $\begin{array}{c}\Delta \mathrm{BT}-19.6 \\
(\star \star)\end{array}$ & $\begin{array}{c}5.73 \pm 1.40 \\
(n=40)\end{array}$ & $\begin{array}{c}\Delta \mathrm{BT}-18.3 \\
(\star \star)\end{array}$ \\
\hline MBR2 & $\begin{array}{c}1.00 \pm 1.00 \\
(n=100)\end{array}$ & $\begin{array}{c}3.84 \pm 0.21 \\
(n=40)\end{array}$ & $\begin{array}{c}\Delta \mathrm{BT}-3.57 \\
(\star)\end{array}$ & $\begin{array}{c}12.4 \pm 5.35 \\
(n=40)\end{array}$ & $\begin{array}{c}\Delta \mathrm{BT}-56.0 \\
(\star \star \star)\end{array}$ & $\begin{array}{c}3.20 \pm 1.34 \\
(\mathrm{n}=40)\end{array}$ & $\begin{array}{c}\Delta \mathrm{BT}-54.4 \\
(\star \star \star)\end{array}$ \\
\hline
\end{tabular}

S2.2.2 Biomarkers for energy reserves (glycogen, protein and lipid content)

Table S15: Energy content as protein, glycogen and lipid in J/mg tissue (mean \pm SD) of Potamopyrgus antipodarum after 28 days of exposure to the negative control (NC), the positive control (PC), the conventional biological treatment (BT) and the eight advanced treatment technologies. $\mathrm{BT}+\mathrm{O}_{3}$ : after ozone system 1, GAC: after non-aerated activated carbon filter treatment, $\mathrm{GAC}_{\mathrm{a}}$ : after aerated activated carbon filter treatment, $\mathrm{BF}$ : after non-aerated biofilter treatment, $\mathrm{BF}_{\mathrm{a}}$ : after aerated biofilter treatment, MBR1/2: after membrane bioreactor $1 / 2, \mathrm{MBR}_{1}+\mathrm{O}_{3}$ : after ozone system 2. The change of the protein, glycogen and lipid content compared to the negative control $(\triangle \mathrm{NC})$ or the conventional biological treatment $(\triangle \mathrm{BT})$ is given in \%. Significant differences compared to $\triangle N C$ and $\Delta B T$ are marked with asterisks: $\star p \leq 0.05, \star \star p \leq 0.01, \star \star \star p \leq 0.001$ (one-way ANOVA with Bonferroni's post-test (energy content as glycogen) or Kruskal-Wallis with Dunn's post-test (energy content as protein and lipid)), n.s. not significant.

\begin{tabular}{|c|c|c|c|c|c|c|}
\hline treatment & protein [J/mg] & $\Delta[\%]$ & glycogen [J/mg] & $\Delta[\%]$ & lipid [J/mg] & $\Delta[\%]$ \\
\hline $\mathrm{NC}$ & $0.29 \pm 0.06(n=19)$ & - & $0.20 \pm 0.03(n=19)$ & - & $1.59 \pm 0.54(n=20)$ & - \\
\hline $\mathrm{PC}$ & $0.26 \pm 0.04(n=20)$ & $\Delta \mathrm{NC}-8.74$ (n.s.) & $0.22 \pm 0.06(n=20)$ & $\Delta \mathrm{NC}+10.5$ (n.s.) & $0.96 \pm 0.42(n=19)$ & $\Delta \mathrm{NC}-39.8(\star \star)$ \\
\hline BT & $0.26 \pm 0.06(n=19)$ & $\Delta \mathrm{NC}-9.56$ (n.s.) & $0.19 \pm 0.04(n=20)$ & $\Delta \mathrm{NC}-3.98$ (n.s.) & $0.95 \pm 0.73(n=20)$ & $\Delta \mathrm{NC}-40.1(\star)$ \\
\hline $\mathrm{BT}+\mathrm{O}_{3}$ & $0.26 \pm 0.06(n=20)$ & $\Delta \mathrm{BT}+0.42$ (n.s.) & $0.19 \pm 0.04(n=20)$ & $\Delta \mathrm{BT}-0.16$ (n.s.) & $1.10 \pm 0.41(n=19)$ & $\Delta \mathrm{BT}+15.0$ (n.s.) \\
\hline GAC & $0.28 \pm 0.04(n=20)$ & $\Delta \mathrm{BT}+6.26$ (n.s.) & $0.24 \pm 0.08(n=20)$ & $\Delta \mathrm{BT}+29.2(\star)$ & $1.09 \pm 0.33(n=19)$ & $\Delta \mathrm{BT}+13.9$ (n.s.) \\
\hline $\mathrm{GAC}_{a}$ & $0.28 \pm 0.05(n=19)$ & $\Delta \mathrm{BT}+7.94$ (n.s.) & $0.21 \pm 0.07(n=20)$ & $\Delta \mathrm{BT}+13.7$ (n.s.) & $1.52 \pm 0.51(n=20)$ & $\Delta \mathrm{BT}+59.7(\star)$ \\
\hline BF & $0.31 \pm 0.07(n=20)$ & $\Delta \mathrm{BT}+17.9$ (n.s.) & $0.19 \pm 0.04(n=20)$ & $\Delta \mathrm{BT}-1.11$ (n.s.) & $2.05 \pm 0.31(n=20)$ & $\Delta \mathrm{BT}+115(\star \star \star)$ \\
\hline $\mathrm{BF}_{\mathrm{a}}$ & $0.26 \pm 0.04(n=20)$ & $\Delta \mathrm{BT}-0.08$ (n.s.) & $0.18 \pm 0.04(n=20)$ & $\Delta \mathrm{BT}-4.78$ (n.s.) & $1.43 \pm 0.47(n=20)$ & $\Delta \mathrm{BT}+49.8$ (n.s.) \\
\hline MBR1 & $0.23 \pm 0.07(n=19)$ & $\Delta \mathrm{BT}-10.3$ (n.s.) & $0.15 \pm 0.05(n=20)$ & $\Delta \mathrm{BT}-19.0$ (n.s.) & $1.40 \pm 0.51(n=18)$ & $\Delta \mathrm{BT}+46.4$ (n.s.) \\
\hline $\mathrm{MBR} 1+\mathrm{O}_{3}$ & $0.25 \pm 0.07(n=20)$ & $\Delta \mathrm{BT}-5.00$ (n.s.) & $0.17 \pm 0.07(n=20)$ & $\Delta \mathrm{BT}-7.80$ (n.s.) & $1.10 \pm 0.38(n=18)$ & $\Delta \mathrm{BT}+14.8$ (n.s.) \\
\hline MBR2 & $0.23 \pm 0.08(n=20)$ & $\Delta \mathrm{BT}-12.7$ (n.s.) & $0.18 \pm 0.11(n=20)$ & $\Delta B T-6.58$ (n.s.) & $1.14 \pm 0.48(n=19)$ & $\Delta \mathrm{BT}+18.9$ (n.s.) \\
\hline
\end{tabular}




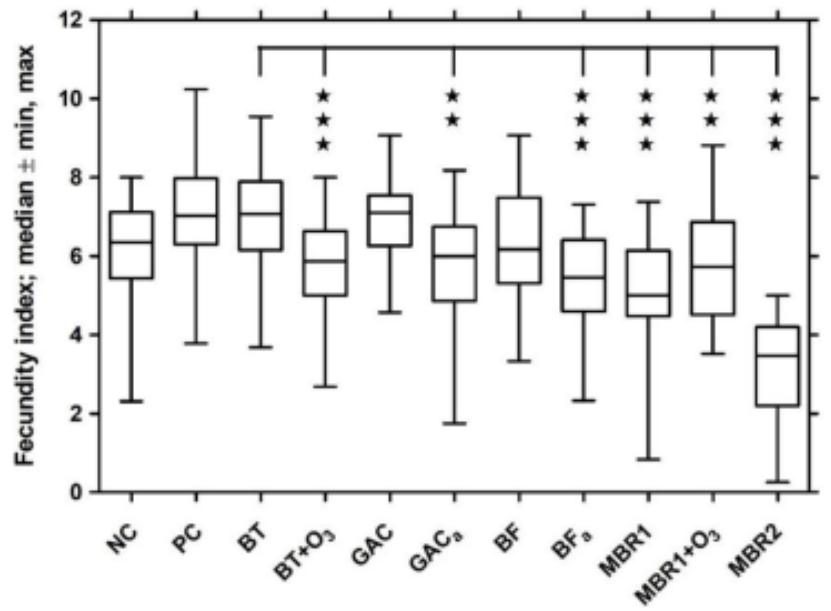

Figure S6: Fecundity index of Potamopyrgus antipodarum after 28 days of exposure to the negative control (NC), the positive control (PC), the conventional biological treatment (BT) and the eight advanced treatment technologies. $\mathrm{BT}+\mathrm{O}_{3}$ : after ozone system 1, GAC: after nonaerated granular activated carbon treatment, $\mathrm{GAC}_{\mathrm{a}}$ : after aerated granular activated carbon treatment, BF: after non-aerated biofilter treatment, $\mathrm{BF}_{\mathrm{a}}$ : after aerated biofilter treatment, MBR1/2: after membrane bioreactor $1 / 2, M B R 1+\mathrm{O}_{3}$ : after ozone system 2. Significant differences to BT are indicated with asterisks: $\star \star p<0.01, \star \star \star p<0.001$ (Kruskal-Wallis with Dunn's post-test), $n=35-40$.

Table S16: Total energy content (protein + glycogen + lipid) in J/mg tissue (mean \pm SD) of Potamopyrgus antipodarum after 28 days of exposure to the negative control (NC), the positive control (PC), the conventional biological treatment (BT) and the eight advanced treatment technologies. $\mathrm{BT}+\mathrm{O}_{3}$ : after ozone system 1, GAC: after non-aerated activated carbon filter treatment, $\mathrm{GAC}_{\mathrm{a}}$ : after aerated activated carbon filter treatment, BF: after non-aerated biofilter treatment, $\mathrm{BF}_{\mathrm{a}}$ : after aerated biofilter treatment, MBR1/2: after membrane bioreactor 1/2, $M B R 1+\mathrm{O}_{3}$ : after ozone system 2. The change of the total energy compared to the negative control $(\triangle N C)$ or the conventional biological treatment $(\Delta B T)$ is given in \%. Significant differences compared to $\triangle \mathrm{NC}$ and $\Delta \mathrm{BT}$ are marked with asterisks: $\star p \leq 0.05, \star \star p \leq 0.01$, $\star \star \star p \leq 0.001$ (one-way ANOVA with Bonferroni's post-test), n.s.: not significant.

\begin{tabular}{|c|c|c|}
\hline treatment & total energy $[\mathrm{J} / \mathrm{mg}]$ & $\Delta[\%]$ \\
\hline $\mathrm{NC}$ & $2.07 \pm 0.56(n=19)$ & - \\
\hline $\mathrm{PC}$ & $1.44 \pm 0.43(n=19)$ & $\Delta \mathrm{NC}-30.6(\star \star \star)$ \\
\hline BT & $1.38 \pm 0.77(n=19)$ & $\Delta \mathrm{NC}-33.2(\star \star \star)$ \\
\hline $\mathrm{BT}+\mathrm{O}_{3}$ & $1.55 \pm 0.42(n=19)$ & $\Delta \mathrm{BT}+12.1$ (n.s.) \\
\hline GAC & $1.61 \pm 0.33(n=19)$ & $\Delta \mathrm{BT}+16.4$ (n.s.) \\
\hline $\mathrm{GAC}_{\mathrm{a}}$ & $1.94 \pm 0.36(n=19)$ & $\Delta \mathrm{BT}+40.2(\star \star)$ \\
\hline BF & $2.54 \pm 0.35(n=20)$ & $\Delta \mathrm{BT}+83.7(\star \star \star)$ \\
\hline $\mathrm{BF}_{\mathrm{a}}$ & $1.87 \pm 0.47(n=20)$ & $\Delta \mathrm{BT}+35.2(\star)$ \\
\hline MBR1 & $1.82 \pm 0.53(n=17)$ & $\Delta \mathrm{BT}+31.6$ (n.s.) \\
\hline $\mathrm{MBR} 1+\mathrm{O}_{3}$ & $1.52 \pm 0.42(n=18)$ & $\Delta \mathrm{BT}+9.47$ (n.s.) \\
\hline MBR2 & $1.55 \pm 0.47(n=19)$ & $\Delta B T+12.1$ (n.s.) \\
\hline
\end{tabular}


S2.2.3 In vitro bioassays for endocrine activity and mutagenicity

Table S17: Estrogenic (YES), anti-estrogenic (YAES), androgenic (YAS) and anti-androgenic (YAAS) activity of the aqueous samples from four

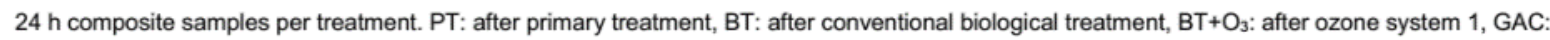
after non-aerated granular activated carbon filter treatment, $\mathrm{GAC}_{\mathrm{a}}$ : after aerated granular activated carbon filter treatment, BF: after non-aerated

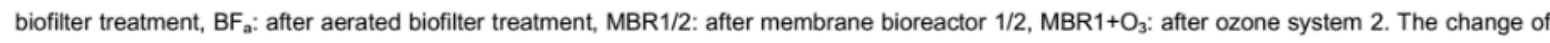
endocrine activity compared to the conventional biological treatment $(\triangle \mathrm{BT})$ is given in \%. n.c.: not calculable.

\begin{tabular}{|c|c|c|c|c|c|c|c|c|}
\hline & YES & $\begin{array}{c}\Delta \mathrm{BT} \\
{[\%]}\end{array}$ & YAES & $\begin{array}{c}\Delta \mathrm{BT} \\
{[\%]}\end{array}$ & YAS & $\begin{array}{c}\Delta \mathrm{BT} \\
{[\%]}\end{array}$ & YAAS & $\begin{array}{r}\mathrm{BT} \\
{[\%]}\end{array}$ \\
\hline PT & $1.60 \pm 0.27(n=8)$ & -58.6 & $95.0 \pm 0.71(n=16)$ & -39.6 & $38.2 \pm 2.30(n=56)$ & -99.8 & $1.03 \pm 0.41(n=40)$ & n.c. \\
\hline BT & $0.66 \pm 0.11(n=32)$ & - & $57.4 \pm 2.83(n=32)$ & - & $0.06 \pm 0.03(n=40)$ & - & $0.00 \pm 0.00(n=32)$ & - \\
\hline $\mathrm{BT}+\mathrm{O}_{3}$ & $0.25 \pm 0.07(n=32)$ & -62.1 & $59.5 \pm 2.89(n=32)$ & +3.62 & $0.03 \pm 0.02(n=40)$ & -53.0 & $0.20 \pm 0.20(n=32)$ & n.c. \\
\hline GAC & $0.33 \pm 0.07(n=32)$ & -50.0 & $66.1 \pm 3.04(n=32)$ & +15.1 & $0.02 \pm 0.01(n=40)$ & -74.0 & $0.00 \pm 0.00(n=32)$ & n.c. \\
\hline $\mathrm{GAC}_{a}$ & $0.24 \pm 0.07(n=32)$ & -63.2 & $71.2 \pm 2.77(n=32)$ & +24.0 & $0.05 \pm 0.03(n=40)$ & -11.4 & $0.00 \pm 0.00(n=32)$ & n.c. \\
\hline BF & $0.28 \pm 0.07(n=32)$ & -57.6 & $60.5 \pm 3.03(n=32)$ & +5.37 & $0.02 \pm 0.02(n=40)$ & -73.2 & $0.26 \pm 0.25(n=32)$ & n.c. \\
\hline $\mathrm{BF}_{\mathrm{a}}$ & $0.33 \pm 0.09(n=32)$ & -50.4 & $64.9 \pm 2.86(n=32)$ & +13.1 & $0.02 \pm 0.02(n=40)$ & -70.9 & $0.00 \pm 0.00(n=32)$ & n.c. \\
\hline MBR1 & $0.21 \pm 0.05(n=24)$ & -68.6 & $61.9 \pm 2.71(n=32)$ & +7.88 & $0.09 \pm 0.03(n=40)$ & +40.3 & $0.84 \pm 0.43(n=31)$ & n.c. \\
\hline $\mathrm{MBR} 1+\mathrm{O}_{3}$ & $0.23 \pm 0.06(n=24)$ & -65.7 & $63.1 \pm 2.90(n=32)$ & +9.91 & $0.07 \pm 0.03(n=40)$ & +21.5 & $0.89 \pm 0.57(n=31)$ & n.c. \\
\hline MBR2 & $0.33 \pm 0.07(n=32)$ & -50.2 & $66.2 \pm 2.94(n=32)$ & +15.3 & $0.04 \pm 0.02(n=40)$ & -42.0 & $0.39 \pm 0.28(n=32)$ & n.c. \\
\hline
\end{tabular}

Table S18: Estrogenic (YES), anti-estrogenic (YAES), androgenic (YAS) and anti-androgenic (YAAS) activity and mutagenicity (Ames YG7108) from four SPE extracts each produced from $24 \mathrm{~h}$ composite samples. $\mathrm{PT}$ : after primary treatment, $\mathrm{BT}$ : after conventional biological treatment, $\mathrm{BT}+\mathrm{O}_{3}$ : after ozone system 1, GAC: after non-aerated granular activated carbon filter treatment, GAC: after aerated granular activated carbon filter treatment, $\mathrm{BF}$ : after non-aerated biofilter treatment, $\mathrm{BF}_{\mathrm{a}}$ : after aerated biofilter treatment, $\mathrm{MBR} 1 / 2$ : after membrane bioreactor $1 / 2, \mathrm{MBR}+\mathrm{O}_{3}$ : after ozone system 2 . The change of endocrine activity and mutagenicity compared to the conventional biological treatment ( $\triangle \mathrm{BT})$ is given in $\%$. 2 : cytotoxic.

\begin{tabular}{|c|c|c|c|c|c|c|c|c|c|}
\hline & YES & $\Delta \mathrm{BT}[\%]$ & YAES & $\Delta \mathrm{BT}[\%]$ & YAS & $\Delta$ BT $[\%]$ & YAAS & $\Delta \mathrm{BT}[\%]$ & Ames YG7108 \\
\hline$\overline{\mathrm{PT}}$ & $\frac{2}{x}$ & - & 2 & - & 2 & - & 2 & - & 2 \\
\hline BT & $\begin{array}{c}16.9 \pm 1.60 \\
(n=32)\end{array}$ & - & $\begin{array}{c}14.1 \pm 1.53 \\
(n=32)\end{array}$ & - & $\begin{array}{c}1.76 \pm 0.31 \\
(n=32)\end{array}$ & - & $\begin{array}{c}72.1 \pm 2.05 \\
(n=32)\end{array}$ & - & $\begin{array}{c}1.82 \pm 0.73 \\
(n=8)\end{array}$ \\
\hline $\mathrm{BT}+\mathrm{O}_{3}$ & $\begin{array}{c}0.59 \pm 0.11 \\
(n=32)\end{array}$ & -96.5 & $\begin{array}{c}37.2 \pm 1.43 \\
(n=32)\end{array}$ & +163 & $\begin{array}{c}0.94 \pm 0.20 \\
(n=32)\end{array}$ & -46.3 & $\begin{array}{c}34.3 \pm 3.79 \\
(n=32)\end{array}$ & -52.5 & $\begin{array}{c}93.2 \pm 1.29 \\
(n=8)\end{array}$ \\
\hline GAC & $\begin{array}{c}0.73 \pm 0.10 \\
(n=32)\end{array}$ & -95.7 & $\begin{array}{c}18.6 \pm 1.90 \\
(n=32)\end{array}$ & +31.8 & $\begin{array}{c}0.59 \pm 0.24 \\
(n=32)\end{array}$ & -66.5 & $\begin{array}{c}19.8 \pm 3.44 \\
(n=32)\end{array}$ & -72.6 & $\begin{array}{c}15.1 \pm 1.56 \\
(n=8)\end{array}$ \\
\hline GACa & $\begin{array}{c}0.69 \pm 0.08 \\
(n=32)\end{array}$ & -95.9 & $\begin{array}{c}23.4 \pm 1.15 \\
(n=32)\end{array}$ & +65.7 & $\begin{array}{c}0.79 \pm 0.16 \\
(n=32)\end{array}$ & -55.1 & $\begin{array}{c}24.5 \pm 4.25 \\
(n=32)\end{array}$ & -66.0 & $\begin{array}{c}14.8 \pm 1.33 \\
(n=8)\end{array}$ \\
\hline BF & $\begin{array}{c}0.78 \pm 0.13 \\
(n=32)\end{array}$ & -95.4 & $\begin{array}{c}35.3 \pm 1.51 \\
(n=32)\end{array}$ & +150 & $\begin{array}{c}0.63 \pm 0.11 \\
(n=32)\end{array}$ & -63.9 & $\begin{array}{c}28.3 \pm 2.39 \\
(n=32)\end{array}$ & -60.8 & $\begin{array}{c}50.8 \pm 2.92 \\
(n=8)\end{array}$ \\
\hline $\mathrm{BF}_{\mathrm{a}}$ & $\begin{array}{c}0.83 \pm 0.15 \\
(n=32)\end{array}$ & -95.1 & $\begin{array}{c}34.5 \pm 1.21 \\
(n=32)\end{array}$ & +144 & $\begin{array}{c}0.28 \pm 0.07 \\
(n=32)\end{array}$ & -84.0 & $\begin{array}{c}32.7 \pm 3.02 \\
(n=32)\end{array}$ & -54.7 & $\begin{array}{c}52.9 \pm 4.87 \\
(n=8)\end{array}$ \\
\hline MBR1 & $\begin{array}{c}1.58 \pm 0.16 \\
(n=32)\end{array}$ & -90.6 & $\begin{array}{c}37.0 \pm 1.66 \\
(n=32)\end{array}$ & +162 & $\begin{array}{c}0.93 \pm 0.16 \\
(n=32)\end{array}$ & -47.0 & $\begin{array}{c}41.2 \pm 3.45 \\
(n=32)\end{array}$ & -42.9 & $\begin{array}{c}2.84 \pm 1.15 \\
(n=8)\end{array}$ \\
\hline $\mathrm{MBR} 1+\mathrm{O}_{3}$ & $\begin{array}{c}0.44 \pm 0.09 \\
(n=32)\end{array}$ & -97.4 & $\begin{array}{c}27.3 \pm 1.64 \\
(n=32)\end{array}$ & +93.3 & $\begin{array}{c}0.55 \pm 0.12 \\
(n=32)\end{array}$ & -68.7 & $\begin{array}{c}21.8 \pm 3.17 \\
(n=32)\end{array}$ & -69.7 & $\begin{array}{c}67.5 \pm 4.62 \\
(n=8)\end{array}$ \\
\hline MBR2 & $\begin{array}{c}3.09 \pm 0.29 \\
(n=32)\end{array}$ & -81.7 & $\begin{array}{c}42.6 \pm 2.95 \\
(n=32)\end{array}$ & +201 & $\begin{array}{c}1.58 \pm 0.29 \\
(n=32)\end{array}$ & -10.1 & $\begin{array}{c}66.6 \pm 3.07 \\
(n=32)\end{array}$ & -7.68 & $\begin{array}{c}0.00 \pm 0.00 \\
(n=8)\end{array}$ \\
\hline
\end{tabular}



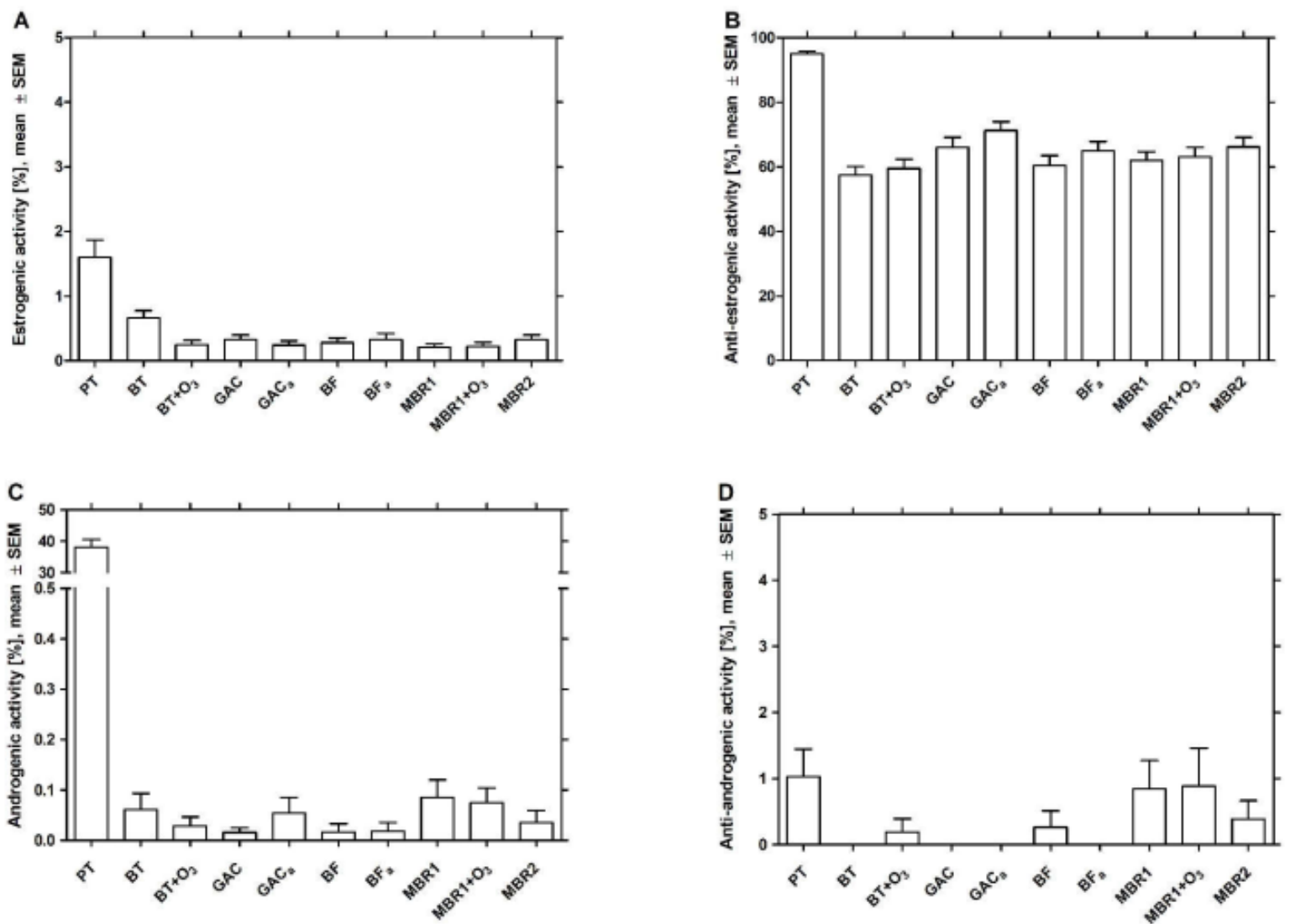

Figure S7: Estrogenic activity (A), anti-estrogenic activity (B), androgenic activity (C) and antiandrogenic activity (D) of the aqueous samples from four $24 \mathrm{~h}$ composite samples per treatment. PT: after primary treatment, BT: after conventional biological treatment, $\mathrm{BT}+\mathrm{O}_{3}$ : after ozone system 1, GAC: after non-aerated granular activated carbon filter treatment, $\mathrm{GAC}_{\mathrm{a}}$ : after aerated granular activated carbon filter treatment, $\mathrm{BF}$ : after non-aerated biofilter treatment, $\mathrm{BF}_{\mathrm{a}}$ : after aerated biofilter treatment, MBR1/2: after membrane bioreactor 1/2, MBR1+O 3 : after ozone system 2. $n=8-32$ (A), $n=16-32(B), n=40-56(C), n=31-40(D)$. 
S2.3 Chemical analysis

Table S19: Concentrations in $\mu \mathrm{g} / \mathrm{L}$ (mean \pm SEM) of chemicals from four $24 \mathrm{~h}$ composite samples in the primary treatment (PT), the conventional biological treatment (BT), the non-aerated granular activated carbon filter treatment (GAC) and the aerated granular activated carbon filter treatment $\left(\mathrm{GAC}_{\mathrm{a}}\right)$. The change of the concentration compared to the primary treatment $(\triangle \mathrm{PT})$ is given in \%. n.d.: not detected.

\begin{tabular}{|c|c|c|c|c|c|c|c|c|c|}
\hline & PT & BT & $\Delta \mathrm{PT}[\%]$ & $\mathrm{BT}+\mathrm{O}_{3}$ & $\Delta \mathrm{PT}[\%]$ & GAC & $\Delta \mathrm{PT}[\%]$ & GAC $_{a}$ & $\Delta \mathrm{PT}[\%]$ \\
\hline $\begin{array}{l}\text { 10,11-Dihydro-10,11- } \\
\text { dihydroxycarbamazepine }\end{array}$ & $\begin{array}{c}3.15 \pm 0.350 \\
(n=4)\end{array}$ & $\begin{array}{c}2.80 \pm 0.252 \\
(n=3)\end{array}$ & -11.1 & $\begin{array}{c}0.353 \pm 0.015 \\
(n=3)\end{array}$ & -88.8 & $\begin{array}{c}0.025 \pm 0.000 \\
(n=4)\end{array}$ & -99.2 & $\begin{array}{c}0.025 \pm 0.000 \\
(n=4)\end{array}$ & -99.2 \\
\hline 1H-Benzotriazol & $\begin{array}{c}25.0 \pm 0.707 \\
(n=4)\end{array}$ & $\begin{array}{c}7.68 \pm 0.544 \\
(n=4)\end{array}$ & -69.3 & $\begin{array}{c}0.650 \pm 0.047 \\
(n=4)\end{array}$ & -97.4 & $\begin{aligned} & 0.026 \pm 0.001 \\
&(n=4)\end{aligned}$ & -99.9 & $\begin{aligned} 0.319 & \pm 0.294 \\
(n & =4)\end{aligned}$ & -98.7 \\
\hline 1-Hydroxy-benzotriazol & $\begin{array}{c}1.31 \pm 0.169 \\
(n=4)\end{array}$ & $\begin{array}{c}0.493 \pm 0.063 \\
(n=4)\end{array}$ & -62.4 & $\begin{array}{c}0.031 \pm 0.006 \\
(n=4)\end{array}$ & -97.6 & $\begin{array}{c}0.025 \pm 0.000 \\
(n=4)\end{array}$ & -98.1 & $\begin{aligned} & 0.025 \pm 0.000 \\
&(n=4)\end{aligned}$ & -98.1 \\
\hline 1-Hydroxy-ibuprofen & $\begin{array}{c}5.83 \pm 0.335 \\
(n=4)\end{array}$ & $\begin{aligned} & 0.187 \pm 0.017 \\
&(n=3)\end{aligned}$ & -96.8 & $\begin{aligned} & 0.050 \pm 0.025 \\
&(n=3)\end{aligned}$ & -99.1 & $\begin{array}{c}0.063 \pm 0.022 \\
(n=4)\end{array}$ & -98.9 & $\begin{aligned} 0.063 & \pm 0.022 \\
(n & =4)\end{aligned}$ & -98.9 \\
\hline 2-Hydroxy-ibuprofen & $\begin{aligned} 47.3 & \pm 4.97 \\
(n & =4)\end{aligned}$ & $\begin{aligned} & 0.618 \pm 0.047 \\
&(n=4)\end{aligned}$ & -98.7 & $\begin{aligned} & 0.265 \pm 0.083 \\
&(n=4)\end{aligned}$ & -99.4 & $\begin{aligned} 0.175 & \pm 0.043 \\
(n & =4)\end{aligned}$ & -99.6 & $\begin{aligned} 0.175 & \pm 0.043 \\
(n & =4)\end{aligned}$ & -99.6 \\
\hline 3-Hydroxy-ibuprofen & $\begin{aligned} 5.98 & \pm 2.42 \\
(n & =4)\end{aligned}$ & $\begin{aligned} 0.375 & \pm 0.072 \\
(n=4) & \end{aligned}$ & -93.7 & $\begin{array}{c}1.50 \pm 1.17 \\
(n=4)\end{array}$ & -74.9 & $\begin{aligned} 0.375 & \pm 0.072 \\
(n=4) & \end{aligned}$ & -93.7 & $\begin{aligned} 0.375 & \pm 0.072 \\
(n & =4)\end{aligned}$ & -93.7 \\
\hline 4-Hydroxy-1H-benzotriazol & $\begin{aligned} & 0.520 \pm 0.047 \\
&(n=4)\end{aligned}$ & $\begin{array}{c}0.044 \pm 0.019 \\
(n=4)\end{array}$ & -91.6 & $\begin{array}{c}0.044 \pm 0.019 \\
(n=4)\end{array}$ & -91.6 & $\begin{aligned} & 0.025 \pm 0.000 \\
&(n=4)\end{aligned}$ & -95.2 & $\begin{array}{c}0.025 \pm 0.000 \\
(n=4)\end{array}$ & -95.2 \\
\hline 4-Hydroxy-diclofenac & $\begin{array}{c}2.38 \pm 0.229 \\
(n=4)\end{array}$ & $\begin{array}{c}1.16 \pm 0.148 \\
(n=3)\end{array}$ & -51.0 & $\begin{array}{c}0.025 \pm 0.000 \\
(n=3)\end{array}$ & -98.9 & $\begin{aligned} & 0.025 \pm 0.000 \\
&(n=4)\end{aligned}$ & -98.9 & $\begin{array}{c}0.025 \pm 0.000 \\
(n=4)\end{array}$ & -98.9 \\
\hline 4-Nitro-sulfamethoxazole & $\begin{array}{c}0.038 \pm 0.007 \\
(n=4)\end{array}$ & $\begin{array}{c}0.033 \pm 0.008 \\
(n=3)\end{array}$ & -11.1 & $\begin{array}{c}0.033 \pm 0.008 \\
(n=3)\end{array}$ & -11.1 & $\begin{array}{c}0.038 \pm 0.007 \\
(n=4)\end{array}$ & \pm 0.0 & $\begin{array}{c}0.038 \pm 0.007 \\
(n=4)\end{array}$ & \pm 0.0 \\
\hline Acyclovir & $\begin{array}{l}6.75 \pm 0.771 \\
(n=4)\end{array}$ & $\begin{array}{c}0.450 \pm 0.069 \\
(n=4)\end{array}$ & -93.3 & $\begin{array}{c}0.031 \pm 0.006 \\
(n=4)\end{array}$ & -99.5 & $\begin{array}{c}0.025 \pm 0.000 \\
(n=4)\end{array}$ & -99.6 & $\begin{array}{c}0.025 \pm 0.000 \\
(n=4)\end{array}$ & -99.6 \\
\hline Amidotrizoic acid & $\begin{array}{c}4.19 \pm 2.97 \\
(n=4)\end{array}$ & $\begin{array}{l}3.19 \pm 1.91 \\
(n=4)\end{array}$ & -24.0 & $\begin{array}{c}2.33 \pm 1.28 \\
(n=4)\end{array}$ & -44.4 & $\begin{array}{l}1.37 \pm 0.302 \\
(n=4)\end{array}$ & -67.4 & $\begin{array}{c}1.43 \pm 0.325 \\
(n=4)\end{array}$ & -65.8 \\
\hline Carbamazepine & $\begin{array}{c}1.20 \pm 0.147 \\
(n=4)\end{array}$ & $\begin{array}{c}1.43 \pm 0.067 \\
(n=3)\end{array}$ & +19.4 & $\begin{aligned} & 0.025 \pm 0.000 \\
&(n=3)\end{aligned}$ & -97.9 & $\begin{aligned} & 0.025 \pm 0.000 \\
&(n=4)\end{aligned}$ & -97.9 & $\begin{aligned} & 0.025 \pm 0.000 \\
&(n=4)\end{aligned}$ & -97.9 \\
\hline Carboxy-acyclovir & $\begin{array}{c}1.04 \pm 0.178 \\
(n=4)\end{array}$ & $\begin{array}{l}4.85 \pm 1.04 \\
(n=4)\end{array}$ & +367 & $\begin{aligned} 0.044 & \pm 0.019 \\
(n=4) & \end{aligned}$ & -95.8 & $\begin{aligned} & 0.025 \pm 0.000 \\
&(n=4)\end{aligned}$ & -97.6 & $\begin{aligned} 0.025 & \pm 0.000 \\
(n=4) & =4\end{aligned}$ & -97.6 \\
\hline Carboxy-ibuprofen & $\begin{aligned} & 74.7 \pm 6.27 \\
&(n=4)\end{aligned}$ & $\begin{aligned} & 0.150 \pm 0.117 \\
&(n=4)\end{aligned}$ & -99.8 & $\begin{array}{c}0.035 \pm 0.006 \\
(n=4)\end{array}$ & -100 & $\begin{aligned} & 0.025 \pm 0.000 \\
&(n=4)\end{aligned}$ & 100 & $\begin{aligned} 0.025 & \pm 0.000 \\
(n & =4)\end{aligned}$ & 100 \\
\hline Caffeine & $\begin{array}{c}162 \pm 23.2 \\
(n=4)\end{array}$ & $\begin{array}{c}0.312 \pm 0.229 \\
(n=3)\end{array}$ & -99.8 & $\begin{array}{c}0.352 \pm 0.224 \\
(n=3)\end{array}$ & -99.8 & $\begin{aligned} & 0.335 \pm 0.180 \\
&(n=4)\end{aligned}$ & -99.8 & $\begin{aligned} & 0.406 \pm 0.190 \\
&(n=4)\end{aligned}$ & -99.7 \\
\hline Dehydrato-erythromycin & n.d. & $\begin{array}{l}0.120 \pm 0.000 \\
(n=1)\end{array}$ & - & $\begin{array}{l}0.050 \pm 0.000 \\
(n=1)\end{array}$ & - & $\begin{array}{l}0.050 \pm 0.000 \\
(n=1)\end{array}$ & - & $\begin{array}{l}0.052 \pm 0.000 \\
(n=1)\end{array}$ & - \\
\hline Diclofenac & $\begin{array}{c}5.08 \pm 0.431 \\
(n=4)\end{array}$ & $\begin{array}{c}4.43 \pm 0.067 \\
(n=3)\end{array}$ & -12.7 & $\begin{aligned} 0.137 & \pm 0.112 \\
(n=3) & =0\end{aligned}$ & -97.3 & $\begin{aligned} 0.025 & \pm 0.000 \\
(n & =4)\end{aligned}$ & -99.5 & $\begin{aligned} 0.025 & \pm 0.000 \\
(n & =4)\end{aligned}$ & -99.5 \\
\hline Erythromycin & n.d. & $\begin{array}{c}0.330 \pm 0.000 \\
(n=1)\end{array}$ & - & $\begin{array}{c}0.025 \pm 0.000 \\
(n=1)\end{array}$ & - & $\begin{array}{c}0.025 \pm 0.000 \\
(n=1)\end{array}$ & - & $\begin{array}{c}0.025 \pm 0.000 \\
(n=1)\end{array}$ & - \\
\hline lomeprol & $\begin{array}{c}9.09 \pm 5.88 \\
(n=4)\end{array}$ & $\begin{array}{c}6.77 \pm 3.14 \\
(n=3)\end{array}$ & -25.6 & $\begin{array}{c}3.07 \pm 1.04 \\
(n=3)\end{array}$ & -66.3 & $\begin{array}{c}0.173 \pm 0.033 \\
(n=4)\end{array}$ & -98.1 & $\begin{array}{c}0.363 \pm 0.059 \\
(n=4)\end{array}$ & -96.0 \\
\hline lopamidol & $\begin{array}{c}0.042 \pm 0.008 \\
(n=3)\end{array}$ & $\begin{array}{c}0.038 \pm 0.013 \\
(n=2)\end{array}$ & -10.0 & $\begin{aligned} & 0.033 \pm 0.008 \\
&(n=3)\end{aligned}$ & -20.0 & $\begin{aligned} & 0.042 \pm 0.008 \\
&(n=3)\end{aligned}$ & \pm 0.0 & $\begin{aligned} 0.042 & \pm 0.008 \\
(\mathrm{n} & =3)\end{aligned}$ & \pm 0.0 \\
\hline lopromide & $\begin{array}{c}8.16 \pm 3.87 \\
(n=4)\end{array}$ & $\begin{aligned} 2.93 & \pm 1.51 \\
(n & =3)\end{aligned}$ & -64.1 & $\begin{aligned} 0.966 & \pm 0.538 \\
(n=3) & =0\end{aligned}$ & -88.2 & $\begin{aligned} 0.025 & \pm 0.000 \\
(n & =4)\end{aligned}$ & -99.7 & $\begin{aligned} 0.054 & \pm 0.018 \\
(n & =4)\end{aligned}$ & -99.3 \\
\hline Mecoprop & $\begin{aligned} 0.038 & \pm 0.007 \\
(n & =4)\end{aligned}$ & $\begin{array}{c}0.033 \pm 0.008 \\
(n=3)\end{array}$ & -11.1 & $\begin{array}{c}0.033 \pm 0.008 \\
(n=3)\end{array}$ & -11.1 & $\begin{aligned} 0.038 & \pm 0.007 \\
(n & =4)\end{aligned}$ & \pm 0.0 & $\begin{aligned} 0.038 & \pm 0.007 \\
(n & =4)\end{aligned}$ & \pm 0.0 \\
\hline N-Acetyl-sulfamethoxazole & $\begin{array}{c}1.25 \pm 0.132 \\
(n=4)\end{array}$ & $\begin{array}{c}0.130 \pm 0.010 \\
(n=3)\end{array}$ & -89.6 & $\begin{aligned} & 0.025 \pm 0.000 \\
&(n=3)\end{aligned}$ & -98.0 & $\begin{array}{c}0.025 \pm 0.000 \\
(n=4)\end{array}$ & -98.0 & $\begin{array}{c}0.025 \pm 0.000 \\
(n=4)\end{array}$ & -98.0 \\
\hline Paracetamol & $\begin{aligned} 14.4 & \pm 2.23 \\
(n & =4)\end{aligned}$ & $\begin{aligned} 0.044 & \pm 0.019 \\
(n & =4)\end{aligned}$ & -99.7 & $\begin{aligned} 0.044 & \pm 0.019 \\
(n=4) & =4\end{aligned}$ & -99.7 & $\begin{aligned} 0.025 & \pm 0.000 \\
(n & =4)\end{aligned}$ & -99.8 & $\begin{aligned} 0.025 & \pm 0.000 \\
(n & =4)\end{aligned}$ & -99.8 \\
\hline Sulfamethoxazole & $\begin{array}{c}1.30 \pm 0.108 \\
(n=4)\end{array}$ & $\begin{aligned} 0.335 & \pm 0.065 \\
(n & =4)\end{aligned}$ & -74.2 & $\begin{aligned} 0.269 & \pm 0.244 \\
(n=4) & =\end{aligned}$ & -79.3 & $\begin{aligned} 0.025 & \pm 0.000 \\
(n & =4)\end{aligned}$ & -98.1 & $\begin{aligned} 0.025 & \pm 0.000 \\
(n & =4)\end{aligned}$ & -98.1 \\
\hline Tolyltriazole & $\begin{array}{c}4.78 \pm 0.58 \\
(n=4)\end{array}$ & $\begin{array}{c}2.32 \pm 0.341 \\
(\mathrm{n}=4)\end{array}$ & -51.5 & $\begin{array}{c}0.032 \pm 0.006 \\
(n=4)\end{array}$ & -99.3 & $\begin{aligned} 0.025 & \pm 0.000 \\
(n & =4)\end{aligned}$ & -99.5 & $\begin{array}{c}0.025 \pm 0.000 \\
(n=4)\end{array}$ & -99.5 \\
\hline Tramadol & $\begin{array}{c}0.998 \pm 0.043 \\
(n=4)\end{array}$ & $\begin{array}{c}0.890 \pm 0.061 \\
(n=3)\end{array}$ & -10.8 & $\begin{array}{c}0.031 \pm 0.006 \\
(n=4)\end{array}$ & -96.9 & $\begin{array}{c}0.025 \pm 0.000 \\
(n=4)\end{array}$ & -97.5 & $\begin{array}{c}0.025 \pm 0.000 \\
(n=4)\end{array}$ & -97.5 \\
\hline Tramadol- $\mathrm{N}$-oxide & $\begin{array}{c}0.025 \pm 0.000 \\
(n=4)\end{array}$ & $\begin{array}{c}0.025 \pm 0.000 \\
(\mathrm{n}=3)\end{array}$ & \pm 0.0 & $\begin{array}{c}0.047 \pm 0.004 \\
(n=3)\end{array}$ & +88.0 & $\begin{array}{c}0.025 \pm 0.000 \\
(n=4)\end{array}$ & \pm 0.0 & $\begin{array}{c}0.025 \pm 0.000 \\
(n=4)\end{array}$ & \pm 0.0 \\
\hline
\end{tabular}


Table S20: Concentrations in $\mu g / \mathrm{L}$ (mean \pm SEM) of chemicals from four $24 \mathrm{~h}$ composite samples in the primary treatment (PT), the non-aerated biofilter treatment $(\mathrm{BF})$, the aerated biofilter treatment $\left(\mathrm{BF}_{\mathrm{a}}\right)$, membrane reactor 1 (MBR1) and membrane reactor 1 after ozone system $2\left(\mathrm{MBR} 1+\mathrm{O}_{3}\right)$. The change of the concentration compared to the primary treatment ( $\triangle \mathrm{PT})$ is given in \%. n.d.: not detected.

\begin{tabular}{|c|c|c|c|c|c|c|c|c|c|}
\hline & PT & $\mathrm{BF}$ & $\Delta \mathrm{PT}[\%]$ & $\mathrm{BF}_{\mathrm{a}}$ & $\Delta \mathrm{PT}[\%]$ & MBR1 & $\Delta \mathrm{PT}[\%]$ & MBR1+O & $\Delta \mathrm{PT}[\%]$ \\
\hline $\begin{array}{l}\text { 10,11-Dihydro-10,11- } \\
\text { dihydroxycarbamazepine }\end{array}$ & $\begin{array}{c}3.15 \pm 0.350 \\
(n=4)\end{array}$ & $\begin{array}{c}0.353 \pm 0.072 \\
(n=4)\end{array}$ & -88.8 & $\begin{array}{c}0.338 \pm 0.043 \\
(n=4)\end{array}$ & -89.3 & $\begin{array}{c}1.98 \pm 0.588 \\
(n=4)\end{array}$ & -37.3 & $\begin{aligned} 0.496 & \pm 0.308 \\
(n & =4)\end{aligned}$ & -84.3 \\
\hline 1H-Benzotriazol & $\begin{array}{c}25.0 \pm 0.707 \\
(n=4)\end{array}$ & $\begin{array}{c}0.650 \pm 0.087 \\
(n=4)\end{array}$ & -97.4 & $\begin{array}{c}0.608 \pm 0.069 \\
(n=4)\end{array}$ & -97.6 & $\begin{array}{c}9.73 \pm 6.76 \\
(n=4)\end{array}$ & -61.1 & $\begin{array}{c}1.17 \pm 0.700 \\
(n=4)\end{array}$ & -95.3 \\
\hline 1-Hydroxy-benzotriazol & $\begin{array}{c}1.31 \pm 0.169 \\
(n=4)\end{array}$ & $\begin{array}{c}0.025 \pm 0.000 \\
(n=4)\end{array}$ & -98.1 & $\begin{array}{c}0.025 \pm 0.000 \\
(n=4)\end{array}$ & -98.1 & $\begin{array}{c}0.423 \pm 0.127 \\
(n=4)\end{array}$ & -67.7 & $\begin{aligned} & 0.099 \pm 0.074 \\
&(n=4)\end{aligned}$ & -92.5 \\
\hline 1-Hydroxy-ibuprofen & $\begin{array}{c}5.83 \pm 0.335 \\
(n=4)\end{array}$ & $\begin{aligned} 0.063 & \pm 0.022 \\
(n & =4)\end{aligned}$ & -98.9 & $\begin{array}{c}0.063 \pm 0.022 \\
(n=4)\end{array}$ & -98.9 & $\begin{array}{c}1.53 \pm 1.46 \\
(n=4)\end{array}$ & -73.7 & $\begin{aligned} 0.090 & \pm 0.044 \\
(n & =4)\end{aligned}$ & -98.5 \\
\hline 2-Hydroxy-ibuprofen & $\begin{aligned} 47.3 & \pm 4.97 \\
(n & =4)\end{aligned}$ & $\begin{aligned} 0.183 & \pm 0.039 \\
(n & =4)\end{aligned}$ & -99.6 & $\begin{aligned} 0.175 & \pm 0.043 \\
(n=4) & \end{aligned}$ & -99.6 & $\begin{aligned} 14.1 & \pm 13.6 \\
(n & =4)\end{aligned}$ & -70.2 & $\begin{aligned} 0.560 & \pm 0.381 \\
(n & =4)\end{aligned}$ & -98.8 \\
\hline 3-Hydroxy-ibuprofen & $\begin{aligned} 5.98 & \pm 2.42 \\
(n & =4)\end{aligned}$ & $\begin{aligned} 0.375 & \pm 0.072 \\
(n & =4)\end{aligned}$ & -93.7 & $\begin{aligned} 0.375 & \pm 0.072 \\
(n & =4)\end{aligned}$ & -93.7 & $\begin{aligned} 2.10 & \pm 1.77 \\
(n & =4)\end{aligned}$ & -64.9 & $\begin{aligned} 1.50 & \pm 1.17 \\
(n & =4)\end{aligned}$ & -74.9 \\
\hline 4-Hydroxy-1H-benzotriazol & $\begin{aligned} & 0.520 \pm 0.047 \\
&(n=4)\end{aligned}$ & $\begin{aligned} 0.025 & \pm 0.000 \\
(n & =4)\end{aligned}$ & -95.2 & $\begin{aligned} 0.025 & \pm 0.000 \\
(n & =4)\end{aligned}$ & -95.2 & $\begin{aligned} 0.411 & \pm 0.363 \\
(n & =4)\end{aligned}$ & -21.1 & $\begin{array}{c}0.051 \pm 0.026 \\
(n=4)\end{array}$ & -90.1 \\
\hline 4-Hydroxy-diclofenac & $\begin{array}{c}2.38 \pm 0.229 \\
(n=4)\end{array}$ & $\begin{aligned} 0.025 & \pm 0.000 \\
(n & =4)\end{aligned}$ & -98.9 & $\begin{array}{c}0.025 \pm 0.000 \\
(n=4)\end{array}$ & -98.9 & $\begin{array}{c}1.03 \pm 0.526 \\
(n=4)\end{array}$ & -56.8 & $\begin{array}{c}0.153 \pm 0.122 \\
(n=4)\end{array}$ & -93.5 \\
\hline 4-Nitro-sulfamethoxazole & $\begin{aligned} 0.038 & \pm 0.007 \\
(n & =4)\end{aligned}$ & $\begin{aligned} & 0.038 \pm 0.007 \\
&(n=4)\end{aligned}$ & \pm 0.0 & $\begin{array}{c}0.038 \pm 0.007 \\
(n=4)\end{array}$ & \pm 0.0 & $\begin{aligned} 0.038 & \pm 0.007 \\
(n & =4)\end{aligned}$ & \pm 0.0 & $\begin{aligned} 0.038 & \pm 0.007 \\
(n & =4)\end{aligned}$ & \pm 0.0 \\
\hline Acyclovir & $\begin{array}{c}6.75 \pm 0.771 \\
(n=4)\end{array}$ & $\begin{aligned} 0.025 & \pm 0.000 \\
(n & =4)\end{aligned}$ & -99.6 & $\begin{aligned} 0.025 & \pm 0.000 \\
(n & =4)\end{aligned}$ & -99.6 & $\begin{aligned} 2.02 & \pm 1.93 \\
(n & =4)\end{aligned}$ & -70.1 & $\begin{array}{c}0.091 \pm 0.066 \\
(n=4)\end{array}$ & -98.6 \\
\hline Amidotrizoic acid & $\begin{aligned} 4.19 & \pm 2.97 \\
(n & =4)\end{aligned}$ & $\begin{aligned} 2.23 & \pm 1.26 \\
(n & =4)\end{aligned}$ & -46.9 & $\begin{array}{c}2.37 \pm 1.33 \\
(n=4)\end{array}$ & -43.5 & $\begin{aligned} 2.89 & \pm 1.66 \\
(n & =4)\end{aligned}$ & -31.2 & $\begin{aligned} 2.22 & \pm 2.09 \\
(n & =4)\end{aligned}$ & -47.0 \\
\hline Carbamazepine & $\begin{array}{c}1.20 \pm 0.147 \\
(n=4)\end{array}$ & $\begin{aligned} 0.025 & \pm 0.000 \\
(n & =4)\end{aligned}$ & -97.9 & $\begin{array}{c}0.025 \pm 0.000 \\
(n=4)\end{array}$ & -97.9 & $\begin{aligned} 0.668 & \pm 0.184 \\
(n & =4)\end{aligned}$ & -44.4 & $\begin{aligned} 0.124 & \pm 0.099 \\
(n & =4)\end{aligned}$ & -89.7 \\
\hline Carboxy-acyclovir & $\begin{array}{c}1.04 \pm 0.178 \\
(n=4)\end{array}$ & $\begin{aligned} 0.025 & \pm 0.000 \\
(n & =4)\end{aligned}$ & -97.6 & $\begin{array}{c}0.025 \pm 0.000 \\
(n=4)\end{array}$ & -97.6 & $\begin{array}{c}2.55 \pm 0.591 \\
(n=4)\end{array}$ & +146 & $\begin{array}{c}1.45 \pm 1.42 \\
(n=4)\end{array}$ & +39.3 \\
\hline Carboxy-ibuprofen & $\begin{array}{c}74.7 \pm 6.27 \\
(n=4)\end{array}$ & $\begin{aligned} 0.028 & \pm 0.003 \\
(n & =4)\end{aligned}$ & -100 & $\begin{array}{c}0.029 \pm 0.004 \\
(n=4)\end{array}$ & -100 & $\begin{aligned} 38.3 & \pm 38.2 \\
(n & =4)\end{aligned}$ & -48.8 & $\begin{aligned} & 0.094 \pm 0.069 \\
&(n=4)\end{aligned}$ & -99.9 \\
\hline Caffeine & $\begin{array}{c}162 \pm 23.2 \\
(n=4)\end{array}$ & $\begin{array}{c}0.338 \pm 0.200 \\
(n=4)\end{array}$ & -99.8 & $\begin{array}{c}0.489 \pm 0.279 \\
(n=4)\end{array}$ & -99.7 & $\begin{array}{c}87.1 \pm 86.3 \\
(n=4)\end{array}$ & -46.2 & $\begin{array}{c}0.491 \pm 0.224 \\
(n=4)\end{array}$ & -99.7 \\
\hline Dehydrato-erythromycin & n.d. & $\begin{aligned} 0.050 & \pm 0.000 \\
(n & =1)\end{aligned}$ & - & $\begin{array}{c}0.050 \pm 0.000 \\
(n=1)\end{array}$ & - & n.d. & - & n.d. & - \\
\hline Diclofenac & $5.08 \pm 0.431$ & $0.025 \pm 0.000$ & -99.5 & $0.025 \pm 0.000$ & -99.5 & $2.35 \pm 0.718$ & -53.7 & $0.269 \pm 0.244$ & -94.7 \\
\hline Erythromycin & $\begin{array}{c}(n=4) \\
\text { n.d. }\end{array}$ & $\begin{array}{c}(n=4) \\
0.025 \pm 0.000 \\
(n=1)\end{array}$ & - & $\begin{array}{c}(n=4) \\
0.025 \pm 0.000 \\
(n=1)\end{array}$ & - & $\begin{array}{c}(n=4) \\
\text { n.d. }\end{array}$ & - & $\begin{array}{c}(n=4) \\
\text { n.d. }\end{array}$ & - \\
\hline lomeprol & $\begin{aligned} 9.09 & \pm 5.88 \\
(n & =4)\end{aligned}$ & $\begin{array}{c}2.45 \pm 0.689 \\
(n=4)\end{array}$ & -73.0 & $\begin{aligned} 2.30 & \pm 0.785 \\
(n & =4)\end{aligned}$ & -74.8 & $\begin{aligned} 4.93 & \pm 2.14 \\
(n=4) & =\end{aligned}$ & -45.8 & $\begin{aligned} 0.718 & \pm 0.153 \\
(n & =4)\end{aligned}$ & -92.1 \\
\hline lopamidol & $\begin{aligned} 0.042 & \pm 0.008 \\
(n=3) & =0\end{aligned}$ & $\begin{array}{c}0.042 \pm 0.008 \\
(n=3)\end{array}$ & \pm 0.0 & $\begin{aligned} 0.042 & \pm 0.008 \\
(n & =3)\end{aligned}$ & \pm 0.0 & $\begin{array}{c}0.042 \pm 0.008 \\
(n=3)\end{array}$ & \pm 0.0 & $\begin{aligned} 0.042 & \pm 0.008 \\
(n=3) & =0\end{aligned}$ & \pm 0.0 \\
\hline lopromide & $\begin{array}{c}8.16 \pm 3.87 \\
(n=4)\end{array}$ & $\begin{array}{c}0.781 \pm 0.397 \\
(n=4)\end{array}$ & -90.4 & $\begin{array}{c}0.515 \pm 0.397 \\
(n=4)\end{array}$ & -93.7 & $\begin{array}{c}1.36 \pm 0.466 \\
(n=4)\end{array}$ & -83.4 & $\begin{array}{c}0.369 \pm 0.115 \\
(n=4)\end{array}$ & -95.5 \\
\hline Mecoprop & $\begin{array}{c}0.038 \pm 0.007 \\
(n=4)\end{array}$ & $\begin{array}{c}0.038 \pm 0.007 \\
(n=4)\end{array}$ & \pm 0.0 & $\begin{aligned} 0.038 & \pm 0.007 \\
(n & =4)\end{aligned}$ & \pm 0.0 & $\begin{aligned} 0.038 & \pm 0.007 \\
(n & =4)\end{aligned}$ & \pm 0.0 & $\begin{aligned} 0.038 & \pm 0.007 \\
(n & =4)\end{aligned}$ & \pm 0.0 \\
\hline N-Acetyl-sulfamethoxazole & $\begin{array}{c}1.25 \pm 0.132 \\
(n=4)\end{array}$ & $\begin{array}{c}0.025 \pm 0.000 \\
(n=4)\end{array}$ & -98.0 & $\begin{aligned} 0.025 & \pm 0.000 \\
(n & =4)\end{aligned}$ & -98.0 & $\begin{aligned} 0.490 & \pm 0.304 \\
(n & =4)\end{aligned}$ & -60.8 & $\begin{aligned} 0.113 & \pm 0.079 \\
(n & =4)\end{aligned}$ & -90.9 \\
\hline Paracetamol & $\begin{aligned} & 14.4 \pm 2.23 \\
&(n=4)\end{aligned}$ & $\begin{array}{c}0.025 \pm 0.000 \\
(n=4)\end{array}$ & -99.8 & $\begin{array}{c}0.025 \pm 0.000 \\
(n=4)\end{array}$ & -99.8 & $\begin{aligned} & 3.04 \pm 3.02 \\
&(n=4)\end{aligned}$ & -78.8 & $\begin{aligned} & 0.030 \pm 0.005 \\
&(n=4)\end{aligned}$ & -99.8 \\
\hline Sulfamethoxazole & $\begin{array}{c}1.30 \pm 0.108 \\
(n=4)\end{array}$ & $\begin{array}{c}0.025 \pm 0.000 \\
(n=4)\end{array}$ & -98.1 & $\begin{aligned} 0.025 & \pm 0.000 \\
(n & =4)\end{aligned}$ & -98.1 & $\begin{aligned} 0.362 & \pm 0.246 \\
(n & =4)\end{aligned}$ & -72.1 & $\begin{aligned} 0.044 & \pm 0.019 \\
(n & =4)\end{aligned}$ & -96.6 \\
\hline Tolyltriazole & $\begin{array}{c}4.78 \pm 0.58 \\
(n=4)\end{array}$ & $\begin{array}{c}0.029 \pm 0.004 \\
(n=4)\end{array}$ & -99.4 & $\begin{array}{c}0.027 \pm 0.002 \\
(n=4)\end{array}$ & -99.4 & $\begin{array}{c}2.50 \pm 1.47 \\
(n=4)\end{array}$ & -47.6 & $\begin{array}{c}0.371 \pm 0.253 \\
(n=4)\end{array}$ & -92.2 \\
\hline Tramadol & $\begin{array}{c}0.998 \pm 0.043 \\
(n=4)\end{array}$ & $\begin{array}{c}0.025 \pm 0.000 \\
(n=4)\end{array}$ & -97.5 & $\begin{array}{c}0.025 \pm 0.000 \\
(n=4)\end{array}$ & -97.5 & $\begin{array}{c}0.665 \pm 0.213 \\
(n=4)\end{array}$ & -33.3 & $\begin{array}{c}0.190 \pm 0.140 \\
(n=4)\end{array}$ & -81.0 \\
\hline Tramadol-N-oxide & $\begin{aligned} 0.025 & \pm 0.000 \\
(n & =4)\end{aligned}$ & $\begin{array}{c}0.043 \pm 0.008 \\
(n=4)\end{array}$ & +71.0 & $\begin{aligned} 0.042 & \pm 0.007 \\
(n & =4)\end{aligned}$ & +68.0 & $\begin{aligned} 0.025 & \pm 0.000 \\
(n & =4)\end{aligned}$ & \pm 0.0 & $\begin{aligned} 0.028 & \pm 0.003 \\
(n & =4)\end{aligned}$ & +10.0 \\
\hline
\end{tabular}


Table S21: Concentrations in $\mu \mathrm{g} / \mathrm{L}$ (mean \pm SEM) of chemicals from four $24 \mathrm{~h}$ composite samples in the primary treatment (PT), the conventional biological treatment (BT) and membrane reactor 2 (MBR2). The change of the concentration of MBR2 compared to the primary treatment ( $\triangle$ PT) is given in \%. n.d.: not detected.

\begin{tabular}{lcccc}
\hline & PT & BT (for comparison) & MBR2 & $\Delta$ PT [\%] \\
\hline 10,11-Dihydro-10,11-dihydroxycarbamazepine & $3.15 \pm 0.350(n=4)$ & $2.80 \pm 0.252(n=3)$ & $2.86 \pm 0.723(n=4)$ & -9.21 \\
1H-Benzotriazol & $25.0 \pm 0.707(n=4)$ & $7.68 \pm 0.544(n=4)$ & $5.55 \pm 1.12(n=4)$ & -77.8 \\
1-Hydroxy-benzotriazol & $1.31 \pm 0.169(n=4)$ & $0.493 \pm 0.063(n=4)$ & $0.660 \pm 0.190(n=4)$ & -49.6 \\
1-Hydroxy-ibuprofen & $5.83 \pm 0.335(n=4)$ & $0.187 \pm 0.017(n=3)$ & $0.128 \pm 0.013(n=4)$ & -97.8 \\
2-Hydroxy-ibuprofen & $47.3 \pm 4.97(n=4)$ & $0.618 \pm 0.047(n=4)$ & $0.700 \pm 0.172(n=4)$ & -98.5 \\
3-Hydroxy-ibuprofen & $5.98 \pm 2.42(n=4)$ & $0.375 \pm 0.072(n=4)$ & $0.375 \pm 0.072(n=4)$ & -93.7 \\
4-Hydroxy-1H-benzotriazol & $0.520 \pm 0.047(n=4)$ & $0.044 \pm 0.019(n=4)$ & $0.082 \pm 0.022(n=4)$ & -84.2 \\
4-Hydroxy-diclofenac & $2.38 \pm 0.229(n=4)$ & $1.16 \pm 0.148(n=3)$ & $1.49 \pm 0.461(n=4)$ & -37.3 \\
4-Nitro-sulfamethoxazole & $0.038 \pm 0.007(n=4)$ & $0.033 \pm 0.008(n=3)$ & $0.038 \pm 0.007(n=4)$ & \pm 0.0 \\
Acyclovir & $6.75 \pm 0.771(n=4)$ & $0.450 \pm 0.069(n=4)$ & $0.127 \pm 0.020(n=4)$ & -98.1 \\
Amidotrizoic acid & $4.19 \pm 2.97(n=4)$ & $3.19 \pm 1.91(n=4)$ & $3.78 \pm 2.45(n=4)$ & -9.78 \\
Carbamazepine & $1.20 \pm 0.147(n=4)$ & $1.43 \pm 0.067(n=3)$ & $1.14 \pm 0.359(n=4)$ & -4.92 \\
Carboxy-acyclovir & $1.04 \pm 0.178(n=4)$ & $4.85 \pm 1.04(n=4)$ & $4.60 \pm 1.18(n=4)$ & +343 \\
Carboxy-ibuprofen & $74.7 \pm 6.27(n=4)$ & $0.150 \pm 0.117(n=4)$ & $0.158 \pm 0.053(n=4)$ & -99.8 \\
Caffeine & $162 \pm 23.2(n=4)$ & $0.312 \pm 0.229(n=3)$ & $0.794 \pm 0.322(n=4)$ & -99.5 \\
Dehydrato-erythromycin & $n . d$. & $0.120 \pm 0.000(n=1)$ & $n . d$. & - \\
Diclofenac & $5.08 \pm 0.431(n=4)$ & $4.43 \pm 0.067(n=3)$ & $3.18 \pm 0.989(n=4)$ & -37.3 \\
Erythromycin & $n . d$. & $0.330 \pm 0.000(n=1)$ & $n . d$. & - \\
lomeprol & $9.09 \pm 5.88(n=4)$ & $6.77 \pm 3.14(n=3)$ & $5.92 \pm 3.50(n=4)$ & -34.9 \\
lopamidol & $0.042 \pm 0.008(n=3)$ & $0.038 \pm 0.013(n=2)$ & $0.042 \pm 0.008(n=3)$ & \pm 0.0 \\
lopromide & $8.16 \pm 3.87(n=4)$ & $2.93 \pm 1.51(n=3)$ & $2.17 \pm 1.37(n=4)$ & -73.4 \\
Mecoprop & $0.038 \pm 0.007(n=4)$ & $0.033 \pm 0.008(n=3)$ & $0.038 \pm 0.007(n=4)$ & \pm 0.0 \\
N-Acetyl-sulfamethoxazole & $1.25 \pm 0.132(n=4)$ & $0.130 \pm 0.010(n=3)$ & $0.173 \pm 0.041(n=4)$ & -86.2 \\
Paracetamol & $14.4 \pm 2.23(n=4)$ & $0.044 \pm 0.019(n=4)$ & $0.025 \pm 0.000(n=4)$ & -99.8 \\
Sulfamethoxazole & $1.30 \pm 0.108(n=4)$ & $0.335 \pm 0.065(n=4)$ & $0.254 \pm 0.069(n=4)$ & -80.4 \\
Tolyltriazole & $4.78 \pm 0.58(n=4)$ & $2.32 \pm 0.341(n=4)$ & $2.25 \pm 0.452(n=4)$ & -52.9 \\
Tramadol & $0.998 \pm 0.043(n=4)$ & $0.890 \pm 0.061(n=3)$ & $0.955 \pm 0.217(n=4)$ & -4.26 \\
Tramadol-N-oxide & $0.025 \pm 0.000(n=4)$ & $0.025 \pm 0.000(n=3)$ & $0.025 \pm 0.000(n=4)$ & \pm 0.0 \\
\hline & & & &
\end{tabular}



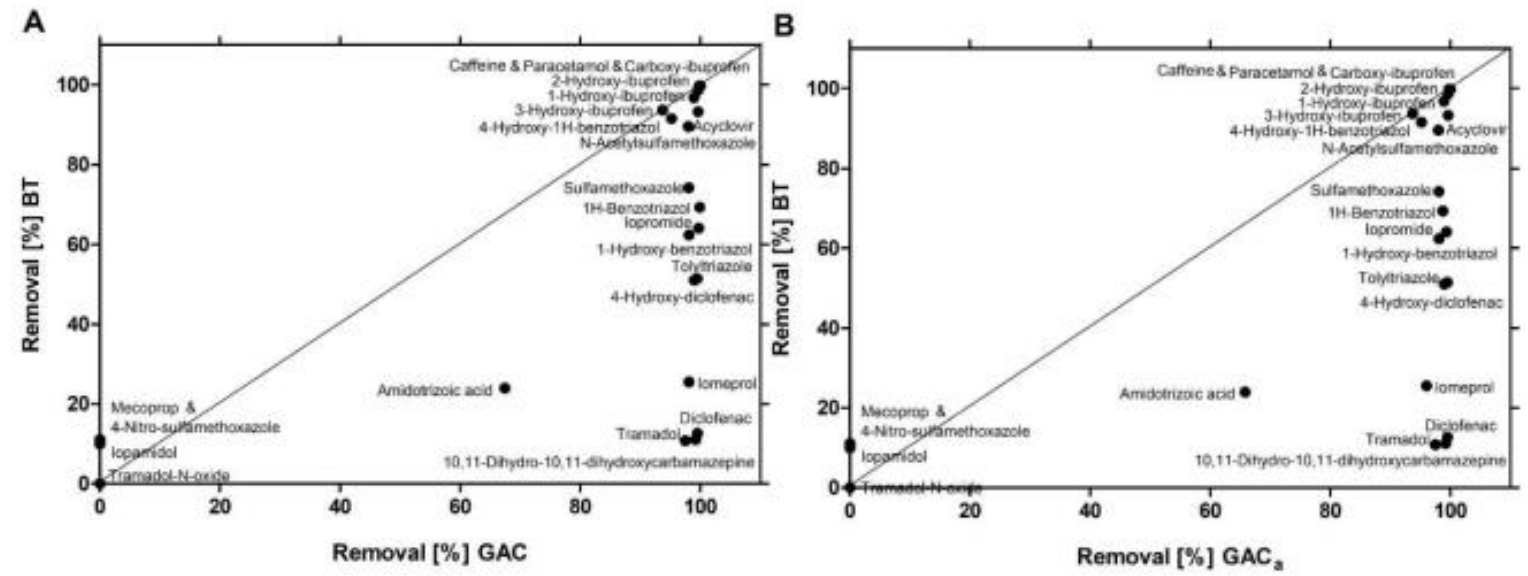

Figure S8: Removal of chemicals in the conventional biological treatment (BT) compared to the non-aerated granular activated carbon filter treatment (GAC, $A$ ) and the aerated granular activated carbon filter treatment $\left(\mathrm{GAC}_{\mathrm{a}}, \mathrm{B}\right) . \mathrm{n}=1-4$.
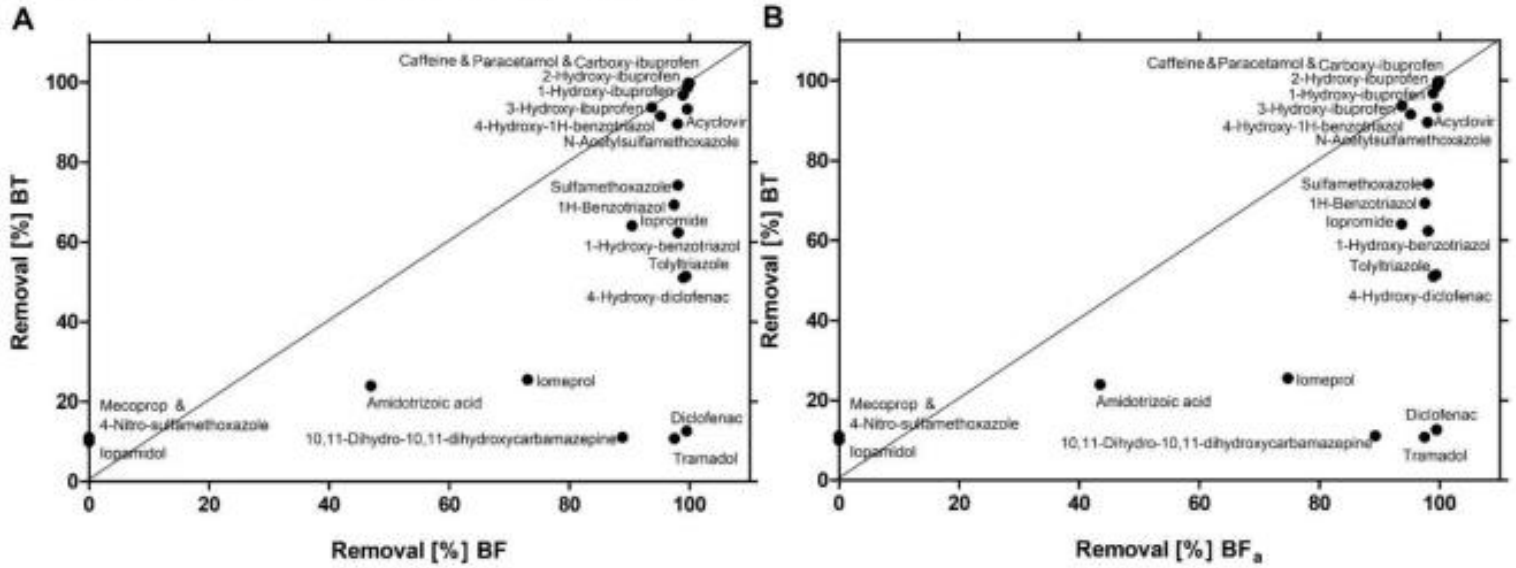

Figure S9: Removal of chemicals in the conventional biological treatment (BT) compared to the non-aerated biofilter treatment $(B F, A)$ and the aerated biofilter treatment $\left(B F_{a}, B\right) . n=1-4$.
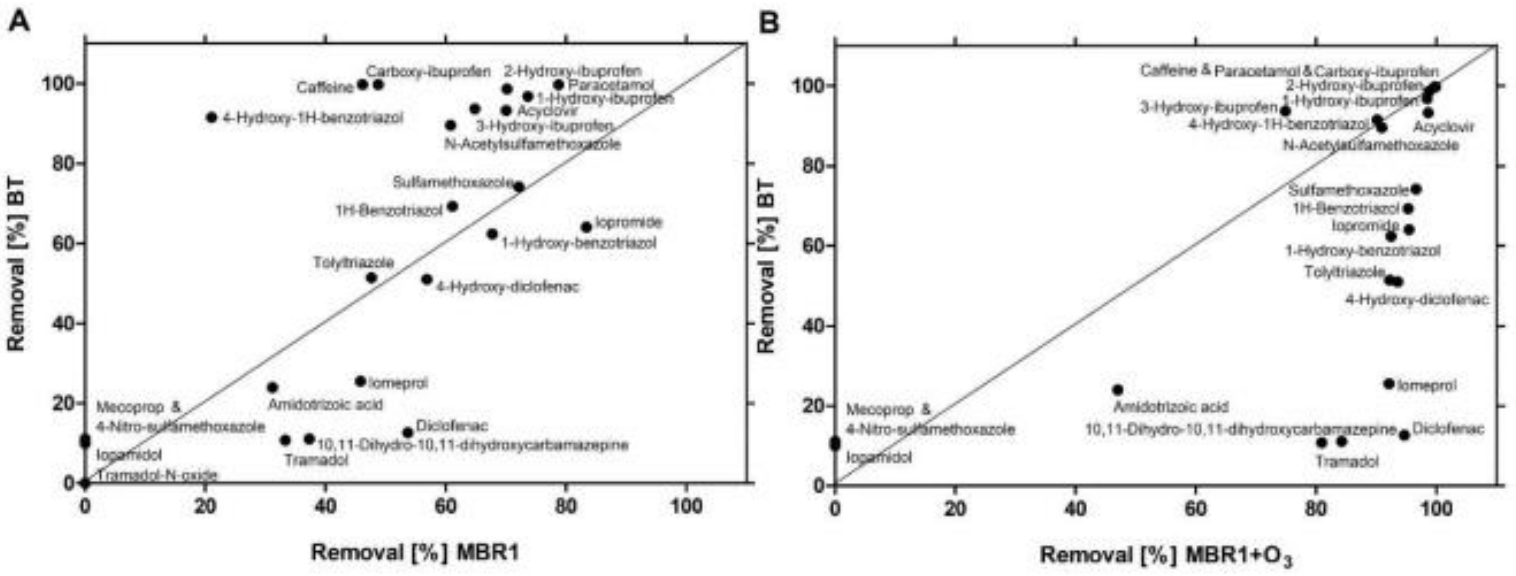

Figure S10: Removal of chemicals in the conventional biological treatment (BT) compared to the membrane bioreactor 1 (MBR1, A) and the membrane bioreactor 1 after ozone system 2 $\left(\mathrm{MBR} 1+\mathrm{O}_{3}, \mathrm{~B}\right) \cdot \mathrm{n}=1-4$. 


\section{S2.4 Physical-chemical parameters}

During the 28 days on-site experiment with Potamopyrgus antipodarum standardised physicalchemical parameters $\left(\mathrm{pH}\right.$, conductivity, oxygen saturation, oxygen content, $\mathrm{NO}_{2}-\mathrm{N}, \mathrm{NO}_{3}-\mathrm{N}$, $\mathrm{NH}_{4}-\mathrm{N}$ and total hardness) were periodically $(n=4)$ determined directly in the exposure vessels using test kits as well as in the course of the almost daily $(n=22)$ surveillance of wastewater parameters at the WWTP (COD, DOC, $\mathrm{NO}_{2}-\mathrm{N}, \mathrm{NO}_{3}-\mathrm{N}, \mathrm{NH}_{4}-\mathrm{N}, \mathrm{P}_{\text {total }}$ and $\mathrm{SAC}_{254}$, compare 2.9). Temperature was measured in the tank. Detailed results are provided in Tables S22-S29 and summarised in the following.

The temperature (set: $16.0 \pm 1.0^{\circ} \mathrm{C}$; measured: $16.3 \pm 0.63^{\circ} \mathrm{C}$, range: $14.2-19.6^{\circ} \mathrm{C} ; n=5544$ ) in the tank and the parameters determined in each exposure vessel met the validity criteria according to OECD (2016) regarding the $\mathrm{pH}$ (set: $8.0 \pm 0.5$, should not fall below $\mathrm{pH} 6.5$, measured: $6.69-8.28, \mathrm{n}=44$ ), oxygen content (set: $>6.0 \mathrm{mg} / \mathrm{L}$, measured: $7.30-13.5 \mathrm{mg} / \mathrm{L}$, $n=44$ ) and oxygen saturation (set: $>60.0 \%$, measured: $72.6-137 \%, n=44$ ). The recommended value for the conductivity was also met in the NC and PC (set: $770 \pm 100 \mu \mathrm{S} / \mathrm{cm}$, measured: $700-800 \mu \mathrm{S} / \mathrm{cm}, \mathrm{n}=8$ ). The conductivity in the exposure vessels of the nine tested wastewater treatments was minimal $947 \mu \mathrm{S} / \mathrm{cm}$ and maximal $1425 \mu \mathrm{S} / \mathrm{cm}(\mathrm{n}=36)$. The total hardness was between $4^{\circ} \mathrm{dH}$ and $77^{\circ} \mathrm{dH}(n=8)$ in the $\mathrm{NC}$ and $\mathrm{PC}$ and between $15^{\circ} \mathrm{dH}$ and $20^{\circ} \mathrm{dH}(\mathrm{n}=36)$ in the wastewater treatment groups.

The concentrations of nitrite $\left(\mathrm{NO}_{2}-\mathrm{N}\right)$ ranged between $0.005 \mathrm{mg} / \mathrm{L}$ and $>0.1 \mathrm{mg} / \mathrm{L}$ in all exposure vessels. The concentrations of ammonium $\left(\mathrm{NH}_{4}-\mathrm{N}\right)$ were $<0.05 \mathrm{mg} / \mathrm{L}$ in all treatments, except once (day 24$)$ in MBR2 $(1.5 \mathrm{mg} / \mathrm{L})$. The concentration of nitrate $\left(\mathrm{NO}_{3}-\mathrm{N}\right)$ was maximal $1.0 \mathrm{mg} / \mathrm{L}$ in $\mathrm{NC}$ and $\mathrm{PC}$ and maximal $10 \mathrm{mg} / \mathrm{L}$ in the $\mathrm{BT}, \mathrm{BT}+\mathrm{O}_{3}$ and the postfiltration systems. The MBR1, MBR1+O $\mathrm{O}_{3}$ and MBR2 showed the highest nitrate concentrations between $40 \mathrm{mg} / \mathrm{L}$ and $80 \mathrm{mg} / \mathrm{L}$ at the end of the experiment.

The results of the physical-chemical analysis as part of the regulative WWTP monitoring were in line with those from the test kits regarding the overlapping nitrogen parameters. In both cases $\mathrm{NO}_{2}-\mathrm{N}, \mathrm{NO}_{3}-\mathrm{N}$ and $\mathrm{NH}_{4}-\mathrm{N}$ exhibited typical concentration trends for activated sludge treatments (that include nitrification-denitrification) and for the present AWWT. COD, DOC, $\mathrm{P}_{\text {total }}$ and $\mathrm{SAC}_{254}$ likewise displayed typical concentration tendencies as reported in the scientific literature (further discussed under 4.3).

Table S22: Chemical oxygen demand (COD) in mg/L measured directly in the ten effluents of the reactors on the pilot wastewater treatment plant during 28 days of exposure. PT: after primary treatment, BT: after conventional biological treatment, BT+ $\mathrm{O}_{3}$ : after ozone system 1, GAC: after nonaerated granular activated carbon filter treatment, $\mathrm{GAC}_{\mathrm{a}}$ : after aerated granular activated carbon filter treatment, $\mathrm{BF}$ : after non-aerated biofilter

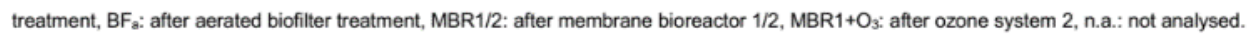

\begin{tabular}{|c|c|c|c|c|c|c|c|c|c|c|}
\hline Time [d] & PT & BT & $\mathrm{BT}+\mathrm{O}_{3}$ & GAC & GAC, & BF & $\mathrm{BF}_{3}$ & MBR1 & $\mathrm{MBR} 1+\mathrm{O}_{3}$ & MBR2 \\
\hline 1 & 123 & 15.0 & 12.5 & n.a. & n.a. & n.a. & n.a. & 12.1 & 10.9 & 22.5 \\
\hline 2 & $311 / 197$ & 17.2 & 16.9 & $<5.00$ & 8.90 & 11.3 & 12.0 & 8.92 & 6.73 & 12.8 \\
\hline 3 & $611 / 312$ & 23.3 & 22.6 & 11.2 & 11.8 & 16.4 & 16.0 & n.a. & n.a. & n.a. \\
\hline 4 & 314 & 23.6 & 20.7 & n.a. & n.a. & n.a. & n.a. & 20.7 & 15.8 & 20.4 \\
\hline 5 & 323 & 25.6 & 21.7 & n.a. & n.a. & n.a. & n.a. & 21.9 & 17.0 & 22.2 \\
\hline 8 & 333 & 29.7 & 24.2 & n.a. & n.a. & n.a. & n.a. & 16.9 & 13.3 & 22.1 \\
\hline 9 & $364 / 633$ & 29.9 & 24.5 & 6.30 & 14.5 & 21.2 & 21.1 & 16.8 & 11.8 & 22.6 \\
\hline 10 & 324 & 29.7 & 25.8 & n.a. & n.a. & n.a. & n.a. & 17.5 & 14.1 & 23.6 \\
\hline 11 & n.a. & 27.7 & 23.6 & n.a. & n.a. & n.a. & n.a. & n.a. & 14.9 & 11.1 \\
\hline 12 & $624 / 314$ & 27.8 & 24.6 & 11.1 & 14.3 & 19.5 & 20.9 & n.a. & n.a. & 29.3 \\
\hline 15 & $664 / 320$ & 26.1 & 22.6 & n.a. & n.a. & n.a. & n.a. & 21.7 & 16.8 & 20.9 \\
\hline 16 & $559 / 291$ & 32.6 & 29.0 & 17.1 & 18.0 & 22.6 & 20.8 & 17.3 & 14.2 & 23.3 \\
\hline 17 & 270 & 28.2 & 23.4 & n.a. & n.a. & n.a. & n.a. & 14.1 & 10.8 & 21.3 \\
\hline 18 & 359 & 29.4 & 24.2 & 12.9 & 13.0 & 19.5 & 20.3 & 15.1 & 11.9 & 22.1 \\
\hline 19 & $282 / 97.9$ & 19.7 & 16.4 & n.a. & n.a. & n.a. & n.a. & 10.2 & 8.66 & 18.0 \\
\hline 22 & 234 & 25.2 & 21.4 & n.a. & n.a. & n.a. & n.a. & 12.2 & 10.2 & 19.2 \\
\hline 23 & $642 / 338$ & 26.3 & 23.1 & 10.9 & $<5.00$ & 17.9 & 18.6 & 14.4 & 10.2 & 19.7 \\
\hline 24 & 250 & 28.0 & 24.4 & n.a. & n.a. & n.a. & n.a. & 14.5 & 12.2 & 21.7 \\
\hline 25 & 397 & 28.1 & 24.1 & 14 & 15.9 & 17.5 & 20.4 & 14.5 & 11.7 & 22.0 \\
\hline 26 & 282 & 30.2 & 26.5 & n.a. & n.a. & n.a. & n.a. & 19.1 & 16.1 & 23.5 \\
\hline 29 & 320 & 30.5 & 25.9 & n.a. & n.a. & n.a. & n.a. & 16.3 & 13.7 & 22.5 \\
\hline 30 & $638 / 349$ & 29.2 & 25.4 & 13.7 & 14.8 & 21.3 & 20.7 & 16.5 & 11.2 & 24.1 \\
\hline
\end{tabular}


Table S23: Dissolved organic carbon (DOC) in $\mathrm{mg} / \mathrm{L}$ measured directly in the ten effluents of the reactors on the pilot wastewater treatment plant during 28 days of exposure. PT: after primary treatment, $\mathrm{BT}$ : after conventional biological treatment, $\mathrm{BT}+\mathrm{O}_{3}$ : after ozone system 1, GAC: after non-aerated granular activated carbon filter treatment, $\mathrm{GAC}_{\mathrm{a}}$ : after aerated granular activated carbon filter treatment, $\mathrm{BF}$ : after non-aerated biofilter treatment, $\mathrm{BF}_{\mathrm{a}}$

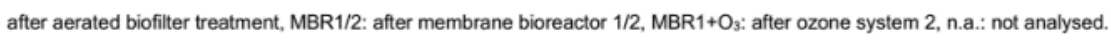

\begin{tabular}{ccccccccccc}
\hline Time [d] & PT & BT & ${\text { BT }+\mathbf{O}_{3}}$ & GAC & GAC & BF & BF & MBR1 & MBR1+O & MBR2 \\
\hline 1 & 39.8 & 6.71 & 6.83 & n.a. & n.a. & n.a. & n.a. & 6.25 & 6.25 & 10.9 \\
2 & 71.0 & 8.89 & 8.22 & 3.01 & 4.76 & 6.68 & 7.41 & 6.41 & 6.07 & 9.43 \\
3 & 101 & 9.85 & 9.42 & 5.41 & 6.14 & 8.13 & 8.07 & n.a. & n.a. & n.a. \\
4 & 96.0 & 9.43 & 9.36 & n.a. & n.a. & n.a. & n.a. & 8.80 & 7.44 & 8.98 \\
5 & 101 & 10.7 & 10.8 & n.a. & n.a. & n.a. & n.a. & 9.23 & 8.45 & 9.76 \\
8 & 101 & 12.0 & 11.6 & n.a. & n.a. & n.a. & n.a. & 7.31 & 6.89 & 9.81 \\
9 & 106 & 11.9 & 11.4 & 4.36 & 6.98 & 9.40 & 9.42 & 8.00 & 6.25 & 9.18 \\
10 & 105 & 12.4 & 11.7 & n.a. & n.a. & n.a. & n.a. & 8.07 & 7.39 & 10.0 \\
11 & n.a. & 12.0 & 11.4 & n.a. & n.a. & n.a. & n.a. & 6.18 & 7.08 & n.a. \\
12 & 98.5 & 11.3 & 11.2 & 5.91 & 7.03 & 8.85 & 9.25 & n.a. & n.a. & 12.7 \\
15 & 96.5 & 10.7 & 10.3 & n.a. & n.a. & n.a. & n.a. & 8.57 & 7.86 & 8.46 \\
16 & 83.5 & 11.4 & 11.6 & 7.42 & 8.29 & 9.8 & 10.5 & 7.16 & 6.54 & 9.12 \\
17 & 82.5 & 11.3 & 16.7 & n.a. & n.a. & n.a. & n.a. & 6.45 & 5.93 & 8.74 \\
18 & 100 & 11.7 & 11.1 & 6.17 & 6.51 & 8.85 & 8.91 & 9.25 & 6.22 & 9.38 \\
19 & 33.9 & 7.80 & 8.05 & n.a. & n.a. & n.a. & n.a. & 5.85 & 4.78 & 7.63 \\
22 & 76.0 & 10.1 & 9.70 & n.a. & n.a. & n.a. & n.a. & 6.14 & 5.54 & 9.02 \\
23 & 98.5 & 10.8 & 10.6 & 5.96 & $<3.00$ & 8.6 & 8.4 & 6.42 & 5.65 & 7.96 \\
24 & 83.5 & 11.0 & 10.8 & n.a. & n.a. & n.a. & n.a. & 6.57 & 8.25 & 8.64 \\
25 & 109 & 11.5 & 11.0 & 6.59 & 6.83 & 9.68 & 8.63 & 6.08 & 5.68 & 8.07 \\
26 & 86.0 & 11.7 & 11.3 & n.a. & n.a. & n.a. & n.a. & 7.72 & 7.21 & 8.81 \\
29 & 102 & 12.8 & 11.7 & n.a. & n.a. & n.a. & n.a. & 9.03 & 6.56 & 9.41 \\
30 & 104 & 12.4 & 11.8 & 6.75 & 7.13 & 9.84 & 9.73 & 6.90 & 6.43 & 8.57 \\
\hline
\end{tabular}

Table S24: Ammonium ( $\mathrm{NH}_{4}-\mathrm{N}$ ) in mg/L measured directly in the ten effluents of the reactors on the pilot wastewater treatment plant during 28 days of exposure. PT: after primary treatment, BT: after conventional biological treatment, BT+O 3 : after ozone system 1, GAC: after non-aerated granular activated carbon filter treatment, $\mathrm{GAC}_{\mathrm{a}}$ : after aerated granular activated carbon filter treatment, $\mathrm{BF}$ : after non-aerated biofilter treatment, $\mathrm{BF}_{\mathrm{a}}$ : after aerated biofilter treatment, MBR1/2: after membrane bioreactor $1 / 2, M B R 1+O_{3}$ : after ozone system 2, n.a.: not analysed.

\begin{tabular}{|c|c|c|c|c|c|c|c|c|c|c|}
\hline Time [d] & PT & BT & $\mathrm{BT}+\mathrm{O}_{3}$ & GAC & GAC, & BF & $\mathrm{BF}_{\mathrm{a}}$ & MBR1 & MBR1+O & MBR2 \\
\hline 1 & 23.8 & n.a. & n.a. & n.a. & n.a. & n.a. & n.a. & $<0.015$ & $<0.015$ & 0.019 \\
\hline 2 & 64.5 & n.a. & n.a. & n.a. & n.a. & n.a. & n.a. & 0.045 & 0.035 & 0.048 \\
\hline 3 & 100 & 0.306 & 0.321 & 0.032 & $<0.015$ & 0.23 & $<0.015$ & n.a. & n.a. & n.a. \\
\hline 4 & 100 & n.a. & n.a. & n.a. & n.a. & n.a. & n.a. & 0.184 & 0.133 & 0.139 \\
\hline 5 & 98.5 & n.a. & n.a. & n.a. & n.a. & n.a. & n.a. & 0.352 & 0.226 & 1.33 \\
\hline 8 & 66.0 & n.a. & n.a. & n.a. & n.a. & n.a. & n.a. & 0.018 & 0.028 & 0.205 \\
\hline 9 & 96.5 & 0.176 & 0.218 & 0.026 & $<0.015$ & 0.212 & 0.018 & 0.058 & 0.047 & 0.117 \\
\hline 10 & 102 & n.a. & n.a. & n.a. & n.a. & n.a. & n.a. & 0.064 & 0.062 & 1.24 \\
\hline 11 & n.a. & n.a. & n.a. & n.a. & n.a. & n.a. & n.a. & n.a. & n.a. & n.a. \\
\hline 12 & 96.0 & n.a. & n.a. & n.a. & n.a. & n.a. & n.a. & n.a. & n.a. & 0.339 \\
\hline 15 & 67.0 & n.a. & n.a. & n.a. & n.a. & n.a. & n.a. & 10.8 & 7.39 & 0.051 \\
\hline 16 & 55.0 & n.a. & n.a. & n.a. & n.a. & n.a. & n.a. & 0.046 & 0.028 & 0.042 \\
\hline 17 & 100 & n.a. & n.a. & n.a. & n.a. & n.a. & n.a. & 0.043 & 0.042 & 1.32 \\
\hline 18 & n.a. & 0.489 & 0.518 & 0.122 & $<0.015$ & 0.361 & 0.017 & n.a. & n.a. & n.a. \\
\hline 19 & 28.0 & n.a. & n.a. & n.a. & n.a. & n.a. & n.a. & $<0.015$ & 0.022 & $<0.015$ \\
\hline 22 & 55.0 & n.a. & n.a. & n.a. & n.a. & n.a. & n.a. & 0.041 & 0.047 & 0.025 \\
\hline 23 & n.a. & n.a. & n.a. & n.a. & n.a. & n.a. & n.a. & n.a. & n.a. & n.a. \\
\hline 24 & 86.5 & n.a. & n.a. & n.a. & n.a. & n.a. & n.a. & 0.106 & 0.111 & 18.9 \\
\hline 25 & n.a. & 0.115 & 0.144 & 0.028 & $<0.015$ & 0.087 & 0.226 & n.a. & n.a. & 16.2 \\
\hline 26 & 97.0 & n.a. & n.a. & n.a. & n.a. & n.a. & n.a. & 6.12 & 5.11 & 26.7 \\
\hline 29 & 90.5 & n.a. & n.a. & n.a. & n.a. & n.a. & n.a. & 4.02 & 3.88 & 39.9 \\
\hline 30 & n.a. & 0.139 & 0.186 & 0.028 & $<0.015$ & 0.088 & 0.019 & n.a. & n.a. & 39.7 \\
\hline
\end{tabular}

Table S25: Nitrite $\left(\mathrm{NO}_{2}-\mathrm{N}\right)$ in $\mathrm{mg} / \mathrm{L}$ measured directly in the ten effluents of the reactors on the pilot wastewater treatment plant during 28 days of exposure. PT: after primary treatment, BT: after conventional biological treatment, $\mathrm{BT}+\mathrm{O}_{3}$ : after ozone system 1, GAC: after non-aerated granular activated carbon filter treatment, $\mathrm{GAC}_{\mathrm{a}}$ : after aerated granular activated carbon filter treatment, $\mathrm{BF}$ : after non-aerated biofilter treatment, $\mathrm{BF}_{\mathrm{a}}$ : after aerated biofilter treatment, MBR1/2: after membrane bioreactor $1 / 2, M B R 1+\mathrm{O}_{3}$ : after ozone system 2, n.a.: not analysed.

\begin{tabular}{|c|c|c|c|c|c|c|c|c|c|c|}
\hline Time [d] & PT & BT & $\mathrm{BT}+\mathrm{O}_{3}$ & GAC & GAC $_{a}$ & BF & $\mathrm{BF}_{\mathrm{a}}$ & MBR1 & ${\mathrm{MBR} 1+\mathrm{O}_{3}}$ & MBR2 \\
\hline 1 & 0.084 & n.a. & n.a. & n.a. & n.a. & n.a. & n.a. & $<0.015$ & $<0.015$ & 0.021 \\
\hline 2 & 0.024 & 0.345 & $<0.015$ & $<0.015$ & $<0.015$ & 0.186 & $<0.015$ & 0.062 & $<0.015$ & 0.051 \\
\hline 3 & 0.031 & 0.336 & $<0.015$ & 0.081 & $<0.015$ & 0.182 & $<0.015$ & n.a. & n.a. & n.a. \\
\hline 4 & 0.035 & n.a. & n.a. & n.a. & n.a. & n.a. & n.a. & 0.403 & 0.020 & 0.138 \\
\hline 5 & 0.026 & n.a. & n.a. & n.a. & n.a. & n.a. & n.a. & 0.999 & 0.056 & 0.279 \\
\hline 8 & 0.032 & n.a. & n.a. & n.a. & n.a. & n.a. & n.a. & 0.076 & $<0.015$ & 0.087 \\
\hline 9 & 0.045 & 0.237 & $<0.015$ & $<0.015$ & $<0.015$ & 0.162 & $<0.015$ & 0.132 & $<0.015$ & 0.220 \\
\hline 10 & 0.027 & n.a. & n.a. & n.a. & n.a. & n.a. & n.a. & 0.328 & $<0.015$ & 0.600 \\
\hline 11 & n.a. & n.a. & n.a. & n.a. & n.a. & n.a. & n.a. & 0.07 & $<0.015$ & n.a. \\
\hline 12 & 0.029 & n.a. & n.a. & n.a. & n.a. & n.a. & n.a. & n.a. & n.a. & 0.406 \\
\hline 15 & 0.032 & n.a. & n.a. & n.a. & n.a. & n.a. & n.a. & 0.342 & 0.049 & 0.085 \\
\hline 16 & 0.027 & n.a. & n.a. & n.a. & n.a. & n.a. & n.a. & 0.027 & $<0.015$ & 0.080 \\
\hline 17 & 0.031 & n.a. & n.a. & n.a. & n.a. & n.a. & n.a. & 0.115 & $<0.015$ & 0.238 \\
\hline 18 & n.a. & 0.538 & $<0.015$ & 0.248 & $<0.015$ & 0.318 & $<0.015$ & n.a. & n.a. & n.a. \\
\hline 19 & 0.157 & n.a. & n.a. & n.a. & n.a. & n.a. & n.a. & 0.024 & $<0.015$ & 0.017 \\
\hline 22 & 0.026 & n.a. & n.a. & n.a. & n.a. & n.a. & n.a. & 0.052 & $<0.015$ & 0.039 \\
\hline 23 & n.a. & n.a. & n.a. & n.a. & n.a. & n.a. & n.a. & n.a. & n.a. & n.a. \\
\hline 24 & 0.28 & n.a. & n.a. & n.a. & n.a. & n.a. & n.a. & 0.087 & $<0.015$ & 0.111 \\
\hline 25 & n.a. & 0.238 & $<0.015$ & 0.032 & $<0.015$ & 0.183 & $<0.015$ & n.a. & n.a. & n.a. \\
\hline 26 & 0.023 & n.a. & n.a. & n.a. & n.a. & n.a. & n.a. & 0.013 & 0.023 & 0.060 \\
\hline 29 & 0.03 & n.a. & n.a. & n.a. & n.a. & n.a. & n.a. & 0.134 & 0.039 & 0.061 \\
\hline 30 & n.a. & 0.265 & $<0.015$ & 0.016 & $<0.015$ & 0.234 & $<0.015$ & n.a. & n.a. & n.a. \\
\hline
\end{tabular}


Table S26: Nitrate $\left(\mathrm{NO}_{3}-\mathrm{N}\right)$ in $\mathrm{mg} / \mathrm{L}$ measured directly in the ten effluents of the reactors on the pilot wastewater treatment plant during 28 days of exposure. PT: after primary treatment, BT: after conventional biological treatment, $\mathrm{BT}+\mathrm{O}_{3}$ : after ozone system 1, GAC: after non-aerated granular activated carbon filter treatment, $\mathrm{GAC}_{\mathrm{a}}$ : after aerated granular activated carbon filter treatment, $\mathrm{BF}$ : after non-aerated biofilter treatment, $\mathrm{BF}_{\mathrm{a}}$ : after aerated biofilter treatment, MBR1/2: after membrane bioreactor $1 / 2, M B R 1+O_{3}$ : after ozone system 2, n.a.: not analysed.

\begin{tabular}{ccccccccccc}
\hline Time [d] & PT & BT & BT+O & GAC & GAC. & BF & BF. & MBR1 & MBR1+O & MBR2 \\
\hline 1 & $<0.230$ & n.a. & n.a. & n.a. & n.a. & n.a. & n.a. & 29.8 & 29.6 & 29.2 \\
2 & 0.295 & n.a. & n.a. & n.a. & n.a. & n.a. & n.a. & 36.6 & 36.2 & 32.8 \\
3 & 0.258 & 1.65 & 2.28 & 2.90 & 3.03 & 2.41 & 2.76 & n.a. & n.a. & n.a. \\
4 & 0.441 & n.a. & n.a. & n.a. & n.a. & n.a. & n.a. & 58.6 & 59.0 & 56.8 \\
5 & 0.332 & n.a. & n.a. & n.a. & n.a. & n.a. & n.a. & 65.4 & 65.6 & 68.0 \\
8 & 0.348 & n.a. & n.a. & n.a. & n.a. & n.a. & n.a. & 49.0 & 50.0 & 66.2 \\
9 & 0.400 & 1.64 & 1.99 & 2.15 & 2.38 & 1.91 & 2.14 & 53.2 & 53.0 & 60.2 \\
10 & 0.367 & n.a. & n.a. & n.a. & n.a. & n.a. & n.a. & 68.0 & 69.0 & 67.8 \\
11 & n.a. & n.a. & n.a. & n.a. & n.a. & n.a. & n.a. & 71.2 & 70.6 & n.a. \\
12 & 0.284 & n.a. & n.a. & n.a. & n.a. & n.a. & n.a. & n.a. & n.a. & 63.6 \\
15 & 0.293 & n.a. & n.a. & n.a. & n.a. & n.a. & n.a. & 42.4 & 51.5 & 50.6 \\
16 & 0.321 & n.a. & n.a. & n.a. & n.a. & n.a. & n.a. & 47.2 & 48.4 & 47.6 \\
17 & 0.421 & n.a. & n.a. & n.a. & n.a. & n.a. & n.a. & 36.8 & 35.8 & 32.2 \\
18 & n.a. & 2.70 & 3.67 & 3.88 & 4.22 & 3.37 & 4.06 & n.a. & n.a. & n.a. \\
19 & 0.232 & n.a. & n.a. & n.a. & n.a. & n.a. & n.a. & 40.4 & 40.6 & 32.4 \\
22 & 0.320 & n.a. & n.a. & n.a. & n.a. & n.a. & n.a. & 37.2 & 37.6 & 27.6 \\
23 & n.a. & n.a. & n.a. & n.a. & n.a. & n.a. & n.a. & n.a. & n.a. & n.a. \\
24 & 0.412 & n.a. & n.a. & n.a. & n.a. & n.a. & n.a. & 50.2 & 51.2 & 24.0 \\
25 & n.a. & 1.63 & 1.99 & 2.42 & 2.55 & 1.96 & 2.21 & n.a. & n.a. & n.a. \\
26 & 0.288 & n.a. & n.a. & n.a. & n.a. & n.a. & n.a. & 27.2 & 29.0 & 7.56 \\
29 & 0.340 & n.a. & n.a. & n.a. & n.a. & n.a. & n.a. & 25.2 & 25.6 & 6.99 \\
30 & n.a. & 1.54 & 2.35 & 2.49 & 2.57 & 2.11 & 2.34 & n.a. & n.a. & n.a. \\
\hline
\end{tabular}

Table S27: Total phosphorus ( $\left.\mathrm{P}_{\mathrm{waxa}}\right)$ in $\mathrm{mg} / \mathrm{L}$ measured directly in the ten effluents of the reactors on the pilot wastewater treatment plant during 28 days of exposure. PT: after primary treatment, BT: after conventional biological treatment, $\mathrm{BT}+\mathrm{O}_{3}$ : after ozone system 1, GAC: after non-aerated granular activated carbon filter treatment, $\mathrm{GAC}_{\mathrm{a}}$ : after aerated granular activated carbon filter treatment, $\mathrm{BF}$ : after non-aerated biofilter treatment, $\mathrm{BF}_{\mathrm{a}}$ : after aerated biofilter treatment, MBR1/2: after membrane bioreactor 1/2, MBR1+O : after ozone system 2, n.a.: not analysed.

\begin{tabular}{|c|c|c|c|c|c|c|c|c|c|c|}
\hline Time [d] & PT & $\overline{B T}$ & $\mathrm{BT}+\mathrm{O}_{3}$ & GAC & GAC, & BF & $\mathrm{BF}_{\mathrm{s}}$ & MBR1 & $\mathrm{MBR} 1+\mathrm{O}_{3}$ & MBR2 \\
\hline 1 & n.a. & n.a. & n.a. & n.a. & n.a. & n.a. & n.a. & n.a. & n.a. & n.a. \\
\hline 2 & 8.29 & n.a. & n.a. & n.a. & n.a. & n.a. & n.a. & 6.04 & 6.07 & 6.48 \\
\hline 3 & n.a. & 0.795 & 0.807 & 0.735 & 0.825 & 0.766 & 0.788 & n.a. & n.a. & n.a. \\
\hline 4 & n.a. & n.a. & n.a. & n.a. & n.a. & n.a. & n.a. & n.a. & n.a. & n.a. \\
\hline 5 & n.a. & n.a. & n.a. & n.a. & n.a. & n.a. & n.a. & n.a. & n.a. & n.a. \\
\hline 8 & n.a. & n.a. & n.a. & n.a. & n.a. & n.a. & n.a. & n.a. & n.a. & n.a. \\
\hline 9 & 11.9 & 0.432 & 0.437 & 0.260 & 0.483 & 0.432 & 0.464 & 5.90 & 5.95 & 7.10 \\
\hline 10 & n.a. & n.a. & n.a. & n.a. & n.a. & n.a. & n.a. & n.a. & n.a. & n.a. \\
\hline 11 & n.a. & n.a. & n.a. & n.a. & n.a. & n.a. & n.a. & n.a. & n.a. & n.a. \\
\hline 12 & n.a. & n.a. & n.a. & n.a. & n.a. & n.a. & n.a. & n.a. & n.a. & n.a. \\
\hline 15 & n.a. & n.a. & n.a. & n.a. & n.a. & n.a. & n.a. & n.a. & n.a. & n.a. \\
\hline 16 & n.a. & n.a. & n.a. & n.a. & n.a. & n.a. & n.a. & n.a. & n.a. & n.a. \\
\hline 17 & n.a. & n.a. & n.a. & n.a. & n.a. & n.a. & n.a. & n.a. & n.a. & n.a. \\
\hline 18 & n.a. & 0.635 & 0.619 & 0.456 & 0.508 & 0.580 & 0.558 & n.a. & n.a. & n.a. \\
\hline 19 & n.a. & n.a. & n.a. & n.a. & n.a. & n.a. & n.a. & n.a. & n.a. & n.a. \\
\hline 22 & 8.76 & n.a. & n.a. & n.a. & n.a. & n.a. & n.a. & 6.64 & 6.51 & 6.98 \\
\hline 23 & n.a. & n.a. & n.a. & n.a. & n.a. & n.a. & n.a. & n.a. & n.a. & n.a. \\
\hline 24 & n.a. & n.a. & n.a. & n.a. & n.a. & n.a. & n.a. & n.a. & n.a. & n.a. \\
\hline 25 & n.a. & 0.485 & 0.476 & 0.557 & 0.496 & 0.438 & 0.576 & n.a. & n.a. & n.a. \\
\hline 26 & n.a. & n.a. & n.a. & n.a. & n.a. & n.a. & n.a. & n.a. & n.a. & n.a. \\
\hline 29 & 12.2 & n.a. & n.a. & n.a. & n.a. & n.a. & n.a. & 7.14 & 7.16 & 7.44 \\
\hline 30 & n.a. & 0.544 & 0.576 & 0.638 & 0.58 & 0.610 & 0.571 & n.a. & n.a. & n.a. \\
\hline
\end{tabular}

Table S28: Spectral absorption coefficient at $254 \mathrm{~nm}\left(\mathrm{SAC}_{254}\right)$ in $\mathrm{cm}^{-1}$ measured directly in the ten effluents of the reactors on the pilot wastewater treatment plant during 28 days of exposure. $\mathrm{PT}$ : after primary treatment, $\mathrm{BT}$ : after conventional biological treatment, $\mathrm{BT}+\mathrm{O}_{3}$ : after ozone system 1, GAC after non-aerated granular activated carbon filter treatment, $\mathrm{GAC}_{\mathrm{a}}$ : after aerated granular activated carbon filter treatment, $\mathrm{BF}$ : after non-aerated biofilter treatment, $B F_{\mathrm{a}}$ : after aerated biofilter treatment, $M B R 1 / 2$ : after membrane bioreactor $1 / 2, M B R 1+\mathrm{O}_{3}$ : after ozone system 2, n.a.: not analysed.

\begin{tabular}{ccccccccccc}
\hline Time [d] & $\mathbf{P T}$ & $\mathbf{B T}$ & $\mathbf{B T}+\mathbf{O}_{3}$ & $\mathbf{G A C}$ & $\mathbf{G A C}$. & $\mathbf{B F}$ & $\mathbf{B F}_{\mathbf{a}}$ & $\mathbf{M B R 1}$ & MBR1+O $_{3}$ & MBR2 \\
\hline 1 & 0.305 & 0.112 & 0.042 & n.a. & n.a. & n.a. & n.a. & 0.126 & 0.093 & 0.217 \\
2 & 0.474 & 0.120 & 0.049 & 0.014 & 0.027 & 0.041 & 0.041 & 0.108 & 0.055 & 0.190 \\
3 & 0.732 & 0.144 & 0.059 & 0.023 & 0.030 & 0.050 & 0.050 & n.a. & n.a. & n.a. \\
4 & 0.706 & 0.162 & 0.068 & n.a. & n.a. & n.a. & n.a. & 0.157 & 0.104 & 0.218 \\
5 & 0.715 & 0.181 & 0.067 & n.a. & n.a. & n.a. & n.a. & 0.170 & 0.110 & 0.225 \\
8 & 0.745 & 0.186 & 0.071 & n.a. & n.a. & n.a. & n.a. & 0.139 & 0.062 & 0.223 \\
9 & 0.796 & 0.193 & 0.082 & 0.022 & 0.037 & 0.069 & 0.070 & 0.131 & 0.017 & 0.224 \\
10 & 0.704 & 0.200 & 0.078 & n.a. & n.a. & n.a. & n.a. & 0.149 & 0.083 & 0.242 \\
11 & n.a. & 0.194 & 0.079 & n.a. & n.a. & n.a. & n.a. & 0.131 & 0.057 & n.a. \\
12 & 0.723 & 0.195 & 0.076 & 0.033 & 0.040 & 0.072 & 0.071 & n.a. & n.a. & 0.292 \\
15 & 0.732 & 0.187 & 0.074 & n.a. & n.a. & n.a. & n.a. & 0.177 & 0.108 & 0.217 \\
16 & 0.609 & 0.190 & 0.079 & 0.038 & 0.038 & 0.065 & 0.066 & 0.125 & 0.058 & 0.214 \\
17 & 0.623 & 0.195 & 0.080 & n.a. & n.a. & n.a. & n.a. & 0.121 & 0.058 & 0.215 \\
18 & 0.935 & 0.200 & 0.085 & 0.038 & 0.040 & 0.071 & 0.074 & 0.126 & 0.055 & 0.226 \\
19 & 0.283 & 0.140 & 0.049 & n.a. & n.a. & n.a. & n.a. & 0.090 & 0.044 & 0.181 \\
22 & 0.586 & 0.171 & 0.071 & n.a. & n.a. & n.a. & n.a. & 0.111 & 0.055 & 0.189 \\
23 & 0.769 & 0.179 & 0.067 & 0.030 & 0.012 & 0.055 & 0.058 & 0.116 & 0.053 & 0.201 \\
24 & 0.589 & 0.190 & 0.077 & n.a. & n.a. & n.a. & n.a. & 0.127 & 0.062 & 0.210 \\
25 & 1.043 & 0.221 & 0.106 & 0.069 & 0.070 & 0.095 & 0.097 & 0.162 & 0.104 & 0.250 \\
26 & 0.676 & 0.205 & 0.080 & n.a. & n.a. & n.a. & n.a. & 0.147 & 0.082 & 0.228 \\
29 & 0.678 & 0.212 & 0.082 & n.a. & n.a. & n.a. & n.a. & 0.132 & 0.074 & 0.219 \\
30 & 0.843 & 0.216 & 0.088 & 0.045 & 0.048 & 0.076 & 0.078 & 0.125 & 0.057 & 0.229 \\
\hline
\end{tabular}


Table S29: Physical-chemical parameters of the negative control (NC), the positive control (PC), the conventional biological treatment (BT) and the eight advanced treatment technologies measured directly in the exposure vessels during 28 days of exposure. BT+ $\mathrm{O}_{3}$ : after ozone system 1, GAC: after non-aerated granular activated carbon filter treatment, GAC : after aerated granular activated carbon filter treatment, BF: after non-aerated biofilter treatment, $\mathrm{BF}_{\mathrm{a}}$ : after aerated biofilter treatment, $\mathrm{MBR} 1 / 2$ : after membrane bioreactor $1 / 2, \mathrm{MBR}_{1}+\mathrm{O}_{3}$ : after ozone system 2, $\mathrm{NO}_{2}-\mathrm{N}:$ nitrite, $\mathrm{NO}_{3}-\mathrm{N}$ : nitrate, $\mathrm{NH}_{4}-\mathrm{N}$ : ammonium.

\begin{tabular}{|c|c|c|c|c|c|c|c|}
\hline & $\mathrm{pH}$ & conductivity $[\mu \mathrm{S} / \mathrm{cm}]$ & oxygen [\%]/[mg/L] & hardness [ $\left.{ }^{\circ} \mathrm{dH}\right]$ & $\mathrm{NO}_{2}-\mathrm{N}[\mathrm{mg} / \mathrm{L}]$ & $\mathrm{NO}_{3}-\mathrm{N}[\mathrm{mg} / \mathrm{L}]$ & $\mathrm{NH}_{4}-\mathrm{N}[\mathrm{mg} / \mathrm{L}]$ \\
\hline \multicolumn{8}{|l|}{ day 4} \\
\hline$\overline{\mathrm{NC}}$ & 7.88 & 782 & $94.0 / 9.55$ & 6 & 0.005 & $<0.5$ & $<0.05$ \\
\hline PC & 7.85 & 783 & $92.1 / 9.28$ & 6 & 0.0 & $<0.5$ & $<0.05$ \\
\hline $\mathrm{BT}+\mathrm{O}_{3}$ & 7.00 & 957 & $129 / 13.0$ & 16 & $>0.1$ & $1-5$ & $<0.05$ \\
\hline GAC & 7.30 & 965 & $112 / 11.3$ & 15 & $>0.1$ & $1-5$ & $<0.05$ \\
\hline $\mathrm{GAC}_{\mathrm{a}}$ & 7.58 & 970 & $93.3 / 9.41$ & 16 & 0.03 & $0.5-1$ & $<0.05$ \\
\hline MBR1 & 6.70 & 1280 & $92.7 / 9.25$ & 19 & $>0.1$ & 40 & $<0.05$ \\
\hline $\mathrm{MBR} 1+\mathrm{O}_{3}$ & 6.74 & 1284 & $105 / 10.5$ & 19 & $>0.1$ & 20 & $<0.05$ \\
\hline MBR2 & 6.69 & 1251 & $91.0 / 9.13$ & 19 & $>0.1$ & 20 & $<0.05$ \\
\hline \multicolumn{8}{|l|}{ day 10} \\
\hline $\mathrm{NC}$ & 8.11 & 800 & $92.7 / 9.40$ & 6 & 0.005 & 0.0 & $<0.05$ \\
\hline $\mathrm{GAC}_{\mathrm{a}}$ & 7.95 & 1212 & $94.4 / 9.28$ & 19 & 0.02 & $0-10$ & $<0.05$ \\
\hline $\mathrm{BF}$ & 7.45 & 1223 & $112 / 11.0$ & 19 & $>0.1$ & $0-10$ & $<0.05$ \\
\hline $\mathrm{BF}_{\mathrm{a}}$ & 7.86 & 1218 & $93.6 / 9.21$ & 19 & 0.02 & $0-10$ & $<0.05$ \\
\hline MBR1 & 7.40 & 1407 & $91.8 / 9.01$ & 20 & $>0.1$ & 50 & - \\
\hline $\mathrm{MBR} 1+\mathrm{O}_{3}$ & 7.26 & 1405 & $121 / 11.9$ & 20 & 0.06 & 50 & - \\
\hline MBR2 & 6.93 & 1425 & $77.9 / 7.65$ & 20 & 0.06 & 50 & - \\
\hline \multicolumn{8}{|l|}{ day 17} \\
\hline $\mathrm{NC}$ & 8.28 & 763 & $90.2 / 8.88$ & 5 & $0.012-0.02$ & $<0.5$ & $<0.05$ \\
\hline PC & 8.0 & 761 & $83.1 / 8.2$ & 4 & 0.012 & $<0.5$ & $<0.05$ \\
\hline BT & 7.81 & 1012 & $87.4 / 8.62$ & 18 & $>0.1$ & 5 & $<0.05$ \\
\hline $\mathrm{BT}+\mathrm{O}_{3}$ & 7.80 & 1007 & $97.7 / 9.67$ & 18 & $>0.1$ & 5 & $<0.05$ \\
\hline GAC & 7.78 & 1003 & $103 / 10.2$ & 18 & $>0.1$ & 5 & $<0.05$ \\
\hline$\overline{N C}$ & 8.14 & 701 & $92.1 / 9.11$ & 4 & 0.012 & $<0.5$ & $<0.05$ \\
\hline $\mathrm{PC}$ & 7.90 & 700 & $83.4 / 8.22$ & 5 & 0.012 & 1.0 & $<0.05$ \\
\hline BT & 7.70 & 947 & $83.0 / 8.22$ & 16 & 0.08 & $1-5$ & $<0.05$ \\
\hline $\mathrm{BT}+\mathrm{O}_{3}$ & 7.63 & 975 & $97.4 / 9.63$ & 16 & $>0.1$ & $1-5$ & $<0.05$ \\
\hline GAC & 7.78 & 976 & $97.9 / 9.65$ & 16 & 0.1 & $1-5$ & $<0.05$ \\
\hline $\mathrm{GAC}_{\mathrm{a}}$ & 7.90 & 970 & $93.2 / 9.19$ & 16 & 0.05 & $1-5$ & $<0.05$ \\
\hline $\mathrm{BF}$ & 7.85 & 974 & $100 / 9.85$ & 16 & $>0.1$ & $1-5$ & $<0.05$ \\
\hline $\mathrm{BF}_{\mathrm{a}}$ & 7.96 & 976 & $92.9 / 9.18$ & 16 & 0.04 & $1-5$ & $<0.05$ \\
\hline MBR1 & 7.70 & 1141 & $91.0 / 9.07$ & 18 & $>0.1$ & $40-80$ & $<0.05$ \\
\hline $\mathrm{MBR} 1+\mathrm{O}_{3}$ & 7.56 & 1147 & $95.3 / 9.42$ & 17 & $>0.1$ & $40-80$ & $<0.05$ \\
\hline MBR2 & 7.57 & 1253 & $83.2 / 8.24$ & 17 & $>0.1$ & 40 & 1.5 \\
\hline
\end{tabular}




\section{A.4 Zusammenfassung (German summary)}

Sauberes Wasser ist eine grundlegende Voraussetzung unseres Lebens. Abwasser-bürtige Emissionen in Form von anthropogenen Mikroschadstoffen gefährden die Resource Wasser und die Integrität des Ökosystems auf verschiedenen Skalen (z.B. Link et al. 2017, Malaj et al. 2014, Pal et al. 2010, Stalter et al. 2013). Bestimmte Kontaminanten und deren Transformationsprodukte verteilen sich weitläufig im Wasserkreislauf und werden kontinuierlich emittiert. Bestimmte Stoffe gelangen so in geringen Konzentrationen bereits ins Trinkwasser (Benotti et al. 2009). Die ökotoxikologische Untersuchung und das Umweltmonitoring von (Mikro-)Schadstoffen ist somit eine essentielle Aufgabe, die in diversen Wasser- und Abwasserdirektiven verankert wurde (z.B. EC 1991, 1998, 2008, US EPA 1972, 2002). Unter anderem widmen sich dieser Aufgabe interdisziplinäre Forschungsprojekte und soziopolitische Plattformen zur Reduktion von Mikroschadstoffen und für ein nachhaltiges Abwassermanagement (z.B. Rizzo 2019). Kläranlagen sind Haupteinleiter für aquatische (Mikro-)Schadstoffe (EEA 2012). In den letzten Jahrzehnten wurden Kläranlagen optimiert und an vielen Standorten um Prozesse wie die Tertiärbehandlung erweitert (Prasse et al. 2015, Reemtsma et al. 2016). Nichtsdestoweniger führt die konventionelle biologische Abwasserbehandlung nur bei einem Bruchteil der bekannten und bedenklichen Substanzen zu einer vollständigen Mineralisierung. Ein Grund hierfür kann eine geringe biologische Abbaubarkeit bestimmter synthetischer Verbindungen sein oder auch eine mangelnde Adsorption an Klärschlammpartikel.

Die wachsende Weltbevölkerung und Verstädterung erhöht die Gesamtmengen des zu behandelnden Abwassers. Ferner werden dank verbesserter Analytik fortwährend neue Mikroschadstoffe nachgewiesen (z.B. Rockström et al. 2009, Stamm et al. 2016, Philip et al. 2011). Gegenwärtig wird daher das Potenzial verschiedener Technologien der weiterführenden Abwasserbehandlung untersucht, um eine zusätzliche Reduktion von Mikroschadstoffen und anderer Abwasserbürtiger Emissionenen zu erreichen (Tchobanoglous and Burton 1991). Die vorliegende Arbeit war in das Forschungsprojekt TransRisk eingebettet, welches sich mit der "Charakterisierung, Kommunikation und Minimierung von Risiken durch neuartige Schadstoffe und Krankheitserreger im Wasserkreislauf" beschäftigt (www.transrisk-projekt.de). Die nachfolgenden ökotoxikologischen 
Herausforderungen, verbunden mit anthropogenen Mikroschadstoffen und den weiterführenden Abwasserbehandlungstechnologien Ozonung und Ozonung gefolgt von körniger Aktivkohle- und Bio-Filtration (Pilot-Maßstab), werden darin detailiert untersucht.

Die Vorbereitung von (Ab-)Wasserproben ist von zentraler Bedeutung für die akkurate und repräsentative ökotoxikologische Bewertung. In der vorliegenden Arbeit wurde untersucht, welche Auswirkung routinemäßig angewandte Vorbereitungstechniken - Ansäuerung $(\mathrm{pH} 2)$, Filtration (1 $\mu \mathrm{m}$ Porengröße) und Festphasenextraktion (SPE) - auf den Nachweis umweltrelevanter endokriner Aktivitäten, Mutagenität, Genotoxizität und Zytotoxizität haben. In früheren Studien zeigte sich, dass diese Methoden in der Lage sind, die Konzentration bioaktiver Verbindungen zu verändern und/oder den Nachweis der Toxizität in (Ab)Wasserproben signifikant zu beeinflussen (z.B. Baker und Kasprzyk-Hordern 2011, Maruya et al. 2016, Neale et al. 2018, Vanderford et al. 2011). Die vorliegende Untersuchung konzentrierte sich auf unbehandeltes, biologisch-behandeltes, ozoniertes und Krankenhaus-Abwasser sowie Grundwasser.

Die Probenansäuerung, die im Allgemeinen zur Inaktivierung von Mikroorganismen angewendet wird, schien zur Stabilisierung der untersuchten In-vitro-Effekte ungeeignet. Letztere waren nach 24 Stunden Lagerung in den meisten angesäuerten Proben im Vergleich zu ihren neutralen Äquivalenten signifikant verändert. Es wurde kein Zusammenhang mit den Aktivitäts-/Toxizitäts-Leveln oder den Sensitivitäten der Bioassays beobachtet. Effekte wie die säurekatalysierte Hydrolyse (Prasse et al. 2015) oder ein mikrobieller Abbau in neutralen Proben (Giebner et al. 2018) konnten anhand der vorliegenden Daten jedoch nicht ausgeschlossen werden. In Folgeexperimenten soll nun geklärt werden, welcher Probentyp (angesäuert/neutral) einer Toxizität im Umweltkontext am Nächsten kommt.

Die Probenfiltration ist für etablierte Vorteile, wie die Reduktion von Schwebstoffen, Mikroorganismen oder des Verstopfens von SPE-Kartuschen, sowie die Stabilisierung bestimmter bioaktiver Verbindungen bekannt (Baker und KasprzykHordern 2011, Gehrmann et al. 2016, Janex-Habibi et al. 2009). In der vorliegenden Arbeit wurden daher mögliche Auswirkungen der Filtration (Glasfaserfilter mit einer 
Porengröße von $1 \mu \mathrm{m}$ ) verglichen, wobei die meisten In-vitro-Effekte unverändert blieben. Mehrfach zeigten gefilterte Proben jedoch auch signifikante Aktivitätsverluste an, welche Schwebstoff-gebundenen Verbindungen zugeschrieben wurden. Ferner wurden in bestimmten Fällen höhere endokrine Aktivitäten im Filtrat nachgewiesen, was ein verschobenes Verhältnis von Agonisten und Antagonisten als Ursache haben könnte (Ihara et al. 2014, Rao et al. 2014).

Derzeitige SPE-Methoden sind vor allem für die chemische Analytik ausgelegt (z.B. Escher et al. 2005, Prasse et al. 2015), während der Anreicherung der Toxizität weit weniger Aufmerksamkeit gewidmet wurde (Bistan et al. 2012, Stalter et al. 2016, Wagner und Oehlmann 2010, Neale et al. 2018). Dies ist jedoch entscheidend für die akkurate und repräsentative Bewertung und das Monitoring von Mikroschadstoffen. Da letztere meist in niedrigen Umweltkonzentrationen auftreten, ist eine Probenanreicherung unter anderem erforderlich, um Bestimmungsgrenzen von Bioassays zu überwinden. Darüber hinaus führen SPE-Methoden zu einem Ausschluss von Verunreinigungen aus Umweltmatrizen, was das Risiko experimenteller Artefakte weiter reduziert (Macova et al. 2010, Neale et al. 2015, Prasse et al. 2015). Die vorliegende Optimierungsstudie untersuchte drei SPESorbentien (Oasis HLB, Supelco ENVI-Carb+, Telos C18/ENV) und zwei pH-Werte zur Probenextraktion ( $\mathrm{pH} 7$ und 2,5). Für die meisten Endpunkte lieferte der Telos C18/ENV Sorbent, gefolgt vom Oasis HLB Sorbent, die besten Ergebnisse. Einige Ausnahmen wurden ebenfalls deutlich, wie der Supelco ENVI-Carb+ Sorbent zum Extrahieren von ozoniertem Abwasser ( $\mathrm{pH} 7$ ). Die höchste Zytotoxizität, welche in der Regel die Gesamt-Schadstoffbelastung einer Probe widerspiegelt, wurde bei einem Proben-pH von 7 extrahiert. Allerdings erleichterte ein $\mathrm{pH}$ von 2,5 den Nachweis der meisten endokrinen Aktivitäten, wodurch eine Probenverdünnung überflüssig wird. Die optimierte SPE-Methode wurde anschließend in einem Bewertungskonzept zur Abwasserqualität eingesetzt (Ternes et al. 2017).

Das Umweltmonitoring von Mikroschadstoffen und möglicher Effekte sind eine wesentliche ökotoxikologische Aufgabe. In-vitro-Bioassays sind wichtige Werkzeuge in diesem Prozess, da sie In-vivo-Methoden und Untersuchungen im Freiland ergänzen können. Im Rahmen dieser Arbeit wurde ein langfristiges In-vitroMonitoring (2012-2015) in einer Wasserschutzregion in Südwestdeutschland durchgeführt. Über 30 Probenahmestellen, welche verschiedene Zonen des 
Wasserkreislaufs widerspiegeln, wurden untersucht. Signifikante und wiederholt auftretende Effekte erlaubten die Klassifizierung und Priorisierung potenzieller Hotspots sowie der Leistung von Kläranlagen. Während in Oberflächengewässern und Grundwasser keine bis minimale Kontaminationen festgestellt wurden, wurden starke Effekte in Krankenhaus-Abwässern und im Rohabwasser gefunden (hauptsächlich östrogene und antiöstrogene, sowie in geringerem Maße androgene Aktivitäten). Die meisten Kläranlagen, darunter mehrere in Wasserschutzzonen, reduzierten diese Effekte auf ein unbedenkliches Niveau (z.B. Langenau, Halzhausen, Steinhäule). Die Reduktion dieser Aktivitäten während des Belebtschlammverfahrens wurde in anderen Studien beobachtet (z.B. Allinson et al. 2011, EC 2012, Sawada et al. 2012). Dies gilt ebenso für die weniger effektiv entfernte antiöstrogene Aktivität (Ihara et al. 2014, Rao et al. 2014).

Einer der Grundwasserhotspots wies wiederholt hohe antiöstrogene Aktivitäten auf. Aufgrund der Trinkwasser-Relevanz dieses Umweltkompartiments wurde ein zusätzliches Monitoring an verschiedenen Probenahmestellen in unmittelbarer Nähe des Hotspots durchgeführt. Diese Standorte umfassten mehrere Grundwasser-entnahmestellen, Brunnen und eine Probe eines Trinkwasserauslasses aus einem nahegelegenen Wasserwerk. Darüber hinaus wurden die Ergebnisse früherer und einer parallelen chemischen Untersuchung in der Modellregion zum Hotspot berücksichtigt (Anna Bollmann unveröffentlichte Ergebnisse, Seitz und Winzenbacher 2017). Das erweiterte Monitoring zeigte, dass sich alle Standorte hinsichtlich der untersuchten In-vitro-Aktivitäten und Toxizitäten unauffällig verhielten. Dieses Ergebnis stimmte mit dem der chemischen Analytik überein. Das fortgesetzte Monitoring der Antiöstrogenität im Grundwasserhotspot und die Untersuchung möglicher Ursachen (z.B. natürliche oder anthropogene Chemikalien) sollte aufgrund der erhöhten Relevanz dieses Umweltkompartiments in Betracht gezogen werden.

Es könnte ebenso von Vorteil sein, Folgeuntersuchungen zur Antiöstrogenität und Antiandrogenität im Krankenhausabwasser durchzuführen, denn das beprobte Krankenhaus (www.uniklinik-ulm.de) könnte unter anderem antiöstrogene (Hu et al. 1993, Knoop et al. 2018, Li et al. 2008, Sohoni und Sumpter 1998) und antiandrogene (Gordon et al. 2017, Grover et al. 2011, Kusk et al. 2011, Sohoni und Sumpter 1998) Chemotherapeutika und andere Arzneimittel in eine 
angeschlossene Kläranlage emittieren. Allerdings wurden in dieser Kläranlage keine erhöhte Antiöstrogenität oder Antiandrogenität detektiert.

Die erhöhte Genotoxizität und Mutagenität des Krankenhausabwassers rät ferner zu einer ereignisorientierten Beprobung im Hinblick auf einen überdurchschnittlich hohen Eintrag während Starkregenereignissen. Für derartige Umweltmonitorings und Folgeuntersuchungen bietet die verwendete Batterie an In-vitro-Bioassays eine geeignete Methodik, die unter anderem mit der Effekt-dirigierten-Analyse kombiniert werden kann (z. B. Burgess et al. 2013, Brack et al. 2017).

In-vivo-Bioassays werden im Allgemeinen für ökotoxikologische Fragestellungen verwendet, welche den gesamten Organismus betreffen. Im vorliegenden Fall wurde der biologische Modellorganismus Caenorhabditis elegans (C. elegans, z.B. Chalfie 2009, Hirschmann 1952, Maupas 1899, Weinhouse et al. 2018) als vielversprechendes Werkzeug zur Charakterisierung der Qualität von Wasser- und Abwasserproben untersucht. Die umwelt- und human-relevanten Endpunkte Reproduktion und Wachstum (Allard et al. 2013, Harlow et al. 2016, Williams et al. 2017) wurden dabei mit einem Biomarkeransatz kombiniert (rekombinanter cyp35A3::GFP-Stamm, Menzel et al. 2007), um mögliche Entwicklungs- und Reproduktionstoxizität (DART) bzw. Cytochrom P450 (Isoform 35A3) induzierenden Verbindungen in diesen Proben zu detektieren. Zu diesem Zweck wurde ein etablierter Nematoden-Bioassay, das International Standard Organization (ISO)Protokoll 10872 (ISO 1996), adaptiert. Neben der standardisierten und regelmäßigen Anwendung dieses Assays in der Umweltforschung (z.B. Höss et al. 2012), zeigten verschiedene Mikroverunreinigungen DART in $C$. elegans bei Konzentrationen im unteren Mikrogramm pro Liter Bereich (z.B. Haegerbäumer et al. 2017, Li et 2016, Zhuang et al. 2014). Diese Studien verdeutlichen die Durchführbarkeit und Sensitivität von C. elegans als ökotoxikologisches Testsystem.

Die vorliegende Arbeit befasst sich mit dem polyzyklischen aromatischen Kohlenwasserstoff $\beta$-Naphthoflavon ( $\beta$-NF), welches als Referenzsubstanz eingesetzt wurde (Leung et al. 2010, Menzel et al. 2001, 2007) sowie mit Abwasserproben aus konventionell-biologischer und weiterführender Abwasserbehandlung (vergleiche zuvor genannte TransRisk-Technologien). Für 
das Abwasser-bürtige $\beta$-NF (Abdel-Shafy und Mansour 2016, Forsgren 2015) wurde mit dem weiterentwickelten Bioassay eine Median-Effektkonzentration ( $\left.\mathrm{EC}_{50}\right)$ für die Brutgröße (96 h) von $114 \mu \mathrm{g} / \mathrm{L}$ berechnet. Mit Hilfe dieses Bioassays wurden anschliessend Experimente mit wässrigen und extrahierten $\beta$-NF-dotierten Oberflächenwasser- und Abwasserproben durchgeführt. Diese Experimente bestätigten die Durchführbarkeit und Sensitivität des angepassten Testsystems. Sie wiesen ferner auf mögliche kumulative Effekte mit anderen Probenbestandteilen und einen Einfluss von Schwebstoffen $(>1 \mu \mathrm{m})$ auf die Reproduktionstoxizität von $\beta-N F$ hin (aufgrund einer möglichen Partikelassoziation). Signifikante DART wurde zudem für einen biologisch-behandelten Kläranlagenablauf beobachtet (4-20x SPE-Extrakte, 96 h). Für die Ozonung und Ozonung in Kombination mit GAC- oder Biofiltration wurde keine Reproduktionstoxizität nachgewiesen (einschließlich des entsprechenden Kläranlagenablaufs). Drei extrahierte Kläranlagenabläufe sowie drei aus vier GAC- bzw. biologischen Nachfiltrationssysteme zeigten darüber hinaus einen signifikanten Wachstumseffekt auf C. elegans-Larven (96 h). Mögliche natürliche (vergleiche Höss et al. 2001) bzw. anthropogene (vergleiche Höss und Weltje 2007) Verursacher dieses Effekts wurden nicht untersucht.

Mit dem Biomarker-Ansatz konnten verschiedene ökotoxikologisch-relevante Beobachtungen gemacht werden. cyp-35A3::GFP wurde durch $\beta$-NF in Abhängigkeit der Expositionskonzentration und -Dauer (0,01-5 mg $\beta-N F / L, 1-48$ h) sowie des Gewebes und des Entwicklungsstadiums (intestinale Expression unmittelbar nach dem Schlüpfen der Eier) induziert. Die höchsten Expressionsraten wurden nach $8 \mathrm{~h}$ Exposition gegenüber 1-5 mg $\beta-N F / L$ (21,3-24 fach über der Kontrolle) mit einem $\mathrm{EC}_{50}-$ Wert von $71,5 \mu \mathrm{g} / \mathrm{L}$ beobachtet. Der zuvor vermutete Einfluss von Schwebstoffen auf die Bioverfügbarkeit von $\beta-N F$ im dotierten Abwasser konnte in diesen Experimenten bestätigt werden. Zusätzlich wurde cyp35A3::GFP signifikant durch einen extrahierten Kläranlagenablauf und dazugehörigen ozonisierten Kläranlagenablauf induziert, während letzterer den Biomarker in höherem Maße induzierte. Es wurde daher angenommen, dass beide Proben cyp-35A3-induzierende Verbindungen enthielten, welche nach deren Reaktion mit Ozon potenziert und/oder bioverfügbarer wurden. Nachfolgende Untersuchungen sollen klären, ob dieser Effekt durch die GAC- und Biofiltrationssysteme eliminiert wird. 
Eine parallele chemische Analyse, die mehrere bekannte Effektoren auf die Reproduktion und das Wachstum von C. elegans integrierte (z.B. Allard et al. 2013, Boyd et al. 2010, Liu et al. 2013), zeigte keine Korrelation zu den detektierten Effekten. Dieser Umstand legt nahe, zukünftige begleitende chemische Analysen hinsichtlich bekannter und zu erwartender Effektoren anzupassen. Diese Anpassung wird der Frage nach der Identität möglicher Verursacher der Effekte dienlich sein. Die erzielten Ergebnisse bestätigten frühere Untersuchungen zur Eignung und Sensitivität von $C$. elegans für die Untersuchung von (Mikro)Schadstoffen und Umweltproben (z.B. Hitchcock et al. 1997, 1998, McLaggan et al. 2012, Roh et al. 2007, Wang et al. 2010, 2015, Xiao et al. 2018, Xiong et al. 2017, Yu et al. 2020). Frühere Untersuchungen an C. elegans, welche meist mehrere Endpunkte auf verschiedenen Ebenen der biologischen Organisation miteinbezogen, waren zudem Teil von Hochdurchsatz-Screenings (z.B. Boyd et al. 2010, Lundby et al. 2016), Umweltrisikobewertungen höherer Ebenen (z.B. Hägerbäumer et al. 2015, Haegerbäumer et al. 2017, Wilson und Khakouli-Duarte 2009), sowie der Konzeption von Adverse Outcome Pathways (Jager und Ashauer 2018). Perspektivisch werden diese Unterfangen zu einem besseren Verständnis der Toxizitätsmechanismen und Umweltrelevanz anthropogener (Mikro)Schadstoffe beitragen.

Abschliesend wurde in dieser Arbeit eine Bewertung der Abwasserqualität im Rahmen eines in TransRisk entwickelten integrierten Bewertungskonzepts durchgeführt (Ternes et al. 2017). Dieses Konzept wurde in einer Pilot-Kläranlage mit den weiterführenden Abwasserbehandlungs-Verfahren Ozonung und Ozonung in Verbindung mit GAC- oder biologischer Nachfiltration (jeweils als belüfteter oder unbelüfteter Filter) etabliert. Die Ozonung im Pilotmaßstab wurde mit konventionellbehandeltem Abwasser (Belebtschlammverfahren) aus einer kommunalen Kläranlage gespeist. Der ökotoxikologische Teil der Bewertung umfasste die beschriebene und optimierte SPE-Methode (Abbas et al. 2019), ausgewählte Invitro-Bioassays (siehe oben), sowie verschiedene In-vivo-Testsysteme (Schneider et al. 2020, Schlüter-Vorberg et al. 2017). Die Auswahl an In-vivo-Bioassays, die hauptsächlich als Durchfluss-On-site-Systeme installiert wurden, erwiesen sich als nützliches und sensitives Werkzeug in früheren Kläranlagenuntersuchungen (z.B. 
da Costa et al. 2014, Gartiser et al. 2010, Giebner et al. 2018, Magdeburg ua 2012, 2014, Maltby et al. 2000, Stalter et al. 2010, 2011).

Ein besonderes Ziel dieser Arbeit war es zu vergleichen, ob der berichtete Nutzen des entwickleten C. elegans Bioassays auf die Untersuchung verschieden behandelter Abwässer aus einer Pilotkläranlage übertragen werden kann und diese Ergebnisse für ein integriertes Bewertungskonzept herangezogen werden können. Die Ergebnisse von fünf In-vitro-Bioassays zur Untersuchung von In-vitro-(Anti)Östrogenität, -(Anti-)Androgenität und Mutagenität, sowie der populationsrelevante Endpunkt Reproduktion von C. elegans, wurden hierbei in eine "Bewertungsmatrix der Abwasserqualität" integriert, um die effektivste weiterführende Abwasserbehandlung auszumachen. In der berechneten Matrix (Daten von

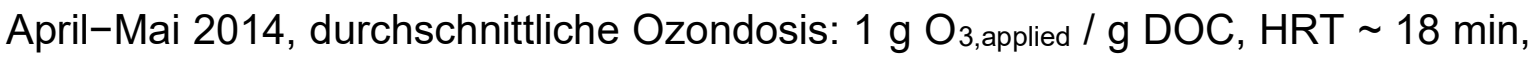
10x SPE-Extrakte aus 24 h-Mischproben) wurde die Ozonung in Kombination mir der unbelüfteten GAC-Filtration als effektivste der fünf Optionen ermittelt. Es konnte ferner gezeigt werden, dass östrogene und (anti-)androgene Aktivitäten zwar durch die weiterführenden Abwasserbehandlungstechnologien stark reduziert, mutagene und antiöstrogene Effekte jedoch nicht vollständig eliminiert werden konnten oder sogar erhöht vorlagen. Diese Beobachtung könnte mit früheren Hypothesen zur Erzeugung toxischer Transformationsprodukten übereinstimmen (da Costa et al. 2014, Jia et al. 2015, Knoop et al. 2018, Magdeburg et al. 2014, Stalter et al. 2010). Geringfügig-nachteilige Auswirkungen der Ozonung auf die Reproduktion von C. elegans (11,7\% verringerte Brutgröße im Vergleich zum Kläranlagenablauf) traten in beiden GAC-gefilterten Proben nicht auf. Beide Proben die der Biofiltration entnommen wurden, zeigten wie zuvor festgestellt einen wachstumsfördernden Effekt (12,5 bzw. 15,1\% Zunahme).

In Übereinstimmung mit diesen (und früheren) mit C. elegans erzielten Ergebnissen, erscheint die weitere Etablierung dieses Nematoden als vielseitiger und sensitiver Modellorganismus für ökotoxikologische Zwecke vielversprechend (einschliessnlich Wasser- und Abwasser-bezogener Untersuchungen). C. elegans könnte hierbei zur Erforschung zusätzlicher bzw. alternativer Testsysteme mit erhöhter Sensitivität und/oder Umweltrelevanz maßgeblich beitragen, wie sie in der wissenschaftlichen Literatur empfohlen wurden (Berger et al. 2016, Schwarzenbach et al. 2006, Völker et al. 2017, Wigh et al. 2016, 2017, 2018, Xiong et al. 2017). 
Im Allgemeinen erwies sich die Kombination von In-vitro- und (vorzugsweise Onsite-)In-vivo-Testsystemen als geeigneter Ansatz für die Bewertung der Abwasserqualität. Idealerweise sollten diese Bewertungen mit einem langfristigen Umweltmonitoring an Vorflutern und betroffenen Gewässern einhergehen (z.B. Auswirkungen auf Indikatortaxa) sowie ferner sozialpolitische Maßnahmen zur Verringerung relevanter Mikroschadstoffe berücksichtigen (z.B. Source control, European Parlament and Council 2013, Lienert et al. 2011, Reichenberger et al. 2007). Ergänzt werden sollten diese Bemühungen mit der Untersuchung weiterer Optimierungspotenziale im Zusammenhang mit der Abwasser-Kanalisation, konventionell-biologischen, tertiären Behandlungen (z.B. Musolff et al. 2010, Phillips et al. 2012, Triebskorn 2017, Völker et al. 2016) und anderer weiterführenden Abwasserbehandlungen (z.B. Barbosa et al. 2016, Becker et al. 2017, Tchobanoglous and Burton 1991, van Hege et al. 2002). Diese Strategien sollten idealerweise die Gesamtheit relevanter Stressoren aquatischer Ökosysteme berücksichtigen (z.B. BMU 2013, EEA 2012, Philip et al. 2011). 


\section{A.5 Acknowledgement}

Included in print version / not included in online version. 


\section{A.6 CV}

Included in print version / not included in online version. 


\section{A.7 Publication list}

Journal articles (peer reviewed)

Schneider I, Abbas A, Bollmann A, Dombrowski A, Knopp G, Schulte-Oehlmann U, Seitz W, Wagner M, Oehlmann J (2020) Water Research 185, 116104

Abbas A*, Schneider I*, Bollmann A, Funke J, Oehlmann J, Prasse C, Schulte-Oehlmann U, Seitz W, Ternes T, Weber M, Weseley H, Wagner M (2018) Water Research 152, 47-60

Abbas A, Valek L, Knopp G, Bollmann A, Seitz W, Schulte-Oehlmann U, Oehlmann J, Wagner M (2018) Environmental Science and Pollution Research 25, 13868-13880

Zhu L, Zhou R, Mettler S, Wu T, Abbas A, Delaney J, Forte JG (2007) American Journal of Physiology - Cell Physiology 293(3), 874-884

Zhu L, Zhou R, Mettler S, Wu T, Abbas A, Forte JG (2007) The FASEB Journal 21(6), A913

Publications (non-peer-reviewed)

Schneider I*, Abbas A*, Wagner M, Schulte-Oehlmann U, Oehlmann J (2015) RiSKWaframework project TransRisk. Final report on projekt $2-$ Ecotoxicological investigations. BMBF 02WRS1275B, 01101785

Abbas $A^{*}$, Andersen $\mathrm{RM}^{*}$, Finnbogadottir GA*, Pasupuleti $\mathrm{P}^{*}$ (2009) Semester project online publication. University of Aalborg, Denmark

\section{Scientific talks}

Schulte-Oehlmann U, Abbas A, Schneider I, Wagner I, Oehlmann J, Coors A, Vorberg L, Bollmann A, Seitz W, Ternes T (2014) IFAT World exhibition and Congress. Munich, Germany

Abbas A, Schneider I, Vorberg L, Bollmann A, Prasse C, Funke J, Wesely H, Schulte-Oehlmann U, OehImann J, Coors A, Seitz W, Ternes T, Wagner M (2013) IWA and Eawag. International Conference Micropol \& Ecohazard. Zurich, Switzerland

Ternes TA, Abbas A, Alexander J, Bollmann A, Coors A, Cornel P, Durmaz V, Funke J, Zhang Y, Zhu H, Geißen S, Götz K, Knopp G, Lütke-Eversloh C, Krausse U, Mückter H, Niethammer M, Prasse C, Schneider I, Schwartz T, Seitz W, Schulte-Oehlmann U, Oehlmann J, Thaler S, Vorberg L, Weber M, Wieland A (2013) BMBF. Statusseminar RiskWa. Karlsruhe, Germany Abbas A speaking for Magdeburg A, Stalter D, Weil W, Knacker T, Oehlmann T (2012) DWA/BfG Workshop. Koblenz, Germany

\section{Poster presentations}

Schulte-Oehlmann U, Abbas A, Alexander J, Bollmann A, Coors A, Durmaz V, Emmert M, Funke J, Mückter H, Oehlmann J, Prasse C, Schneider I, Schwarz T, Seitz W, Weber M, Vorberg L, Wagner M, Ternes T (2015) RiSKWa-Abschlusskonferenz, Berlin, Germany

Schneider I*, Abbas A*, Vorberg L, Knopp G, Wagner M, Schulte-Oehlmann U, Oehlmann J, Coors A, Cornel P, Ternes T (2014) DWA, Wasserchemische Gesellschaft and German Agency of Hydrologe (BfG) Workshop. Koblenz, Germany

Vorberg L, Abbas A, Schneider I, Schulte-Oehlmann U, Knopp G, Cornel P, Ternes T, Coors A (2014) Effects of differently treated wastewater effluents observed with selected in vivo test systems. 24th SETAC Europe annual Meeting. Basel, Switzerland

Abbas A, Valek L, Oehlmann J, Wagner M (2013) GSA C. elegans meeting. Los Angeles, USA

Knopp G, Cornel P, Schneider I, Abbas A, Schulte-Oehlmann U, Alexander J, Schwartz T, Wiland A, Funke J, Ternes T, Vorberg L, Coors A (2013) RiSKWa-Statusseminar, Karlsruhe, Germany

Abbas A, Hegemann B, Ahlf W (2012) SETAC World Conference, Berlin, Germany

Abbas A, Oehlmann J, Ahlf W (2012) $2^{\text {nd }}$ International Symposium on Nematodes as Environmental Bioindicators. Associated of Applied Biologists. Gent, Belgium

Zhu L, Abbas A*, Wu T*, Patel S, Karvar S, Yun CC, Forte JG (2006) ASCB conference. San Diego, USA

*authors indexed with asterisk contributed equally to the respective work 